FHWA/IN/JTRP-2000/16

Final Report

EXAMINATION OF POST-TENSIONED STEEL BRIDGES IN INDIANA

Hung-I Wu

Mark D. Bowman

July 2000 
Final Report

FHWA/IN/JTRP-2000/16

\title{
EXAMINATION OF POST-TENSIONED STEEL BRIDGES IN INDIANA
}

\author{
By \\ Hung-I Wu \\ and \\ Mark D. Bowman \\ School of Civil Engineering \\ Purdue University \\ Joint Highway Research Program \\ Project Number: C-36-5600 \\ File Number: 7-4-41 \\ SPR-2151
Conducted in Cooperation with the
Indiana Department of Transportation
and the
U.S. Department of Transportation
Federal Highway Administration

The contents of this report reflect the view of the authors, who are responsible for the facts and the accuracy of the data presented herein. The contents do not necessarily reflect the official views or policies of the Indiana Department of Transportation or the Federal Highway Administration. This report does not constitute a standard, specification or regulation.

Purdue University

West Lafayette, IN 47907

July 2000 


\begin{tabular}{|c|c|c|}
\hline $\begin{array}{l}\text { 1. Report No. } \\
\text { FHWA/IN/JTRP-2000/16 }\end{array}$ & 2. Government Accession No. & 3. Recipient's Catalog No. \\
\hline \multirow{2}{*}{\multicolumn{2}{|c|}{$\begin{array}{l}\text { 4. Title and Subtitle } \\
\text { Examination of Post-Tensioned Steel Bridges in Indiana }\end{array}$}} & $\begin{array}{l}\text { 5. Report Date } \\
\text { July } 2000\end{array}$ \\
\hline & & 6. Performing Organization Code \\
\hline \multicolumn{2}{|l|}{$\begin{array}{l}\text { 7. Author(s) } \\
\text { Hung-I Wu and Mark D. Bowman }\end{array}$} & $\begin{array}{l}\text { 8. Performing Organization Report No. } \\
\text { FHWA/IN/JTRP-2000/16 }\end{array}$ \\
\hline \multirow{2}{*}{\multicolumn{2}{|c|}{$\begin{array}{l}\text { 9. Performing Organization Name and Address } \\
\text { Joint Transportation Research Program } \\
1284 \text { Civil Engineering Building } \\
\text { Purdue University } \\
\text { West Lafayette, Indiana } 47907-1284\end{array}$}} & 10. Work Unit No. \\
\hline & & $\begin{array}{l}\text { 11. Contract or Grant No. } \\
\text { SPR-2151 }\end{array}$ \\
\hline \multirow{2}{*}{\multicolumn{2}{|c|}{$\begin{array}{l}\text { 12. Sponsoring Agency Name and Address } \\
\text { Indiana Department of Transportation } \\
\text { State Office Building } \\
100 \text { North Senate Avenue } \\
\text { Indianapolis. IN } 46204\end{array}$}} & $\begin{array}{l}\text { 13. Type of Report and Period Covered } \\
\text { Final Report }\end{array}$ \\
\hline & & 14. Sponsoring Agency Code \\
\hline \multicolumn{3}{|l|}{ 15. Supplementary Notes } \\
\hline
\end{tabular}

The purpose of the research study is to conduct an investigation to understand the performance of a relatively new type of bridge construction that involves prestressed (post-tensioned) steel-concrete composite bridge members. Strictly speaking, the technical and economical advantages of this type of structure have been understood for several decades. However, the application of this concept to practice is still very limited due to difficulties associated with the post-tensioning anchorage. The study was thus intended to explore the mechanical behavior of such construction. Both the short-term and long-term bridge performance were evaluated in this study.

The investigation included experimental as well as analytical evaluations of the structural behavior. The experimental part involved a series of field measurements of a newly constructed steel-concrete composite bridge which was post-tensioned with high-strength steel tendons. This bridge, which is part of the I-90 Indiana Toll Road, is located in Elkhart County, Indiana. Strain distributions in the composite girders and the elongation of a selected post-tensioning tendon were measured at certain crucial phases of construction. The field monitoring was periodically conducted over a period of about one year after the post-tensioning operation. Analytical solutions were developed to evaluate the structural response due to various types of instantaneous loads, temperature effects and time-dependent factors. The validity and effectiveness of the analytical model were examined by comparing the calculated results with the data collected from the field. Further prediction of the long-term structural response was also performed based on the proposed methodology. A cost comparison for the structural components between the Elkhart post-tensioned bridge and a nearby conventional steel bridge was also conducted.

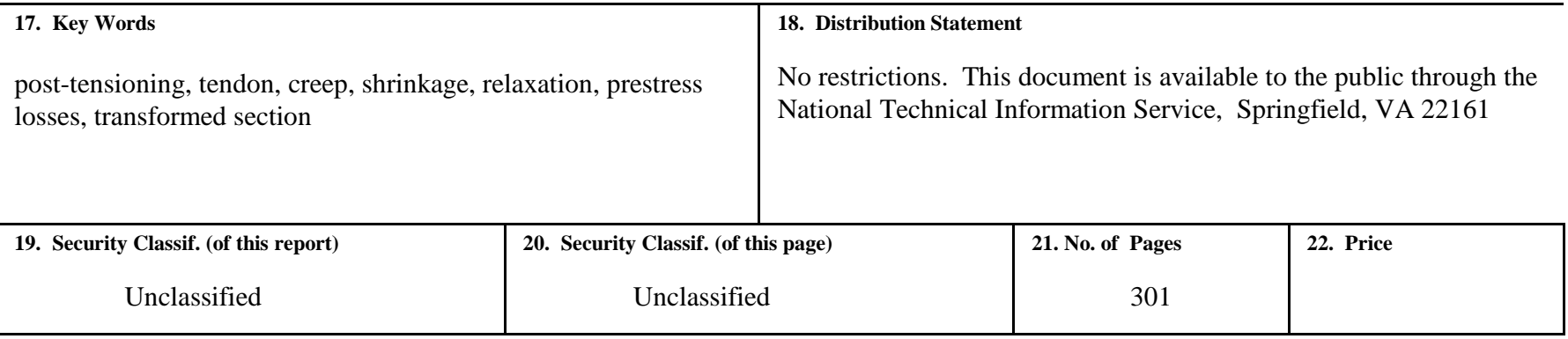




\section{ACKNOWLEDGMENTS}

The research project was financially supported by the Federal Highway Administration, the Indiana Department of Transportation and the Toll Road Division. The authors would like to express their grateful acknowledgments for the sponsorship toward the completion of the final report.

The valuable suggestions and comments provided by the Study Advisory Committee are sincerely appreciated. The members of the Study Advisory Committee include Mr. David Ward, Mr. Samuel E. Wolfe and Mr. Hasmukh Patel from Indiana Department of Transportation; Mr. Rick Drumm from Federal Highway Administration; Mr. Joseph Day from Vincennes Steel Corporation and Professor Julio A. Ramirez from Purdue University.

Sincere thanks are also due to the personnel from Vincennes Steel Corporation, Walsh Construction Company and the Toll Road Division of INDOT for their assistance and cooporation in preparing and conducting the field measurements. Appreciation is also extended to individuals from Purdue University who have provided enthusiastic and timely help for the experimental portion of the study. Among them, in particular, are Mr. Harry Tidrick, Dr. Amy Grider Barth, Dr. Brian Malone, Dr. Asnee Pochanart, Mr. JiunGuan Lin, and Mr. Chien-Po Chen. 


\section{TABLE OF CONTENTS}

Page

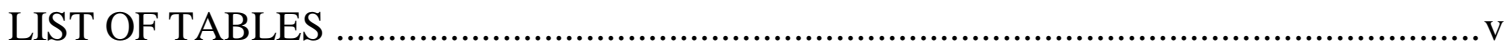

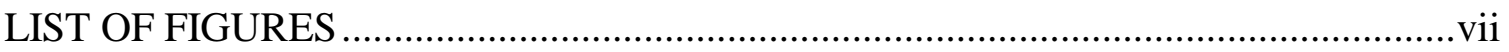

IMPLEMENTATION REPORT .......................................................................

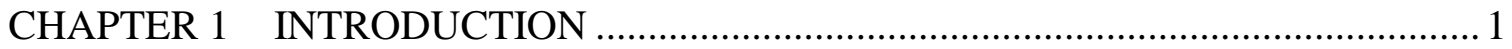

1.1 Background and Motivation of Investigation ............................................ 1

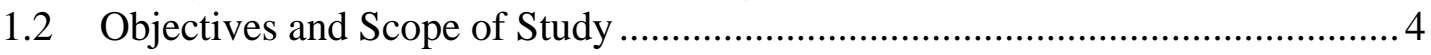

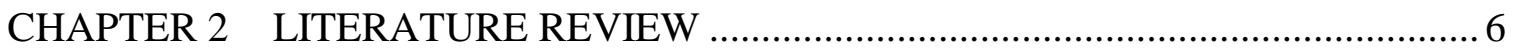

2.1 Construction Methods........................................................................... 6

2.2 Predicted Structural Response and Load-Carrying Capacity ............................ 8

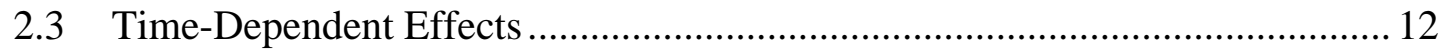

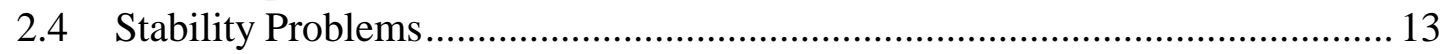

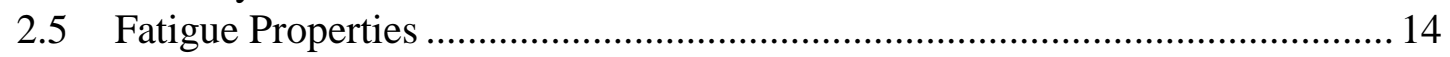

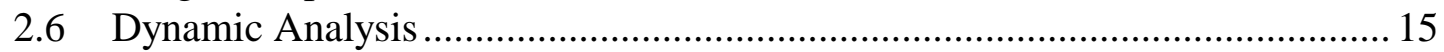

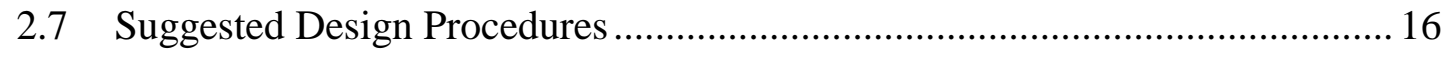

CHAPTER 3 EXPERIMENTAL PROGRAM AND RESULTS ............................... 21

3.1 Profile of Elkhart County I-90 Toll Road Bridge .......................................... 21

3.2 Description of Experimental Program ........................................................... 23

3.3 Results from Bridge Testing ................................................................ 25

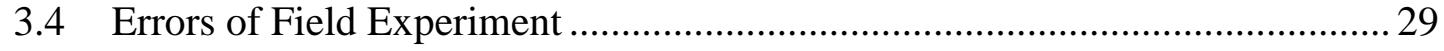

\section{CHAPTER 4 ANALYSIS OF SHORT-TERM AND THERMAL}

BRIDGE RESPONSE................................................................ 66

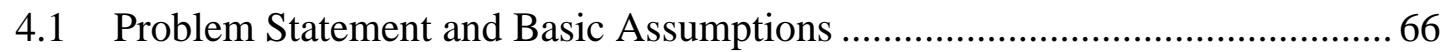

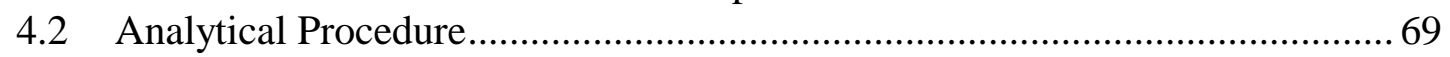

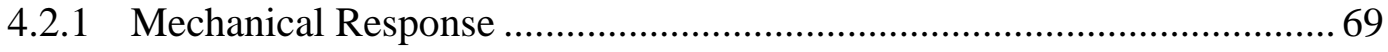

4.2.2 Thermal Response ................................................................... 74 


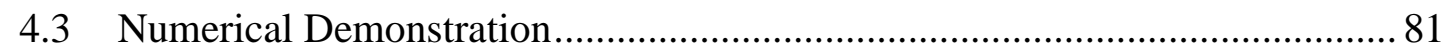

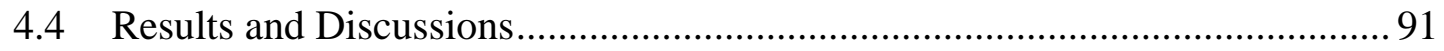

4.4.1 Mechanical Response ……………………....................................... 91

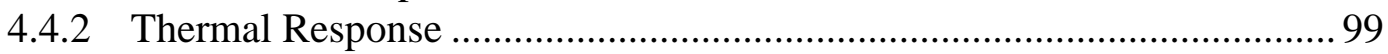

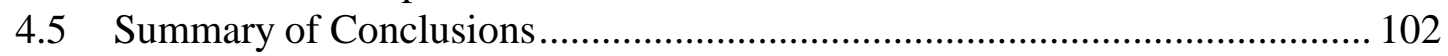

CHAPTER 5 PREDICTION OF LONG-TERM BRIDGE PERFORMANCE ............ 140

5.1 Time-Dependent Material Behavior ........................................................... 140

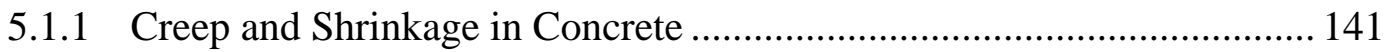

5.1.2 Relaxation in Steel Tendon................................................................ 147

5.2 Problem Statement and Basic Assumptions .................................................. 148

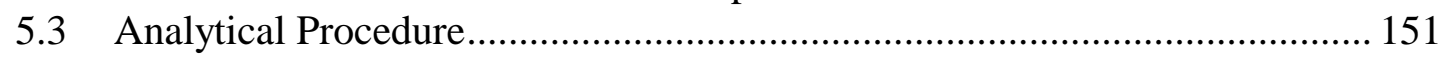

5.3.1 Equivalent Axial Force and Bending Moment ...................................... 152

5.3.2 Structural Response Functions due to Shrinkage and Creep .................... 154

5.3.3 Calculation of Long-Term Structural Response ..................................... 161

5.3.4 Summary of Analytical Procedure............................................................ 166

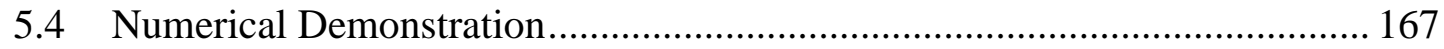

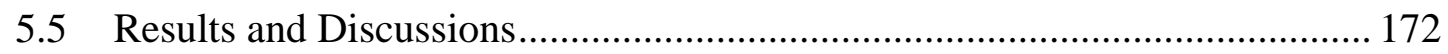

5.5.1 Comparison of Analytical and Experimental Results............................. 172

5.5.2 Further Prediction of Structure Performance....................................... 175

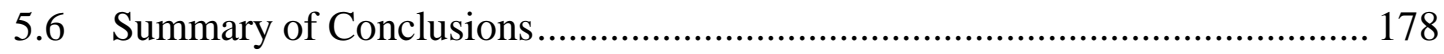

\section{CHAPTER 6 COMPARISON OF COST DATA FOR TWO COMPOSITE}

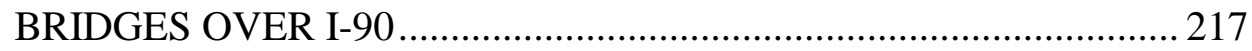

6.1 Major Structural Features of Two Bridges ……………………………....... 217

6.2 Comparison of Cost .............................................................................. 219

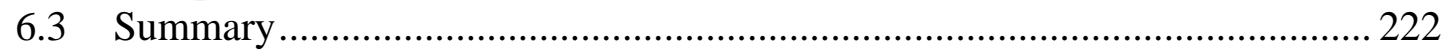

CHAPTER 7 CONCLUSIONS AND RECOMMENDATIONS ………………….... 230

7.1 Conclusions......................................................................................... 230

7.2 Recommendations for Use of Analytical Model …………........................... 233

7.3 Recommendations for Implementation........................................................ 235

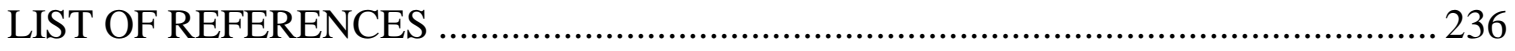

APPENDIX A PHOTOGRAPHS OF ELKHART COUNTY BRIDGE...................... 241

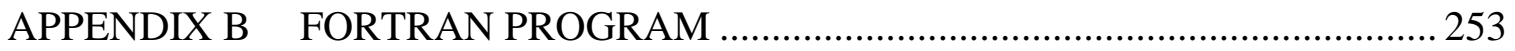

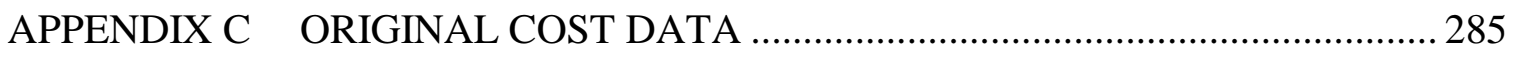




\section{LIST OF TABLES}

Table

Page

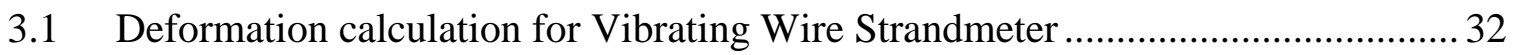

3.2 Sequential order of strain measurement for Figs. 3.11 to 3.17 ............................ 33

3.3 Sequential order of strain measurement for Figs. 3.18 and 3.19 .......................... 34

4.1 Estimated stresses in concrete deck due to post-tensioning............................... 104

4.2 Strain increments in post-tensioning tendons along girder G3

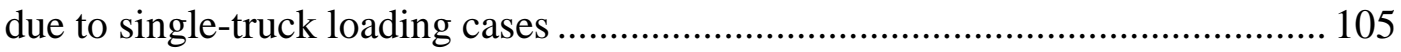

4.3 Calculated strain values at gage locations G3 2 and G3 6

(at the level of the top surface of the bottom flange) ......................................... 106

4.4 Estimated thermal stresses in the interior girder G3 due to

assumed temperature variation .............................................................. 107

5.1 Factor $k_{h}$ (from AASHTO LRFD Specifications) used to calculate

shrinkage strain (for Eqs. 5.10 a \& b) ............................................................... 180

5.2 Aging coefficient $\chi\left(t, t_{0}\right)$ calculated by Bazant (1972) .................................... 181

5.3 Stress distributions due to time-dependent effect during the

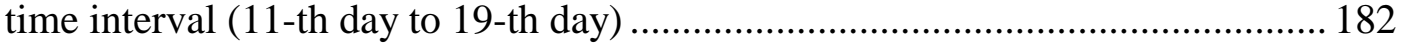

5.4 Instantaneous strains at the level of concrete gages..................................... 183

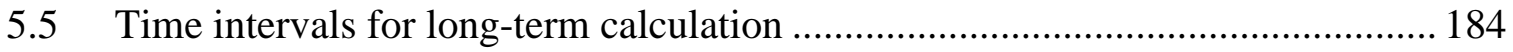

6.1 Costs of structural components of bridge (CV) in English unit......................... 224

6.2 Costs of structural components of bridge (CV) in metric unit.......................... 225 
6.3 Costs of structural components of bridge (PT) in metric unit ............................ 226

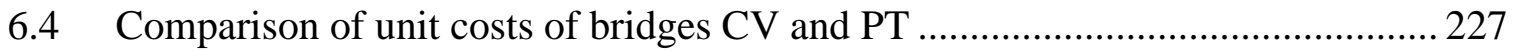

B.1 Source code of FORTRAN program POSTSTEL ........................................... 255

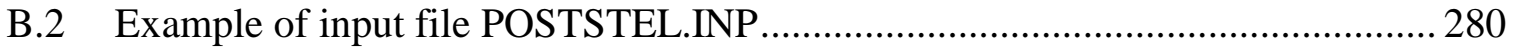

B.3 Example of input file GAGELOPS.INP ...................................................... 281

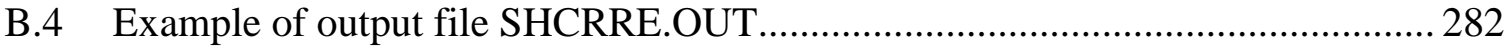

B.5 Example of output file STRESUM.OUT .................................................... 283

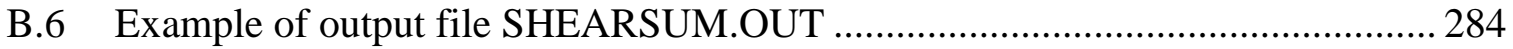

C.1 Original cost data for construction items of bridge (CV) .............................. 286

C.2 Original cost data for construction items of bridge (PT) ................................. 292 


\section{LIST OF FIGURES}

Figure $\quad$ Page

2.1(a) Construction method (By using high-strength steel tendons) ............................ 18

2.1(b) Construction method (By stressing components of hybrid girders) .................... 19

2.1(c) Construction method (By utilizing the composite action of concrete slab) ........ 20

3.1 Elkhart County I-90 Toll Road Bridge...................................................... 35

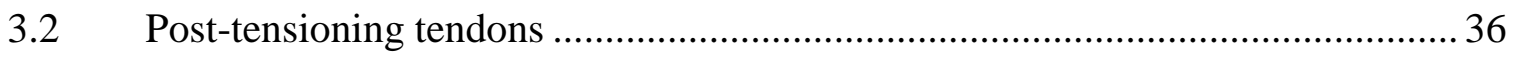

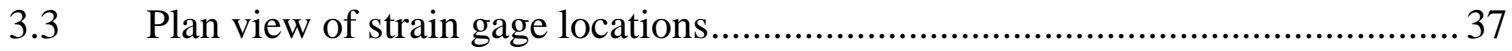

3.4(a) Positions of strain gages on steel plate girder (G1 1, G1 2, G1 5, G1 6) ............ 38

3.4(b) Positions of strain gages on steel plate girder (G1 4) .................................... 39

3.4(c) Positions of strain gages on steel plate girder (G1 7) ................................. 40

3.4(d) Positions of strain gages on steel plate girder (G3 1, G3 2, G3 6, G3 7) ........... 41

3.4(e) Positions of strain gages on steel plate girder (G3 5) ...................................... 42

3.5(a) Positions of strain gages in concrete deck (G1 1, G1 4, G3 1) ......................... 43

3.5(b) Positions of strain gages in concrete deck (G1 2, G1 5, G3 2) ......................... 44

3.5(c) Positions of strain gages in concrete deck (G1 6, G1 7, G3 5, G3 6, G3 7)....... 45

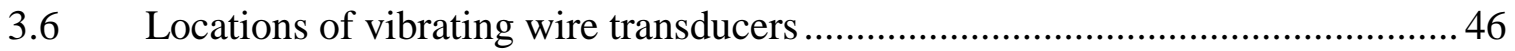

3.7 Components and installation of Model 4410 Vibrating Wire Strandmeter ......... 47 
Figure

3.8 Front panel of GK-403 Vibrating Wire Readout ......................................... 48

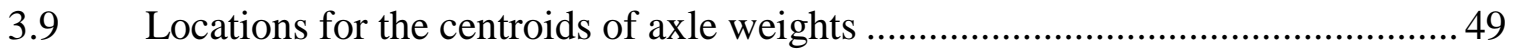

3.10 Axle weights and dimensions of dump truck ............................................. 50

3.11 Measure strain distributions in steel girder G1 at location $2 \ldots \ldots \ldots \ldots \ldots \ldots \ldots \ldots \ldots . . . .51$

3.12 Measure strain distributions in steel girder G3 at location 2 ..........................52

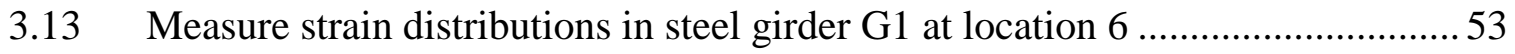

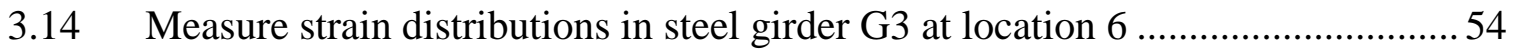

3.15 Measure strain distributions in steel girder G1 at location 7 ............................55

3.16 Measure strain distributions in steel girder G3 at location 7 ............................56

3.17 Measure strain distributions in steel girder G3 at location 1 ............................57

3.18 Measured strain distributions in concrete deck along girder G1 .......................58

3.19 Measured strain distributions in concrete deck along girder G3 ......................59

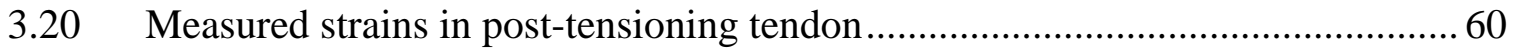

3.21 Measured strain distributions in steel girder G3 at location 2

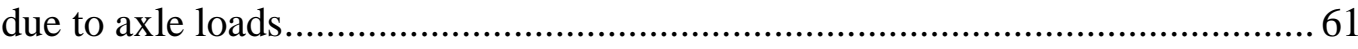

3.22 Measured strain distributions in steel girder G3 at location 6

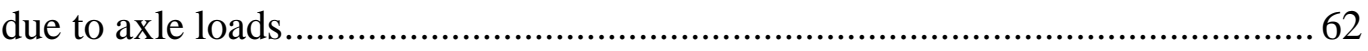

3.23 Measured strain distributions in concrete deck at location G3 2 due to axle loads.

3.24 Measured strain distributions in concrete deck at location G3 6 due to axle loads 64

3.25 Measured strains in post-tensioning tendon due to axle loads. 65 
Figure

4.1 Strain distributions in simplified beam model (in positive moment region) ..... 108

4.2 Equivalent forces and moments produced by post-tensioning force 109

4.3 Functions of moment $m(x)$ and axial force $a(x)$ due to unit post-tensioning force.

4.4 Typical pattern of temperature variation along composite girder section.....

4.5 Decomposition of approximate bi-linear temperature variation

4.6 Mechanical effect of non-uniform temperature variation in concrete deck ....... 113

4.7 Response moments due to non-uniform temperature variation ....................... 114

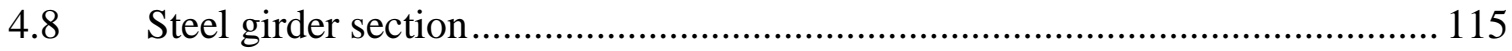

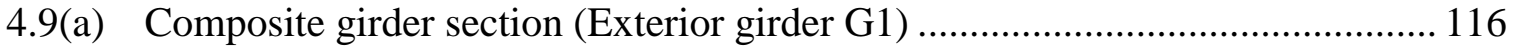

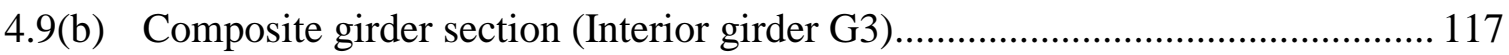

4.10(a) Transformed steel section with $f_{c}^{\prime}=58.61 \mathrm{MPa}($ Exterior girder G1) .............. 118

4.10(b) Transformed steel section with $f_{c}^{\prime}=58.61 \mathrm{MPa}$ (Exterior girder G3) .............. 119

4.11 Unit moment and axial force functions (when $\left.f_{c}^{\prime}=58.61 \mathrm{MPa}\right) \ldots \ldots \ldots \ldots \ldots \ldots \ldots . . . . . . . .120$

4.12(a) Transformed steel section with $f_{c}{ }^{\prime}=68.95 \mathrm{MPa}$ (Exterior girder G1) .............. 121

4.12(b) Transformed steel section with $f_{c}{ }^{\prime}=68.95 \mathrm{MPa}$ (Exterior girder G3) .............. 122

4.13 Unit moment and axial force functions (when $\left.f_{c}^{\prime}=68.95 \mathrm{MPa}\right) \ldots \ldots \ldots \ldots \ldots \ldots \ldots . . . . . . . .123$

4.14 Procedure to determine distributed axle loads for girders ............................. 124

4.15 Distributed axle loads on girders G1 and G3 ............................................ 125

4.16 Assumed bi-linear temperature variation in composite section of girder G3 .... 126

4.17 Response moments due to assumed temperature variation............................. 127 
4.18 Measured and calculated strain distributions in steel girder G1 at location $2 \ldots 128$

4.19 Measured and calculated strain distributions in steel girder G3 at location 2 ... 129

4.20 Measured and calculated strain distributions in steel girder G1 at location $6 \ldots 130$

4.21 Measured and calculated strain distributions in steel girder G3 at location 6 ... 131

4.22 Measured and calculated strain distributions in steel girder G1 at location 7 ... 132

4.23 Measured and calculated strain distributions in steel girder G3 at location 7 ... 133

4.24 Measured and calculated strain distributions in steel girder G3 at location $1 \ldots 134$

4.25 Measured and calculated strain distributions in concrete deck along girders (a) G1 and (b) G3 due to post-tensioning

4.26 Effect of concrete shrinkage................................................................... 136

4.27 Measured and calculated strain distributions in steel girder G1 due to due to load case (b)

4.28 Measured and calculated strain distributions in steel girder G3 due to due to load case (h)

4.29 Estimated thermal stresses in composite girder G3 based on assumed temperature variation.

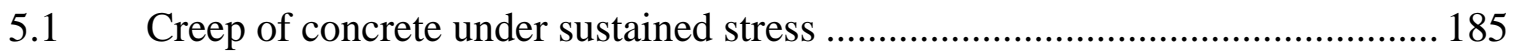

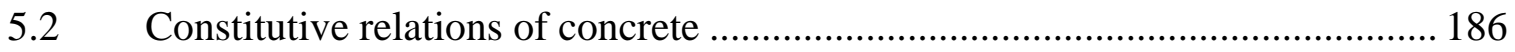

5.3 Application of equivalent axial force and bending moment .......................... 187

5.4 Structural response functions due to shrinkage effect over the length of a two-span bridge structure

5.5 Structural response functions due to creep effect over the length of a two-span bridge structure 
5.6 Structural response functions due to prestress losses over the length of a two-span bridge structure

5.7 Structural response due to shrinkage effect during the time interval (11-th day to 19-th day)

5.8(a,b) Structural response due to creep effect during the time interval (11-th day to 19-th day)

5.8(c,d) Structural response due to creep effect during the time interval

(11-th day to 19-th day)

5.9 Structural response due to prestress losses during the time interval (11-th day to 19-th day)

5.10 Traffic barriers of Elkhart County Bridge................................................... 195

5.11 Measured and calculated prestress levels..................................................... 196

5.12 Measured and calculated strain distributions in steel girder G1 at location $2 \ldots 197$

5.13 Measured and calculated strain distributions in steel girder G3 at location $2 \ldots 198$

5.14 Measured and calculated strain distributions in steel girder G1 at location 6 ... 199

5.15 Measured and calculated strain distributions in steel girder G3 at location 6 ... 200

5.16 Measured and calculated strain distributions in steel girder G1 at location 7 ... 201

5.17 Measured and calculated strain distributions in steel girder G3 at location 7 ... 202

5.18 Shrinkage functions suggested by AASHTO specification ............................ 203

5.19 Creep function suggested by AASHTO specification................................... 204

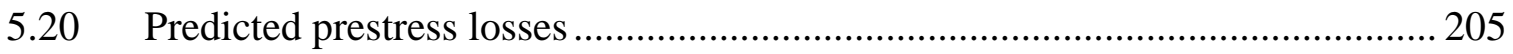

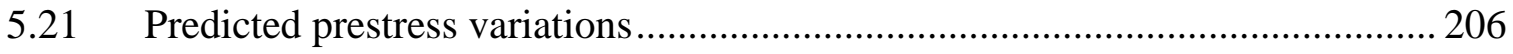


Figure

5.22(a) Predicted long-term stress distributions along exterior girders (G1)

(a) At internal support (pier)

5.22(b) Predicted long-term stress distributions along exterior girders (G1)

(b) At drape location

5.22(c) Predicted long-term stress distributions along exterior girders (G1)

(c) At midspan.

5.22(d) Predicted long-term stress distributions along exterior girders (G1)

(d) At 0.4 span from end

5.22(e) Predicted long-term stress distributions along exterior girders (G1)

(e) At bridge end

5.23(a) Predicted long-term stress distributions along exterior girders (G3)

(a) At internal support (pier)

5.23(b) Predicted long-term stress distributions along exterior girders (G3)

(b) At drape location

5.23(c) Predicted long-term stress distributions along exterior girders (G3)

(c) At midspan.

5.23(d) Predicted long-term stress distributions along exterior girders (G3)

(d) At 0.4 span from end....

5.23(e) Predicted long-term stress distributions along exterior girders (G3)

(e) At bridge end 216

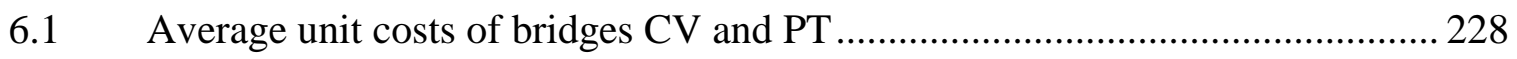

6.2 Percentages of total unit costs of structural components ...............................229

A.1 Overview of Elkhart County I-90 Toll Road Bridge..................................... 242

A.2 View of middle pier towers and exterior girder during erection....................... 242

A.3 Strain gage on bottom flange with protective coating ................................. 243

A.4 Post-tensioning duct and strain gages at location 7 ................................... 243 
Figure

A.5 Steel cross frame, concrete diaphragm and strain gages at location 6

A.6 View of girder end cast integrally into abutment wall and longitudinal stiffeners

A.7 View of girders framing to the south abutment and the conventional steel bridge in the background. (Refer to the economic analysis in Chapter 6.)

A.8 Strands for transverse post-tensioning of the abutment wall

A.9 View from under the bridge before post-tensioning. (Note that PVC ducts are draped prior to prestressing.)

A.10 View of the bridge deck before placing steel reinforcement. (Note the metal deck ribs are perpendicular to the girders.)

A.11 Shear stud connectors and reinforcing bars. (Note the strain gages on the rebars placed above the steel girder flange.)

A.12 Duct in outside abutment wall for post-tensioning tendons

A.13 Hydraulic-operated device to feed strand from the strand spool into the post-tensioning duct

A.14 Strand being fed into the duct

A.15 Twelve strands placed in the post-tensioning ducts prior to post-tensioning.

(Note the wedges placed over the strands on the right side.)

A.16 Construction workers guiding a hydraulic ram onto the strands

A.17 Ram used to tension the twelve strands in one duct. (Note the pressure gage used to control the post-tensioning force.) 250

A.18 Overhanging forming used to support wet concrete 250

A.19 Longitudinal post-tensioning ducts passing through the concrete diaphragm ... 251

A.20 Boxes for connecting strain gage wires and data acquisition system .251

A.21 Operation of data acquisition system used in the field measurement 252 


\section{IMPLEMENTATION REPORT}

In the past few years, bridge construction in Indiana has been strongly dominated by prestressed bulb tee girders with a composite deck. This system has been very popular because it is economical and easy to construct. Recently, however, cost comparison studies have suggested that post-tensioned steel girders with composite concrete decks may provide an economical alternative to prestressed bulb tee girders. The system that has been proposed for use in Indiana involves steel girders with reinforced concrete diaphragms between the girders that are used for anchorage and draping of the posttensioning tendons.

Besides possible economic advantages and increasing the load-carrying capacity, the use of post-tensioning in steel-concrete composite bridge structures also offers several benefits:

(1) The range of elastic behavior is expanded. The expansion of elastic material behavior eventually results in a procrastination or even elimination of the occurrence of plastic deformation.

(2) The deformation due to service loading is reduced.

(3) The probability of the formation of cracks in the concrete deck is lowered. 
(4) Fatigue strength is improved by virtue of the introduction of post-tensioning, as well as the use of uniform cross sections.

(5) Future repair costs are lowered as a result of reduced deck cracking and improved fatigue characteristics.

The purpose of the research was to explore the mechanical behavior and economy of such type of bridge construction. Consequently, a related goal of the investigation was to develop a reliable and practical analytical model to predict the instantaneous (shortterm), time-dependent (long-term) and thermal performance of the bridge structures. The bridge response was evaluated by using both experimental and analytical methods. The performance of a new bridge in Elkhart County over the I-90 Indiana Toll Road was monitored by collecting the strain values at particular critical locations in the steel and concrete components, along with the elongation of a selected post-tensioning tendon. Analysis was performed to estimate the overall structure response, and the results were compared to the experimental data. The validity and effectiveness of the analytical model were examined by comparing the calculated results with the data collected from the field.

Several important observations from the experimental and analytical investigation are summarized as below:

(1) The experimental measurements confirmed the presence of compressive stresses throughout the deck shortly after post-tensioning. The bridge deck may be subjected to some tensile stresses due to restrained shrinkage and creep at particular locations. However, the magnitude of the predicted long-term tensile stresses are not sufficient to cause cracks in the concrete slab. 
(2) The short-term stresses can be reliably predicted using simple analytical models.

(3) Prestress losses in post-tensioned composite bridges are less significant compared with losses that occur in conventional prestressed concrete bridge structures.

(4) Time-dependent factors can introduce significant compressive stresses in the steel girder section. As a result, the web of the plate girder may be susceptible to the possibility of local buckling, and must be designed accordingly.

(5) The cost of the post-tensioned steel bridge was not found to be more economical than conventional steel bridge construction. However, no final conclusion on the economical viability is postulated because the construction expenses of posttensioned steel-concrete composite bridges could be reduced further.

Based upon the observations and conclusions from the study described herein, the following recommendations for implementing the research are provided:

(1) Post-tensioned, composite steel bridges appear to be a viable alternative to conventional steel bridges. Advantages of the post-tensioned bridges include greater ease in fabrication of the steel members and the elimination of fatigue sensitive details, providing for greater durability and economy.

(2) The analytical model described herein can be used to evaluate and design posttensioned steel composite deck bridges. Both immediate and long-term effects due to creep and shrinkage should be included to prevent local buckling of the steel girder web.

(3) The total cost of the post-tensioned steel composite bridge at Elkhart was roughly equivalent to the cost of a comparable conventional steel bridge. Improved 
economies are expected as additional post-tensioned steel bridges are built. As experience is gained with the construction of this bridge type, then the cost of forming and building the concrete diaphragms will be reduced. Additional cost data should be gathered as other post-tensioned steel bridges are constructed.

(4) The use of HPS-70W steel should be considered for future post-tensioned steel bridges. Hybrid designs that use both grades 50 and 70 steels can provide for uniform cross sections and greater economies in fabrication and construction. 


\section{CHAPTER 1}

\section{INTRODUCTION}

\subsection{Background and Motivation of Investigation}

During the past few decades, two types of structures have intensively been adopted for the field of bridge construction, namely, prestressed concrete beam (pretensioned or post-tensioned) and composite steel beam (concrete deck with steel girders underneath). The purposes of exerting prestress to the former type of structure is to provide compressive loading to overcome the inherent deficiency of tensile strength of concrete prior to the application of anticipated service loads. The philosophy behind the design of composite steel bridge is that steel is a better material for flexural elements than concrete due to its equal strength in tension and compression.

In recent years, another concept, combining the major benefits for both of the aforementioned structures, has brought attention to the designers of bridge systems, i.e., prestressed composite steel bridge. This type of construction is customarily divided into two categories, pre-tensioned and post-tensioned structures. Such way of classification is identical to that used in prestressed concrete structural elements. The terms "pretensioning" and "post-tensioning" sometimes cause confusion. In a pre-tensioned 
structure the prestress force is employed before the concrete slab is poured, while for a post-tensioned structure the prestress force is applied after deck casting.

The primary reason for employing prestress forces is to counteract the stresses caused by the concrete slab and the vehicle live loads. Moreover, since the load-carrying capacity is increased, there is an increased potential saving of material costs. The use of prestressed composite steel construction may save from $3 \%$ up to $25 \%$ of total expenses of materials when compared with those of conventional composite steel bridge structures without prestressing (Anand and Talesstchi 1973; Densford et al. 1990; Anderson 1995; Snyder 1995, 1996). The amount of savings depends upon factors such as bridge span length, construction method (with or without using falsework during the casting of concrete deck), method of prestressing (pre-tensioning or post-tensioning) and arrangement for the application of prestress.

Aside from the major advantages of increasing the ultimate capacity and saving material costs by reducing the weight of the structural elements, prestressed composite steel structures also offer several additional benefits:

(1) The range of elastic behavior is expanded. The reason is similar to that described above. Since some favorable stresses are induced in the structural components, especially the residual compressive stress in steel girder, the required stress to reach yielding in the bottom flange, which is proportional to the externally applied loads, is higher than that of the composite steel structure without prestressing. The expansion of elastic material behavior eventually results in a delay or even elimination of the occurrence of plastic deformation. 
(2) The deformation due to service loading is also reduced. The prestress force produces some counter-acting curvatures in advance. For example, if high-strength steel tendons are used, the jacking force, combined with arranged eccentricity creates certain cambered moments along bridge spans in the opposite direction to those generated by future service loads.

(3) The probability of the formation of cracks in the concrete deck is lower. The concrete deck of a composite girder may crack if the stress in the concrete exceeds the level of tensile strength of concrete. The prestress force can be applied so that the whole slab is subject to compressive stresses. For simply supported bridges with single span, this is almost true for any case. For multi-span bridges, tensile stresses may still exist in the negative moment regions near the internal supports. However, with a sophisticated design, it is possible to eliminate those tensile stress regions, or at least make them as small as possible, so that the chance of concrete cracking is largely decreased. Prevention of concrete cracking also reduces the chance of invasion of moisture, humidity and salt by-products which are the dominant causes of reinforcement corrosion.

(4) Fatigue strength is improved by virtue of the introduction of prestressing. For bridge structures, the repeated or cyclic loading comes from the traffic flow passing above. With the presence of initial compressive prestresses, the mean values of stress variations in steel sections tend to shift to the negative direction. As a result the maximum tensile stresses are lowered and consequently the fatigue life of the structural steel is extended. 
Since the aforementioned advantages are generally acknowledged, more detailed information is then desired for a better understanding of the performance of this type of bridge construction. The task can be accomplished by conducting well-organized field measurements to provide useful data and developing a reasonable analytical methodology as the basis of design procedure.

\subsection{Objectives and Scope of Study}

The goal of this study is to develop an analytical model that can be used to predict the mechanical behavior and performance of steel-concrete composite bridges posttensioned with high-strength steel tendons, especially for those with draped tendon profiles. Since prestressed structures are still not popular in the United States, and almost no corresponding design specifications have been formulated, it is then important to continue any relevant research activity so that the their characteristics can be more clearly understood.

The research includes both experimental and analytical parts. The experimental portion of the study involves the field monitoring of a newly constructed post-tensioned steel-concrete composite bridge. The bridge is located in Elkhart County and spans across I-90 on the Indiana Toll Road. The on-site testing was performed during and after the period of construction in order to understand the structural performance of the bridge system at certain critical construction stages as well as the long-term behavior. The analytical work examined the overall mechanical behavior of prestressed composite steel bridges by using proposed analytical approaches. Evaluation of stress and strain 
distributions was based on small deformation assumption. The analytical results were compared with data collected from the field to examine the effectiveness of the analytical model.

A review of related research studies conducted during the last four decades is presented in Chapter 2. Those technical reports are classified into seven categories according to topical subject area. In Chapter 3 the experimental program is described. Test results are demonstrated and discussed. Chapter 4 and Chapter 5 deal with the instantaneous and long-term structural response, respectively. In both chapters, analytical procedures are presented first, and then the solutions based on the proposed analytical approaches are compared with the results of field measurements. Temperature effects are included in Chapter 4. A comparative cost analysis is conducted in Chapter 6 in order to visualize the benefit of this type of bridge system from an economic point of view. Conclusions from the experimental and analytical studies are summarized in Chapter 7. 


\section{CHAPTER 2}

\section{LITERATURE REVIEW}

Even though the concept of prestressed composite steel structure is not new and the technical advantages have been recognized, the applications to bridge construction are very rare in the United States and other parts of the world in contrast to other much more

prevalent construction methods. A few examples were described in technical reports by Saadatmanesh et al. (1989), Montens and O'Hagan (1992), Muller and Lockwood (1992), Weaver and Bonasso (1994) and Anderson (1995). Those successfully built structures, mostly bridge constructions, show the feasibility of designs for diverse lengths of span, geometric configurations and construction methods. Research has been conducted concerning about this subject and it is summarized in the following categories.

\subsection{Construction Methods}

Three construction approaches, in terms of the methods of prestressing, have been mentioned in the existing literature: (a) By using high-strength steel strands or bars; (b) by stressing components of hybrid beams and (c) by utilizing the composite action of a concrete slab (Eckberg 1968; Densford and Hendrick 1990). The three types of construction methods are illustrated in Fig. 2.1. 
The first method, applying prestress forces through high-strength steel tendons, is the most commonly used of the three. Most of the related studies, conducted analytically or experimentally, were based on this construction method. The prestressing procedure and equipment are similar to those used for prestressed concrete structures. Prestress forces can be applied either before or after the casting of concrete deck depending on the judgment of designers.

In the second method, a couple of jacking forces are applied vertically on one of the flanges of the steel girder, then with the girder in the loaded position, a high-strength cover plate is welded to the flanges. The jacking forces then are released and the prestressed girder is erected in a hog position. Finally the concrete deck is cast and the composite section is ready for the external loads.

The concept of the third method is actually similar to that of the second. The steel girder is first fabricated with predetermined camber, then transported to construction site for erection. The next step is to apply downward jacking forces to the girder, and then cast a high-strength concrete slab to the bottom flange. The jacking forces are maintained until the concrete reaches the required strength.

Perhaps because of the complexity of construction procedures, the last two methods are not attractive to current designers as the first approach Detailing considerations may stymie the application in practice. Among them, for example, are the welding and concrete work necessary under applied flexural loading for the second and third methods, respectively. 


\subsection{Predicted Structural Response and Load-Carrying Capacity}

Most of the research regarding the behavior of prestressed composite steel structures were focused on the topic of the calculation of stress and strain and the evaluation of loading capacity. Szilard (1959) published a paper demonstrating a general principle for analyzing and designing prestressed composite steel structures. The study case was a single-span composite steel bridge which was simply supported, and posttensioned by high-strength steel cables with a parabolic profile along the span. Treatments to account for the effects of concrete creep and shrinkage, prestress losses and uneven temperature changes were included in the computation of stress distribution and flexural strength.

Hoadley (1963) studied the behavior of simply supported, single-span composite steel beams with a constant eccentric pre-stressing force applied along the entire span. He used the strain energy principle to derive an expression for the increase in tendon forces due to external load and adopted the concept of transformed section to calculate the stresses in the composite section. Comparisons were made between the moment capacities of conventional and prestressed composite steel beams, leading to the conclusion that the strength and ductility of a composite steel bridge could be increased by the efficient use of prestressing.

Kennedy and Grace (1982) employed a convergent series solution based on the elastic plate theory to analyze continuous composite steel bridges under static loading. They compared the performance of two bridge models, the first one was a traditional composite steel structure and the second one was prestressed with tendons placed in the 
concrete slab but was identical to the first one in dimensions. Experiment and analysis were both carried out in their study. Results of their investigation indicated that prestressing the deck slab substantially increased the cracking load as well as the stiffness of the bridge.

Dunker et al. (1987) surveyed the feasibility of repairing or strengthening existing composite steel bridges by means of eccentric post-tensioning. In their study, four typical single-span composite steel bridges were post-tensioned with certain eccentricities along their interior steel girders. They calculated the resulting axial forces as well as midspan moments in all girders for each bridge and proposed some simplified procedure for the evaluation of those qualities.

Basu et al. (1987a, 1987b) conducted an investigation on the mechanical behavior of partially prestressed continuous composite steel beams. In their study, critical positive and negative moments in a two-span beam were evaluated analytically through applying several concentrated loads along the spans. They also designed an equivalent experiment, involving a double-span composite steel beam with the prestressing tendons embedded in the concrete slab, to verify the validity of their analytical work.

Saadatmanesh et al. (1989a, 1989b) performed an experiment to trace the loaddeformation curves for two single-span and simply supported composite steel beams containing straight high-strength steel tendons running through the entire span lengths. One of the beams was subjected to a bending positive moment and the tendons were located below the bottom flange. The other one was subjected to a negative bending moment and the tendons were placed just beneath the top flange. Both of the beams were 
free from initial prestress forces. A pair of concentrated loads of the same magnitude were applied to produce a constant-moment region at mid-span for each beam. Afterward, they developed an analytical model to predict the maximum stress and moment capacity under elastic and inelastic deformation based on sectional equilibrium and compatibility requirements. The results obtained through their computational scheme showed satisfactory agreement with the measured data even though the self-weights of the structures were neglected in their computation. They also demonstrated the advantages of prestressed beams over conventional ones by listing a contrast of the calculated yielding and ultimate loads for both structures.

Ayyub et al. (1990) analytically and experimentally inspected the moment capacities of three pre-tensioned composite steel beams by applying a pair of equal downward concentrated forces all the way to the initial yielding of tension flange and structural failure. The three beams differed in tendon profiles and tendon material (highstrength bar or low-relaxation strand). The analytical method which they termed as "strain compatibility method" was used to calculate the stresses and deformations at all levels of loading. Aside from demonstrating sufficient reliability of their analytical procedure, they also discovered that the use of strands as prestressing tendons were superior to that of high-strength bars, and that draped tendons provided more ductility than straight tendons.

Troitsky et al. (1989) explored the spatial variations of stress intensity analytically and experimentally for loaded pre-tensioned and post-tensioned composite steel girders within the elastic ranges of material properties. They used a virtual work method to calculate the increment in the initial tendon force under the application of external load. 
With the assumption of small elastic deformation, they superimposed the stresses due to various sources of loading, such as dead load, live load, original prestress force and increased tendon forces to attain the total stresses at several selected locations along the span. Cases for straight and bent-up tendon profiles were both included in their investigation. Those computational procedures then were followed to calculate the stress distributions of a prestressed composite steel girder specimen, and the results were compared with the data collected from the corresponding experiment. Reasonable similarity was found according to their comparison.

Tong and Saadatmanesh (1992) carried out a parametric study for continuous prestressed composite steel girders. First they formulated two analytical models for the elastic analysis of composite steel girders with straight and draped prestressing tendon profiles, then they examined the effects of those factors such as the level of prestress force, eccentricity, tendon arrangement, tendon length, prestressing sequence and girder span. The knowledge gained in the research was used to probe the feasibility of upgrading the loading capacity of an existing two-span, continuous composite highway bridge. The results from a combination of the aforementioned design variables showed the possibility of enhancing the strength of a structure by the use of prestressing.

Ayyub et al. (1992a, 1992b) indicated that the negative moment region could be a critical problem around the internal supports of continuous girders. They conducted a study to understand the performance of prestressing tendons under the action of negative bending moment. Both analytical and experimental programs were utilized to evaluate composite steel girders with tendons placed right below top flanges and inside the 
concrete slab. Their results showed that placement of strands in the concrete deck was a more efficient designing alternative.

Additional investigations were conducted by Reagan and Krahl (1967), Klaiber et al. (1982), Anand and Fennell (1974), Troitsky et al. (1989, 1991). In summary, all of those research studies indicated that prestressed composite steel structures provide a competitive option over other types of structures due to promising superiority in loadcarrying capacity, strength, and economy.

\subsection{Time-Dependent Effects}

Generally, several time-dependent effects which are related to material properties and history of loading may affect the long-term performance of a prestressed composite steel structure. Those effects include the shrinkage and creep of concrete, and relaxation of prestress in the steel tendon. Few research papers have been found that effectively dealt with this topic. The difficulties for precise calculation arises from the complicated interrelation between those effects. However, reasonable decoupling of those effects is probably sufficient for general design purposes.

In 1959, Szilard suggested several empirical and semi-empirical formulae to account for the effects of creep and shrinkage in the concrete decks of post-tensioned composite steel bridges. The modulus of concrete for long-term loading history was then evaluated according to those formulae. Hoadley (1963) and Saadatmanesh et al. (1989c) used a simple procedure to include the effect of concrete creep in calculating the stress distributions in a composite section subjected to a superimposed dead load. Their 
approach was to triple the modulus ratio of steel to concrete which was used to determine the sectional properties of the corresponding transformed section. Dezi et al. $(1995,1996)$ conducted a more elaborated research focused on the effect of concrete creep on continuous composite steel beams with prestressed slabs. Their analysis was based on the theory of linear viscoelasticity (used to describe the material behavior of concrete) and a step-by-step numerical integration procedure.

Pochanart (1999) investigated time-dependent effects on the performance of steelconcrete composite bridges post-tensioned by high-strength steel tendons. He adopted a viscoelastic model proposed by Bazant and Wittmann (1982) to simulate the creep behavior of the concrete deck of the bridge. In his study, the elastic modulus of concrete was represented by an exponential series in terms of time, and other structural components were assumed to be linear elastic. Shrinkage in concrete deck and relaxation in steel tendons were also involved. The analysis was performed by using ABAQUS, a general-purpose finite element program. Parametric studies were conducted to investigate how long-term bridge performance is affected by critical factors such as bridge geometry, material properties and environmental conditions. His study showed that time-dependent factors will cause prestress losses as well as stress redistribution in structural components.

\subsection{Stability Problems}

Very little attention has been given to the buckling problems which might occur in prestressed structural beam elements. Present cases of design tend to build the girders or beams compact enough so that the possibility of the occurrence of any type of buckling is 
minimized. More research in regard to this topic are expected to be conducted. Bradford (1991a) studied the elastic buckling behavior of prestressed steel girders with single span. Those girders under investigation were simply supported, and the tendon profiles were parallel to the neutral axes of the girders (i.e., constant eccentricities). The author indicated that according to previous studies of the stability for thin-web plate girders, the critical buckling would occur in the web. He then assumed reasonable functions to describe the deformed shape of the steel plate girder under the action of constant axial compression and bending moment. The buckling load and mode shape could be obtained by solving the corresponding eigenvalue problem. Parametric study was performed for girders with various dimensional ratios and prestress eccentricities to establish design charts. Tochacek and Ferjencik (1992) provided new design formulae for checking the stability of steel beams prestressed with straight tendons in comparison with the specifications in Czechoslovak National Standard for steel design.

\subsection{Fatigue Properties}

Fatigue of steel structures results from repeated or cyclic loading. For bridge structures, fatigue strength is of particular importance when compared with other types of construction due to the moving vehicles that are the frequent sources of repetitive loading. So far, few technical reports have been published on the fatigue behavior of prestressed composite steel bridges. Kennedy and Grace (1990) experimentally studied the effect of prestressing on the fatigue strength for composite steel bridges. They applied periodically repeated loads to two $1 / 4$-scale, double-span composite steel bridge models, 
one of them was prestressed in the concrete deck around interior supports and the other was a conventional composite steel structure. The periodic loads were set to be nearly tuned to the fundamental frequencies of both bridge models to produce responses as large as possible. The test results showed remarkable improvement of fatigue properties with the presence of prestressing.

Albrecht et al. (1995) conducted an experimental study to determine the fatigue strength of several prestressed composite steel beam specimens. The stresses in the structural components, including strands, shear connectors and cover plates welded to the central potions of the tension flanges were monitored during the application of repeated loads in certain magnitudes. Their test results showed that the welded cover plate ends controlled the structural fatigue strength, and no fatigue failures were observed in the shear studs and tendons during their testing. Li et al. (1995) analyzed the fatigue test data for separate structural components, such as seven-wire strands, shear studs and cover plates from previous studies they had collected. Those collected test data were fitted with log-log linear S-N curves, and the fatigue limit for each individual component was then estimated accordingly.

\subsection{Dynamic Analysis}

Kennedy and Grace (1990) investigated the dynamic response of continuous composite steel beams with prestressed concrete slabs (prestressing in the vicinity of internal supports) through theoretical analysis and experimental study. They modeled the dynamic behavior of the composite steel bridge by a partial differential equation in terms 
of the time-dependent deflection function based on orthotropic plate theory. By assuming a sinusoidal series solution for the deflection function satisfying specified boundary conditions, mode shapes and the vibrational frequencies for free vibration were then obtained accordingly. They then designed an experiment to sweep out those physical qualities (mode shapes and natural frequencies) for the first four modes by applying periodical loading with varying frequencies over a range from 0 to $80 \mathrm{~Hz}$. Results of the comparison between their analytical and experimental tasks showed acceptable similarity. The stiffness of continuous composite steel bridge was found to be enhanced in the presence of prestress force in the negative moment region due to the reduction of crack formation.

\subsection{Suggested Design Procedures}

A couple of publications have been found that are pertinent to the design of prestressed structures. Szilard (1959) presented design equations for statically determinate composite steel beams with prestressing tendons in a parabolic configuration along the spans. Those equations dealt with the calculation of the stress distribution and deflection considering effects such as concrete shrinkage and creep, prestress losses and uneven temperature changes.

In 1971, Tochacek and Amrhein (1971) published a paper to indicate the that the concept of limit states design is more suitable than that of allowable stress in designing prestressed steel structures. Anand and Talesstchi (1973) developed several equations for designing simply supported prestressed composite steel beams. The prestressing was 
achieved by welding a high-strength steel plate to the bottom of the tension flange of the already jacked steel beam (the second construction method described previously). They further simplified their design equations for the case of wide-flange sections to get approximate but more concise expressions without losing too much precision. The moment-carrying capacity is governed by the permissible bending stress of the steel beam or the high-strength steel plate. Several design examples were also presented to demonstrate the advantages of prestressed structures over their non-prestressed counterparts.

In 1981 Johnson et al. (1981) published a report to discuss the limit state design for four different types of prestressed composite steel structures which had been studied by The European Joint Committee for Composite Structures. The four types of structures differed in construction methods such as the way and time of prestressing and the usage of temporary shoring. Saadatmanesh et al. (1989c) proposed a procedure for flexural design of prestressed composite steel beams. Cases for the positive moment as well as negative moment regions were both involved and treated separately according to working stress and load factor design philosophy. 


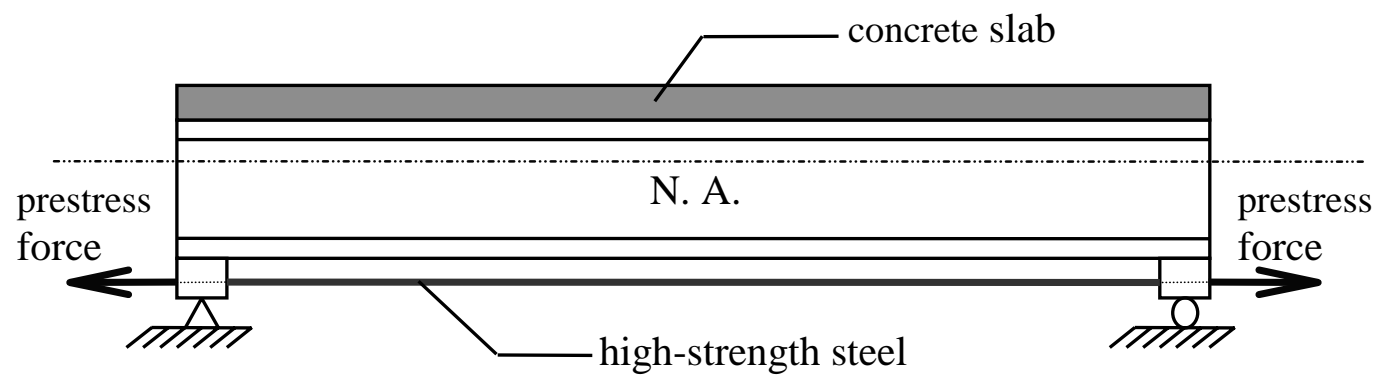

Straight-line tendon profile

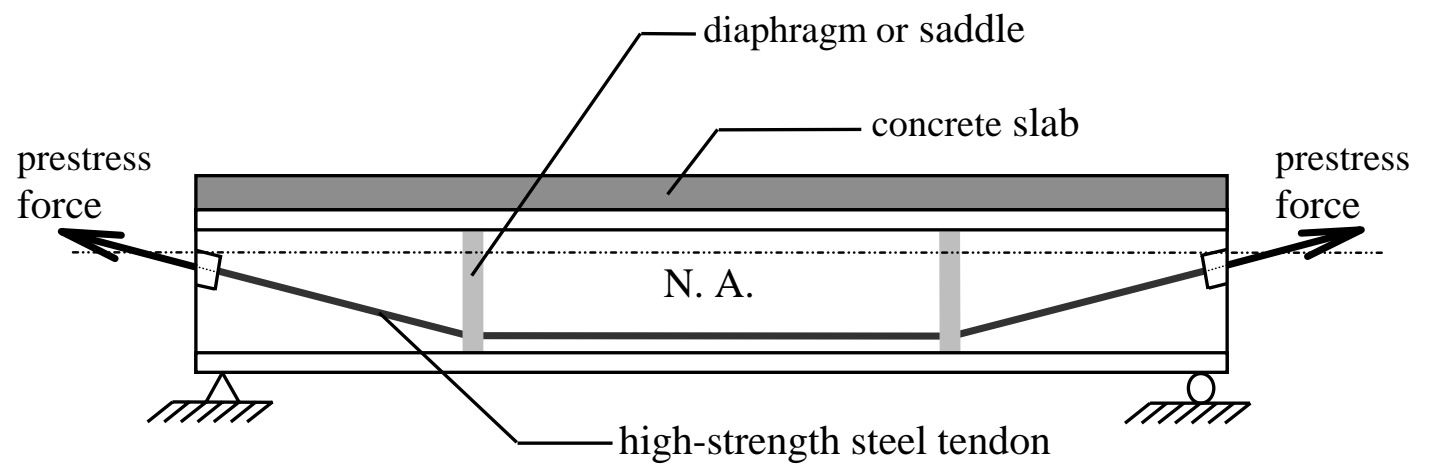

Draped tendon profile

(a) By using high-strength steel strands.

Figure 2.1 Construction method. 

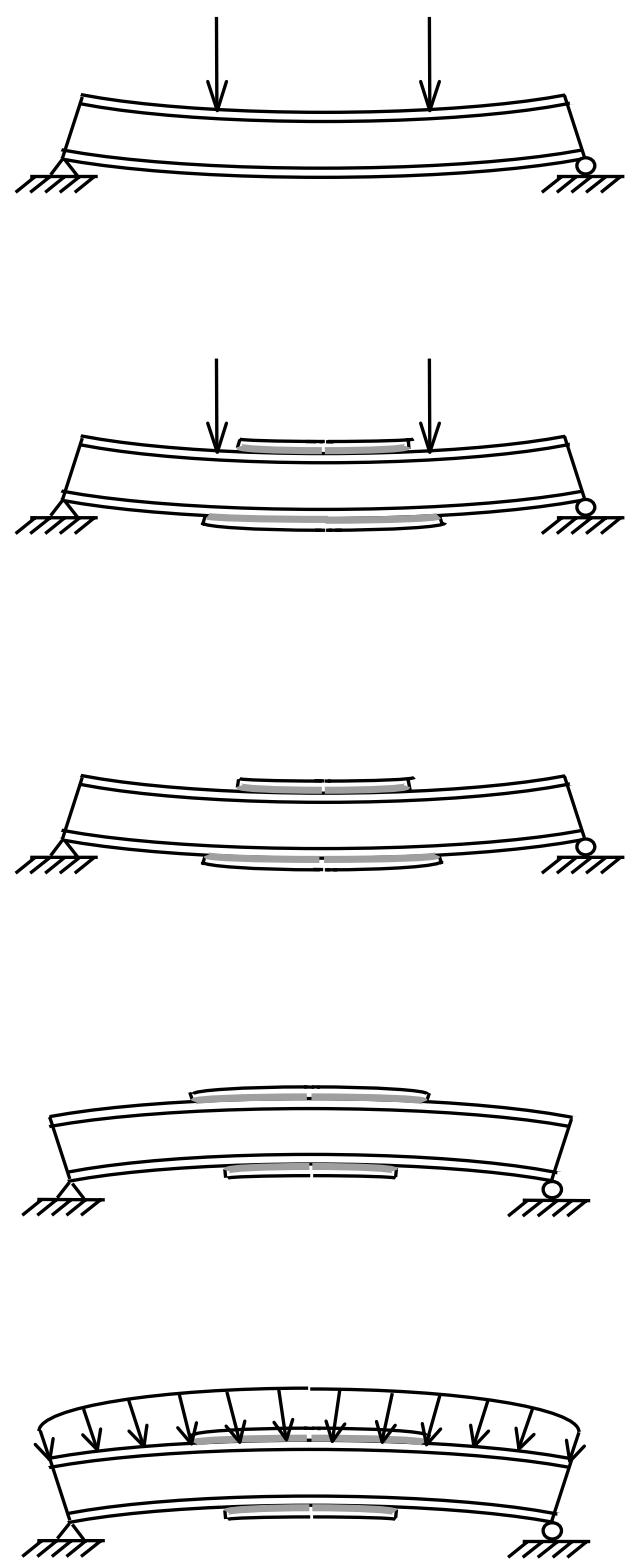

(1) Apply jacking forces on the top flange.

(2) Weld high-strength cover plates to the flanges with the jacking forces maintained.

(3) Remove the jacking forces.

(4) Turn the girder upside down.

(5) Cast concrete slab and impose loads.

(b) By stressing components of hybrid girders.

Figure 2.1 Construction method. 

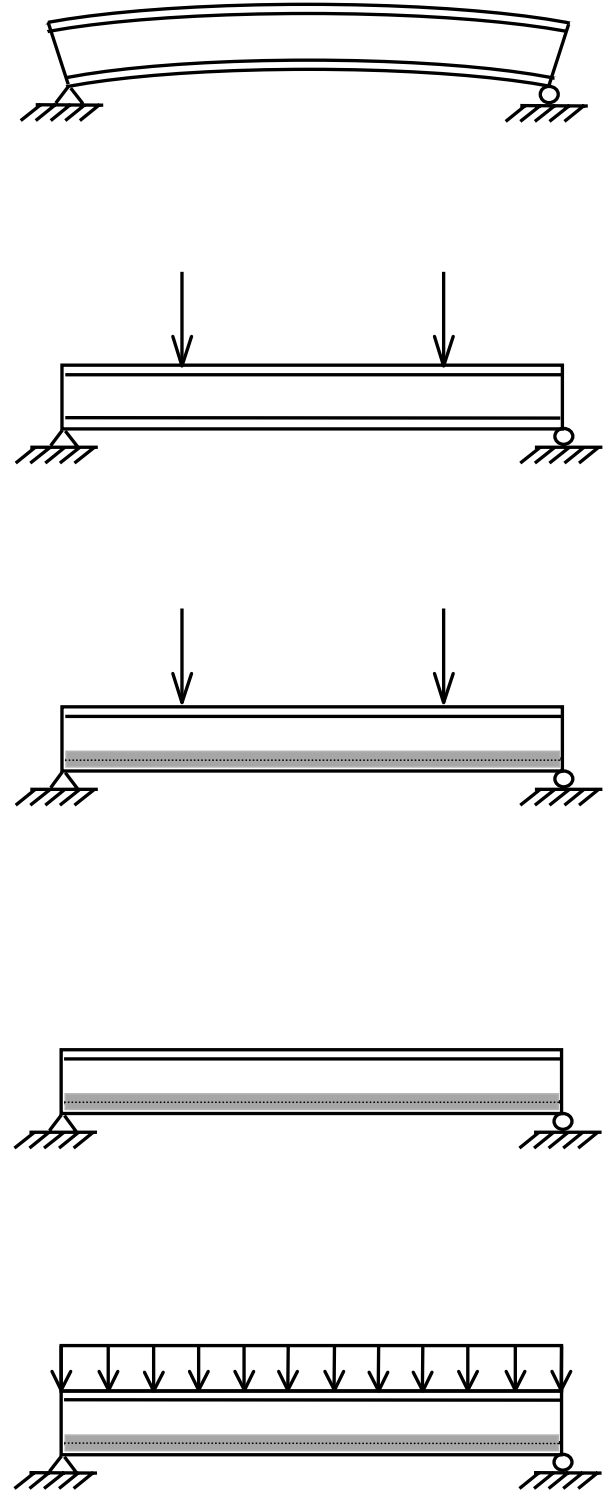

(1) Erect the girder which is fabricated in a predetermined camber shape.

(2) Apply downward jacking forces to the girder.

(3) Cast high-strength concrete to the bottom flange with the jacking forces persisted during the concrete curing.

(4) Remove the jacking forces when the concrete reaches the design strength.

(5) Cast concrete slab and impose loads.

(c) By utilizing the composite action of concrete slab.

Figure 2.1 Construction method. 


\section{CHAPTER 3 \\ EXPERIMENTAL PROGRAM AND RESULTS}

\subsection{Profile of Elkhart County I-90 Toll Road Bridge}

The experimental portion of the proposed research involves the field testing of the Elkhart County I-90 Toll Road Bridge which is now open to traffic. Depicted in Fig. 3.1 are the profile and sectional views of the bridge. It is a steel-concrete composite bridge with two spans of equal length. The concrete deck is supported by seven built-up steel plate girders. Shear stud connectors were used to prevent relative sliding between the concrete slab and the steel stringers along the contact surface. The Elkhart County bridge was designed to be built with the steel girders cast together with the walls at the abutments. The prestress was applied to the bridge after the concrete deck had achieved sufficient strength; therefore, the structure is called post-tensioned. Fourteen posttensioning tendons, stressed in particular order, run along the bridge length, with the ends anchored at both abutments and the longitudinal tendon profile deflected at two drape points, as shown in Fig. 3.2.

The concrete diaphragms (Fig. 3.2) were designed to serve as drape supports for post-tensioning tendons. This feature provides some additional possible saving over the 
design in which the tendon drapes or saddles are assembled together with the plate girders in the steel fabrication shop.

The introduction of post-tensioning provided initial stresses in the structural components to counteract the gravity loads imposed earlier (the weight of concrete deck and steel girders) and other loading applied later. Therefore, as for prestressed steelconcrete composite bridges, the flexural capacity of the bridge was enhanced. Moreover, the elastic range of bridge performance was also extended, and hence the occurrence of plastic deformation will be deferred or even eliminated.

The bridge design has two additional advantages: a reduced probability of crack formation in the concrete slab and an improvement of the fatigue properties of the structure. The post-tensioning force was designed to primarily subject the concrete deck to compressive stresses. The compression stress will result in less cracking of the concrete deck and will significantly decrease the invasion of salt and moisture into the concrete. Consequently, corrosion of the reinforcement will be lessened considerably. Accordingly, the potential for reducing deck repair costs in the future is significant. The improvement of fatigue structural behavior attributes to the fact that the composite section is exposed to a lower mean stress level of cyclic or dynamic loading from traffic due to compressive prestress forces. As a result, it is also likely that fracture and fatigue failures at the welded bridge details will be decreased.

Several photographs are provided in Appendix A to illustrate the construction and structural configuration of the Elkhart County Bridge. 


\subsection{Description of Experimental Program}

Strain measurements were taken at certain locations along an exterior and an interior girders for both steel and concrete elements. A total of eleven locations, six in the exterior girder (Girder 1) and five in the interior girder (Girder 3), were selected to attach electrical resistance strain gages for field investigation (Fig. 3.3). Since the bridge is symmetric about the piers, strain gages were placed on the south span only for each girder. Gage positions at each location were depicted in Fig. 3.4 and Fig. 3.5. In those figures, the numbering system of strain gages is also demonstrated. With the knowledge of the elastic modulus of the high-strength steel strand, the post-tensioning force can be determined through measuring the deformation of a selected tendon. Four locations at the monitored tendon were selected: two near the piers and one at each abutment (Fig. 3.6).

The strain gages on steel plate girders were installed in the steel fabrication shop at Vincennes, Indiana before they were transported to the construction site. Hence, the level of difficulty associated with attaching the strain gages in the field was significantly reduced.

To monitor strain levels in the concrete bridge deck, strain gages were attached to reinforcing bars that were embedded in the slab. The strain gages were attached to the rebars in the Kettlehut Structure Engineering Laboratory at Purdue University. After the gages were attached, the rebars were then delivered to the construction site for final installation in the concrete deck.

Four basic procedures were involved in the installation of strain gages, including surface preparation, gage bonding, wire attachment and protective coating. The last 
procedure is of particular importance for the electrical resistance strain gages which are exposed to high levels of moisture and humidity in a field environment. All the strain gages were aligned to be parallel to the longitudinal bridge span. OPTIM's MEGADAC 200 Data Acquisition System, accompanied with the software OPUS, was adopted for the field experiment to convert electrical signals into digital strain readings.

The tendon deformations were evaluated by using vibrating wire transducers. (Model No. 4410) and a corresponding readout device (Model No. GK-403), which are products of Gook Corporation. The transducer is designed to be clamped around the wire strand at both ends, and connected to the readout box while the readings were taken. The components of the Model 4410 Vibrating Wire Strandmeter and the front panel of the GK-403 Readout unit are illustrated in Fig. 3.7 and Fig. 3.8, respectively. The deformation in the post-tensioning tendon was not obtained directly but converted from the reading called "digit" via the formulae provided by the manufacturer (Table 3.1). Temperature was also shown through the readout box which was used for the correction of thermal effect.

Field measurement and testing were arranged to be taken at certain decisive phases of the construction period and continued over about one year. There were three critical construction stages during which data were collected: (1) after the steel plate girders were erected and before the concrete deck was poured; (2) soon after the concrete deck was poured and before longitudinal post-tensioning; and (3) during and right after longitudinal post-tensioning. Live load testing was coordinated and performed in cooperation with the Toll Road Division. A dump truck with known axle weights was 
positioned at each of nine selected locations along the bridge span where strain gages were placed (Fig. 3.9). Dimensions including the front-to-rear and right-to-left tire spacing as well as the axle weights of dump truck are described in Fig. 3.10.

\subsection{Results from Bridge Testing}

Some selected but representative results from the field experiment are presented and discussed in this section. The strain distributions shown thereafter are relative to the zero readings which were taken before the pouring of concrete deck and served as the baseline for all the later measurements. It is worth noting that the strain distribution due to the weight of steel girder is therefore not included in the experimental readings. Listed in Table 3.2 are the dates when strain measurements were taken, labeled as stage a to stage g. These stages are utilized in presenting the data in Figs. 3.11 to 3.17, which are the strain distributions across the depth of the web of the steel girder. In those figures the scale of vertical axis corresponds to the gage position relative to the top surface of the bottom flange (the lower end of web). Tensile and compressive strains are represented by positive and negative numbers, respectively.

Strain distributions in the steel girders G1 (the exterior girder) and G3 (the interior girder) at location 2 are respectively shown in Fig. 3.11 and Fig. 3.12. This location is $1,220 \mathrm{~mm}$ away from the center of the bridge and is within the negative moment region. Linear strain distributions were approximately obtained at this longitudinal location. The dead load created negative bending moment, and as the post-tensioning forces were applied, the curvature of the girder was decreased since the slope of sectional strain 
distribution became less steep. The magnitudes of strain values for G1 and G3 at this location are fairly comparable. Strain readings collected after post-tensioning were continuously decreasing.

Figs. 3.13 and 3.14 are for the strain distributions in the steel girders G1 and G3 at location 6 , which is $14,110 \mathrm{~mm}$ away from the end of bridge. This location is near the position of maximum positive moment due to downward loading. It can be seen that as the post-tensioning forces were imposed (at stages $b$ and c), the sections were then subjected to some compressive stresses since the strain distribution shifted to the negative direction. The linearity of strain distribution in G1 was nearly preserved at every stage of measurement. However, in girder G3, after the weight of concrete slab was superimposed, the strain values obtained from the gages on both sides of the girder web resulted in a strain distribution that was not linear. Furthermore, after the tendons were stressed, not only the linearity was no longer maintained, but the strain readings on both sides of the web were far from consistent. Some out-of-plane deformation could have been introduced in G3 at this location. Deterioration of certain strain gages is also a possible cause of abnormal strain readings. Another observation is that as time progressed, the strains tended to keep decreasing very uniformly.

Figs. 3.15 and 3.16 are for the strain distributions in the steel girders G1 and G3 at location 7 which is $1,365 \mathrm{~mm}$ from the south end of the bridge. At this location the girder sections were likely distorted since the strain distributions were not linear. It was probably due to the deformation resulting from the out-of-plane bending other than the inplane action. The effect of stress concentration by post-tensioning operation near the 
bridge end and shrinkage in the concrete slab might also be a contribution to these nonlinear strain distributions. Inconsistency for strain reading on both sides of steel girder was also found in G3. That might be a consequence of sectional distortion.

Shown in Fig. 3.17 are the strain distributions in the steel girder G3 at location 1. The strain gages placed here were embedded in the central concrete diaphragm where one of the interior drape points is situated. Many strain readings at this location were unreasonable, most of which were excessively high. It is believed that most of the strain gages were seriously perturbed during the transverse post-tensioning, and perhaps also during the casting of the diaphragms.

Observing the strain distributions after post-tensioning operation in Figs. 3.11 to 3.16 (plots $\mathrm{d}$ to $\mathrm{h}$ ), it is found that the steel section was subjected to additional compressive stresses which is considered as the result of the factor due to time-dependent material behavior. The long-term effects are discussed later in Chapter 5.

Figs. 3.18 and 3.19 illustrate the strain variations at different locations in the concrete deck along the girders G1 and G3, respectively. The scale on the horizontal axis has no physical meaning, but rather corresponds to different stages of construction or moments while strain readings were taken (the sequential order of strain measurement) which can be referred in Table 3.3. In these figures, the larger the number, the later instant it corresponds to. Continuous decreasing in strain values (shift to negative values) with respect to time was observed at each gage position, which is believed to be due to the combined effect of long-term behavior and temperature change. For the pair of gages at the same longitudinal location along each girder, although the strain readings were 
diverse in numerical values, the trend of variations with regard to time were rather similar for most cases. Ideally, since no temporary shoring was used during construction, the initial strain for each gage placed in the concrete deck should be null at the "stage zero" which was designated as "four days after concrete deck was poured" The non-zero initial strain values are believed to be the consequence of the bending of the gage-affixed rebars during the concrete pouring.

Fig. 3.20 is the strain variation in the monitored post-tensioning tendon. The scale of horizontal axis represents the time in days. The zero refers to the date when the vibrating wire transducers were installed. The post-tensioning forces were applied at the date two. Since the readings were taken right after complete stressing, the tendon force at this moment could be regarded as the initial prestress force. One can observe that after nearly two months, the strain or stress in the tendon had approached a nearly constant value. Most of the steel relaxation was believed to have occurred at that time.

The selected results of live load test are demonstrated in Fig. 3.21 to Fig. 3.25. The truck positions and the magnitudes of axle weights for the test can be referred back to Figs. 3.9 and 3.10, respectively. Illustrated in Figs. 3.21 and 3.22 are the strain distributions in the steel girder G3 at locations 2 and 6 due to the truck weight positioned at all the nine chosen locations. The results of the test showed that the bridge responses due to this level of live loading were small compared to those resulting from the application of dead loads and post-tensioning.

In Figs. 3.23 and 3.24 the strain distributions in concrete due to axle loads, at locations 2 and 6 along the girder G3 are demonstrated. Those figures show the strain 
variations as the truck moved longitudinally (the left figure) or transversely (the right figure). Reference to Fig. 3.9 would make it easier to understand the figures. The strain values were found to be not significant since they were so small.

The strains in post-tensioning tendons induced by the truck weight are illustrated in Fig. 3.25, and they are presented in the same manner as those in Figs. 3.23 and 3.24. They were even more insignificant than those found in the steel girders and the concrete slab. It seems that the increment of tendon force due to the live load at the magnitude for this testing is negligible since the strains values were barely perceptible, only a few microstrains $\left(10^{-6}\right)$.

The strains in the steel and concrete components and the deformation in highstrength steel strand were the information gathered in the bridge testing. Other qualities, such as the deflections along the bridge spans, reactions, rotations and relative movements at supports may be attainable in a laboratory, but are virtually impossible or very difficult to observe in the field environment.

\subsection{Errors of Field Experiment}

Any experiment is inevitably susceptible to errors arising from diverse reasons. For this bridge test, the inherent causes of errors include leadwire effect, transverse sensitivity in strain gages and moisture.

The leadwire effect is generally negligible for experiment conducted indoors since the leadwires connecting the strain gages and the data collecting device are usually short enough to produce noticeable errors. However, in this research project some leadwires are 
as long as $50,000 \mathrm{~mm}$ and the error due to wire length need to be examined. The percentage of error, based on the three-wire hookup adopted in the in-site experiment, was estimated by the formula:

$$
\% \text { of error }=\frac{2 R_{L} / R_{g}}{1+2 R_{L} / R_{g}}
$$

where $R_{g}$ is the electrical resistance of the strain gages. $R_{L}$ is the electrical resistance in a single leadwire of a particular length. In the conducted investigation, $R_{g}=$ $350 \Omega$, and the maximum leadwire resistance was measured no larger than $5 \Omega$ for two wires $\left(2 R_{L}\right)$. Therefore, the percentage of error due to leadwire effect is estimated no greater than $1.5 \%$.

Transverse sensitivity in a strain gage refers to the behavior of gage in responding to strains which are perpendicular to the primary sensing axis of the gage (TECH NOTE no. TN-509, Micro-Measurements Division, Measurement Group). The error due to transverse sensitivity is a result of the Poisson's ratios of the gage and the measured material. It is an approximate linear function of the transverse sensitivity of gage foil and the ratio of axial strain to transverse strain in the gage. The transverse sensitivity is determined by the type of strain gage and is provided by the manufacturers (usually a few percentages). Since all the strain gages were oriented in the direction of the major principal stress and essentially no stresses were exerted in the perpendicular directions for the case of study, the errors due to this factor are believed to be insignificant and can be neglected. 
Moisture and humidity may induce tremendous errors in a long-term observation by introducing significant zero shifting to the gage reading. It is, however, very hard to justify the errors caused by this factor. The invasion of dampness is believed to be the most threatening obstacle for successful field measurements when using electrical resistance strain gages. Strain readings may also be subjected to errors due to factors such as Wheatstone bridge nonlinearity, misalignment and temperature variation. Efforts had been made to control the level of those errors within a minimum extent.

The vibrating wire strandmeters are a more sophisticated and delicate transducer than strain gages, and they were expected to provide more reliable information than the strain gages if properly installed. The most possible error resulted from the thermal expansion (or contraction) of the gage itself. However, temperature correction could be performed by employing the correction equation listed in the technical manual to almost eliminate the errors. 


\section{Table 3.1 Deformation calculation for Vibrating Wire Strandmeter}

1. Formula without temperature correction:

$$
\begin{aligned}
& D=\left(R_{1}-R_{0}\right) \times C \times F \\
& D: \text { deformation } \\
& R_{1}: \text { current reading } \\
& R_{0}: \text { initial reading obtained at installation } \\
& C \quad: \text { calibration factor provided by manufacturer } \\
& F \quad: \text { engineering unit conversion factor }
\end{aligned}
$$

2. Formula with temperature correction:

$$
\begin{aligned}
& D=\left[\left(R_{1}-R_{0}\right) \times C+\left(T_{1}-T_{0}\right) \times K\right] \times F \\
& D: \text { deformation } \\
& R_{1}: \text { current reading } \\
& R_{0}: \text { initial reading obtained at installation } \\
& C: \text { calibration factor provided by manufacturer } \\
& F \quad: \text { engineering unit conversion factor } \\
& T_{1}: \text { current temperature } \\
& T_{0}: \text { initial temperature obtained at installation } \\
& K \quad \text { thermal coefficient }\left[=\left(R_{1} \times 0.000295+1.724\right) \times C\right]
\end{aligned}
$$


Table 3.2 Sequential order of strain measurement for Figs. 3.11 to 3.17 .

Before post-tensioning

a. $6 / 23,1997,4$ days after concrete deck was poured.

During post-tensioning $(6 / 30,1997)$ :

b. T1 T14 100\% (from south abutment).

c. $\quad \mathrm{T} 1 \sim \mathrm{T} 14100 \%$ (from north abutment)

After post-tensioning

d. $7 / 8,1997$, approximately 1 week after post-tensioning.

e. $\quad 7 / 15,1997$, approximately 2 weeks after post-tensioning.

f. $7 / 23,1997$, approximately 3 weeks after post-tensioning.

g. $8 / 21,1997$, approximately 7 weeks after post-tensioning.

h. 4/2, 1998, approximately 9 months after post-tensioning. 
Table 3.3 Sequential order of strain measurement for Figs. 3.18 and 3.19.

Before post-tensioning

0. $6 / 23,1997,4$ days after concrete deck was poured.

During post-tensioning $(6 / 30,1997)$ :

1. T1, T2 100\%, T3 20\% (from south abutment).

2. T1 T6 100\%, (from south abutment).

3. T1 T7 100\%, T8 20\% (from south abutment).

4. T1 T7 100\%, T8 60\% (from south abutment).

5. T1 T7 100\%, T8 100\% (from south abutment).

6. T1 T14 100\% (from south abutment).

7. T1 T8 100\% (from north abutment).

8. T1 T14 100\% (from north abutment)

After post-tensioning

9. $7 / 8,1997$, approximately 1 week after post-tensioning.

10. 7/15, 1997, approximately 2 weeks after post-tensioning.

11. 7/23, 1997, approximately 3 weeks after post-tensioning.

12. $8 / 21,1997$, approximately 7 weeks after post-tensioning.

13. 4/2, 1998, approximately 9 months after post-tensioning. 


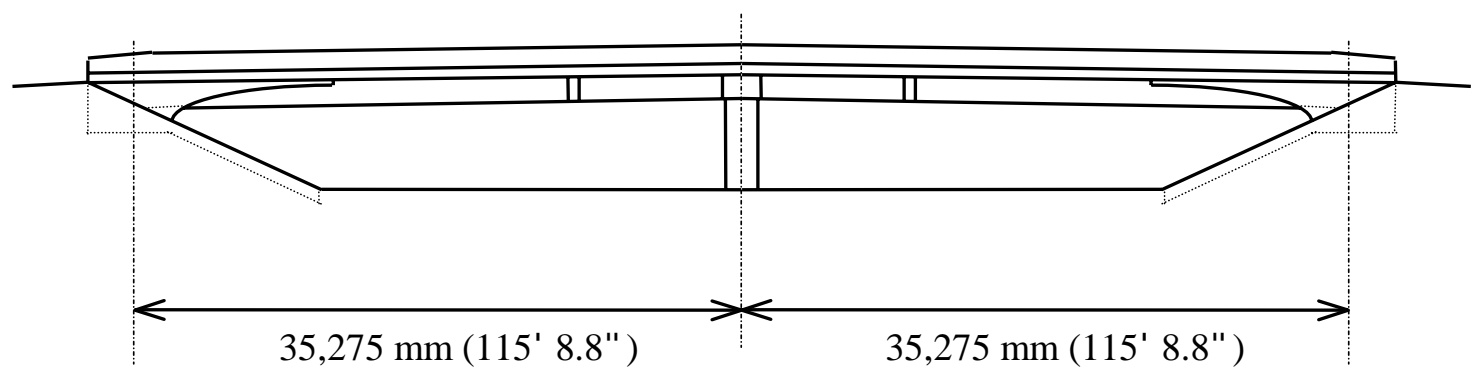

West East

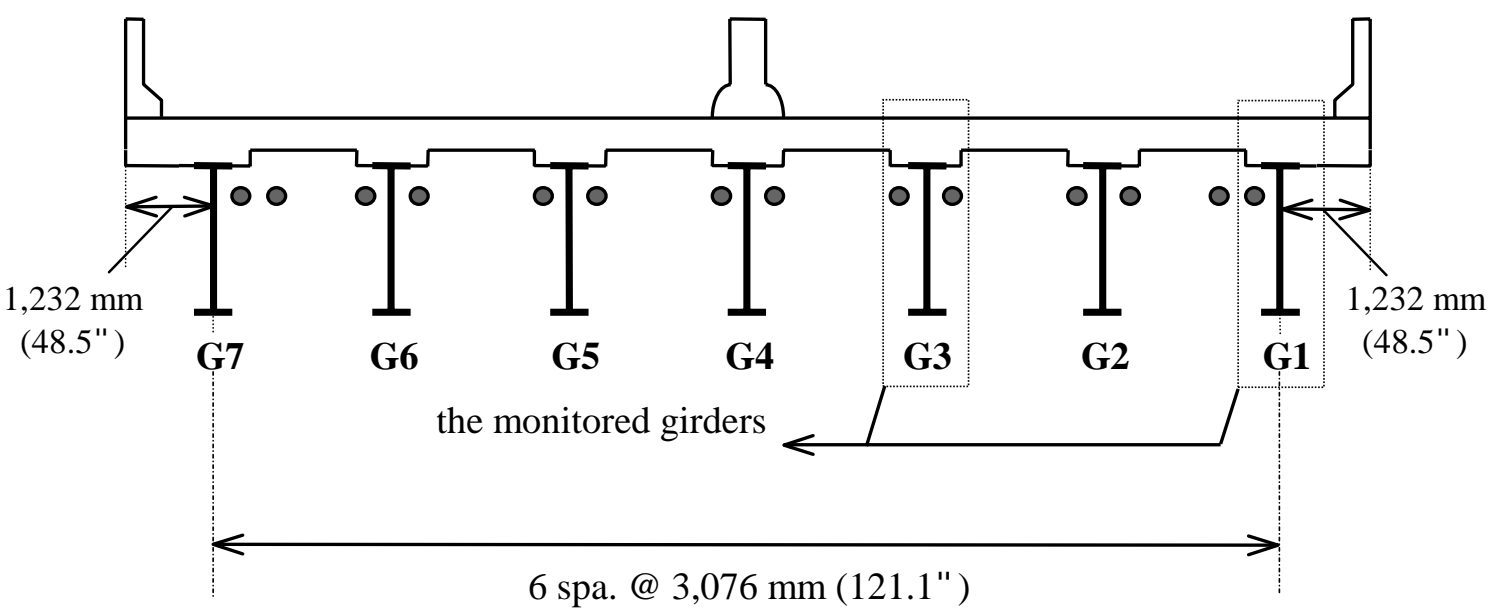

Figure 3.1 Elkhart County I-90 Toll Road Bridge. 

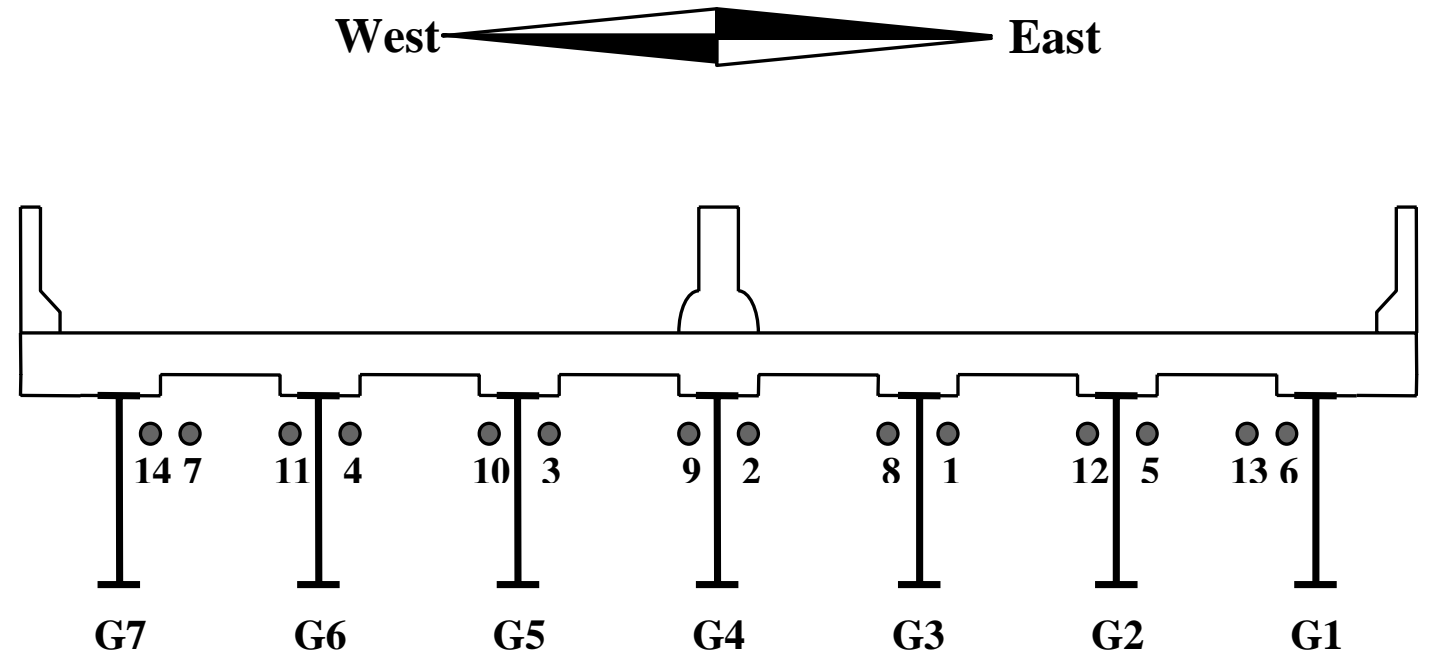

Stressing sequence of longitudinal post-tensioning tendons

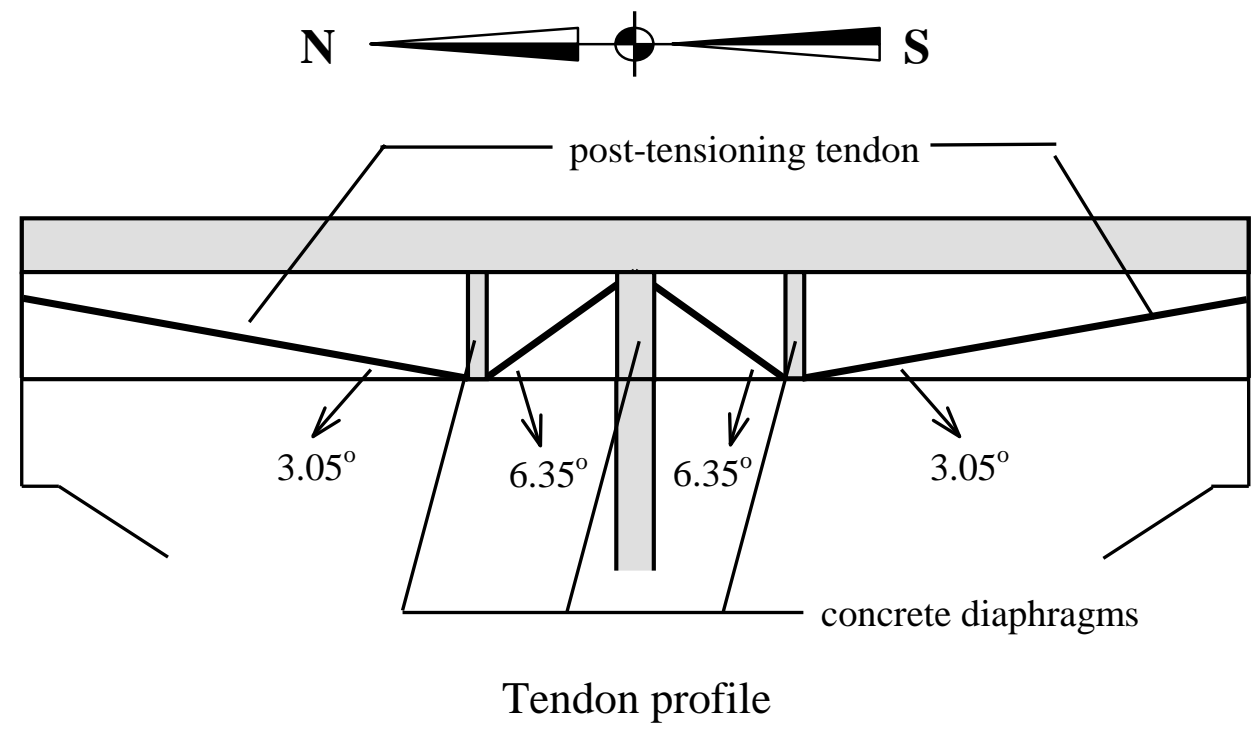

Figure 3.2 Post-tensioning tendons. 

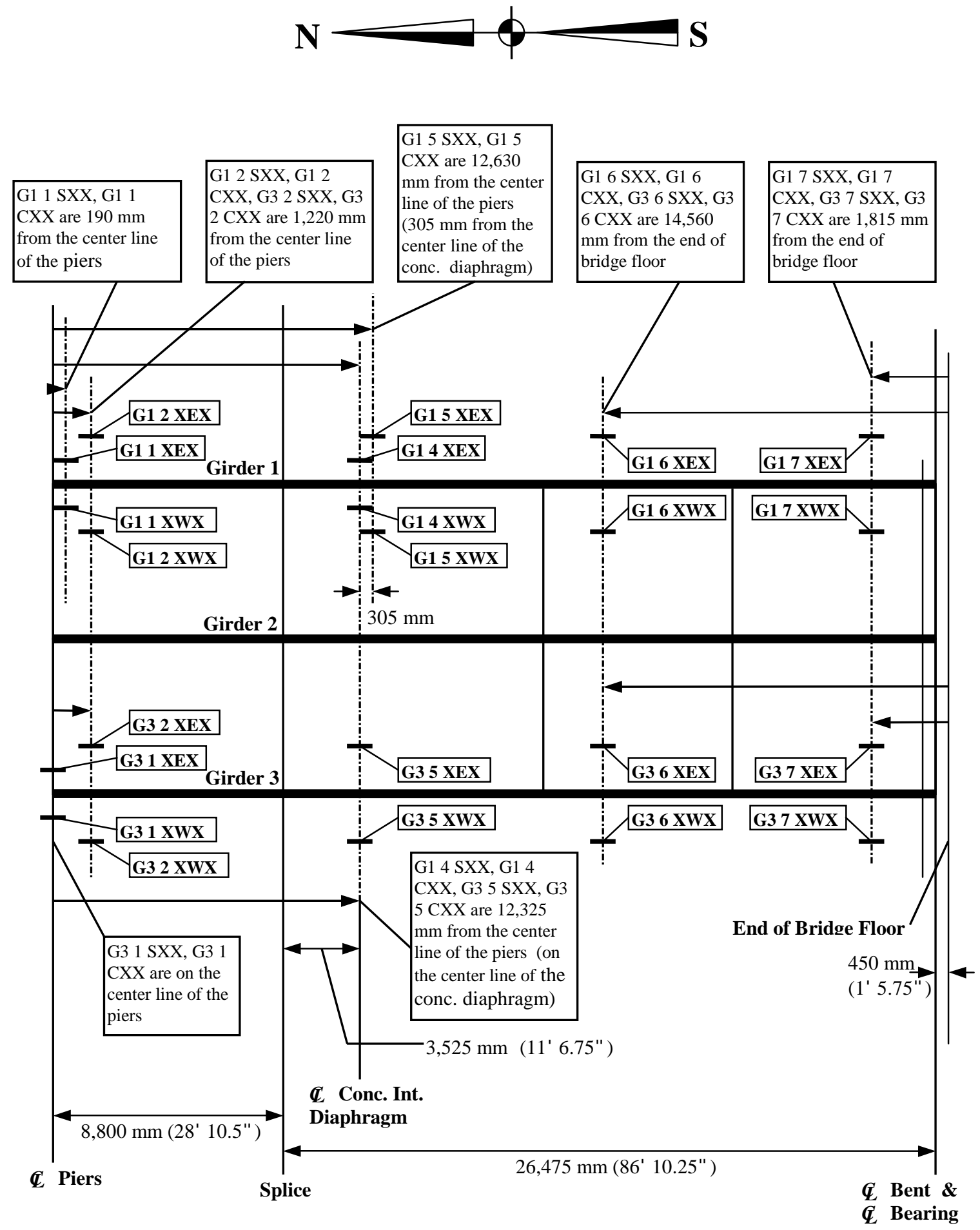

Figure 3.3 Plan view of strain gage locations. 


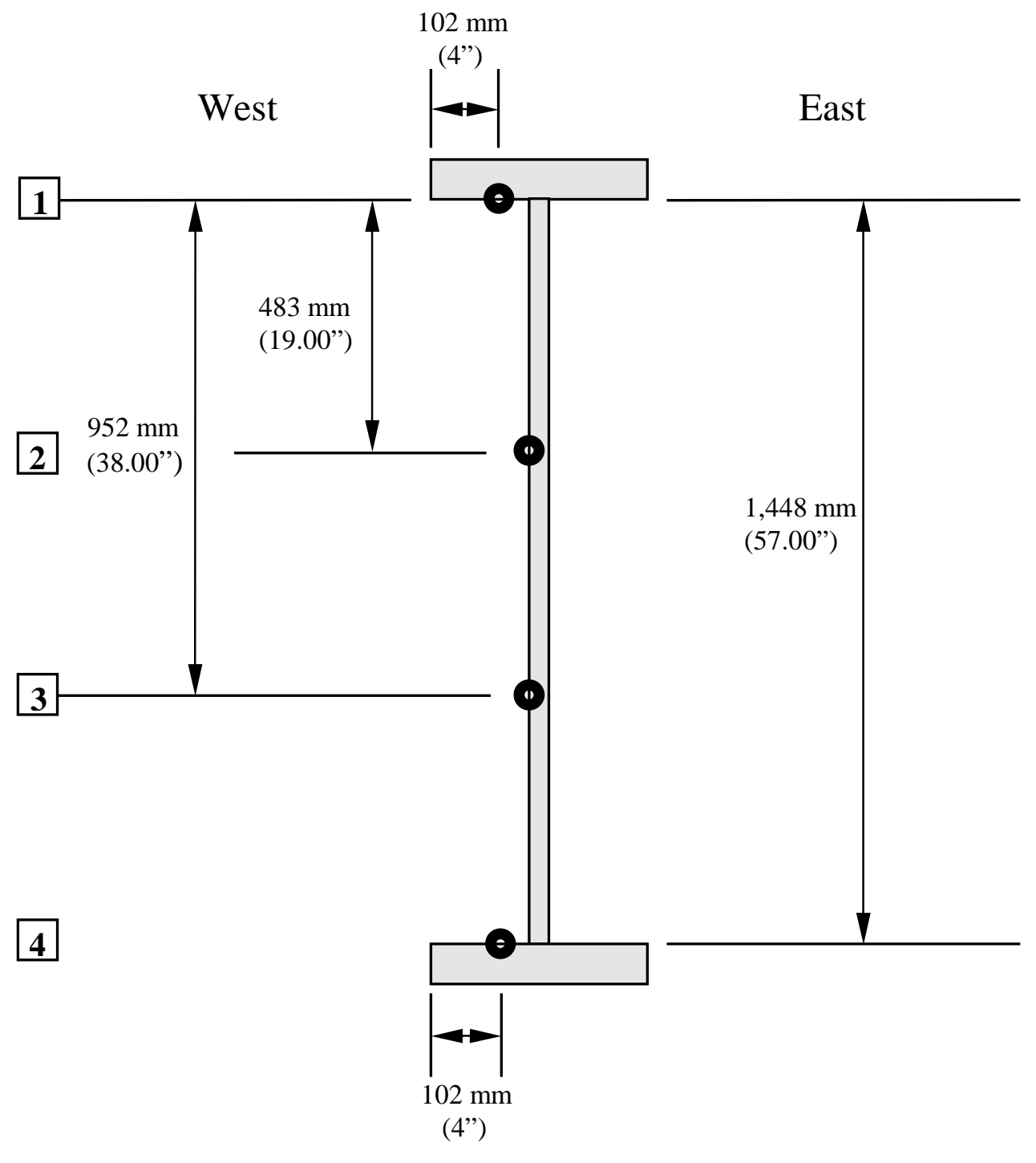

(a) G1 1 SXX, G1 2 SXX, G1 5 SXX, G1 6 SXX; $\mathrm{XX}=\mathrm{W} 1, \mathrm{~W} 2, \mathrm{~W} 3, \mathrm{~W} 4$

Figure 3.4 Positions of strain gages on steel plate girder. 


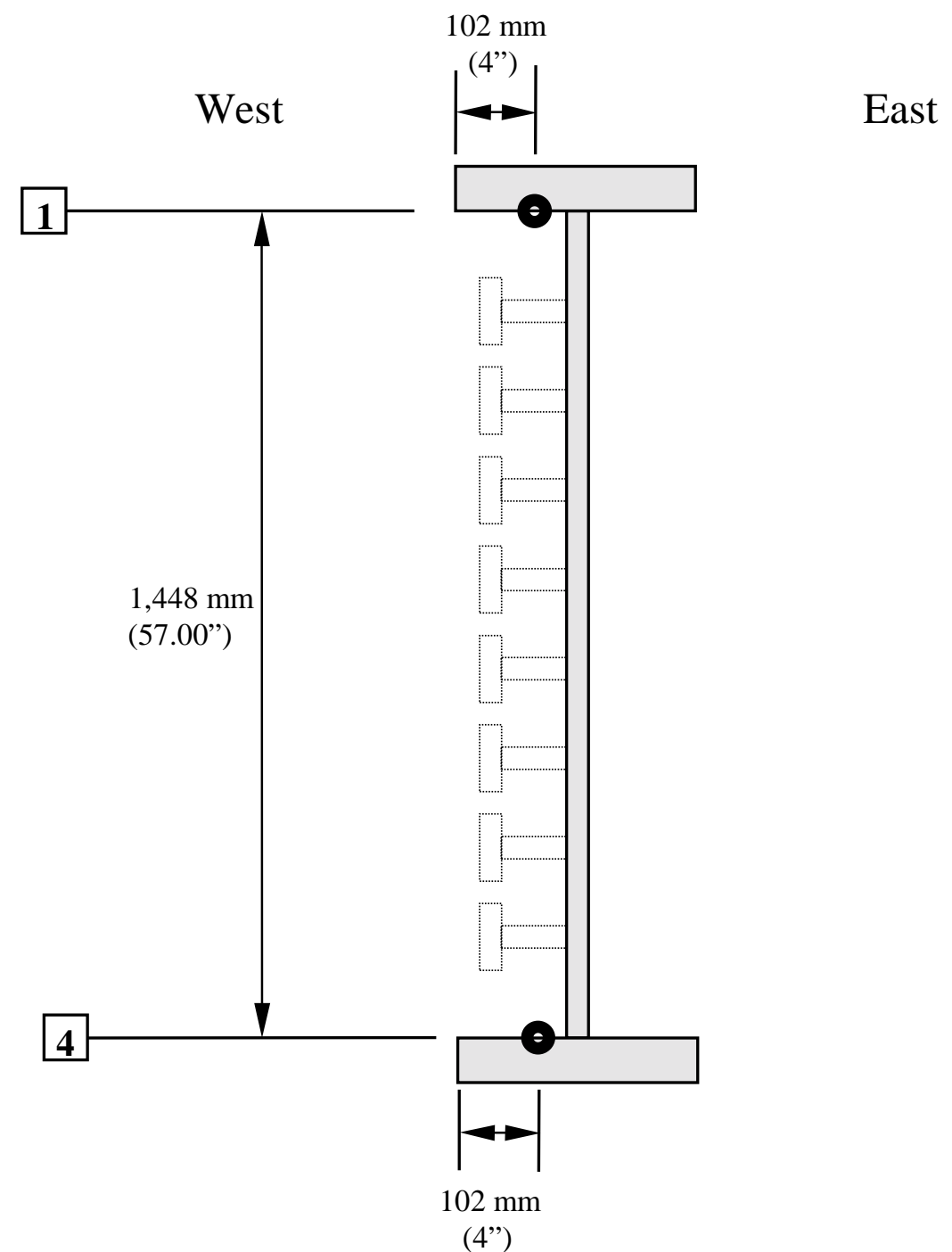

(b) G1 4 SXX; XX = W1, W4

Figure 3.4 Positions of strain gages on steel plate girder. 


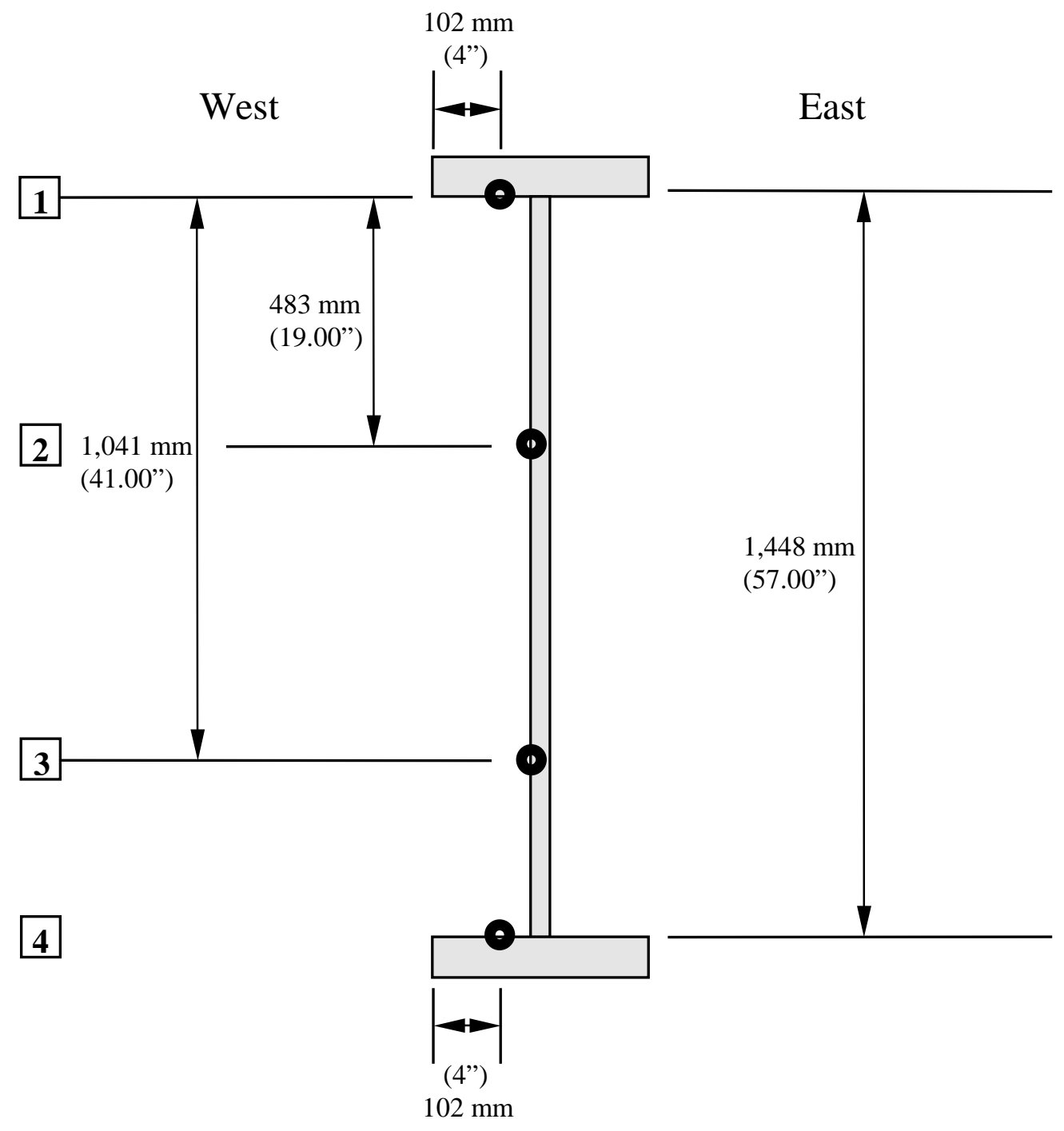

(c) G1 $7 \mathrm{SXX}$; XX = W1, W2, W3, W4

Figure 3.4 Positions of strain gages on steel plate girder. 


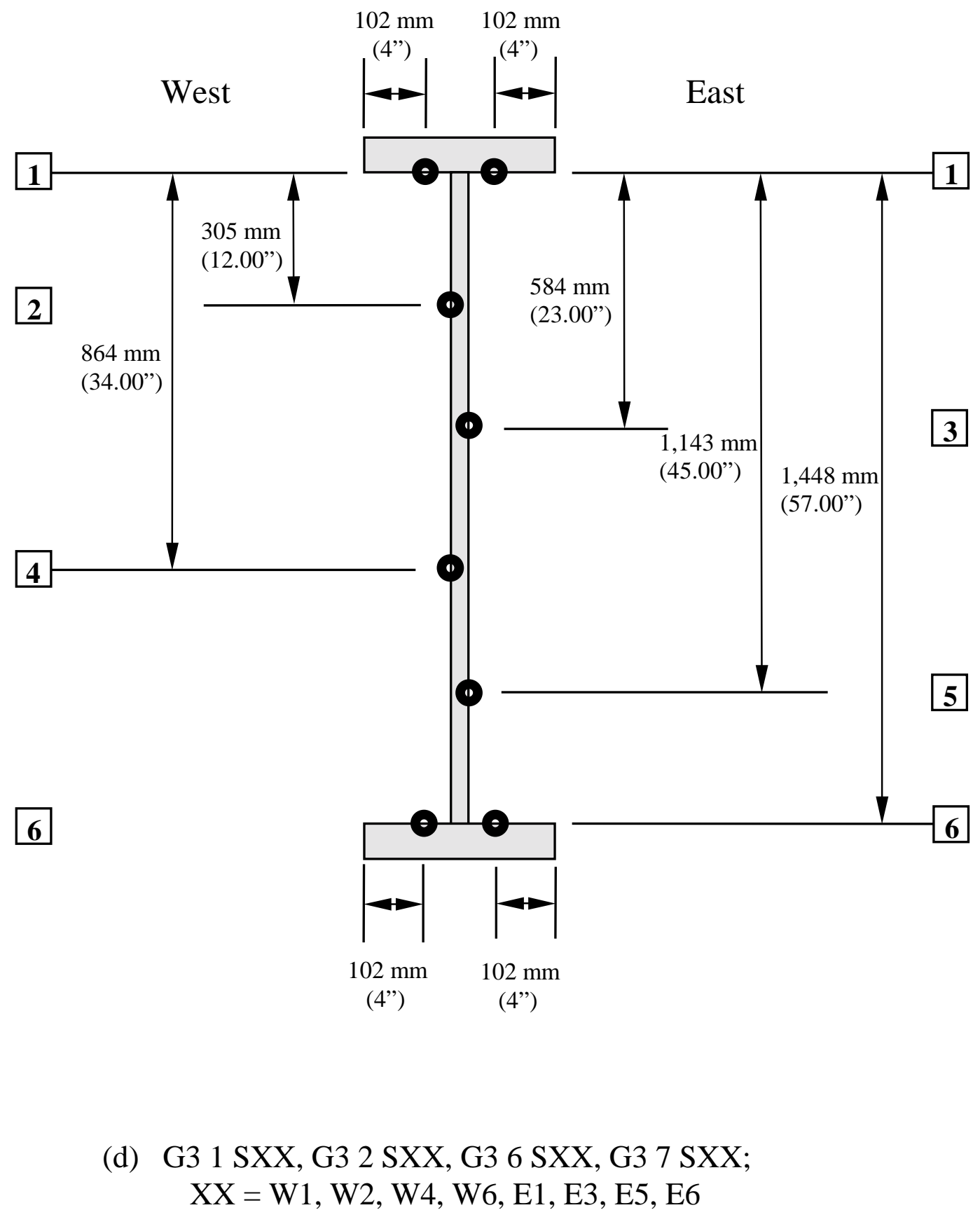

Figure 3.4 Positions of strain gages on steel plate girder. 


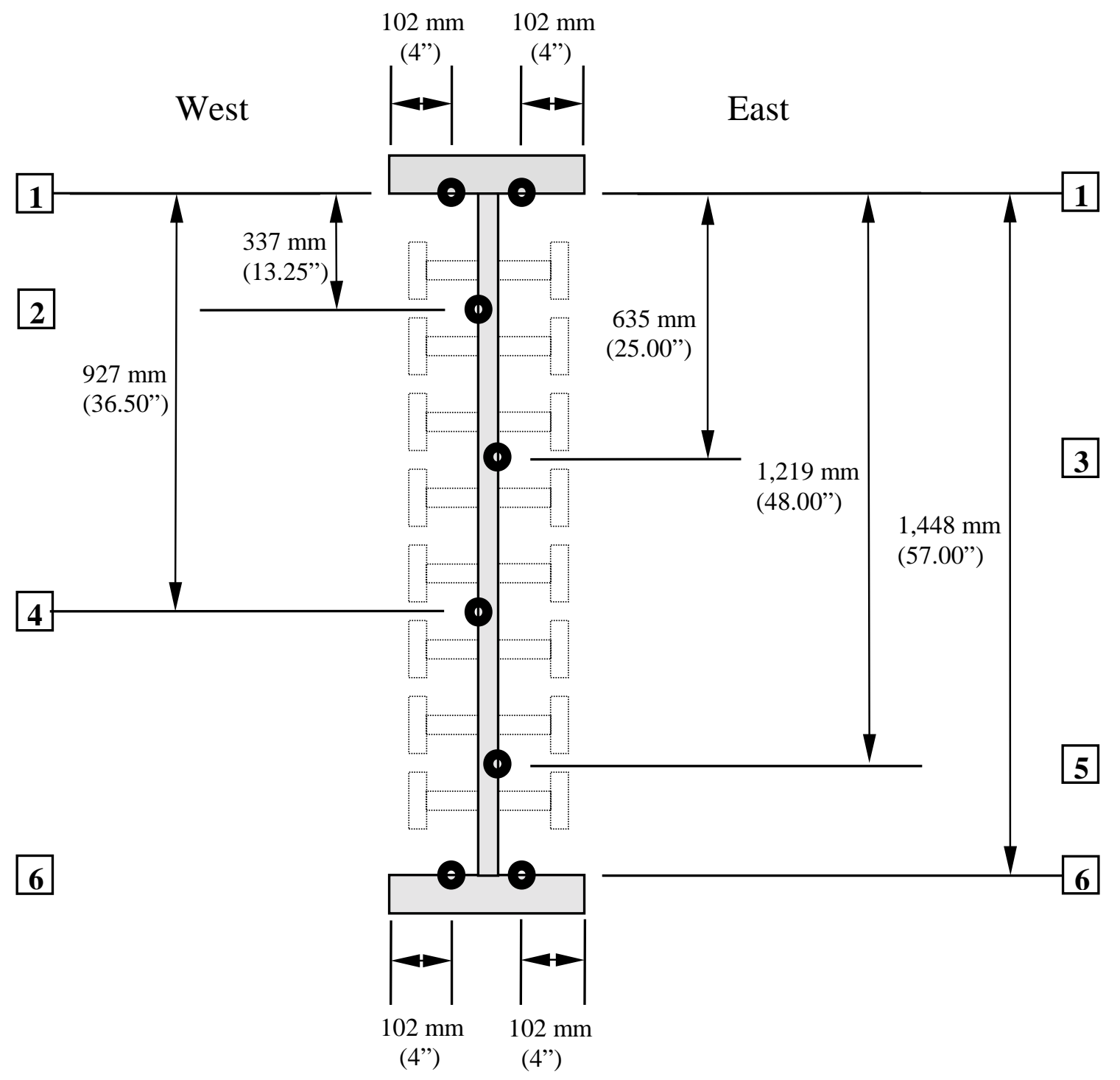

(e) G3 5 SXX; XX = W1, W2, W4, W6, E1, E3, E5, E6

Figure 3.4 Positions of strain gages on steel plate girder. 


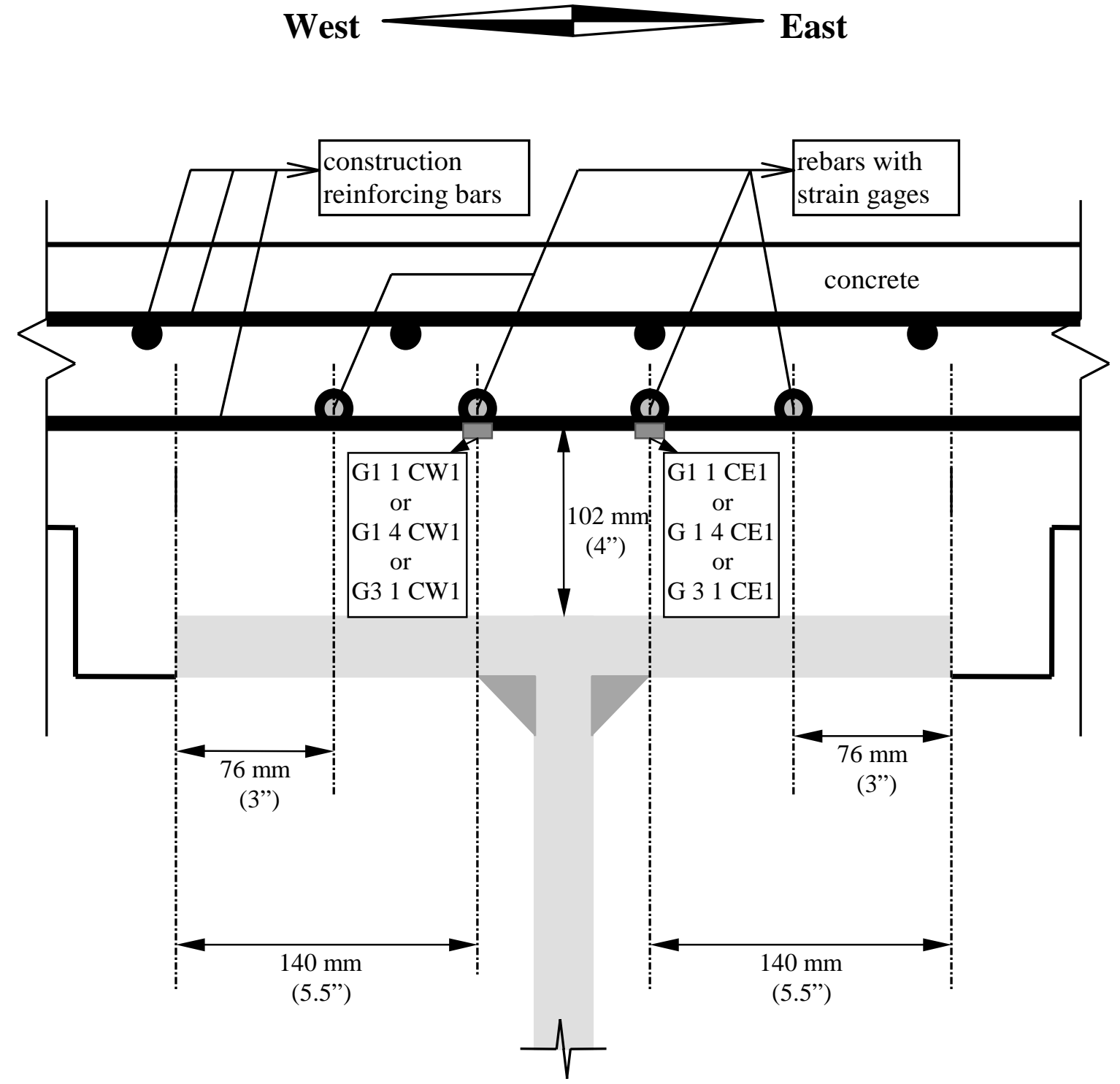

(a) G1 1 CE1 \& G1 1 CW1, G1 4 CE1 \& G1 4 CW 1 and G3 1 CE1 \& G3 1 CW 1

Figure 3.5 Positions of strain gages in concrete deck. 


\section{West East}

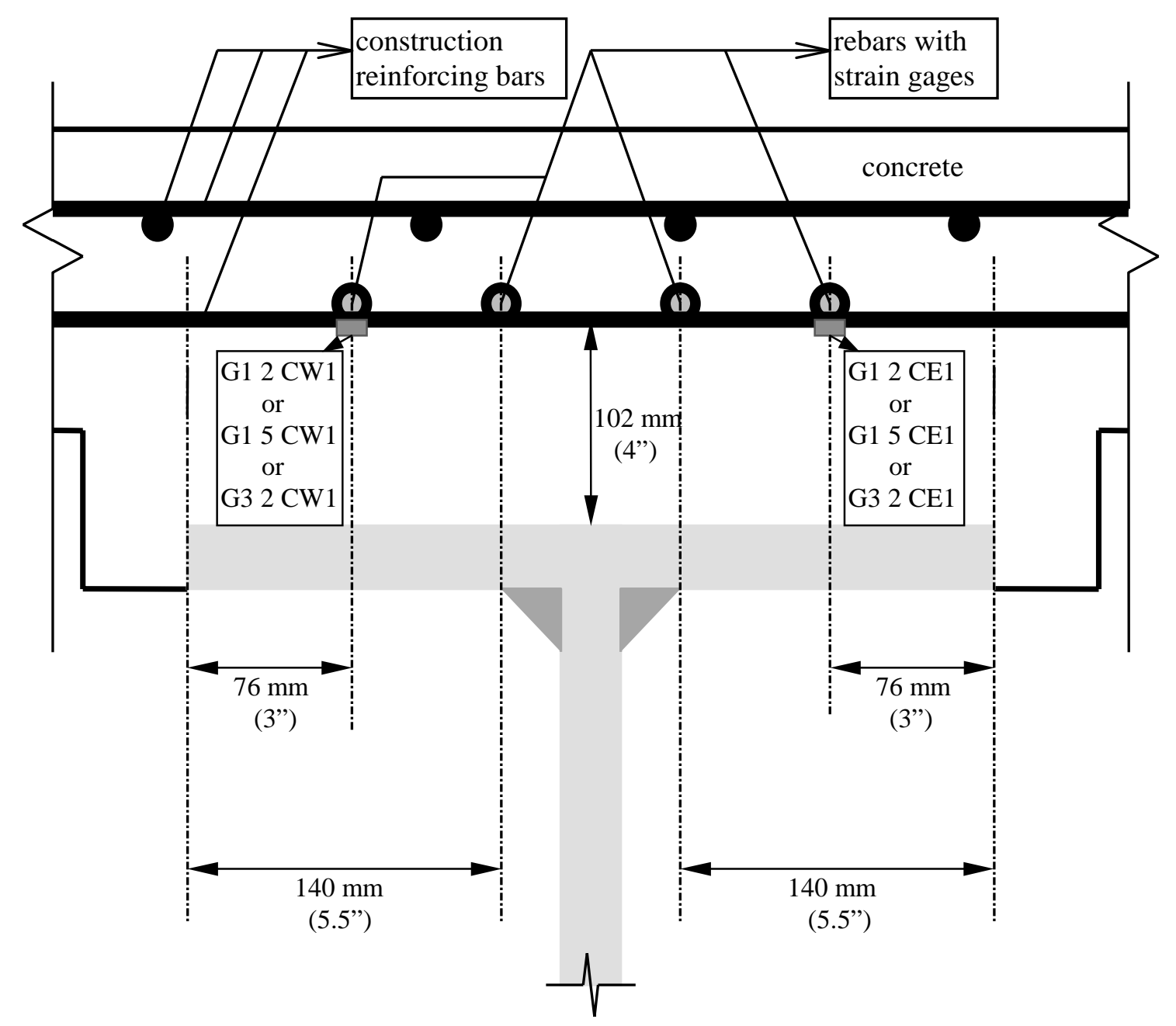

(b) G1 2 CE1 \& G1 2 CW1, G1 5 CE1 \& G1 5 CW1 and G3 2 CE1 \& G3 2 CW1

Figure 3.5 Positions of strain gages in concrete deck. 

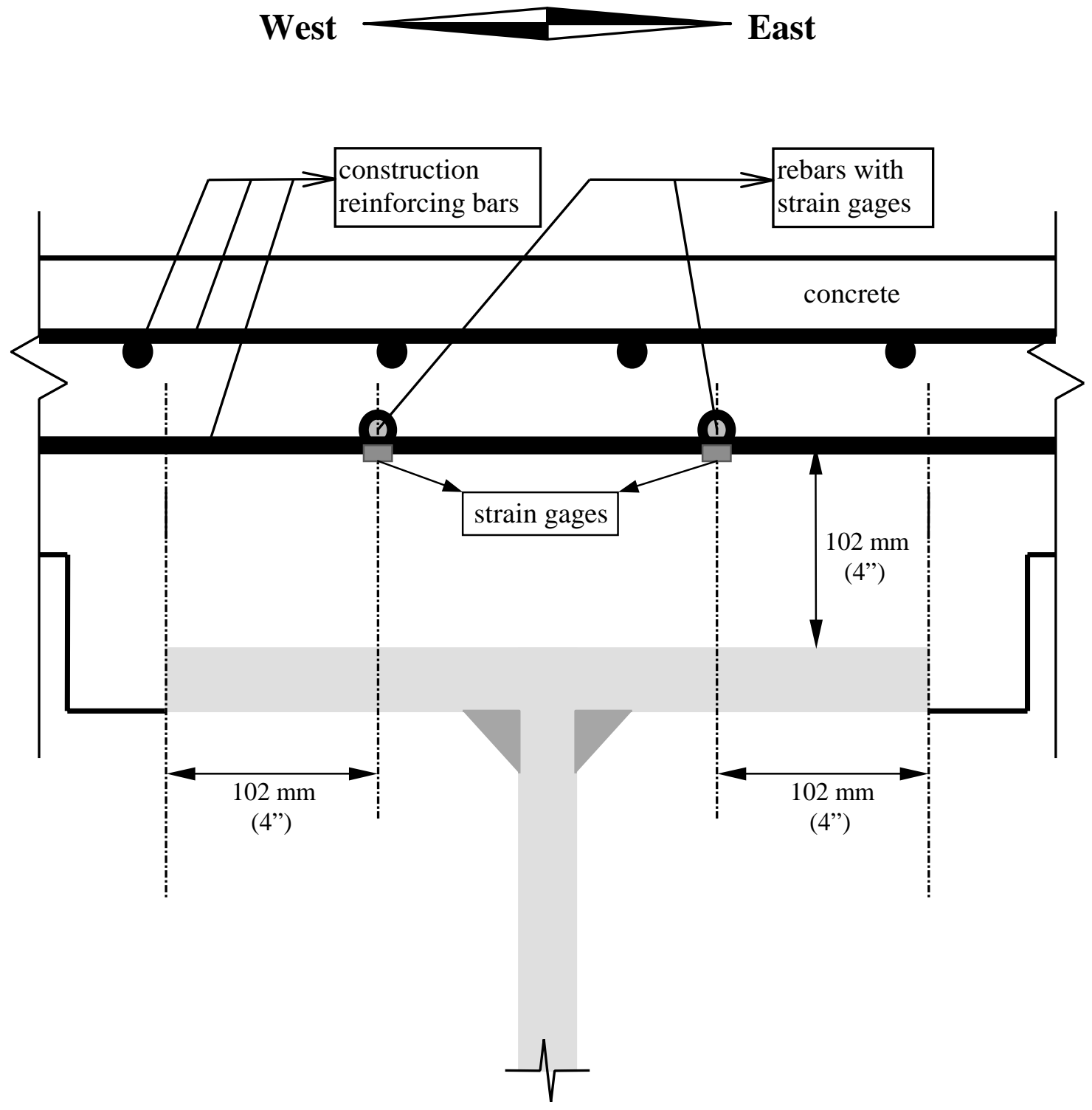

(c) G1 6 CX1, G1 7 CX1, G3 5 CX1, G3 6 CX1, G3 7 CX1

Figure 3.5 Positions of strain gages in concrete deck. 


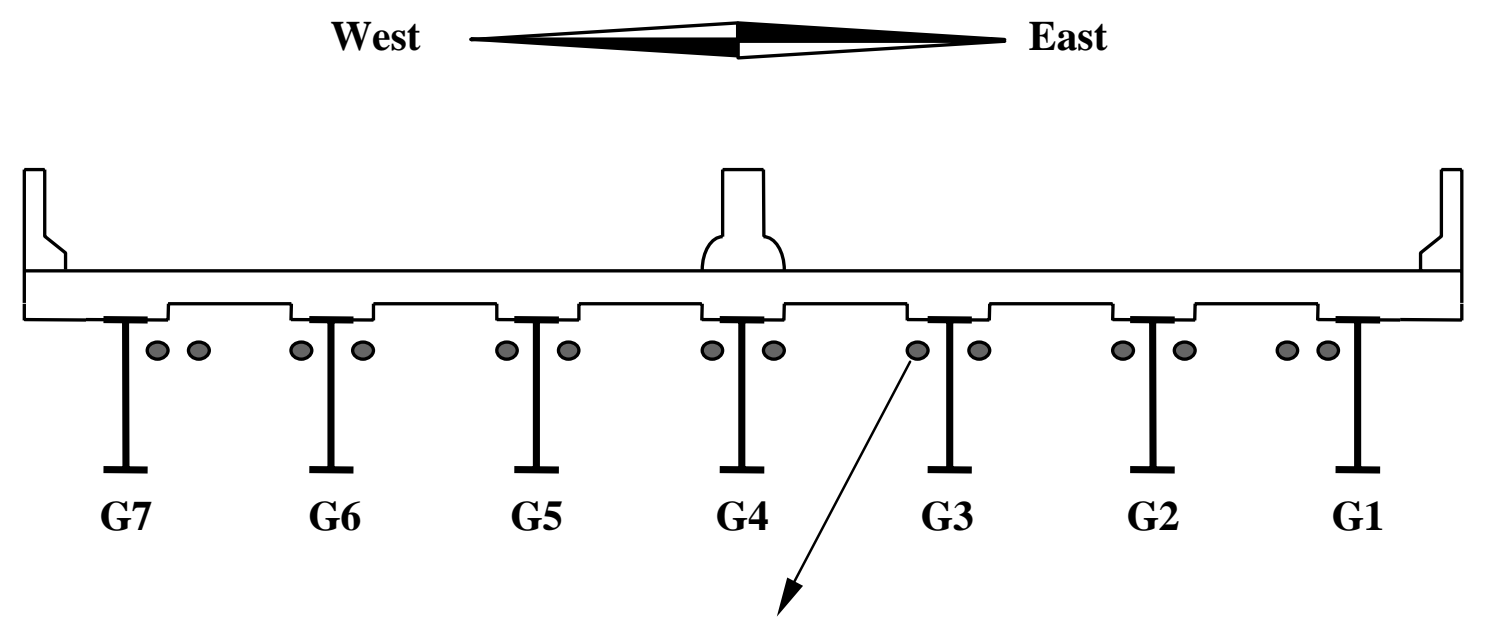

the tendon installed with Vibrating Wire Strandmeters

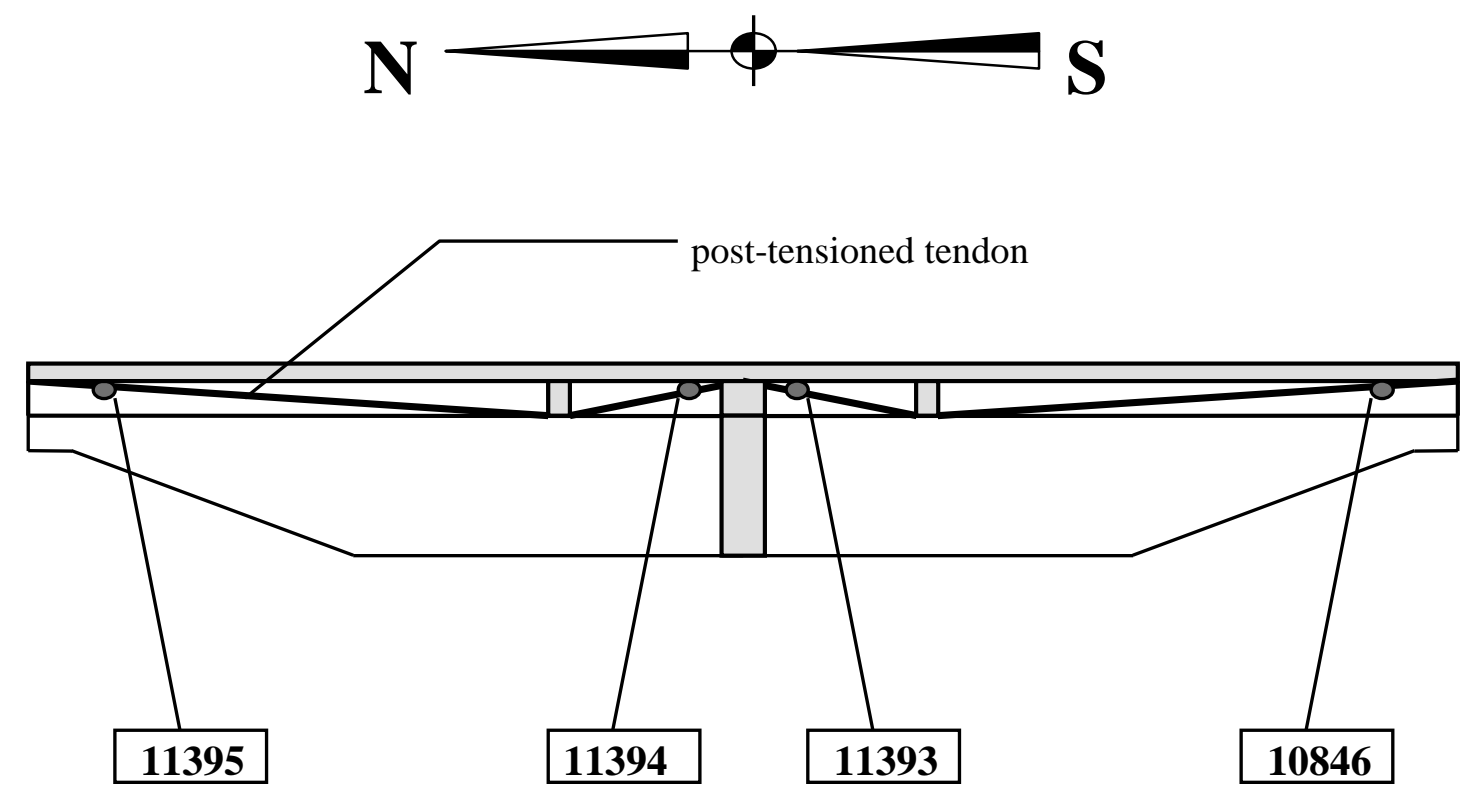

Figure 3.6 Locations of vibrating wire transducers. 

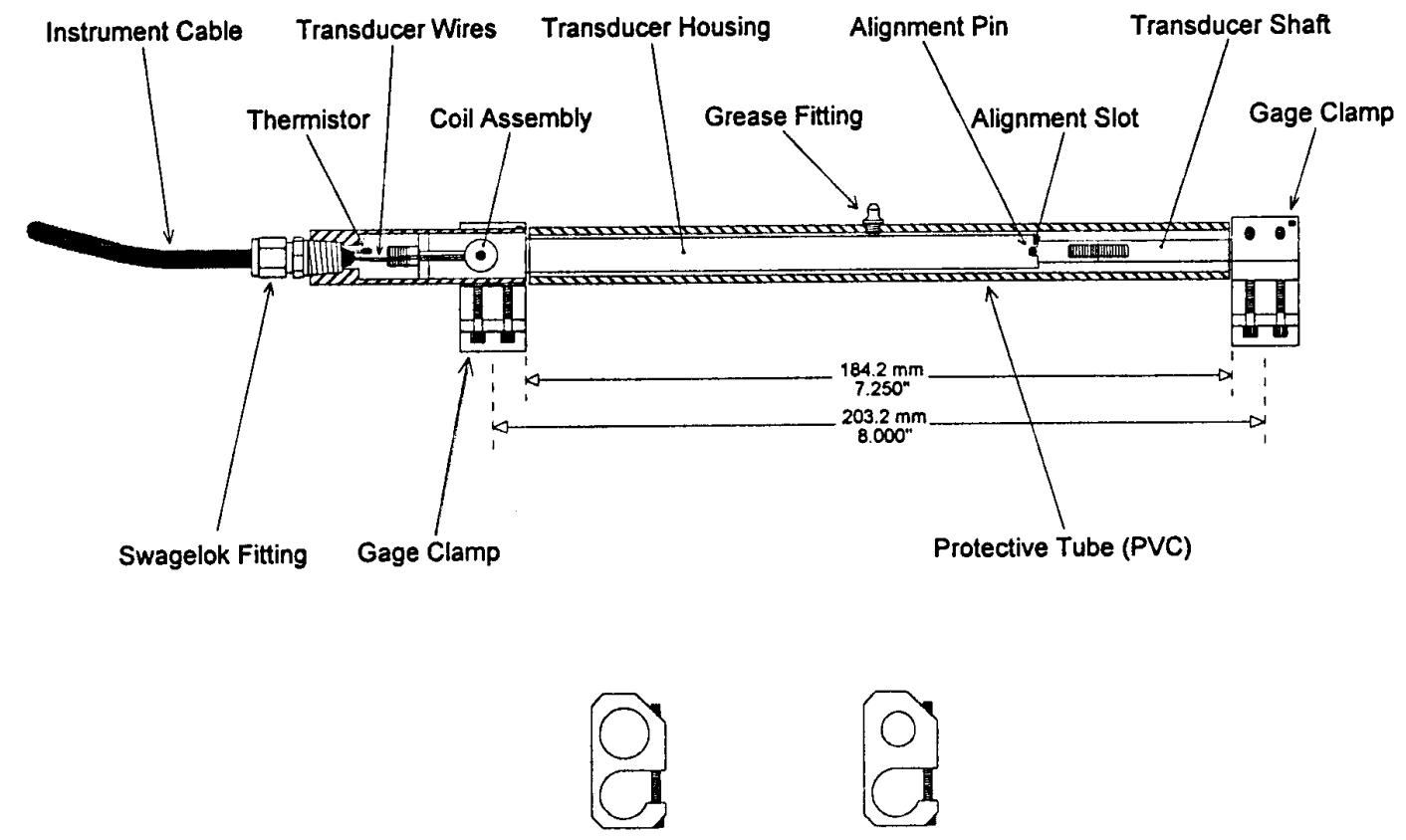

Gage Clamp (cable end)

Gage Clamp (shaft end)

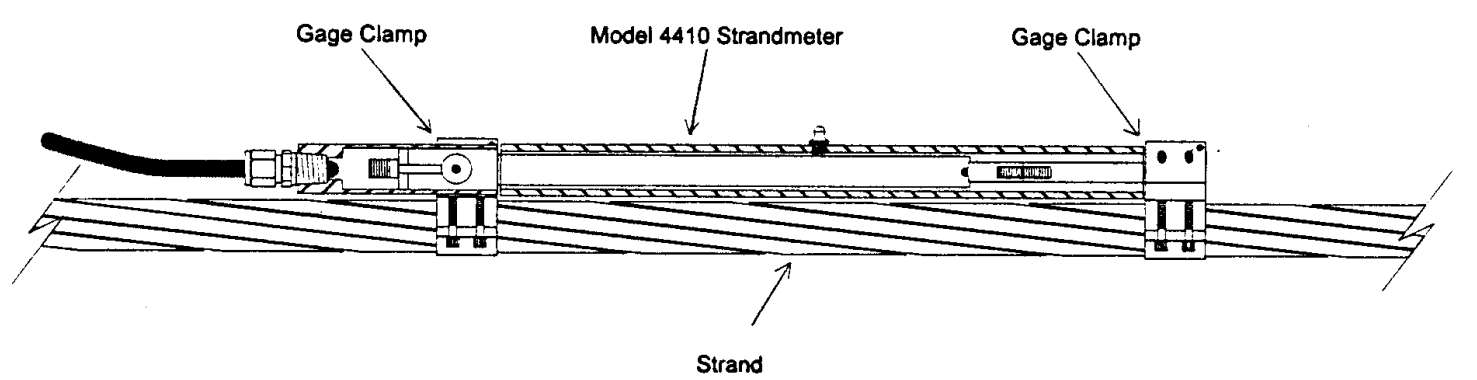

Figure 3.7 Components and installation of Model 4410 Vibrating Wire Strandmeter. 


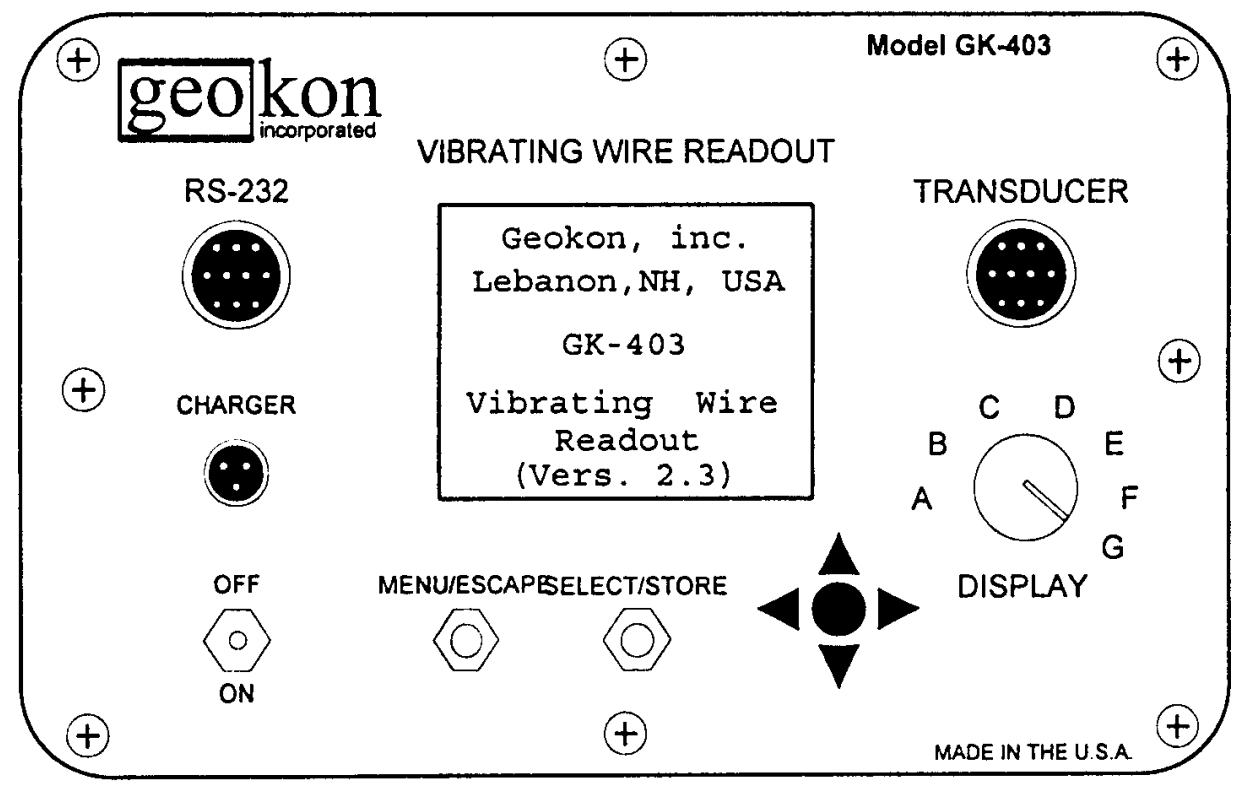

Figure 3.8 Front panel of GK-403 Vibrating Wire Readout. 


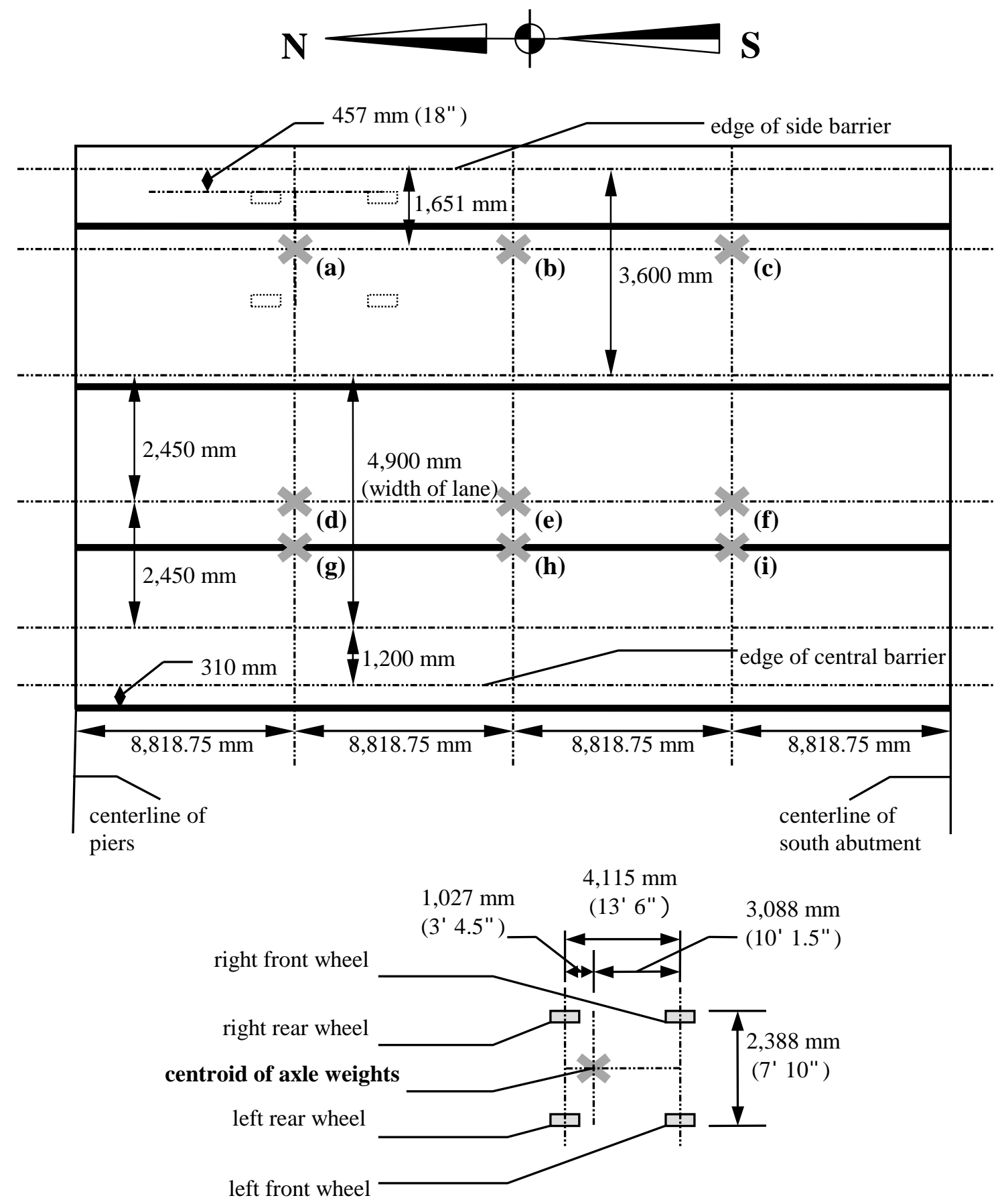

Figure 3.9 Locations for the centroids of axle weights. 


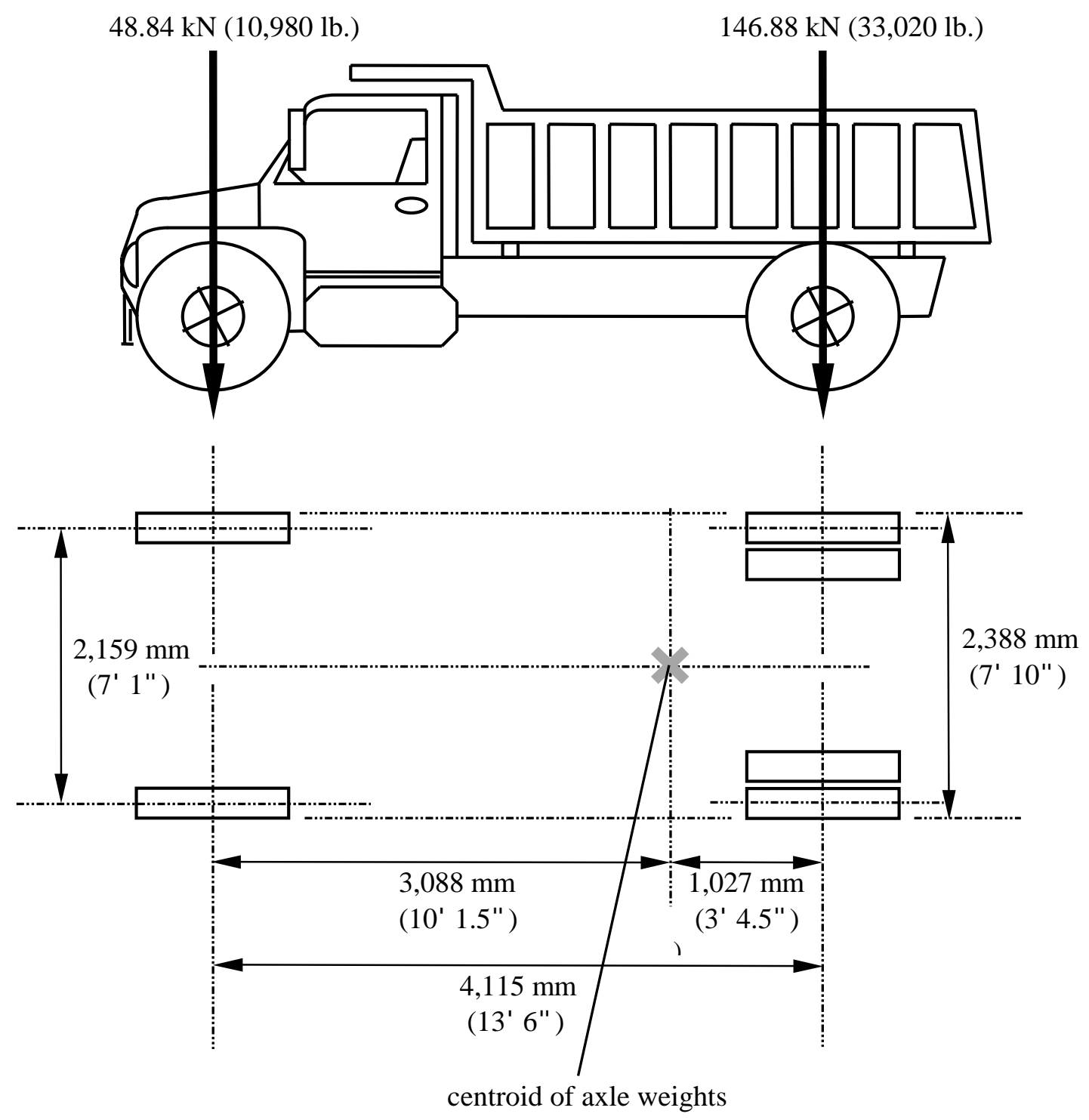

Figure 3.10 Axle weights and dimensions of dump truck. 

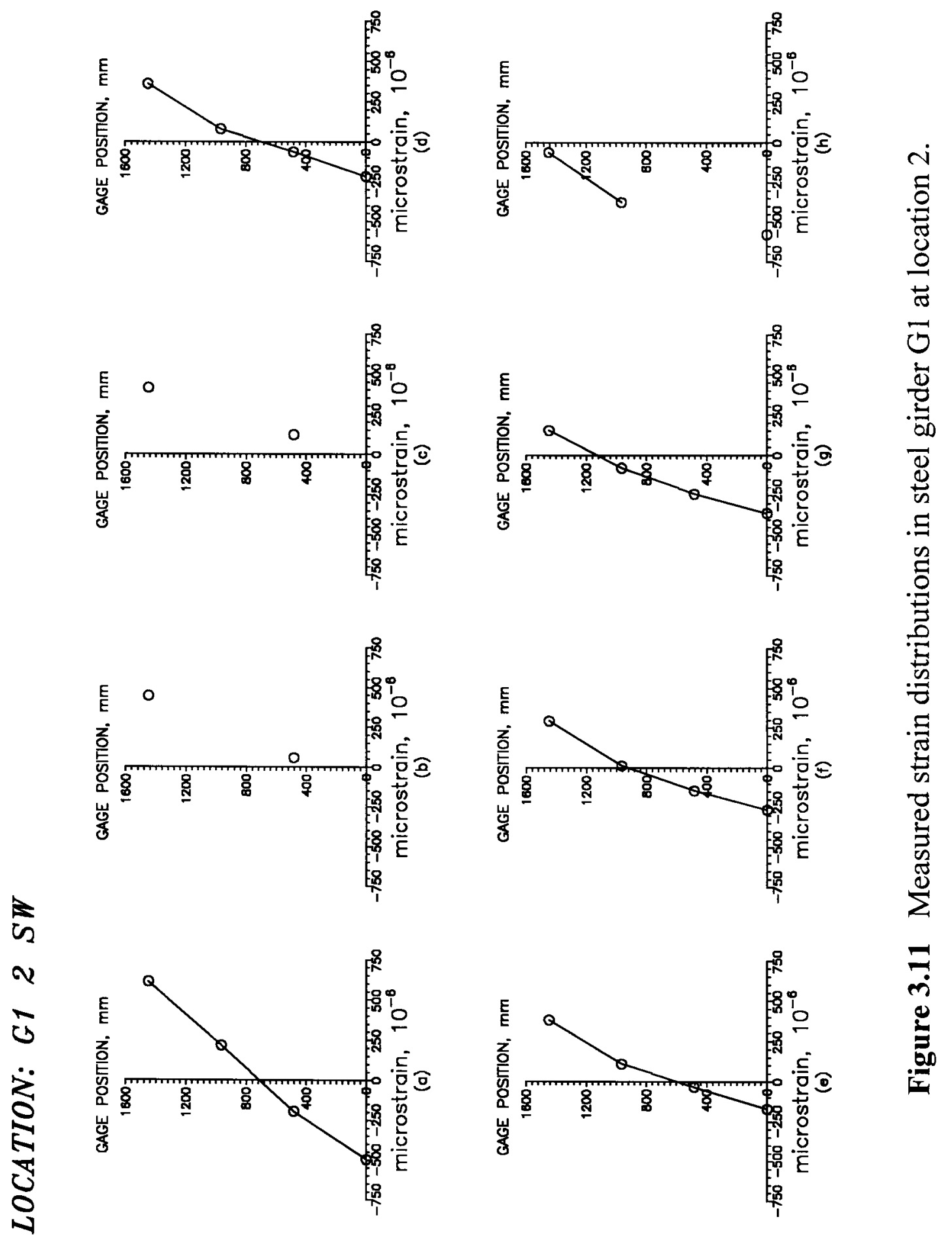

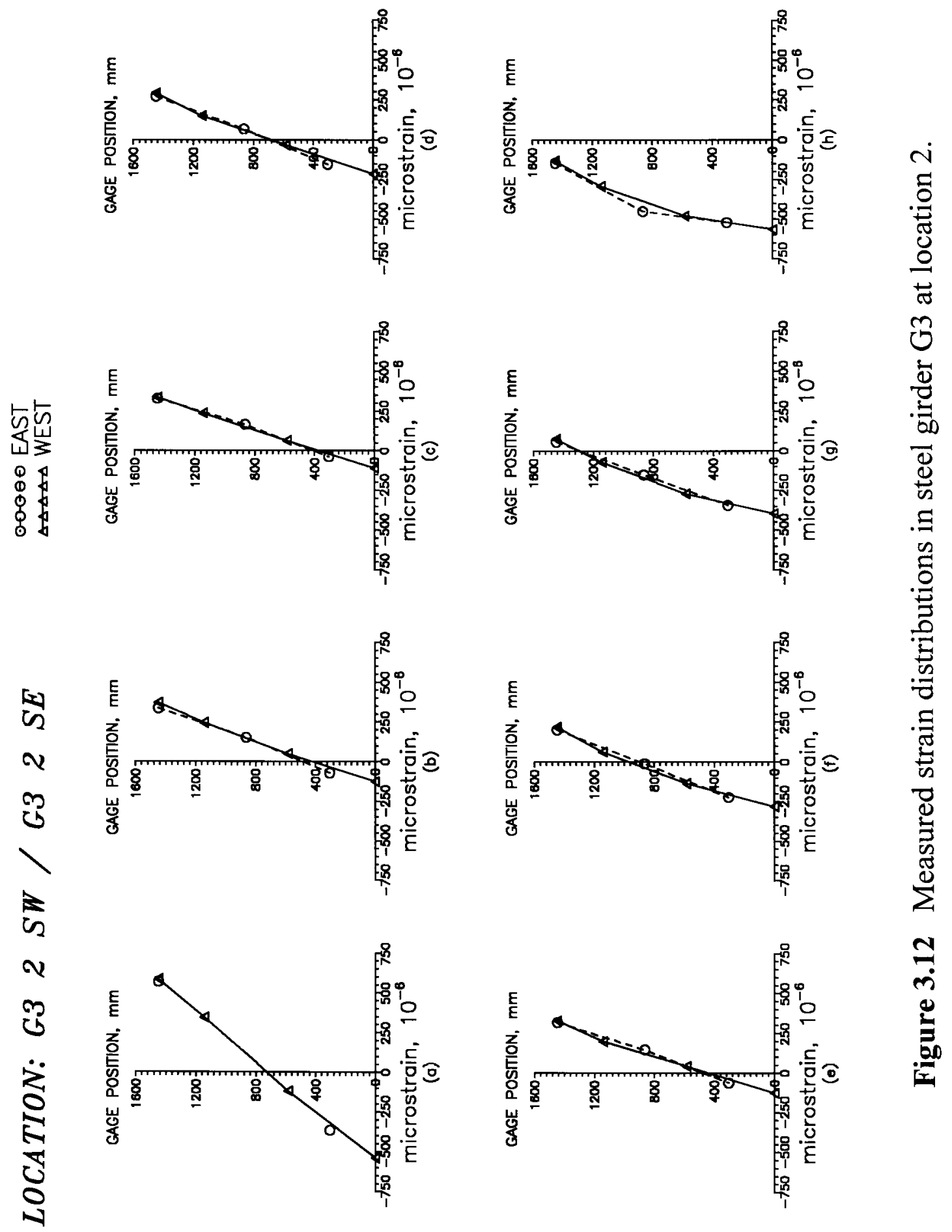

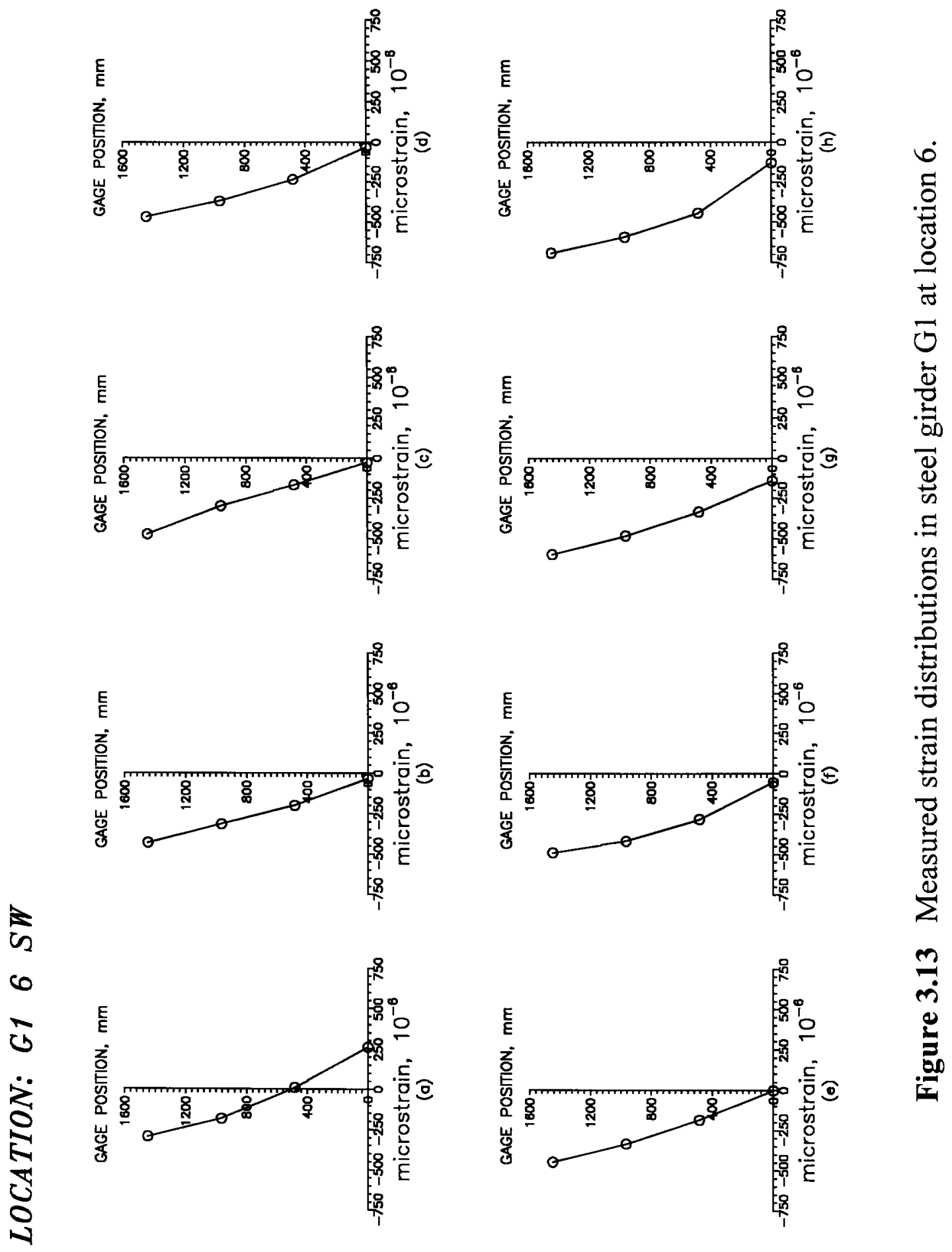

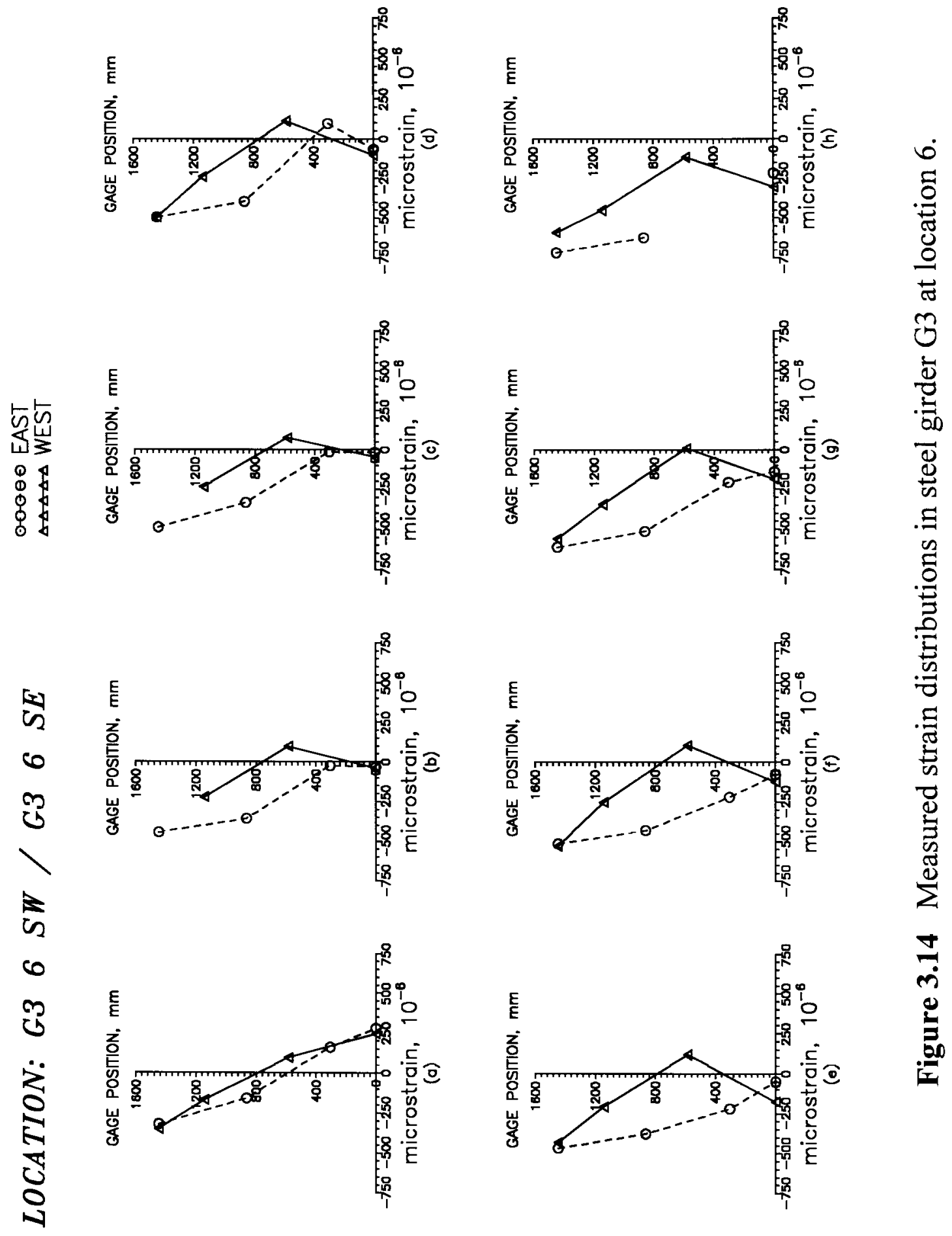

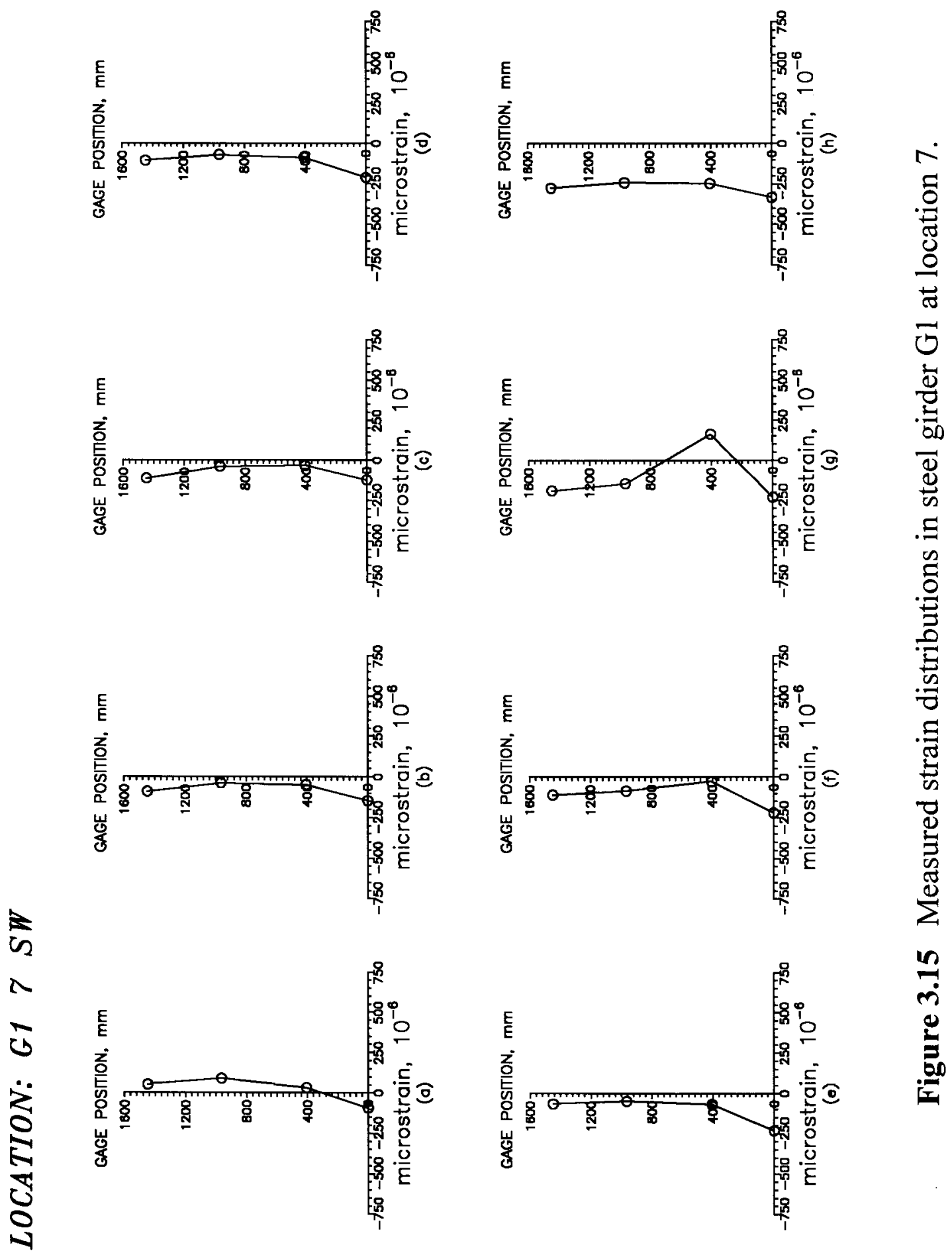

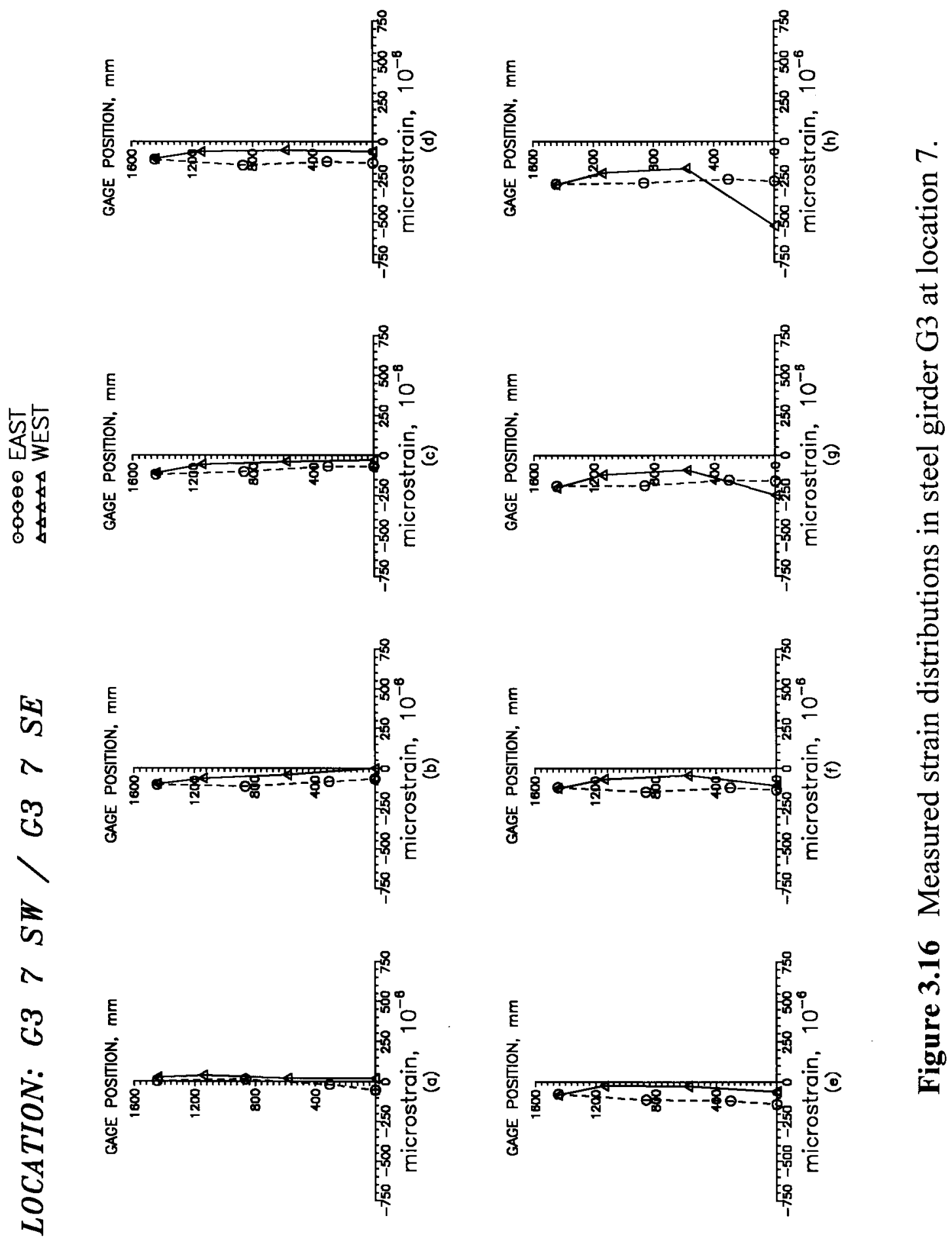

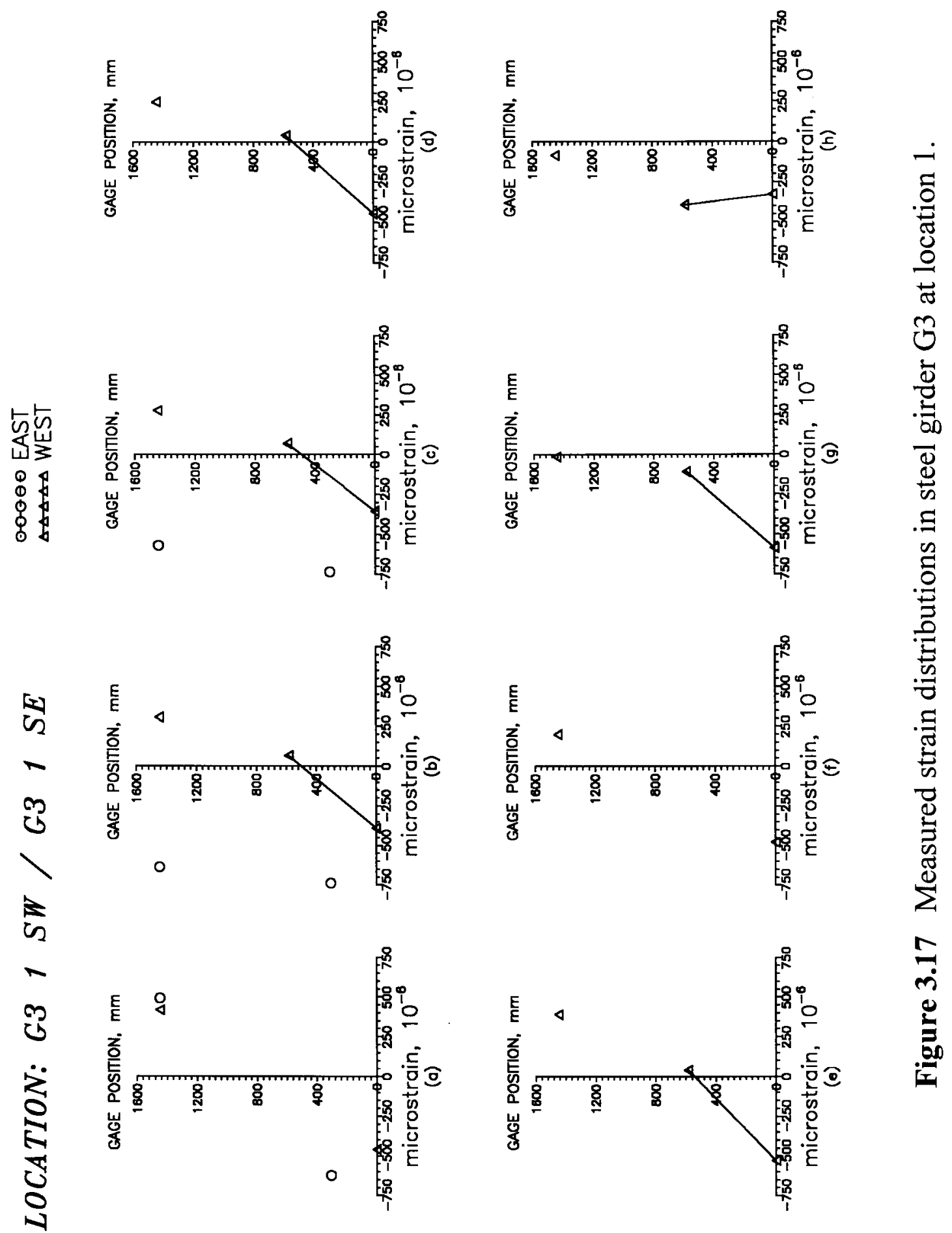


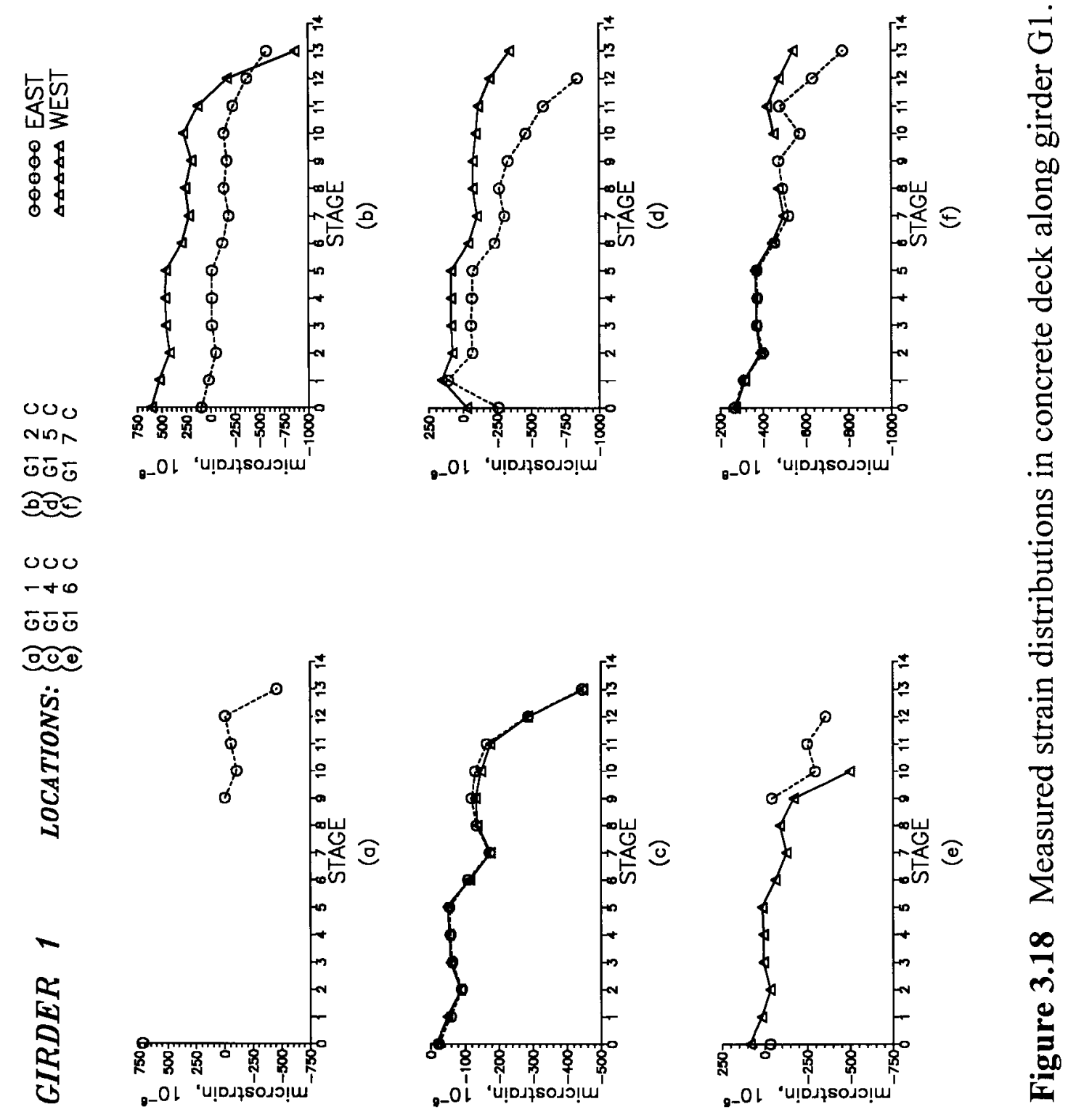



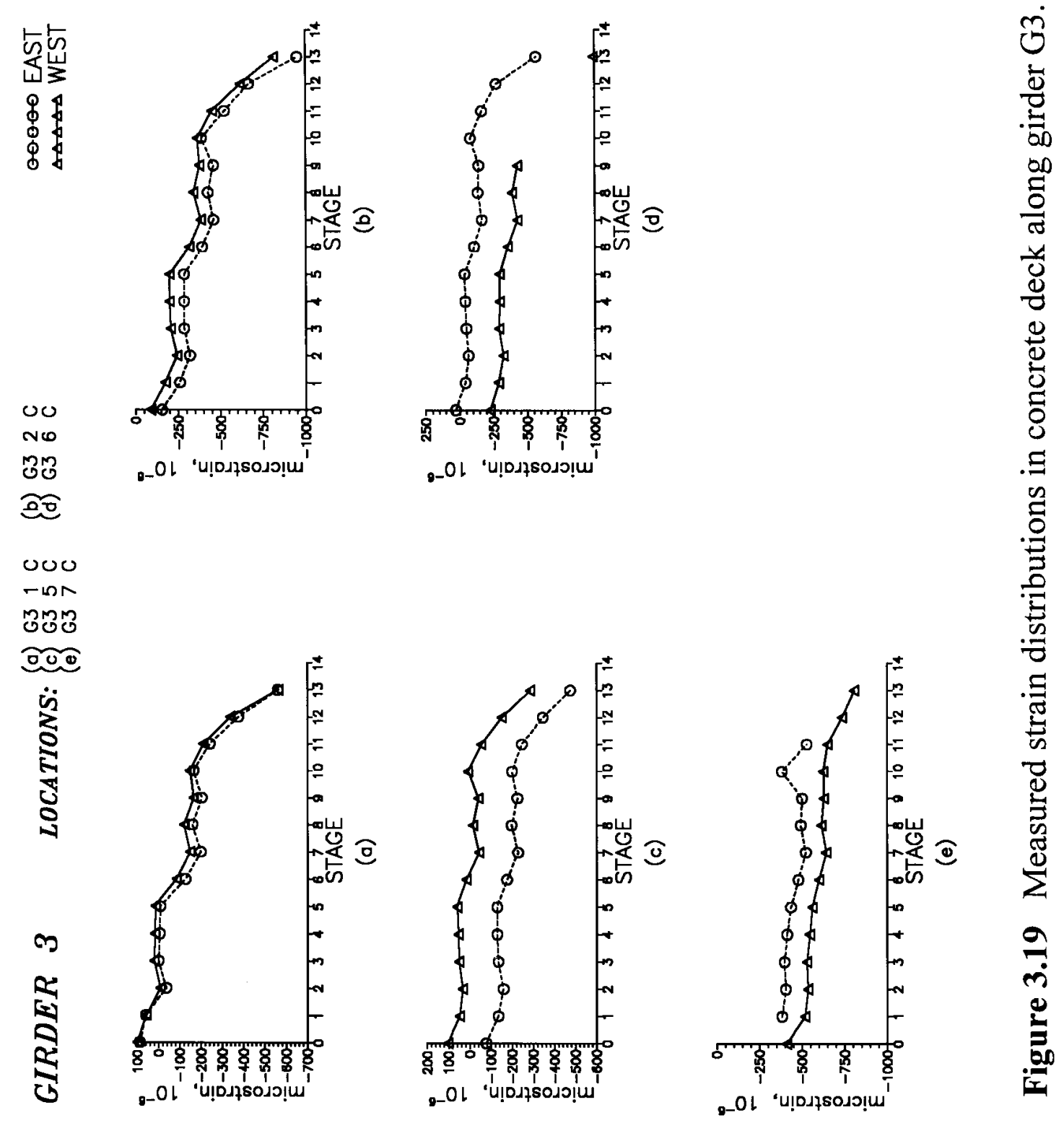

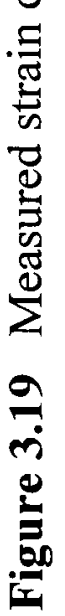




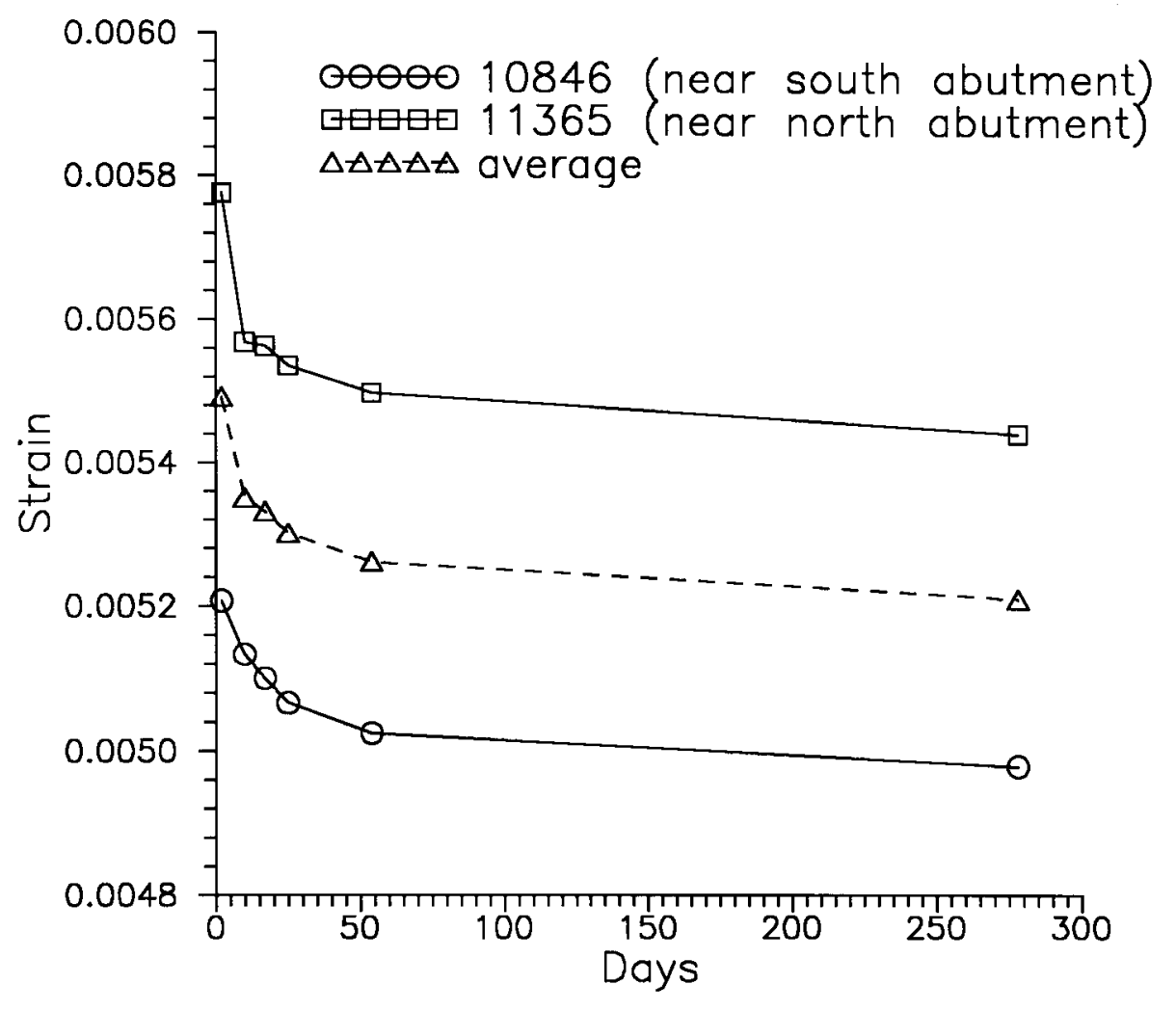

Figure 3.20 Measured strains in post-tensioning tendon. 


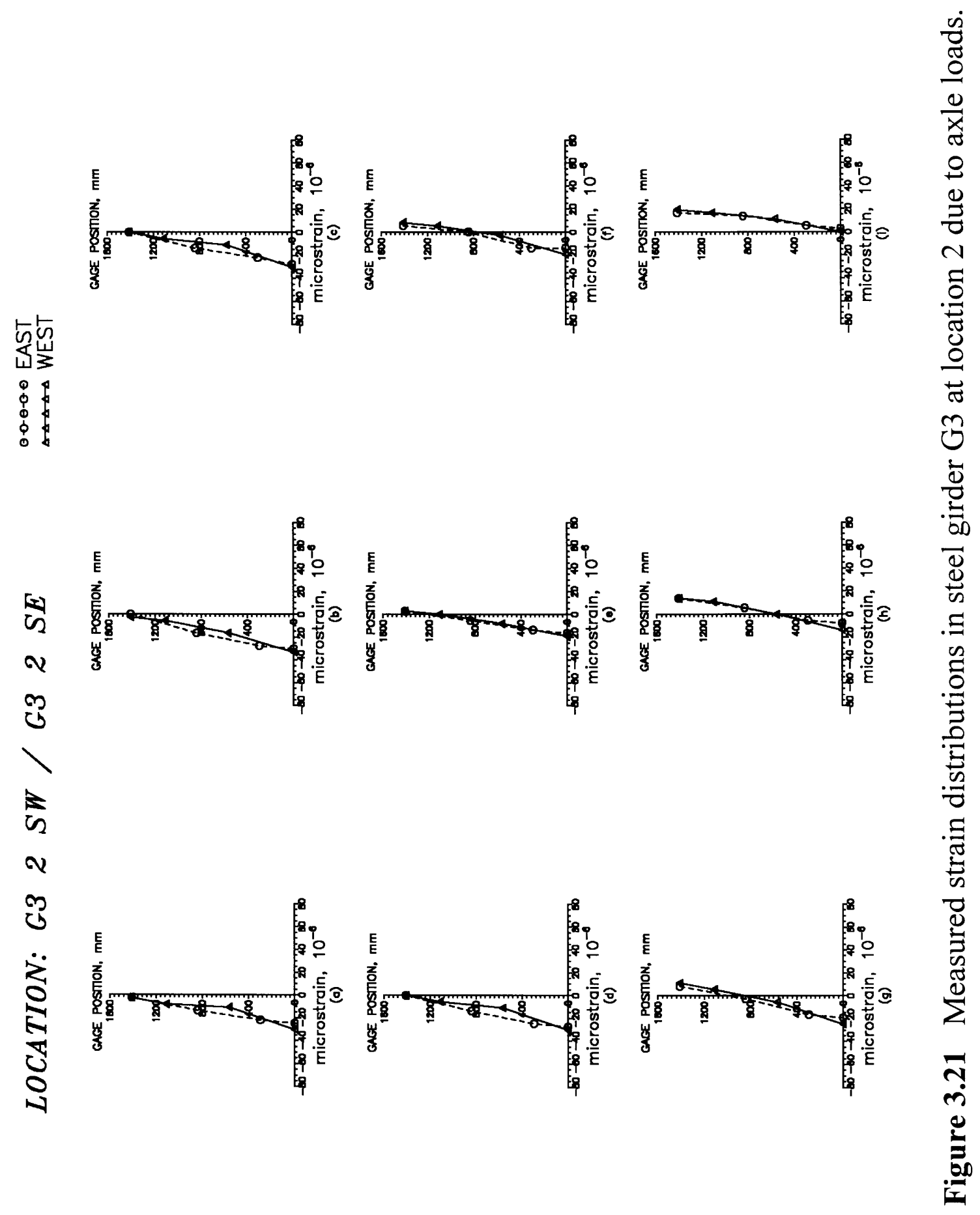




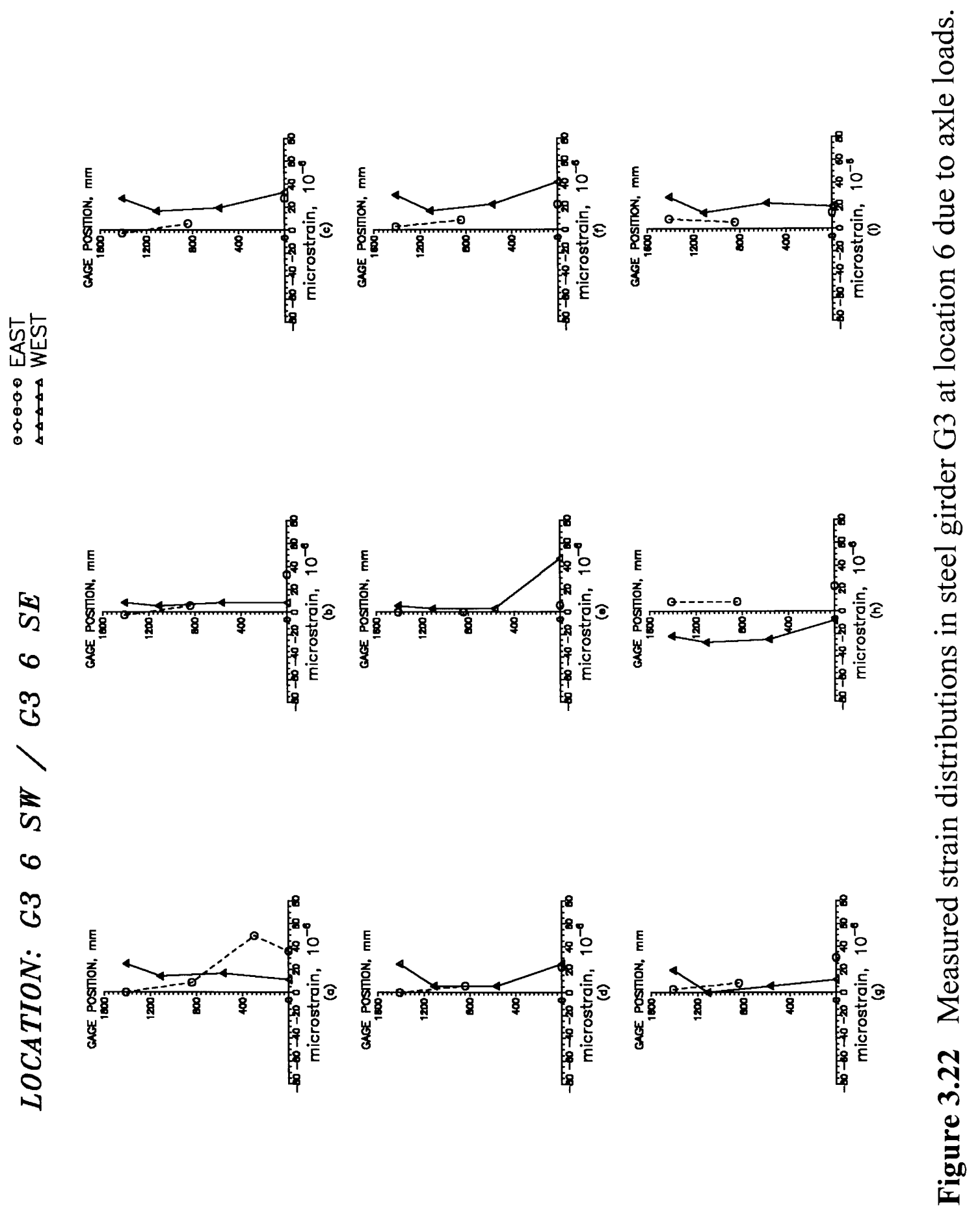




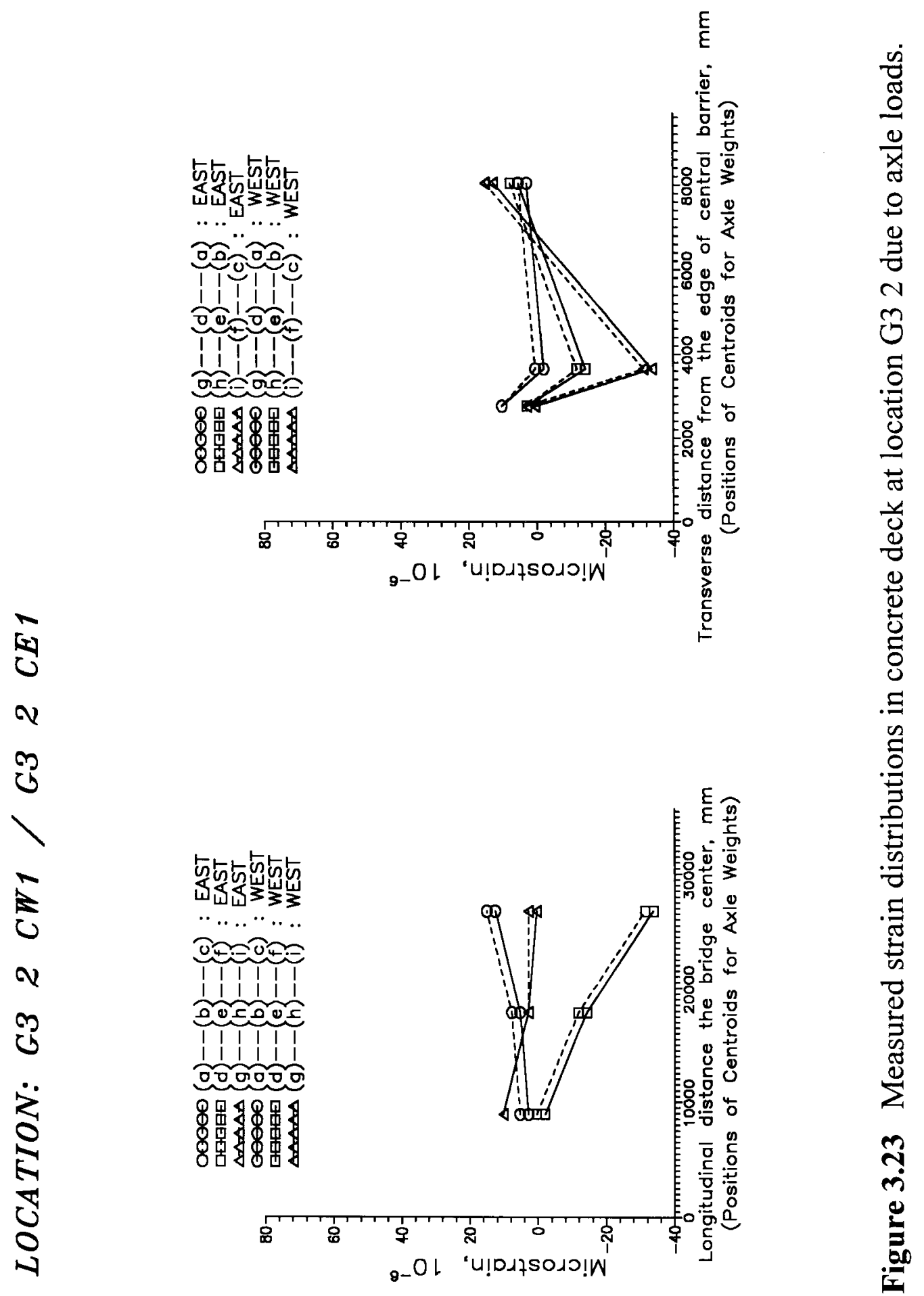




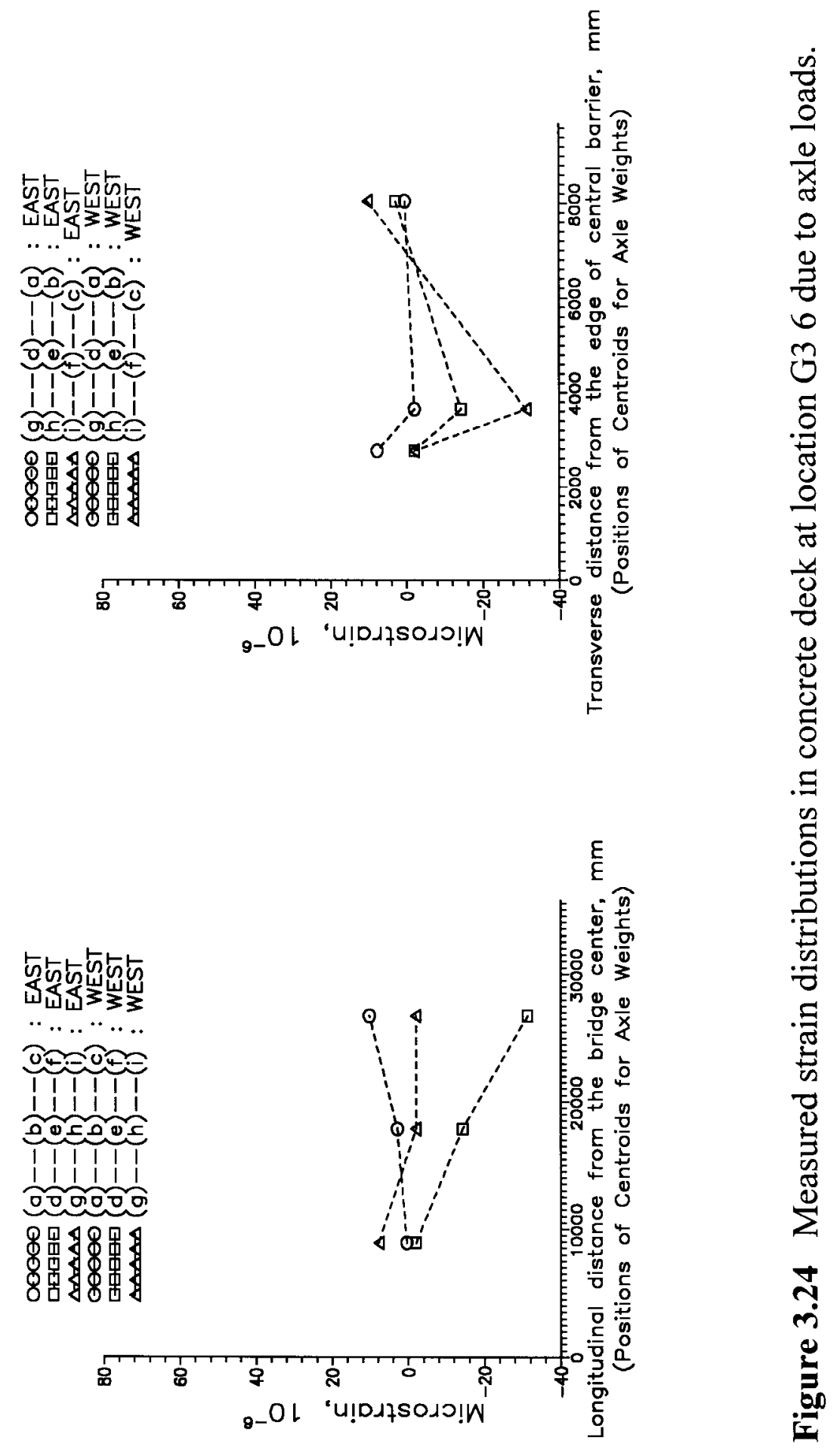




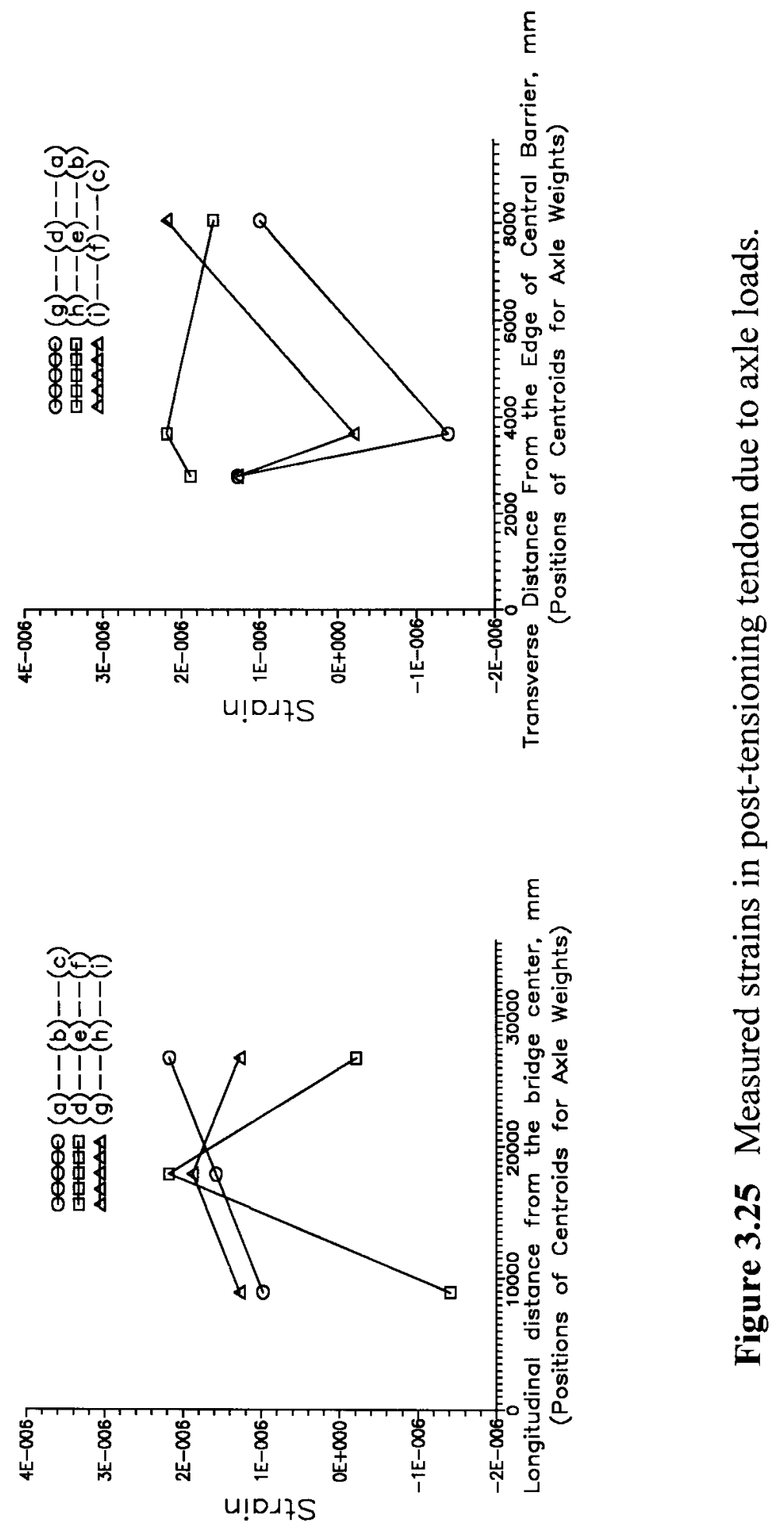




\section{CHAPTER 4}

\section{ANALYSIS OF SHORT-TERM AND THERMAL BRIDGE RESPONSE}

An analysis to investigate the short-term (instantaneous) structural behavior caused by dead load, post-tensioning force and live loads is presented in this chapter. Evaluation of thermal response due to non-uniform temperature variation in the composite section is also involved. The calculations are based upon the linear elastic assumption which is considered to be sustained in this case of bridge design under the service loading condition. The analytical solutions are eventually compared with the test results to verify the applicability of the proposed methodology.

\subsection{Problem Statement and Basic Assumptions}

The general bridge performance due to various types of loading including dead load, live load and prestress is examined based on first-order analysis. The purpose of the analytical work is to develop a systematic analytical process that could be utilized in bridge design.

Beam elements are used in the analysis to model the structural behavior. Moreover, five simplifying assumptions are made: 
(1) Small and linear elastic deformation: The small deformation assumption implies the applicability of the principle of superposition. It greatly reduces the complexity of engineering problems but still preserves the required precision. Linear elastic material behavior is presumed because the design bridge loading will not cause inelastic deformations. The virtual work principle, associated with the compatibility postulate, can be used to estimate the increment in tendon force due to loads applied after post-tensioning based on the assumption of linear elasticity.

(2) Bending planes remain plane: The classical beam theory for bending is applied. It is assumed that no warping deformation exists along the cross section of the beam.

(3) Negligence of shear deformation: Shear deformation along the beam section is assumed to have a very small effect on the calculation of flexural response. In using energy method to evaluate the tendon force increment, the strain energy due to shear strain is neglected.

(4) Fully composite action: The shear studs are effective in mobilizing composite behavior and, thereby, preventing relative movements at the level of the interface between concrete and steel after the concrete is hardened. Discontinuity of strain on the contact surface due to relative slide is not considered in the computation.

(5) Uniform tendon force: This assumption reflects the fact that the post-tensioning tendons are not bonded to the surrounding concrete or other structural components. The resultant strain or stress in the tendon is not identical to that which exists at the same level in steel girder or concrete deck, as would be the case for fully bonded tendons. The resultant tendon force, as well as the post-tensioning force, tend to be 
fairly constant along the entire length even though some friction forces are expected at drape points because of the changes of the direction of tendon profile.

(6) Uncracked concrete deck: Cracking in concrete may result in a significant reduction in sectional stiffness to resist flexural deformation. The possibility of the formation of concrete cracking is enhanced by the introduction of potential tensile stresses developed in the concrete element. With the introduction of initial compressive stresses caused by post-tensioning, the concrete deck should be reasonably assumed to remain uncracked. Later in this chapter this assumption is to be justified through showing that the resultant stress in the concrete deck under service loading is found to be negative everywhere with a magnitude which is much lower than that of nominal concrete strength $\left(f_{c}^{\prime}\right)$.

The concept of transformed section is adopted at certain stages of loading after the composite section becomes effective. The reinforcement in the concrete deck alters the sectional properties of the transformed section by approximately only $1.5 \%$, and therefore are ignored in calculation. With the assumption of uncracked concrete deck, the sectional properties are assumed to be constant longitudinally regardless of the local presence of concrete diaphragms.

Construction sequences and methods are taken into account in the computation of strain or stress distributions. For a post-tensioned composite steel-concrete bridge which is not supported by temporary shoring during the entire construction period (the example for the Elkhart County Bridge), the stress due to the total self weight (the steel girder and the concrete deck) is carried by the steel section, while the stresses resulting from the 
post-tensioning force, the superimposed dead load (e.g. concrete traffic barriers) and the live load (e.g. vehicles) are resisted by the composite section. Sectional properties of the transformed section are evaluated correspondingly for the composite cross section. A concise depiction of the strain distributions due to various sources of loading is shown in Fig. 4.1. Also shown are the appropriate sections used to resist the corresponding types of loading.

\subsection{Analytical Procedure}

The bridge response is categorized according to the types of loading which is either mechanical or thermal. Mechanical response results from the loading patterns including dead load, post-tensioning force, live load and superimposed dead load. Thermal response is induced by the change of temperature distribution in the structure.

\subsubsection{Mechanical Response}

Fig. 4.1 demonstrates the patterns of strain distribution along the steel and composite girder sections due to various types of loading. The first significant load applied to the bridge structure is the weight of the steel girders and the concrete deck. As aforementioned and also demonstrated in Fig 4.1, the gravity load is carried by the steel section only since no falsework was used during construction. The strain distribution for any cross section at any location along bridge length can be obtained simply through the following equation: 


$$
\varepsilon_{g}\left(x, y_{s}\right)=\frac{M_{g}(x) y_{s}}{E_{s} I_{s}}
$$

where $M_{g}$ is the bending moment at that longitudinal location due to the gravity loads of steel girder and concrete deck; $y_{s}$ is the vertical distance from the neutral axis of the steel section (positive for positions below the neutral axis and negative for positions above); $E_{s}$ is the elastic modulus of steel; and $I_{s}$ is the moment of inertia of the steel section.

The moment diagram expressed as a function of the distance away from one of the bridge end $(x)$ is denoted as $M_{g}(x)$. To determine $M_{g}(x)$, the boundary conditions must first be specified. As in the case of the Elkhart County Bridge, the girder is not actually simply supported because it is not allowed to rotate freely as a hinge in the major bending plane at either end. The end of the steel girder is designed to be cast into the abutment wall for the purpose of carrying additional moment. This feature should cause some redistribution of bending moment beyond that from the simply-supported-beam assumption. However, it is very difficult to quantify this effect precisely, since it depends upon the abutment stiffness relative to that of the bridge structure, as well as the interaction of the superstructure with the foundation.

As the concrete hardens and the compressive strength reaches an acceptable level, the post-tensioning operation is ready to be performed. In the calculation, the eccentrically applied post-tensioning force is decomposed into sets of externally applied axial compressive force, concentrated loads at the drape locations, and bending moments at drape points and bridge ends, as presented in Fig. 4.2. It should be noted that although in Fig. 4.2 the same symbol " $P$ " (representing effective prestress force) is used in expressing equivalent force and moment quantities applied along the entire length of 
bridge, the real prestress force is not constant at different drape locations due to the existence of friction. The composite steel-concrete section is converted into a pure steel section with uniform material properties, and then the sectional properties are computed according to the transformed geometry. The reinforcement in the concrete slab is neglected in computing the transformed section. Also it is assumed that the transformed section for the positive moment region (Fig. 4.1) applies along the entire bridge length since it is presumed that no cracks in concrete slab is to develop during post-tensioning..

In forming the transformed section, the modular ratio is first evaluated:

$$
n=E_{s} / E_{c}
$$

in which $E_{c}$ is the elastic modulus of concrete at the time of post-tensioning. The transverse dimension of the concrete deck, which is perpendicular to the bending plane, is shrunk by a factor equal to the modular ration $n$ to constitute a uniform steel section. The strain distribution at a given cross section is computed as follows:

$$
\varepsilon_{p}\left(x, y_{c p}\right)=\frac{[P \times m(x)] y_{c p}}{E_{s} I_{c p}}+\frac{P \times a(x)}{E_{s} A_{c p}}
$$

where $y_{c p}$ is the relative vertical distance to the position of the neutral axis of the composite section; $E_{s}$ again is the elastic modulus of steel; $I_{c p}$ and $A_{c p}$ are the moment of inertia and the sectional area of the transformed section, respectively; $P$ is the initial effective post-tensioning force (positive value); $m(x)$ and $a(x)$ are the unit moment and axial force functions resulting from a unit tendon force. A conceptual portrait of $m(x)$ and $a(\mathrm{x})$ for the Elkhart County Bridge is illustrated in Fig. 4.3. They are, however, not 
presented in exact scale. Eq. (4.3) indicates that the prestress load is resisted by both steel and concrete.

Upon the completion of post-tensioning operation, the high-strength steel tendons become part of the structure and begin to provide resistance to external loads applied afterwards. The procedure of the calculation for the response due to later applied live load or superimposed dead load is different from conventional beam analysis because of the introduction of the additional static indeterminacy, i.e. the increment in tendon force. This physical quantity can be estimated by the principle of virtual work associated with the compatibility condition. The analytical procedure is termed as flexibility method or force method, since it starts with choosing the tendon force increment $\Delta P$ as a redundant force and then formulating the compatibility equation as the following:

$$
\delta_{p}+\Delta P \delta_{1}=0
$$

where $\delta_{p}$ and $\delta_{1}$ are the amounts of changes of the total length of tendon in the direction of tendon profile due to external load and unit tendon force, respectively. With the unit moment and axial force functions developed, $\delta_{p}$ and $\delta_{1}$ can be calculated via the virtual work principle:

$$
1 \times \delta_{p}=\frac{\int_{0}^{L} M(x) \times m(x) d x}{E_{s} I_{c p}}
$$

and

$$
1 \times \delta_{1}=\frac{\int_{0}^{L} m(x) \times m(x) d x}{E_{s} I_{c p}}+\frac{1 \times 1 \times L_{t}}{E_{t} A_{t}}+\frac{\int_{0}^{L} a(x) \times a(x) d x}{E_{s} A_{c p}}
$$


where $M(x)$ is the moment due to the load externally applied after post-tensioning; and $E_{t}$, $A_{t}$ and $L_{t}$ are the elastic modulus, sectional area and total length of the high-strength steel tendon. The integration is taken over the full bridge length $L$. The substitution of Eqs. (4.5) and (4.6) into Eq. (4.4) gives

$$
\Delta P=\frac{-\frac{\int_{0}^{L}[M(x) \times m(x)] d x}{L_{t}}}{\frac{\int_{0}^{L} m^{2}(x) d x}{L_{t}}+\frac{E_{s} I_{c p}}{E_{t} A_{t}}+\frac{\left[\int_{0}^{L} a^{2}(x) d x\right] \times I_{c p}}{L_{t} A_{c p}}}
$$

Eq. (4.7) is a general expression which can be applied to continuous steel-concrete composite girders with an arbitrary tendon profile, as long as $m(x)$ and $a(x)$ are obtained and elastic deformation is assured. It should be noted that according to Eq. (4.7), the elongation (stretching) of tendon gives a positive value of $\Delta P$. The resulting strain distribution in the composite section at a given location then is

$$
\varepsilon_{e}\left(x, y_{c p}\right)=\frac{[M(x)+\Delta P \times m(x)] y_{c p}}{E_{s} I_{c p}}+\frac{\Delta P \times a(x)}{E_{s} A_{c p}}
$$

Based on the assumption of linear elastic material properties, the strains are related to stresses by the elastic moduli of the materials. In general, the values of $I_{c p}, A_{c p}$ and $m(x)$ in Eqs. (4.5) to (4.8) are different from those in Eq. (4.3) because of the increase in the elastic modulus of concrete. Due to the same reason, for a fixed point at the same cross section, the value of $y_{c p}$ in Eq. (4.8) is also not identical to that in Eq. (4.3). 


\subsubsection{Thermal Response}

Bridge structures are subjected to complex environmental changes with time under field conditions. Among the most influential environmental factors are the ambient air temperature, solar radiation, air velocity and relative humidity. While all of these factors play some role in the performance and response of a bridge, temperature change is probably one of the most significant factors. This section will examine the response of a bridge structure to thermal fluctuations.

For a statically determinant bridge, a linear temperature change along the depth of girder section results in thermal displacement without causing thermal stresses. Thermal stresses may be induced in a statically determinant girder if the temperature variation is not linearly distributed. Such non-linear thermal stresses, sometimes referred to as eigenstresses (Ghali and Favre 1994; Collins and Mitchell 1991), are self-equilibrating across the cross-section. On the other hand, temperature change in a bridge structure with static indeterminacy, whether linear or non-linear, produces not only thermal deformation but also stresses. In many occasions the stresses resulting from temperature effect are comparable to or even larger than those caused by vehicles passing over the bridge. Due to this reason, the effect of temperature-related factors of continuous bridges, such as the Elkhart County Bridge, needs to be investigated. The effort is concentrated on developing a procedure to estimate the thermal stresses in a composite bridge section and the variations in tendon force due to thermal effect based on assumed temperature variations.

The first step to evaluate the thermal stresses in a bridge structure is to determine the distribution of temperature variation along the section. A few research has been 
conducted on the topic of temperature distributions along steel-concrete composite sections, analytically and experimentally (Emanuel and Hulsey 1978; Fu et al. 1991). Temperature variation in a bridge structure caused by ambient air temperature and solar radiation is expected to follow a diurnal cycle, or it is viewed as the cause of the expansion or contraction of bridge components relative to the state corresponding to the time of erection. In either way, consider a general non-uniform distribution of temperature change in a composite girder as depicted in Fig. 4.4. Because the concrete deck has a higher specific heat and volume-to-surface ratio than the steel girder, the nonuniform distribution of temperature change (and also temperature distribution at particular time) exhibits a higher gradient in the concrete component than in the steel element. It is recognized that the transverse temperature distribution is not necessarily uniform, especially for the exterior girder with a larger portion of surface directly exposed to the sunlight and the air. In order to reduce the complexity of the problem, the transverse non-uniformity in temperature changes in the composite section is not considered in the analysis.

The nonlinear distribution of temperature variation can be approximately replaced by two line-segments as also shown in Fig. 4.4. Furthermore, for the convenience of analysis, the bi-linear distribution is decomposed into three parts, each of which is represented by a linear function in terms of the vertical position in the composite section $y_{c p}$. Those functions include a uniform and linear distributions across the entire composite section [ $\Delta T_{1}\left(y_{c p}\right)$ and $\Delta T_{2}\left(y_{c p}\right)$ respectively], and another linear distribution in the concrete section only $\left[\Delta T_{3}\left(y_{c p}^{C}\right)\right]$. The decomposition of the original distribution and the 
determination of the magnitude of each component are demonstrated in Fig. 4.5. The distribution of temperature change across the composite section is assumed to be unaltered along the bridge length.

The intensity of the uniform distribution $\Delta T_{1}\left(y_{c p}\right)$, is taken as the temperature variation at the level of the neutral axis of the composite section, which is symbolized as $\Delta T_{N A}$. This component is the cause of uniform axial contraction or extension. The linear distribution $\Delta T_{2}\left(y_{c p}\right)$ results in changes of curvature. Its influence on the bridge deflection coexists with the existence of structural indeterminacy. The mechanical effect of the third component of thermal distribution $\Delta T_{3}\left(y_{c p}^{C}\right)$ can be regarded as an eccentric compressive or tensile force acting at the centroid of the triangular distribution, or equivalently, a uniform axial force and a constant bending moment acting on the center of the composite section (Fig. 4.6), The structural response due to $\Delta T_{3}\left(y_{c p}^{C}\right)$ depends on the restraint conditions of the bridge system.

Since the distribution of temperature change across the composite section is assumed to be continuous, discontinuity of strain distribution is therefore expected at the concrete-steel interface because of the difference in the coefficients of thermal expansion of the two different materials. Nevertheless, the difference is not significant $\left(10.0 \times 10^{-6}\right.$ ${ }^{\circ} \mathrm{C}$ for normal weight concrete vs. $11.5 \times 10^{-6} /{ }^{\circ} \mathrm{C}$ for structural steel). It is hence reasonable to assign an identical value for the coefficient of thermal expansion for both materials in analysis to avoid the problem of strain discontinuity.

The bi-linear curve of the non-uniform temperature variation is determined by three parameters, which are the temperature changes at the levels of the top surface of the 
concrete deck $\left(\Delta T_{T C}\right)$, the interface of the concrete deck and the steel girder $\left(\Delta T_{T S}\right)$, and the bottom of the steel girder $\left(\Delta T_{B S}\right)$. Accordingly, the factors used for further defining the decomposed components of the original distribution function, $\Delta T_{1}\left(y_{c p}\right), \Delta T_{2}\left(y_{c p}\right)$ and $\Delta T_{3}\left(y_{c p}^{C}\right)$ are correspondingly found to be

$$
\begin{gathered}
\Delta T_{N A}=\frac{\Delta T_{T S} \times h_{N A}+\Delta T_{B S} \times\left(h_{s}-h_{N A}\right)}{h_{s}} \\
\Delta T_{d 2}=\left(\Delta T_{T S}-\Delta T_{B S}\right) \times \frac{h_{s}+h_{c}}{h_{s}} \\
\Delta T_{d 3}=\Delta T_{T C}-\Delta T_{B S}-\left(\Delta T_{T S}-\Delta T_{B S}\right) \times \frac{h_{s}+h_{c}}{h_{s}}
\end{gathered}
$$

in which $h_{s}$ and $h_{c}$ are the depth of the steel girder and the thickness of the concrete deck; $h_{N A}$ is the depth of the sectional centroid, i.e. the vertical distance from the bottom of the girder to the position of the neutral axis of the composite section (or transformed steel section). A clear geometric comprehension for those quantities in Eqs. (4.9) can be achieved by referring Fig. 4.5 .

In a composite girder with a simply supported single span, the thermal distribution $\Delta T_{2}\left(y_{c p}\right)$ will result in a constant curvature which is determined by the following expression:

$$
\kappa=-\frac{\alpha \Delta T_{d 2}}{h}
$$

where $h$ is the depth of the composite section which equals $h_{s}+h_{c} ; \alpha$ is the coefficient of thermal expansion which may be taken as that for concrete. The minus sign is used in Eq. (4.10) in order to make the concave deflection correspond to positive curvature. Next, if additional restraints are imposed to make the structure become statically indeterminate, 
then the introduction of structural redundancy ensues. For instance, if an internal support is imposed at midspan of the original simply supported girder to restrain vertical deflection at that location (as for the case of Elkhart County Bridge), the following response moment is then expected to develop along the girder length:

$$
\begin{aligned}
M_{r 2}(x) & =\frac{3 \alpha \Delta T_{d 2} E_{s} I_{c p}}{2 h} \times \frac{x}{L / 2} & \text { for } & 0 \leq x \leq L / 2 \\
& =\frac{3 \alpha \Delta T_{d 2} E_{s} I_{c p}}{2 h} \times \frac{L-x}{L / 2} & \text { for } & L / 2 \leq x \leq L
\end{aligned}
$$

where $L$ is the bridge length. The function of response moment $M_{r 2}(x)$ is symmetric about the internal restraint due to the symmetry of the girder. It is derived by means of the flexibility method in which the reaction at the internal support is chosen as the redundant force.

As mentioned earlier, the effect of the thermal distribution function $\Delta T_{3}\left(y_{c p}^{C}\right)$ with non-zero values only within the range of concrete deck is mechanically visualized as a uniform axial force and bending moment applied to the center of the composite section. The magnitude of the uniform axial force is calculated as

$$
N_{\Delta T_{3}}=\frac{\alpha \Delta T_{d 3}}{2} E_{c} A_{c}=\frac{\alpha \Delta T_{d 3}}{2} E_{s} \frac{A_{c}}{n}
$$

in which $E_{c}$ and $E_{s}$ are the elastic moduli of concrete and steel, respectively; $n$ is the steelto-concrete modular ratio which is defined by Eq. (4.2); and $A_{c}$ is the sectional area of concrete deck. The corresponding uniformly applied bending moment is obtained by multiplying the quantity $N_{\Delta T_{3}}$ with the eccentricity of the applied force $\bar{y}_{c p}^{C}$, which is the relative distance from the neutral axis to the center of the triangular distribution $\Delta T_{3}\left(y_{c p}^{C}\right)$. 
Specifically, for a rectangular cross-section of concrete deck, the value of $\bar{y}_{c p}^{C}$ is given by the following:

$$
\bar{y}_{c p}^{C}=h_{s}-h_{N A}+2 h_{c} / 3
$$

Once again with the existence of the internal restraint at the middle of the bridge length, the responding moment is found to be

$$
M_{r 3}(x)=-N_{\Delta T_{3}} \times\left(\bar{y}_{c p}^{C}\right) \times(1-3 x / L) \quad \text { for } \quad 0 \leq x \leq L / 2
$$

Like $M_{r 2}(x)$, the function $M_{r 3}(x)$ is also symmetric about the location $x=0.5 L$ due to the same reason. In Eq. (4.14) the minus sign before $N_{\Delta T_{3}}$ is inserted so that the value of $M_{r 3}$ is able to conform to the sign convention for curvature stated above. Response moments $M_{r 2}(x)$ and $M_{r 3}(x)$ are conceptually drawn in Fig. 4.7.

Now consider the following compatibility relation which is similar to that shown in Eq. (4.4):

$$
\delta_{p, \Delta T}+\Delta P_{\Delta T} \delta_{1}=0
$$

where the quantity $\delta_{1}$, as given in Eq. (4.6), is the shortening in the direction of tendon configuration due to the application of unit tendon force. The tendon deformation due to the uneven temperature change along its axial direction can be computed according to the principle of virtual work:

$$
\begin{array}{r}
1 \times \delta_{P, \Delta T}=\frac{\int_{0}^{L}\left[M_{r 2}(x)+M_{r 3}(x)\right] m(x) d x}{E_{s} I_{c p}}+\frac{\int_{0}^{L} N_{\Delta T_{3}} \times a(x) d x}{E_{s} A_{c p}} \\
+\int_{0}^{L}\left[\kappa \times m(x)+\alpha \Delta T_{N A} \times a(x)\right] d x
\end{array}
$$

The definitions of the variables in Eq. (4.16) are given in previously presented equations. By substituting Eqs. (4.6) and (4.16) into Eq. (4.15), $\Delta P_{\Delta T}$ is obtained subsequently: 


$$
\Delta P_{\Delta T}=-\delta_{P, \Delta T} / \delta_{1}
$$

Additionally, if the thermal properties of the tendon itself is taken into account, Eq. (4.17a) is then modified as

$$
\Delta P_{\Delta T}=-\delta_{P, \Delta T} / \delta_{1}-\alpha_{t} \Delta T_{t} E_{t} A_{t}
$$

where $\alpha_{t}$ is the coefficient of thermal expansion of the steel tendon; and $\Delta T_{t}$ is the temperature change in the tendon.

The stress distributions in the girder can be computed with the above information. Since it is assumed that no restraint against axial deformation exists in the bridge construction, then the uniform distribution of temperature variation $\Delta T_{1}\left(y_{c p}\right)$ has no direct contribution in constituting thermal stresses in the girder. In other words, the girder is allowed to expand or shorten almost uniformly and freely. The axial thermal deformation of the composite girder is only somewhat reduced by the existence of the steel tendons. The thermal response in the girder is a combined result from the uneven temperature variation, the resistance of the internal restraint against statically determinant displacement, and the change in tendon force. Respectively, the thermal stresses in the steel and concrete section are determined by

$$
\begin{aligned}
\sigma_{\Delta T}^{S}\left(x, y_{c p}\right) & =\frac{\left[M_{r 2}(x)+M_{r 3}(x)+\Delta P_{\Delta T} \times m(x)\right] y_{c p}}{I_{c p}}+\frac{\left[N_{\Delta T_{3}}+\Delta P_{\Delta T} \times a(x)\right]}{A_{c p}} \\
\sigma_{\Delta T}^{C}\left(x, y_{c p}\right)=\frac{\left[M_{r 2}(x)+M_{r 3}(x)+\Delta P_{\Delta T} \times m(x)\right] y_{c p}}{n \times I_{c p}}+\frac{\left[N_{\Delta T_{3}}+\Delta P_{\Delta T} \times a(x)\right]}{n \times A_{c p}} & -E_{c} \times \bar{\varepsilon}_{\Delta T_{3}}\left(y_{c p}\right)
\end{aligned}
$$

where the restraining thermal strain $\bar{\varepsilon}_{\Delta T_{3}}$, as a function of vertical position $y_{c p}$, is calculated according to 


$$
\bar{\varepsilon}_{\Delta T_{3}}\left(y_{c p}\right)=\alpha \Delta T_{d 3} \times\left[\frac{\left|y_{c p}\right|-\left(h_{s}-h_{N A}\right)}{h_{c}}\right]
$$

The restraining thermal stress $E_{c} \times \bar{\varepsilon}_{\Delta T_{3}}$ results from the bonding between the steel girder and concrete slab. Eqs. (4.18) and (4.19) are the general approximate expressions to estimate the thermal stresses in a steel-concrete composite due to the non-uniform distribution of temperature variation with the pattern shown in Fig. 4.4. It is emphasized again that the above derivation is based on the assumption of simply supported end condition. If, however, the end rigidity is properly quantified so that the additional resulting moments are able to be estimated, the calculation of the thermal stresses $\Delta \sigma_{\Delta T}^{S}$ and $\Delta \sigma_{\Delta T}^{C}$ can thus be refined by including the additionally induced moments in the response moments $M_{r 2}(x)$ and $M_{r 3}(x)$ in Eqs. (4.18) and (4.19).

\subsection{Numerical Demonstration}

In this section, the calculation of the strain and stress distributions for the Elkhart County Bridge is demonstrated numerically. The locations of the cross sections chosen for the demonstration are G1 2, G1 6, G3 2 and G3 6 (Fig. 3.3). Throughout the calculation the SI (International System of Units, metric units) are used in order to be consistent with the dimensional units presented in the drawings of the Elkhart County Bridge.

The dead load, which includes the weight of steel girder and concrete is solely supported by the steel plate girder. The dimensions of the steel plate girder and the corresponding sectional and material properties are illustrated in Fig. 4.8. The unit 
weights of steel and concrete are taken as $D_{s}=7.682 \times 10^{-8} \mathrm{kN} / \mathrm{mm}^{3}$ and $D_{c}=2.356 \times 10^{-8}$ $\mathrm{kN} / \mathrm{mm}^{3}$ (150 pcf, normal weight concrete). To estimate the weight of concrete to be carried by an individual girder, the "effective width" of the concrete slab for that girder section must be determined. This essential information is shown in Fig. 4.9.

According to Fig. 4.9, The intensity of the uniformly distributed dead loads for girders G1 and G3 can be computed:

$$
\begin{aligned}
w_{G 1} & =\left(A_{s} \times D_{s}+A_{c} \times D_{c}\right) \\
& =42,164 \mathrm{~mm}^{2} \times 7.682 \times 10^{-8} \mathrm{kN} / \mathrm{mm}^{3}+662,150 \mathrm{~mm}^{2} \times 2.356 \times 10^{-8} \mathrm{kN} / \mathrm{mm}^{3} \\
& =0.01884 \mathrm{kN} / \mathrm{mm} \\
w_{G 3} & =\left(A_{s} \times D_{s}+A_{c} \times D_{c}\right) \\
& =42,164 \mathrm{~mm}^{2} \times 7.682 \times 10^{-8} \mathrm{kN} / \mathrm{mm}^{3}+68,0240 \mathrm{~mm}^{2} \times 2.356 \times 10^{-8} \mathrm{kN} / \mathrm{mm}^{3} \\
& =0.01927 \mathrm{kN} / \mathrm{mm}
\end{aligned}
$$

As noted earlier, the girder ends are encased within the concrete abutments and, consequently, develop some degree of flexural moment due to corresponding restraint. If, however, the bridge is assumed to be simply supported at the ends, then the moments that act on the cross-sections of interest are given as follows:

$$
\begin{array}{ll}
M_{g @ G 12}=-2.4377 \times 10^{6} \mathrm{kN}-\mathrm{mm} & M_{g @ G 16}=1.6410 \times 10^{6} \mathrm{kN}-\mathrm{mm} \\
M_{g @ G 32}=-2.4933 \times 10^{6} \mathrm{kN}-\mathrm{mm} & M_{g @ G 36}=1.6785 \times 10^{6} \mathrm{kN}-\mathrm{mm}
\end{array}
$$

Referring back to Fig. 4.8 for finding the elastic modulus of steel $E_{s}=200 \mathrm{kN} / \mathrm{mm}^{2}(200$ GPa) and the moment of inertia $I_{s}=1.52637 \times 10^{10} \mathrm{~mm}^{4}$, the strain values for the above bending moments are attained by applying Eq. (4.1)

$$
\begin{array}{ll}
\varepsilon_{g @ G 12}^{S_{\text {WTOP }}}=636.3 \times 10^{-6} & \varepsilon_{g @ G 12}^{S_{\text {WBOT }}}=-520.3 \times 10^{-6} \\
\varepsilon_{g @ G 16}^{S_{\text {WTOP }}}=-428.3 \times 10^{-6} & \varepsilon_{g @ G 16}^{S_{\text {WBOT }}}=350.3 \times 10^{-6}
\end{array}
$$




$$
\begin{array}{ll}
\varepsilon_{g @ G 32}^{S_{W T O P}}=650.8 \times 10^{-6} & \varepsilon_{g @ G 32}^{S_{W B O T}}=-532.2 \times 10^{-6} \\
\varepsilon_{g @ G 36}^{S_{W T O P}}=-438.1 \times 10^{-6} & \varepsilon_{g @ G 36}^{S_{W B O T}}=358.3 \times 10^{-6}
\end{array}
$$

In the above symbols for strain, the superscripts $S_{W T O P}$ and $S_{W B O T}$ correspond to the vertical positions at the levels of the bottom surface of the top flange (the upper edge of the web, $y_{s G 1}^{S_{W T O P}}=y_{s G 3}^{S_{W T O P}}=-796.6 \mathrm{~mm}$ ), and the top surface of bottom flange (the lower edge of the web, $y_{s G 1}^{S_{W B O T}}=y_{s G 3}^{S_{W B O T}}=651.4 \mathrm{~mm}$ ), respectively. Those superscript representations are also adopted in the subsequent paragraphs for indicating the same locations in the steel plate girder.

Next the response caused by post-tensioning is estimated. The first step is to compute the modular ratio $n=E_{s} / E_{c}$ as defined by Eq. (4.2) for developing the transformed sections for composite girders G1 and G3. Composite action was assumed to be fully effective when the tendons were stressed.

The compressive strength of concrete $f_{c}^{\prime}$ at the day of post-tensioning operation is estimated to be $58.61 \mathrm{MPa}(8,500 \mathrm{psi})$. This value is obtained by interpolating the data from cylindrical concrete tests. The modulus of elasticity of the concrete is then further estimated by using the following empirical equation (Farny and Panarese, 1994):

$$
E_{c}=3,320 \times \sqrt{f_{c}^{\prime}}+6,900 \mathrm{MPa}
$$

which yields $E_{c}=32,320 \mathrm{MPa}\left(32.32 \mathrm{kN} / \mathrm{mm}^{2}\right)$; and the corresponding modular ratio $n=$ $200 / 32.32=6.19$. Subsequently, the sectional properties are calculated according to the geometry and dimensions of the transformed section (Fig. 4.10).

The initial jacking force is $2,308 \mathrm{kN}$ (518.87 kips) for each tendon. However, the initial effective post-tensioning force $P$ is roughly $18 \%$ less than that because of the 
mechanical losses due to friction, anchorage set and elastic shortening. For each individual composite girder, the average effective prestress force is evaluated as

$$
P=\varepsilon \times E_{t} \times A_{t}=(0.005491) \times\left(202.71 \mathrm{kN} / \mathrm{mm}^{2}\right) \times\left(141.16 \mathrm{~mm}^{2} \times 2 \times 12\right)=3,771 \mathrm{kN}
$$

In the above $\varepsilon=0.005491$ is the average strain in the monitored tendon converted from the original elongation reading taken right after post-tensioning. Each tendon is composed of twelve seven-wire steel strands with a modulus of elasticity equal to $202.71 \mathrm{kN} / \mathrm{mm}^{2}$ and an area equal to $141.16 \mathrm{~mm}^{2}$. The factor 2 indicates that each girder is prestressed by two adjacent tendons.

The unit moment function $m(x)$ and axial force function $a(\mathrm{x})$ must be developed before applying Eq. (4.3) to obtain the responding strains due to post-tensioning. In Fig. 4.11 $m(x)$ and $a(x)$ are plotted over the entire bridge length. The function $m(x)$ is not exactly the same for composite girders G1 and G3 because of the slight difference in the vertical location of neutral axis. However, the difference is insignificant. Like $M_{g}(x)$ in the previous discussion, $m(x)$ is developed based on the assumption of hinge end supports. The values of $m(x)$ at those selected gage locations G1 2, G1 6, G3 2 and G3 6 are found as follows:

$$
\begin{array}{ll}
m_{@ G 12}=930.55 \mathrm{kN}-\mathrm{mm} / \mathrm{kN} & m_{@ G 16}=-387.48 \mathrm{kN}-\mathrm{mm} / \mathrm{kN} \\
m_{@ G 32}=935.98 \mathrm{kN}-\mathrm{mm} / \mathrm{kN} & m_{@ G 36}=-392.33 \mathrm{kN}-\mathrm{mm} / \mathrm{kN}
\end{array}
$$

The unit axial force function $a(x)$ is identical for both girders, and is uniform for the entire bridge length. It is calculated:

$$
a(x)=-1 \times \cos \theta_{1}=(1 \mathrm{kN}) \times\left[\cos \left(3.05^{\circ}\right)\right] / \mathrm{kN}=-0.9986 \mathrm{kN} / \mathrm{kN}
$$


where $\theta_{1}=3.05^{\circ}$ is the degree of inclination of the tendon profile between the bridge end and the intermediate concrete diaphragm (Fig. 3.2).

The strain distribution due to post-tensioning can now be evaluated using Eq (4.3). For the girder G1, $I_{c p}=4.25810 \times 10^{10} \mathrm{~mm}^{4}, A_{c p}=149,200 \mathrm{~mm}^{2}, y_{c p G 1}^{S_{\text {WTOP }}}=-121.6$ $\mathrm{mm}$, and $y_{c p G 1}^{S_{W B O T}}=1,326.4 \mathrm{~mm}$. The following results are obtained for $\mathrm{G} 1$ :

$$
\begin{array}{ll}
\varepsilon_{p @ G 12}^{S_{W T O P}}=-176.3 \times 10^{-6} & \varepsilon_{p @ G 12}^{S_{W B O T}}=420.5 \times 10^{-6} \\
\varepsilon_{p @ G 16}^{S_{W T O P}}=-105.4 \times 10^{-6} & \varepsilon_{p @ G 16}^{S_{W B O T}}=-353.9 \times 10^{-6}
\end{array}
$$

The strain in the concrete deck at the level of the embedded strain gage is also calculated using Eq. (4.3) with the same numbers, except that $y_{c p}^{C}{ }_{G 1}=-248.6 \mathrm{~mm}$ :

$$
\varepsilon_{p @ G 12}^{C}=-228.7 \times 10^{-6} \quad \quad \varepsilon_{p @ G 16}^{C}=-83.6 \times 10^{-6}
$$

Similarly for girder G3, the substitution of $I_{c p}=4.32607 \times 10^{10} \mathrm{~mm}^{4}, A_{c p}=152,124 \mathrm{~mm}^{2}$, $y_{c p G 3}^{S_{W T O P}}=-109.5 \mathrm{~mm}, y_{c p G 3}^{S_{W B O T}}=1,338.5 \mathrm{~mm}$, and $y_{c p G 3}^{C}=-236.5 \mathrm{~mm}$ into Eq. (4.3) yields:

$$
\begin{array}{ll}
\varepsilon_{p @ G 32}^{S_{W T O P}}=-168.5 \times 10^{-6} & \varepsilon_{p @ G 32}^{S_{W B O T}}=422.4 \times 10^{-6} \\
\varepsilon_{p @ G 36}^{S_{W T O P}}=-105.1 \times 10^{-6} & \varepsilon_{p @ G 36}^{S_{W B O T}}=-352.7 \times 10^{-6}
\end{array}
$$

and

$$
\varepsilon_{p @ G 32}^{C}=-220.3 \times 10^{-6} \quad \varepsilon_{p @ G 36}^{C}=-83.4 \times 10^{-6}
$$

A live load test was conducted by placing a dump truck (Fig. 3.10) at particular locations on the Elkhart County Bridge (Fig. 3.9) to measure the flexural response of the composite girders and the elongation of the tendon. The analysis for the truck loading is performed according to the procedure described in the preceding section 4.2.1, and is illustrated numerically in the ensuing paragraphs. 
The analysis starts with the evaluation of the elastic modulus of concrete $E_{c}$ at the time of testing. The empirical formula Eq. (4.21) relating the compressive strength of concrete $f_{c}^{\prime}$ to $E_{c}$, is used for such purpose. The value of $f_{c}^{\prime}$ is estimated to be $68.95 \mathrm{MPa}$ $(10,000 \mathrm{psi})$ according to the results of cylindrical tests. $E_{c}=34,470 \mathrm{MPa}(34.47$ $\mathrm{kN} / \mathrm{mm}^{2}$ ) and the modular ratio $n=200 / 34.47=5.80$ are computed through sequential substitutions of Eqs. (4.20) and (4.2). The sectional properties of the transformed sections with a modular ratio $n$ equal to 5.80 is illustrated in Fig. 4.12.

The unit moment function $m(x)$ (for both $\mathrm{G} 1$ and G3, under the condition that $E_{c}=$ 34,470 MPa) and axial force function $a(x)$ are portrayed in Fig. 4.13. For the chosen gage locations,

$$
\begin{array}{ll}
m_{@ G 12}=936.02 \mathrm{kN}-\mathrm{mm} / \mathrm{kN} & m_{@ G 16}=-392.37 \mathrm{kN}-\mathrm{mm} / \mathrm{kN} \\
m_{@ G 32}=941.40 \mathrm{kN}-\mathrm{mm} / \mathrm{kN} & m_{@ G 36}=-397.18 \mathrm{kN}-\mathrm{mm} / \mathrm{kN}
\end{array}
$$

and $a(x)=-0.9986 \mathrm{kN} / \mathrm{kN}$ for both composite girders G1 and G3. The calculation of the moment $M(x)$ due to the truck loading relies on how the bridge deck disperses the axle weights to individual girders. However, it is difficult to precisely find out the analytical solution for the moment caused by the truck loading. A simplified method is proposed to estimate the concentrated loads distributed to each girder. The procedure is described in the following:

(1) Apply a unit concentrated load at the spot corresponding to the longitudinal axle location in the two-span steel girder, and then find the reciprocal of the value of the deflection at that loaded point. 
(2) Model a transverse concrete beam with a length equal to the width of the bridge, a thickness equal to that of the deck of the bridge, and a width equal to $500 \mathrm{~mm}$. Rest this beam on seven elastic supports with an identical spring constant which is taken as the deflection reciprocal calculated in the first step. Each elastic support represents a steel stringer.

(3) Load the elastically supported beam by the transverse axle weights which correspond to the locations of either the front or the rear wheels. Solve for the reaction forces in the simulated springs. The results are regarded as the concentrated forces for portions of truck weight applied to individual girders.

(4) Employ the procedure (1) through (3) for both front and rear axle loading. For the girder to be investigated (G1 or G3), the assemblage of the computed concentrated loads is the source to produce the resulting moment $M(x)$ which is to be used in Eqs. (4.5), (4.7) and (4.8) for the calculation of the tendon force increment and strain distribution due to the truck loading.

The procedure described above is demonstrated graphically in Fig. 4.14 for a better understanding. In the second step for modeling the transverse concrete beam, the width of the beam section is a factor that affects the results of subsequent calculations. Consequently, a sensitivity study was conducted to determine the variation in the load distributed to adjacent girders when the axle weight was applied to the multiple-span concrete beam. It was found that the load distributed to adjacent girders was not sensitive to the variation of the beam width over a wide range of widths $(200 \mathrm{~mm}$ to $800 \mathrm{~mm}$ ). Hence, a value of $500 \mathrm{~mm}$ was selected for all computations. This approximate 
procedure, however, does not consider the stiffness of the transverse concrete diaphragm, which may potentially make the deck system more rigid.

In the following the response at gage locations G1 2 and G1 6 resulting from truck loading case (b), and the response at locations G3 2 and G3 6 due to loading case (h) are examined. Truck locations (b) and (h) (see Fig. 3.9) are expected to produce the largest response at the designated gage locations. The axle loads imparted to the girders are estimated by using the aforementioned method, and the results are shown in Fig. 4.15. The moments $M(x)$ then are obtained accordingly. With the knowledge of the values of all the required variables in Eq. (4.7), the tendon force increment $\Delta P$ can be calculated by numerical substitution and integration:

For loading case (b):

$$
\Delta P_{G 1}=6.944 \mathrm{kN}
$$

For loading case $(\mathrm{h})$ :

$$
\Delta P_{G 3}=5.509 \mathrm{kN}
$$

The moments $M(x)$ at those indicated locations are found as follows:

For loading case (b):

$$
M_{@ G 12}=-3.3870 \times 10^{5} \mathrm{kN}-\mathrm{mm} \quad M_{@ G 16}=7.6267 \times 10^{5} \mathrm{kN}-\mathrm{mm}
$$

For loading case (h):

$$
M_{@ G 32}=-2.7022 \times 10^{5} \mathrm{kN}-\mathrm{mm} \quad M_{@ G 36}=6.0845 \times 10^{5} \mathrm{kN}-\mathrm{mm}
$$

The final substitution of the preceding calculated values and other given constants into Eq. (4.8) gives the following results:

For loading case (b): 


$$
\begin{array}{lll}
\varepsilon_{e @ G 12}^{C}=7.964 \times 10^{-6} & \varepsilon_{e @ G 12}^{S_{W T O P}}=3.995 \times 10^{-6} & \varepsilon_{e @ G 12}^{S_{W B O T}}=-51.824 \times 10^{-6} \\
\varepsilon_{e @ G 16}^{C}=-18.640 \times 10^{-6} & \varepsilon_{e @ G 16}^{S_{W T O P}}=-9.870 \times 10^{-6} & \varepsilon_{e @ G 16}^{S_{W B O T}}=117.826 \times 10^{-6}
\end{array}
$$

For loading case (h):

$$
\begin{array}{lll}
\varepsilon_{e @ G 32}^{C}=5.984 \times 10^{-6} & \varepsilon_{e @ G 32}^{S_{W T O P}}=2.777 \times 10^{-6} & \varepsilon_{e @ G 32}^{S_{W B O T}}=-41.068 \times 10^{-6} \\
\varepsilon_{e @ G 36}^{C}=-14.036 \times 10^{-6} & \varepsilon_{e @ G 36}^{S_{W T O P}}=-6.919 \times 10^{-6} & \varepsilon_{e @ G 36}^{S_{W B O T}}=93.374 \times 10^{-6}
\end{array}
$$

The calculation of thermal response starts with specifying a sectional distribution of temperature variation. Shown in Fig. 4.16 are the assumed components of the nonuniform temperature change along the depth of the interior girder G3. The transformed section of G3, with a modular ratio based upon the concrete strength at 28 days, is 5.80 , as previously depicted in Fig. 4.12(b). For the purposes of demonstration, the three initial parameters to define the approximate bi-linear temperature variation are given as follows:

$$
\Delta T_{B S}=15^{\circ} \mathrm{C} \quad \Delta T_{T S}=20^{\circ} \mathrm{C} \quad \Delta T_{T C}=40^{\circ} \mathrm{C}
$$

Although the cross-section of the concrete deck is not exactly a rectangle, as that assumed to establish Eqs. (4.9) as well as (4.13), it is appropriate to substitute $h_{c}=240 \mathrm{~mm}, h_{s}=$ $1505 \mathrm{~mm}$ and $h=1745 \mathrm{~mm}$ (see Fig. 4.9b), and the specified values of $\Delta T_{T C}, \Delta T_{T S}$ and $\Delta T_{B S}$ into Eqs (4.9) to obtain the next three factors as in the following:

$$
\Delta T_{N A}=19.59^{\circ} \mathrm{C} \quad \Delta T_{d 2}=5.80{ }^{\circ} \mathrm{C} \quad \Delta T_{d 3}=19.20{ }^{\circ} \mathrm{C}
$$

The value of the coefficient of thermal expansion $\alpha$ is taken as that of concrete which equals to $10.0 \times 10^{-6} /{ }^{\circ} \mathrm{C}$. The curvature $\kappa$ is calculated according to Eq. (4.10):

$$
\kappa=-\frac{\alpha \Delta T_{d 2}}{h}=-\frac{\left(0.00001 /{ }^{\circ} \mathrm{C}\right) \times\left(5.80{ }^{\circ} \mathrm{C}\right)}{1,745 \mathrm{~mm}}=-3.322 \times 10^{-8}
$$


The response moment $M_{r 2}(x)$ is obtained by using Eq. (4.11), and $M_{r 3}(x)$ is obtained by using Eq. (4.14) associated with Eqs. (4.12) and (4.13). The values of the uniform axial force $N_{\Delta T_{3}}$, and the peak values of $M_{r 2}(x)$ and $M_{r 3}(x)$ occurring at the pier and at the bridge end are calculated as, respectively

$$
\begin{aligned}
& N_{\Delta T_{3}}=2,251.3 \mathrm{kN} \\
& M_{r 2}(x=35,275 \mathrm{~mm})=436,200 \mathrm{kN} / \mathrm{mm} \\
& M_{r 3}(x=0 \mathrm{~mm})=-635,700 \mathrm{kN} / \mathrm{mm}
\end{aligned}
$$

$M_{r 2}(x)$ and $M_{r 3}(x)$ are graphically presented in Fig. 4. 17.

The estimation for the change of tendon force due to thermal effect is carried out based on Eqs. (4.6), (4.16) and (4.17). Without considering the thermal expansion of the steel tendons, Eq. (4.17a) is employed, yielding

$$
\Delta P_{\Delta T}=-\delta_{P, \Delta T} / \delta_{1}=170.3 \mathrm{kN}
$$

Otherwise, by considering the thermal deformation in the steel tendon due to a temperature rise $\Delta T_{t}=15{ }^{\circ} \mathrm{C}$, and also adopting the value $\alpha_{t}=1.15 \times 10^{-5} /{ }^{\circ} \mathrm{C}$ as the coefficient of thermal expansion for the steel tendon, the application of Eq. (4.17b) reaches

$$
\begin{aligned}
\Delta P_{\Delta T} & =-\delta_{P, \Delta T} / \delta_{1}-\alpha_{t} \Delta T_{t} E_{t} A_{t} \\
& =170.3 \mathrm{kN}-\left(0.0000115 /{ }^{\circ} \mathrm{C}\right) \times\left(15{ }^{\circ} \mathrm{C}\right) \times\left(202.71 \mathrm{kN} / \mathrm{mm}^{2}\right) \times\left(3,388 \mathrm{~mm}^{2}\right) \\
& =51.8 \mathrm{kN}
\end{aligned}
$$

The thermal stresses in the steel and concrete components are computed according to Eqs. (4.18) and (4.19). For example, if Eq. (4.17b) is involved in calculation, the stresses (or the stress variations) in the girder G3 at location 1 (longitudinal bridge center) on the top 
and the bottom surfaces of the concrete deck, and at the upper and the lower edges of the steel web are, respectively, found to be

$$
\begin{aligned}
& \sigma_{\triangle T @ G 31}^{C_{T O P}}=-0.00540 \mathrm{kN} / \mathrm{mm}^{2}=-5.40 \mathrm{MPa} \\
& \sigma_{\triangle T @ G 31}^{C_{B O T}}=0.00199 \mathrm{kN} / \mathrm{mm}^{2}=1.99 \mathrm{MPa} \\
& \sigma_{\triangle T @ G 31}^{S_{W T O P}}=0.01199 \mathrm{kN} / \mathrm{mm}^{2}=11.99 \mathrm{MPa} \\
& \sigma_{\triangle T B G 31}^{S_{W B O T}}=0.03884 \mathrm{kN} / \mathrm{mm}^{2}=38.84 \mathrm{MPa}
\end{aligned}
$$

\section{4 $\underline{\text { Results and Discussions }}$}

A comparison between the experimental an analytical results for instantaneous structural response is made in this section. Discrepancies from the comparison are also discussed. It is intended to inspect the validity of the analytical approaches and the importance of factors which are neglected during the calculation. Thermal response is evaluated based on an assumed bi-linear distribution of temperature variation. The induced thermal stresses are compared with those resulting from the application of dead load, truck load and post-tensioning.

\subsubsection{Mechanical Response}

The strain distributions in the steel plate girder due to gravity loading and posttensioning are selectively shown in Fig. 4.18 to Fig. 4.24. Each of the figures is comprised of three plots labeled from (a) to (c). The results of theoretical calculation and field measurement are drawn together in each plot for the convenience of comparison. In those plots, the vertical scale stands for the strain gage position measured from the top 
surface of the bottom flange, and the horizontal scale is the quantity of elastic strain. The strain values are positive for tension and negative for compression.

In each one of the figures from Fig. 4.18 to Fig. 4.24, plot (a) represents the bridge response due to the dead load. However, the weight of steel plate girder is excluded. The zero readings of the strain values were taken in the field after the erection of the steel girders. Consequently, the gravity weight of the concrete deck was then the only portion of dead load that is reflected in the dead load plot. The field readings were collected four days after the concrete deck was poured. The resulting stresses of dead load are resisted by the steel section, as demonstrated previously.

Plot (b) is for the response due to the application of post-tensioning, The strain distributions of test results shown in the plot are obtained by subtracting the strain values in plot (a) from the data collected right at the completion of post-tensioning, which are the numbers for the experiment results in plot (c). For analytical results, strain distributions due to dead load [presented in plot (a)] and prestress [presented in plot (b)] are calculated first and then superimposed [in plot (c)]. The stresses caused by posttensioning are carried by the composite section. For all the analytical results shown in the figures, the calculations are based on the assumption that the girder is pinned at the abutment, therefore no capacity to carry bending moment is allowed at the ends.

Fig. 4.18 and Fig. 4.19 are for the strain distributions in steel girders G1 and G3 respectively at the same longitudinal location 2 , which is $1,220 \mathrm{~mm}$ (four feet) from the center pier of the bridge. At this location, the steel section is subjected to negative moment due to dead load, and the composite section is subjected to positive moment due 
to post-tensioning. Linear strain distributions are almost obtained from the field measurements. The curvature of the girder is decreased at this location with the application of post-tensioning, since the slope of sectional strain distribution becomes less steep [from plot (a) to plot (c)]. The comparison of experimental and analytical results shows fairly good agreement. It seems that the bridge behavior at this location can be very well predicted. Since the bridge section at location 2 is exposed to a higher level of stresses, the detected response is less susceptible to measurement errors which are not reflected in the analysis.

Shown in Fig. 4.20 and Fig. 4.21 are the strain distributions in steel sections at locations G1 6 and G3 6. This longitudinal location is $14,110 \mathrm{~mm}$ away from the end of the bridge, which is near the spot where maximum positive moment occurs due to distributed downward loading. The sign of bending curvature at this location is opposite to that in location 2 at every stage of loading. The linearity of strain distribution is not very well preserved with the introduction of post-tensioning, especially for girder G3. Some out-of-plane bending might have taken place other than the in-plane deformation. In general, the analytical procedure provides a successful estimation of structural response at the location G1 6. On the other hand, for some strain gages attached on the web of girder G3 at location 6, the measured strains do not fit the predicted values too well.

Fig. 4.22 and Fig. 4.23 represent the structural response in steel components at the locations G1 7 and G3 7, which are both only 1,365 $\mathrm{mm}$ from the south end of the bridge. Some significant inconsistency is found between the experimental and analytical results. 
Since the actual response near the bridge abutment is small and sensitive to end condition, it is believed that the idealization of hinge supports at bridge ends in calculation is the major cause of such discrepancy. For instance, according to this assumption, the section at this location is stressed under a positive bending action due to dead load [plot (a)], yet the measured strain readings shows that the section seems to be deformed in a negative curvature. The response of girders at locations 2 and 6 should also be affected somewhat by the end rigidity. However, its influence is comparatively less notable. The strain observations at locations 2 and 6 suggest that end rigidity, while present, plays a small role in the response. For those critical locations, the assumption of pinned ends does not lead to significant errors and, instead, results in reasonable predictions of the structural behavior.

Unless kept in an environment with a relative humidity of $100 \%$, concrete losses moisture with the progress of time and decreases in volume. Such phenomenon is known as shrinkage. Despite that some curing process was undertaken, the concrete slab was inevitably subjected to the effect of shrinkage. This factor, however, is neglected in the analytical procedure for the reason that it is very difficult to quantify, otherwise its influence would be reflected in plots (a) as well as in plots (b) and (c). On the other hand, the experimental strain values in those plots should involve the shrinkage effect. However, the analytical results are not significantly deviated from the test results by neglecting this factor.

Additionally shown in Fig. 4.24 is the strain distributions at location G3 1, which is $191 \mathrm{~mm}$ from the bridge center pier. The strain gages placed here are embedded in the 
central concrete diaphragm where the interior saddle between the two drape points is situated. Many strain readings at this location are not in a reasonable range. Those readings are excessively high, and then are excluded from the plots. As stated in Chapter 3 , it is inferred that the performance of most of the strain gages was deteriorated by the introduction of transverse post-tensioning at the interior pier and the casting of diaphragm itself. In fact, poor strain readings were regularly obtained from those gages located inside the concrete diaphragms.

Fig. 4.25 illustrates the strain distributions in concrete deck due to post-tensioning along girders G1 and G3 at the level of strain gages. The analytical results are obtained via the previously described procedure, without considering the possible rotational restraints at bridge ends and the effect of concrete shrinkage. The experimental strain values shown in this figure are obtained by taking the difference between strain readings taken immediately after post-tensioning and four days after the casting of the concrete deck. The two sets of measurements were seven days apart. Two general trends are found through comparing the results of analysis and experiment. First, the analytical method tends to underestimate the response (the analytical results are "less negative"), and second, the difference seems to be most significant near the bridge end, and decreases constantly toward the piers (the bridge center). The deviation of the analytical results from the experimental results is probably a consequence of concrete shrinkage. A qualitative explanation for the cause of the discrepancy due to concrete shrinkage is presented in Fig. 4.26. 
The first two figures ( $a$ and $b$ ) in Fig. 4.26 indicate that the effect of concrete shrinkage can be regarded as a uniform compressive force applied eccentrically at the level of the center of concrete slab. Therefore, a compressive axial force applied uniformly on the composite section and equal bending moments applied at both girder ends can be equivalently assumed. The third figure (c) is the moment diagram due to the equivalent moment. The strain (or stress) distributions in the concrete at the level of gages along the girder are shown in the next three figures (d, e and f). Figures $d$, e and $f$ are, respectively, strain distributions due to the equivalent bending moment, compressive force, and the combined effect. The last sketch (figure f) then represents the results of ignoring the factor of concrete shrinkage in analysis. In other words, it represents the difference between the calculated and observed results. Theoretically, the amount of difference is most significant at the end, and varies linearly to reach the minimum at the bridge center. This explanation matches quite well the observation in Fig. 4.25, particularly for girder G3. Additionally listed in Table 4.1 are the estimated concrete stresses due to post-tensioning at several critical locations.

The induced strain distributions in steel girders due to truck loading are selectively shown in Fig. 4.27 [for loading case (b) at locations G1 2 and G1 6] and Fig. 4.28 [for loading case (h) at locations G3 2 and G3 6]. The structural response is much smaller than that caused by dead load or post-tensioning. The analytical solutions are attained by employing the methods proposed in the preceding sections. First, the portion of axle weights distributed to each girder is estimated. Then, with that information, the strain or stress distributions at those designated locations are calculated through a series 
of substitution and integration. The comparison of the analyzed and measured results shows that the theoretical prediction tends to be conservative. The main reason is perhaps that the influence of the stiffness of the transverse diaphragms and the transverse posttensioning in the concrete deck are overlooked in determining the distribution factors for truck loading. The axle loads are more uniformly distributed to adjacent girders than expected with the help of the diaphragms and transverse post-tensioning.

Listed in Table 4.2 are the increments of strain in the tendon monitored along girder G3 caused by the nine truck loading cases. The experimental values are obtained by substituting the measured elongation readings into the conversion equation provided by the vibrating wire gage manufacturer. The analytical values are obtained through dividing the computed increased tendon forces $[\Delta P$ in Eq. (4.7)] by the product of the elastic modulus and total area of high-strength tendons $\left(E_{t} A_{t}\right)$. In contrast to the test results, the calculated numbers are distinctly larger. However, both of them are minute, with magnitudes under 10 microstrains $\left(10 \times 10^{-6}\right)$. For physical quantities (analyzed and measured) so small in magnitude that they almost hit the resolution capacity of the adopted sensor, such difference is not too meaningful.

Since it is noticed that the truck-induced tendon force $\Delta P$ is usually pretty small, an attempt is made to re-estimate the structure response without considering the effect of $\Delta P$. Eq. (4.8) used to calculate the strain distributions in the composite section is then reduced to

$$
\varepsilon_{e}\left(x, y_{c p}\right)=\frac{[M(x)] y_{c p}}{E_{s} I_{c p}}
$$


Eq. (4.22) is much simpler then Eq. (4.8) and involves no complicated integration in the computation. The strains at the locations G3 2 and G3 6, as computed by using Eqs. (4.8) and (4.22), are listed and compared in Table 4.3. The sectional level for calculation is the top surface of the bottom flange. A survey of the comparison in the table reveals that the simplification in computation is practical since the deviation originated from the use of Eq. (4.22) is constantly less than $2 \%$.

One of the assumptions of the analytical model is that the concrete deck is free from cracking under service loading conditions. By observing the strain values at the level of concrete gages shown in Fig. 4.25, it is found that the concrete deck seems to be subjected to compressive stress at every location. However, a more thorough examination is performed by checking the stresses at the levels of the top and bottom of the concrete slab along the girders G1 and G3 at critical locations. The analyzed results listed in Table 4.1 show that the whole concrete slab is free from any tensile stresses under the application of post-tensioning. According to Table 4.1, the highest and lowest compressive stress levels on the top surface of the concrete deck are found to be -10.16 $\mathrm{MPa}$ (at the bridge center near G1) and $-0.69 \mathrm{MPa}$ (at the drape point near G1). At the bottom of the concrete slab, the extreme stresses are $-6.39 \mathrm{MPa}$ (at the bridge center near G1) and -2.79 $\mathrm{MPa}$ (at the drape point near G1). In addition, the most critical stresses that the axle load test produces include:

-1.21 MPa (on the top of concrete deck; due to load case b; at bridge center; near G1) $-0.46 \mathrm{MPa}$ (at the bottom of concrete deck; due to load case b; at bridge center; near G1) $0.54 \mathrm{MPa}$ (on the top of concrete deck; due to load case b; at midspan; near G1) 
0.19 MPa (at the bottom of concrete deck; due to load case b; at midspan; near G1)

In any case, the combined stress is expected to be compressive, and the magnitude is much lower than the compressive concrete strength $f_{c}^{\prime}$ which is estimated to be -68.95 $\mathrm{MPa}$. The assumption of uncracked concrete deck is then proven to be appropriate. Moreover, since that the stress level under service loading condition is less than $0.5 f_{c}^{\prime}$, which is generally regarded as the linear elastic limit of concrete, the use of constant modular ratio $n$ in determining the properties of transformed section is also considered to be a reasonable approach.

\subsubsection{Thermal Response}

Because no thermal sensors were installed within and on the surface of the composite girder section in the field, the thermal response is not able to be accurately evaluated. Shown in Fig. 4.16 is the assumed sectional temperature variations for estimating the corresponding thermal response, which is the one used in the numerical demonstration. The temperature change in the tendon, $\Delta T_{t}$, is assumed to be the same as that occurring at the bottom of the steel girder, $\Delta T_{B S}$, implying that the Eq. (4.17b) is used for calculating the tendon force variation.

Following the proposed analytical procedure, the distributions of thermal stress are estimated at particular longitudinal locations along the interior girder G3, including (a) the internal support, (b) the drape location, (c) the midspan, (d) the 0.4 span from end, and (e) the bridge end. The analytical results are demonstrated in Fig. 4.29. In this figure, stress values are presented versus the corresponding elevation measured from the base of 
the composite girder. It is observed that the thermal stresses in the concrete slab exhibit fairly small variations at different longitudinal locations. In contrast, the sectional distribution of thermal stresses in the steel section depends on location. The gradient of the distribution of thermal stress in the steel girder reaches the maximum positive value at the bridge end; and the largest negative value at the pier. Somewhere around the midspan a uniform distribution of thermal stress in the steel section may be predicted. The estimated thermal stresses at those critical locations are also listed in Table 4.4.

The greatest thermal stress is expected to occur at the bottom of the steel girder around the pier. The magnitude of stress, for the assumed case $\left(\Delta T_{B S}=15^{\circ} \mathrm{C}, \Delta T_{T S}=20\right.$ ${ }^{\circ} \mathrm{C}, \Delta T_{T C}=40{ }^{\circ} \mathrm{C}, \Delta T_{t}=15{ }^{\circ} \mathrm{C}$ ), is found to be equal to $39.43 \mathrm{MPa}$ in tension. The minimum (most negative or compressive) thermal stress in steel occurs at the bottom of the girder at the end of the bridge $(-6.28 \mathrm{MPa})$. For the concrete deck, the spots where the greatest compressive and tensile thermal stresses occur, respectively, are the upper surface at the location of the pier (the bridge center), and the bottom at the bridge abutment (the bridge end). The corresponding magnitudes are found to be $-5.40 \mathrm{MPa}$ and 2.68 MPa. The above analyzed stress values are compared with the following quantities:

The yield stress of the steel: $344.7 \mathrm{MPa}$ (50 ksi)

The stress on the top of the steel girder at the location G3 1:

due to dead load: $161.29 \mathrm{MPa}$

due to post-tensioning: $-37.71 \mathrm{MPa}$

due to truck loading case $<\mathrm{h}>$ (most critical case): $0.86 \mathrm{MPa}$

The stress at the bottom of the steel girder at the location G3 1: 
due to dead load: $-134.17 \mathrm{MPa}$

due to post-tensioning: $107.31 \mathrm{MPa}$

due to truck loading case $<\mathrm{h}>$ (most critical case): $-10.09 \mathrm{MPa}$

The compressive strength of concrete at 28 days $\left(f_{c}^{\prime}\right):-68.95 \mathrm{MPa}$

The tensile strength of concrete $\left(\cong 0.1 f_{c}^{\prime}\right): 6.90 \mathrm{MPa}$

The stress on the top of the concrete deck at the location G3 1:

due to post-tensioning: $-9.83 \mathrm{MPa}$

due to truck loading case $<\mathrm{h}>$ (most critical case): $0.44 \mathrm{MPa}$

The stress at the bottom of the concrete deck at the location G3 1:

due to post-tensioning: $-6.10 \mathrm{MPa}$

due to truck loading case $<\mathrm{h}>$ (most critical case): $0.14 \mathrm{MPa}$

The comparison suggests that under drastic temperature change, the induced thermal stresses could be larger than those due to traffic loading, and almost comparable to those caused by post-tensioning at certain locations.

Through the numerical operation, it is found that the third component of the decomposed temperature variation $\left(\Delta T_{3}\right)$ is the dominant cause of thermal stresses, meaning that the temperature variation in the concrete deck is the major factor of the thermal effect. Uniform axial deformation (the effect of $\Delta T_{1}$ ) and constant bending (the effect of $\Delta T_{2}$ ) due to temperature change make a comparatively smaller contribution to thermal response. The calculated thermal stresses or stress variations may differ from the actual values due to the resistance in axial and bending actions at the abutments which are assumed to be nonexistent in the numerical analysis. 
The thermal response in a bridge girder relies on the sectional distribution of temperature variation which is a relative quantity to a certain temperature level. If the formerly assumed distribution is reversed (i.e., let $\Delta T_{B S}=-15^{\circ} \mathrm{C}, \Delta T_{T S}=-20{ }^{\circ} \mathrm{C}, \Delta T_{T C}=$ $-40{ }^{\circ} \mathrm{C}$, and $\Delta T_{t}=-15{ }^{\circ} \mathrm{C}$ ), the thermal stresses obtained differ from those previously calculated at the same locations only in sign.

\subsection{Summary of Conclusions}

Based upon the observations of the strain data collected at the Elkhart County I-90 Toll Road bridge and predicted structural response, the following conclusions can be drawn:

(1) The analytical procedure described herein provides a satisfactory prediction for the real structural response. The assumption of pin-supported end condition in moment calculation does not result in significant discrepancy between analyzed and measured strain values at most critical locations.

(2) When not accounted for in the analysis, shrinkage in the concrete deck could be a factor that causes some noticeable difference between the measured and predicted results. In the study case, the negligence of shrinkage leads to an underestimation of strains in the concrete component, yet in steel components, this effect is not significant.

(3) Truck loading generally induces some increment in tendon force, however its magnitude is minute. In practice this quantity can be ignored, and the analytical procedure will be much simpler. 
(4) The assumption of an uncracked concrete deck is proven to be valid throughout the entire analysis procedure. The initial compressive stresses introduced by the application of post-tensioning enables the concrete deck to be free from tensile stresses in the longitudinal direction, and thereby prevents cracking in the deck perpendicular to the girder.

(5) Estimation of thermal response needs detailed information of the temperature distributions in the structure components. Precise determination of thermal response is not anticipated for most practical situations. The numerical results of the proposed analytical approach, which is based on the use of a bi-linear approximate function to simulate the actual temperature variation, suggests that the thermal stresses (or the variations of thermal stresses) could be greater than those caused by vehicle loading, and comparable to those due to post-tensioning around the interior support (pier). 
Table 4.1 Estimated stresses in concrete deck due to post-tensioning

G1

Longitudinal distance from end

Stresses in concrete deck (MPa)

$(\mathrm{mm})$

$$
\sigma^{C_{\text {TOP }}} \quad \sigma^{C_{\text {BOT }}}
$$

35,275.0 (internal support)

$-10.16$

$-6.39$

$22,950.0 \quad$ (drape point)

$-0.69$

$-2.79$

17,637.5 (midspan)

$-1.44$

$-3.08$

14,110.0 (0.4 span from end)

$-1.94$

$-3.27$

$0.0 \quad$ (bridge end)

$-3.92$

$-4.02$

\section{G3}

Longitudinal distance from end

(mm)

35,275.0 (internal support)

22,950.0 (drape point)

17,637.5 (midspan)

$14,110.0 \quad$ (0.4 span from end)

$0.0 \quad$ (bridge end)
Stresses in concrete deck (MPa)

$\sigma^{C_{\text {TOP }}}$

$\sigma^{C_{B O T}}$

$-9.83$

$-6.10$

$-0.77$

$-2.84$

$-1.47$

$-3.09$

$-1.93$

$-3.26$

$-3.79$

$-3.92$

Note:

$\sigma^{C_{T O P}}:$ stress at the level of the top surface of the concrete deck $\sigma^{C_{B O T}}:$ stress at the level of the bottom surface of the concrete deck 
Table 4.2 Strain increments in post-tensioning tendons along girder G3 due to single-truck loading cases

\begin{tabular}{ccc} 
Load case & Experimental results & Analytical resu \\
\hline a & $1.347 \times 10^{-6}$ & $-0.003 \times 10^{-6}$ \\
b & $2.019 \times 10^{-6}$ & $0.312 \times 10^{-6}$ \\
c & $2.688 \times 10^{-6}$ & $0.074 \times 10^{-6}$ \\
d & $-1.340 \times 10^{-6}$ & $4.606 \times 10^{-6}$ \\
e & $2.696 \times 10^{-6}$ & $7.614 \times 10^{-6}$ \\
f & $0.008 \times 10^{-6}$ & $5.761 \times 10^{-6}$ \\
g & $1.680 \times 10^{-6}$ & $4.924 \times 10^{-6}$ \\
h & $2.356 \times 10^{-6}$ & $8.022 \times 10^{-6}$ \\
i & $1.682 \times 10^{-6}$ & $6.127 \times 10^{-6}$
\end{tabular}


Table 4.3 Calculated strain values $\left(10^{-6}\right)$ at gage locations G3 2 and G3 6 (at the level of top surface of the bottom flange)

Location G3 2:

Load case

(1)

$\begin{array}{lr}\mathrm{a} & 0.069 \\ \mathrm{~b} & -1.622 \\ \mathrm{c} & -0.360 \\ \mathrm{~d} & -31.122 \\ \mathrm{e} & -39.577 \\ \mathrm{f} & -27.547 \\ \mathrm{~g} & -33.290 \\ \mathrm{~h} & -41.695 \\ \mathrm{i} & -29.295\end{array}$

(2)

$\begin{array}{rr}0.0685 & 0.359 \\ -1.598 & 1.528 \\ -0.354 & 1.642 \\ -30.762 & 1.172 \\ -38.981 & 1.528 \\ -27.096 & 1.664 \\ -32.905 & 1.171 \\ -41.068 & 1.528 \\ -28.816 & 1.664\end{array}$

(3)

.359

528

1.172

1.528

.664

1.528

Location G3 6:

Load case

$\begin{array}{lr}\mathrm{a} & -0.036 \\ \mathrm{~b} & 3.653 \\ \mathrm{c} & 0.992 \\ \mathrm{~d} & 40.501 \\ \mathrm{e} & 89.116 \\ \mathrm{f} & 73.438 \\ \mathrm{~g} & 43.301 \\ \mathrm{~h} & 93.884 \\ \mathrm{i} & 78.084\end{array}$

(1)

$-0.036$

.653

0.992

89.116

3.438

3.884
(2)

$-0.036$

3.633

0.987

40.208

88.632

73.071

42.988

93.374

77.695
(3)

0.560

0.546

0.479

0.728

0.546

0.501

0.728

0.546

0.502

(1): Strains calculated by Eq. (4.22) [neglect the effect of $\Delta P$ ]

(2): Strains calculated by Eq. (4.8) [consider the effect of $\Delta P$ ]

(3): $\frac{(1)-(2)}{(2)} \times 100 \%$ 
Table 4.4 Estimated thermal stresses in the interior composite girder G3 due to assumed temperature variation

\begin{tabular}{ccccc} 
Longitudinal distance & \multicolumn{5}{c}{ Stresses in composite girder section (MPa) } \\
from bridge end (mm) & $\sigma_{\Delta T}^{C_{T O P}}$ & $\sigma_{\Delta T}^{C_{B O T}}$ & $\sigma_{\Delta T}^{S_{T O P}}$ & $\sigma_{\Delta T}^{S_{B O T}}$ \\
$35,275.0$ & -5.40 & 1.99 & 11.53 & 39.43 \\
$22,950.0$ & -4.58 & 2.26 & 13.13 & 21.28 \\
$17,637.5$ & -4.29 & 2.36 & 13.70 & 14.89 \\
$14,110.0$ & -4.10 & 2.43 & 14.07 & 10.66 \\
0.0 & -3.33 & 2.68 & 15.57 & -6.28
\end{tabular}

Note:

$\sigma_{\Delta T}^{C_{T O P}}:$ thermal stress at the level of the top surface of the concrete deck $\sigma_{\Delta T}^{C_{B O T}}:$ thermal stress at the level of the bottom surface of the concrete deck $\sigma_{\Delta T}^{S_{T O P}}:$ thermal stress at the level of the top surface of the top steel flange $\sigma_{\Delta T}^{S_{B O T}}:$ thermal stress at the level of the bottom surface of the bottom steel flange 


\section{Loading type}

weight of steel girder

weight of concrete slab

post-tensioning force

superimposed dead load

live load

increment in tendon force due to load applied after post-tensioning
Section to carry load

Strain distribution
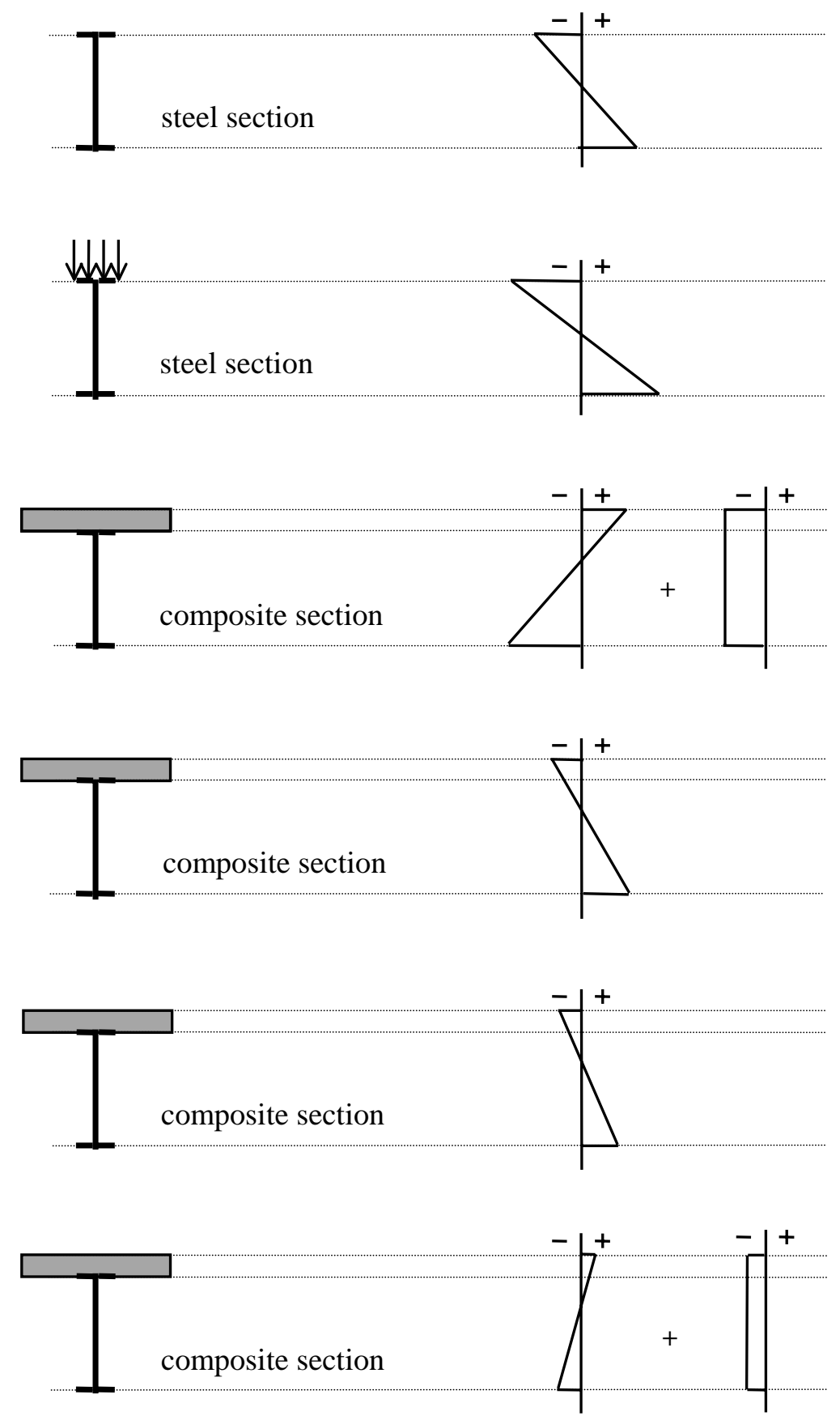

Figure 4.1 Strain distributions in simplified beam model (in positive moment region). 

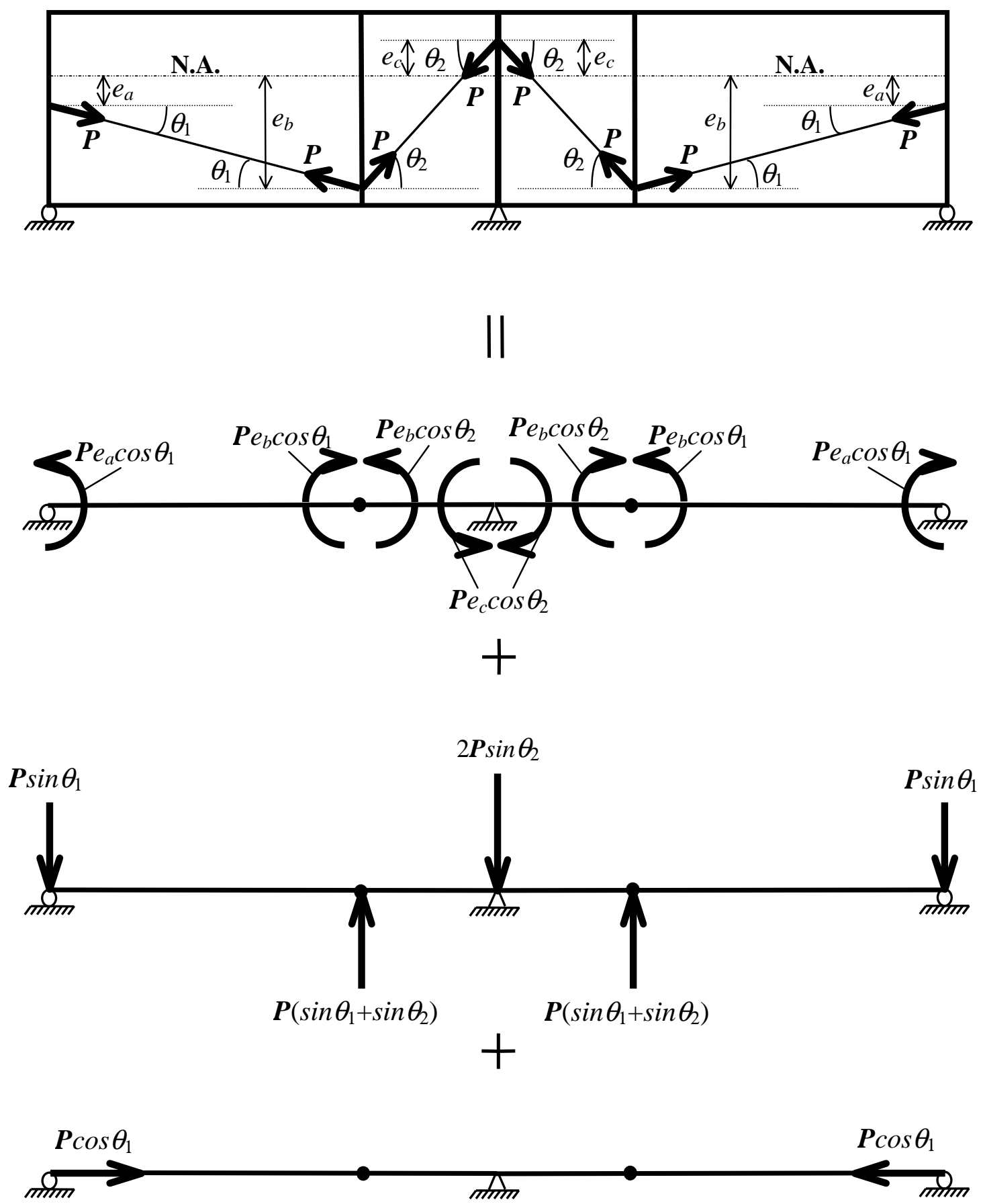

Figure 4.2 Equivalent forces and moments produced by post-tensioning force. 

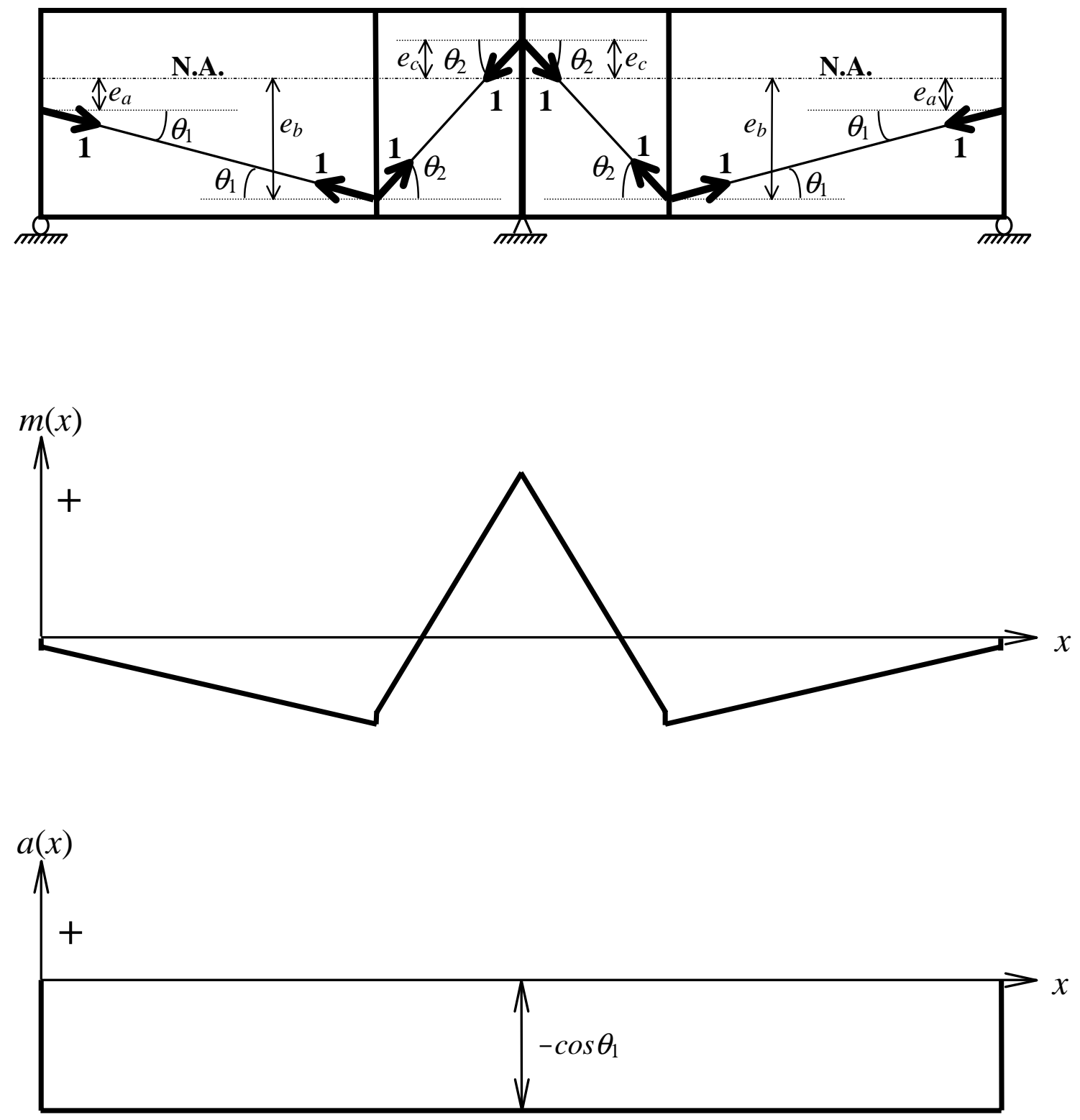

Figure 4.3 Functions of moment $m(x)$ and axial force $a(x)$ due to unit post-tensioning force. 


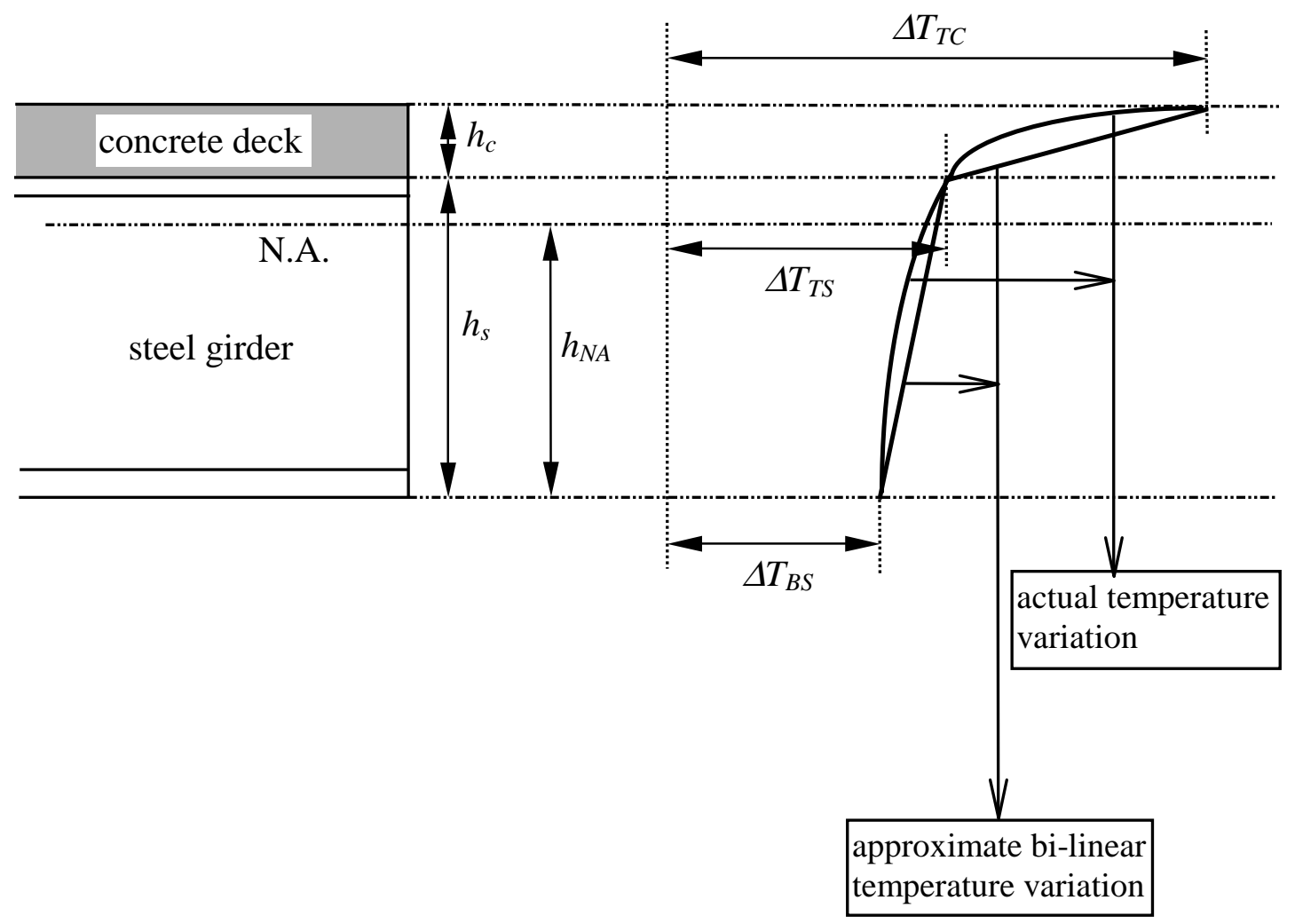

Figure 4.4 Typical pattern of temperature variation along composite girder section. 

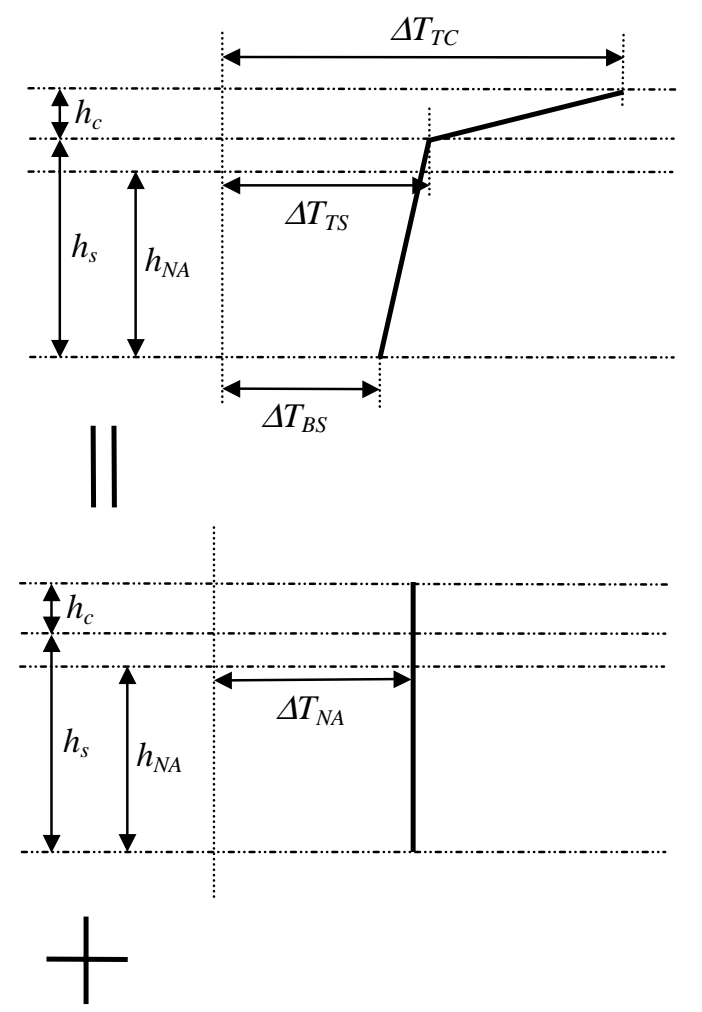

$$
\Delta T_{N A}=\frac{\Delta T_{T S} \times h_{N A}+\Delta T_{B S} \times\left(h_{s}-h_{N A}\right)}{h_{s}}
$$

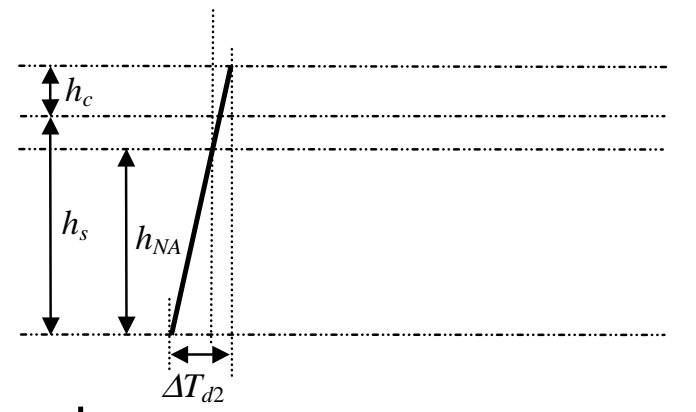

$$
\Delta T_{d 2}=\left(\Delta T_{T S}-\Delta T_{B S}\right) \times \frac{h_{s}+h_{c}}{h_{s}}
$$
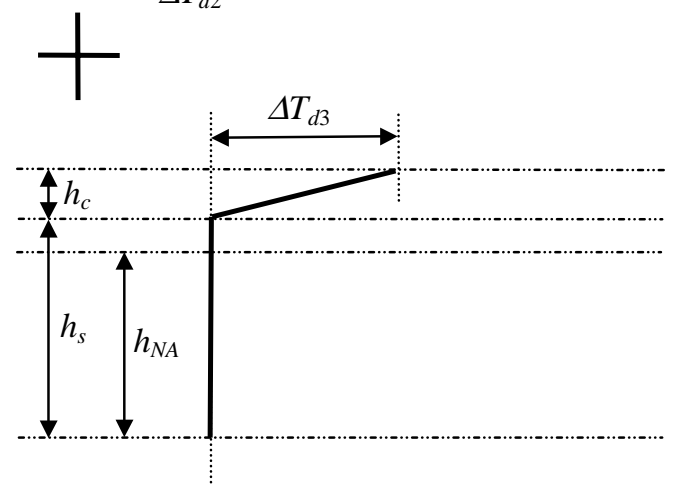

$\Delta T_{d 3}=\Delta T_{T C}-\Delta T_{B S}-\left(\Delta T_{T S}-\Delta T_{B S}\right) \times \frac{h_{s}+h_{c}}{h_{s}}$

Figure 4.5 Decomposition of approximate bi-linear temperature variation. 


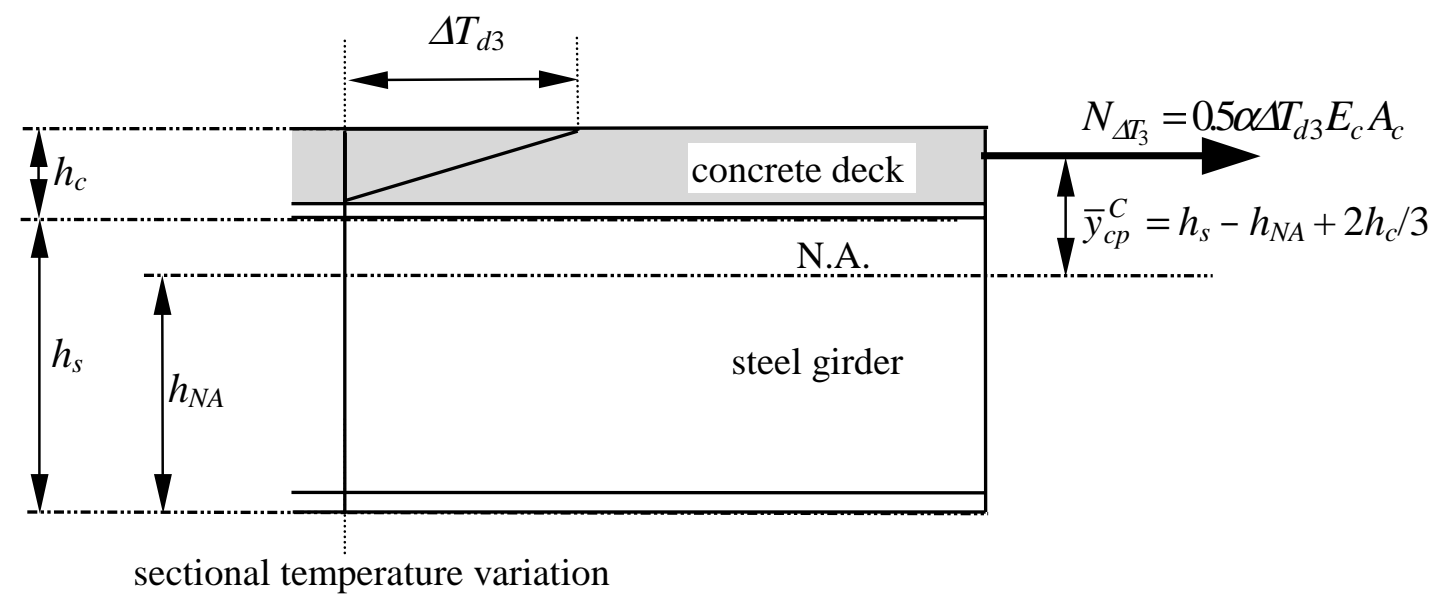

(a) Eccentrically applied axial force.

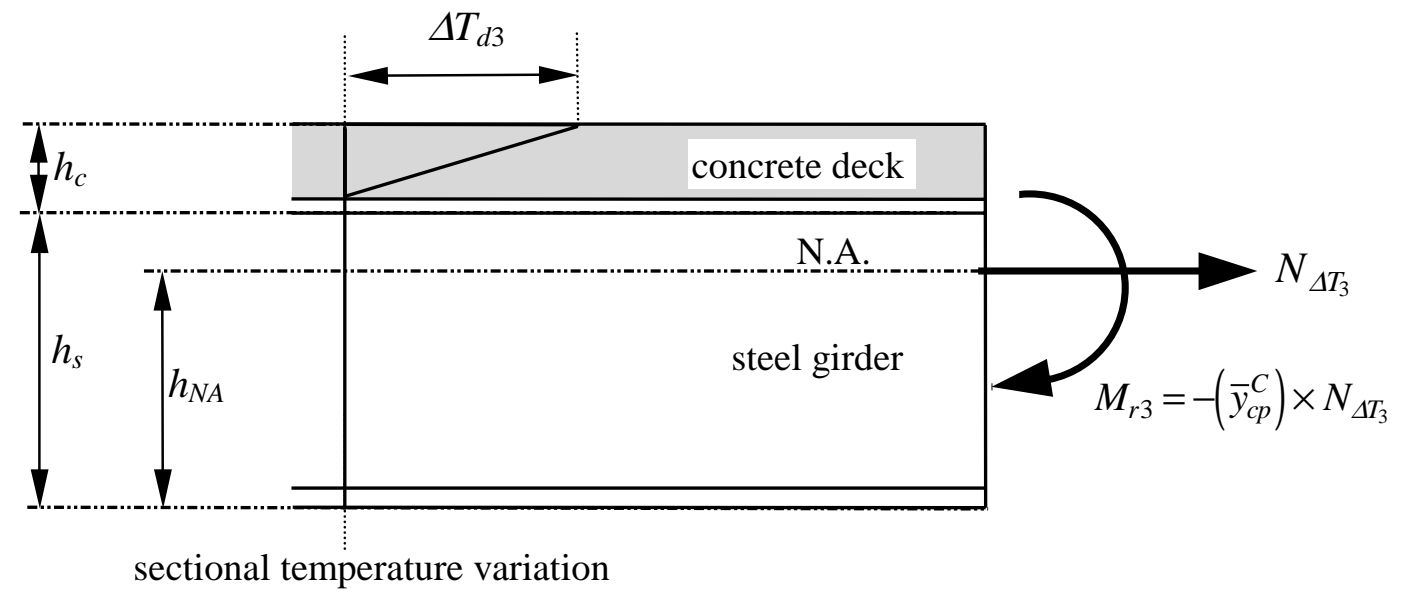

(b) Equivalent axial force and bending moment.

Figure 4.6 Mechanical effect of non-uniform temperature variation in concrete deck. 


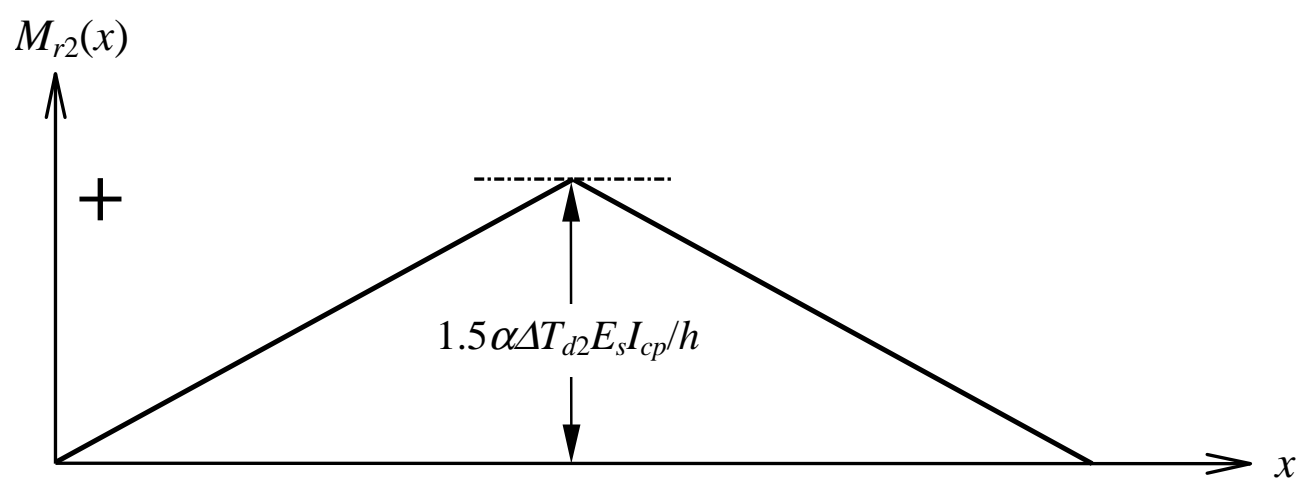

(a)

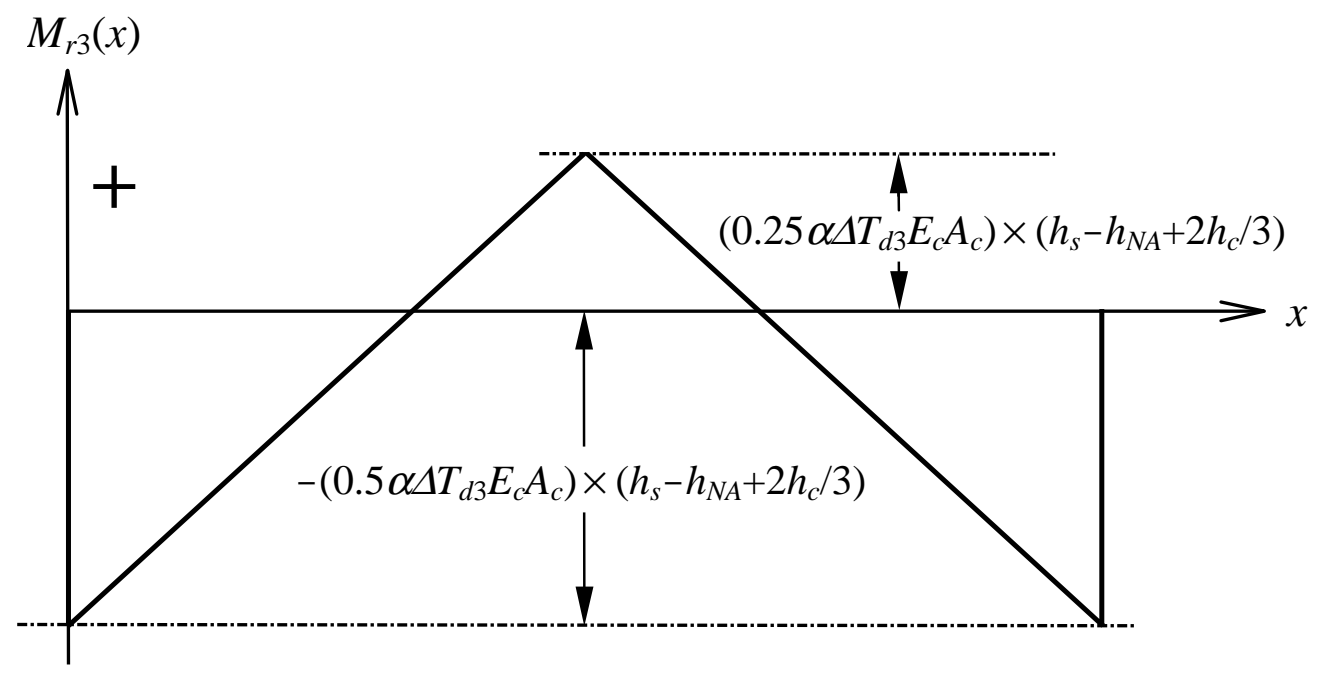

(b)

Figure 4.7 Response moments due to non-uniform temperature variation. 


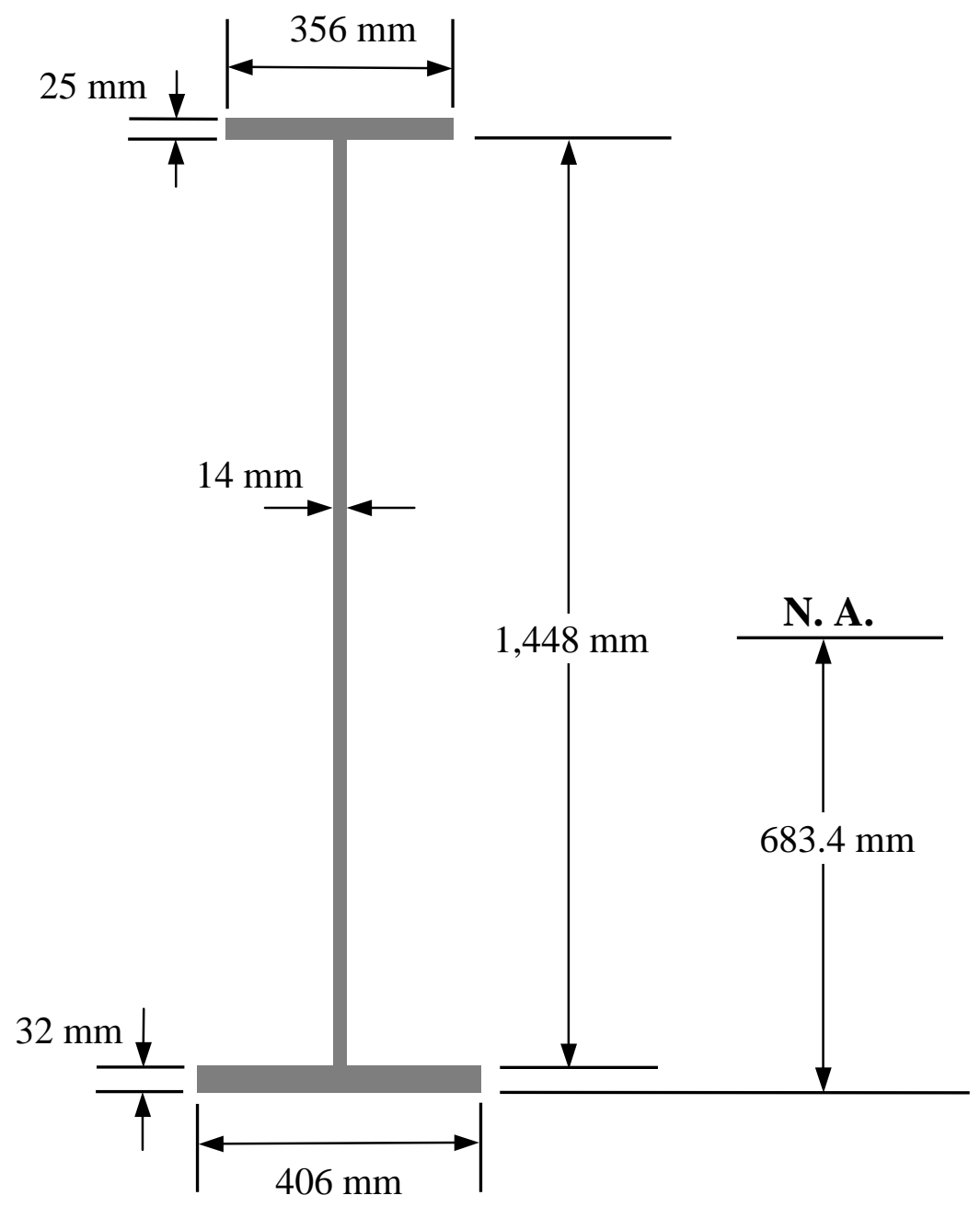

sectional properties:

$$
\begin{aligned}
A_{s} & =42,164 \mathrm{~mm}^{2} \\
I_{s} & =1.52637 \times 10^{10} \mathrm{~mm}^{4} \\
\bar{y}_{s} & =683.4 \mathrm{~mm}
\end{aligned}
$$

material properties:

$$
E_{s}=200 \mathrm{kN} / \mathrm{mm}^{2}
$$

Figure 4.8 Steel girder section. 


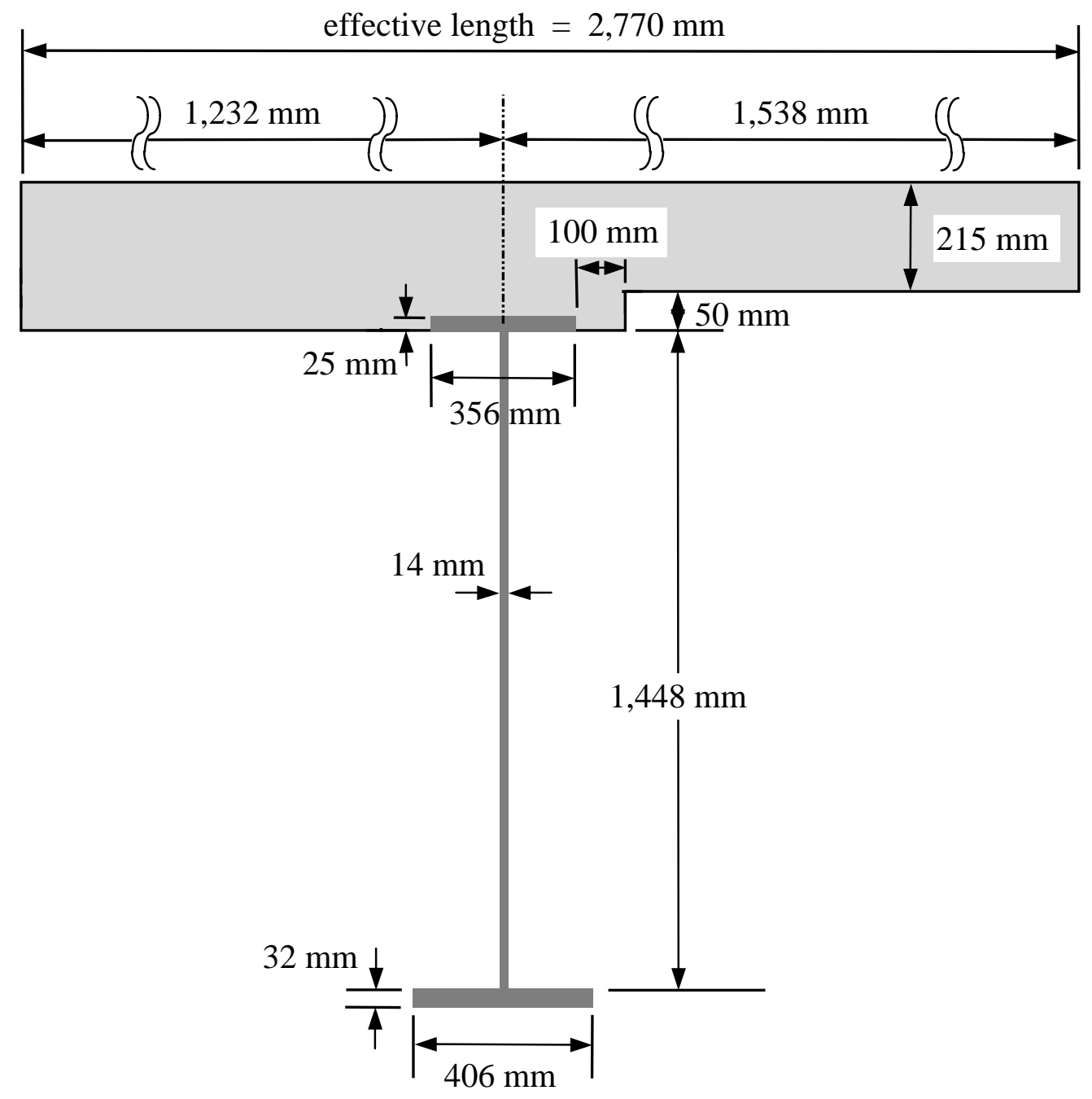

(a) Exterior girder G1.

Figure 4.9 Composite girder section. 


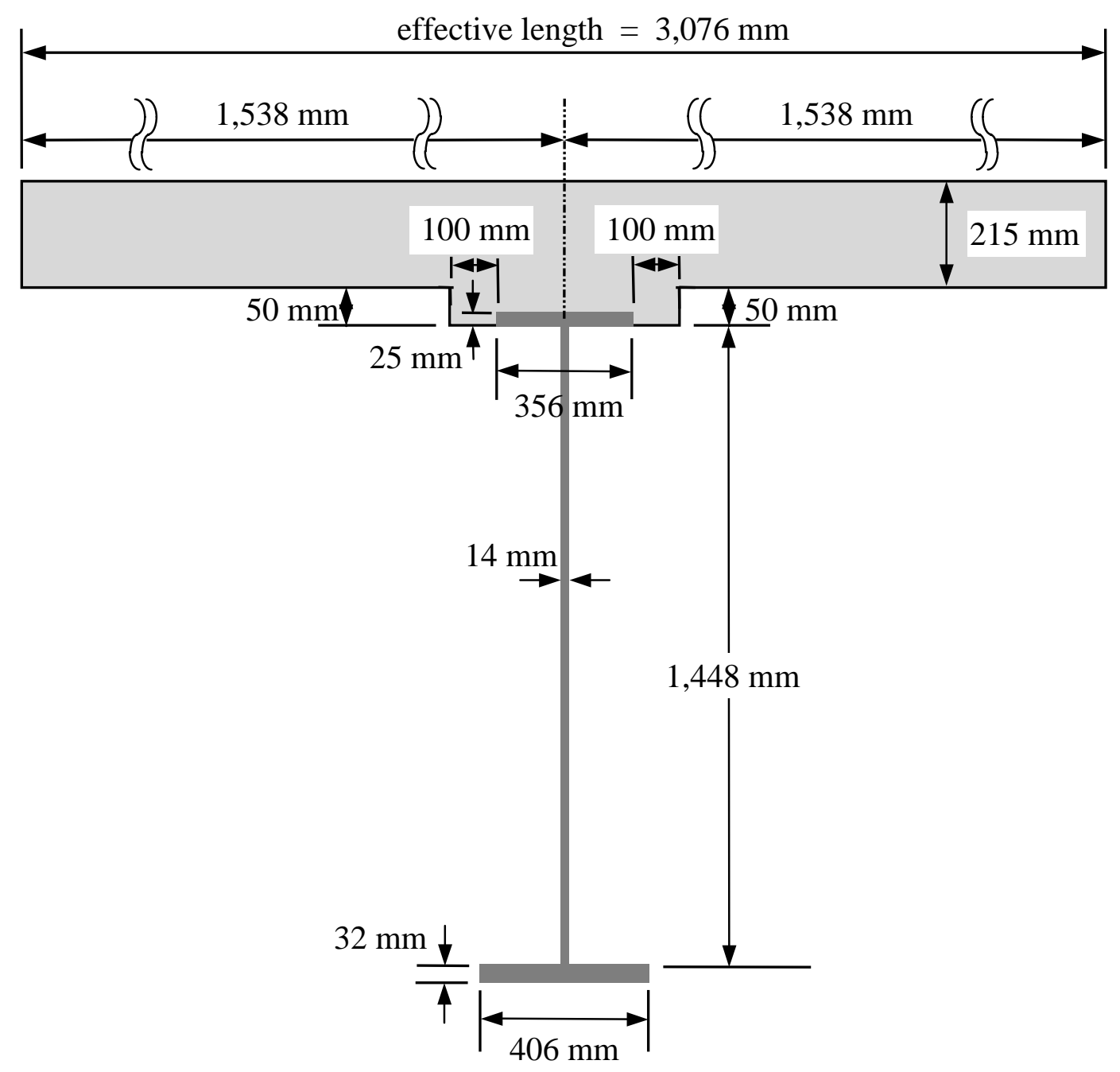

(b) Interior girder G3.

Figure 4.9 Composite girder section. 


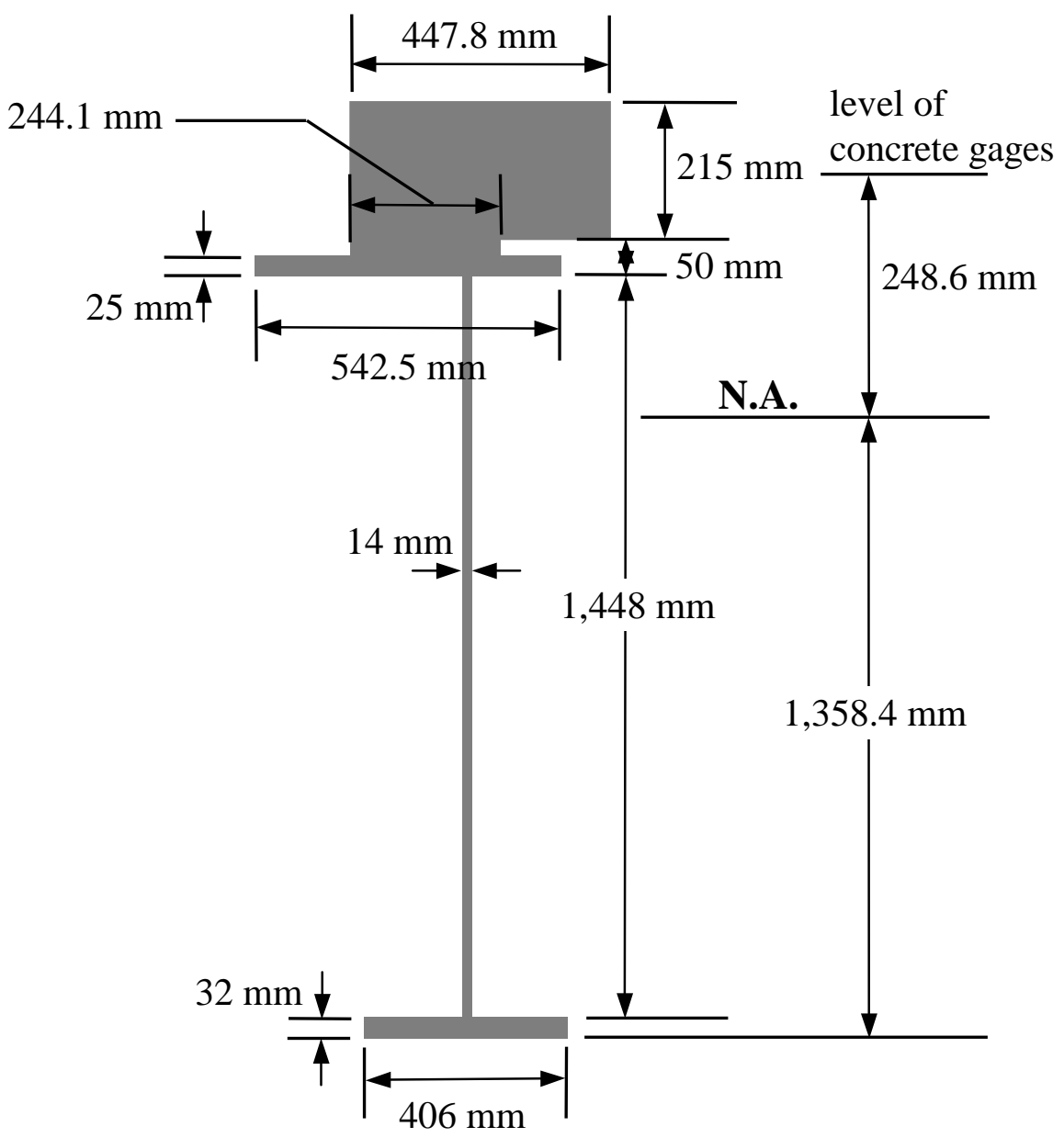

sectional properties:

material properties:

$$
\begin{aligned}
A_{c p} & =149,200 \mathrm{~mm}^{2} \\
I_{c p} & =4.25810 \times 10^{10} \mathrm{~mm}^{4} \\
\bar{y}_{c p} & =1,358.4 \mathrm{~mm}
\end{aligned}
$$$$
E_{s}=200 \mathrm{kN} / \mathrm{mm}^{2}
$$$$
E_{c}=32.32 \mathrm{kN} / \mathrm{mm}^{2}
$$$$
n=6.19
$$

(a) Exterior girder G1.

Figure 4.10 Transformed steel section (with $f_{c}^{\prime}=58.61 \mathrm{MPa}$ ). 


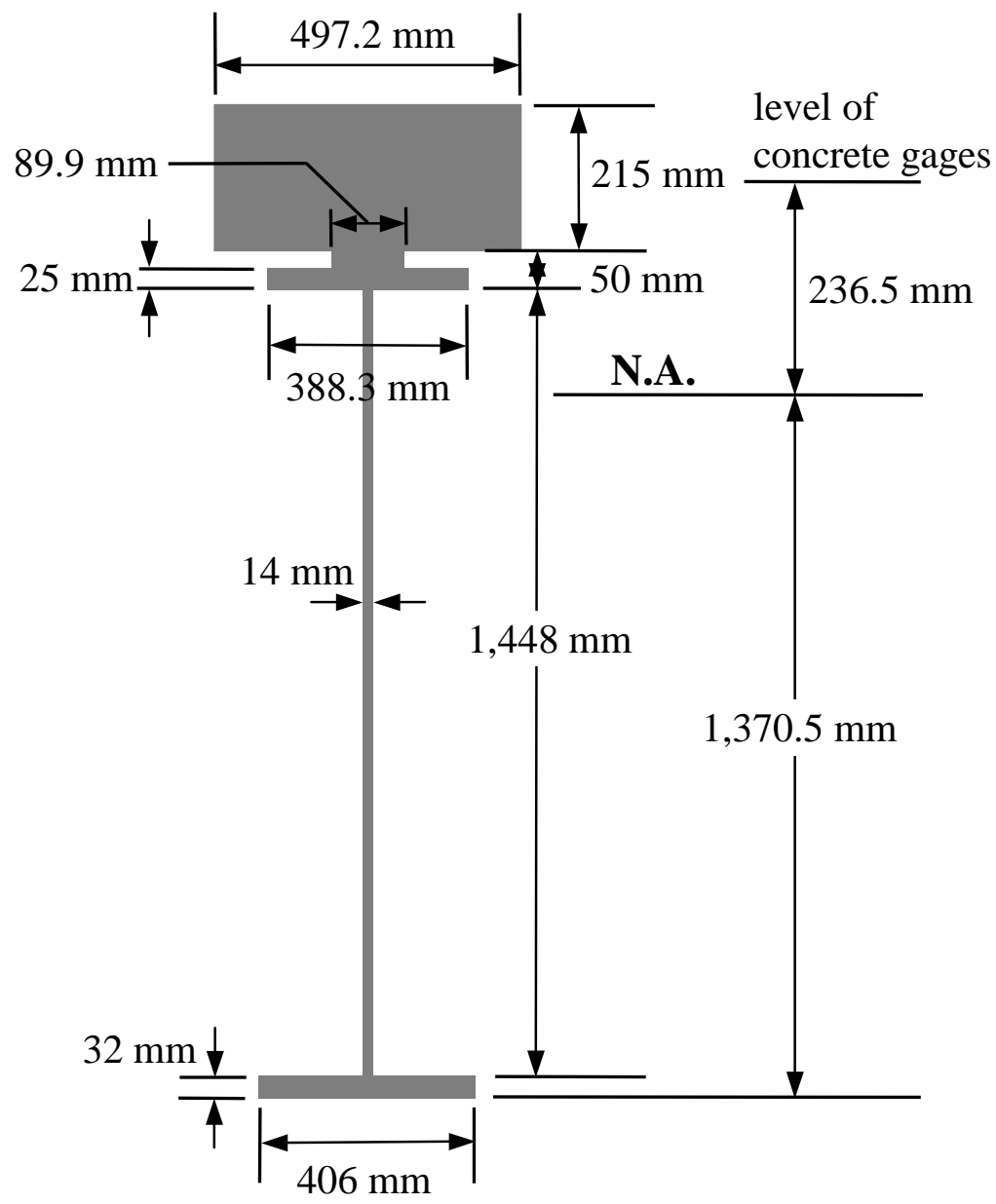

sectional properties:

material properties:

$$
\begin{aligned}
A_{c p} & =152,124 \mathrm{~mm}^{2} \\
I_{c p} & =4.32607 \times 10^{10} \mathrm{~mm}^{4} \\
\bar{y}_{c p} & =1,370.5 \mathrm{~mm}
\end{aligned}
$$$$
E_{s}=200 \mathrm{kN} / \mathrm{mm}^{2}
$$$$
E_{c}=32.32 \mathrm{kN} / \mathrm{mm}^{2}
$$$$
n=6.19
$$

(b) Interior girder G3.

Figure 4.10 Transformed steel section (with $f_{c}^{\prime}=58.61 \mathrm{MPa}$ ). 


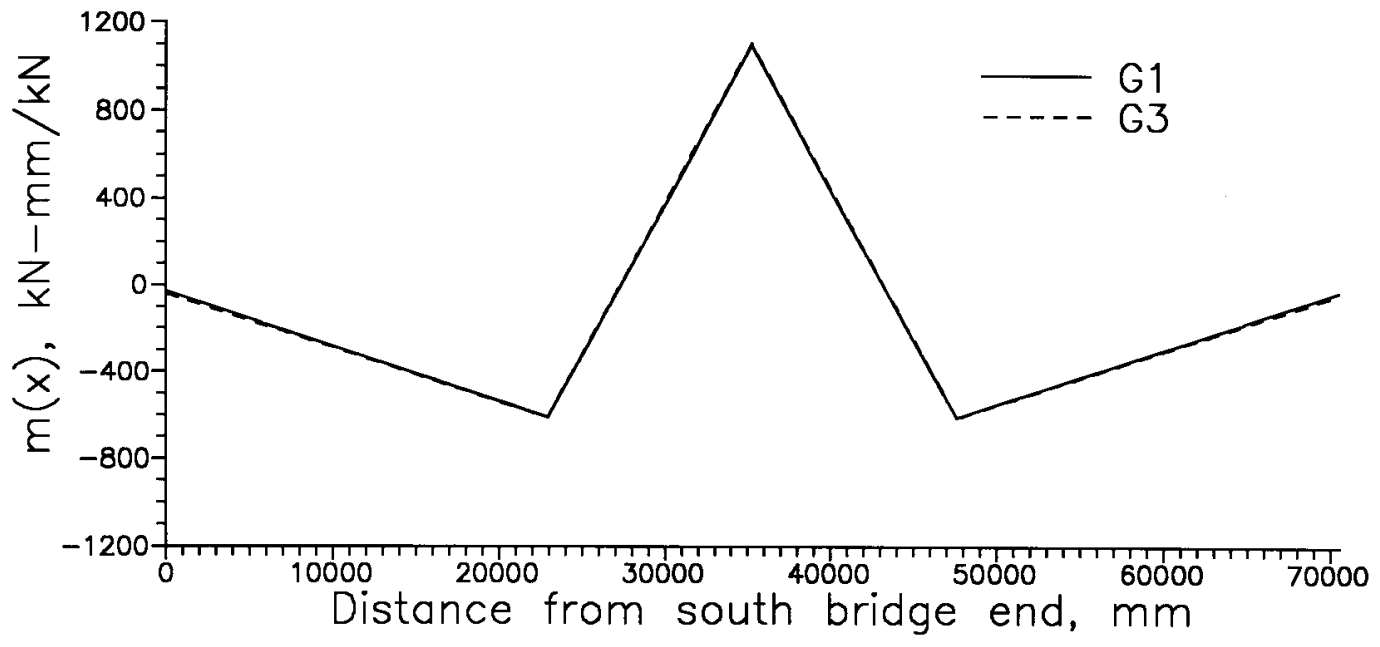

(a) Unit moment function

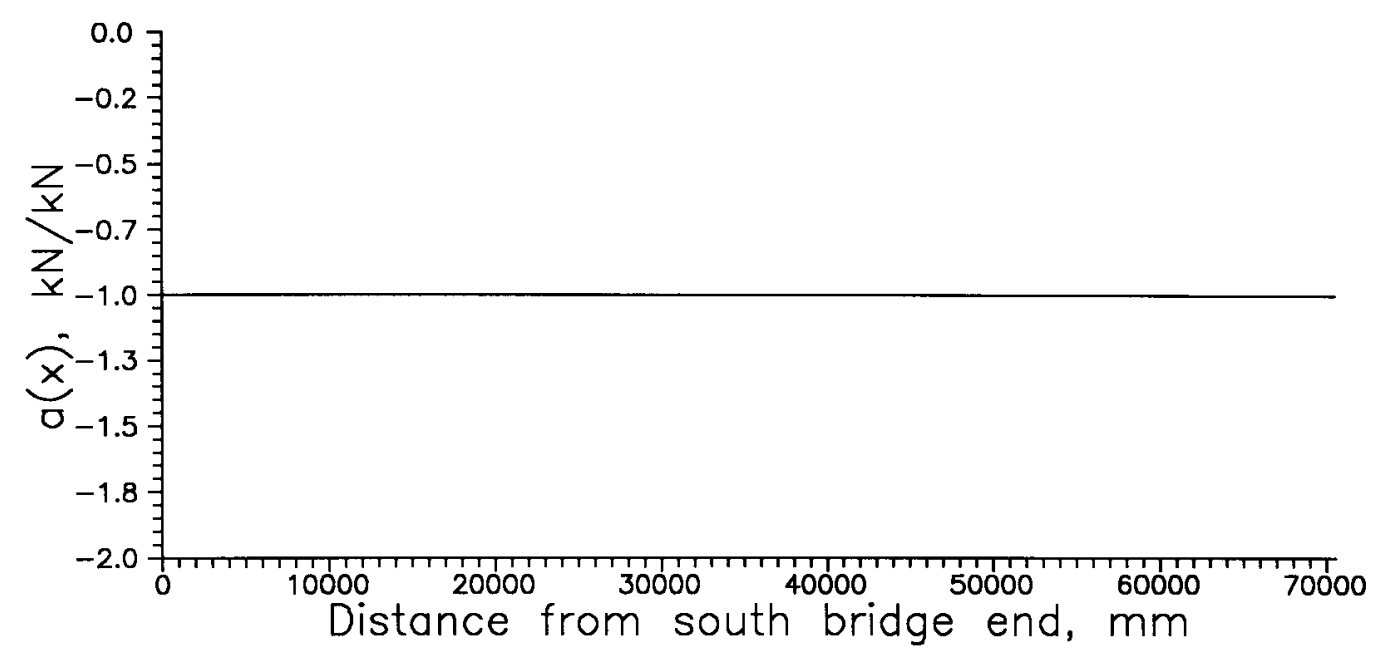

(b) Unit axial force function

Figure 4.11 Unit moment and axial force functions (when $f_{c}^{\prime}=58.61 \mathrm{MPa}$ ). 


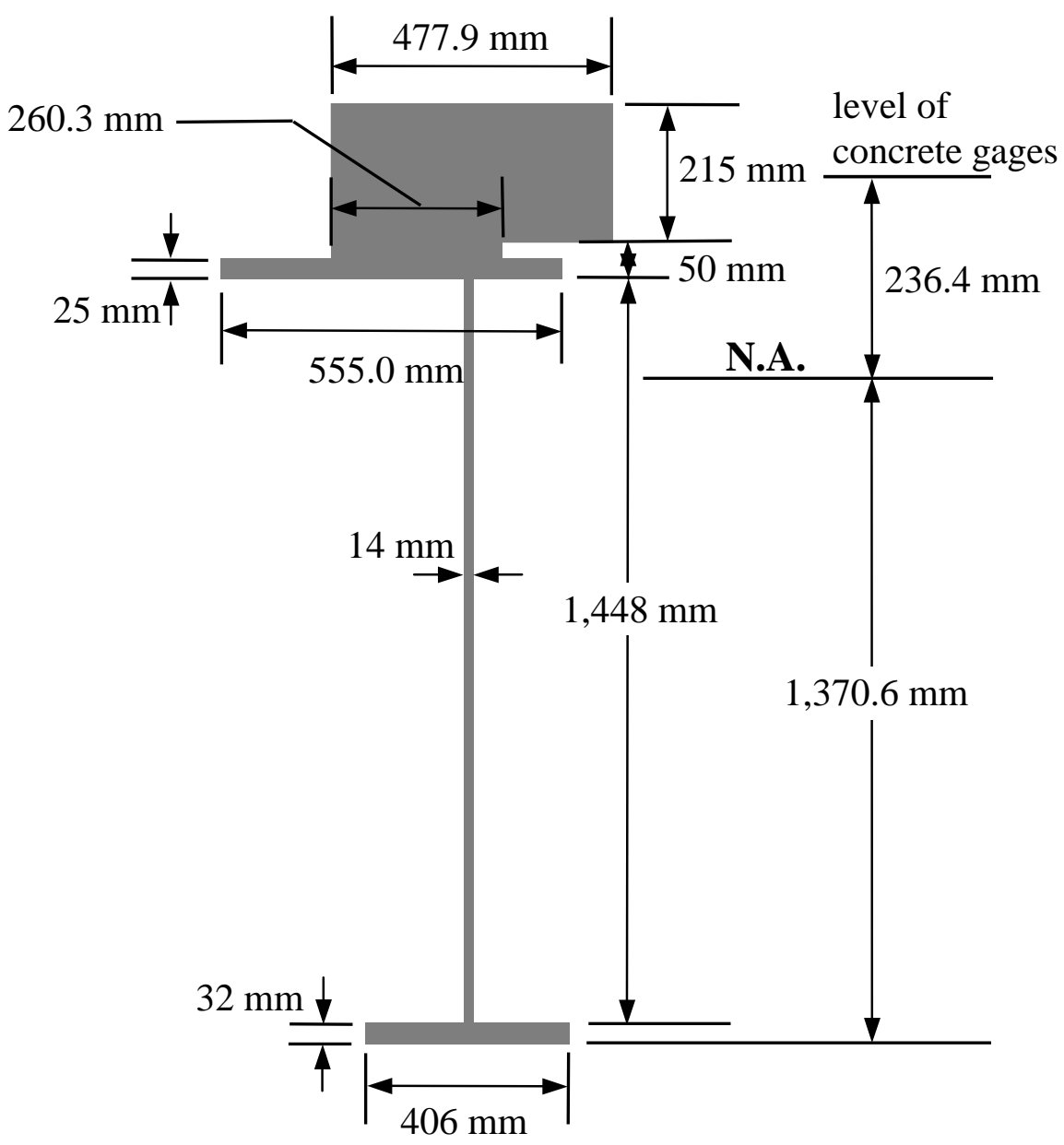

sectional properties:

material properties:

$$
\begin{aligned}
A_{c p} & =156,328 \mathrm{~mm}^{2} \\
I_{c p} & =4.30979 \times 10^{10} \mathrm{~mm}^{4} \\
\bar{y}_{c p} & =1,370.6 \mathrm{~mm}
\end{aligned}
$$$$
E_{s}=200 \mathrm{kN} / \mathrm{mm}^{2}
$$$$
E_{c}=34.47 \mathrm{kN} / \mathrm{mm}^{2}
$$$$
n=5.80
$$

(a) Exterior girder G1.

Figure 4.12 Transformed steel section (with $f_{c}^{\prime}=68.95 \mathrm{MPa}$ ). 


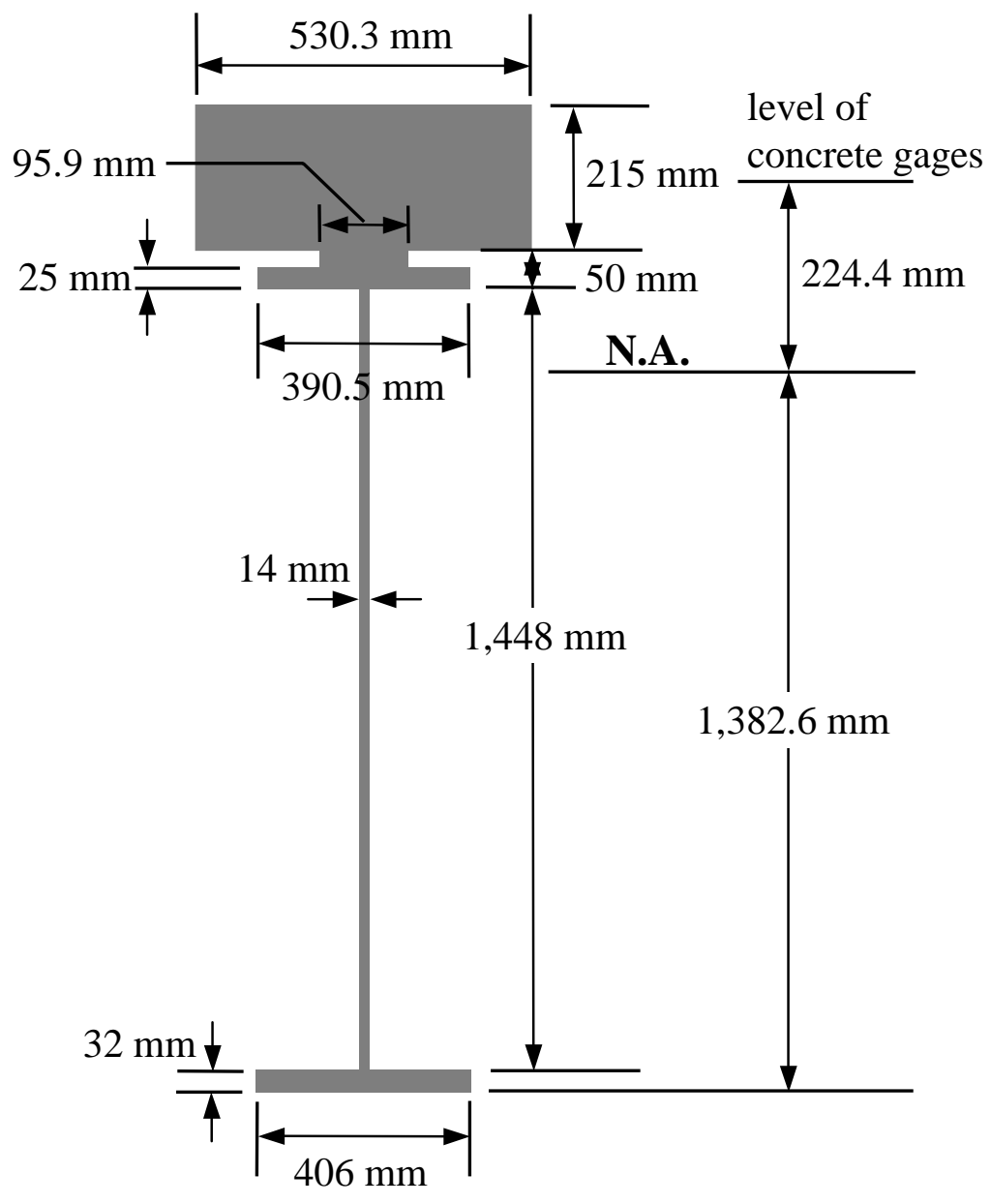

sectional properties:

material properties:

$$
\begin{aligned}
A_{c p} & =159,447 \mathrm{~mm}^{2} \\
I_{c p} & =4.37763 \times 10^{10} \mathrm{~mm}^{4} \\
\bar{y}_{c p} & =1,382.6 \mathrm{~mm}
\end{aligned}
$$$$
E_{s}=200 \mathrm{kN} / \mathrm{mm}^{2}
$$$$
E_{c}=34.47 \mathrm{kN} / \mathrm{mm}^{2}
$$$$
n=5.80
$$

(b) Interior girder G3.

Figure 4.12 Transformed steel section (with $f_{c}^{\prime}=68.95 \mathrm{MPa}$ ). 


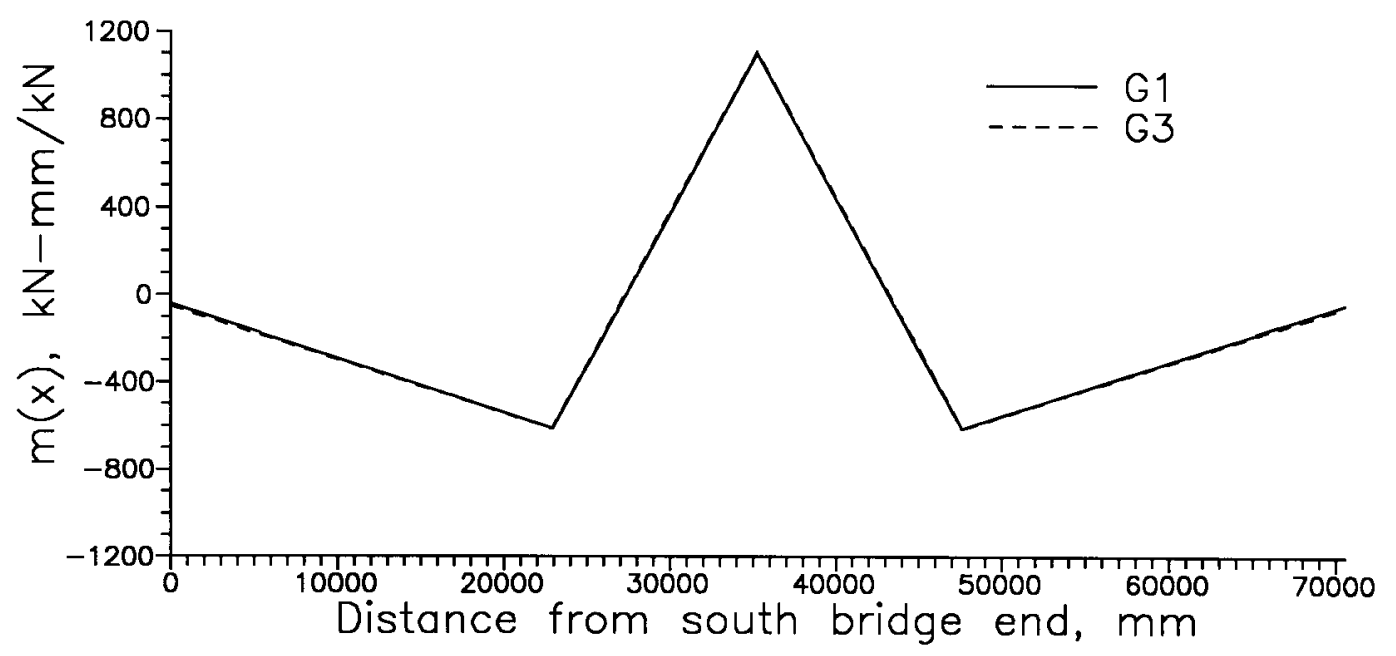

(a) Unit moment function

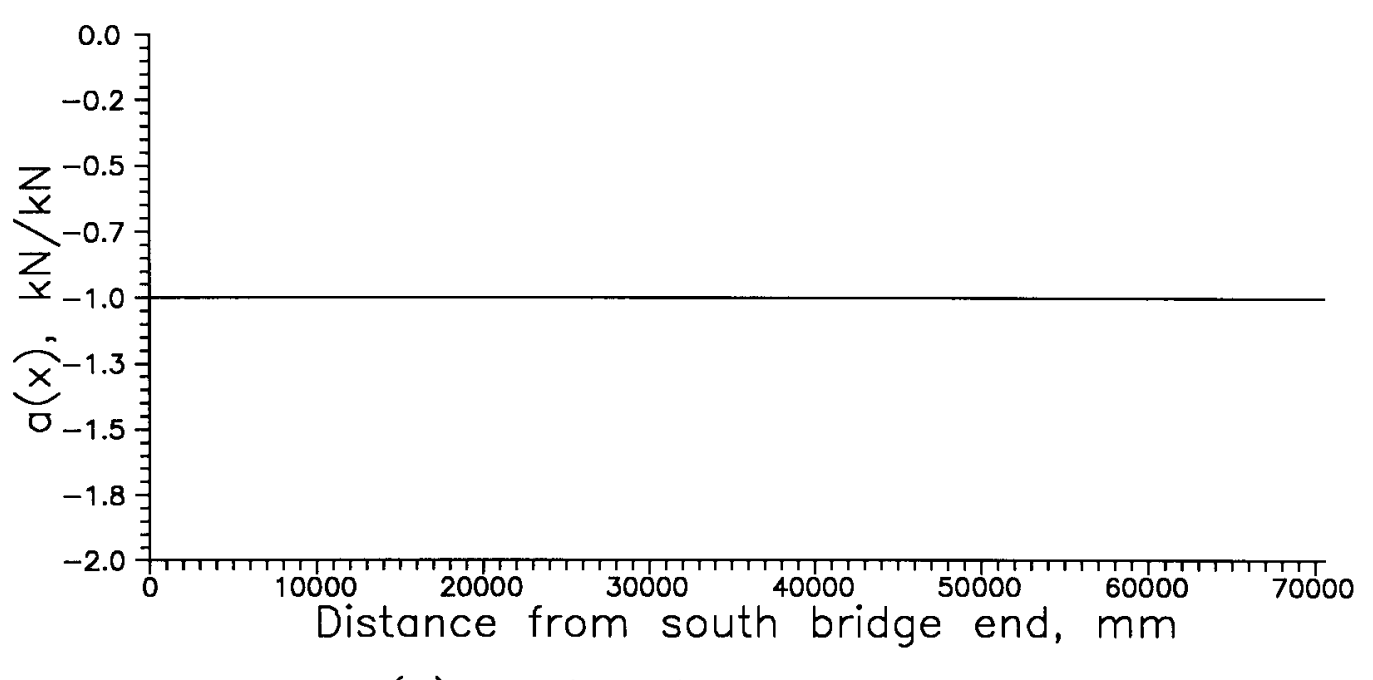

(b) Unit axial force function

Figure 4.13 Unit moment and axial force functions (when $f_{c}^{\prime}=68.95 \mathrm{MPa}$ ). 

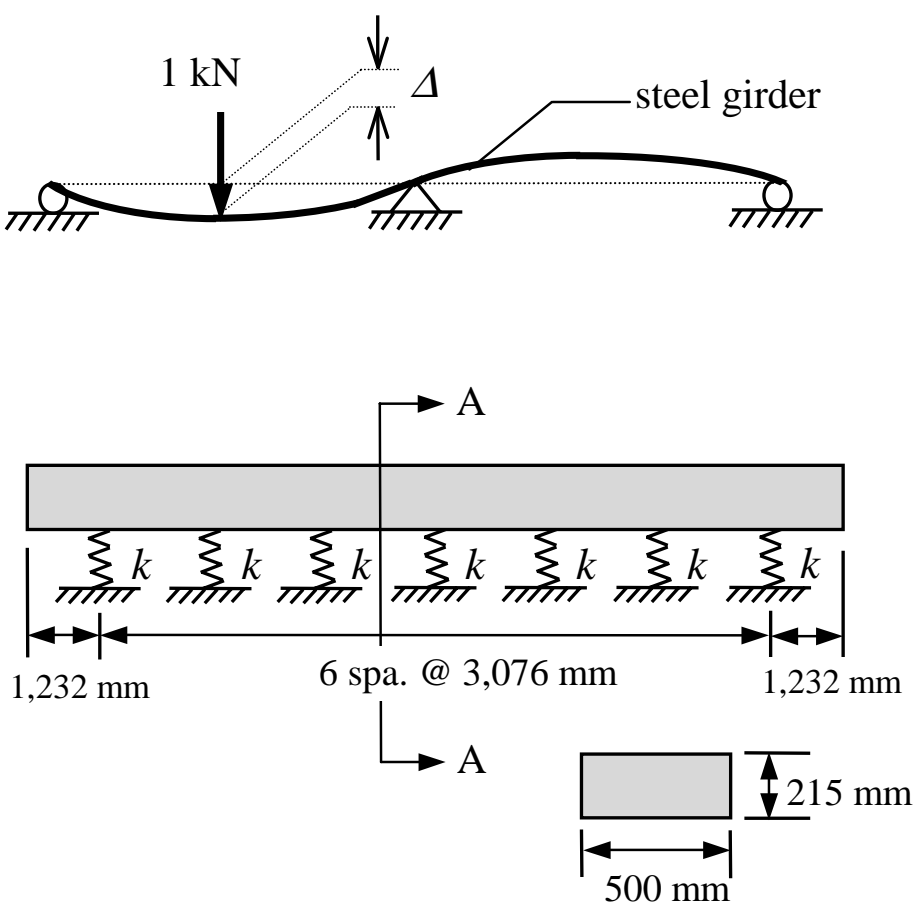

$\underline{\text { Section A-A }}$
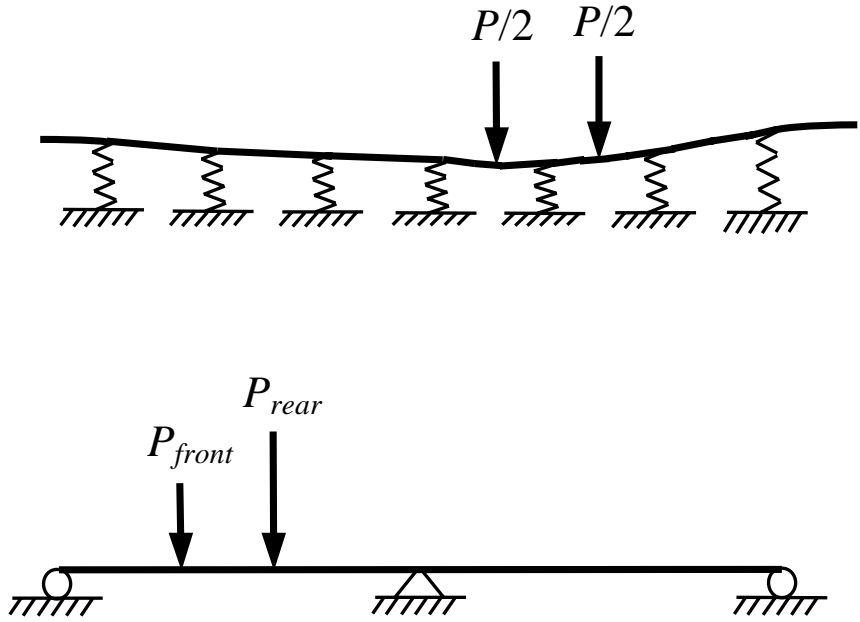

(1) Find spring constant by applying unit concentrated force at longitudinal axle (front or rear) location: $k=1 / \Delta$

(2) Model the equivalent transverse beam for the designated longitudinal location.
(3) Apply the axle loads (front or rear) to the transverse beam to find the reaction forces in the springs which are regarded as the forces distributed to the girders.

(4) Employ the procedure (1) through to (3) for each longitudinal axle position. Accumulate the computed spring forces.

Figure 4.14 Procedure to determine distributed axle loads for girders. 


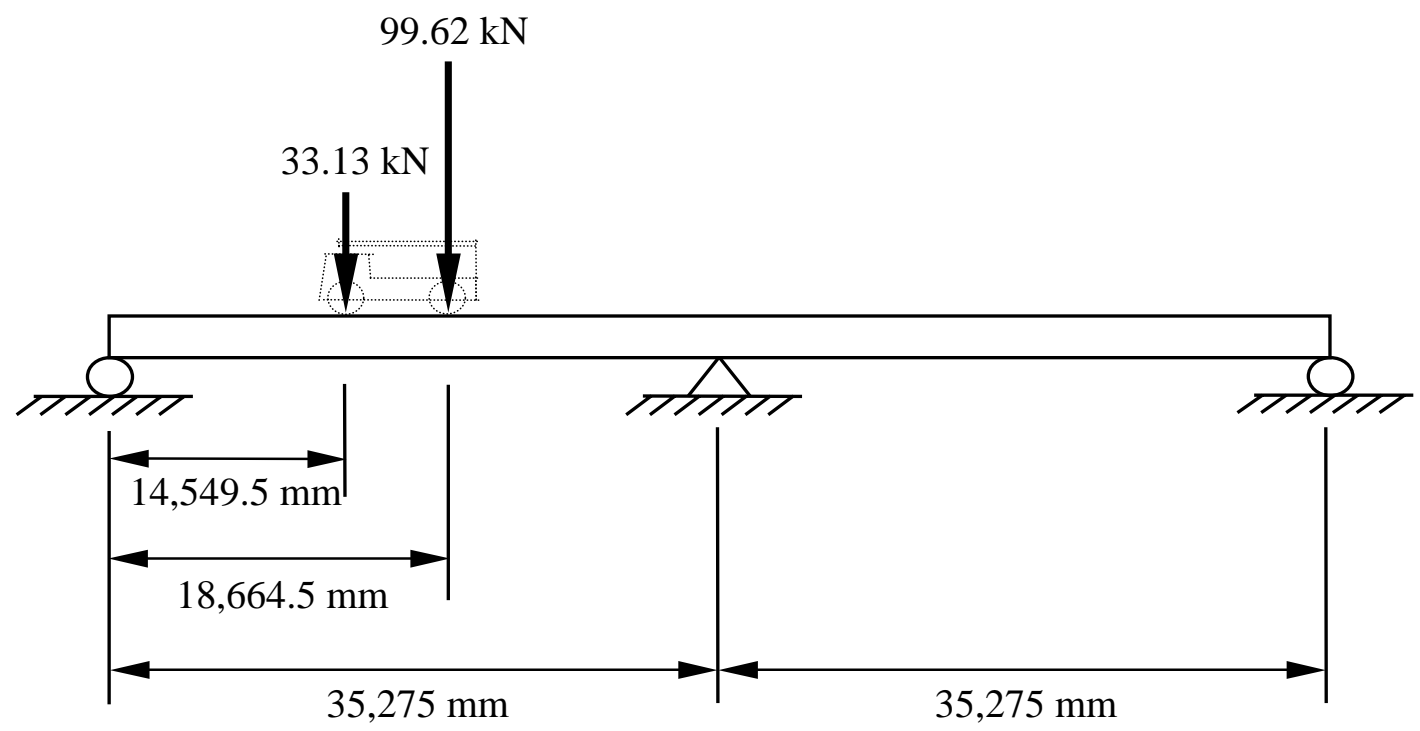

(a) Load case $\langle$ b $>$ for girder G1.

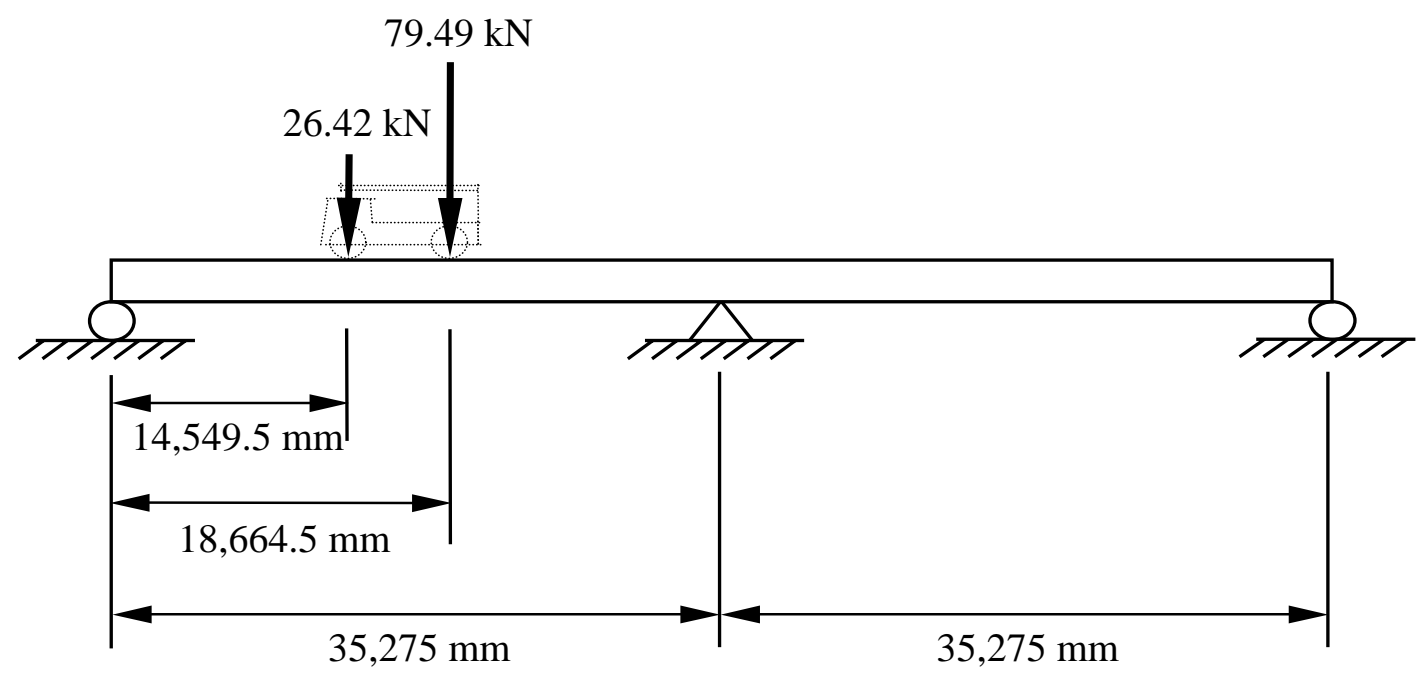

(b) Load case $<\mathrm{h}>$ for girder G3.

Figure 4.15 Distributed axle loads on girders G1 and G3. 


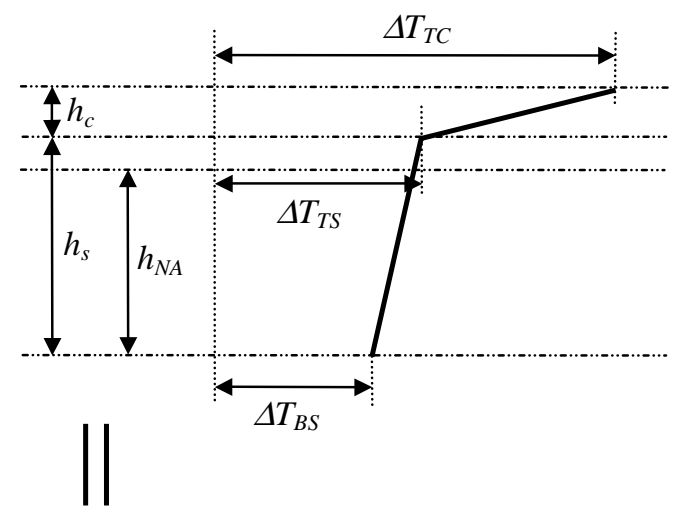

$$
\begin{aligned}
\Delta T_{T C} & =40^{\circ} \mathrm{C} \\
\Delta T_{T S} & =20^{\circ} \mathrm{C} \\
\Delta T_{B S} & =15^{\circ} \mathrm{C}
\end{aligned}
$$

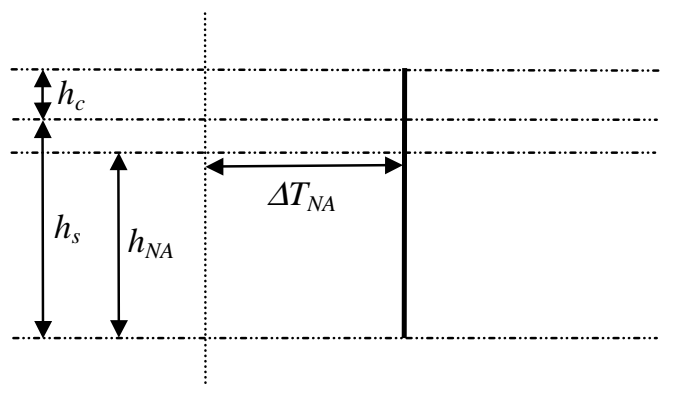

$$
\Delta T_{N A}=19.59^{\circ} \mathrm{C}
$$
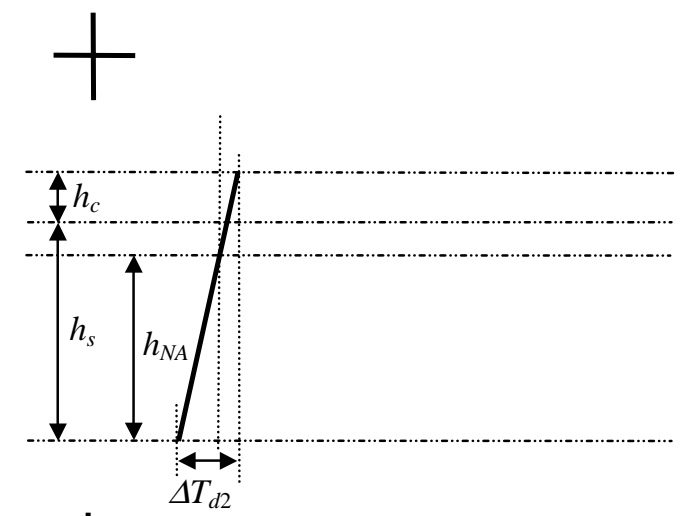

$$
\Delta T_{d 2}=5.80^{\circ} \mathrm{C}
$$
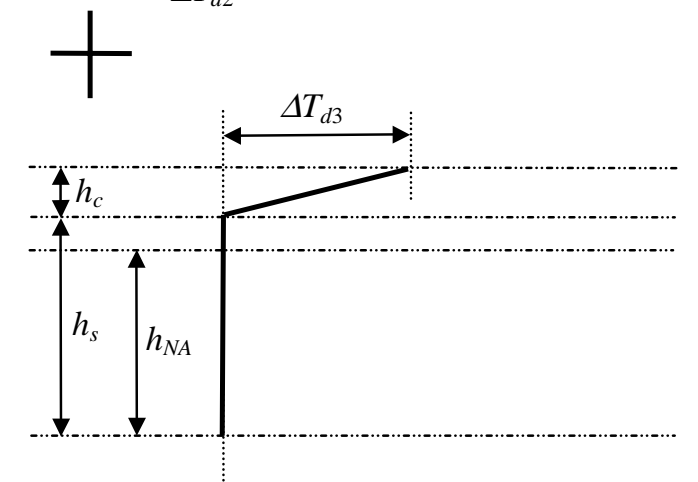

$\Delta T_{d 3}=19.20^{\circ} \mathrm{C}$

Figure 4.16 Assumed bi-linear temperature variation in composite section of girder G3. 


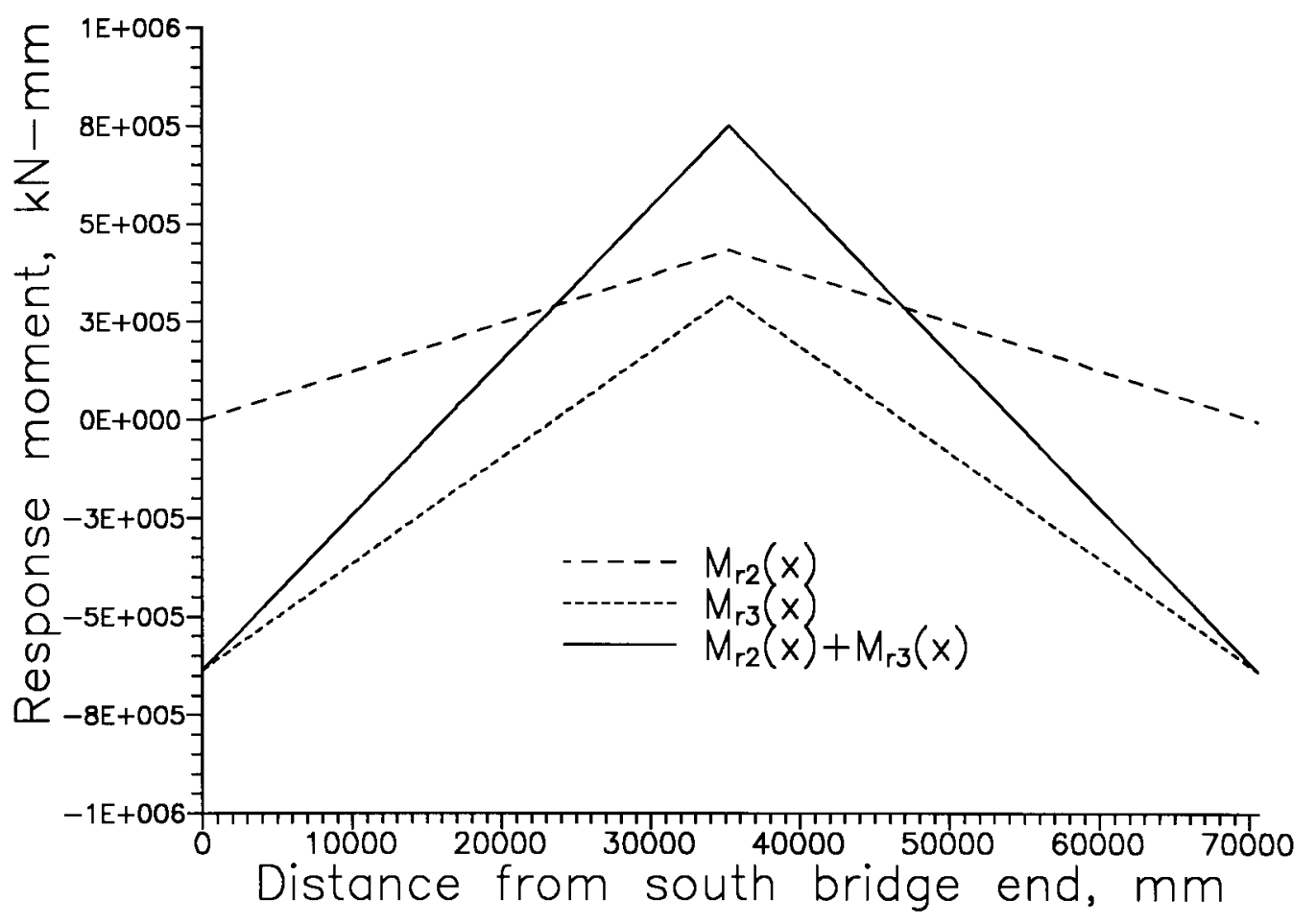

Figure 4.17 Response moments due to assumed temperature variation. 


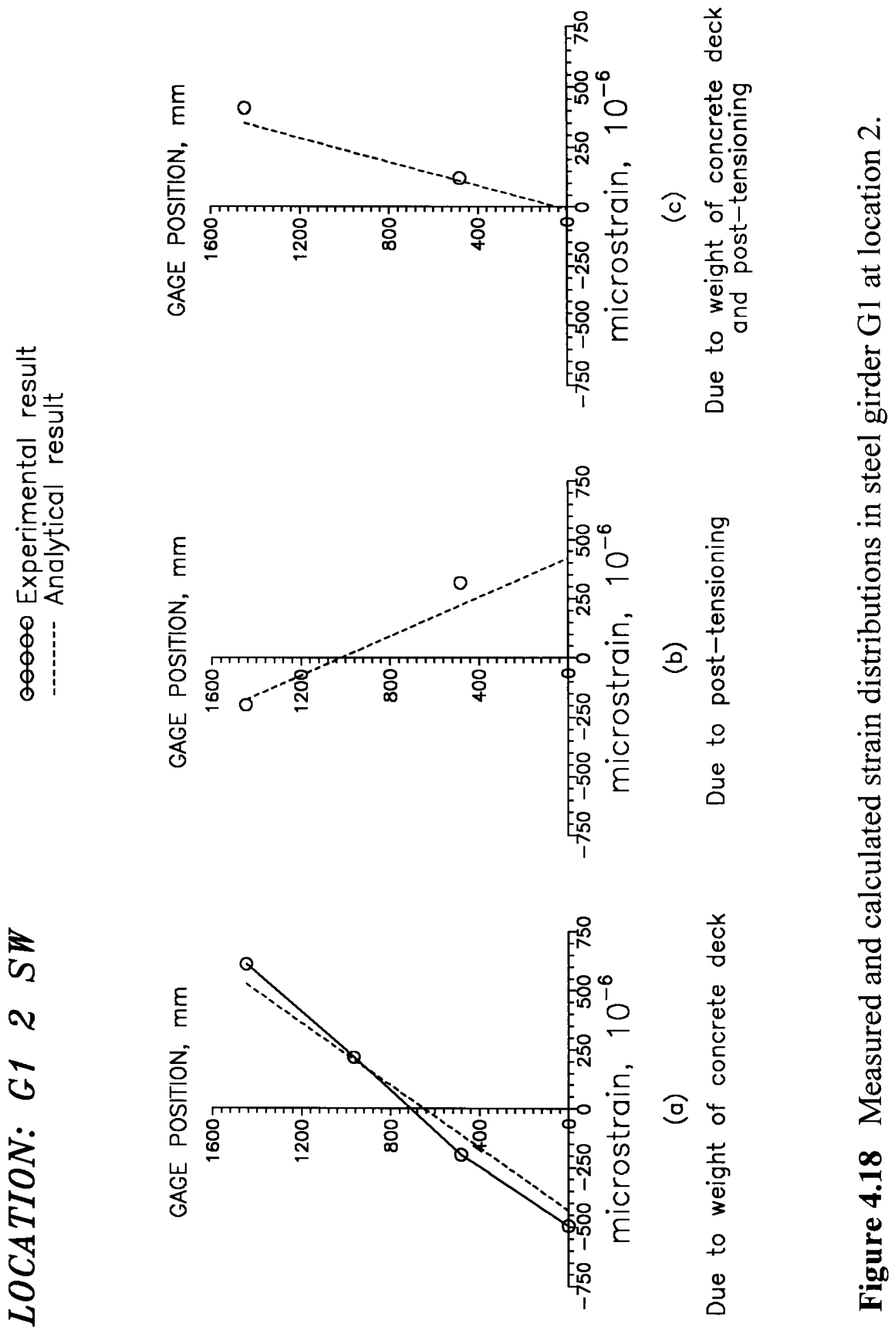




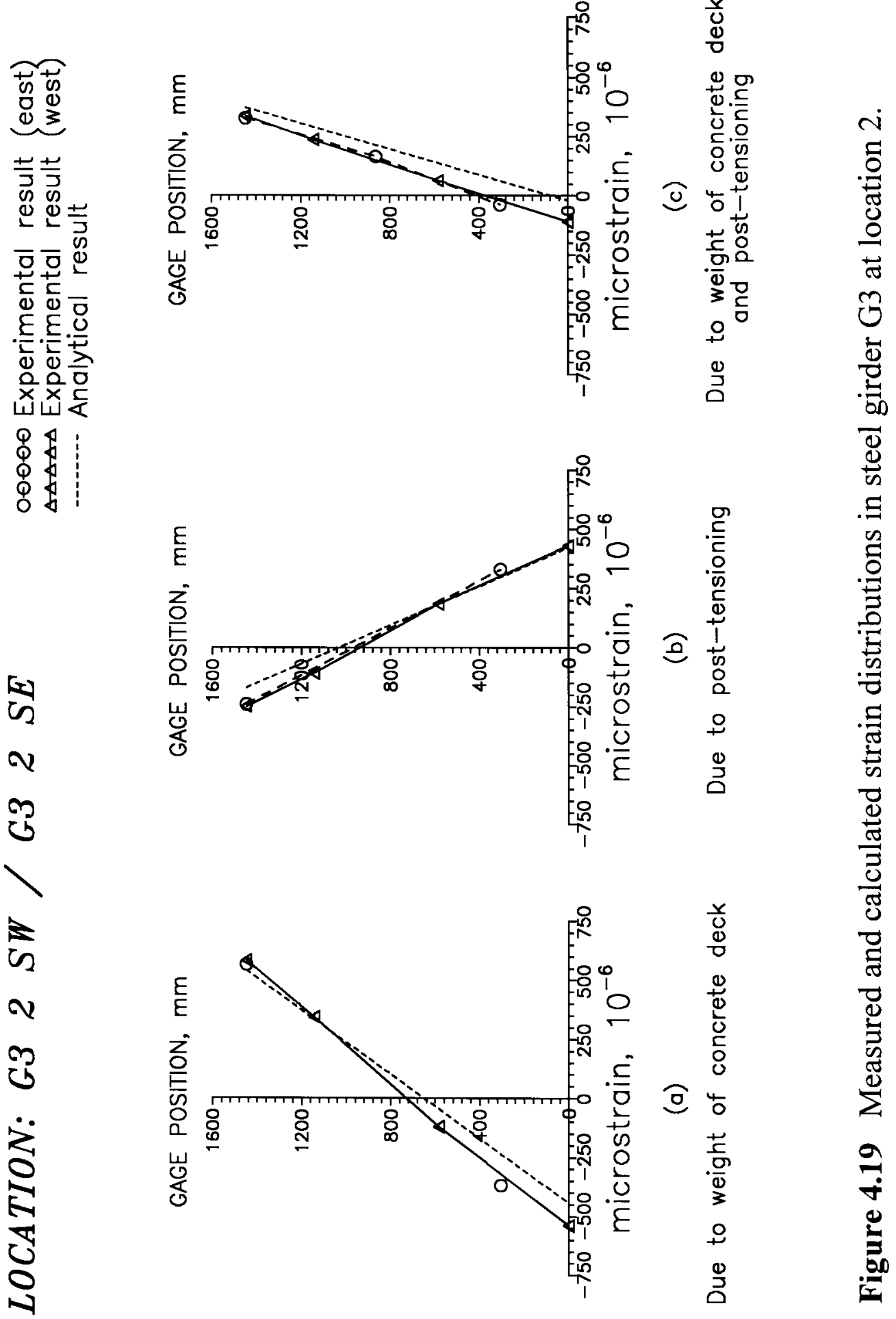




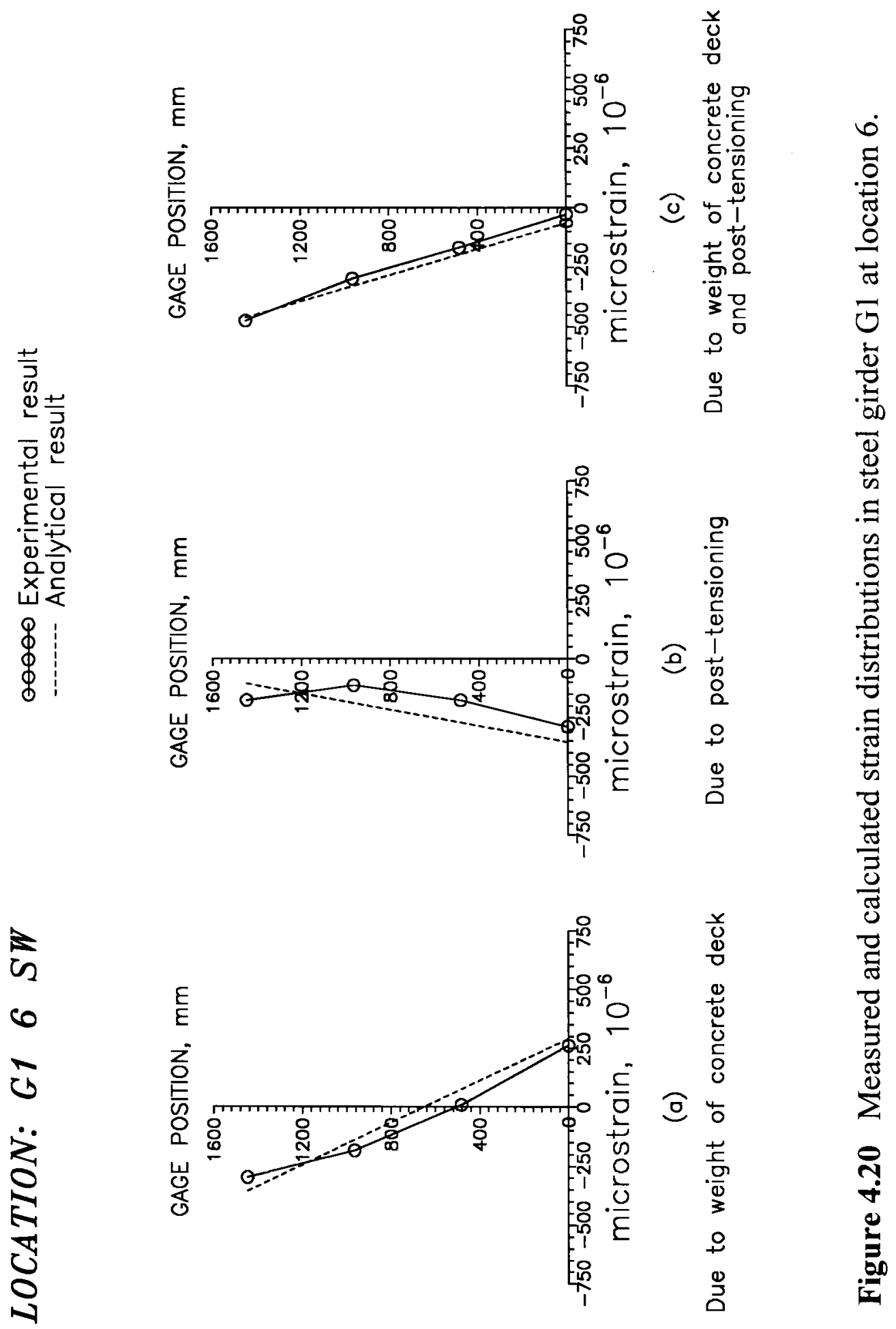




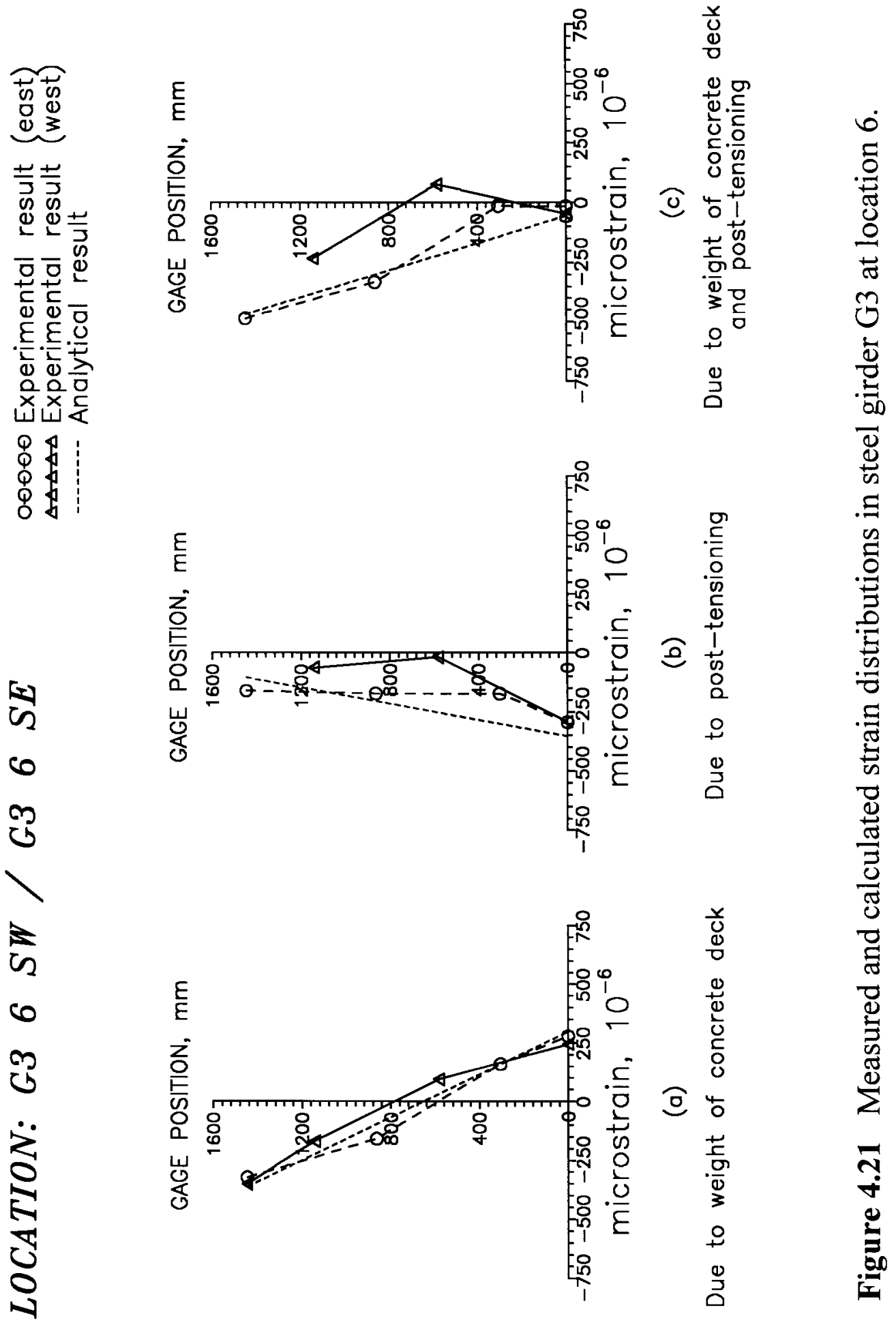




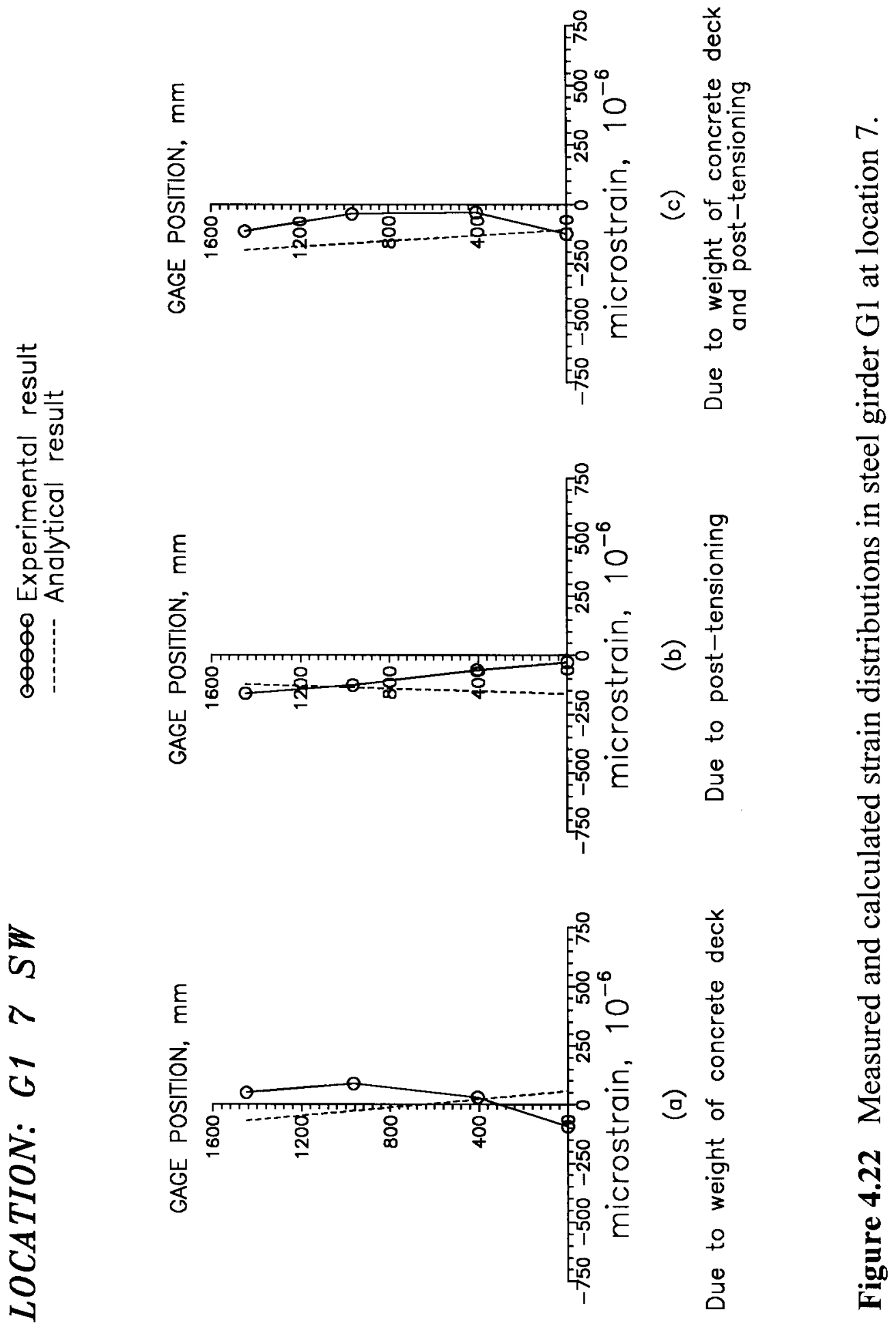




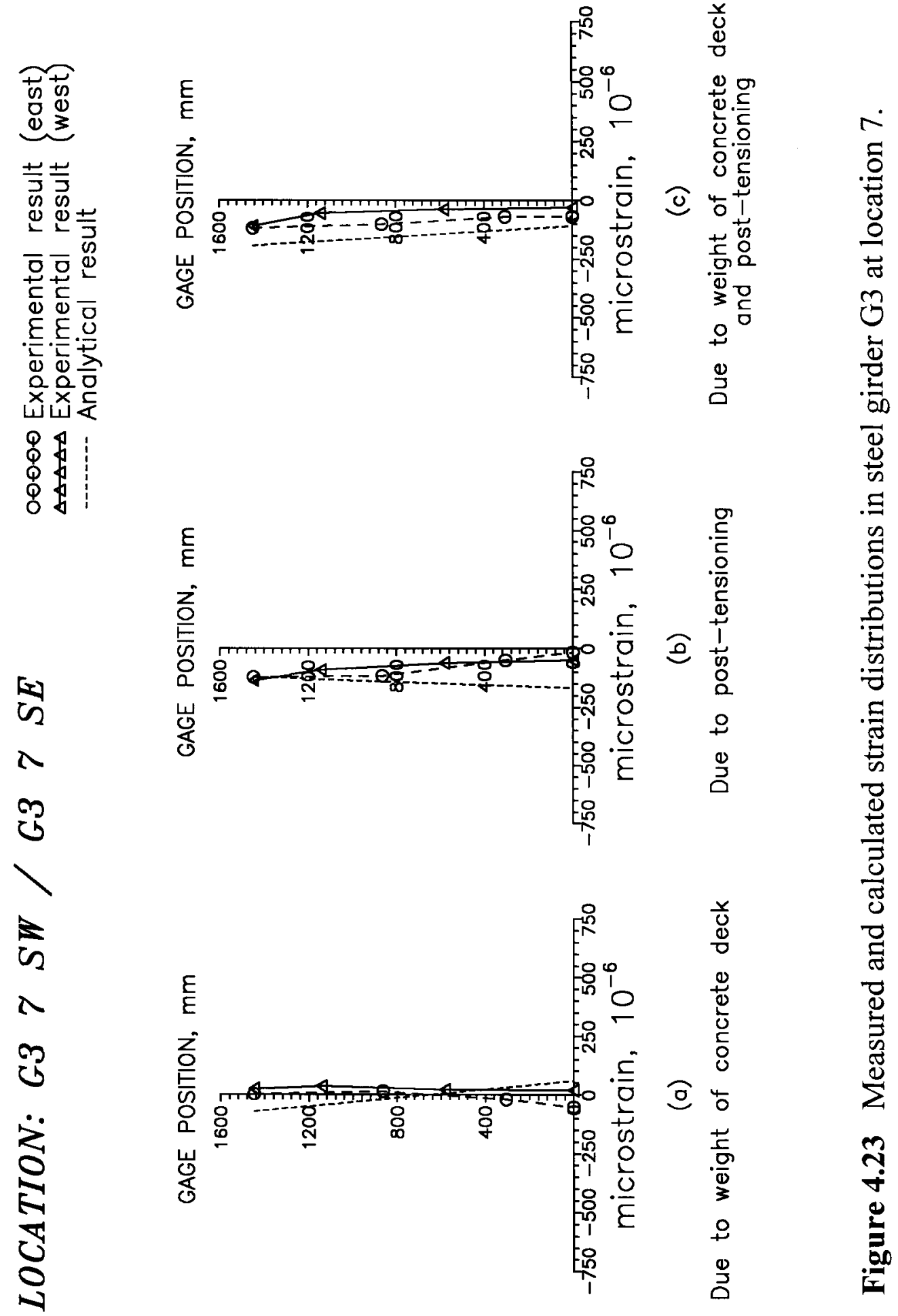




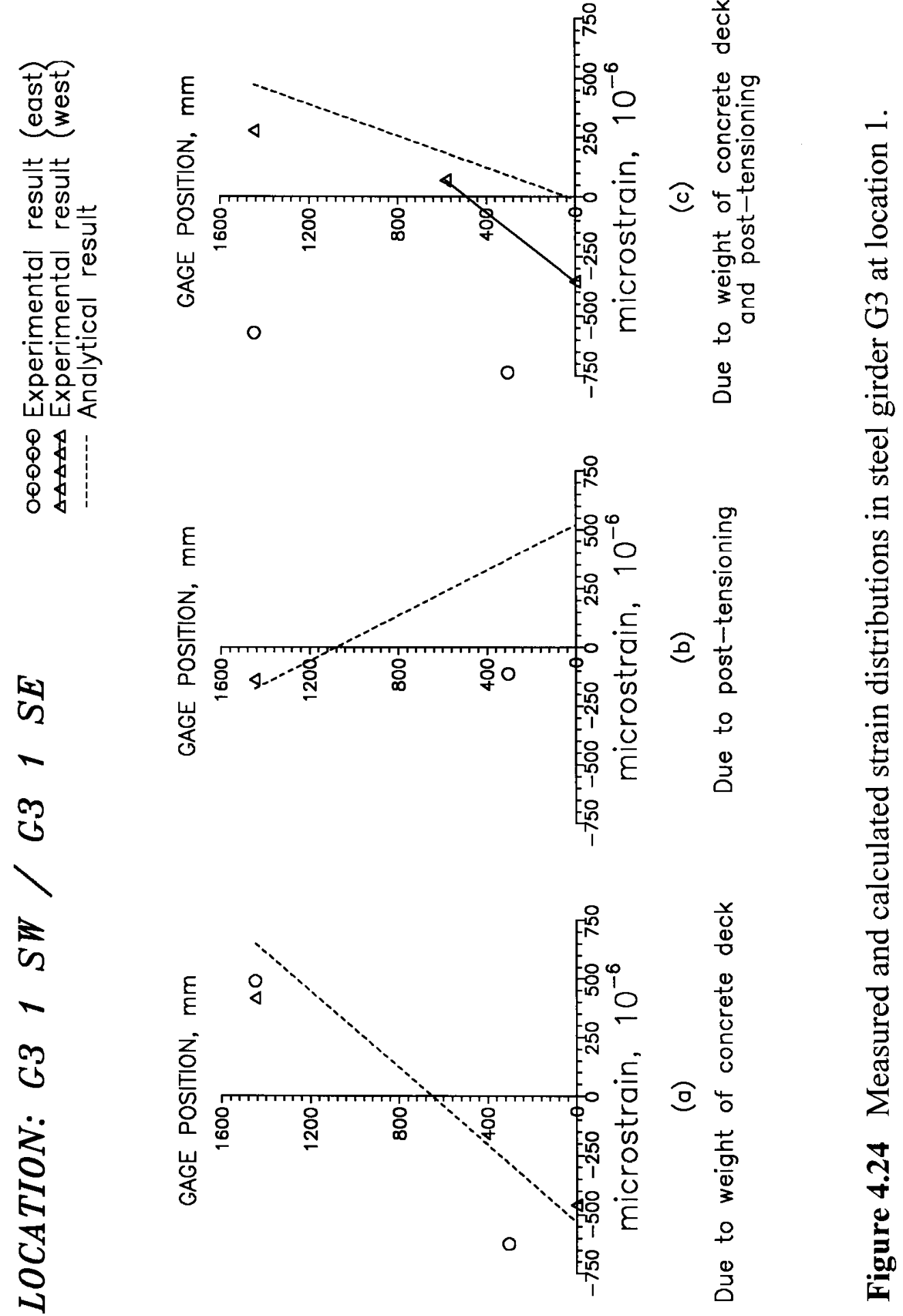


00000 Experimental result (east)

$\Delta \Delta \Delta \Delta \Delta$ Experimental result (west)

Analytical result

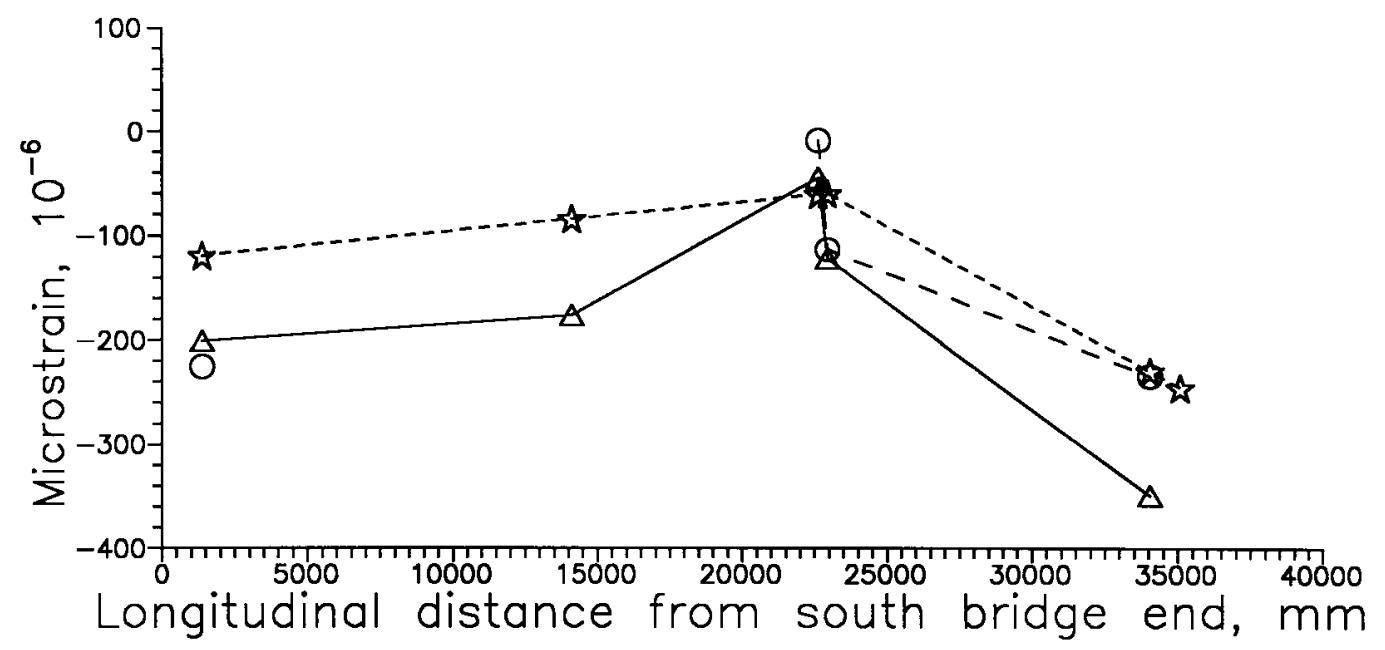

(a)

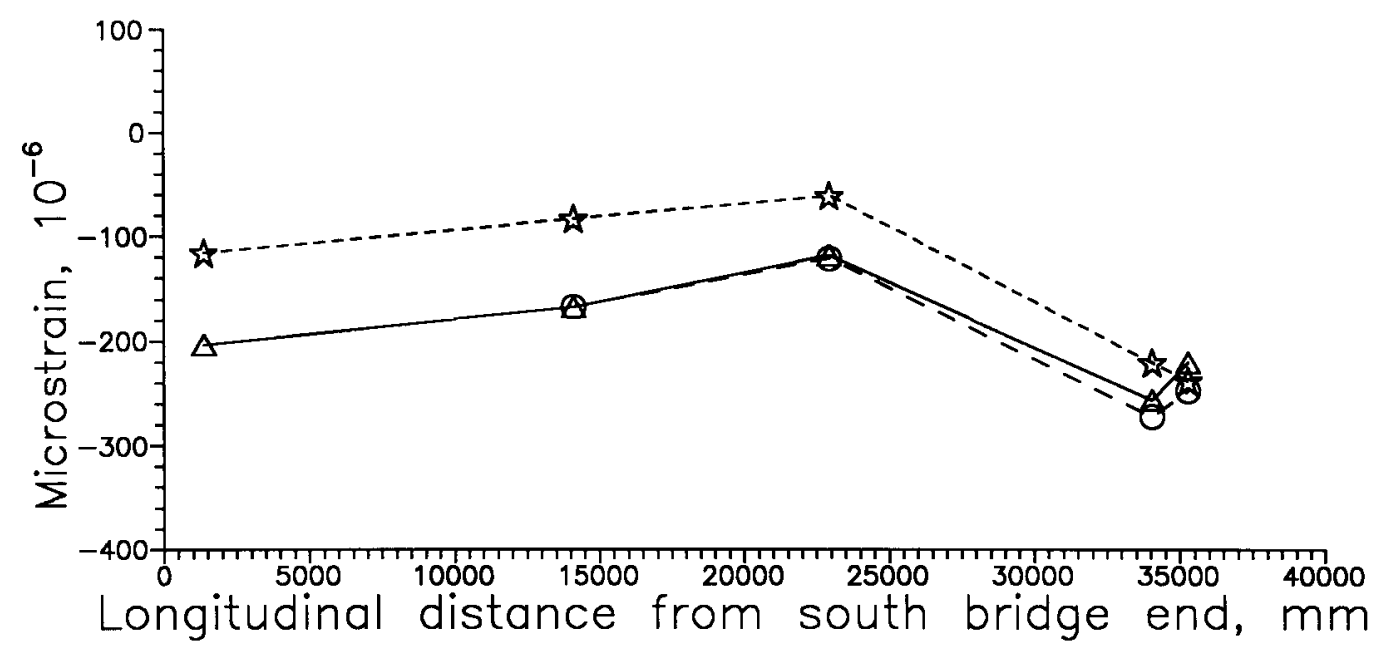

(b)

Figure 4.25 Measured and calculated strain distributions in concrete deck along girders (a) G1 and (b) G3 due to post-tensioning. 


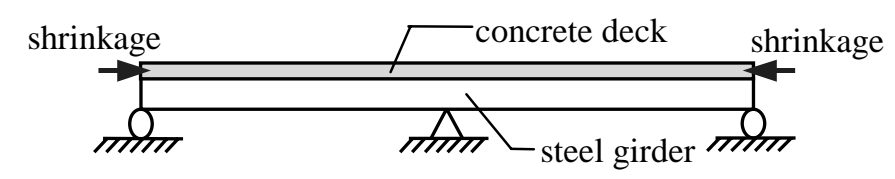

(a) Composite girder with concrete deck subjected to shrinkage.

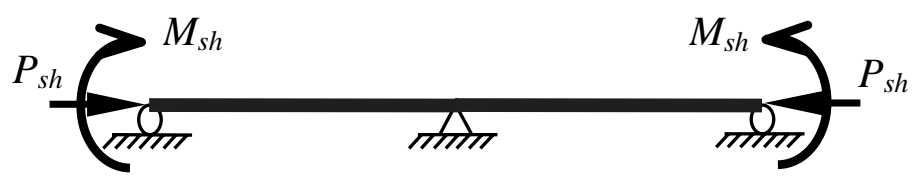

(b) Equivalent axial compressive force and bending moments for representing shrinkage effect.

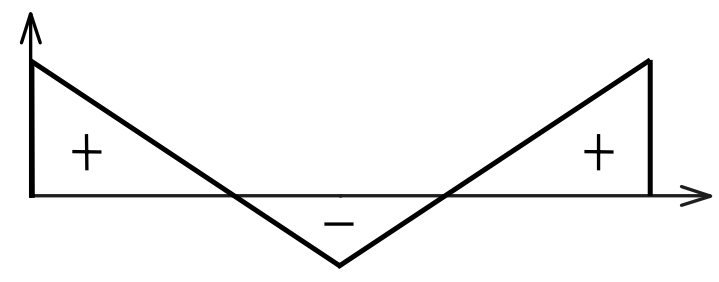

(c) Moment diagram of equivalent bending moments.

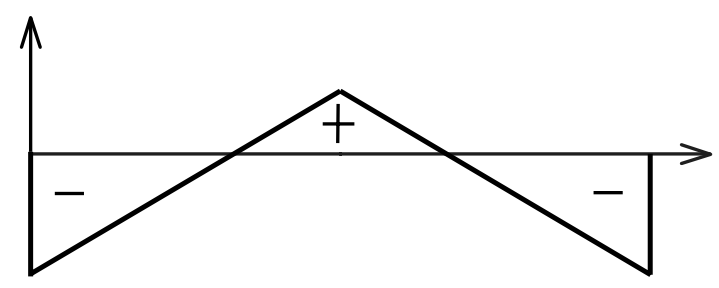

(d) Strain distribution in concrete deck due to equivalent bending moments.
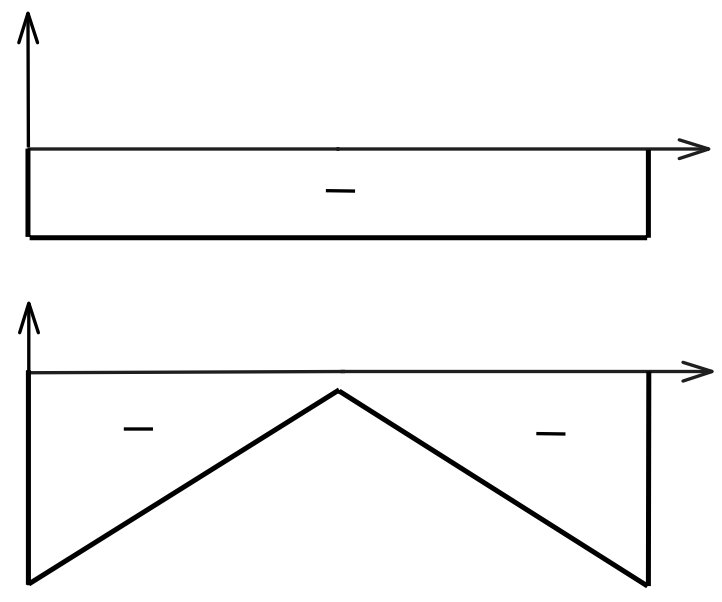

(e) Strain distribution in concrete deck due to equivalent axial compressive force.

(f) Strain distribution in concrete deck due to combined effect (bending moment + axial compressive force).

Figure 4.26 Effect of concrete shrinkage. 


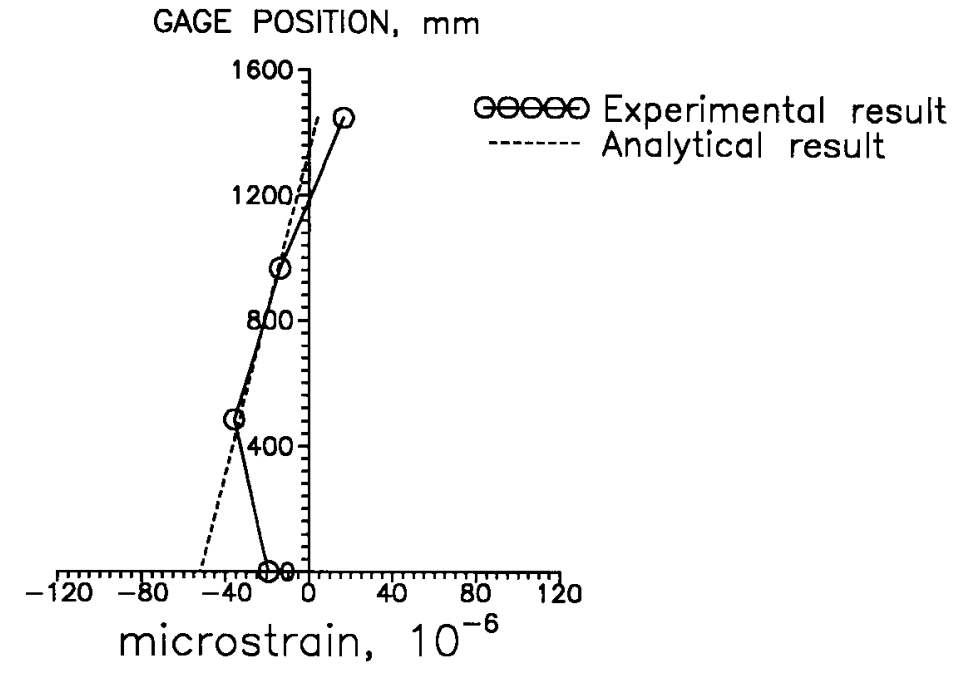

(a) LOCATION G1 2

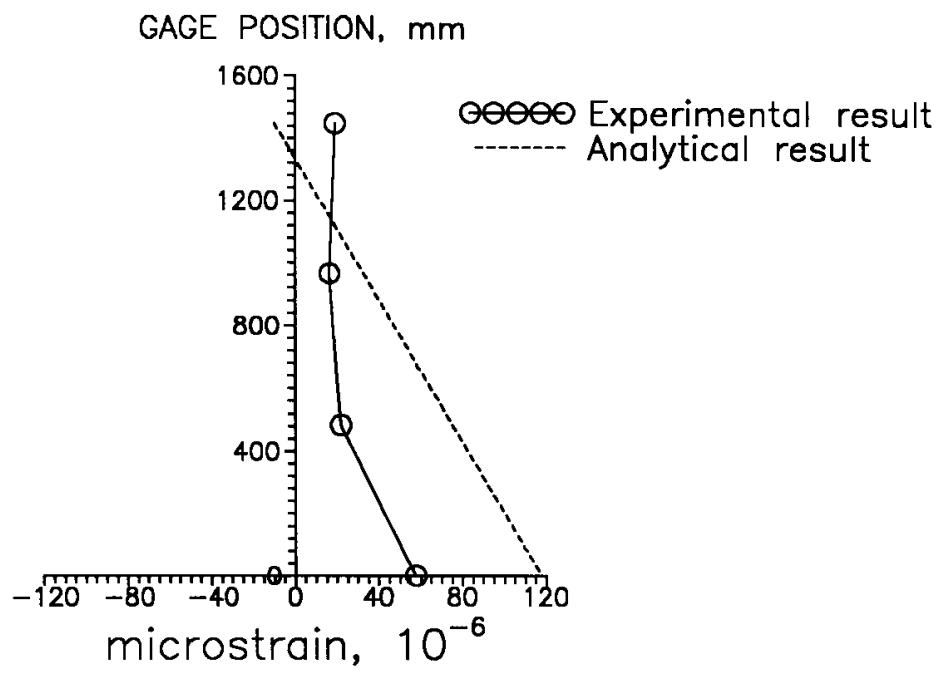

(b) LOCATION G1 6

Figure 4.27 Measured and calculated strain distributions in steel girder G1 due to truck loading case (b). 


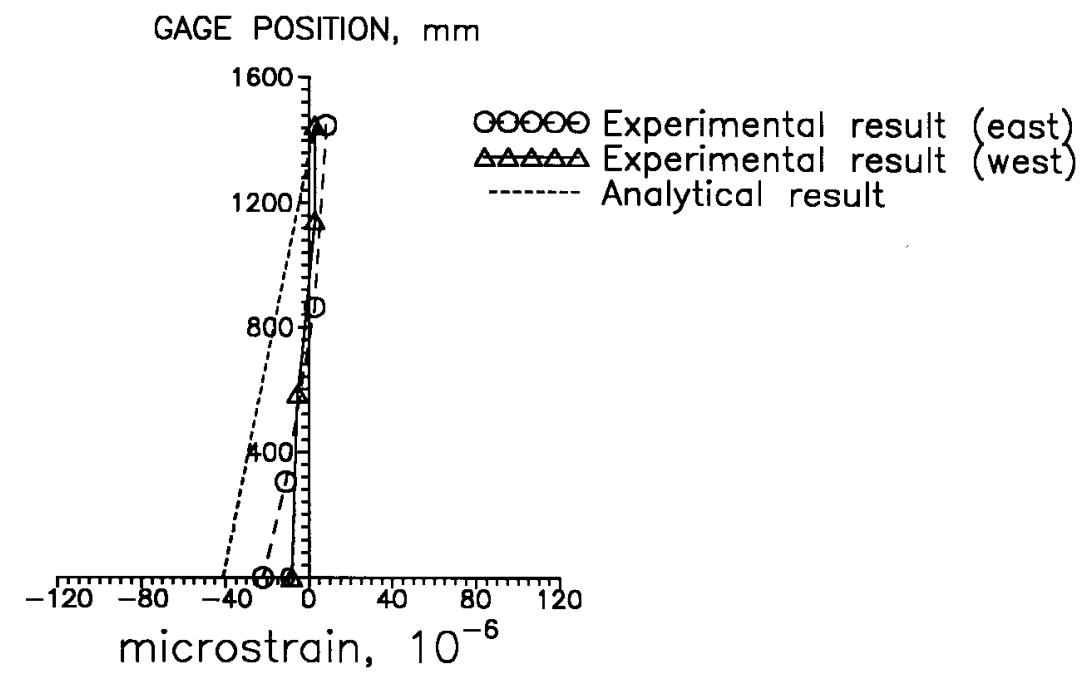

(a) LOCATION G3 2

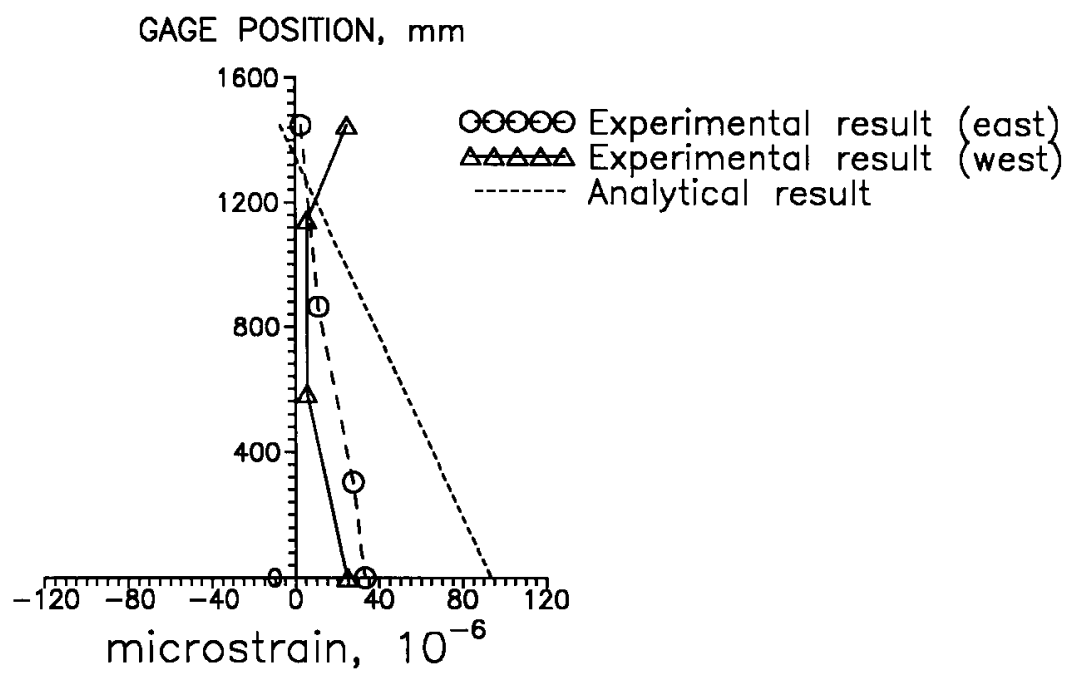

(b) LOCATION G3 6

Figure 4.28 Measured and calculated strain distributions in steel girder G3 due to truck loading case (h). 


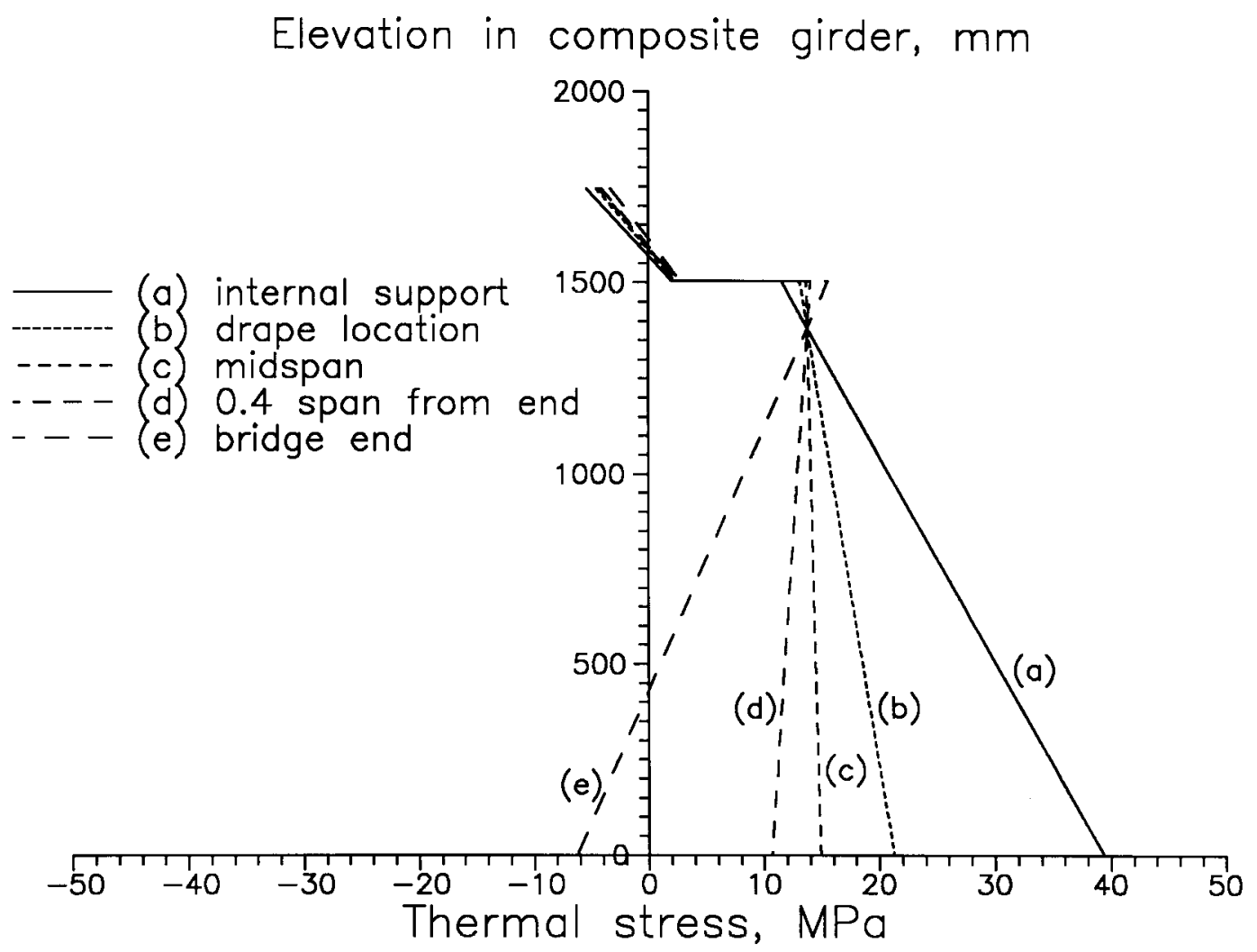

Figure 4.29 Estimated thermal stresses in composite girder G3 based on assumed temperature variation. 


\section{CHAPTER 5}

\section{PREDICTION OF LONG-TERM BRIDGE PERFORMANCE}

The effects of time-dependent factors, including the shrinkage as well as creep in the concrete deck and the relaxation in the high-strength steel tendons, are discussed in this chapter. An analytical procedure is proposed to predict the long-term structural response and the results are compared with the data of the field measurements of the Elkhart County I-90 Toll Road Bridge.

\subsection{Time-Dependent Material Behavior}

The stress and strain in a prestressed structure which is partially composed of concrete elements are inevitably subject to change over a long period of time, during which the creep as well as shrinkage in concrete and the relaxation in steel develop gradually. These factors, rather than being independent to each other, are interrelated. However, in dealing with practical engineering problems, it is more expedient to treat these factors separately. The idealization of decoupling these time-dependent effects is not simply for avoiding the complexity of the nature of the problems. In fact, since there are usually many uncertainties for material properties (especially for concrete) involved, a simplified analytical approach is acceptable as long as it can provide reasonable results. 
The structural response due to the effect of different type of time-dependent material property is usually evaluated individually and then superimposed.

\subsubsection{Creep and Shrinkage in Concrete}

The strain-stress relation of concrete depends upon the rate and the time history of loading. When a concrete specimen is loaded, the response consists of both instantaneous and time-dependent parts. The stain in concrete increases if the applied stress is sustained for a particular duration of time. Such phenomenon is referred to as creep. The amount of creep deformation, aside from the magnitude and the duration of applied stress level, is also influenced by several other factors. Some of them are directly related to the properties of the concrete mix, while others depend on environmental and loading conditions.

High strength concrete normally exhibits smaller creep deformation. Moreover, creep may be reduced by increasing the aggregate content, enlarging the maximum aggregate size, and using a stiffer aggregate type. It also depends on the water-cement ratio and the cement type since those are the key factors to determine the strength of concrete (Gilbert, 1988). The maturity of concrete (in other words, the degree of hydration) at the time of loading may also affect the creep behavior. The long-term deformation decreases as the age of concrete at first loading increases. Lower environmental humidity, smaller concrete member size and higher temperature are the other factors that contribute to the increase of creep deformation. 
Although the stress-strain relation for concrete is not perfectly linear, it is commonly assumed that the stress in concrete is proportional to strain under service loading condition. Therefore, the immediate strain occurring when the load is initially imposed can be obtained by the following equation according to Hooke's law:

$$
\varepsilon_{c}\left(t_{0}\right)=\frac{\sigma\left(t_{0}\right)}{E_{c}}
$$

where $t_{0}$ represents the age of concrete at first loading, $\sigma\left(t_{0}\right)$ is the sustained stress level imposed at time $t_{0}$, and $E_{c}$ is the elastic modulus for instantaneous loading. Under the application of constant sustained stress, the total strain at time $t$ (the age of concrete when strain value is calculated) is expressed as

$$
\varepsilon_{c}(t)=\frac{\sigma\left(t_{0}\right)}{E_{c}}\left[1+\varphi\left(t, t_{0}\right)\right]
$$

where $\varphi\left(t, t_{0}\right)$ is a dimensionless parameter, which is usually termed the creep coefficient, that is a function of $t_{0}$ and $t$. The strain that occurs upon initial loading is called the elastic strain, while the additional strain develops with the progress of time is called creep strain. The creep coefficient is then the ratio of creep strain to elastic strain. Fig. 5.1 is a graphic demonstration of elastic strain $\mathcal{E}_{c}\left(t_{0}\right)$ and creep strain $\varepsilon_{c}(t)$.

Fig. 5.2 shows the stress-strain curves for both fast (short-term) and slow (longterm) loading processes. The component of creep strain can be observed in this figure. In many occasions of practical application, the concept of effective modulus is adopted (Gilbert, 1988; Collins and Mitchell 1991). In contrast to the short-term elastic modulus $E_{c}$, the following equation is used to calculate the long-term effective modulus: 


$$
E_{c, e f f}=\frac{E_{c}}{1+\varphi\left(t, t_{0}\right)}
$$

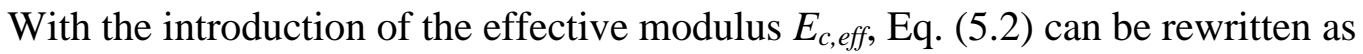

$$
\varepsilon_{c}(t)=\frac{\sigma\left(t_{0}\right)}{E_{c, e f f}}
$$

The above equation indicates that the long-term strain (sum of elastic and creep strains) can be obtained by using the reduced elastic stiffness $E_{c, \text { eff. }}$

If, in addition to the constant sustained stress $\sigma\left(t_{0}\right)$ which is instantaneously applied at time $t_{0}$, a stress increment is also introduced and its magnitude gradually varies form zero at concrete age $t_{0}$ to a final value $\Delta \sigma(t)$ at age $t$, the strain at age $t$ can be expressed as follows (Gilbert, 1988; Collins and Mitchell 1991; Chali and Favre 1994):

$$
\varepsilon_{c}=\frac{\sigma\left(t_{0}\right)}{E_{c, e f f}}+\frac{\Delta \sigma(t)}{E_{c, a d j}}
$$

in which

$$
E_{c, a d j}=\frac{E_{c}}{1+\chi\left(t, t_{0}\right) \varphi\left(t, t_{0}\right)}
$$

is termed as age-adjusted effective modulus. The factor $\chi\left(t, t_{0}\right)$ is referred to as the aging coefficient or relaxation coefficient. The value of aging coefficient varies from about 0.5 to 1.0 , depending on the age of concrete and the duration of loading. The adjustment of the effective modulus was first proposed and employed to account for creep strain by Trost (1967), and was later more rigorously formulated and developed by Bazant (1972).

Shrinkage in concrete is a reduction in volume resulting from the loss of moisture during the drying process. Chemical reactions, such as carbonation also cause shrinkage deformation. Although drying and chemical shrinkage are quite different in nature, there 
is no reason to distinguish them in practice from a structural engineering point of view (Gilbert, 1988). In a reinforced concrete element the deformation due to shrinkage is reduced by the embedded reinforcement, since it provides some resistance to the shrinkage action which only exists in concrete. A similar mechanism also exists between steel and concrete structural components in steel-concrete composite bridges.

Unlike creep, shrinkage is basically independent of applied stress level. However, it is affected by several factors which also influence the creep behavior. The amount of shrinkage depends largely upon the composition of concrete, and can vary over a wide range. In general, high ambient relative humidity, and the use of hard, dense, stiff aggregates with low absorption result in a reduction of shrinkage. Other influential factors include the water-cement ratio, the type of cure, the size and geometry of concrete element, and the duration of drying period. Each of the above factors plays a role in determining the total amount water in the concrete mix.

A key factor involved in evaluating time-dependent deformations in concrete is the use of adequate creep and shrinkage functions. Numerous expressions can be found from various sources of technical reports and publications. A representative list of those sources includes: British Standard (1985); CEB-FIP Model Code (1990); Collins and Mitchell (1991); ACI Committee 209 (1992); AASHTO LRFD Specifications (1994). Those publications provide different empirical formulae for estimating creep and shrinkage coefficients based on various sources of test results. In reality, the amount of creep and shrinkage in a particular concrete structure is very difficult to estimate accurately since there are so many uncertainties in the construction field, which are 
related to material properties, environmental conditions, or quality of construction. Without specific physical test, the expectation of a result with errors less than $\pm 30 \%$ seems not practical. Viewing from this point, it is reasonable to use a simplified method involving existing empirical equations to evaluate the time-dependent structural response. In analyzing the long-term performance of the Elkhart County Bridge, the creep and shrinkage functions suggested in the AASHTO LRFD Specifications (1994) are adopted. Those empirical equations are also appeared in the book by Collins and Mitchell (1991). In the following those equations are presented.

The creep coefficient $\varphi$ in a concrete can be estimated as

$$
\varphi\left(t, t_{0}\right)=3.5 k_{c} k_{f}\left(1.58-\frac{H}{120}\right) t_{0}^{-0.118} \frac{\left(t-t_{0}\right)^{0.6}}{10+\left(t-t_{0}\right)^{0.6}}
$$

for which

$$
k_{f}=\frac{1}{0.67+\left(\frac{f_{c}^{\prime}}{62}\right)}
$$

and

$$
k_{c}=\left[\frac{\frac{\left(t-t_{0}\right)}{26 e^{0.01417(V / S)}+\left(t-t_{0}\right)}}{\frac{t-t_{0}}{45+\left(t-t_{0}\right)}}\right]\left[\frac{1.80+1.77 e^{-0.0213(V / S)}}{2.587}\right]
$$

where

$$
\begin{aligned}
& t \quad \text { :age of concrete at time of calculation (day) } \\
& t_{0} \quad \text { : age of concrete at time of initial loading (day) } \\
& H \quad \text { : relative humidity }(\%)
\end{aligned}
$$


$f_{c}^{\prime}$ : specified compressive strength of concrete at 28 days (MPa)

$V / S$ : volume-to-surface ratio of concrete component (mm)

$k_{c} \quad$ : factor to account for the effect of the geometry of concrete ( $V / S$ ratio)

$k_{f}:$ factor for the effect of concrete strength

On the other hand, the amount of shrinkage $\varepsilon_{s h}$ as a function of time may be taken as in the following:

For moist-cured concrete:

$$
\varepsilon_{s h}\left(t, t_{d}\right)=-k_{s} k_{h}\left[\frac{\left(t-t_{d}\right)}{35+\left(t-t_{d}\right)}\right] 0.51 \times 10^{-3}
$$

For steam-cured concrete:

$$
\varepsilon_{s h}\left(t, t_{d}\right)=-k_{s} k_{h}\left[\frac{\left(t-t_{d}\right)}{55+\left(t-t_{d}\right)}\right] 0.56 \times 10^{-3}
$$

in both of which

$$
k_{s}=\left[\frac{\frac{\left(t-t_{d}\right)}{26 e^{0.01417(V / S)}+\left(t-t_{d}\right)}}{\frac{t-t_{d}}{45+\left(t-t_{d}\right)}}\right]\left[\frac{1064+3.7(V / S)}{923}\right]
$$

where

$t \quad$ : age of concrete at time of calculation (day)

$t_{d} \quad$ : age of concrete when concrete starts to be exposed to drying (day)

$k_{s} \quad:$ size factor determined by the volume-to-surface ratio

$k_{h}$ : coefficient to include the influence of ambient humidity (Table 5.1) 
The above empirical expressions can qualitatively reflect the effects of several important influential factors on the time-dependent material characteristics as mentioned earlier.

\subsubsection{Relaxation in Steel Tendon}

The force in a stressed steel tendon will gradually reduce with time as the elongation of the tendon is kept constant. This physical phenomenon is referred to as relaxation. Relaxation in a prestressed tendon depends primarily on the prestress level and the type of the steel material. Tendons subjected to tensile stresses of lower magnitudes exhibit less relaxation. Use of low-relaxation steel strands is an effective way to reduce the prestress losses due to relaxation. Like creep and shrinkage in concrete, precise evaluation of the relaxation in the steel in the field is not possible unless information for specific material properties under specific prestress conditions are well defined. Due to this reason, existing empirical equations are assumed to be applicable for general purposes. One of the most commonly used models for stress relaxation were suggested by the PCI Committee on Prestress Losses (1975). For low-relaxation steel, the prestress losses $\Delta \sigma_{r e}$ in the tendon due to relaxation can be estimated as

$$
\Delta \sigma_{r e}=f_{s t}\left\{\left[\log 24 t_{2}-\log 24 t_{1}\right] / 45\right\} \times\left[f_{s t} / f_{p y}-0.55\right]
$$

where

$$
\begin{aligned}
& f_{s t} / f_{p y}-0.55 \geq 0.05 \\
& f_{p y}=0.90 f_{p u}
\end{aligned}
$$


For stress-relieved steel:

$$
\Delta \sigma_{r e}=f_{s t}\left\{\left[\log 24 t_{2}-\log 24 t_{1}\right] / 10\right\} \times\left[f_{s t} / f_{p y}-0.55\right]
$$

where

$$
\begin{aligned}
& f_{s t} / f_{p y}-0.55 \geq 0.05 \\
& f_{p y}=0.85 f_{p u}
\end{aligned}
$$

The above equations are used to calculate the prestress losses caused by steel relaxation over the time interval $t_{1}$ to $t_{2}$ (in days) in which the time $\left(t_{1}\right.$ and $\left.t_{2}\right)$ is counted from the day the prestress force is applied. The value of $t_{1}$ at the time of anchorage of prestressed tendon shall be taken as $1 / 24$ to avoid mathematical incorrectness. In Eqs. (5.12), $f_{s t}$ is the stress in tendon at time $t_{1} ; f_{p y}$ and $f_{p u}$ are the stresses corresponding to $1 \%$ of elongation ( $1 \%$ strain offset), and the ultimate tensile strength of the prestressed steel, respectively.

\subsection{Problem Statement and Basic Assumptions}

The long-term bridge behavior is conducted by first-order analysis. It is intended to develop an analytical procedure to predict how the structural response, as measured by factors such as the longitudinal stress or strain distributions in the composite girders and the prestress losses in high-strength steel tendons, varies with time. Creep and shrinkage are assumed to be the exclusive time-dependent material characteristics of concrete, while relaxation behavior is presumed to occur only in post-tensioned tendons. The creep behavior is assumed to be linear since compressive stresses rarely exceed $50 \%$ of concrete strength at service loads (a very true statement for the Elkhart County Bridge). No time-dependent material behavior is expected in the steel girders. The time-dependent 
creep, shrinkage and relaxation functions recommend by the AASHTO LRFD Specifications and the PCI Committee on Prestress Losses are adopted for the numerical evaluation of the Elkhart County Bridge. The analyzed results are compared with that obtained from the field test data. In essence, the purpose of the analysis is to study the long-term interaction effects between different structural components, which are the concrete deck, the steel girders, and the steel tendons.

The assumptions made for evaluating the instantaneous structural response also prevail for analyzing the long-term bridge performance, including: (1) small and linear elastic deformation; (2) bending planes remain plane; (3) fully composite action; (4) uniform tendon force; and (5) uncracked concrete deck (see Chapter 4). These assumptions are reasonably made to enable the application of simplified analytical approaches such as the method of superposition, the principle of virtual work associated with compatibility equation, and the concept of transformed section.

The last assumption in the preceding paragraph (uncracked concrete deck) is proven to be eligible in analyzing the short-term response (Chapter 4) since both analytical and experimental results indicate that when the bridge is post-tensioned, the stress value in the concrete is negative (compressive) at every location and is well below the compressive concrete strength $f_{c}^{\prime}$. In the long-term analysis, since the steel girders provide certain resistance against the concrete shortening due to shrinkage and creep, some additional tensile stresses may be generated at certain longitudinal locations in the concrete deck such as the drape point (which is actually the most critical location). However, later in this chapter the analyzed results will show that the magnitude is still 
much lower than that of the nominal tensile concrete strength (about $0.1 f_{c}^{\prime}$ ), and thus the validity of this assumption is verified.

In forming a transformed section with an elastic modulus equal to that of steel, the local presence of concrete diaphragm and the reinforcement are neglected for the same reason stated in the preceding chapter. Since no cracks are assumed to exist anywhere in the concrete deck, along with the ignoring of the existence of concrete diaphragm and reinforcement, the sectional properties of the transformed section are assumed to be uniform along the bridge span.

The Elkhart County Bridge is the subject for applying the proposed analytical method. For the bridge structure without using the temporary shoring during construction, the post-tensing force is considered to be the only source to cause significant sustained loading. Although the traffic barriers create additional stresses in the concrete deck, the magnitude is insignificant compared with that due to post-tensioning. This argument will be justified later by a numerical demonstration.

The ultimate goal of the analysis is not to attempt to calculate the time-dependent structural response with high degree of accuracy. It is, however, anticipated to develop a rational approach so that the long-term performance of the post-tensioned composite bridge can be predicted within certain reasonable range. Efforts are made by first comparing the calculated results with the field measurements, then discussing the effectiveness of the analytical model, and finally extending the prediction to the service life cycle of a conventional bridge which is about 30 years. 


\subsection{Analytical Procedure}

The long-term structural response is a result of the interaction between different types of time-dependent material behavior, namely the creep and shrinkage in concrete and the relaxation in steel tendon. Those effects are continuously existing and developing with the progress of time. The structure response is therefore a continuous function in terms of time. At the earlier stage of concrete drying and period after post-tensioning operation, the interaction is more prominent, and then it declines with time.

Despite the fact that the long-term effect is a continuous process, for practical purposes, several discrete time intervals may be taken during the time period of analysis. The step-by-step analytical procedure is performed through the assumption that the timedependent response develops under uniform loading conditions during each time interval. The response computed in each interval is accumulated to attain the total long-term response which is then added to the instantaneous response to constitute the total response. The duration of time interval should be chosen to provide adequate results. In the early phase of service life the interval lengths should not be taken too long since the actual response varies more drastically with time, especially when significant changes in loading are expected. Longer time intervals can be used for later time steps. A step-bystep analytical procedure is recommend by the PCI Committee on Prestress Losses (1975) for estimating the prestress losses in conventional prestressed concrete structures. 


\subsubsection{Equivalent Axial Force and Bending Moment}

The analysis to account for the creep and shrinkage effects is conducted based on an approach which is similar to that adopted by several researchers (Gilbert 1988; Bradford 1991b; Ghali and Favre 1994; Gilbert and Bradford 1995; Bradford 1997) to evaluate the time-dependent response in either conventional prestressed concrete or steelconcrete composite beams. The application of this analytical model involves a series of artificial restraining-and-releasing procedures in each time interval, during which the loading condition is considered to be unaltered.

During a specific period of time (from $t_{1}$ to $t_{2}$ ) which is after the day of drying $\left(t_{d}\right)$ and the day of initial loading $\left(t_{0}\right)$, if the strain distribution at a given cross section is prevented form further change beyond the instantaneous component of strain, a restraining axial force $(-\Delta N)$ and a bending moment $(-\Delta M)$ must be applied to the centroid of the composite section to prevent the concrete from free deformation. Subsequently, the restraining force and moment are removed by imposing a pair of equal and opposite force $\Delta N$ and moment $\Delta M$. The magnitudes of $\Delta N$ and $\Delta M$ at a given cross section are calculated as

$$
\begin{gathered}
\Delta N=\int_{0}^{h_{c}}\left[\Delta \varepsilon_{s h}\left(t_{2}, t_{1}, y_{c p}^{C}\right)+\Delta \varphi\left(t_{2}, t_{1}\right) \times \varepsilon_{0}\left(y_{c p}^{C}\right)\right] \times\left[E_{c, a d j} \times b\left(y_{c p}^{C}\right)\right] d y_{c p}^{C} \\
\Delta M=\int_{0}^{h_{c}}\left[\Delta \varepsilon_{s h}\left(t_{2}, t_{1}, y_{c p}^{C}\right)+\Delta \varphi\left(t_{2}, t_{1}\right) \times \varepsilon_{0}\left(y_{c p}^{C}\right)\right] \times\left[E_{c, a d j} \times b\left(y_{c p}^{C}\right) \times y_{c p}^{C}\right] d y_{c p}^{C}
\end{gathered}
$$

where $y_{c p}^{C}$ is the vertical distance measured from the position of the neutral axis of the composite section to the point of interest in the concrete, which is taken as negative in algebraic value to give a consistent sign convention for flexural deformation as that 
defined in the foregoing chapter; $h_{c}$ is the thickness of the concrete deck; $b\left(y_{c p}^{C}\right)$ is the width of the concrete element at the vertical position $y_{c p}^{C} ; \varepsilon_{0}\left(y_{c p}^{C}\right)$ is the strain distribution along the depth of concrete deck caused by instantaneous loading. The age-adjusted elastic modulus of concrete $E_{c, a d j}$ is defined by Eq. (5.6). It is adopted because that the artificial restraining-and-releasing procedure is considered as a gradual loading process (Gilbert 1988; Ghali and Favre 1994). Replacing $t_{0}$ and $t$ with $t_{1}$ and $t_{2}$ respectively in Eq (5.6) yields

$$
E_{c, a d j}=\frac{E_{c}}{1+\chi\left(t_{2}, t_{1}\right) \varphi\left(t_{2}, t_{1}\right)}
$$

where $E_{c}$ is the short-term elastic modulus of concrete. The components $\Delta \varepsilon_{s h}\left(t_{2}, t_{1}, y_{c p}^{C}\right)$ and $\Delta \varphi\left(t_{2}, t_{1}\right)$ can be obtained by

$$
\begin{aligned}
\Delta \varepsilon_{s h}\left(t_{2}, t_{1}, y_{c p}^{C}\right) & =\varepsilon_{s h}\left(t_{2}, t_{d}, y_{c p}^{C}\right)-\varepsilon_{s h}\left(t_{1}, t_{d}, y_{c p}^{C}\right) \\
\Delta \varphi\left(t_{2}, t_{1}\right) & =\varphi\left(t_{2}, t_{0}\right)-\varphi\left(t_{1}, t_{0}\right)
\end{aligned}
$$

which represent the fractions of shrinkage and creep developing in concrete during the period $\left(t_{1}, t_{2}\right)$. The use of subscript " $c p$ " implies that $\Delta N$ and moment $\Delta M$ are applied to the composite section, referring to the sectional centroid.

Eqs. (5.13) and (5.14) imply that the equivalent incremental axial force $\Delta N$ and bending moment $\Delta M$ are the combined results of the creep and shrinkage deformations developed in the unrestrained concrete deck and the composite action between the concrete deck and the steel girder. By assuming that the shrinkage is uniform in the concrete deck and using the stress value at the center of concrete $\left(\bar{y}_{c p}^{C}\right)$ as the average stress in the concrete, Eqs (5.13) and (5.14) can be approximated as follows: 


$$
\begin{gathered}
\Delta N=E_{c, a d j} A_{c}\left[\Delta \varepsilon_{s h}\left(t_{2}, t_{1}\right)+\Delta \varphi\left(t_{2}, t_{1}\right) \times \varepsilon_{0}\left(\bar{y}_{c p}^{C}\right)\right] \\
\Delta M=E_{c, a d j} A_{c}\left[\Delta \varepsilon_{s h}\left(t_{2}, t_{1}\right)+\Delta \varphi\left(t_{2}, t_{1}\right) \times \varepsilon_{0}\left(\bar{y}_{c p}^{C}\right)\right] \times \bar{y}_{c p}^{C}
\end{gathered}
$$

in which $A_{c}$ is the cross-sectional area of concrete. The mechanism of the restraining-andreleasing process is also illustrated in Fig. 5.3.

\subsubsection{Structural Response Functions due to Shrinkage and Creep}

In the next step, the equivalent axial force $\Delta N$ and bending moment $\Delta M$ are applied to the composite girder during an individual time interval. An "age-adjusted transformed section" with a uniform elasticity modulus equal to that of steel $E_{s}$ is constituted through the introduction of the modular ratio:

$$
n^{\prime}=E_{s} / E_{c, a d j}
$$

which reflects the nature of the assumption that the loading process is gradual during the specific time interval. The sectional properties, such as the moment of inertia, gross area and position of neutral axis of the age-adjusted transformed section are computed according to the equivalent modular ratio defined in Eq. (5.20).

If the equivalent force $\Delta N$ and moment $\Delta M$ are to be applied to a statically determinant structure, the axial force and bending moment diagrams for the structural reactions can be obtained easily. For example, suppose that a pair of specified axial force $\Delta N(x)$ and bending moment $\Delta M(x)$ are applied to a single-span, simply-supported beam, the resultant diagrams in structure for axial and bending actions as functions of the longitudinal position $x$ are identical correspondingly to $\Delta N(x)$ and $\Delta M(x)$. However, for structures in which a certain degree of static indeterminacy exists, the solutions are not so 
direct. The internal restraints in a statically indeterminate structure have to be considered in determining the stress and strain distributions.

To demonstrate the procedure for estimating the time-dependent response in a post-tensioned steel-concrete composite bridge girder, the example of Elkhart County Bridge is used in the following. The analytical model, however, is not restricted to this particular construction, but is applicable to similar structures: continuous composite bridge systems which are eccentrically post-tensioned with drape points located within spans. The effects of different types of time-dependent factors are treated separately for the convenience of discussion.

The shrinkage in the concrete deck and the composite action between the steel girder and the concrete slab is the cause of the equivalent axial force $\Delta N_{s h}$ and bending moment $\Delta M_{s h}$. The reinforcement in the concrete deck also provides some resistance against shrinkage deformation but is negligible in calculation since that the area of reinforcement constitutes only a small portion of the gross cross-section. The amount of shrinkage, as aforementioned, is assumed to be uniform in the concrete. For a specific duration of time interval $\left(t_{1}, t_{2}\right), \Delta N_{s h}$ and $\Delta M_{s h}$ are obtained according to the following:

$$
\begin{gathered}
\Delta N_{s h}\left(t_{2}, t_{1}, x\right)=E_{c, a d j} A_{c} \Delta \varepsilon_{s h}\left(t_{2}, t_{1}\right) \\
\Delta M_{s h}\left(t_{2}, t_{1}, x\right)=-E_{c, a d j} A_{c} \Delta \varepsilon_{s h}\left(t_{2}, t_{1}\right)\left[\left|y_{c p}^{C_{T O P}}\right|-h_{c 1} / 2\right]
\end{gathered}
$$

where $y_{c P}^{C_{T O P}}$ is the distance measured from the neutral axis of the age-adjusted transformed section to the outermost concrete fiber; $h_{c 1}$ is the thickness of the major block of the concrete deck with the sectional geometry similar to that of the Elkhart County Bridge (see Fig. 4.9a and Fig. 4.9b). Eq. (5.22) is based on the assumption that 
$\Delta N_{s h}$ is applied at the mid-depth of the major block of the concrete deck to induce $\Delta M_{s h}$, which should be a reasonable approximation.

For the same time interval $\left(t_{1}, t_{2}\right)$, the structural response due to the application of the shrinkage-equivalent axial force and bending moment, as functions in terms of longitudinal location $x$, are qualitatively depicted in Fig. 5.4 and also algebraically presented as follows:

$$
\begin{gathered}
\Delta N_{s h}^{*}\left(t_{2}, t_{1}, x\right)=E_{c, a d j} A_{c} \Delta \varepsilon_{s h}\left(t_{2}, t_{1}\right) \\
\Delta M_{s h}^{*}\left(t_{2}, t_{1}, x\right)=-E_{c, a d j} A_{c} \Delta \varepsilon_{s h}\left(t_{2}, t_{1}\right)\left[\left|y_{c p}^{C_{T O P}}\right|-h_{c 1} / 2\right]\left(1-1.5 x / L_{1 / 2}\right) \\
\text { for } 0 \leq x \leq L_{1 / 2}
\end{gathered}
$$

where $L_{1 / 2}$ is the span length of bridge, which is equal to half of the entire bridge length $L$ $\left(L_{1 / 2}=0.5 L\right)$. In order to distinguish the applied load and the structural reaction, the symbol "**" is used as a superscript. The axial force response function $\Delta N_{s h}^{*}(x)$ and the applied axial force function $\Delta N_{s h}(x)$ are identical because there is no restraint against axial deformation; and its value is constant along the bridge span since the shrinkage strain is uniform. In contrast, even though the equivalent moment $\Delta M_{s h}(x)$ is uniformly applied to the girder (constant value over span), the response moment $\Delta M_{s h}^{*}(x)$ is a linear function between the bridge end and the pier. The reason is that the internal bridge support at pier provides a restraint for vertical deflection, introducing a vertical reaction force which eventually makes $\Delta M_{s h}(x)$ and $\Delta M_{s h}^{*}(x)$ different. The response moment diagram $\Delta M_{s h}^{*}(x)$ is determined by means of traditional force method (flexibility method) which is described in standard textbooks for mechanics of materials or structural analysis. The derivation of Eq. (5.24) involves the assumption that the girder is simply supported 
by rollers at both ends, which also prevails in the analysis for instantaneous response (Chapter 4). Both of $\Delta N_{s h}^{*}(x)$ and $\Delta M_{s h}^{*}(x)$ are symmetric about the pier because of the symmetry of the bridge structure.

The creep-equivalent axial force $\Delta N_{c r}(x)$ and bending moment $\Delta M_{c r}(x)$ applied to the composite section during the time interval $\left(t_{1}, t_{2}\right)$ exhibit similar characteristics to those due to $\Delta N_{s h}(x)$ and $\Delta M_{s h}(x)$. The influence of reinforcement is also neglected and full composite action is assumed. The major difference between these two timedependent factors is that the shrinkage strain is independent of stress and location, while the creep strain depends on both the initial stress level and the location in the concrete. In developing the response functions $\Delta N_{c r}^{*}(x)$ and $\Delta M_{c r}^{*}(x)$ along the composite girder due to the application of $\Delta N_{c r}(x)$ and $\Delta M_{c r}(x)$, internal structural restraints also must be taken into account. For the convenience of calculation and discussion, the unrestrained creep strain in the concrete is divided into two parts, resulting from the initial axial stress and the flexural stress due to post-tensioning.

For each analyzed time interval $\left(t_{1}, t_{2}\right)$, the strain distribution developed in the unrestrained concrete due to creep is estimated according to the following equations:

$$
\Delta \varepsilon_{c r}^{C}=\Delta \varepsilon_{c r a}^{C}+\Delta \varepsilon_{c r m}^{C}
$$

in which

$$
\begin{gathered}
\Delta \varepsilon_{c r a}^{C}\left(t_{2}, t_{1}, x\right)=\frac{P\left(t_{1}\right) \times a_{0}(x)}{E_{s} A_{c p 0}} \Delta \varphi\left(t_{2}, t_{1}\right) \\
\Delta \varepsilon_{c r m}^{C}\left(t_{2}, t_{1}, x, y_{c p}^{C}\right)=\frac{\left[P\left(t_{1}\right) \times m_{0}(x)\right] y_{c p 0}^{C}}{E_{s} I_{c p 0}} \Delta \varphi\left(t_{2}, t_{1}\right)
\end{gathered}
$$


where the use of " 0 " in subscript is to indicate the initial loading condition and sectional properties, and therefore $a_{0}(x)$ and $m_{0}(x)$ are the initial unit axial force and moment functions as defined in Chapter $4 ; A_{c p 0}$ and $I_{c p 0}$ are the initial gross sectional area and moment of inertia of the transformed section at the time of post-tensioning; $E_{s}$ is the elastic modulus of steel; $y_{c p 0}^{C}$ stands for the vertical position in concrete referring to the initial neutral axis. $P\left(t_{1}\right)$ is the magnitude of prestress force in steel tendon at time $t_{1}$. For the $n$-th time step of analysis,

$$
P^{n}\left(t_{1}\right)=P_{0}+\sum_{i}^{n-1} \Delta P^{i}\left(t_{2}, t_{1}\right)
$$

in which $P_{0}$ is the initial post-tensioning force; $\Delta P^{i}\left(t_{2}, t_{1}\right)$ is the calculated loss of prestress force in the previous $i$-th time step, with a negative algebraic value. The use of Eqs. (5.25), (5.26a) and (5.27a) implies that the stress condition is assumed to be unchanged during the time interval $\left(t_{1}, t_{2}\right)$. The components of strain increments $\Delta \varepsilon_{c r a}^{C}$ and $\Delta \varepsilon_{c r m}^{C}$ represent the effects of axial compressive force and bending moment due to initial application of post-tensioning force. Moreover, since $a_{0}(x)$ is a uniform function of longitudinal location (see Chapter 4), then the magnitude of $\Delta \varepsilon_{c r a}^{C}$ is also a constant value over the bridge length. The value of $\Delta \varepsilon_{c r}^{C}$ (the sum of $\Delta \varepsilon_{c r a}^{C}$ and $\Delta \varepsilon_{c r m}^{C}$ ) is negative because the initial stress due to post-tensioning at any location in concrete is found to be compressive (Chapter 4).

Eqs. (5.26a) and (5.27a) associated with Eq. (5.28) are actually conservative approximations of the following more accurate expressions:

$$
\Delta \varepsilon_{c r a}^{C}\left(t_{2}^{n}, t_{1}^{n}, x\right)=\frac{P_{0} \times a_{0}(x)}{E_{s} A_{c p 0}} \Delta \varphi\left(t_{2}^{n}, t_{1}^{n}\right)+\sum_{i}^{n-1} \frac{\Delta P^{i} \times a_{0}^{i}(x)}{E_{s} A_{c p 0}^{i}}\left[\varphi\left(t_{2}^{n}, t_{2}^{i}\right)-\varphi\left(t_{1}^{n}, t_{2}^{i}\right)\right]
$$




$$
\begin{aligned}
\Delta \varepsilon_{c r m}^{C}\left(t_{2}^{n}, t_{1}^{n}, x, y_{c p}^{C}\right)= & \frac{\left[P_{0} \times m_{0}(x)\right] y_{c p 0}^{C}}{E_{s} I_{c p 0}} \Delta \varphi\left(t_{2}^{n}, t_{1}^{n}\right) \\
& +\sum_{i}^{n-1} \frac{\left[\Delta P^{i} \times m_{0}^{i}(x)\right] y_{c p}^{C i}}{E_{s} I_{c p 0}^{i}}\left[\varphi\left(t_{2}^{n}, t_{2}^{i}\right)-\varphi\left(t_{1}^{n}, t_{2}^{i}\right)\right]
\end{aligned}
$$

where the superscripts " $n$ " and " $i$ " stand for the current $n$-th and previous $i$-th time intervals of analysis (time steps). In these two expressions, $a_{0}^{i}(x), m_{0}^{i}(x), A_{c p 0}^{i}, I_{c p 0}^{i}$ and $y_{c p}^{C i}$ are calculated based on the concrete properties at the end of the $i$-th time step. The use of Eqs. (5.26b) and (5.27b) leads to less conservative results, which, however, exhibit only insignificant difference compared to those obtained according to the much simpler expressions Eqs. (5.26a) and (5.27a). Due to this fact, the approximate Eqs. (5.26a) and (5.27a) associated with Eq. (5.28) can be reasonably employed.

The creep-equivalent axial force and bending moment can be obtained through using Eqs. (5.26) and (5.27). For the effect of $\Delta \varepsilon_{c r a}^{C}$ :

$$
\begin{gathered}
\Delta N_{c r a}\left(t_{2}, t_{1}, x\right)=E_{c, a d j} A_{c} \Delta \varepsilon_{c r a}^{C}\left[t_{2}, t_{1}, x,\left(y_{c p}^{C}=\left|y_{c p 0}^{C_{T O P}}\right|-h_{c 1} / 2\right)\right] \\
\Delta M_{c r a}\left(t_{2}, t_{1}, x\right)=-\Delta N_{c r a}\left(t_{2}, t_{1}, x\right)\left(\left|y_{c p}^{C_{T O P}}\right|-h_{c 1} / 2\right)
\end{gathered}
$$

Similarly, for the effect of $\Delta \varepsilon_{c r m}^{C}$ :

$$
\begin{gathered}
\Delta N_{c r m}\left(t_{2}, t_{1}, x\right)=E_{c, a d j} A_{c} \Delta \varepsilon_{c r m}^{C}\left[t_{2}, t_{1}, x,\left(y_{c p}^{C}=\left|y_{c p 0}^{C_{T O P}}\right|-h_{c 1} / 2\right)\right] \\
\Delta M_{c r m}\left(t_{2}, t_{1}, x\right)=-\Delta N_{c r m}\left(t_{2}, t_{1}, x\right)\left(\left|y_{c p}^{C_{T O P}}\right|-h_{c 1} / 2\right)
\end{gathered}
$$

where the age-adjusted elastic modulus $E_{c, a d j}$ is defined in Eq. (5.15); $y_{c p 0}^{C_{T O P}}$ and $y_{c p}^{C_{T O P}}$ are the distances from the top surface of concrete deck to the neutral axes of the initial and the age-adjusted transformed sections, respectively; and $h_{c 1}$ is the thickness of the major 
block of concrete deck, which can be referred in Figs. 4.9a and 4.9b. Like Eq. (5.22), Eqs. (5.30) and (5.32) are also formulated through the assumption that $\Delta N_{c r a}$ and $\Delta N_{c r m}$ are applied approximately at the center of the concrete deck.

To develop the response axial force and moment diagrams used to calculate the time-dependent stress or strain distribution, the factor of structural redundancy must be taken into account. The following expressions are obtained as the functions of the structural reactions due to the imposing of creep-equivalent forces $\left(\Delta N_{c r a}\right.$ and $\left.\Delta N_{c r m}\right)$ and moments $\left(\Delta M_{\text {cra }}\right.$ and $\left.\Delta M_{\text {crm }}\right)$ during the period $\left(t_{1}, t_{2}\right)$ :

$$
\begin{gathered}
\Delta N_{c r a}^{*}\left(t_{2}, t_{1}, x\right)=E_{c, a d j} A_{c} \Delta \varepsilon_{c r a}^{C}\left[t_{2}, t_{1}, x,\left(y_{c p}^{C}=\left|y_{c p 0}^{C_{T O P}}\right|-h_{c 1} / 2\right)\right] \\
\Delta M_{c r a}^{*}\left(t_{2}, t_{1}, x\right)=-\Delta N_{c r a}^{*}\left(t_{2}, t_{1}, x\right)\left(\left|y_{c p}^{C_{T O P}}\right|-h_{c 1} / 2\right)\left(1-1.5 x / L_{1 / 2}\right) \\
\Delta N_{c r m}^{*}\left(t_{2}, t_{1}, x\right)=E_{c, a d j} A_{c} \Delta \varepsilon_{c r m}^{C}\left[t_{2}, t_{1}, x,\left(y_{c p}^{C}=\left|y_{c p 0}^{C_{T o P}}\right|-h_{c 1} / 2\right)\right] \\
\Delta M_{c r m}^{*}\left(t_{2}, t_{1}, x\right)=-\Delta N_{c r m}^{*}\left(t_{2}, t_{1}, x\right)\left(\left|y_{c p}^{C_{T o P}}\right|-h_{c 1} / 2\right) \\
\text { for } 0 \leq x \leq L_{1 / 2}
\end{gathered}
$$

The functions $\Delta N_{c r a}^{*}$ and $\Delta N_{c r m}^{*}$ are identical to $\Delta N_{c r a}$ and $\Delta N_{c r m}$ respectively because no restraint is provided in the axial direction. The value of $\Delta N_{c r a}^{*}$ is a constant which is independent of the longitudinal location since that $\Delta \varepsilon_{c r a}^{C}$ is a single-value function of $x$. The derivation for $\Delta M_{c r a}^{*}$ is similar to that for $\Delta M_{s h}^{*}$, which in fact is to determine the response moment diagram in a continuous girder system with two equal spans when subjected to a constant bending action. The response function $\Delta M_{c r m}^{*}$ is obtained simply by multiplying the function $\Delta N_{c r m}^{*}$ with the eccentricity $\left(\left|y_{c p}^{C_{T o P}}\right|-h_{c 1} / 2\right)$. Like $\Delta M_{s h}^{*}$, $\Delta M_{c r a}^{*}$ and $\Delta M_{c r m}^{*}$ are derived based on the simply supported end condition. A conceptual 
depiction for $\Delta N_{c r a}^{*}, \Delta N_{c r m}^{*}, \Delta M_{c r a}^{*}$ and $\Delta M_{c r m}^{*}$ is given in Fig. 5.5. All of those functions are symmetric about the bridge center due to the symmetry of the structure configuration as well as the loading condition.

Any other source of sustained loading to cause creep effects can be handled by the same procedure described above. For practice, if the immediate stress developed in the concrete is not notable compared with that due to post-tensioning, the inclusion of shortterm response alone is sufficient.

\subsubsection{Calculation of Long-Term Structural Response}

With the knowledge of the long-term (creep and shrinkage) response functions for the structure, the structural response due to time-dependent effects can be evaluated accordingly. One of the most interested response quantities is the prestress losses. Although several suggestions on estimating prestress losses are found in existing specifications or design recommendations such as those from the PCI Committee on Prestress Losses (1975) and the AASHTO LRFD Specifications (1994), those approaches were developed for conventional prestressed concrete structures, and hence are not applicable to a post-tensioned steel structure. A method based on the virtual work principle associated with a compatibility equation is proposed to estimate the loss of prestress force due to shrinkage and creep effects. The procedure of analysis is similar to that described in Chapter 4 which is used to estimate the increment in tendon force due to externally applied load within the range of elastic material behavior. The prestress losses 
resulting from different time-dependent factors are calculated separately in order to visualize their contributions.

Consider the following compatibility condition for a specific time interval $\left(t_{1}, t_{2}\right)$ :

$$
\delta_{p, s h}+\Delta P_{s h}\left(t_{2}, t_{1}\right) \times \delta_{1}=0
$$

where $\delta_{p, s h}$ is the elongation in the direction of tendon profile due to the application of shrinkage-equivalent axial force and bending moment; $\delta_{1}$ is the tendon shortening due to unit tendon force; and $\Delta P_{s h}$ is the losses of prestress force due to shrinkage effect. The quantities of $\delta_{p, s h}$ and $\delta_{1}$ are computed by employing the principle of virtual work:

$$
\begin{gathered}
1 \times \delta_{p, s h}=\frac{\int_{0}^{L} \Delta N_{s h}^{*}\left(t_{2}, t_{1}, x\right) \times a^{\prime}(x) d x}{E_{s} A_{c p}^{\prime}}+\frac{\int_{0}^{L} \Delta M_{s h}^{*}\left(t_{2}, t_{1}, x\right) \times m^{\prime}(x) d x}{E_{s} I_{c p}^{\prime}} \\
1 \times \delta_{1}=\frac{\int_{0}^{L} m^{\prime}(x) \times m^{\prime}(x) d x}{E_{s} I_{c p}^{\prime}}+\frac{1 \times 1 \times L_{t}}{E_{t} A_{t}}+\frac{\int_{0}^{L} a^{\prime}(x) \times a^{\prime}(x) d x}{E_{s} A_{c p}^{\prime}}
\end{gathered}
$$

in which $A_{c p}^{\prime}$ and $I_{c p}^{\prime}$ are the gross area and moment of inertia of the age-adjusted transformed section; $a^{\prime}(x)$ and $m^{\prime}(x)$ are the unit axial force and unit moment functions; $E_{t}, A_{t}$, and $L_{t}$ are the elastic modulus, area and total length of the high-strength steel tendon; $E_{S}$ is the elastic modulus of the steel girder. The quantities associated with prime notation are evaluated based on the age-adjusted modular ratio $n^{\prime}$. The integration is taken over the full bridge length $L$. The substitution of Eqs. (5.38) and (5.39) into Eq. (5.37) results in 


$$
\Delta P_{s h}=\frac{-\frac{\int_{0}^{L} \Delta N_{s h}^{*}(x) a^{\prime}(x) d x}{L_{t} A_{c p}^{\prime}} I_{c p}^{\prime}-\frac{\int_{0}^{L} \Delta M_{s h}^{*}(x) m^{\prime}(x) d x}{L_{t}}}{\frac{\int_{0}^{L} m^{\prime 2}(x) d x}{L_{t}}+\frac{E_{s} I_{c p}^{\prime}}{E_{t} A_{t}}+\frac{\int_{0}^{L} a^{\prime 2}(x) d x}{L_{t} A_{c p}^{\prime}} I_{c p}^{\prime}}
$$

Eq. (5.40) is the expression for estimating the prestress losses due to shrinkage effect during the time interval $\left(t_{1}, t_{2}\right)$. The losses of prestress force due to creep effect during the same period can be estimated by following the same procedure, which finally yields

$$
\Delta P_{c r}=\frac{-\frac{\int_{0}^{L}\left[\Delta N_{c r a}^{*}(x)+\Delta N_{c r m}^{*}(x)\right] a^{\prime}(x) d x}{L_{t} A_{c p}^{\prime}} I_{c p}^{\prime}-\frac{\int_{0}^{L}\left[\Delta M_{c r a}^{*}(x)+\Delta M_{c r m}^{*}(x)\right] m^{\prime}(x) d x}{L_{t}}}{\frac{\int_{0}^{L} m^{\prime 2}(x) d x}{L_{t}}+\frac{E_{s} I_{c p}^{\prime}}{E_{t} A_{t}}+\frac{\int_{0}^{L} a^{\prime 2}(x) d x}{L_{t} A_{c p}^{\prime}} I_{c p}^{\prime}}
$$

Since all the functions of location $x$ are symmetric about the longitudinal center of the bridge in this case of study, the integration may be taken only over half of the bridge length $L_{1 / 2}(=0.5 L)$, along with the replacement of $L_{t}$ by $0.5 L_{t}$ in Eqs. (5.40) and (5.41).

Calculation of the losses of prestress force $\Delta P_{s h}$ and $\Delta P_{c r}$ via Eqs. (5.40) and (5.41) requires performing numerical integration which sometimes is not desirable in practice. It may, however, be approximated by using simpler expressions. Based on the results of numerical operation of those two equations, it is found that the most dominant term in the denominator (same for both equations) is the second one, i.e. the term $E_{s} I_{c p}^{\prime} / E_{t} A_{t}$. Furthermore, it is also observed that the most prominent terms of integration in the numerators are the ones associated with the terms $\Delta N_{s h}^{*}$ in Eq. (5.40) and $\Delta N_{c r a}^{*}$ in Eq. (5.41). In addition, by taking advantage of the argument that the angle of inclination of the tendon profile is small, the following approximations can be used: 


$$
\begin{gathered}
a_{0}(x)=a^{\prime}(x) \cong-1.0 \\
L \cong L_{t}
\end{gathered}
$$

Through a series of substitutions, the simplified versions of Eqs. (5.40) and (5.41) are attained as follows:

$$
\begin{gathered}
\Delta P_{s h}\left(t_{2}, t_{1}\right) \cong\left[\Delta \varepsilon_{s h}\left(t_{2}, t_{1}\right) E_{c} A_{c}\right] \times\left[\frac{1}{E_{s} A_{c p}^{\prime}}\right] \times\left[E_{t} A_{t}\right] \\
\Delta P_{c r}\left(t_{2}, t_{1}\right) \cong\left[\frac{-\Delta \varphi\left(t_{2}, t_{1}\right) P\left(t_{1}\right)}{E_{s} A_{c p 0}} E_{c} A_{c}\right] \times\left[\frac{1}{E_{s} A_{c p}^{\prime}}\right] \times\left[E_{t} A_{t}\right]
\end{gathered}
$$

The above approximate formulae reveal that the most important component of time-dependent factors to cause prestress losses is the action of uniform compression. With that conclusion, the estimation of $\Delta P_{s h}$ and $\Delta P_{c r}$ can be envisioned as three steps, each of which corresponds to a bracket in either expression (5.43) or (5.44). To begin with, the uniformly applied compressive force due to shrinkage or creep is generated in the first bracket by multiplying the shrinkage or creep strain, the elastic modulus and area of the concrete deck. The second step is to apply the compressive force calculated in the first step to the age-adjusted transformed section, which, in view of algebraic meaning, is to divide the force by the product of the elastic modulus and area of the age-adjusted transformed section. Finally, by consenting the consistency of the strains in the composite section and steel tendon, the losses of prestress force can be obtained through the multiplication of the result form the first two steps and the product of the elastic modulus and area of the steel tendon. Later the effectiveness of using the expressions (5.43) and (5.44) is examined numerically by comparing the computed results based on the approximate formulae and the more elaborate equations [Eqs. (5.40) and (5.41)]. 
The prestress losses due to the relaxation in steel tendon can be estimated by using any existing empirical formula such as that presented as Eq. (5.12). The total losses of prestress force during the period $\left(t_{1}, t_{2}\right)$ then is the sum of those caused by shrinkage, creep and relaxation effects:

$$
\Delta P\left(t_{2}, t_{1}\right)=\Delta P_{s h}\left(t_{2}, t_{1}\right)+\Delta P_{c r}\left(t_{2}, t_{1}\right)+\Delta P_{r e}\left(t_{2}, t_{1}\right)
$$

It is noted that the quantities $\Delta P_{s h}$ and $\Delta P_{c r}$ calculated according to Eqs. (5.40) and (5.41) or (5.43) and (5.44) are negative in values. The value of $\Delta P_{r e}$ is also managed to be negative. The mechanism of the losses in prestress force can be equivalent by applying a compressive force $\Delta P$ along the direction of tendon profile. As a result, a set of structural resultant reactions: $\Delta P \times a^{\prime}(x)$ for axial and $\Delta P \times m^{\prime}(x)$ for bending actions are to develop. An illustration for both $\Delta P \times a^{\prime}(x)$ and $\Delta P \times m^{\prime}(x)$ is provided in Fig. 5.6.

The time-dependent structural response in the composite girder is now attainable with all the required information of $\Delta N_{s h}^{*}, \Delta M_{s h}^{*}, \Delta N_{c r a}^{*}, \Delta N_{c r m}^{*}, \Delta M_{c r a}^{*}, \Delta M_{c r m}^{*}$ and $\Delta P$. The stress distribution developed in the steel girder and concrete deck due to the timedependent effects during the time interval $\left(t_{1}, t_{2}\right)$ can be calculated respectively:

$$
\begin{aligned}
& \Delta \sigma^{S}\left(x, y_{c p}^{\prime}\right)=\frac{\Delta M_{t o t}^{*}(x) y_{c p}^{\prime}}{I_{c p}^{\prime}}+\frac{\Delta N_{t o t}^{*}(x)}{A_{c p}^{\prime}} \\
& \Delta \sigma^{C}\left(x, y_{c p}^{\prime}\right)= {\left[\frac{\Delta M_{t o t}^{*}(x) y_{c p}^{\prime}}{I_{c p}^{\prime}}+\frac{\Delta N_{t o t}^{*}(x)}{A_{c p}^{\prime}}\right] \times \frac{1}{n^{\prime}} } \\
&-\left[\Delta \varepsilon_{s h}+\Delta \varepsilon_{c r a}^{C}\left(x, y_{c p 0}^{C}\right)+\Delta \varepsilon_{c r m}^{C}\left(x, y_{c p 0}^{C}\right)\right] \times E_{c, a d j}
\end{aligned}
$$

where

$$
\Delta M_{\text {tot }}^{*}(x)=\Delta M_{s h}^{*}(x)+\Delta M_{c r a}^{*}(x)+\Delta M_{c r m}^{*}(x)+\Delta P \times m^{\prime}(x)
$$




$$
\Delta N_{\text {tot }}^{*}(x)=\Delta N_{s h}^{*}(x)+\Delta N_{c r a}^{*}(x)+\Delta N_{c r m}^{*}(x)+\Delta P \times a^{\prime}(x)
$$

and $\Delta \varepsilon_{c r a}^{C}$ and $\Delta \varepsilon_{c r m}^{C}$ are given in Eqs. (5.26) and (5.27).

\subsubsection{Summary of Analytical Procedure}

The analytical procedure described in the above paragraphs is concisely summarized in the following for clarity. For a time interval $\left(t_{1}, t_{2}\right)$ :

(1) Calculate the prestress force for the current time step, $P\left(t_{1}\right)$ [use Eq. (5.28)].

(2) Calculate shrinkage strain and creep coefficient, $\Delta \varepsilon_{s h}\left(t_{2}, t_{1}\right)$ [may use Eqs. (5.10), (5.11), (5.16) and Table 5.1], and $\Delta \varphi\left(t_{2}, t_{1}\right)$ [may use Eqs. (5.7), (5.8), (5.9) and $(5.17)]$

(3) Calculate the age-adjusted elastic modulus, $E_{c, \text { adj }}$ [use Eq. (5.15)].

(4) Calculate the sectional properties of the age-adjusted transformed section, including the gross area $A_{c p}^{\prime}$, moment of inertia $I_{c p}^{\prime}$ and position of neutral axis $\bar{y}_{c p}^{\prime}$ based on the modular ratio, $n^{\prime}$ [use Eq. (5.20)].

(5) Develop the structural resultant functions due to shrinkage effect, $\Delta N_{s h}^{*}(x)$ and $\Delta M_{s h}^{*}(x)$ [use Eqs. (5.23) and (5.24)].

(6) Calculate the losses of prestress force due to shrinkage, $\Delta P_{s h}$ [use Eq. (5.40) or $(5.43)]$

(7) Develop the structural resultant functions due to creep effect, $\Delta N_{c r a}^{*}(x), \Delta M_{c r a}^{*}(x)$, $\Delta N_{c r m}^{*}(x)$ and $\Delta M_{c r m}^{*}(x)$ [use Eqs. (5.26), (5.27), (5.33), (5.34), (5.35) and (5.36)].

(8) Calculate the losses of prestress force due to creep, $\Delta P_{s h}$ [use Eq. (5.41) or (5.44)].

(9) Calculate the losses of prestress force due to relaxation, $\Delta P_{r e}$ [may use Eq. (5.12)]. 
(10) Calculate the total losses of prestress force, $\Delta P\left(t_{2}, t_{1}\right)$ [use Eq. (5.45)].

(11) Calculate the stress distribution in steel girder due to time-dependent effects, $\Delta \sigma^{S}$ [use Eqs. (5.46), (5.48) and (5.49)].

(12) Calculate the stress distribution in steel girder due to time-dependent effects, $\Delta \sigma^{C}$ [use Eqs. (5.26), (5.27), (5.47), (5.48) and (5.49)].

\subsection{Numerical Demonstration}

The estimation of structural response by employing the proposed procedure is demonstrated numerically by using the Elkhart County Bridge as the example. The functions of shrinkage strain and creep coefficient for concrete are adopted from the AASHTO LRFD Specifications (1994), which are presented in Section 5.1.1. The estimation of prestress losses due to the relaxation in steel tendon is based on the recommendations provided by the PCI Committee on Prestress Losses (1975), which suggests some empirical equations [Eq. (5.12)]. The demonstration is performed for the first time interval of analysis, which spans a period of eight days starting form the day of post-tensioning. To avoid repetition, the following presentation is made only for the interior bridge girders (G3).

The time-dependent analysis involves several functions in terms of time, with the units in days. The day that the concrete deck was poured $(6 / 19 / 97)$ is set to be day zero, therefore the age of concrete coincides with the time for the long-term calculation. According to that, the day of post tensioning (6/30/97) corresponds to $t_{0}=11$-th days, and therefore the time interval for numerical demonstration is $\left(t_{1}, t_{2}\right)=(11,19)$. The initial 
prestress level after the instant mechanical losses is estimated as $P\left(t_{1}=t_{0}=11\right.$-th day $)=$ $E_{t} \times \varepsilon_{t 0}=(0.005491) \times\left(202.71 \mathrm{kN} / \mathrm{mm}^{2}\right)=1.1131 \mathrm{kN} / \mathrm{mm}^{2}=1,113.1 \mathrm{MPa}$ (Chapter 4) The day of concrete drying $t_{d}$ is difficult to determine. Curing process had been undertaken over certain period of time and it is not practical to ascertain when the concrete started to shrink notably. In the analysis $t_{d}$ is taken to be equal to $t_{0}$, the day of post-tensioning. This number is considered to be both reasonable and practical.

Several other factors need to be specified to estimate the shrinkage strain and creep coefficient, including

volume to surface ratio $V / S=109.7 \mathrm{~mm}(\mathrm{G} 1) ; 101.1 \mathrm{~mm}(\mathrm{G} 3)$

concrete strength at 28 days $f_{c}^{\prime}=68.95 \mathrm{MPa}$

relative humidity $H=70 \%$

The value of $f_{c}^{\prime}$ is determined from the interpolation of the cylindrical concrete test data provided by the contractor. To estimate the unrestrained shrinkage strain in concrete, the empirical equation for moist-cured concrete [Eq. (5.10a)] is adopted since this type of curing process is probably similar to that taken in the field. The creep coefficient for unrestrained concrete is calculated based on Eq. (5.7). For the interior girders (G3), the following results are obtained:

$$
\begin{aligned}
& \Delta \varepsilon(19,11)=-32.2 \times 10^{-6} \\
& \Delta \varphi(19,11)=1.341 \times 10^{-1}
\end{aligned}
$$

The age-adjusted elastic modulus $E_{c, a d j}$ is determined by using Eq. (5.15). In that equation, $E_{c}$ represents the short-term elastic modulus of the concrete which can be evaluated according to Eq. (4.21) if the concrete strength is specified. However, during 
this time period $\left(t_{1}, t_{2}\right)=(11,19)$, the concrete has not yet reached its design strength $f_{c}^{\prime}$ which is taken as the compressive strength at 28 days. The mean elastic modulus is thus taken for the calculation in this time interval by averaging the values of $E_{c}$ at $t_{1}\left(E_{c}=\right.$ $32,320 \mathrm{MPa}$ for $f_{c}=58.61 \mathrm{MPa}$ at 11-th day $)$ and $t_{2}\left(E_{c}=33,050 \mathrm{MPa}\right.$ for $f_{c}=62.05$ MPa at 19-th day), yielding a value of $\bar{E}_{c}=32,690 \mathrm{MPa}$. For those time intervals with $t_{1}$ $\geq 28$-th day, the averaging process is not necessary since the concrete strength is assumed to be constant. The value of aging coefficient $\chi(19,11)$ is obtained by two-way interpolation from the tabulated data found in Bazant's paper (1972), and which are listed in Table 5.2. The value of $\chi(19,11)$ is found to be equal to 0.6439 , and therefore

$$
E_{c, a d j}=30,090 \mathrm{MPa}
$$

Accordingly, the values of $A_{c p}^{\prime}, I_{c p}^{\prime}$ and $\bar{y}_{c p}^{\prime}$ of the age-adjusted transformed section are calculated based on the modular ratio $n^{\prime}=E_{s} / E_{c, a d j}=200 / 32.69=6.12$ :

$$
A_{c p}^{\prime}=144,539 \mathrm{~mm}^{2} \quad I_{c p}^{\prime}=4.26749 \times 10^{10} \mathrm{~mm}^{4} \quad \bar{y}_{c p}^{\prime}=1,356.7 \mathrm{~mm}
$$

Following the proposed analytical procedure, the structural response functions due to shrinkage and creep effects are developed with the acquisition of the above information. The results are shown in Fig. 5.7 and Fig. 5.8. All of these functions are symmetric about the center of the bridge. The loss of post-tensioning force in the steel tendon due to shrinkage and creep are estimated according to the derived equations. If Eqs. (5.40) and (5.41) are used, the following results for prestress losses are obtained:

$$
\begin{aligned}
& \Delta \sigma_{s h}=-3.912 \mathrm{MPa} \\
& \Delta \sigma_{c r}=-2.143 \mathrm{MPa}
\end{aligned}
$$


Alternatively, to avoid numerical integration, approximate formulae [Eqs. (5.43) and (5.44)] are used to give the following stress increments:

$$
\begin{aligned}
& \Delta \sigma_{s h}=-4.619 \mathrm{MPa} \\
& \Delta \sigma_{c r}=-2.387 \mathrm{MPa}
\end{aligned}
$$

Stress losses due to relaxation is calculated according to the formula for low-relaxation steel [Eq. (5.12a)] which is the material type of the tendon used for this bridge structure $\left(f_{p u}=1,862 \mathrm{MPa} ; f_{p y}=0.9 f_{p u}=1,675 \mathrm{MPa}\right)$. The estimated result is

$$
\Delta \sigma_{r e}=-6.459 \mathrm{MPa}
$$

The functions $\Delta P \times m^{\prime}(x)=A_{t} \times \Delta \sigma \times m^{\prime}(x)$ and $\Delta P \times a^{\prime}(x)=A_{t} \times \Delta \sigma \times a^{\prime}(x)$ used for calculating structural response are plotted in Fig. 5.9. The total time-dependent prestress losses during this time interval is the sum of $\Delta \sigma_{s h}, \Delta \sigma_{c r}$ and $\Delta \sigma_{r e}$ :

$$
\begin{aligned}
& \Delta \sigma^{T}(19,11)=-3.912 \mathrm{MPa}-2.143 \mathrm{MPa}-6.459 \mathrm{MPa}=-12.513 \mathrm{MPa} \text { (exact), or } \\
& \Delta \sigma^{T}(19,11)=-4.619 \mathrm{MPa}-2.387 \mathrm{MPa}-6.459 \mathrm{MPa}=-13.464 \mathrm{MPa} \text { (approximate). }
\end{aligned}
$$

It is observed that at this very early phase after the anchorage of tendons, the effect of steel relaxation is the major source to cause prestress loss. Furthermore, the estimation based upon the approximate method seems more conservative since it yields a larger value of calculated total prestress losses.

The stress distributions at certain longitudinal gage locations due to timedependent factors evaluated by considering the total effect [using Eqs. (5.46) and (5.47) associated with Eqs. (5.48) and (5.49)] are listed in Table 5.3. The vertical positions for presentation are: the levels of the embedded concrete gages, the bottom surface of the top steel flange, and the top surface of the bottom steel flange. It is found that at almost all of 
the locations in the steel girder, additional compressive stresses are introduced by the time-dependent effects. In the concrete deck some tensile stresses are generated because of the resistance from the steel girder to prevent the concrete from free shrinkage and creep.

Besides the post-tensioning force, there are some other sustained loads that could cause creep in concrete. For the Elkhart County Bridge, the most significant one comes from the weight of the traffic barriers which are shown in Fig. 5.10. The weight of one central and two side barriers are assumed to be evenly distributed to each of the seven girders since the concrete slab is very rigid. The barriers were cast during this time period. The instant strains caused by the weight of the barriers at the level of concrete gages at each longitudinal gage locations are listed in Table 5.4, together with the instantaneous strains due to the application of post-tensioning. The comparison between the two sets of data suggests that the factor due to the weight of the barriers to cause creep deformation is negligible if compared with that resulting from post-tensioning. The weight of barriers imposed on the bridge also induced some increment in tendon force which theoretically might also have some effects in the long term. However the magnitude (immediately induced tendon force) is minute according to calculation $(6.67 \mathrm{kN}$ vs. $3,771 \mathrm{kN}$ of initial tendon force), and therefore this quantity is also neglected. In summary, to calculate the total stress or strain, only the instantaneous response in the composite section is considered. 


\subsection{Results and Discussions}

The analytical results for the subsequent time steps are presented in this section. The calculation is carried out by a computer program coded with FORTRAN language. Comparison between the theoretical solutions and field measurements is first made to inspect the effectiveness of the analytic model, and then further evaluation on the longterm bridge performance beyond the last day of test data collection is conducted according to the same analytical procedure. The time intervals for calculation are specified by the ages of concrete and are listed in Table 5.5. The concrete strengths at the ages of 11,19 and 26 days are 58.61, 62.05 and $68.95 \mathrm{MPa}$, respectively. After 28 days the concrete is assumed to have a constant strength equal to $68.95 \mathrm{MPa}$.

\subsubsection{Comparison of Analytical and Experimental Results}

Fig. 5.11 shows the variation of prestress with respect to time in days. Results for the gages near the north and south abutments (the only two active gages) are presented in addition to the average values of these two. The process of analytical calculation starts with assigning an initial prestress level which is obtained from the data collected on the construction site at the time immediately following the post-tensioning operation. Compared with the experimental results, the analytical results tend to underestimate the prestress losses at the beginning, but match the later test data well. The diversity for the earlier time intervals is perhaps due to the nature of the adopted shrinkage and creep functions. Another observation reveals that the simplified analytical method which uses the approximate formulae Eqs. (5.43) and (5.44) gives more conservative results. 
However, it is concluded that those simplified formulae are able to provide satisfactory approximation, since the deviation from the exact solutions [based on Eqs. (5.40) and (5.41) to account for shrinkage and creep effects] is not very significant.

The total strain distributions in the steel girder at the longitudinal gage locations 2 (near pier), 6 (near midapan) and 7 (near abutment) at the ends of several time intervals are presented in Figs. 5.12 to 5.17. The elevation of gage position is measured from the top surface of the bottom flange. Each of the figure contains four plots which correspond to the following times: (a) right after post-tensioning (Post/T), (b) 15 days after Post/T, (c) 52 days after Post/T and (d) 276 days after Post/T. Both of the results from analysis and experiment are demonstrated in each plot for the purposes of comparison. The calculation includes the instantaneous strain distributions caused by the weight of traffic barriers, but excludes the creep effect due to the barrier load. The equations with full integration for estimating $\Delta P_{s h}$ and $\Delta P_{c r}$ [Eqs. (5.40) and (5.41)] are used instead of the approximate formulae [Eqs. (5.43) and (5.44)]. As for the strain distributions presented in Chapter 4, the calculated structural response does not include the part caused by the selfweight of steel girder in order to be compared with the collected test data. In short, the analytical strain distributions shown in Figs. 5.12 to 5.17 are obtained by superimposing those due to dead load (weight of concrete only), post-tensioning (instantaneous loading), time-dependent factors (shrinkage, creep and relaxation), and additionally imposed dead load (weight of barriers, instantaneous effect only). For the strain distributions presented in plots (b), (c) and (d), those induced by the barrier weight comprise only a small portion compared with other sources of loading. 
Observing Figs. 5.12 to 5.17, the conclusion is drawn that the time-dependent effects can introduce additional significant compressive stresses in the steel section. The strain distributions at the critical locations (locations 2 and 6) seem to shift to the negative (compressive) direction fairly uniformly, which means that the axial deformation is more prominent than the flexural deformation at those locations. Moreover, the amount of shifting at location 2 is more than that at location 6 . The changes of slope of strain distributions at location 7 , on the other hand, are more obvious than those at locations at locations 2 and 6 according to the numerical calculation, indicating that a more notable local variation of curvature may be expected around the end region.

In general, the analytical model provides a reasonable estimate of the actual structural response. The predicted results are particular satisfactory at location 6 which is about the midspan of the bridge. In fact, this is the location where the calculated response is less sensitive to the input parameters such as the end condition of girder, elastic modulus of concrete and other variables in time-dependent functions. The agreement between the analytical and experimental results at location 2 is not as good as that at location 6 , but is still in a reasonable range. For both girders at location 2 , the estimated strain values at the bottom of the flanges are roughly about $70 \%$ to $90 \%$ of the corresponding measured results. Once again, as that discussed in Chapter 4, the analysis does not result in a good prediction at location 7 around the abutment, since the analytical model is based upon the assumption of simply supported end conditions. However, the response near the bridge end is not the most crucial. It is hence rational to neglect the end rigidity inasmuch as that structural behavior at the critical locations is able to be properly 
evaluated with tolerable precision. At all of those locations, the discrepancies between the analyzed and test results increase as time progresses. Part of the reason may possibly be attributed to the instability of the electrical resistance strain gages due to the degradation of the protective coating for preventing the invasion of moisture and humidity toward the time of latter field testing.

The diversity between the analytical and experimental results may partially be ascribed to temperature changes. The analysis performed herein does not involve the calculation of thermal response, which, however, is present in the test data. To evaluate this factor effectively, detailed information about the distributions of temperature variations in structural components including the steel girders, the concrete deck and high-strength steel tendons must be acquired.

\subsubsection{Further Prediction of Structural Performance}

Since the reliability of the proposed analytical procedure is found to be acceptable throughout the comparison between the calculated and test results, further evaluation of the structural performance beyond the last field measurement can be conducted analytically with confidence. The prediction is extended for 30 years, which is considered to be a sufficient period of time such that most time-dependent effects will have occurred.

The shrinkage strain is plotted as a function of time according to Eqs. (5.10a) (moist-cured concrete) and (5.10b) (steam-cured concrete) for both of the exterior (G1) and interior (G3) girders in Fig. 5.18. It is shown that the difference between the two functions is very small at the early stage of drying. At the 30 -year service life of the 
bridge, the shrinkage strains reach approximately 400 microstrains. The creep coefficient for unrestrained concrete based on Eq. (5.7) is plotted in Fig. 5.19 as a function of time, for both the exterior and interior girders. It is shown that the ultimate values of creep coefficient are around 1.1.

The estimated prestress losses in the tendons along G1 (the exterior girder) and G3 (the interior girder) due to the three types of time-dependent factors are demonstrated in Fig. 5.20. Ultimately, the shrinkage effect plays the most dominant role in the longterm behavior. Initially, relaxation in the steel tendon is the major source of prestress losses. However, the influence of relaxation decays very rapidly and eventually becomes the smallest fraction of the total prestress losses in the long run. The variation of prestress is shown in Fig. 5.21 as a supplement, The value of prestress in the figure is obtained by subtracting the total prestress losses at the corresponding time in Fig. 5.20 from the initial prestress (1,113.1 MPa). The predicted ultimate prestress losses (72.52 MPa for G1, and 74.45 MPa for G3) are about $7 \%$ of the initial prestress.

Time-dependent variations in the predicted stresses in the steel and concrete portions of the composite girder are evaluated at particular critical locations along the bridge span. Those longitudinal locations include: (a) internal support, (b) drape location, (c) midspan, (d) 0.4 span from end, and (e) bridge end. The vertical positions for the calculation are the top of steel girder, the bottom of steel girder (for stress in steel), and the top of concrete deck. The stress variations are depicted graphically in Fig. 5.22 for the exterior bridge girder (G1) and in Fig. 5.23 for the interior girder (G3). The stress value shown in each of those figures is the sum of stresses due to all sources of loading, 
exclusive of that caused by live load (Unlike the strain distributions shown in Figs. 5.12 to 5.17, the contribution from the self-weight of steel girder is included). It is apparent that the time-dependent factors combine to introduce significant compressive forces in the steel section. Those forces seem to be applied with certain uniformity except for that at the bridge end. The most crucial section is considered to be that at the drape location. Since for this section the stress distribution that develops over a long time period is almost uniform and the magnitude is significant (even though not the greatest), it is potentially the most likely location where the web may buckle locally. Since additional compressive stresses are introduced into the beam due to time-dependent effects, then the design stability check for the beam web should account for these additionally induced stresses.

Also portrayed in Figs. 5.22 and 5.23 are the stresses on the top surface of the concrete deck at the corresponding longitudinal locations along the exterior and interior girders. The critical locations are considered to be those positions where concrete cracking might exist. It is observed that some tensile stresses are gradually generated in the concrete deck, which is regarded as the result of the resistance from the steel girders acting against the unrestrained shrinkage and creep deformations in concrete. At certain locations, the total concrete stresses eventually become tensile after 30 years of service. Among them the most critical one is that at the drape point. The stresses at that location after 30 years in G1 and G3 are calculated as $2.31 \mathrm{MPa}$ and $2.40 \mathrm{MPa}$, respectively. Compared with the tensile concrete strength which is roughly estimated as $0.1 f_{c}^{\prime}=$ $0.1 \times 68.95 \mathrm{MPa}=6.985 \mathrm{MPa}$, the estimated maximum service stresses in the concrete are 
still within the range of the uncracked condition even if the live load stresses are included (The truck loading test presented in Chapter 4 produces a maximum tensile stress in the concrete equal to $0.54 \mathrm{MPa}$ according to the previous calculation). This finding also justifies the legitimacy of the assumption of uncracked concrete which is made at the very first beginning to ensure the use of uniform sectional properties of the composite girder.

\subsection{Summary of Conclusions}

The long-term performance of post-tensioned steel-concrete composite bridges was investigated by comparing predicted long-term response with that exhibited by the Elkhart County I-90 Toll Road Bridge. The following conclusions are summarized from the above discussions:

(1) Based on the concept of equivalent axial force and bending moment associated with the adoption of the age-adjusted effective modulus method, the proposed analytical model is capable of providing a reasonable prediction of the long-term structural behavior. Use of the shrinkage and creep functions suggested by the AASHTO LRFD Specifications and the empirical formula for steel relaxation recommended by the PCI Committee on Prestress Losses appears to produce reasonable results.

(2) The most important component to cause prestress losses due to shrinkage and creep effects is the action of uniform compression. According to that conclusion, the calculation of the prestress losses due to those factors can be simplified by avoiding numerical integration. Compared with the exact solutions, the approximate results tend to be slightly more conservative, with only insignificant difference. 
(3) Time-dependent effects potentially introduce significant compressive stresses in the steel girder section. The possibility of web local buckling is therefore an essential consideration in design process. The most crucial location may be the region near the drape point, since the stress distribution is rather uniform at that location.

(4) The concrete deck is subjected to tensile stresses at particular locations, since the free shrinkage and creep deformations that develop in the deck are effectively prevented by interconnection of the concrete deck and the top flange of the steel girder. The tensile stress magnitude, however, is not considered to be large enough to cause cracks in the concrete slab. 
Table 5.1 Factor $k_{h}$ (from AASHTO LRFD Specifications) used to calculate shrinkage strain (for Eqs. $5.10 \mathrm{a} \& \mathrm{~b}$ )

$\begin{array}{cc}\text { average ambient relative humidity }(\%) & k_{h} \\ 40 & 1.43 \\ 50 & 1.29 \\ 60 & 1.14 \\ 70 & 1.00 \\ 80 & 0.86 \\ 90 & 0.43 \\ 100 & 0.00\end{array}$


Table 5.2 Aging coefficient $\chi\left(t, t_{0}\right)$ calculated by Bazant (1972)

\begin{tabular}{|c|c|c|c|c|c|}
\hline \multirow[b]{3}{*}{$t-t_{0}$ (days) } & \multirow[b]{3}{*}{$\varphi\left(t_{\infty}, 7\right)$} & \multicolumn{4}{|c|}{ Value of $\chi\left(t, t_{0}\right)$} \\
\hline & & \multicolumn{4}{|c|}{$t_{0}$ (days) } \\
\hline & & 10 & $10^{2}$ & $10^{3}$ & $10^{4}$ \\
\hline \multirow[t]{4}{*}{10} & 0.5 & 0.525 & 0.804 & 0.811 & 0.809 \\
\hline & 1.5 & 0.720 & 0.826 & 0.825 & 0.820 \\
\hline & 2.5 & 0.774 & 0.842 & 0.837 & 0.830 \\
\hline & 3.5 & 0.806 & 0.856 & 0.848 & 0.839 \\
\hline \multirow[t]{4}{*}{$10^{2}$} & 0.5 & 0.505 & 0.888 & 0.916 & 0.915 \\
\hline & 1.5 & 0.739 & 0.919 & 0.932 & 0.928 \\
\hline & 2.5 & 0.804 & 0.935 & 0.943 & 0.938 \\
\hline & 3.5 & 0.839 & 0.946 & 0.951 & 0.946 \\
\hline \multirow[t]{4}{*}{$10^{3}$} & 0.5 & 0.511 & 0.912 & 0.973 & 0.981 \\
\hline & 1.5 & 0.732 & 0.943 & 0.981 & 0.985 \\
\hline & 2.5 & 0.795 & 0.956 & 0.985 & 0.988 \\
\hline & 3.5 & 0.830 & 0.964 & 0.987 & 0.990 \\
\hline \multirow[t]{4}{*}{$10^{4}$} & 0.5 & 0.461 & 0.887 & 0.956 & 0.965 \\
\hline & 1.5 & 0.702 & 0.924 & 0.966 & 0.972 \\
\hline & 2.5 & 0.770 & 0.940 & 0.972 & 0.976 \\
\hline & 3.5 & 0.808 & 0.950 & 0.977 & 0.980 \\
\hline
\end{tabular}


Table 5.3 Stress distributions due to time-dependent effect during the time interval (11-th day to 19-th day)

Longitudinal distance from end

\begin{tabular}{rr}
\multicolumn{2}{c}{$(\mathrm{mm})$} \\
35,275 & $(\mathrm{G} 31)$ \\
34,055 & $(\mathrm{G} 32)$ \\
22,950 & $(\mathrm{G} 35)$ \\
14,110 & $(\mathrm{G} 36)$ \\
1,365 & (G3 7)
\end{tabular}

\begin{tabular}{ccc}
\multicolumn{3}{c}{ Stress value $(\mathrm{MPa})$} \\
\hline \multicolumn{3}{c}{ Vertical position } \\
$y^{C}$ & $y^{S_{\text {WTOP }}}$ & $y^{S_{\text {WBOT }}}$ \\
0.65 & -8.77 & -11.86 \\
0.63 & -8.42 & -11.27 \\
0.44 & -5.19 & -5.87 \\
0.36 & -6.00 & -2.79 \\
0.24 & -7.18 & 1.65
\end{tabular}

Note: $y^{C} \quad$ : the level of the embedded concrete gages

$y^{S_{\text {WTOP }}}:$ the level of the bottom of the top flange (upper edge of the web)

$y^{S_{\text {WBOT }}}:$ the level of the top of the bottom flange (lower edge of the web) 
Table 5.4 Instantaneous strains at the level of concrete gages

\begin{tabular}{ccc} 
& \multicolumn{2}{c}{ Instantaneous strain $\left(10^{-6}\right)$} \\
\cline { 2 - 3 } Longitudinal distance from end & \multicolumn{2}{c}{ Cause of strain } \\
$35,275(\mathrm{~mm})$ & Post-tensioning & Weight of barriers \\
$34,055(\mathrm{G} 3 \mathrm{2})$ & -237.8 & 12.7 \\
$22,950(\mathrm{G} 35)$ & -220.3 & 10.6 \\
$14,110(\mathrm{G} 36)$ & -60.6 & -3.3 \\
$1,365(\mathrm{G} 3$ 7) & -83.4 & -7.2 \\
\end{tabular}


Table 5.5 Time intervals for long-term calculation

\begin{tabular}{|c|c|c|}
\hline time step no. & start (days) & end (days) \\
\hline 1 & 11 & 19 \\
\hline 2 & 19 & 26 \\
\hline 3 & 26 & 34 \\
\hline 4 & 34 & 50 \\
\hline 5 & 50 & 63 \\
\hline 6 & 63 & 100 \\
\hline 7 & 100 & 150 \\
\hline 8 & 150 & 200 \\
\hline 9 & 200 & 250 \\
\hline 10 & 250 & 287 \\
\hline 11 & 287 & 376 \\
\hline 12 & 376 & 741 \\
\hline 13 & 741 & 1,106 \\
\hline 14 & 1,106 & 1,471 \\
\hline 15 & 1,471 & 1,836 \\
\hline 16 & 1,836 & 2,201 \\
\hline 17 & 2,201 & 2,566 \\
\hline 18 & 2,566 & 2,931 \\
\hline 19 & 2,931 & 3,661 \\
\hline 20 & 3,661 & 4,756 \\
\hline 21 & 4,756 & 5,851 \\
\hline 22 & 5,851 & 7,311 \\
\hline 23 & 7,311 & 9,136 \\
\hline 24 & 9,136 & 10,961 \\
\hline
\end{tabular}

Note: each time interval is specified by the ages of concrete. 


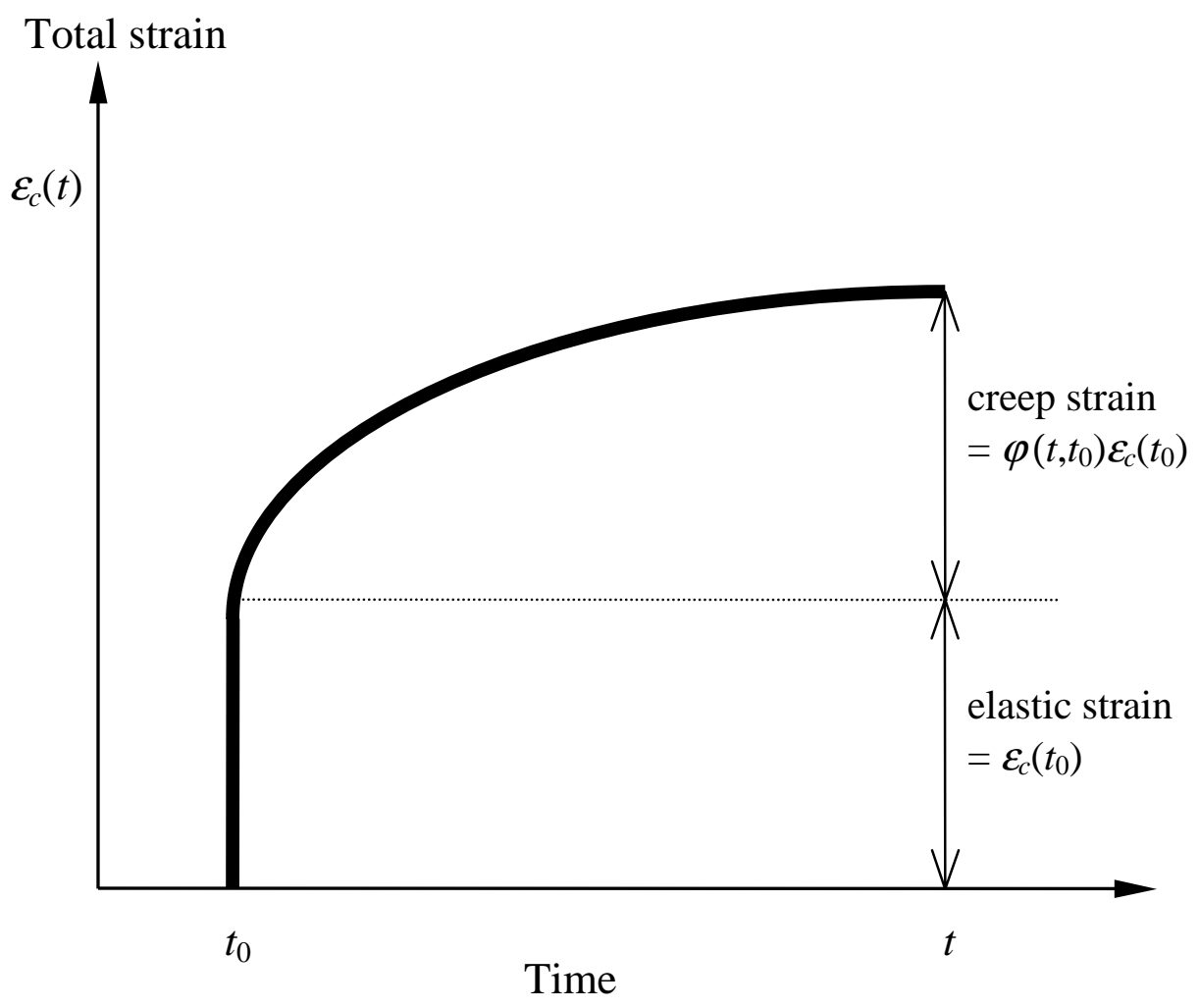

Figure 5.1 Creep of concrete under sustained stress. 


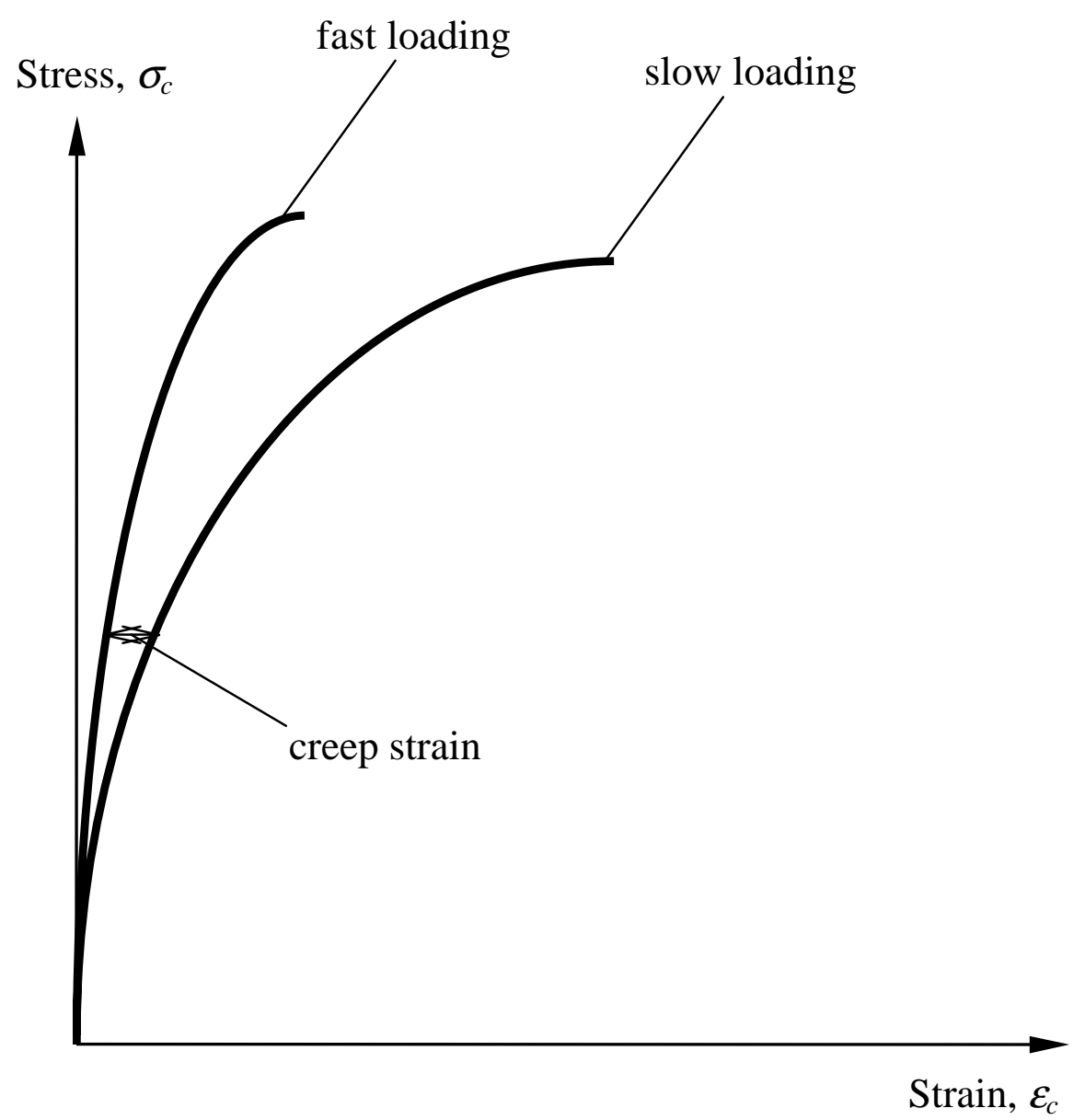

Figure 5.2 Constitutive relations of concrete. 


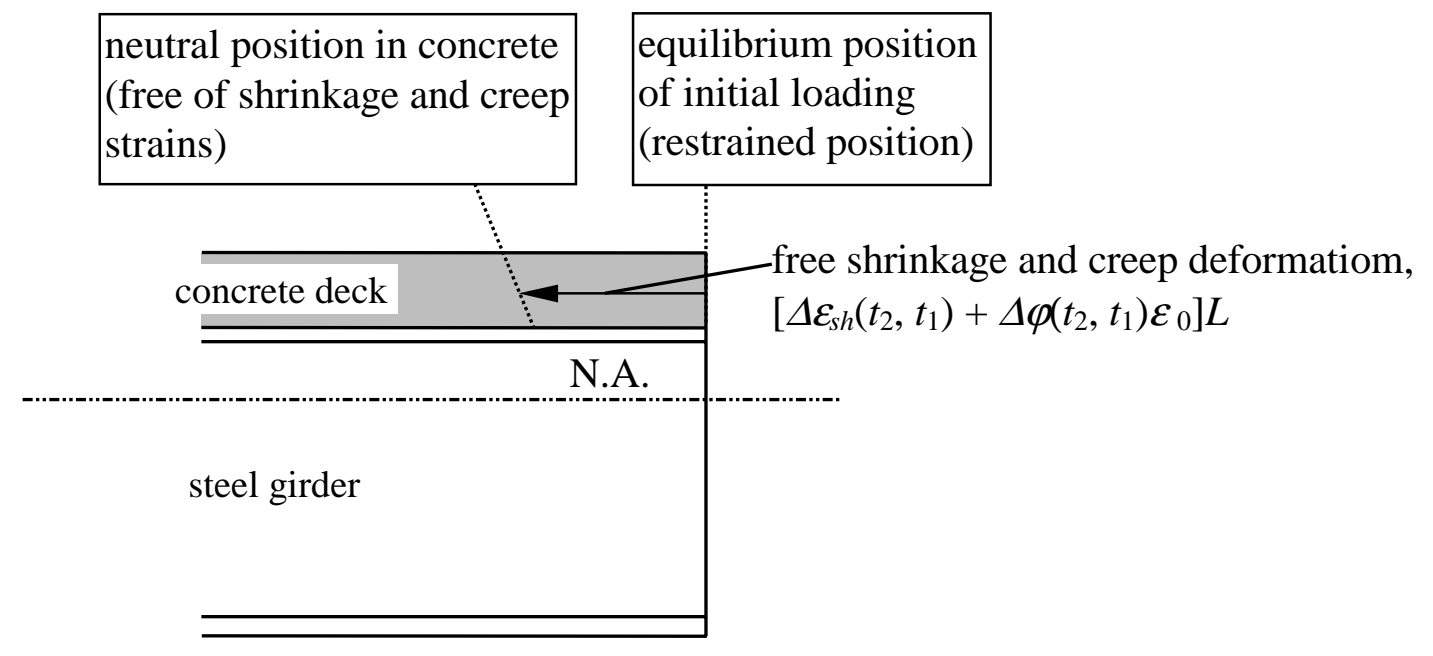

(a) Restraining process.

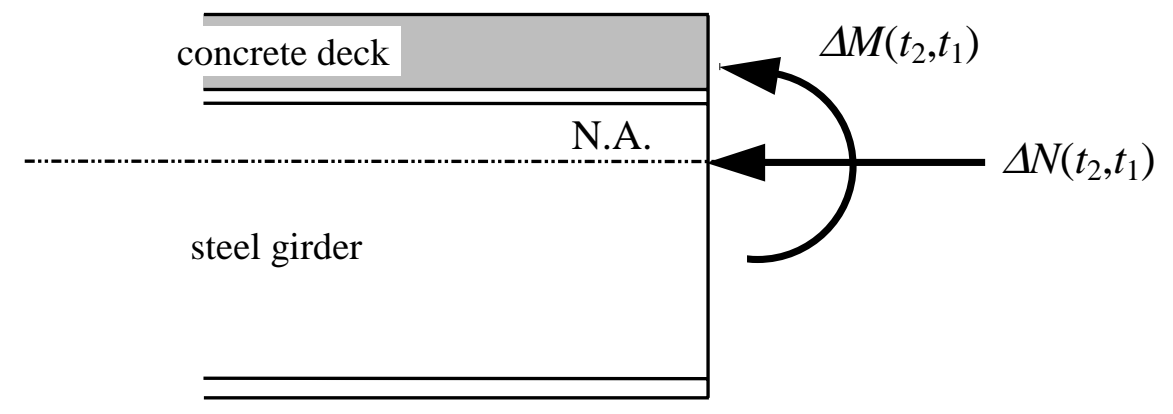

(b) Releasing process.

Figure 5.3 Application of equivalent axial force and bending moment. 


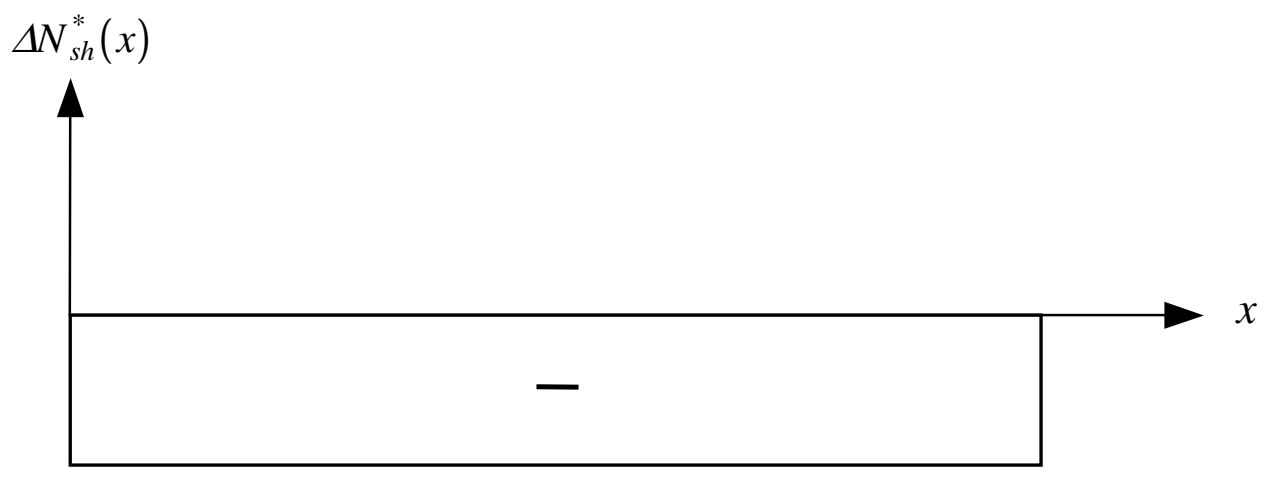

(a) Axial force response function due to shrinkage.

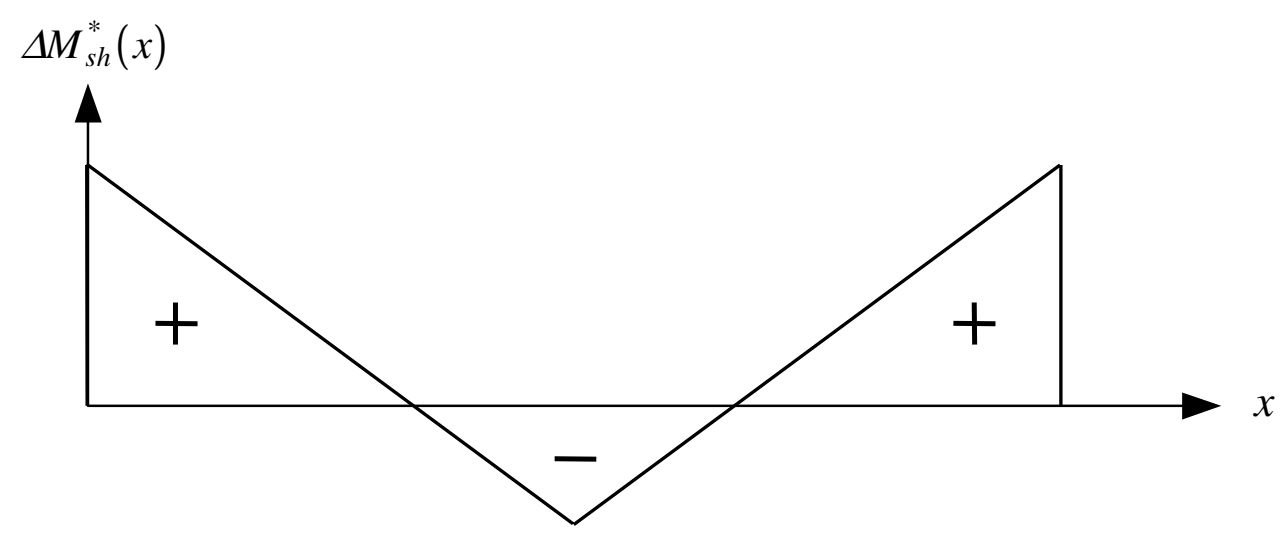

(b) Bending moment response function due to shrinkage.

Figure 5.4 Structural response functions due to shrinkage effect over the length of a two-span bridge structure. 


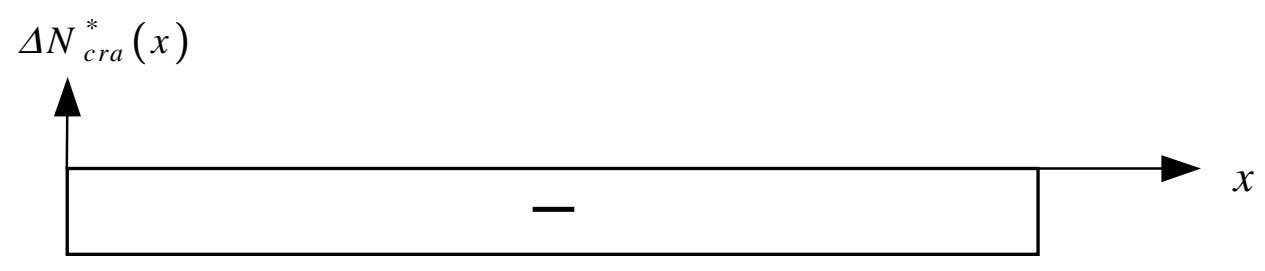

(a) Axial force response function due to creep ( $\Delta \varepsilon_{c r a}^{C}$ effect).

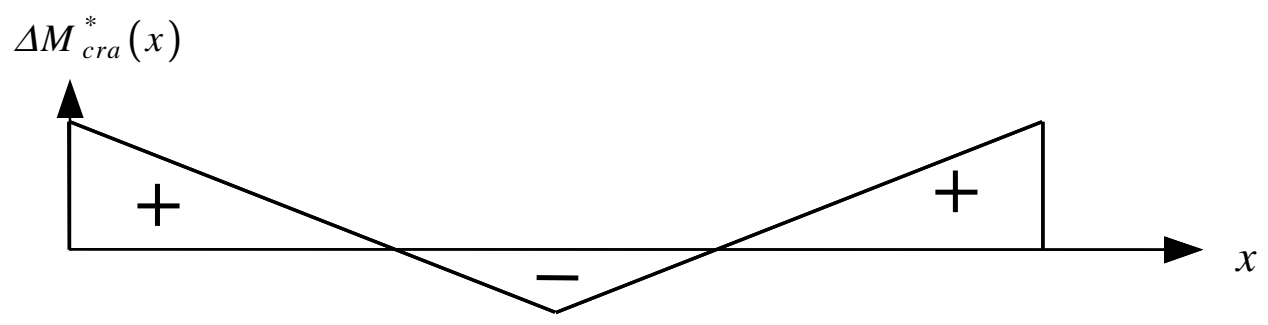

(b) Bending moment response function due to creep ( $\Delta \varepsilon_{c r a}^{C}$ effect).

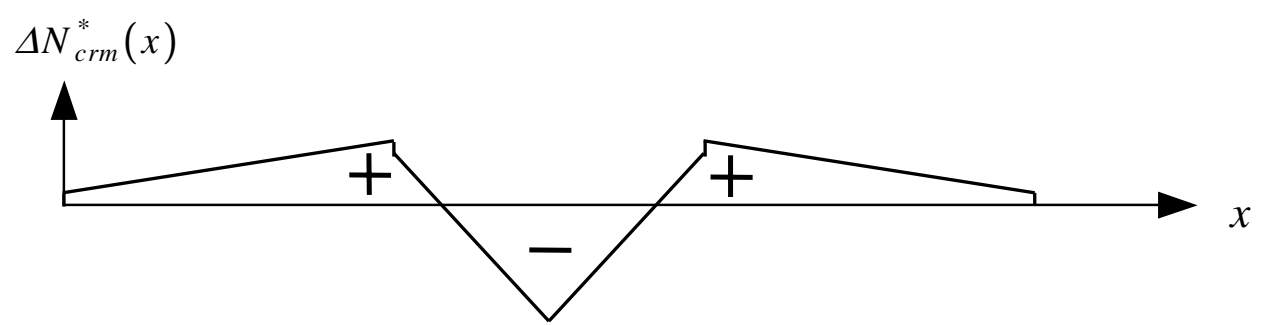

(c) Axial force response function due to creep $\left(\Delta \varepsilon_{c r m}^{C}\right.$ effect).

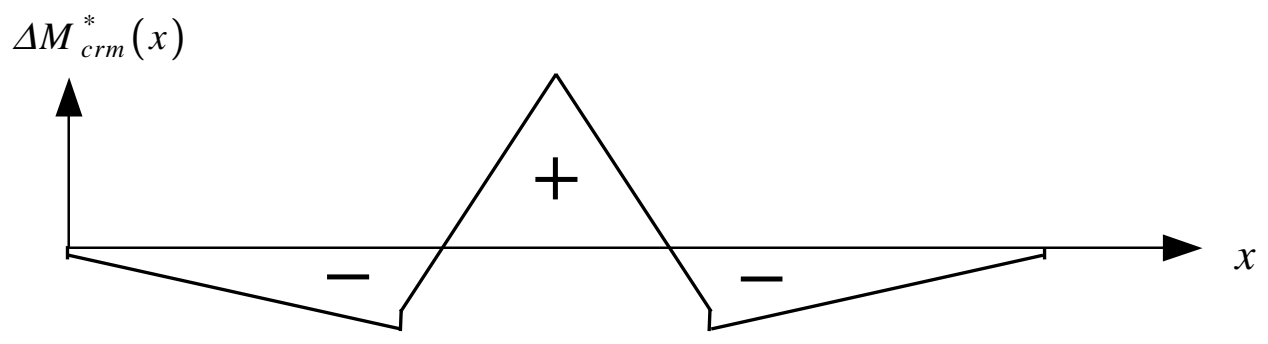

(d) Bending moment response function due to creep ( $\Delta \varepsilon_{c r m}^{C}$ effect).

Figure 5.5 Structural response functions due to creep effect over the length of a two-span bridge structure. 


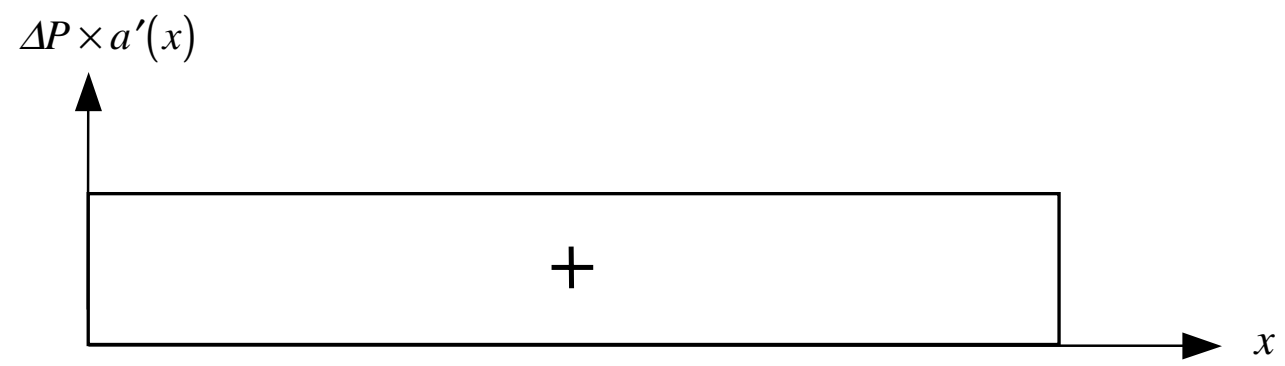

(a) Axial force response function due to prestress losses.

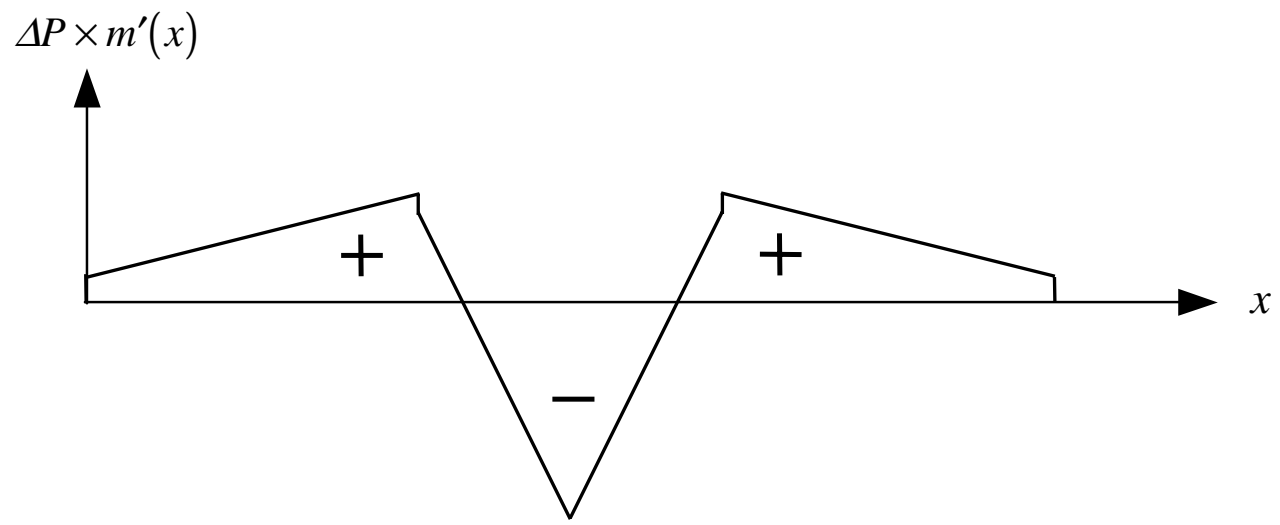

(b) Bending moment response function due to prestress losses.

Figure 5.6 Structural response functions due to prestress losses over the length of a two-span bridge structure. 


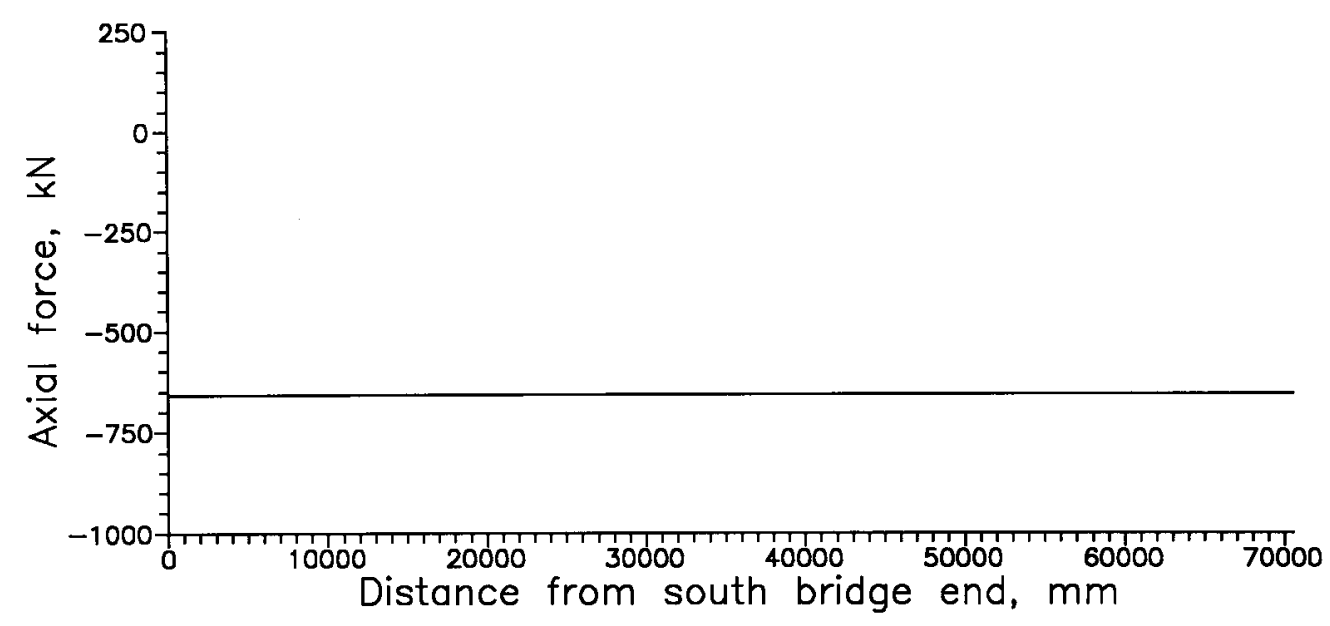

(a) Axial force response due to shrinkage

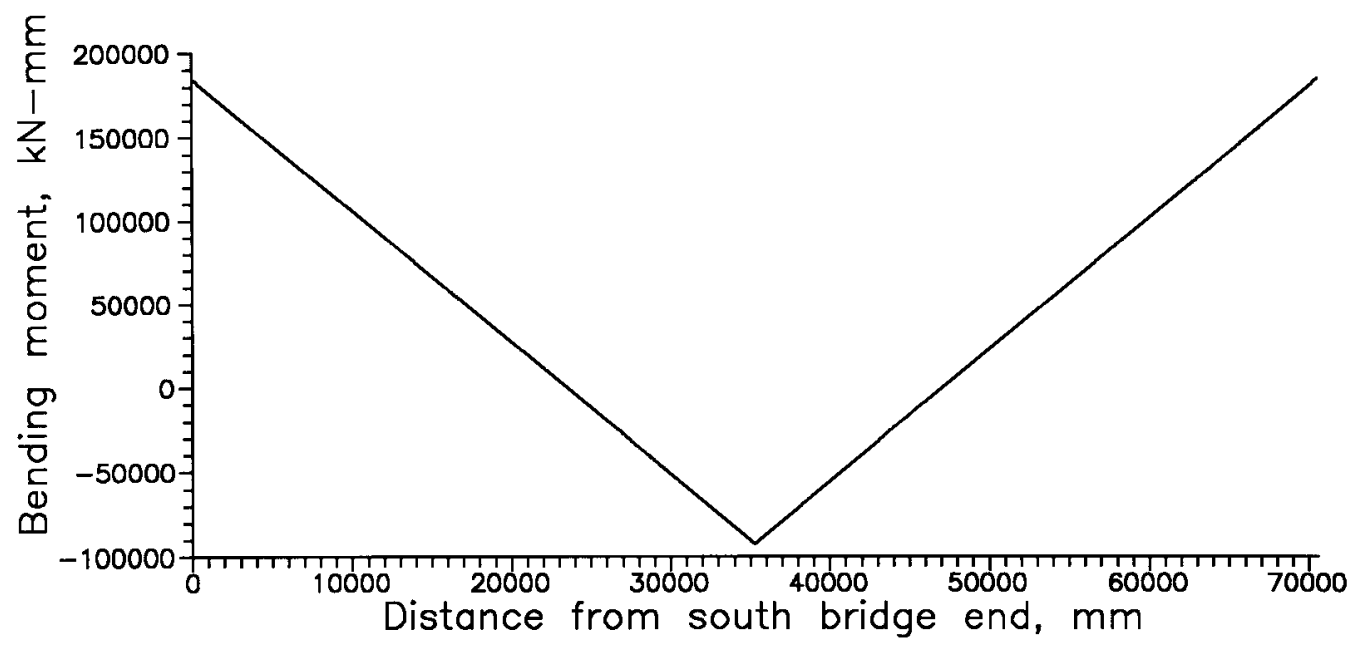

(b) Bending moment response due to shrinkage

Figure 5.7 Structural response due to shrinkage effect during the time interval (11-th day to 19-th day). 


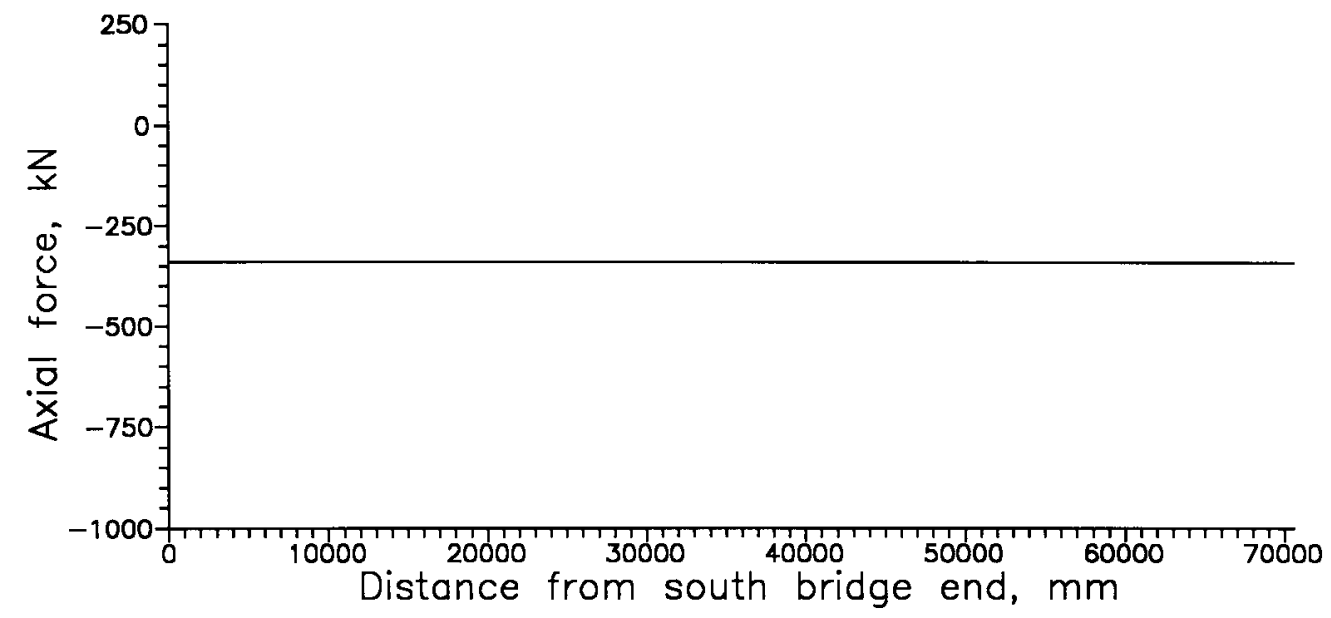

(a) Axial force response due to $\operatorname{creep}\left(\Delta \varepsilon_{\text {ora }}^{\mathrm{c}}\right)$

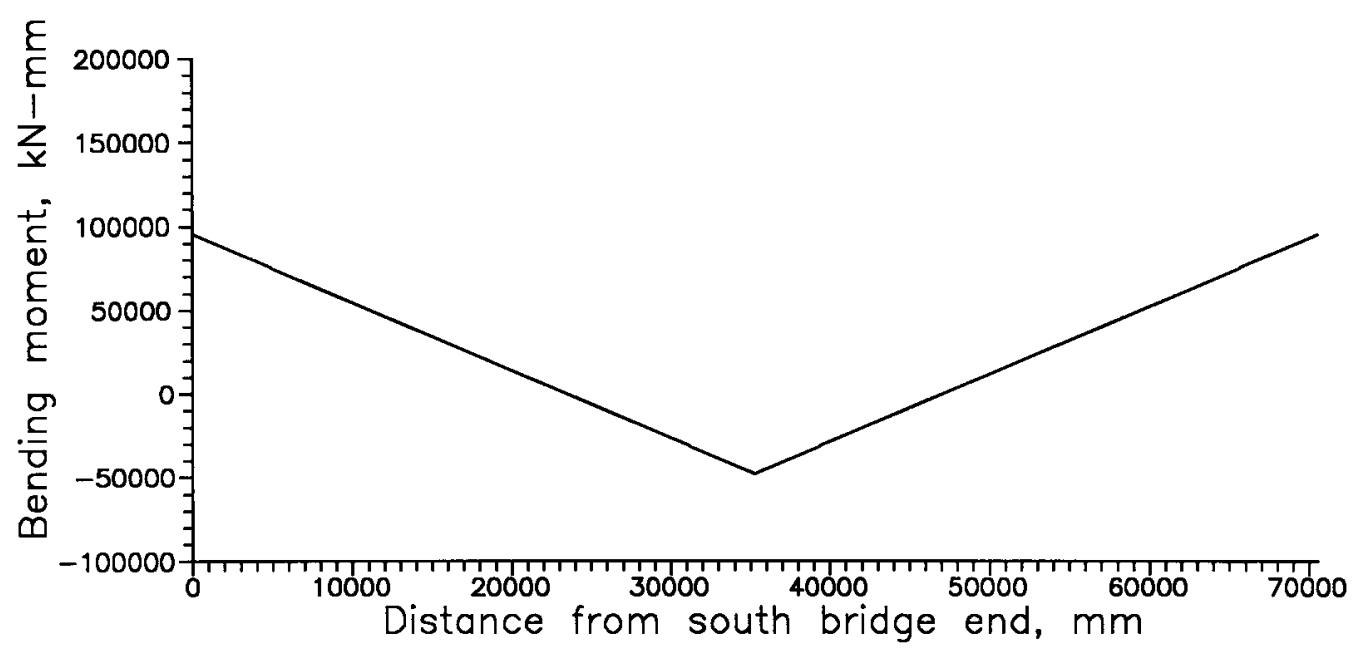

(b) Bending moment response due to creep $\left(\Delta \varepsilon_{\text {era }}^{c}\right)$

Figure 5.8 Structural response due to creep effect during the time interval (11-th day to 19-th day). 


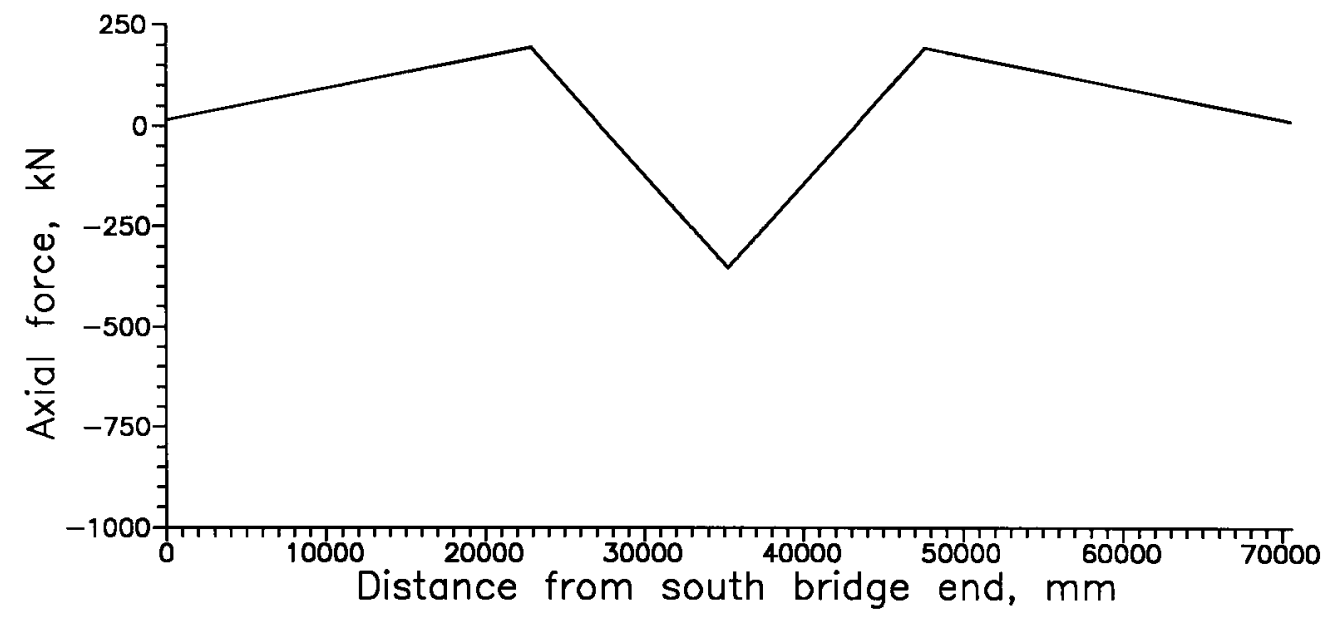

(c) Axial force response due to creep $\left(\Delta \varepsilon_{\text {crm }}^{c}\right)$

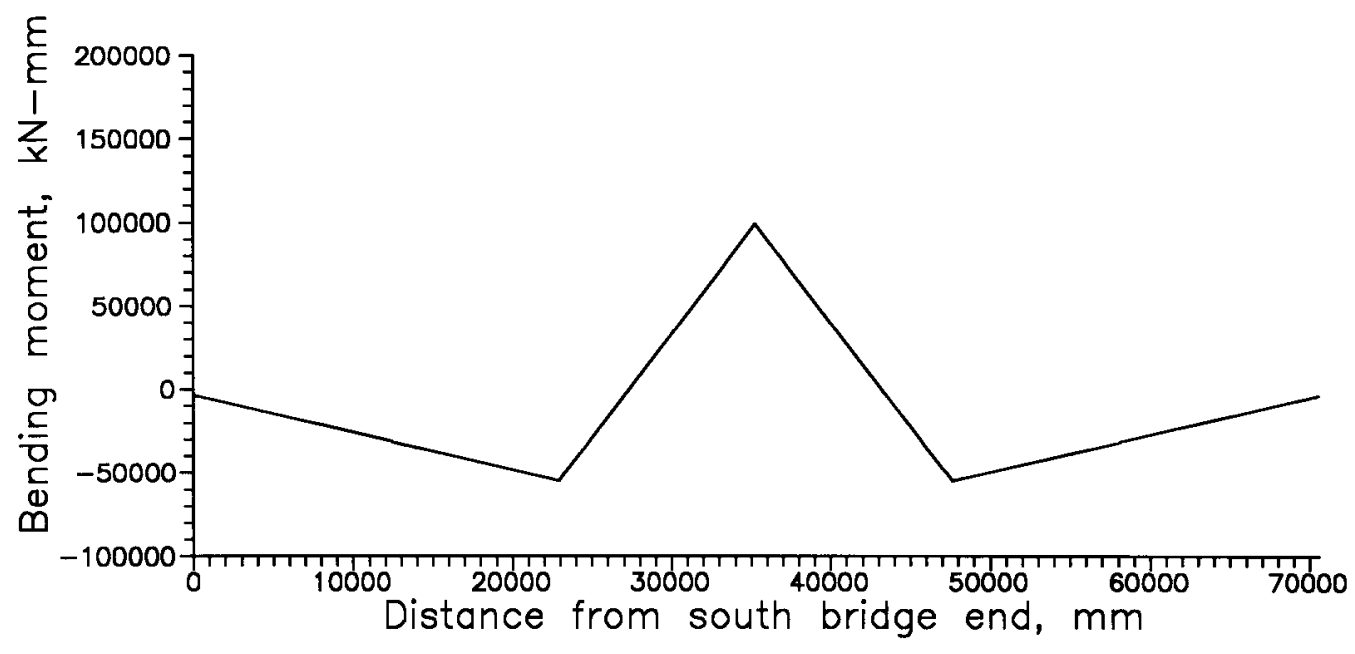

(d) Bending moment response due to creep $\left(\Delta \varepsilon_{\text {crm }}^{c}\right)$

Figure 5.8 Structural response due to creep effect during the time interval (11-th day to 19-th day). 


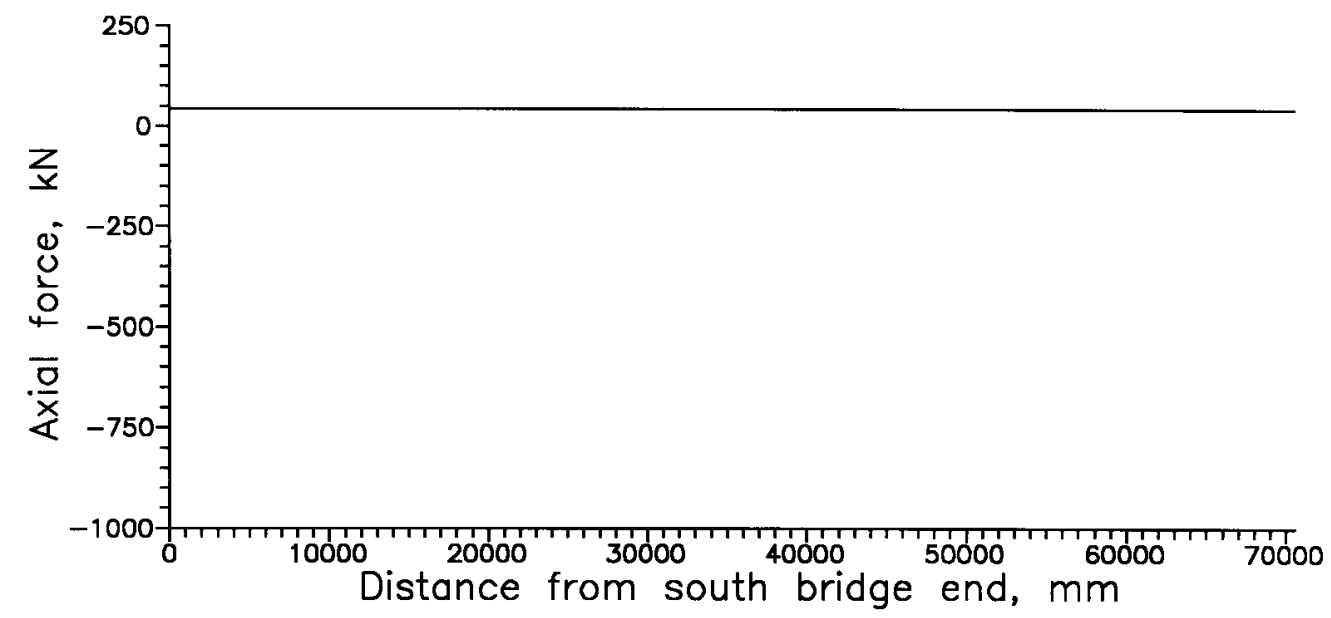

(a) Axial force response due to prestress losses

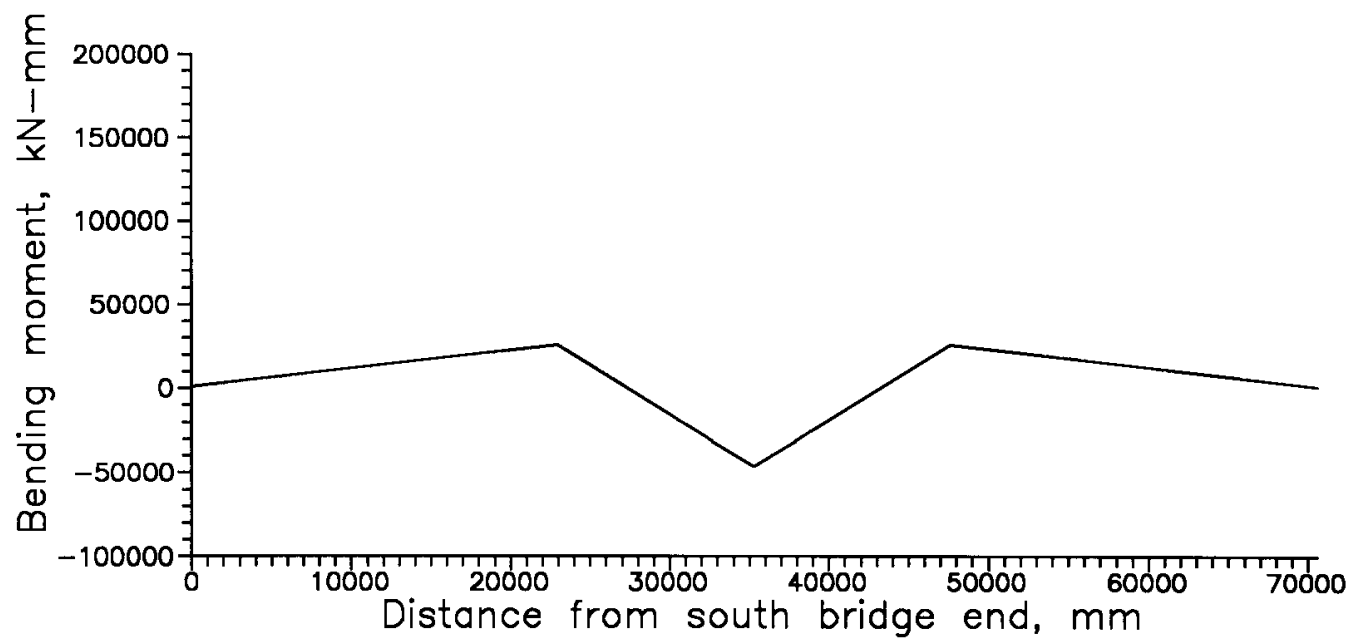

(b) Bending moment response due to prestress losses

Figure 5.9 Structural response due to prestress losses during the time interval (11-th day to 19-th day). 

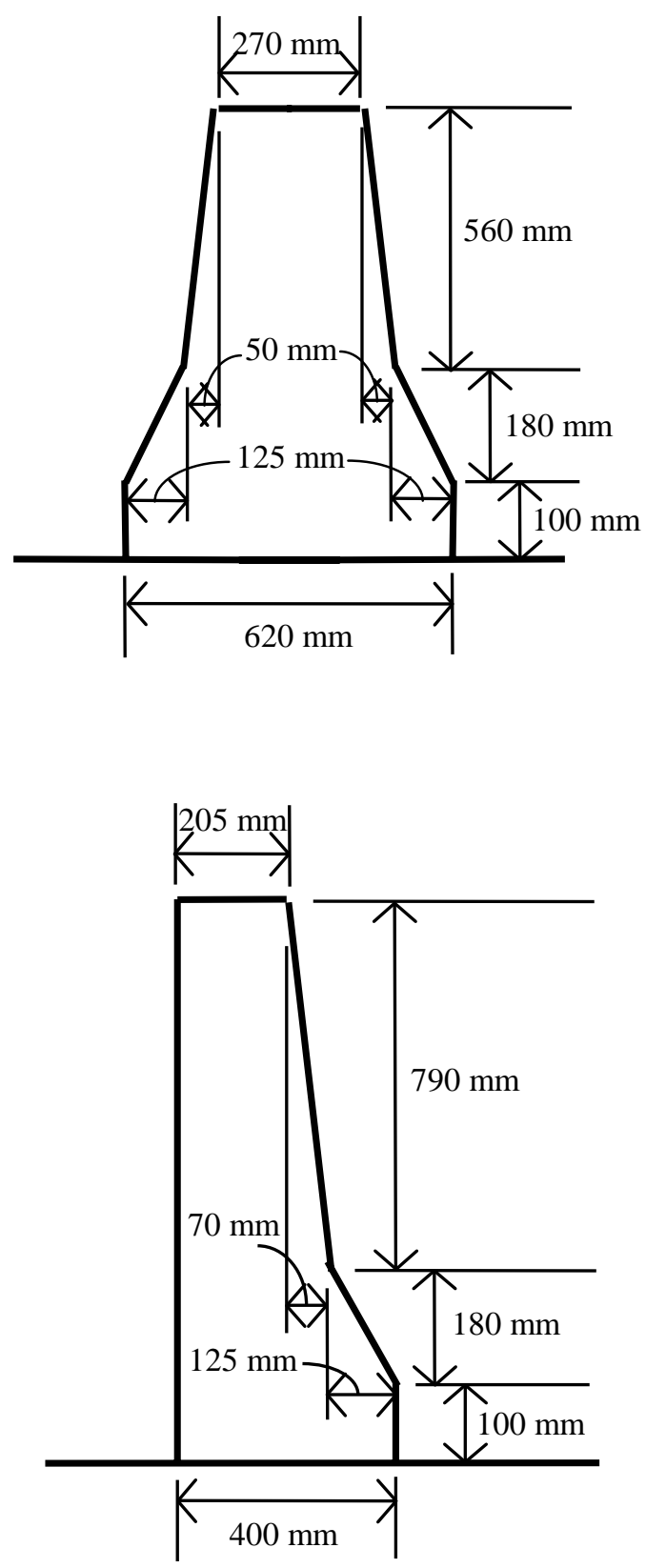

(a) Central barrier: sectional area $=$

$$
\begin{aligned}
& (620)(100)+ \\
& (0.5)(600+370)(180)+ \\
& (0.5)(370+270)(560) \\
& =330,300 \mathrm{~mm}^{2}
\end{aligned}
$$

(b) Side barrier:

sectional area $=$

$(400)(100)+$

$(0.5)(400+275)(180)+$ $(0.5)(275+205)(790)$

$=290,350 \mathrm{~mm}^{2}$

Unit weight for each girder:

$$
\begin{aligned}
{\left[330,300 \mathrm{~mm}^{2}\right.} & \left.+2\left(290,350 \mathrm{~mm}^{2}\right)\right]\left(2.356 \times 10^{-8} \mathrm{kN} / \mathrm{mm}^{3}\right) / 7 \\
& =0.003066 \mathrm{kN} / \mathrm{mm}
\end{aligned}
$$

Figure. 5.10 Traffic barriers of Elkhart County Bridge. 


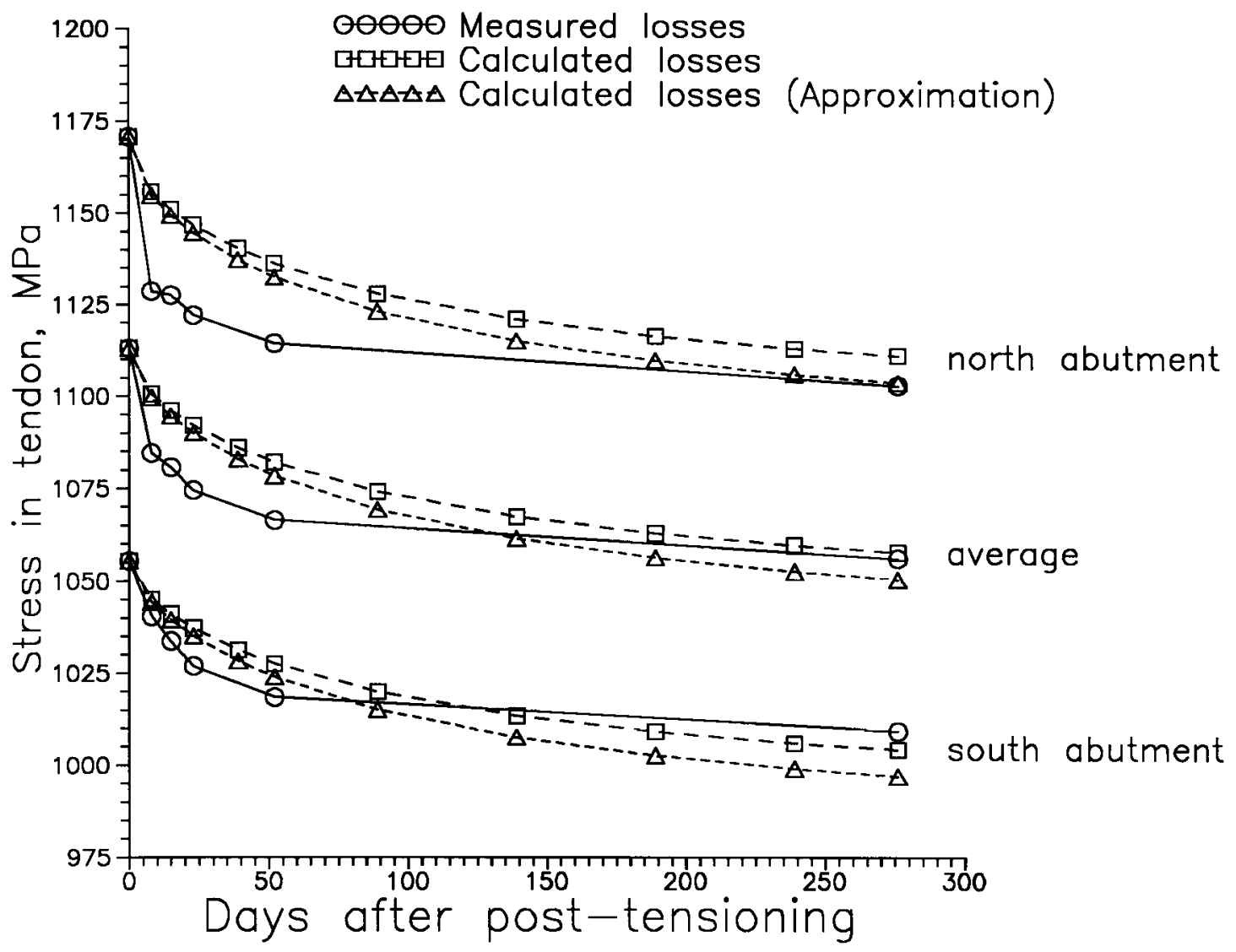

Figure 5.11 Measured and calculated prestress levels. 


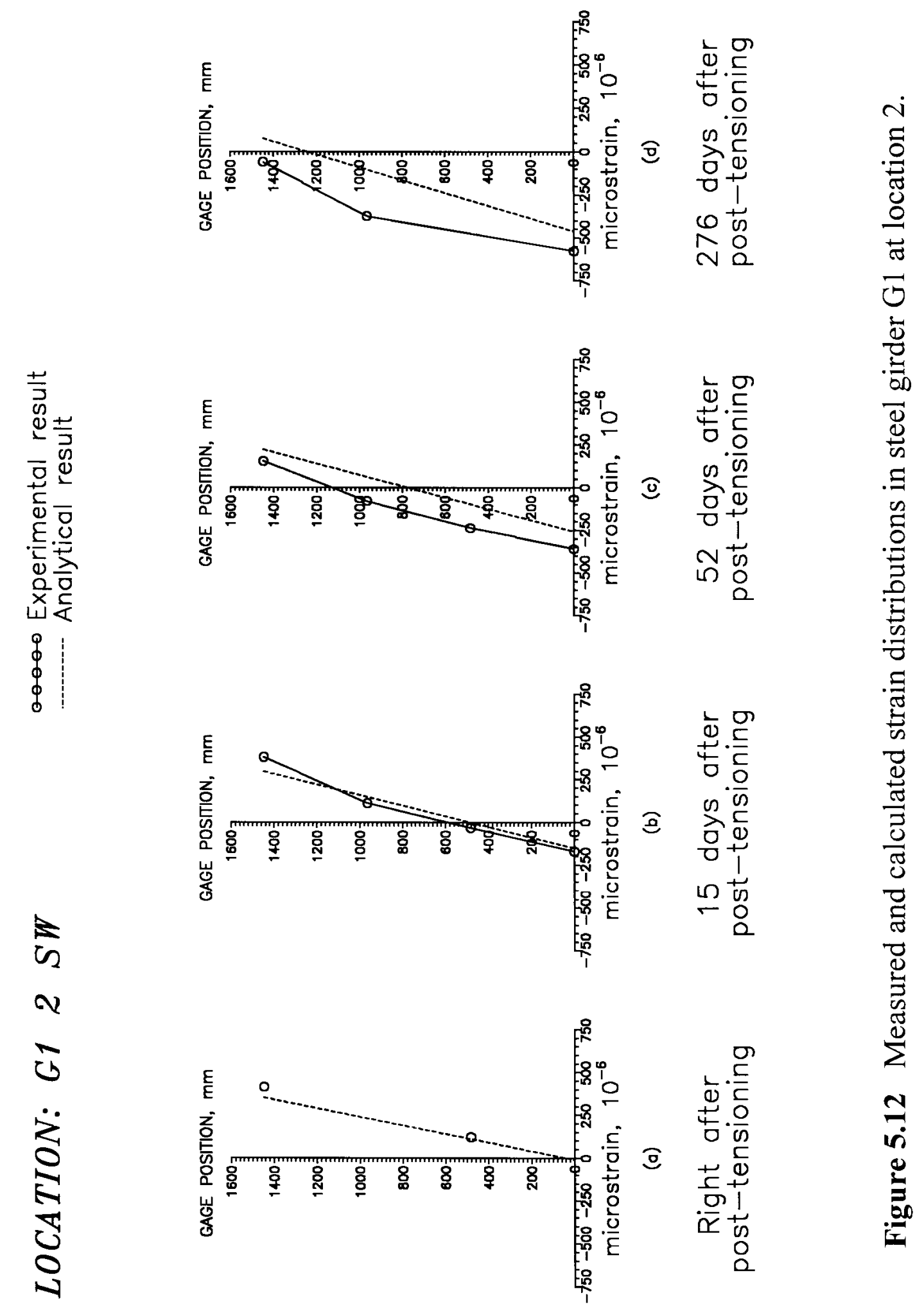




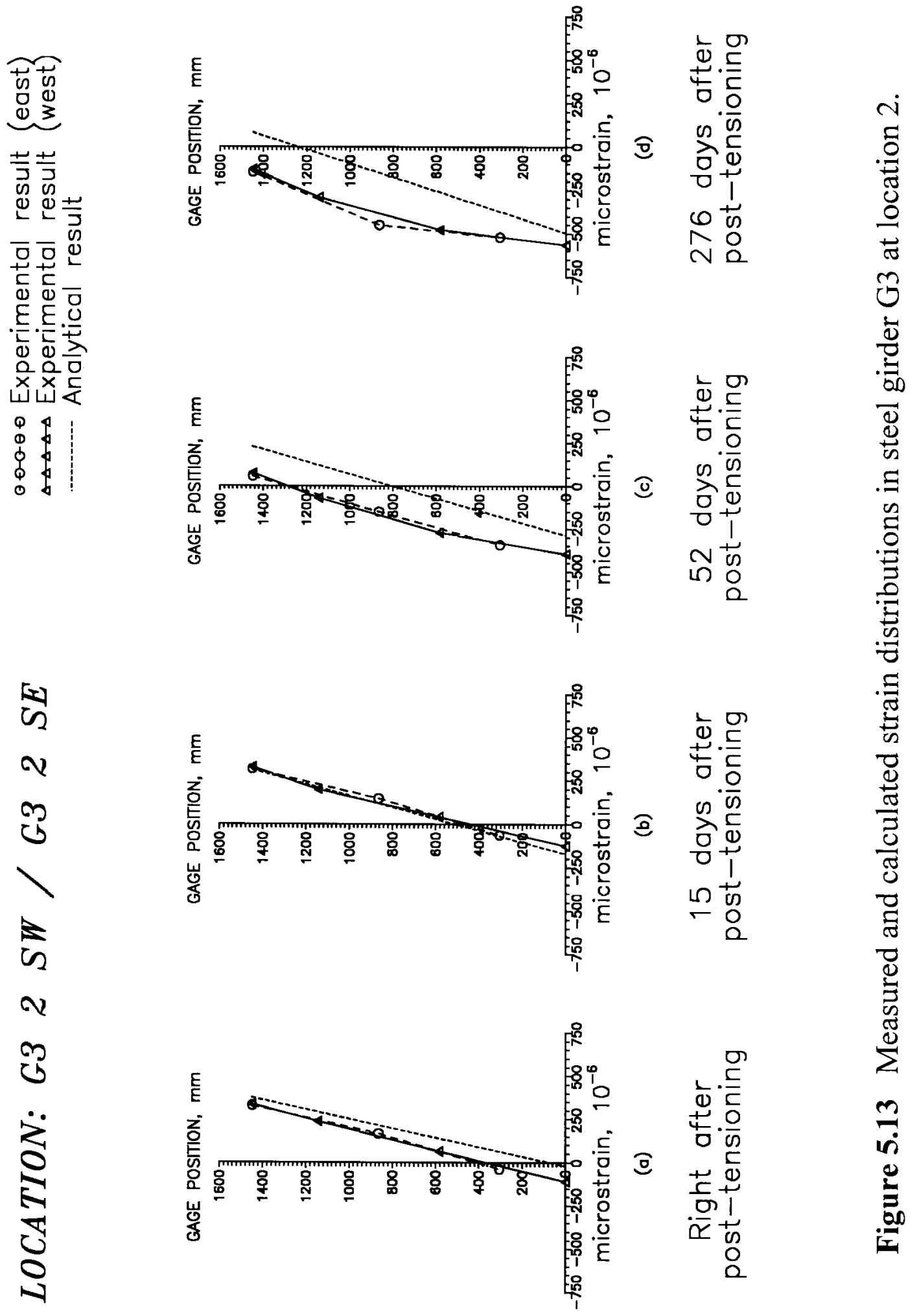




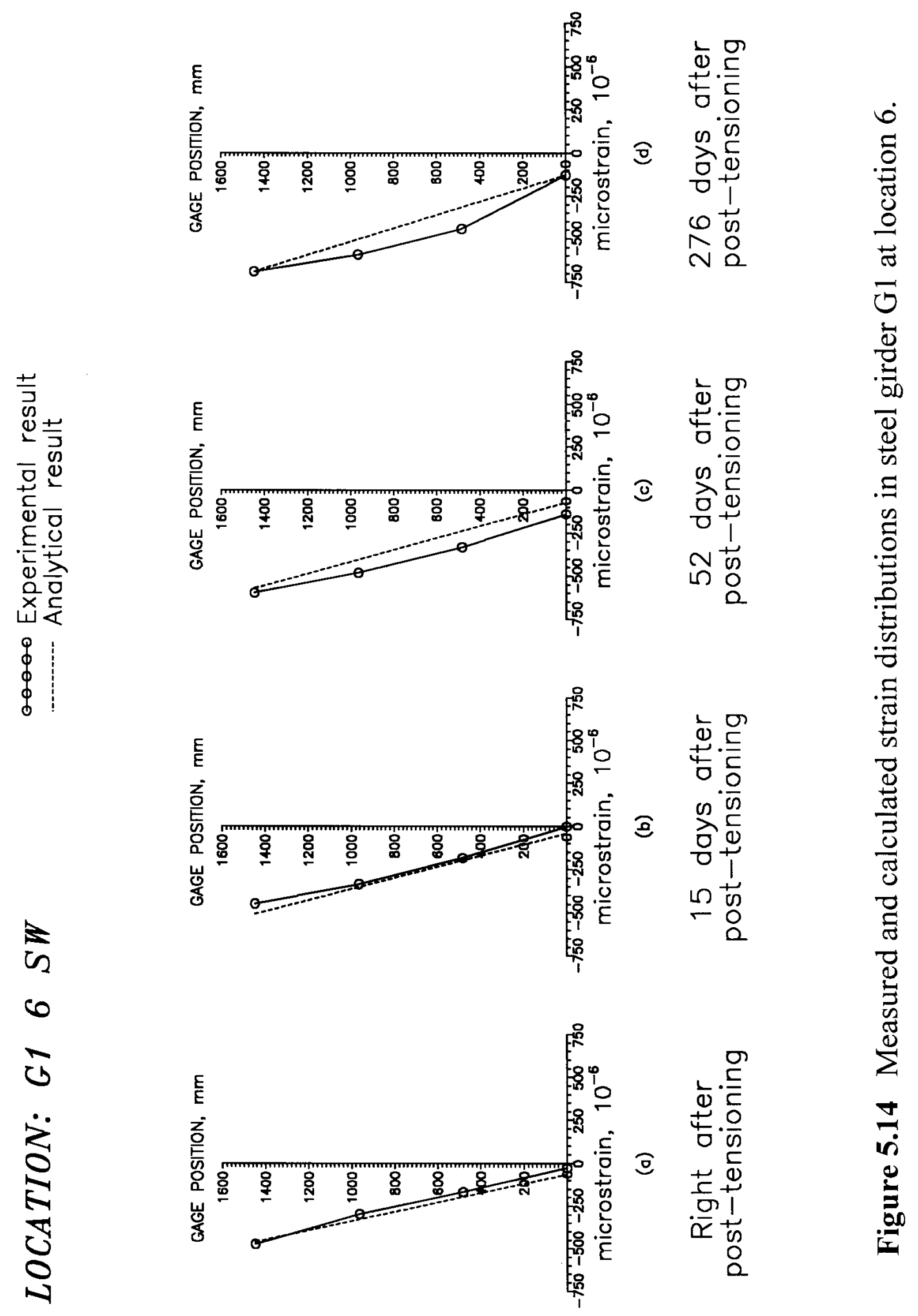




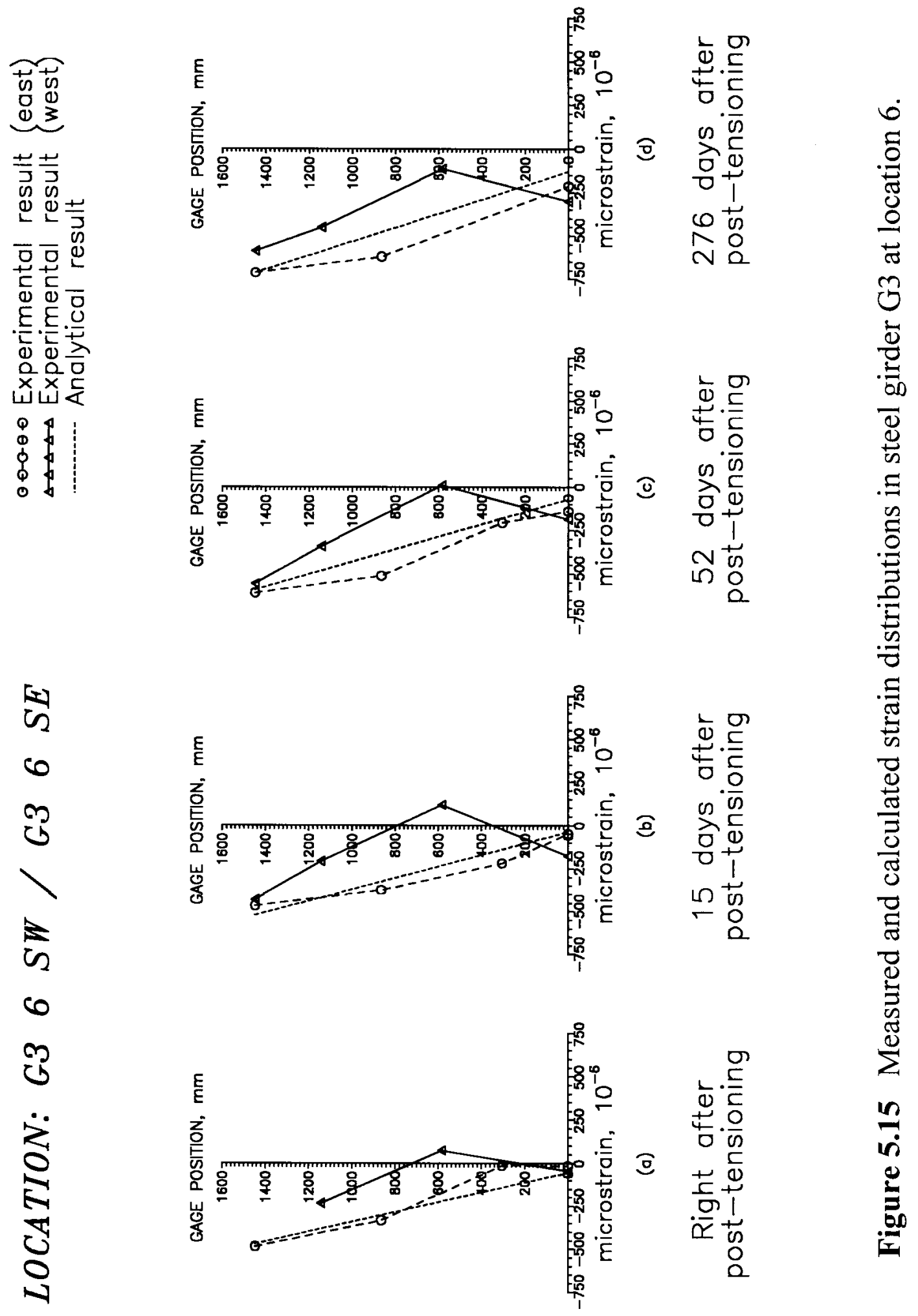




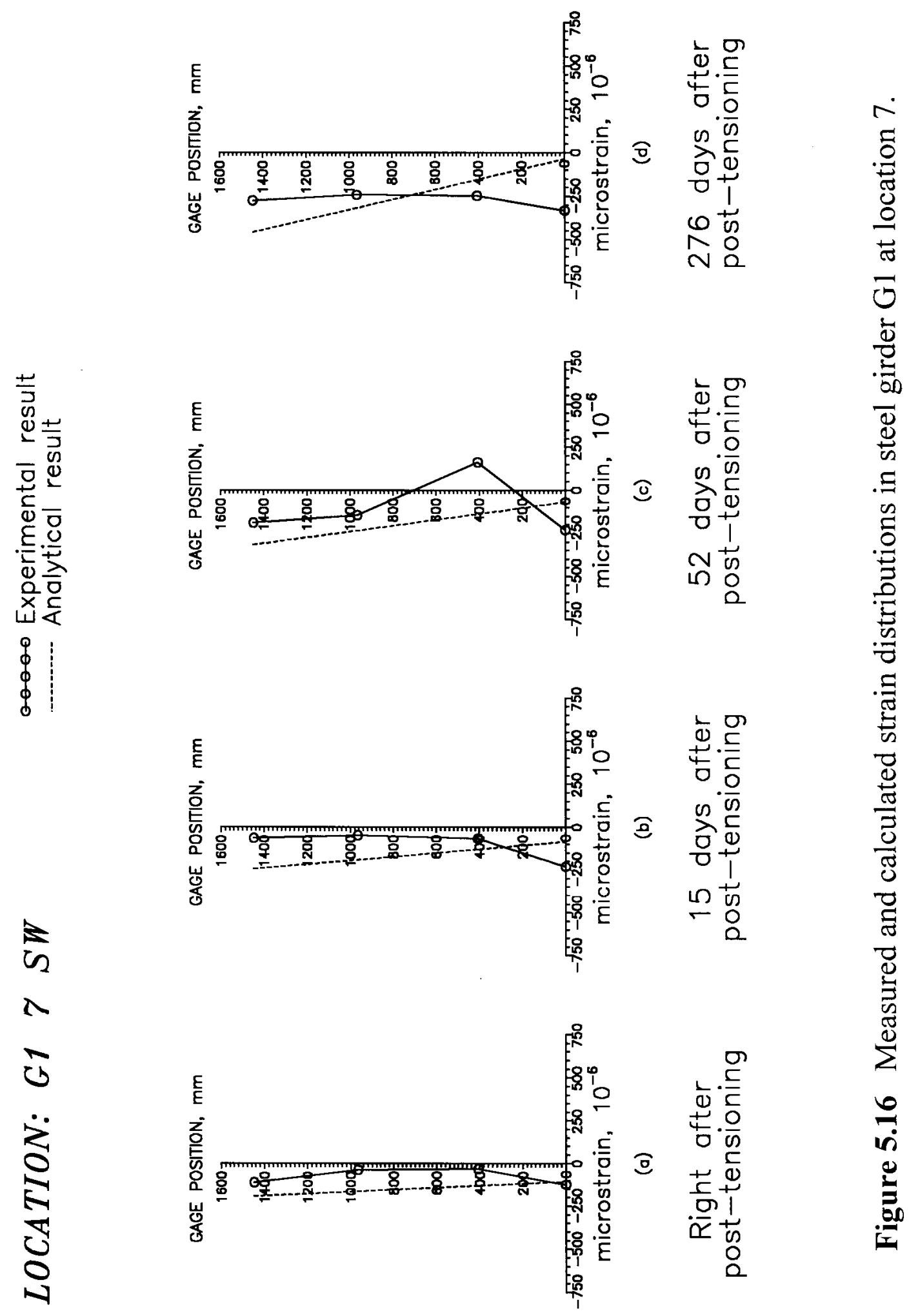




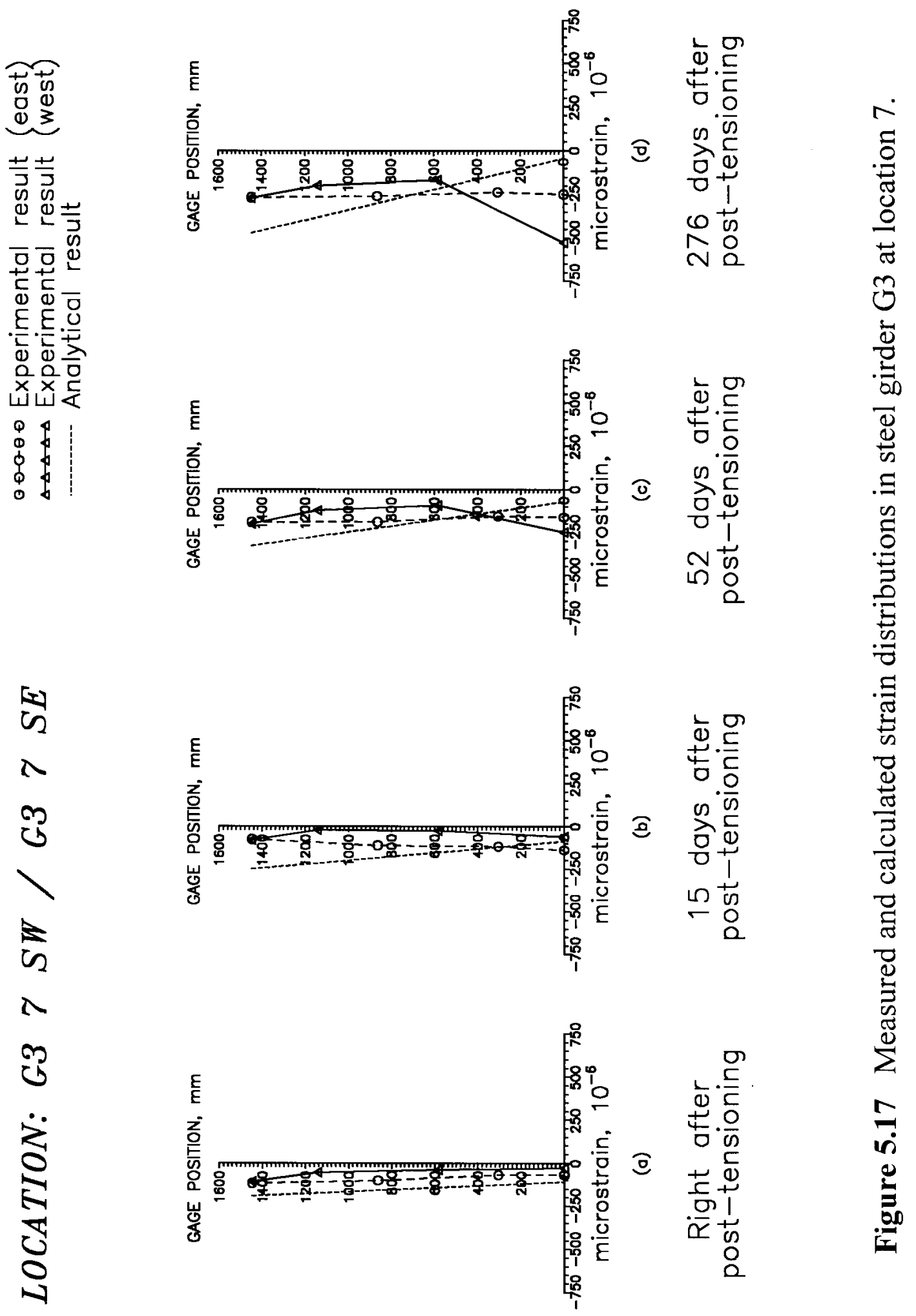




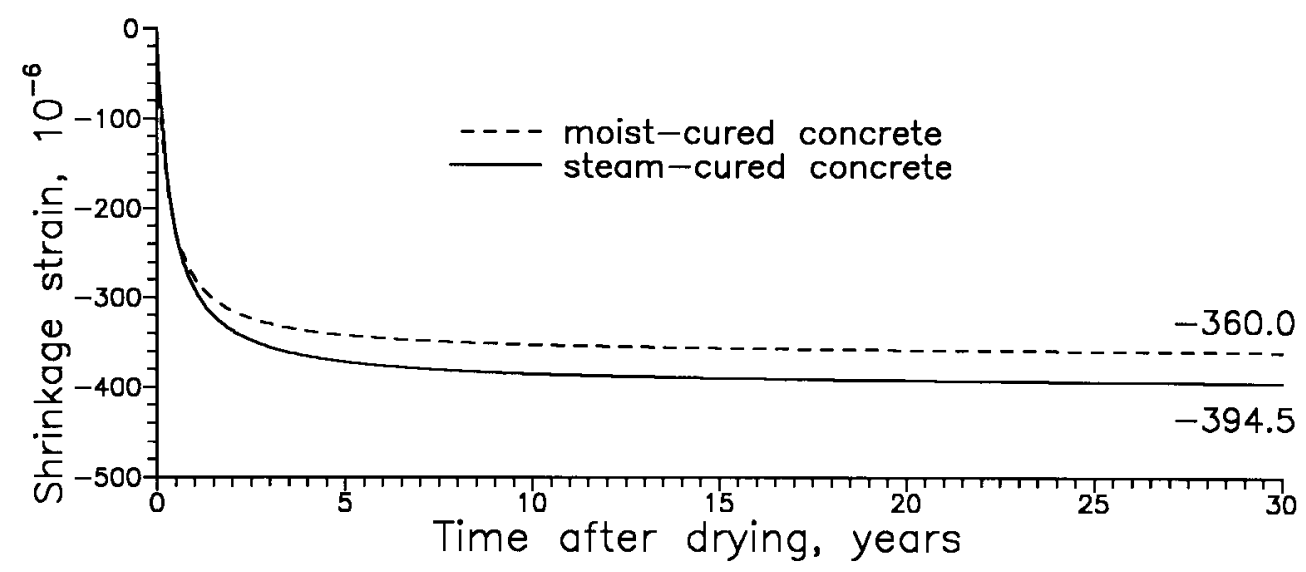

(a) For concrete of exterior girders

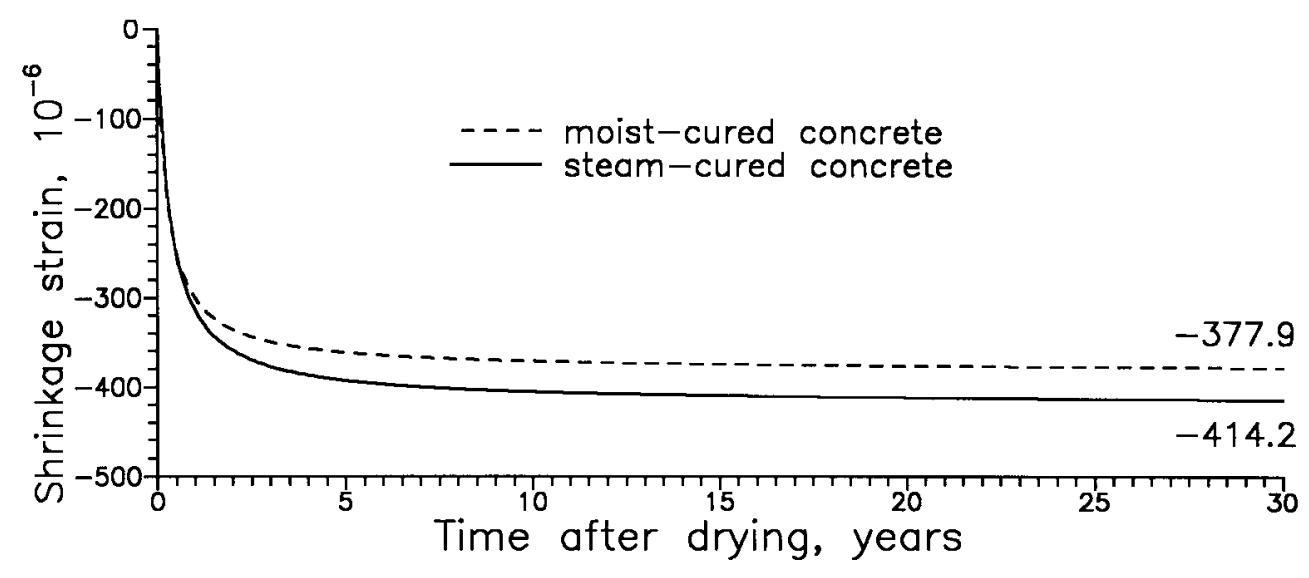

(b) For concrete of interior girders

Figure 5.18 Shrinakge functions suggested by AASHTO Specifications. 


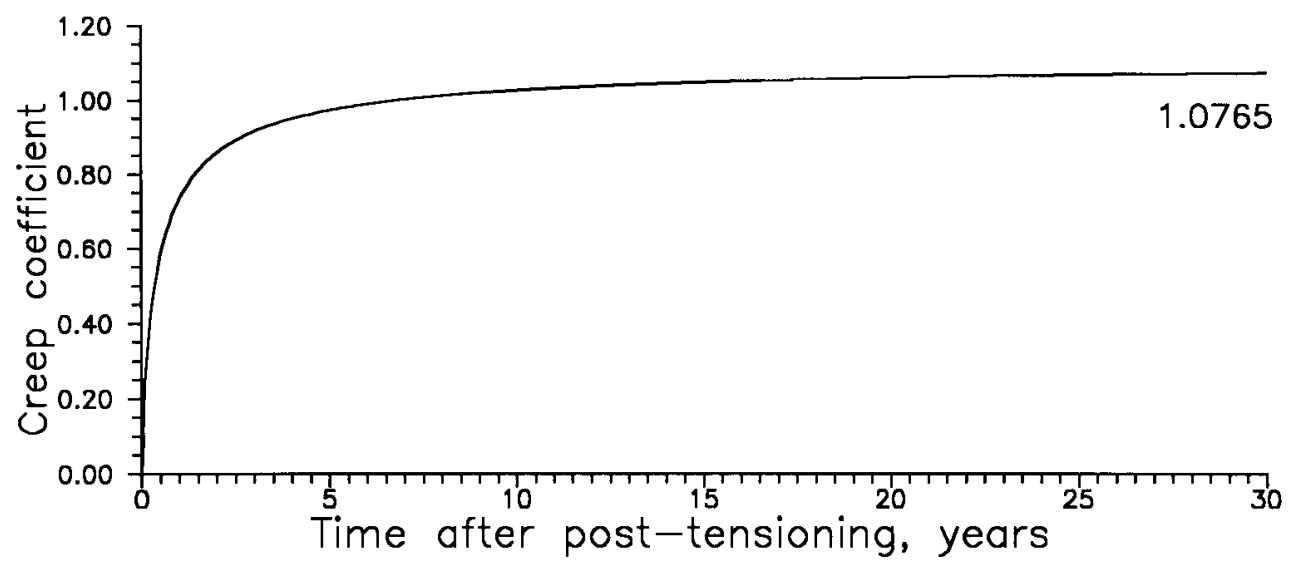

(a) For concrete of exterior girders

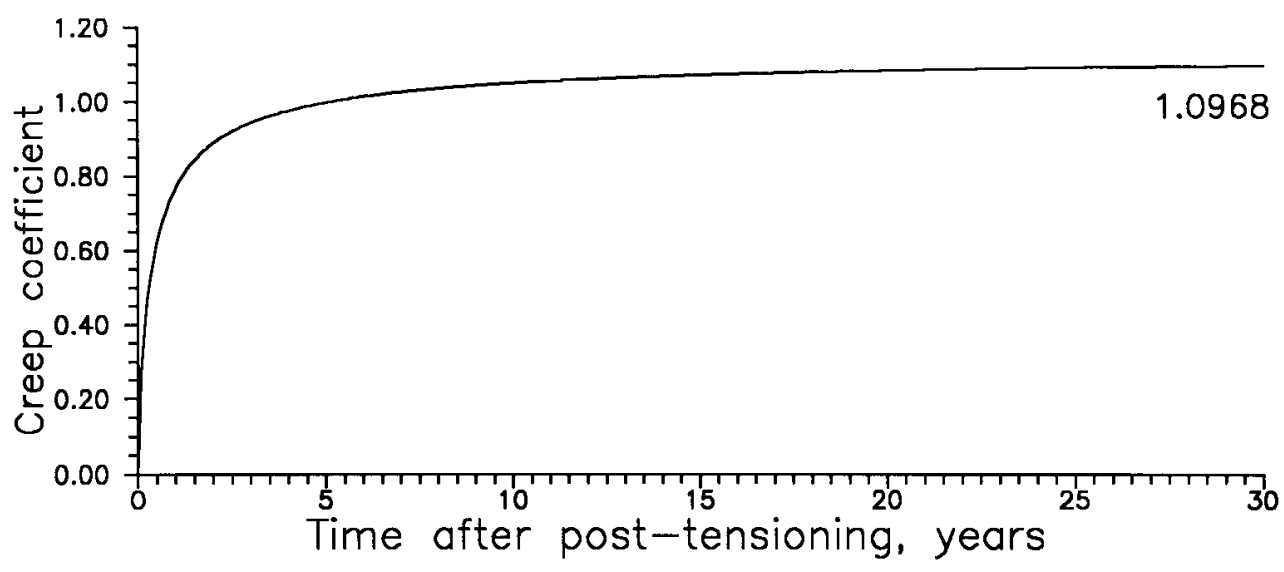

(b) For concrete of interior girders

Figure 5.19 Creep function suggested by AASHTO Specifications. 


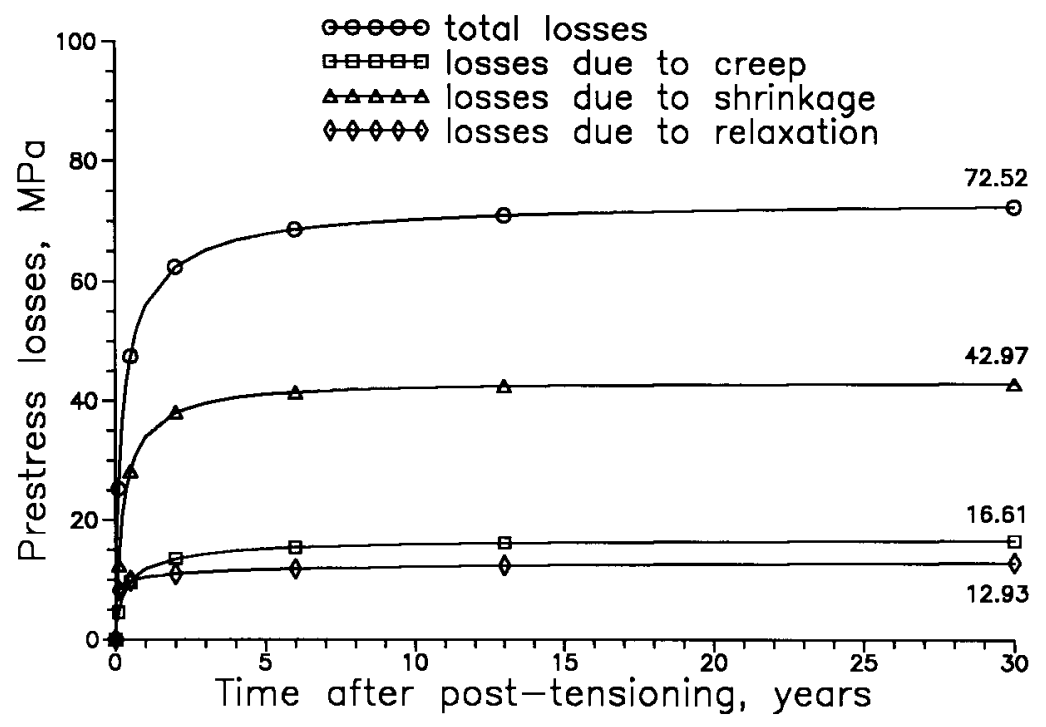

(a) For tendons along exterior girders

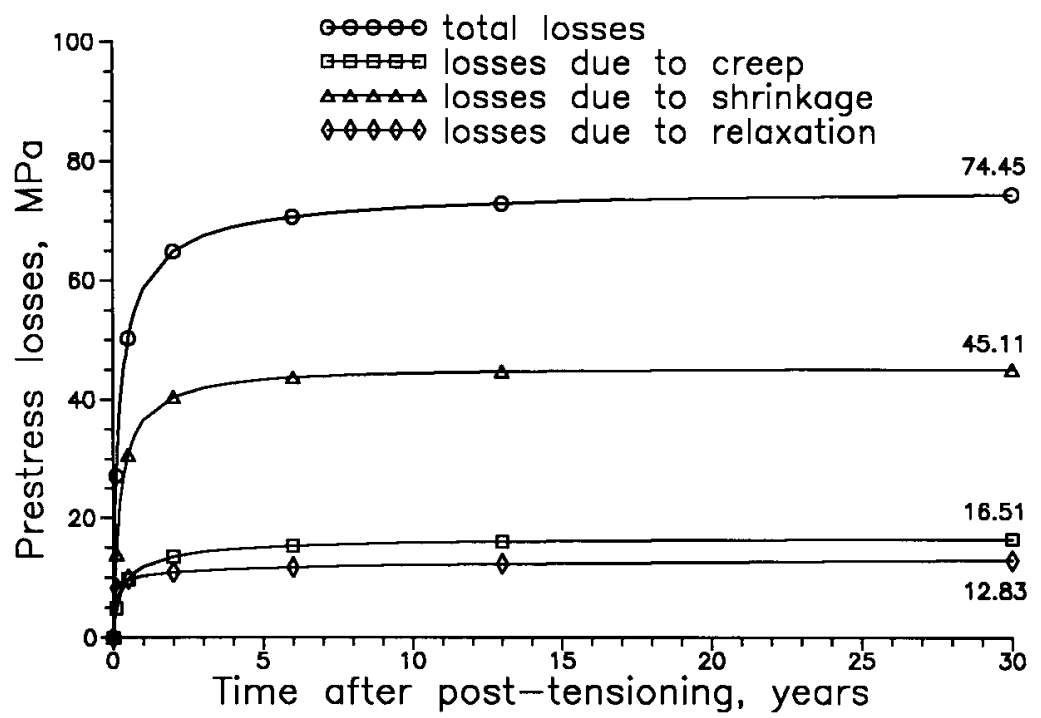

(b) For tendons along interior girders

Figure 5.20 Predicted prestress losses. 


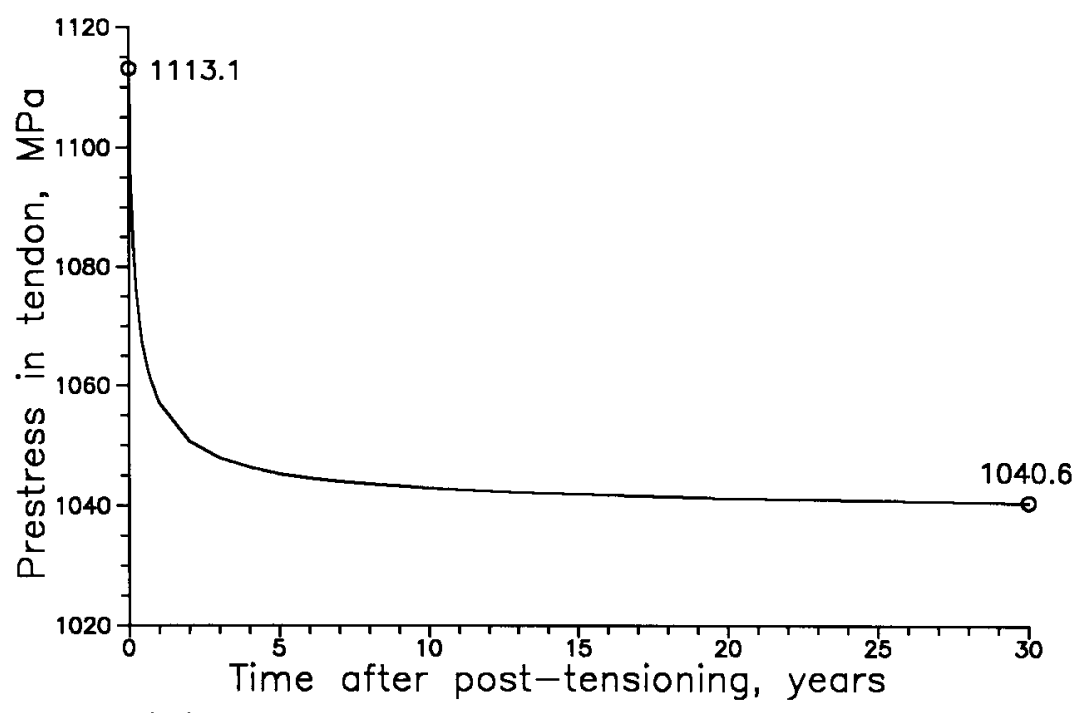

(a) For tendons along exterior girders

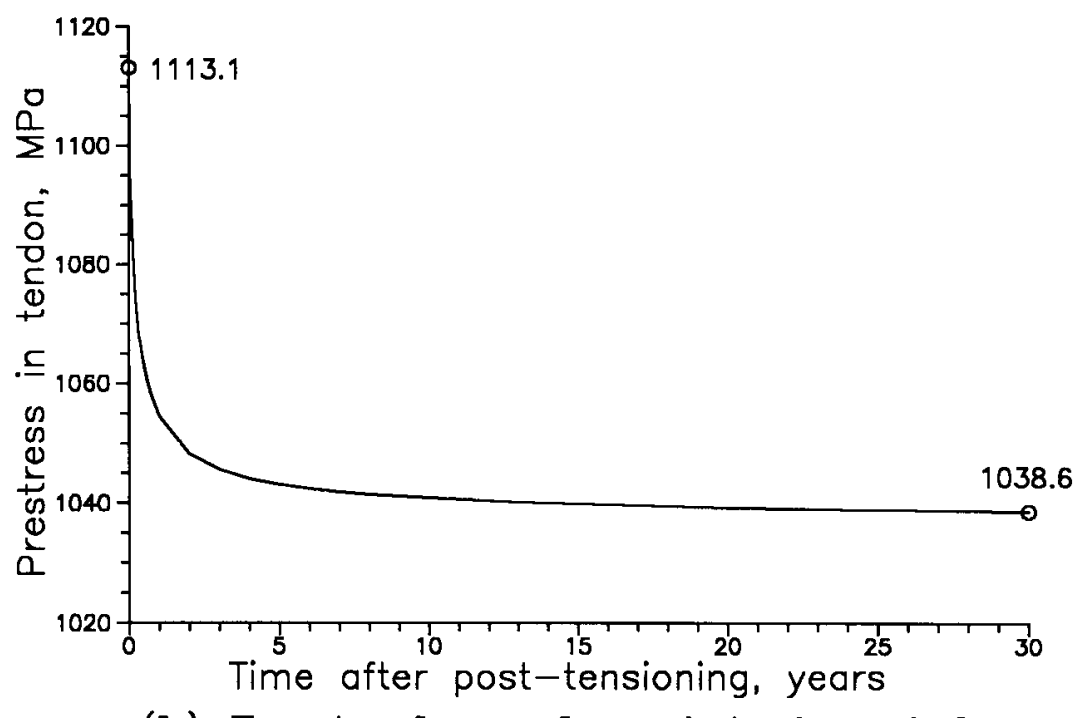

(b) For tendons along interior girders

Figure 5.21 Predicted prestress variations. 


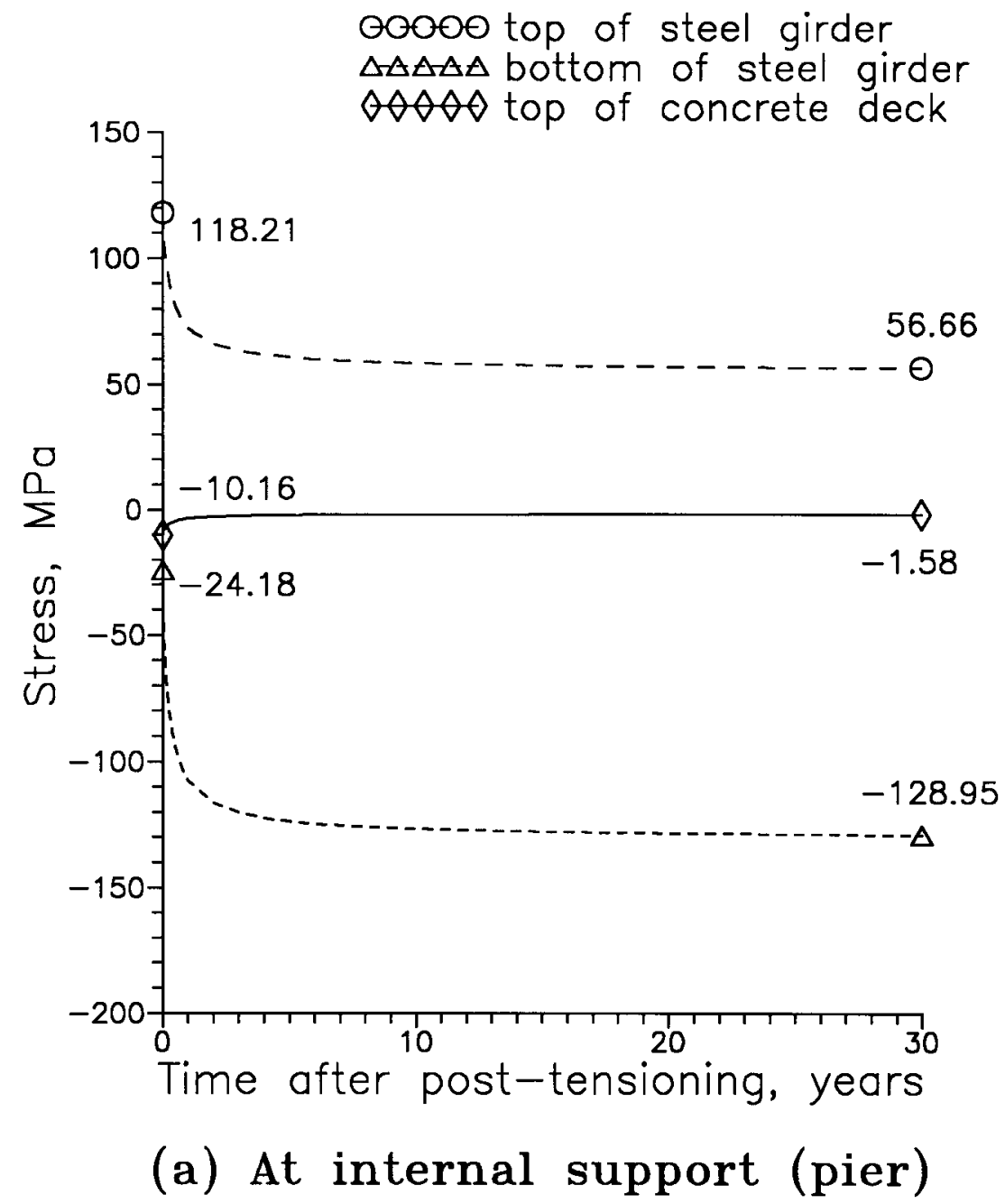

Figure 5.22 Predicted long-term stress distributions along exterior girders (G1). 


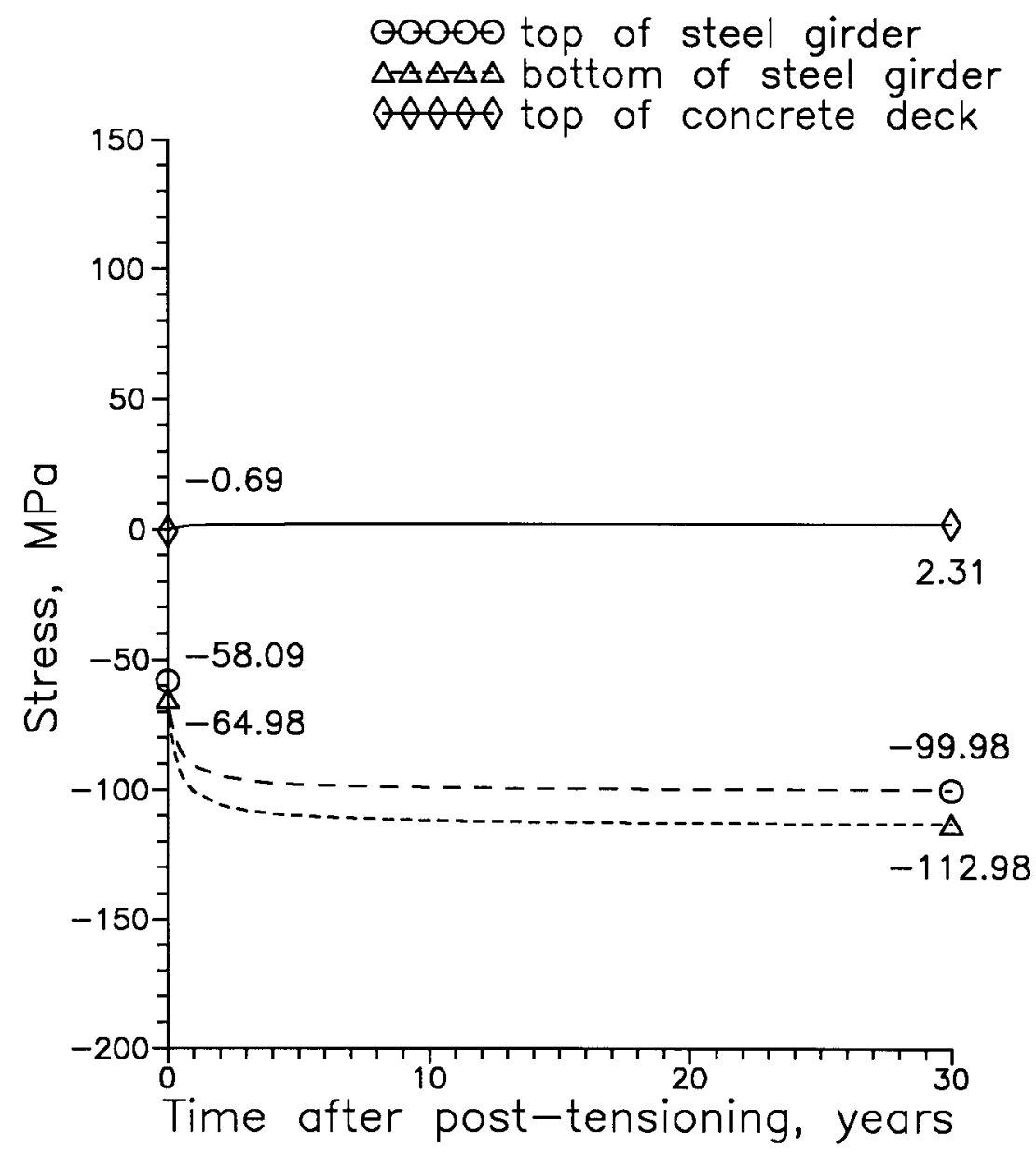

(b) At drape location

Figure 5.22 Predicted long-term stress distributions along exterior girders (G1). 


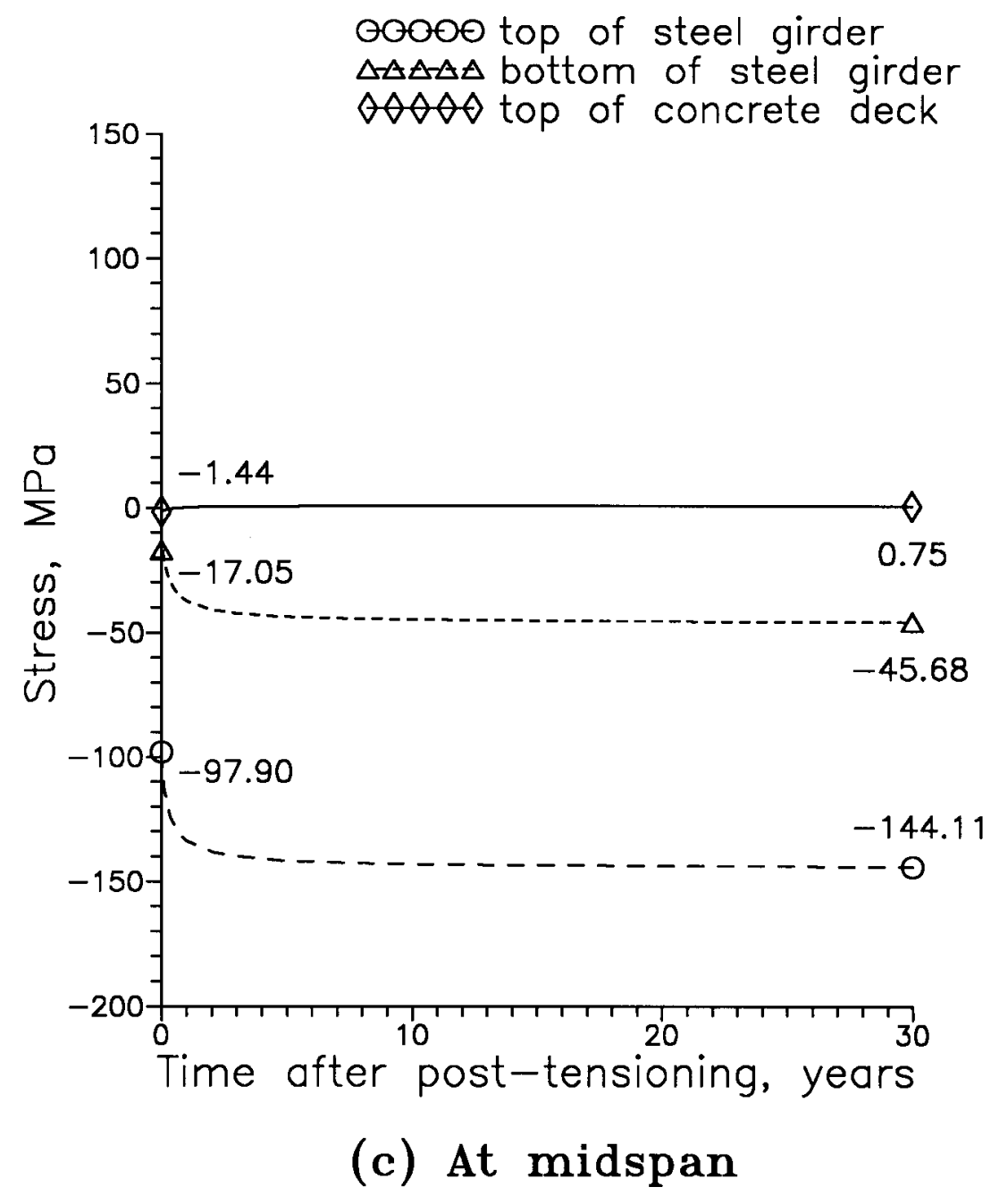

Figure 5.22 Predicted long-term stress distributions along exterior girders (G1). 


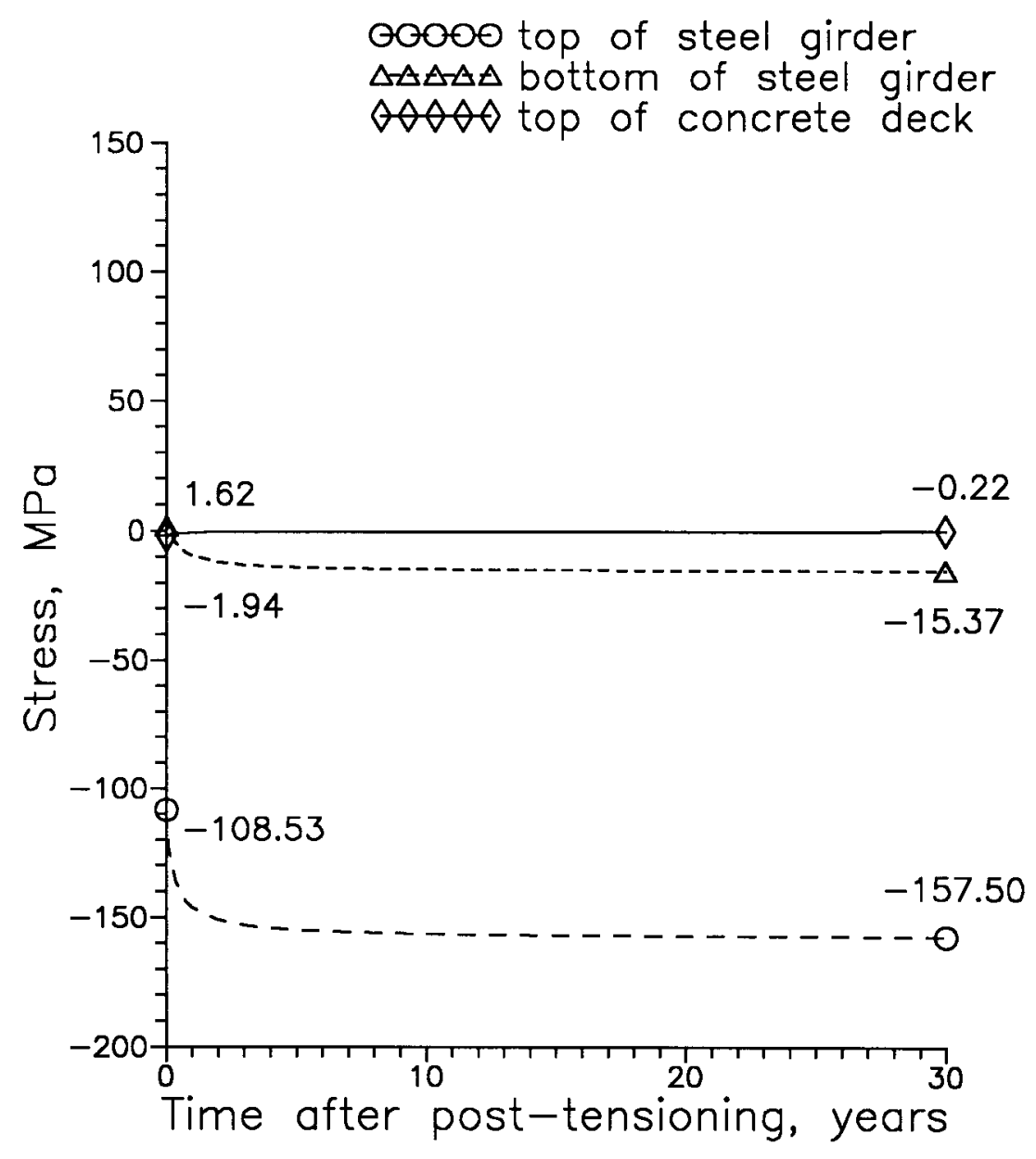

(d) At 0.4 span from end

Figure 5.22 Predicted long-term stress distributions along exterior girders (G1). 


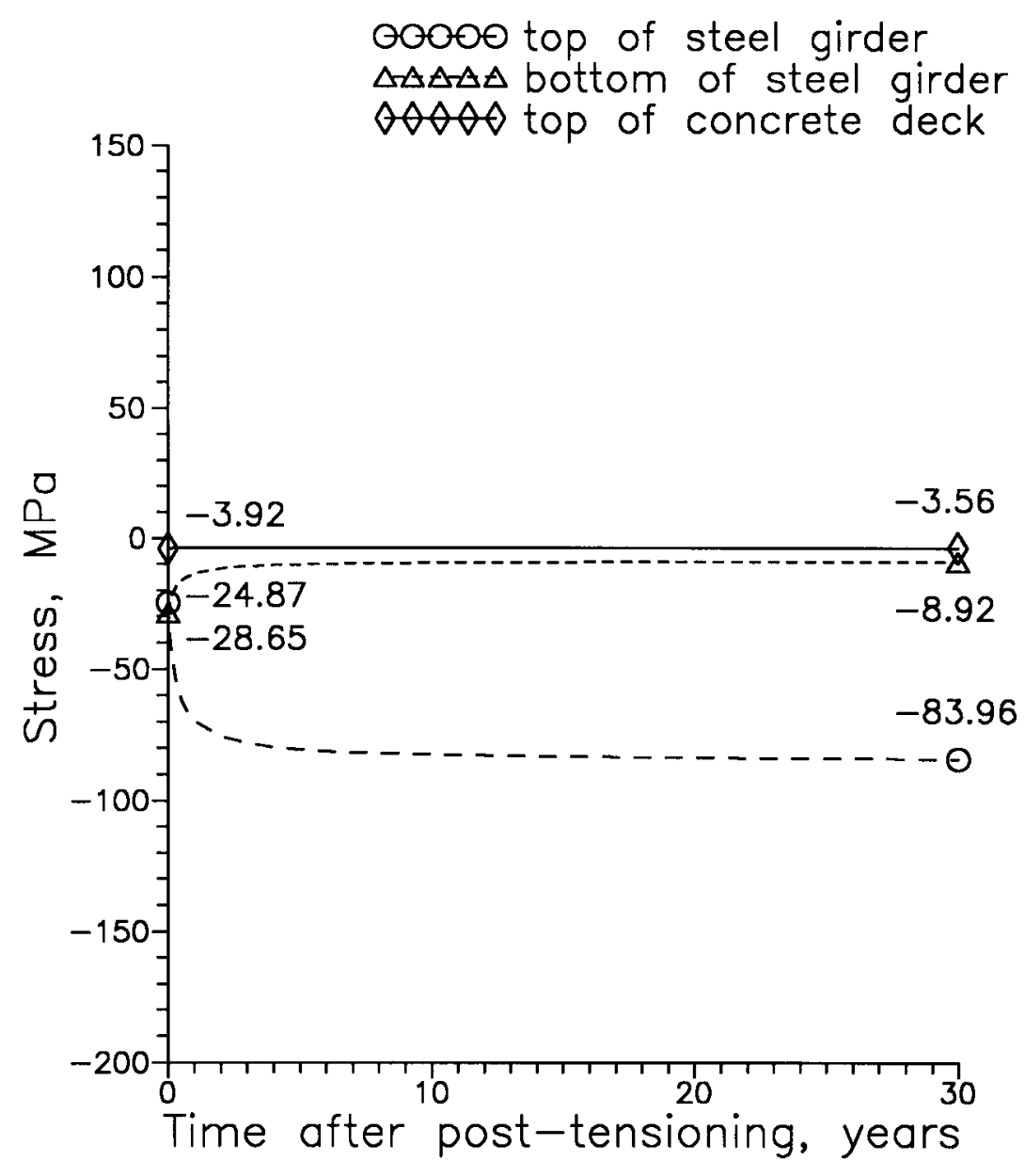

(e) At bridge end

Figure 5.22 Predicted long-term stress distributions along exterior girders (G1). 


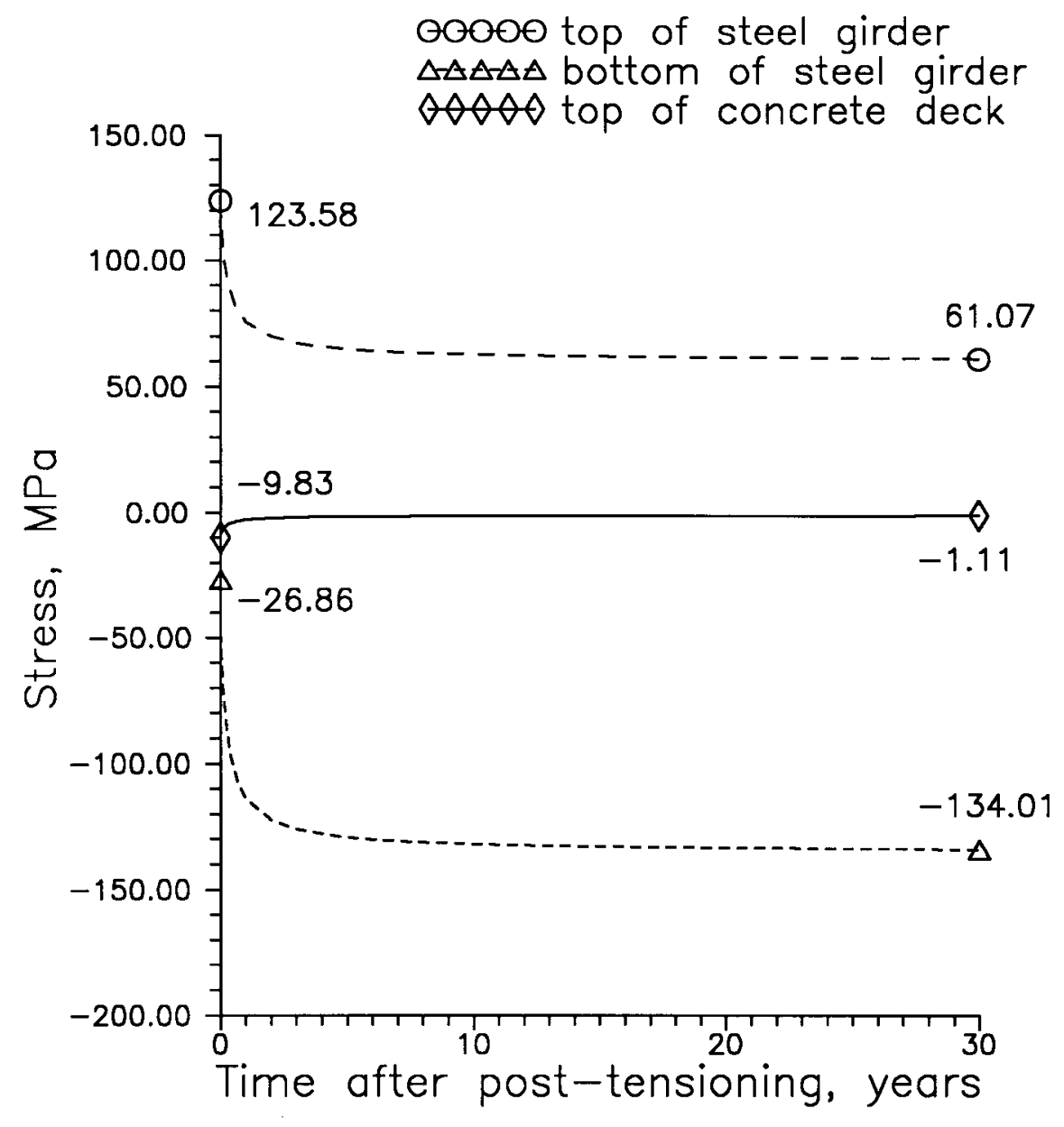

\section{(a) At internal support (pier)}

Figure 5.23 Predicted long-term stress distributions along interior girders (G3). 


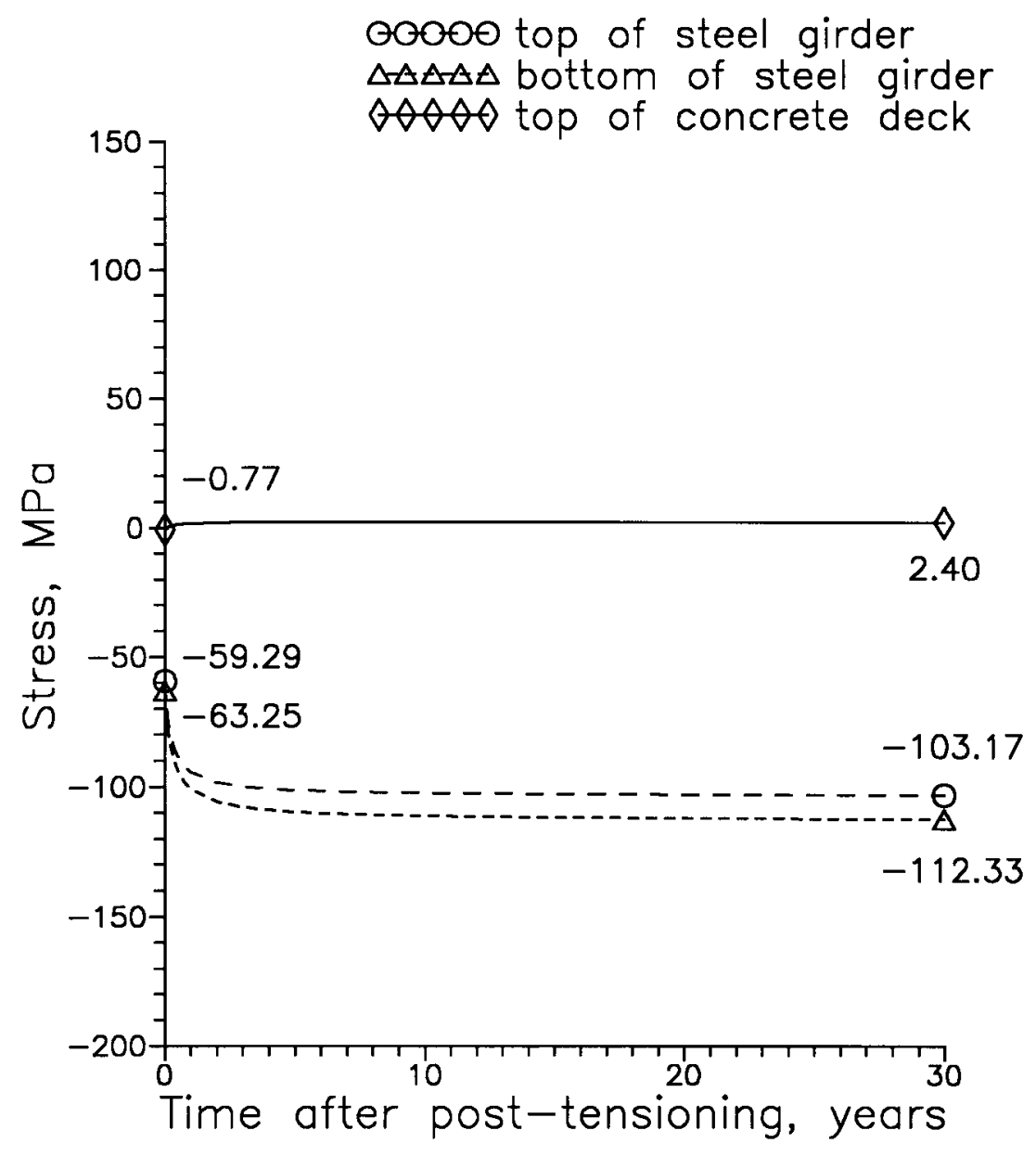

(b) At drape location

Figure 5.23 Predicted long-term stress distributions along interior girders (G3). 


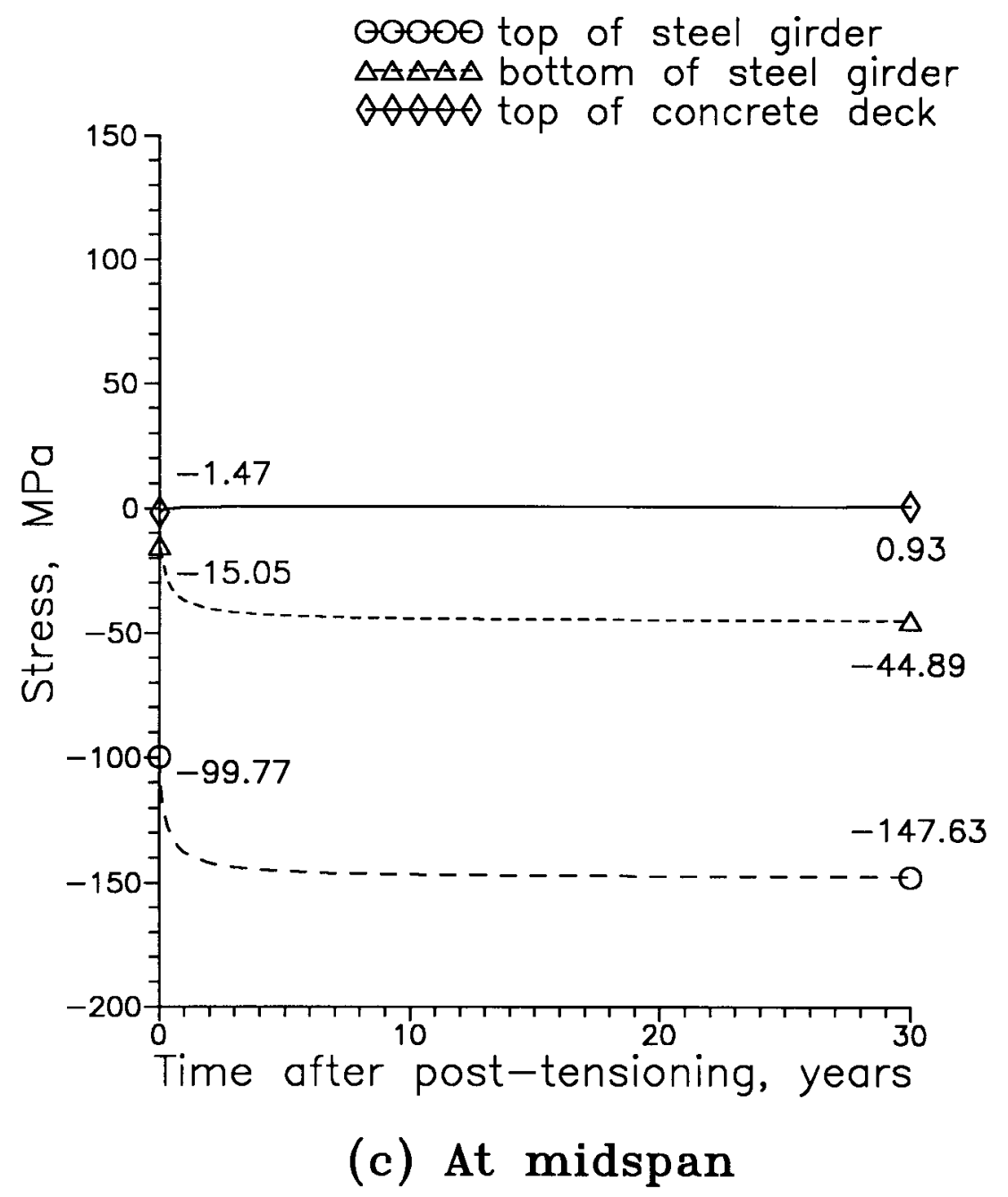

Figure 5.23 Predicted long-term stress distributions along interior girders (G3). 


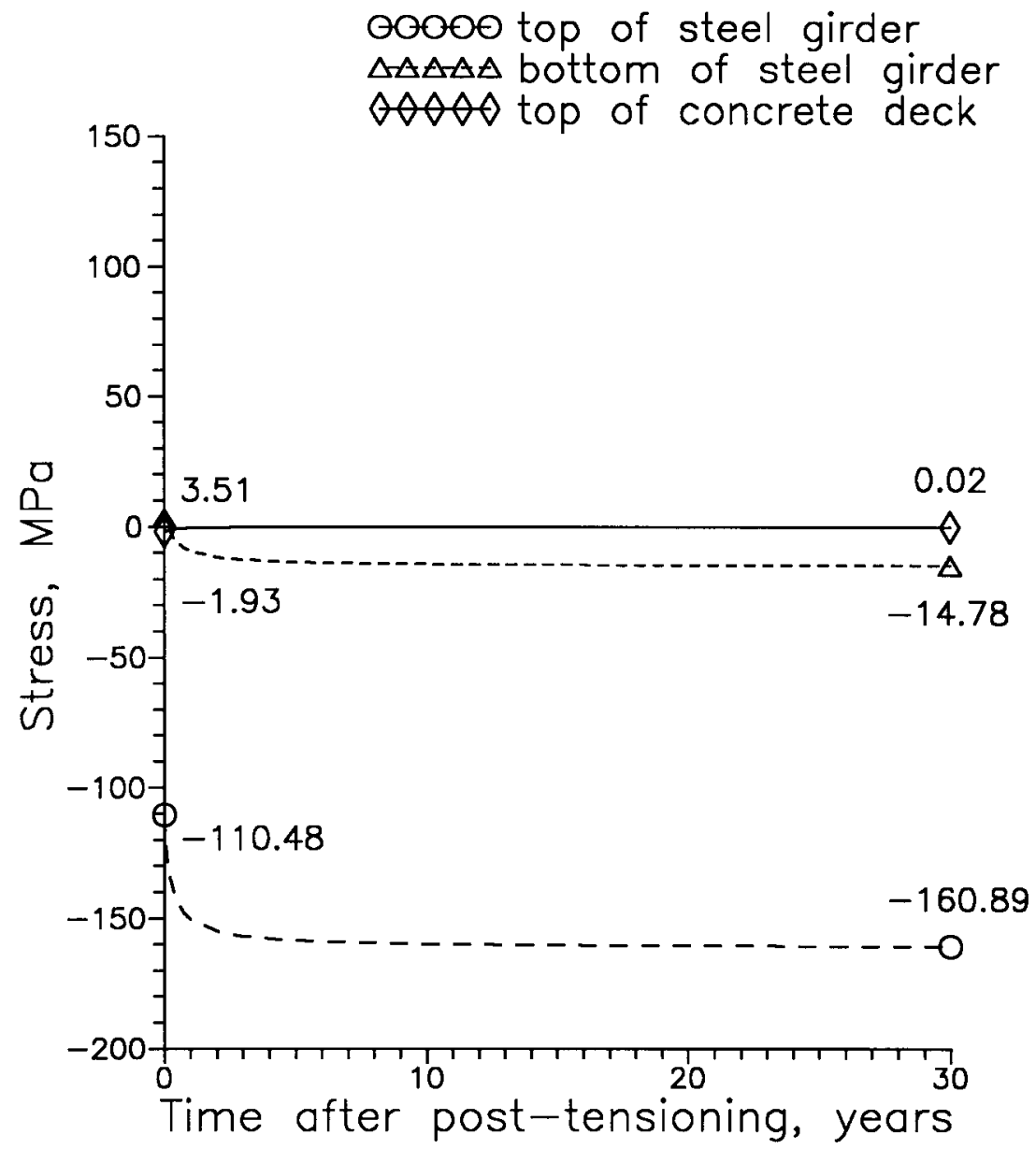

(d) At 0.4 span from end

Figure 5.23 Predicted long-term stress distributions along interior girders (G3). 


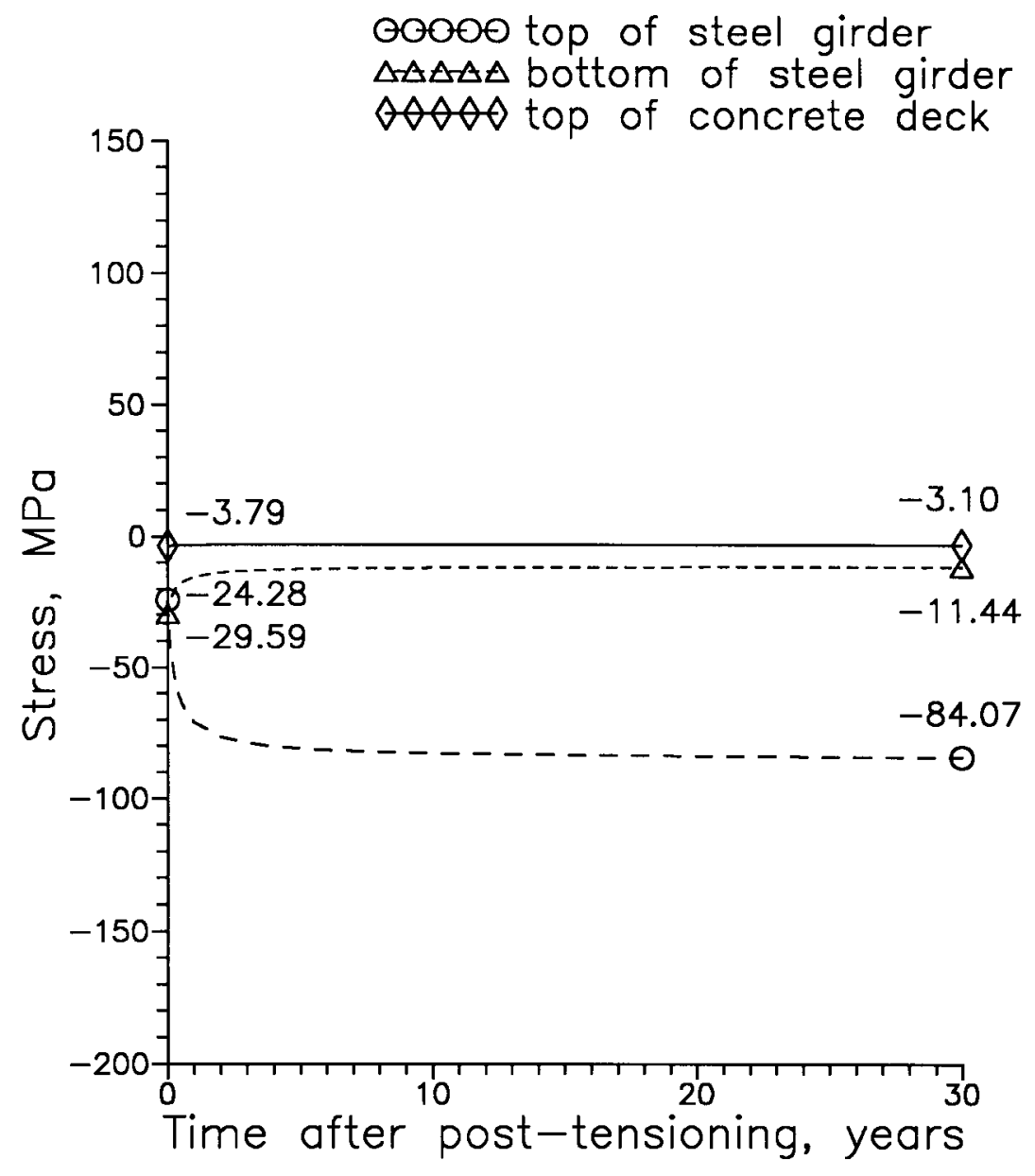

(e) At bridge end

Figure 5.23 Predicted long-term stress distributions along interior girders (G3). 


\section{CHAPTER 6 \\ COMPARISON OF COST DATA FOR TWO COMPOSITE BRIDGES OVER I-90}

A cost comparison between the post-tensioned Elkhart County Toll Road Bridge and another steel girder composite deck bridge over highway I-90 is undertaken in this chapter. The two bridge structures were built at nearly the same time, and they are located very close to one another. While the composite bridge evaluated as part of this project was post-tensioned during construction, the other bridge was built as a conventional composite structure with steel girders and a composite concrete deck. In the following discussion the conventional and the post-tensioned bridges are denoted as CV and PT, respectively.

\subsection{Major Structural Features of Two Bridges}

Despite of the major difference in the superstructures of the CV and PT bridges, there are similarities between the two structures. Both bridges have two spans over highway I-90, and they are of about the same width. Both bridge decks are supported by seven plate girders and all bridge deck reinforcement is epoxy coated. The widths of the concrete decks of the CV and PT bridges are 21,438 $\mathrm{mm}$ and 20,920 mm respectively. 
The CV bridge, with a length of $85,954 \mathrm{~mm}$, is $14,504 \mathrm{~mm}$ longer than the PT bridge $(71,450 \mathrm{~mm})$. The thickness of the deck is almost the same: $203 \mathrm{~mm}$ for the CV bridge and $215 \mathrm{~mm}$ for the PT bridge. Although the volume of the concrete deck of the CV bridge is larger than that of the PT bridge, the total amount of reinforcement used in the two bridge decks is almost identical. Expressed in cubic millimeters, the volumes of the reinforcing steel bars in the decks of the CV and PT bridges are about $9.79 \times 10^{9} \mathrm{~mm}^{3}$ and $9.84 \times 10^{9} \mathrm{~mm}^{3}$ accordingly. If the reinforcement per unit area of the bridge deck is computed, then it is found that the deck reinforcement is $41.70 \mathrm{~kg} / \mathrm{m}^{2}$ for the $\mathrm{CV}$ bridge versus $51.67 \mathrm{~kg} / \mathrm{m}^{2}$ for the PT bridge. By comparing these values, it is found that $23.9 \%$ more reinforcement was used in the PT bridge than in the CV bridge deck.

The substructure for the two bridges also share some similar features. The abutments of both bridges contain several steel encased concrete piles which are surrounded by a concrete wall on the top portions. The CV bridge contains twelve piles at each abutment, whereas the PT bridge contains thirteen piles. Furthermore, the ends of the steel girders of both bridges were cast into the abutment walls.

The primary difference in the superstructures of the two bridges is the use of posttensioning in the PT bridge. The post-tensioning forces are applied longitudinally along the entire length and transversely at the locations of concrete diaphragms and abutments. However, a few other special features were incorporated into the design of the PT bridge. The PT bridge utilized a hybrid design with Grade 70 over the interior supports and Grade 50 used elsewhere. Also, the post-tensioning was designed such that the concrete deck was subjected to a compressive force throughout the entire length to control deck 
cracking. In addition, the PT steel girders were designed to be of the same dimensions and shear connectors were used along the entire span. On the other hand, the CV bridge was a traditional design, with the concrete deck subjected to tensile stresses around the internal support (conventionally considered as the negative moment region), and composite behavior not considered around this location. As a result, steel flanges with comparatively larger thickness were adopted in this region for the $\mathrm{CV}$ bridge. Another difference in geometry between the two structures is that the CV bridge is skewed at an angle of $5^{\circ} 32^{\prime} 4^{\prime \prime}$, whereas the orientation of the girders of the PT bridge is perpendicular to the centerline of the abutment (no skewness).

The piers of the two bridges are also constructed differently. The pier of the CV bridge is a column frame pier. Seven columns are encased in a continuous thin concrete wall with a thickness of about the diameter of the column. The wall rests upon a footing supported by fifty-one concrete piles. The PT bridge contains two solid wall piers, with a fluted facia for architectural relief, on a raft footing.

\subsection{Comparison of Cost}

The comparison of the costs between the two bridges is based on the cost items for the major structural-related components. Listed in Tables 6.1 and 6.2 are the cost items for the CV bridge in English and metric units, respectively. The original list of cost items for the CV bridge was reported in English units as presented in Table 6.1. Table 6.2 is a simple conversion of unit costs from Table 6.1. Table 6.3 lists the cost items of structural components for the PT bridge. 
The total amount of concrete, as well as structural steel, used in the superstructure of the $\mathrm{CV}$ bridge is greater than that used in the PT bridge, since the $\mathrm{CV}$ bridge has a wider deck and a longer span than the PT bridge. The quantities of reinforcement listed in Table 6.2 and 6.3 are not distinguished for superstructure and substructure according to the information provided by INDOT. It seems that more reinforcement was used in the substructure of the PT bridge than in that of the CV bridge. Moreover, in addition to two side traffic barriers, the PT bridge also has a median barrier.

Two superstructure items are not commonly shared by the two bridge structures. In the CV bridge the girders are placed upon several bearings at the abutments and the pier, which added 11,200 dollars (Item Nos. 8 and 9 in Table 6.2) to the total cost. Also, the last five items of the superstructure category in Table 6.3 (B19 and EW) for the PT bridge correspond to the cost of post-tensioning, for a total amount of $\$ 157,345.77$. The total substructure costs for the two bridges are not very different: $\$ 184,642.24$ for the $C V$ bridge versus $\$ 158,906.12$ for the PT bridge. By observing Tables 6.2 and 6.3 , it is found that the unit prices of most corresponding items for the two structures are approximately comparable.

The cost data are categorized into several items: (1) concrete for the superstructure; (2) reinforcement; (3) structural steel (including bearings for the CV bridge); (4) railing concrete; (5) post-tensioning (only for the PT bridge); and (6) substructure. In order to develop a fair comparison, the amount $(\$)$ for each item is divided by the total area (in square meters) of the bridge deck $\left(1,842.68 \mathrm{~m}^{2}\right.$ for the $\mathrm{CV}$ bridge and $1,494.73 \mathrm{~m}^{2}$ for the PT bridge) to obtain the average cost. The results are listed 
in Table 6.4 and depicted graphically in Figs. 6.1 and 6.2. It should be noted that the amount of reinforcement in item (2) refers to that used in the whole structure, because the original cost data do not separate the reinforcing bars used in superstructure and substructure.

The average cost $\left(\$ / \mathrm{m}^{2}\right)$ of concrete used for the superstructure in the PT bridge is about $44 \%$ higher than that in the CV bridge. The significant difference is due primarily to a higher unit price for the concrete used in the PT bridge. The average cost of the steel reinforcement for the PT bridge is also higher than that for the CV bridge, since the PT bridge structure is more heavily reinforced (comparatively more reinforcement was used) than the $\mathrm{CV}$ bridge. Also, the use of transverse and longitudinal post-tensioning added an additional $\$ 105.27 / \mathrm{m}^{2}$ to the total cost. The average costs of other common items of the two bridges show relatively small diversity. Finally, the total average cost for the PT bridge is about $33 \%$ higher than that for the CV bridge.

A note should be made regarding the unit cost of the structural steel for the two bridges. Fig. 6.1 shows that the two unit costs are almost identical. Nevertheless, the base price for the steel may have been just slightly high due to the fabrication simplicity of the PT bridge. There were no changes in the cross section due to flange thickness transitions. In fairness, however, additional plates were attached to the web of the PT bridge in the vicinity of the concrete diaphragms, and holes had to be drilled in the web at the concrete diaphragm and the middle pier locations to permit transverse post-tensioning. Moreover, the structural steel unit cost was influenced somewhat by the cost of painting the 
structure. The PT bridge was constructed with weathering steel which is not painted, while the CV bridge steel was painted.

Since the CV bridge was built about one year prior to the PT bridge, some adjustment could be made to reflect inflation. By multiplying the cost of the CV bridge by 1.05 to account for this factor, the total adjusted average cost of the $\mathrm{CV}$ bridge turns out to be $\$ 735.19 / \mathrm{m}^{2}$. Based on the adjusted unit cost of the CV bridge, the average cost $\left(\$ / \mathrm{m}^{2}\right)$ of the PT bridge is roughly $27 \%$ higher than that of the CV bridge. Although the cost of the PT bridge is quite a bit higher than the CV bridge, it should be remembered that this is the first PT bridge built in Indiana. The PT bridge construction required extra forming and labor costs in the regions of the post-tensioning drape points at the concrete diaphragms and middle pier. This is part of the reason the concrete unit costs are higher. Undoubtedly, this cost would decrease as the contractor gained experience by building a few bridges of the PT type.

\subsection{Summary}

The average unit costs of a conventional and a post-tensioned steel bridges are compared. The comparison, however, is not intended to draw a final conclusion on the economical benefits or disadvantages of post-tensioned composite bridges over conventional composite bridges. Besides the application of post-tensioning, there are still several notable differences in the designs of superstructure as well as substructures between the two bridges. Moreover, the unit prices for certain corresponding construction 
items also show some significant disparities. The two bridge structures should be better viewed as particular cases representing two possible options for highway bridge design. 
Table 6.1 Costs of structural components of bridge (CV) in English units

\begin{tabular}{|c|c|c|c|c|}
\hline & Superstructure & & & \\
\hline Item No. & Contract Items & Amount (\$) & Quantity & Unit Price $(\$)$ \\
\hline 2 & Superstructure, Concrete, $\mathrm{C}$ with Microsilica & $197,700.00$ & 659.00 & 300.00 \\
\hline 6 & Reinforcing Steel & $5,058.72$ & $10,539.00$ & 0.48 \\
\hline 7 & Reinforcing Steel, Epoxy Coated & $97,194.93$ & $198,357.00$ & 0.49 \\
\hline 12 & Structural Steel & $766,217.99$ & 1.00 & $766,217.99$ \\
\hline 5 & Railing, Concrete, $\mathrm{C}$, with Microsilica & $28,200.00$ & 564.00 & 50.00 \\
\hline 8 & Bearing Assembly, Fixed & $7,000.00$ & 7.00 & $1,000.00$ \\
\hline \multirow[t]{2}{*}{9} & Bearing Assembly, Ends & $4,200.00$ & 14.00 & 300.00 \\
\hline & Substructure & & & \\
\hline Item No. & Contract Items & Amount (\$) & Quantity & Unit Price $(\$)$ \\
\hline 2 & Superstructure, Concrete, $\mathrm{C}$ with Microsilica & $197,700.00$ & 659.00 & 300.00 \\
\hline 3 & Concrete, A, Substructure & $19,800.00$ & 49.50 & 400.00 \\
\hline 4 & Concrete, B, Footing & $32,910.00$ & 109.70 & 300.00 \\
\hline 13 & Pile, Concrete, Steel Shell, Encased 0.203", 14" & $74,995.50$ & $2,550.00$ & 29.41 \\
\hline \multirow[t]{2}{*}{14} & Pile, Concrete, Steel Shell, Encased 0.312", 14" & $56,936.74$ & $1,781.50$ & 31.96 \\
\hline & Total & $1,290,213.88$ & & \\
\hline
\end{tabular}

Note:

Unit prices and quantities of items 2, 3, and 4 are based on cubic yards.

Unit price and quantity of item 5 are based on linear feet.

Unit prices and quantities of items 6 and 7 are based on pounds.

Unit prices and quantities of items 13 and 14 are based on feet. 
Table 6.2 Costs of structural components of bridge (CV) in metric units

\begin{tabular}{|c|c|c|c|c|}
\hline & Superstructure & & & \\
\hline Item No. & Contract Items & Amount (\$) & Quantity & Unit Price (\$) \\
\hline 2 & Superstructure, Concrete, C with Microsilica & $197,700.00$ & 503.84 & 392.39 \\
\hline 6 & Reinforcing Steel & $5,058.72$ & $4,781.00$ & 1.06 \\
\hline 7 & Reinforcing Steel, Epoxy Coated & $97,194.93$ & $89,995.00$ & 1.08 \\
\hline 12 & Structural Steel & $766,217.99$ & 1.00 & $766,217.99$ \\
\hline 5 & Railing, Concrete, C, with Microsilica & $28,200.00$ & 46.23 & 609.99 \\
\hline 8 & Bearing Assembly, Fixed & $7,000.00$ & 7.00 & $1,000.00$ \\
\hline 9 & Bearing Assembly, Ends & $4,200.00$ & 14.00 & 300.00 \\
\hline & Substructure & & & \\
\hline Item No. & Contract Items & & & \\
\hline 3 & Concrete, A, Substructure & $19,800.00$ & 37.85 & 523.18 \\
\hline 4 & Concrete, B, Footing & $32,910.00$ & 83.87 & 392.39 \\
\hline 13 & Pile, Concrete, Steel Shell, Encased 0.203", 14" & $74,995.50$ & 777.20 & 96.49 \\
\hline 14 & Pile, Concrete, Steel Shell, Encased 0.312", 14" & $56,936.74$ & 543.00 & 104.86 \\
\hline & Total & & Unit Price (\$) \\
\hline & Suantity & & \\
\hline
\end{tabular}

Note:

Unit prices and quantities of items 2, 3, 4 and 5 are based on cubic meters.

Unit prices and quantities of items 6 and 7 are based on kilograms.

Unit prices and quantities of items 13 and 14 are based on meters. 
Table 6.3 Costs of structural components of bridge (PT) in metric units

\begin{tabular}{|c|c|c|c|c|}
\hline & Superstructure & & & \\
\hline Item No. & Contract Items & Amount (\$) & Quantity & Unit Price $(\$)$ \\
\hline B10 & Concrete, C, Superstructure & $56,229.15$ & 56.90 & 988.21 \\
\hline B11 & Concrete, Microsilica, C, Superstructure & $174,606.39$ & 385.70 & 452.70 \\
\hline B13 & Reinforcing Steel & $17,589.52$ & $16,913.00$ & 1.04 \\
\hline B14 & Reinforcing Steel, Epoxy Coated & $142,605.00$ & $114,084.00$ & 1.25 \\
\hline B18 & Structural Steel & $622,521.25$ & 1.00 & $622,521.25$ \\
\hline B12 & Concrete, Microsilica, C, Railing & $62,540.33$ & 73.80 & 847.32 \\
\hline B19 & Post-Tensioning Tendon and Thread Bars & $149,581.77$ & 1.00 & $149,581.77$ \\
\hline EW & Gouting of Tendon after Testing & $4,584.00$ & 1.00 & $4,584.00$ \\
\hline EW & Labor to Install Supports for Long P.T. & $1,800.00$ & 1.00 & $1,800.00$ \\
\hline EW & Material to Install Supports for Long P.L. & 600.00 & 1.00 & 600.00 \\
\hline \multirow[t]{2}{*}{ EW } & Traffic Main, for Install supports for P.T. & 780.00 & 1.00 & 780.00 \\
\hline & Substructure & & & \\
\hline Item No. & Contract Items & Amount (\$) & Quantity & Unit Price $(\$)$ \\
\hline B9 & Concrete, C, Substructure & $90,023.22$ & 206.48 & 435.99 \\
\hline B8 & Concrete, A, Footing & $13,724.37$ & 99.00 & 138.63 \\
\hline \multirow[t]{2}{*}{ B7 } & Pile, Concrete, Steel Shell, Encased $7.92 \mathrm{~mm}, 356 \mathrm{~mm}$ & $55,158.53$ & 609.15 & 90.55 \\
\hline & Total & $1,392,343.53$ & & \\
\hline
\end{tabular}

Note:

Unit prices and quantities of items B8, B9, B10, B11 and B12 are based on cubic meters. Unit prices and quantities of items B13 and B14 are based on kilograms.

Unit prices and quantities of item B7 are based on meters. 
Table 6.4 Comparison of costs of bridges (CV) and (PT)

\begin{tabular}{|c|c|c|}
\hline \multicolumn{3}{|l|}{ Bridge $(\mathrm{CV})$} \\
\hline Item & $\$ /\left(\mathrm{m}^{2}\right)$ & $\%$ \\
\hline Concrete for superstructure & 107.29 & 15.30 \\
\hline Reinforcement & 55.49 & 7.90 \\
\hline Structural steel \& bearings & 421.89 & 60.30 \\
\hline Railing concrete & 15.30 & 2.20 \\
\hline Post-tensioning & 0.00 & 0.00 \\
\hline Substructure & 100.20 & 14.30 \\
\hline Total & 700.18 & 100.00 \\
\hline \multicolumn{3}{|l|}{ Bridge (PT) } \\
\hline Item & $\$ /\left(\mathrm{m}^{2}\right)$ & $\%$ \\
\hline Concrete for superstructure & 154.43 & 16.60 \\
\hline Reinforcement & 107.17 & 11.50 \\
\hline Structural steel & 416.48 & 44.70 \\
\hline Railing concrete & 41.84 & 4.50 \\
\hline Post-tensioning & 105.27 & 11.30 \\
\hline Substructure & 106.31 & 11.40 \\
\hline Total & 931.50 & 100.00 \\
\hline
\end{tabular}




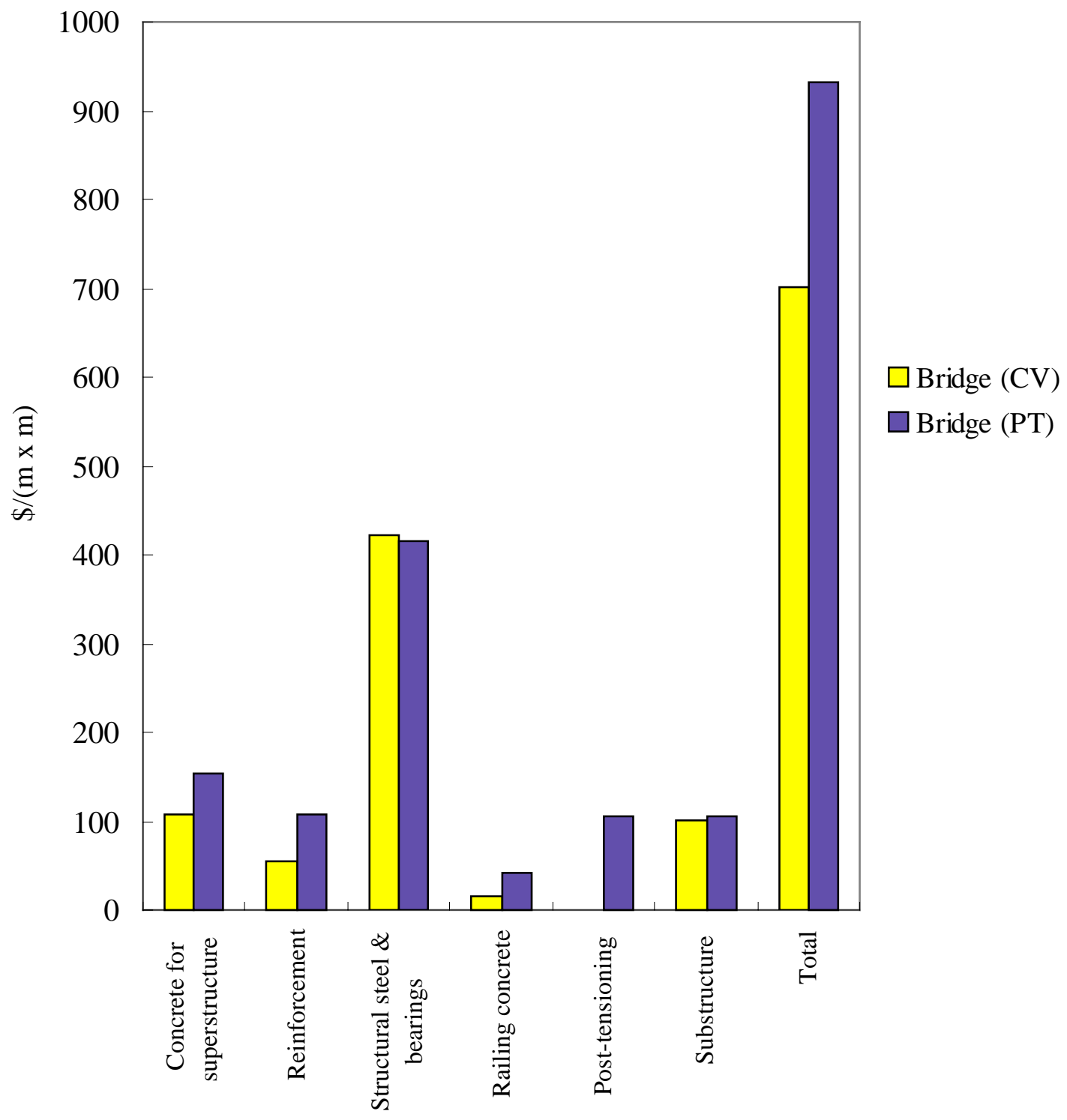

Figure 6.1 Average unit costs of bridges (CV) and (PT). 


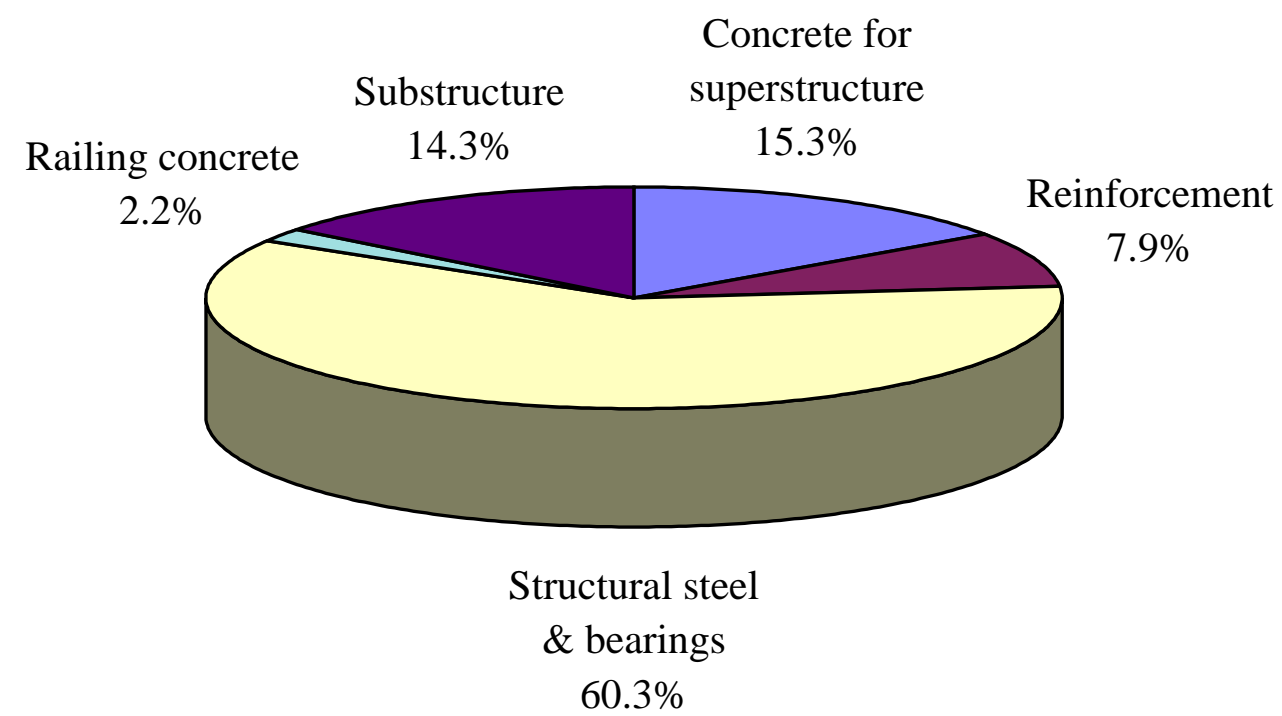

(a) Bridge (CV)

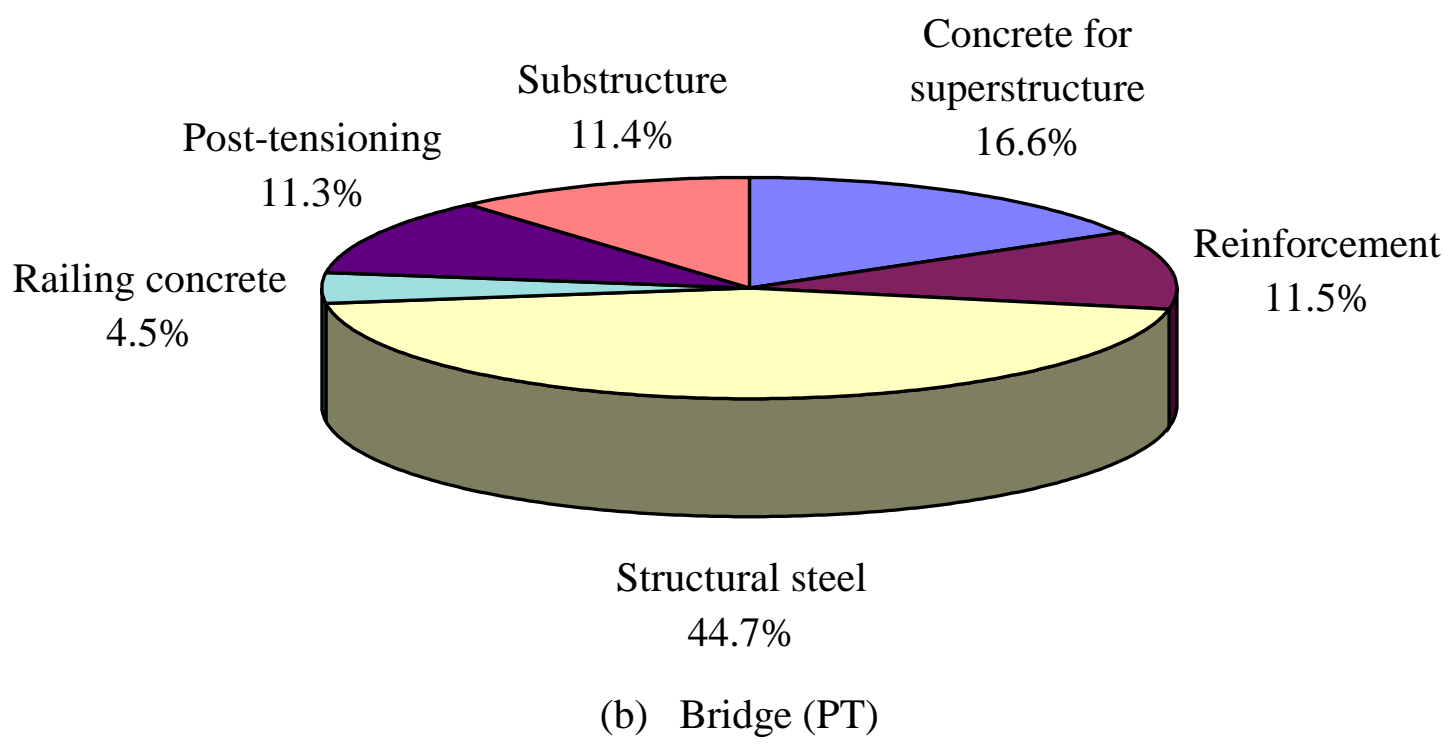

Figure 6.2 Percentages of total unit costs of structural components. 


\section{CHAPTER 7}

\section{CONCLUSIONS AND RECOMMENDATIONS}

\subsection{Conclusions}

The objective of the research project is to develop a reliable and practical methodology to predict the instantaneous (short-term) and time-dependent (long-term) performance of post-tensioned steel-concrete composite bridges. The bridge response was evaluated by using both experimental and analytical methods. The performance of a new bridge in Elkhart County over the I-90 Indiana Toll Road was monitored by collecting the strain readings at certain locations in the steel and concrete components, along with the elongation of a post-tensioning tendon. Analysis was performed to estimate the overall structure response, and the results were compared to the experimental data. The bridge response based on the analytical methodology was predicted over a long time period (30 years, well beyond the duration of field monitoring which was about 10 months) to evaluate long-term performance.

The response of post-tensioned composite bridges can be appropriately evaluated by utilizing a combination of classical beam theory, the concept of transformed section, the virtual work principle and compatibility equations. The proposed analytical approach is suitable for design purposes since it is capable of providing reasonable results without 
time-consuming computation or complicated finite element formulations. In addition, the physical meanings of temperature and time-dependent effects can be easily visualized through the analytical procedure. Several important conclusions drawn from the experimental and analytical results presented in previous chapters are noted in the following:

(1) Comparison of the analyzed results and experimental data suggests that a relatively simple approach to predict the performance of post-tensioned composite bridges is possible. Both calculated instantaneous and long-term structural response based on the proposed method show a satisfactory agreement with field test results at the most critical locations.

(2) The experimental measurements confirmed the presence of compressive stresses throughout the deck shortly after post-tensioning. The initial compressive force introduced by post-tensioning enables an analysis conducted on the basis of an uncracked concrete deck. In other words, transformed sections can be assumed to be valid along the entire bridge span for long-term analysis. Considering timedependent effects, the bridge deck may be subjected to some tensile stresses due to restrained shrinkage and creep at particular locations, such as those near the drape points. However, the magnitude of the tensile stresses were not sufficient to cause cracking in the concrete slab.

(3) The increment of tendon force due to truck loading can be estimated by using the principle of virtual work in conjunction with appropriate compatibility equations. However, the magnitude of the additional tendon force is insignificant and, 
therefore, can be ignored in computing the stress or strain distribution along the girder section.

(4) The precise thermal response is not able to be determined because detailed information of temperature distribution or variation in the structural components is required for accurate calculation. However, thermal stresses along a composite girder section can be estimated by using an assumed bi-linear distribution of temperature variation. The most critical thermal stress is expected to occur near the bridge pier.

(5) Prestress losses in post-tensioned composite bridges caused by time-dependent factors, including shrinkage and creep in the concrete deck and relaxation in the steel tendon, are less significant compared with losses that occur in conventional prestressed concrete bridge structures. For this study, the total prestress losses after thirty years of service are predicted to be about $7 \%$ of the initial effective prestress.

(6) Time-dependent factors can introduce significant compressive stresses in the steel girder section. This phenomenon is confirmed from both experimental observation and theoretical calculation. As a result, the web of the plate girder is susceptible to the possibility of local buckling. According to the analysis, the most crucial spot is near the drape location where the stress distribution is expected to be most uniform.

(7) The benefit of a simplified solution technique is particularly appropriate in the timedependent analysis. Since the time-dependent material characteristics involve considerable uncertainty, elaborate computational procedures such as those based 
on very complicated viscoelastic concrete models and finite element formulations with high precision, are probably not warranted.

(8) The cost comparison between the investigated bridge and another existing conventional steel bridge did not demonstrate an economical advantage for posttensioned steel bridge construction. However, no final conclusion on the economic viability of post-tensioned steel bridge is postulated because the construction expenses of post-tensioned steel bridges could be reduced further.

\subsection{Recommendations for Use of Analytical Model}

Application of the proposed analytical procedure is recommended for calculating the short-term and long-term bridge response. Based on the findings reported herein, the following suggestions may be used for future design and analysis of prestressed composite steel bridge structures:

(1) The girders can be assumed to be simply supported at the integral bridge abutments. The adequacy of this hypothesis was examined by comparing the analytical solutions with the test data. Although this assumption does not result in a particularly good estimation of the strain distribution in the girder section near the abutment (which is not a critical location for flexural response), the bridge response at the critical locations such as midspan and pier is reasonably predicted based on this assumption.

(2) The postulate of an uncracked concrete deck can be made in the analysis. For practical bridge design, this presumption should be valid in calculating the stresses 
due to dead load and post-tensioning, and possibly time-dependent effects in unshored bridge structures. This argument implies that transformed sections could be used in both positive and negative moment regions for the design associated with those types of loading. However, for live load design, it is suggested that different types of sections be used for calculating the capacities for resisting positive and negative moments. The method of transformed section can be applied in the positive moment regions where the concrete is subjected to compressive stresses due to live loads. On the other hand, the concrete is assumed to be ineffective in the negative moment regions.

(3) As shown previously in the results of experiment and analysis, the prestress increment due to the truck loading is insignificant and it can be neglected. Also, in practice the prestress increment resulting from the external loading applied after the operation of post-tensioning is very small and can be reasonably ignored.

(4) The long-term bridge performance is controlled by various factors, which can be qualitatively predicted by using the AASHTO and PCI empirical equations for creep function, shrinkage strain function, and prestress losses function. Prestress losses due to shrinkage and creep in concrete can be estimated using a simplified procedure which involves no numerical integration. Significant compressive stresses caused by time-dependent effects are expected to develop in the steel girder near the drape locations. Consequently, the possibility of web local buckling should be evaluated and prevented in the design process. 


\subsection{Recommendations for Implementation}

Based upon the observations and conclusions from the study described herein, the following recommendations for implementing the research are provided:

(1) Post-tensioned, composite steel bridges appear to be a viable alternative to conventional steel bridges. Advantages of post-tensioned bridges include greater ease in fabrication of the steel members, compressive stress in the concrete deck, and the elimination of fatigue sensitive details, providing for greater durability and economy.

(2) The analytical model described herein can be used to evaluate and design posttensioned steel composite deck bridges. Both immediate and long-term effects due to creep and shrinkage should be included to prevent local buckling of the steel girder web.

(3) The total cost of the post-tensioned steel composite bridge was roughly equivalent to the cost of a comparable conventional steel bridge. Improved economies are expected as additional post-tensioned steel bridges are built. As experience is gained with the construction of this bridge type, then the cost of forming and building the concrete diaphragms will be reduced. Additional cost data should be gathered as other post-tensioned steel bridges are constructed.

(4) The use of HPS-70W steel should be considered for future post-tensioned steel bridges. Hybrid designs that use both grades 50 and 70 steels can provide for uniform cross sections and greater economies in fabrication and construction. 


\section{LIST OF REFERENCES}

AASHTO (1998). Standard Specifications for Highway Bridges (LRFD), 2-nd ed., American Association of State Highway and Transportation Officials, Washington, D.C.

ACI Committee 209 (1992). Prediction of Creep, Shrinkage and Temperature Effects in Concrete Structures. 209R-92, American Concrete Institute, Detroit, MI.

Albrecht, P., Li, W., and Saadatmanesh, H. ( (1995). "Fatigue strength of prestressed composite steel-concrete beams." Journal of Structural Engineering, ASCE, 121(12), $1850-1856$.

Anand, S. C., and Fennell, C. (1975). "Prestressed composite steel beam design." Technical Notes, Journal of the Structural Division, ASCE, 102(1), 313-318.

Anand, S. C., and Talesstchi, A. (1973). "Prestressed composite steel beam design." Journal of the Structural Division, ASCE, 99(3), 301-319.

Anderson, R. B. (1995). "Post-tensioned box girders." Modern Steel Construction, AISC, 35(11), 44-51.

Ayyub, B. M., Sohn, Y. G., and Saadatmanesh, H. (1990). "Prestressed composite girders under positive moment." Journal of Structural Engineering, ASCE, 116(11), 29312950.

Ayyub, B. M., Sohn, Y. G., and Saadatmanesh, H. (1992a). "Prestressed composite girders. I: experimental study for negative moment." Journal of Structural Engineering, ASCE, 118(10), 2743-2762.

Ayyub, B. M., Sohn, Y. G., and Saadatmanesh, H. (1992b). "Prestressed composite girders. II: analytical study for negative moment." Journal of Structural Engineering, ASCE, 118(10), 2763-2783.

Basu, P. K., Sharif, A. M., and Ahmed, N. U. (1987a). "Partially prestressed composite beams. II.” Journal of Structural Engineering, ASCE, 113(9), 1909-1925. 
Basu, P. K., Sharif, A. M., and Ahmed, N. U. (1987b). "Partially prestressed composite beams. II.” Journal of Structural Engineering, ASCE, 113(9), 1926-1938.

Bazant, Z. P. (1972). "Prediction of concrete creep effects using age-adjusted effective modulus method." 69(4), 212-217.

Bazant, Z. P., and Whittmann, F. H. (1972). Creep and Shrinkage in Concrete Structures. John Wiley \& Sons, New York, NY., 163-256.

Bradford, M. A. (1991a). "Buckling of prestressed steel girders." Engineering Journal, AISC, 3rd Quarter, 98-101.

Bradford, M. A. (1991b). "Deflections of composite steel-concrete beams subject to creep and shrinkage." ACI Structural Journal, 88(5), 610-614.

Bradford, M. A. (1997). "Shrinkage behavior of steel-concrete composite beams." ACI Structural Journal, 94(6), 625-632.

British Standards Institution (1985). "British standard, structural use of concrete, part 2, code of practice for special circumstances.” BS 8110: Part 2.

CEB-FIP Model Code (1990). "Model code for concrete structures. (MC-90)" Comite Euro-International du Beton (CEB)-Federation Internationale de la Precontrainte (FIP), Paris-Londen.

Collins, M. P., and Mitchell, D. (1991). Prestressed Concrete Structures. Prentice-Hall, Englewood Cliffs, NJ.

Densford, T. A., Hendrick, T. L., and Murray, T. M. (1990). "Short span prestressed steel bridges.” Engineering Journal, AISC, 3rd Quarter, 114-120.

Dezi, L., Leoni, G., and Tarantino, A. M. (1995). "Time-dependent analysis of prestressed composite beams." Journal of Structural Engineering, ASCE, 121(4), 621633.

Dezi, L., Leoni, G., and Tarantino, A. M. (1996). "Algebraic methods for creep analysis of continuous composite beams." Journal of Structural Engineering, ASCE, 122(4), 423-430.

Dunker, K. F., and Klaiber, F. W. (1986). "Post-tensioning distribution in composite bridges.” Journal of Structural Engineering, ASCE, 112(11), 2540-2553.

Eckberg, C. E. (1968). "Development and use of prestressed steel flexural members." Journal of the Structural Division, ASCE, 94(9), 2033-2060. 
Emanuel, J. H. and Hulsey, J. L. (1978). "Temperature Distributions in Composite Bridges.” Journal of the Structural Division, ASCE, 104(1), 65-78.

Fu, H.C., Ng. S. F., and Cheung, M. S. (1989). "Thermal Behavior of Composite Bridges." Journal of Structural Engineering, ASCE, 116(12), 3302-3323.

Ghali, A., and Favre, R. (1994). Concrete Structures: Stresses and Deformations. 2-nd ed., E \& FN SPON, London, UK.

Gilbert, R. I. (1988). Time Effects in Concrete Structures. Elsevier Science Publishers, Amsterdam, The Netherlands.

Gilbert, R. I., and Bradford, M. A. (1995). "Time-dependent behavior of continuous composite beams at service loads." Journal of Structural Engineering, ASCE, 121(2), 319-327.

Hoadley, P. G. (1963). "Behavior of prestressed composite steel beams." Journal of the Structural Division, ASCE, 89(3), 21-34.

Johnson, R. P., Roik, K., and Wolfel, E. (1981). "Limit state design of prestressed composite structures." Journal of Constructional Steel Research, 1(3), 24-27.

Johnson, C. P., and Will, K. M. (1974). "Beam buckling by finite element procedure." Journal of the Structural Division, ASCE, 100(3), 669-685.

Kennedy, J. B., and Grace, N. F. (1982). "Prestressed decks in continuous composite bridges." Journal of the Structural Division, ASCE, 108(11), 2394-2410.

Kennedy, J. B., and Grace, N. F. (1990). "Prestressed continuous composite bridges under dynamic load.” Journal Structural Engineering, ASCE, 116(6), 1660-1678.

Klaiber, F. W., Dunker, K. F., and Sanders, W. W. (1982). "Strengthening of single-span steel-beam bridges." Journal of the Structural Division, ASCE, 108(12), 2766-2780.

Li, W., Albrecht, P., and Saadatmanesh, H. ( (1995). "Strengthening of composite steelconcrete bridges.” Journal of Structural Engineering, ASCE, 121(12), 1842-1849.

Montens, S., and O' Hagan, D. (1992). "Bringing bridges design into the next century: the construction of the Roize bridge." Modern Steel Construction, AISC, 32(9), 53-54.

Muller, J., and Lockwood, J. D. (1992). "Innovations in composite bridge structures." Modern Steel Construction, AISC, 32(9), 47-52. 
PCI Committee on Prestress Losses(1975). "Recommendations for estimating prestress losses." Journal of Prestressed Concrete Institute, 20(4), 4-35.

Pochanart, A. (1999). "Time dependent effects in post-tensioned concrete-steel composite girder bridge.” Ph.D. Thesis. Purdue University.

Reagan, R. S., and Krahl, N. W. (1967). "Behavior of prestressed composite beams." Journal of the Structural Division, ASCE, 93(6), 87-108.

Saadatmanesh, H., Albrecht, P., and Ayyub, B. M. (1989a). "Experimental study of prestressed composite beams." Journal of Structural Engineering, ASCE, 115(9), 2348-2363.

Saadatmanesh, H., Albrecht, P., and Ayyub, B. M. (1989b). "Analytical study of prestressed composite beams." Journal of Structural Engineering, ASCE, 115(9), 2364-2381.

Saadatmanesh, H., Albrecht, P., and Ayyub, B. M. (1989c). "Guidelines for flexural design of prestressed composite beams." Journal of Structural Engineering, ASCE, 115(11), 2944-2961.

Snyder, R.D. (1995). "Prestressed steel girders for single span bridges." Engineering Journal AISC, 3rd Quarter, 83-86.

Snyder, R.D. (1996). "Prestressed steel girders for single span bridges." Engineering Journal AISC, 2nd Quarter, 116-117.

Szilard, R. (1959). "Design of prestressed composite steel structures." Journal of the Structural Division, ASCE, 85(9), 97-123.

Tochacek, M., and Amrhein, F. G. (1971). "Which design concept for prestressed steel?" Engineering Journal AISC, 8(1), 18-30.

Tochacek, M., and Ferjencik, P. (1992). "Further stability problems of prestressed steel structures." Journal of Constructional Steel Research, 22, 79-86.

Tong, W., and Saadatmanesh, H. (1992). "Parametric study of continuous prestressed composite girders.” Journal of Structural Engineering, ASCE, 118(1), 186-206.

Troitsky, M. S., Zielinski, Z. A., and Nouraeyan, A. (1989). "Pre-tensioned and posttensioned composite girders." Journal of Structural Engineering, ASCE, 115(12), 3142-3153. 
Troitsky, M. S., Zielinski, Z. A., and Rabbani, N. F. (1989). "Prestressed-steel continuous-span girders." Journal of Structural Engineering, ASCE, 115(6), 13571370 .

Troitsky, M. S., Zielinski, Z. A., and Rabbani, N. F. (1991). "Prestressing force increment of two-span steel girder." Canadian Journal of Civil Engineering, Vol. 18, 163-170.

Trost, H. (1967). "Auswirkungen des superpositionsprinzips auf kriech- und relaxationsprobleme bei beton and spannbeton." (Effects of the principle of superposition on creep and relaxation problems for concrete and prestressed concrete). Beton- und Stahlbetonbau, 62(10), 230-238; 62(11), 261-269.

Weaver, D. L., and Bonasso, S. G. (1994). "Exploring new bridge designs." Modern Steel Construction, AISC, 34(9), 50-53.

TECH NOTE, Micro-Measurements Division, Measurement Group. 


\section{APPENDIX A PHOTOGRAPHS OF ELKHART COUNTY BRIDGE}

Several photographs are illustrated here to demonstrate the construction and structural configuration of the Elkhart County Bridge. 


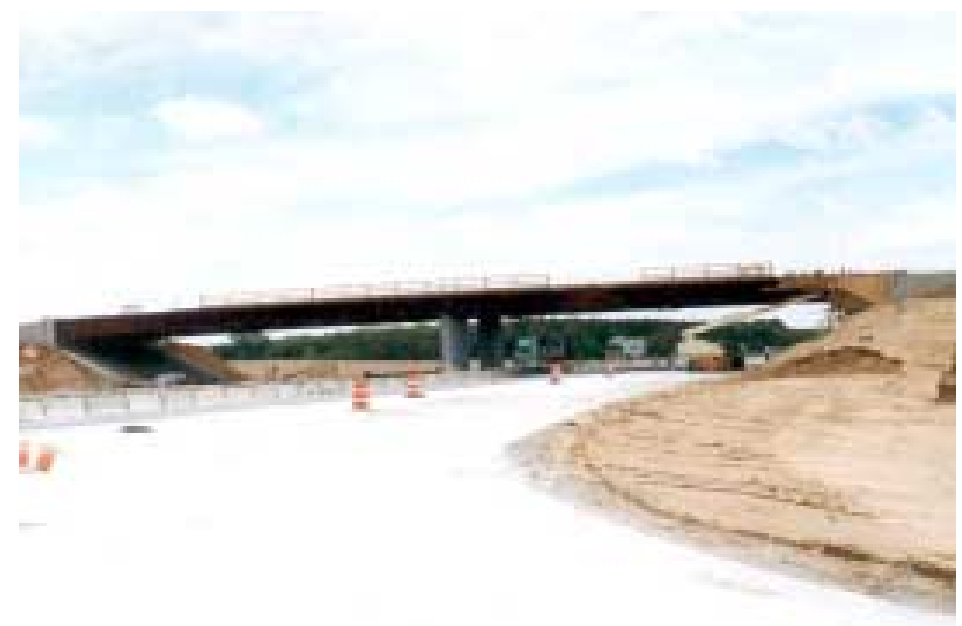

Figure A.1 Overview of Elkhart County I-90 Toll Road Bridge.

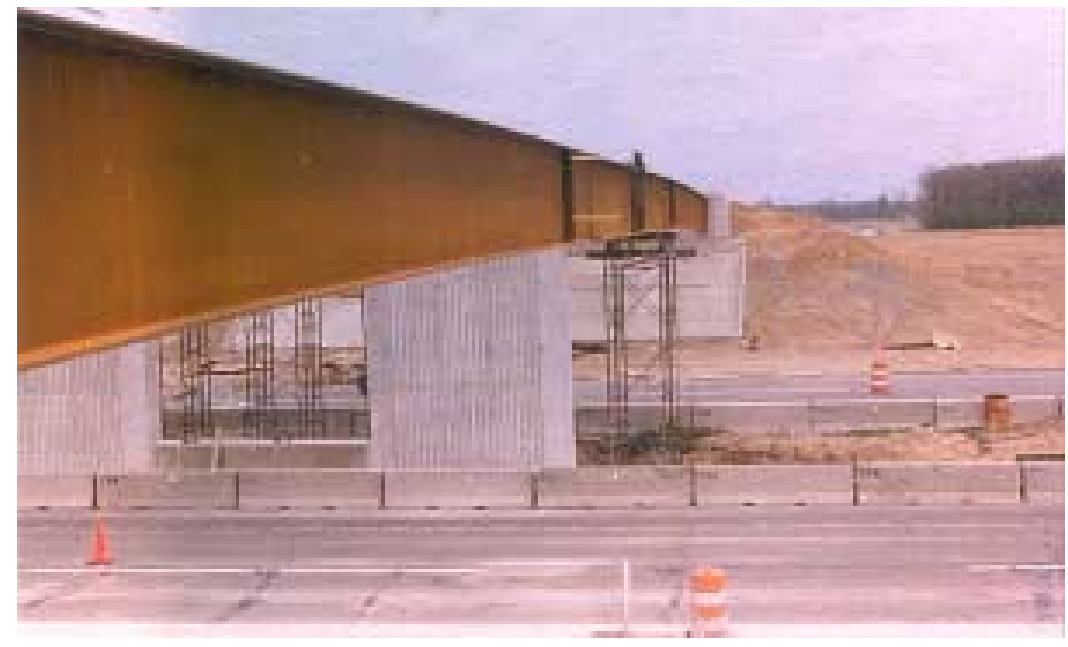

Figure A.2 View of middle pier towers and exterior girder during erection. 


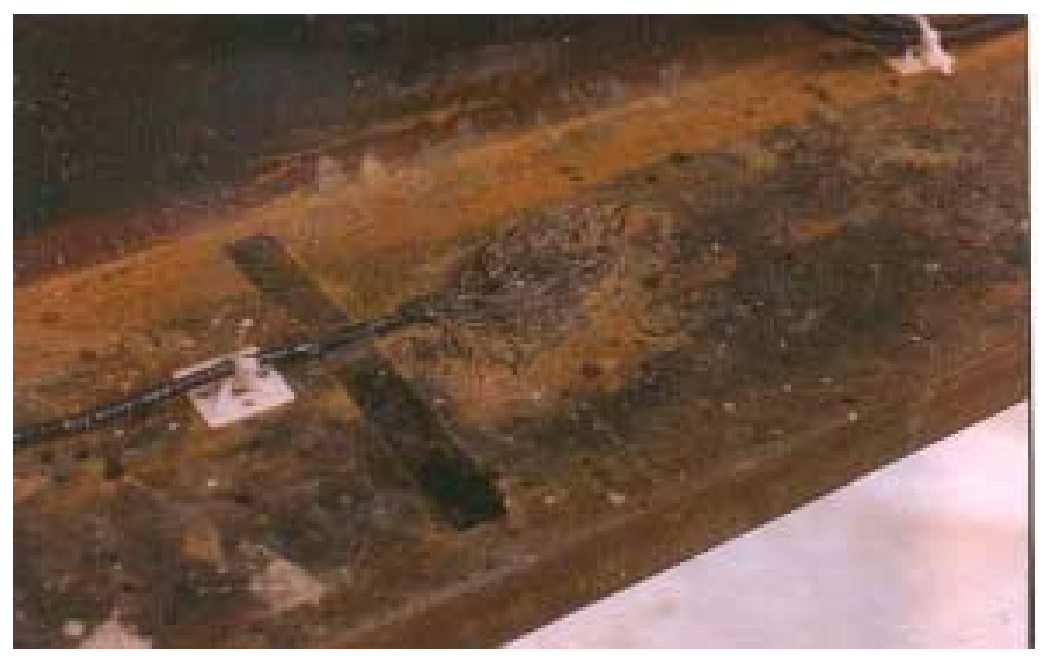

Figure A.3 Strain gage on bottom flange with protective coating.

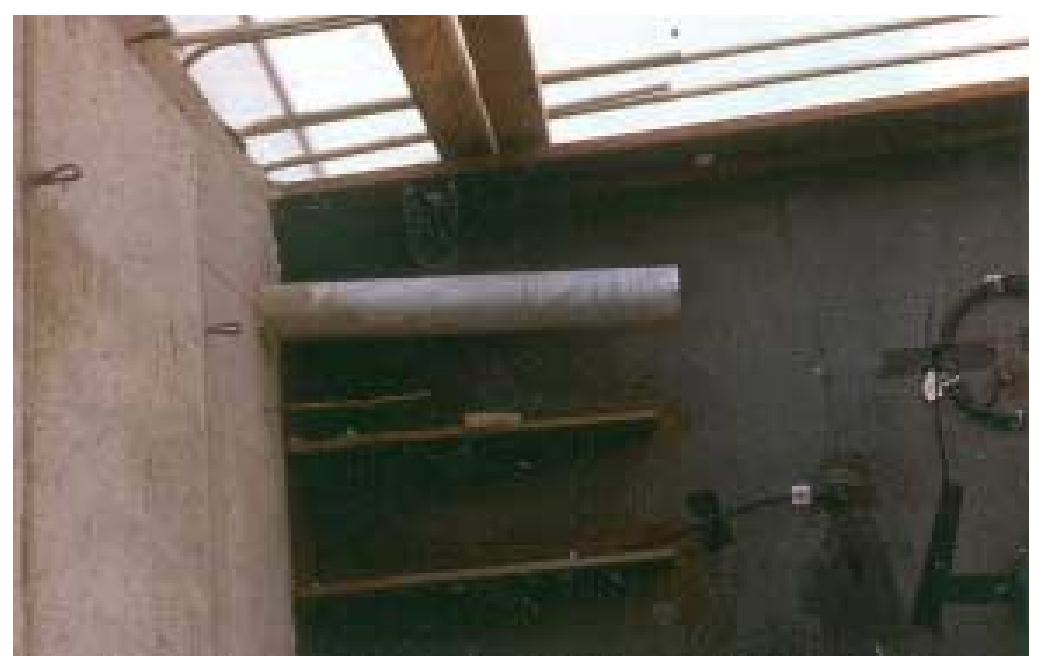

Figure A.4 Post-tensioning duct and strain gages at location 7. 


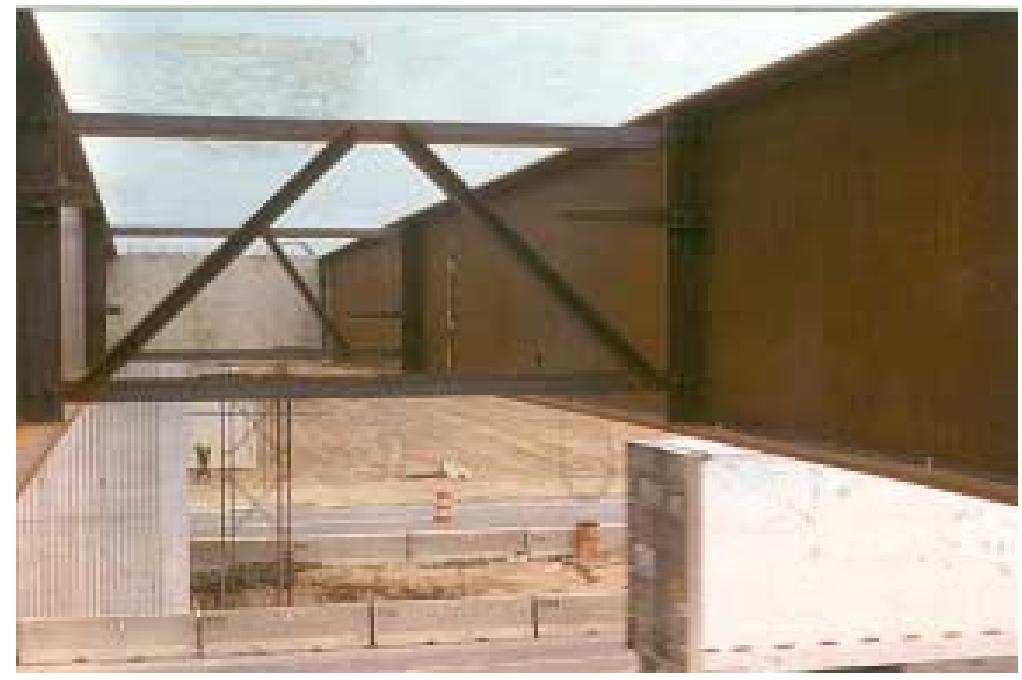

Figure A.5 Steel cross frame, concrete diaphragm and strain gages at location 6 .

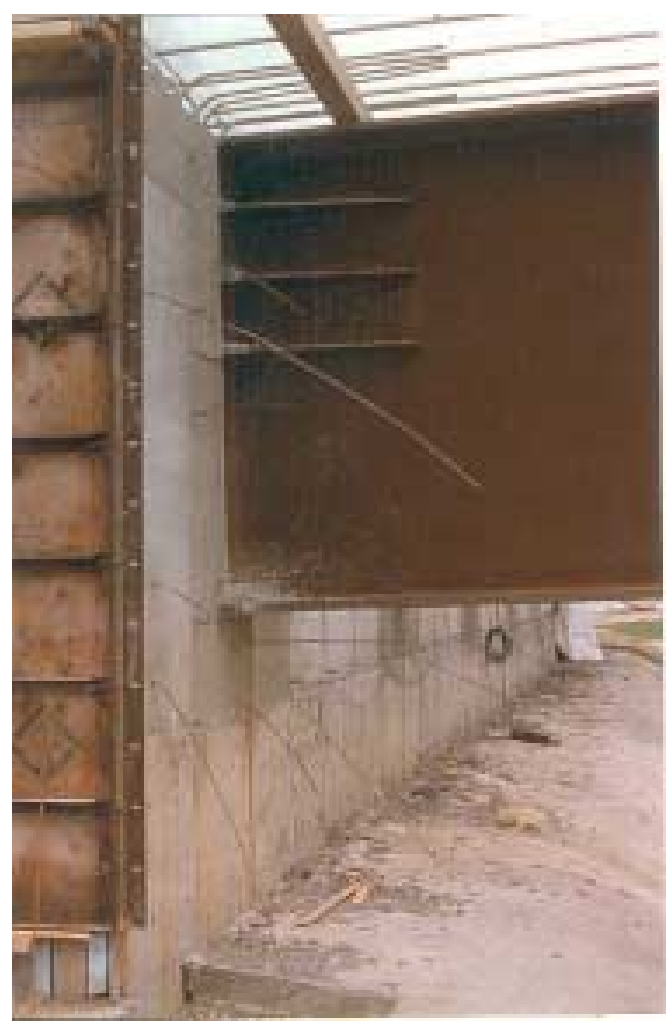

Figure A.6 View of girder end cast integrally into abutment wall and longitudinal stiffeners. 


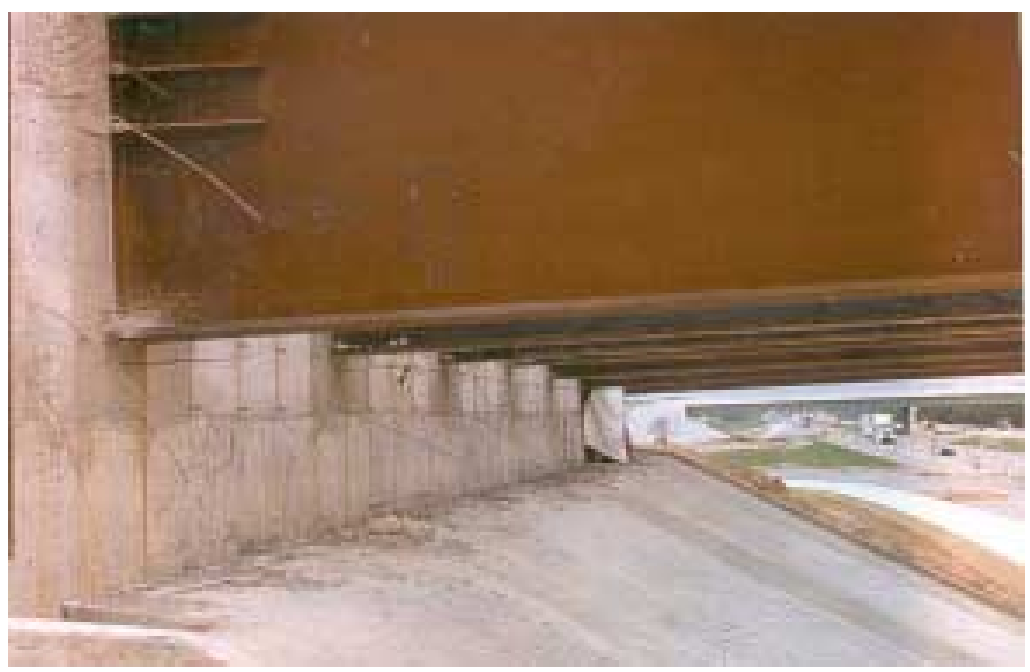

Figure A.7 View of girders framing to the south abutment and the conventional steel bridge in the background. (Refer to the economic analysis in Chapter 6.)

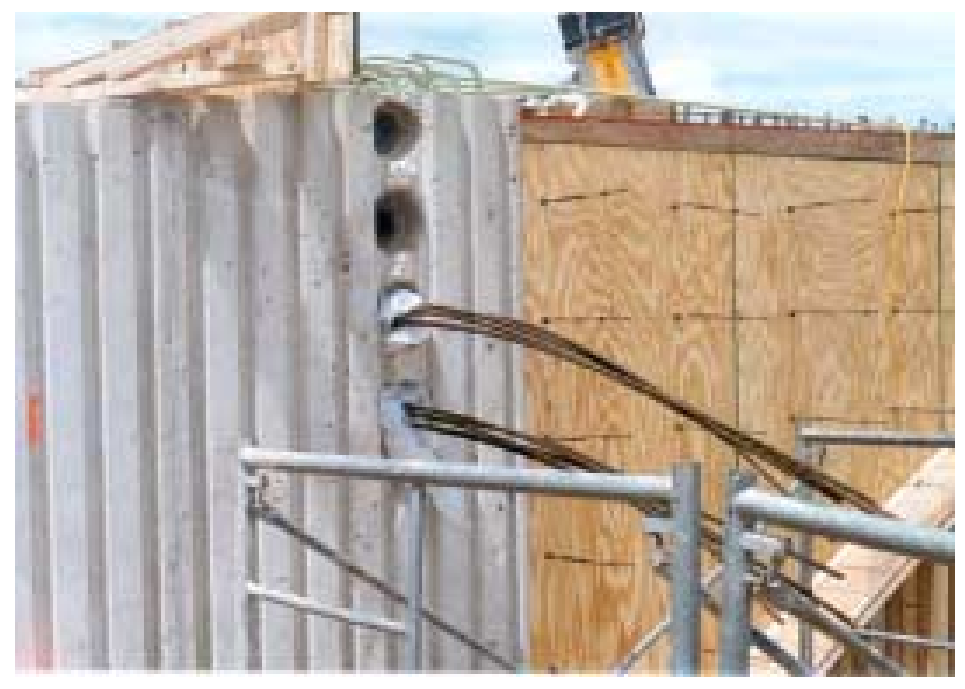

Figure A.8 Strands for transverse post-tensioning of the abutment wall. 


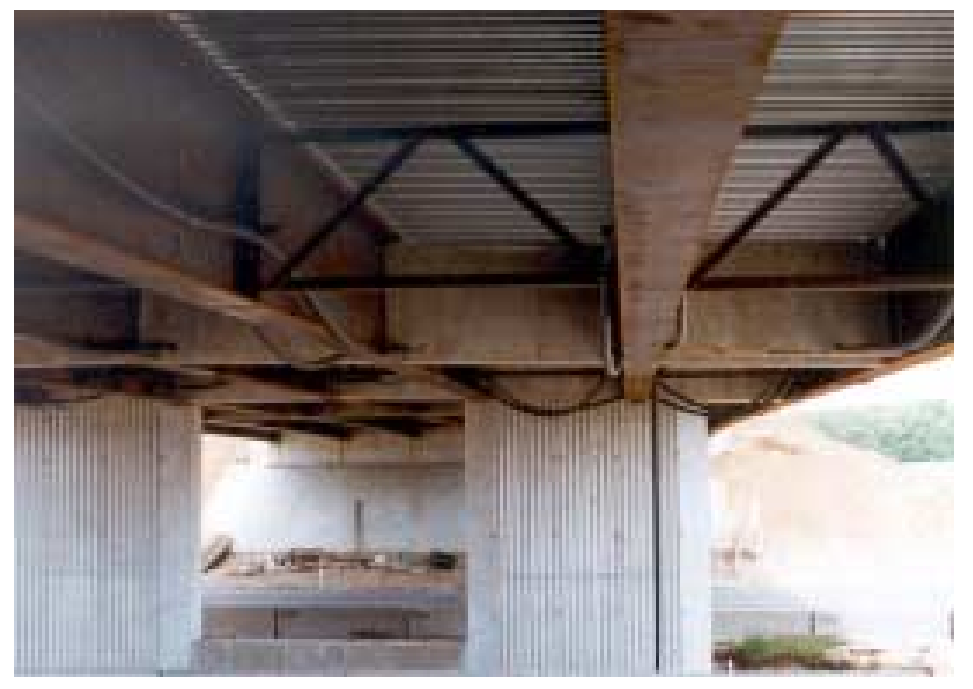

Figure A.9 View from under the bridge before post-tensioning. (Note that PVC ducts are draped prior to prestressing.)

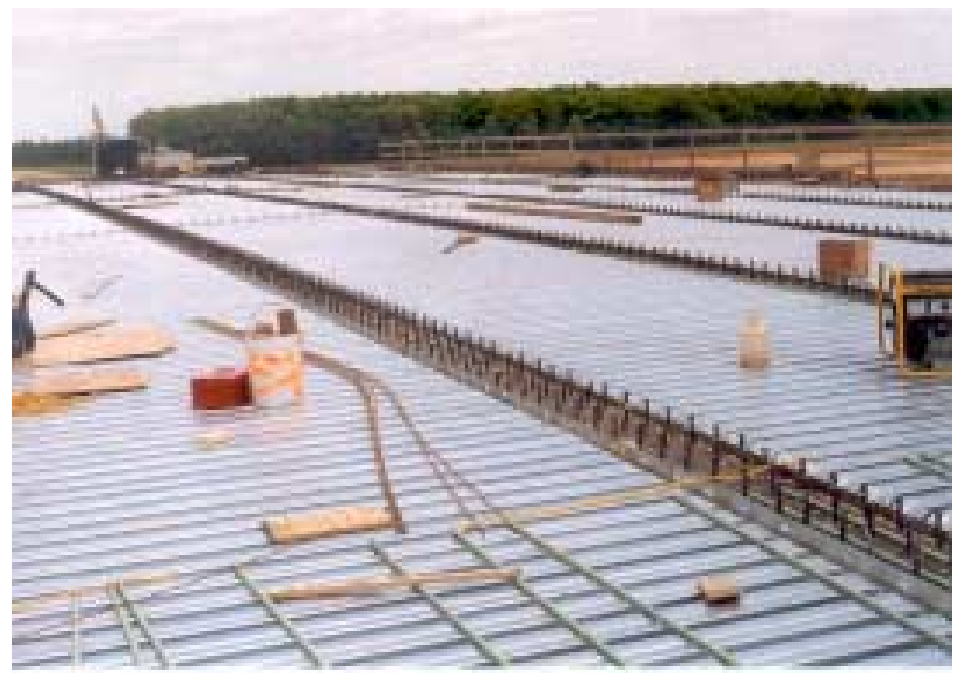

Figure A.10 View of the bridge deck before placing steel reinforcement. (Note the metal deck ribs are perpendicular to the girders.) 


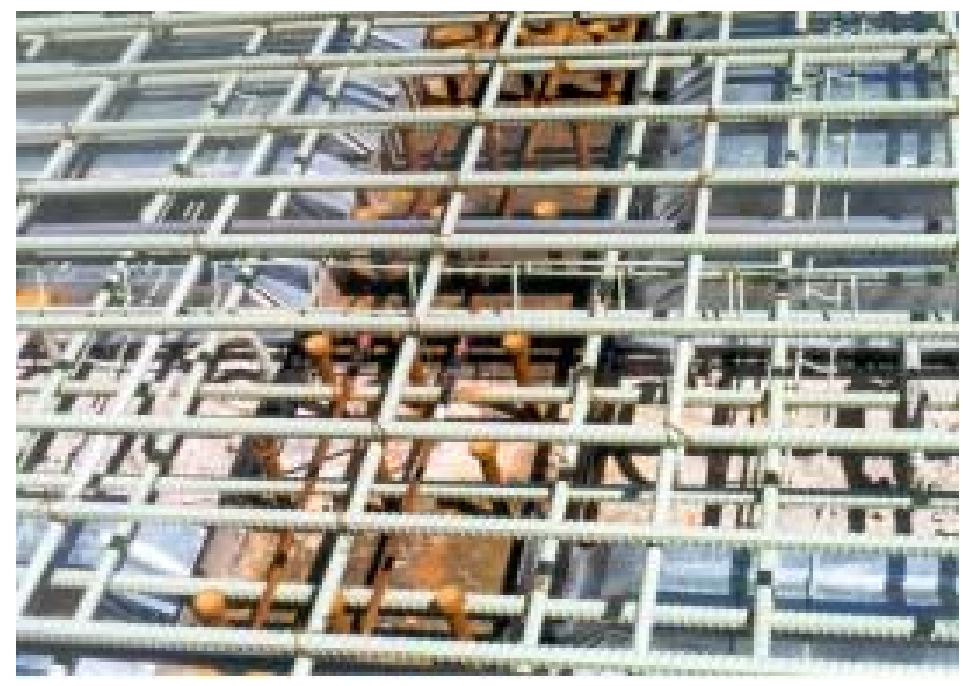

Figure A.11 Shear stud connectors and reinforcing bars. (Note the strain gages on the rebars placed above the steel girder flange.)

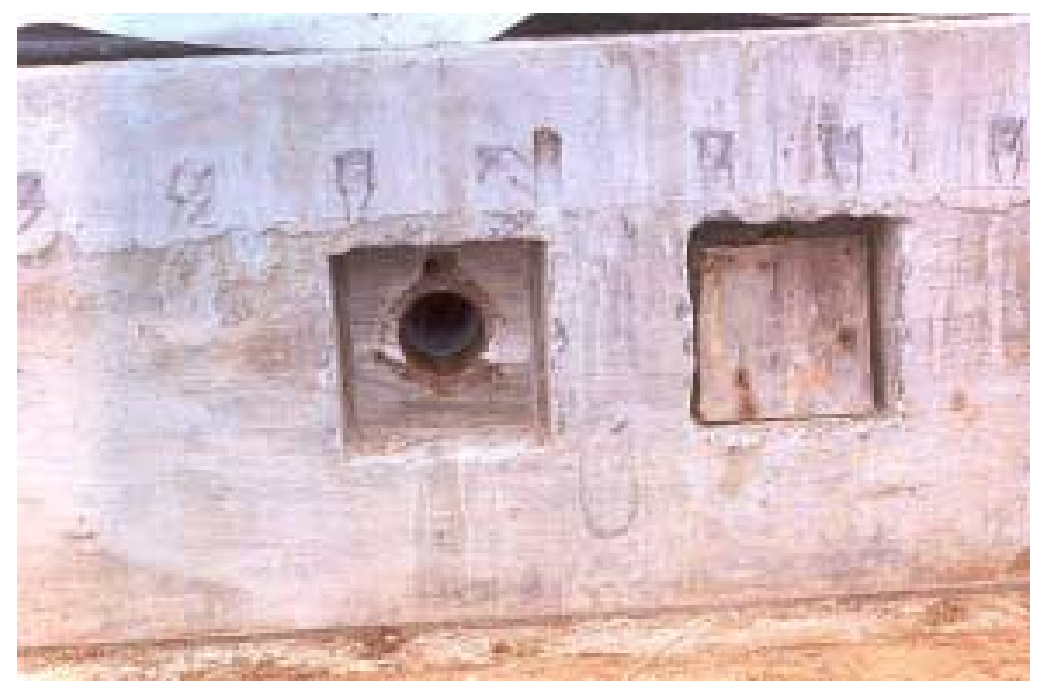

Figure A.12 Duct in outside abutment wall for posttensioning tendons. 


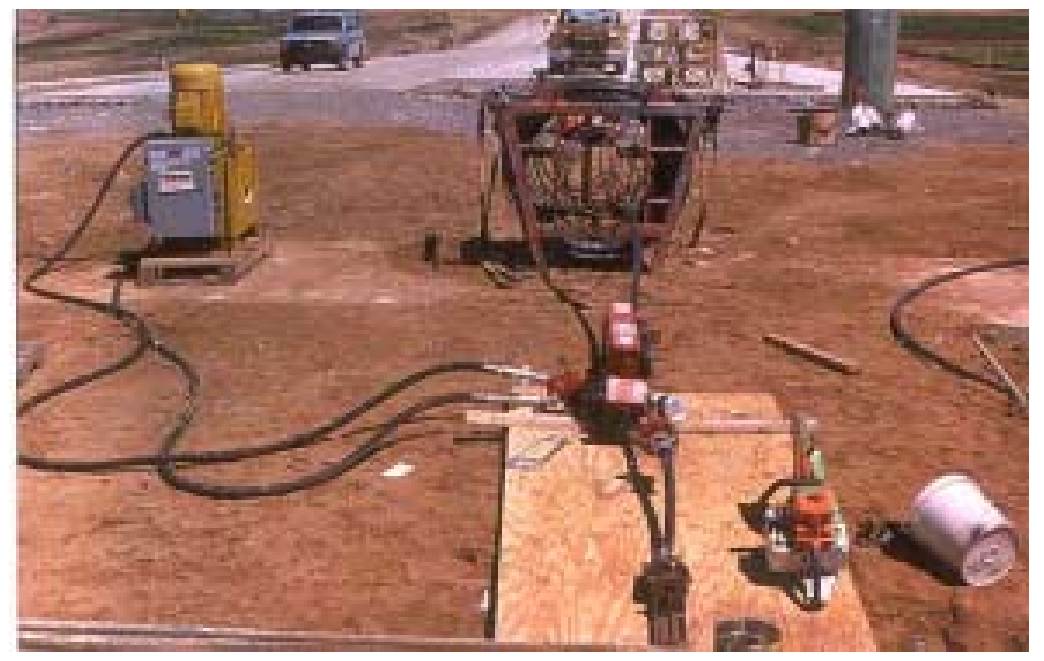

Figure A.13 Hydraulic-operated device to feed strand from the strand spool into the post-tensioning duct.

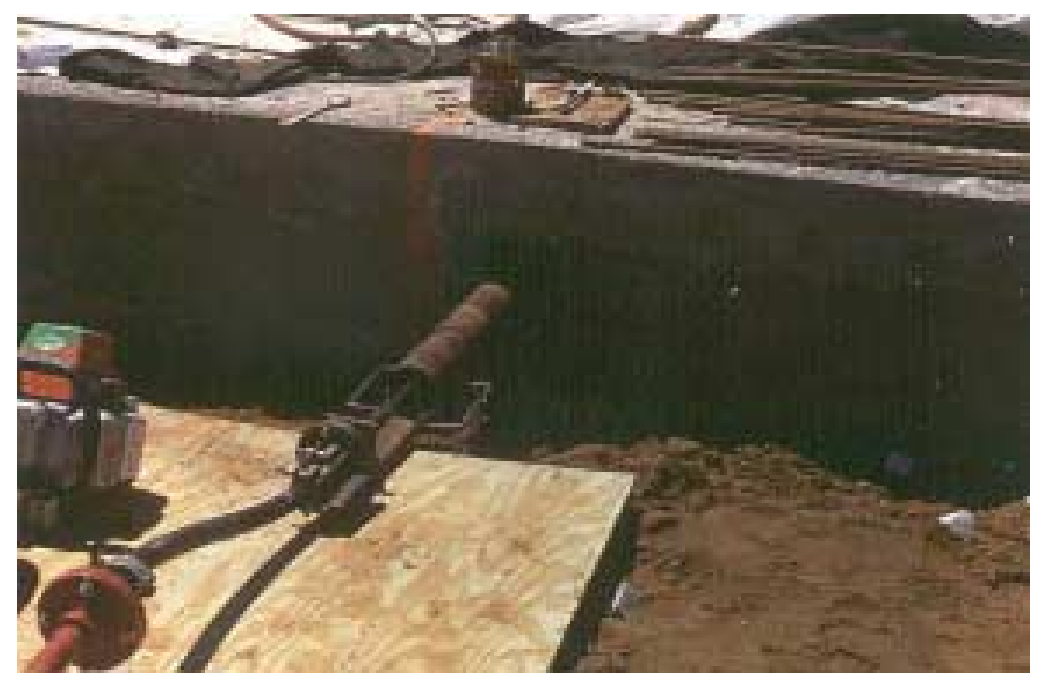

Figure A.14 Strand being fed into the duct. 


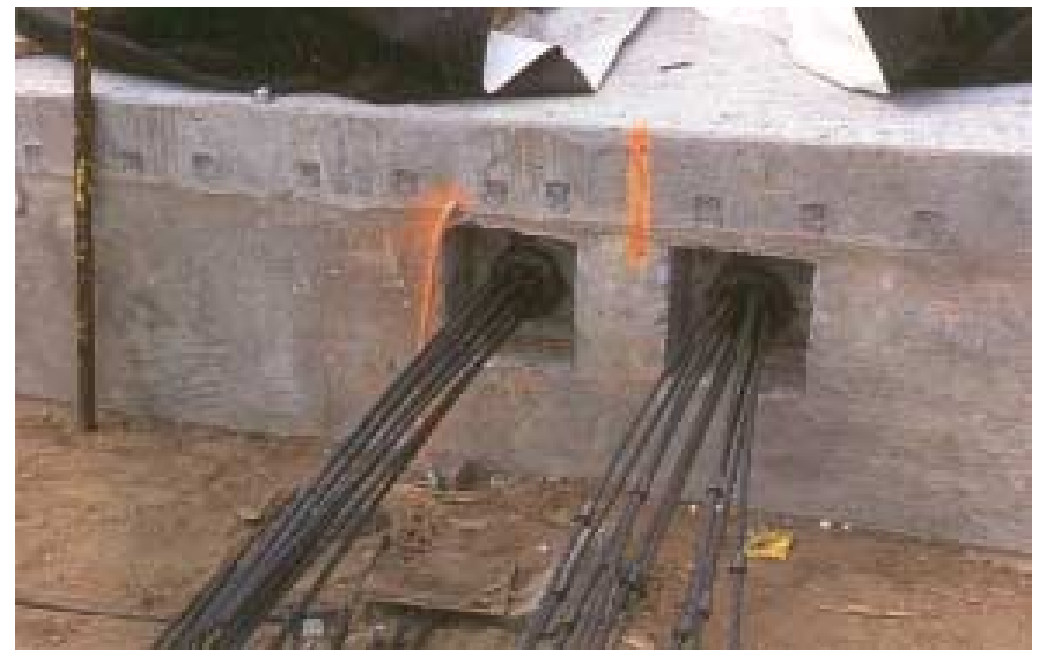

Figure A.15 Twelve strands placed in the post-tensioning ducts prior to post-tensioning. (Note the wedges placed over the strands on the right side.)

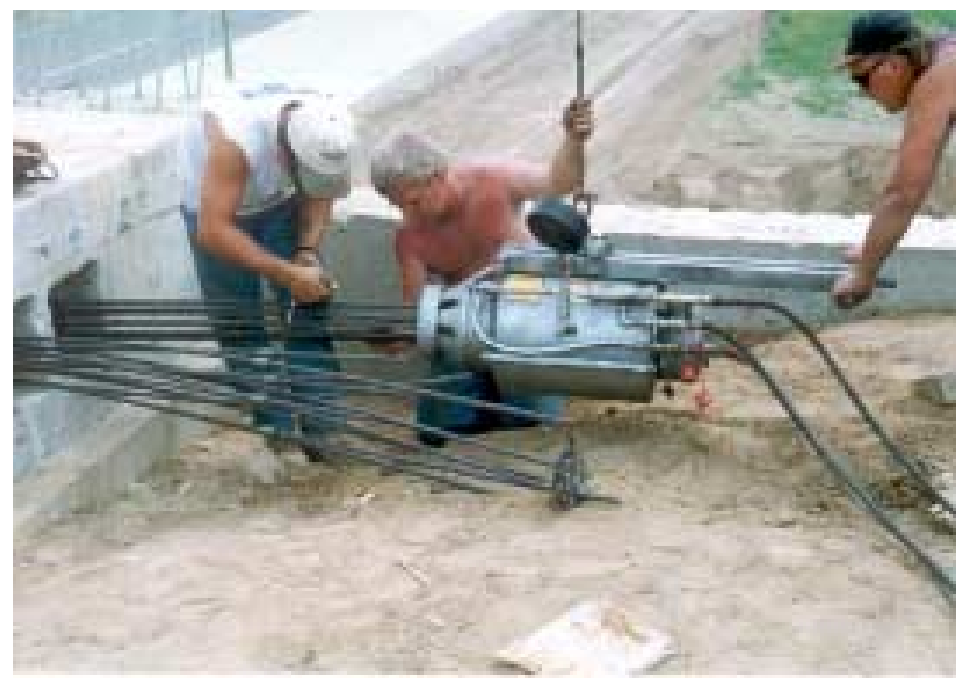

Figure A.16 Construction workers guiding a hydraulic ram onto the strands. 


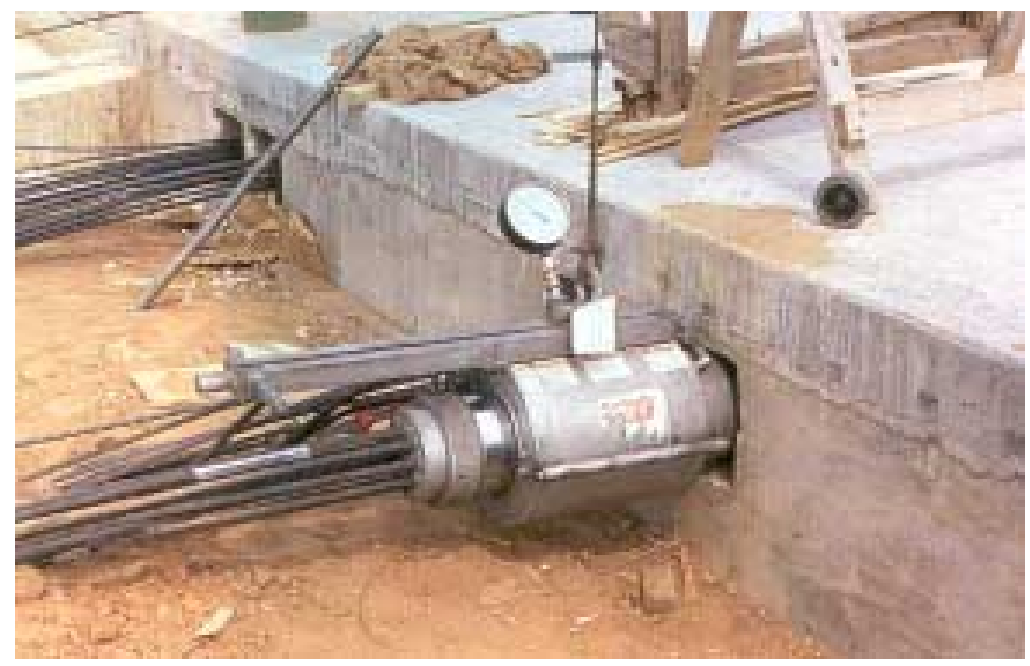

Figure A.17 Ram used to tension the twelve strands in one duct. (Note the pressure gage used to control the posttensioning force.)

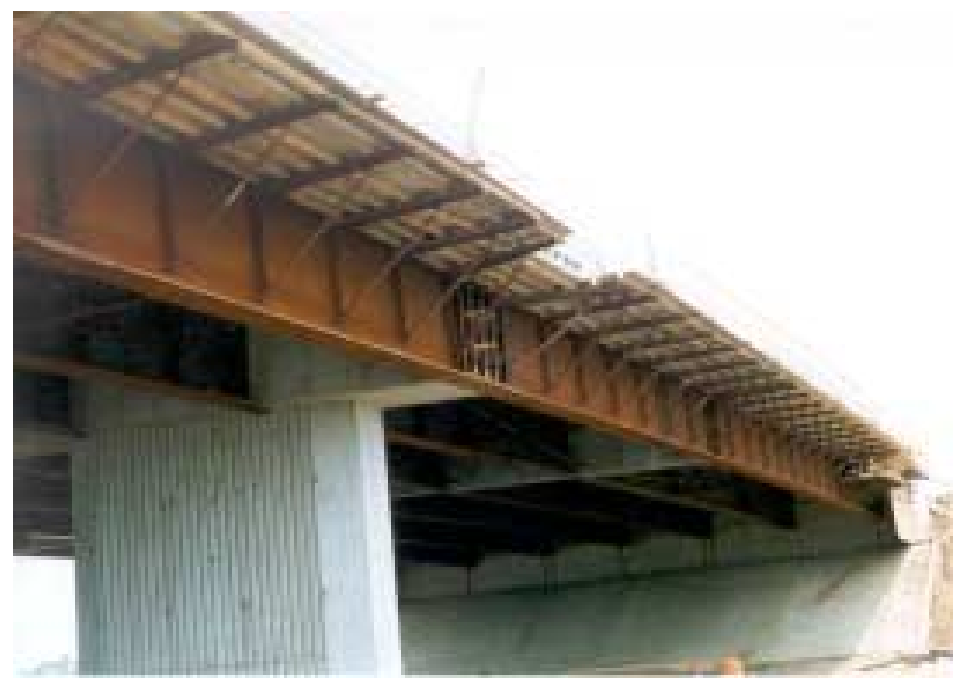

Figure A.18 Overhanging forming used to support wet concrete. 


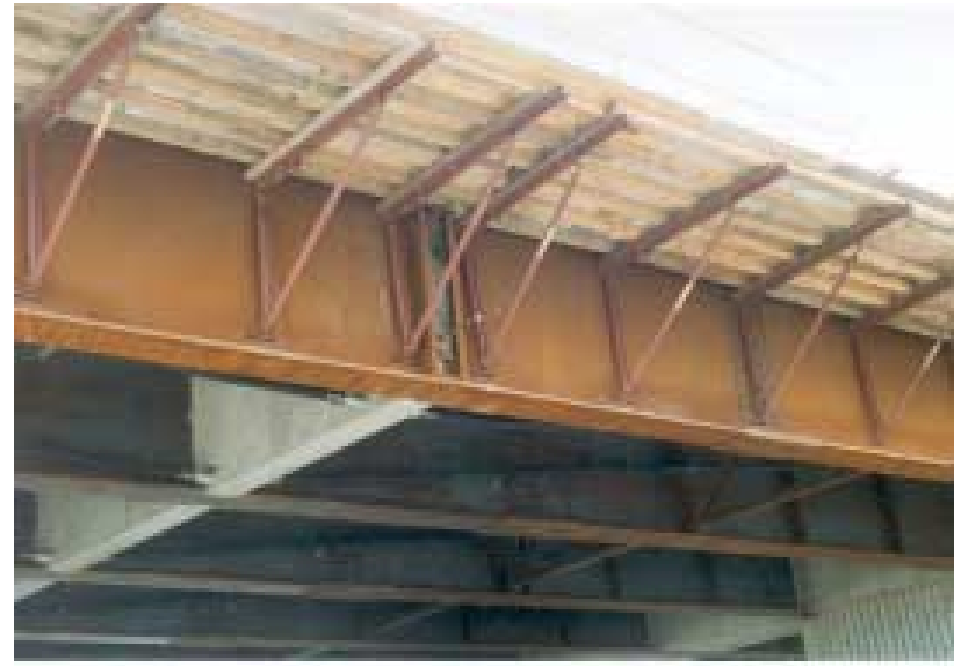

Figure A.19 Longitudinal post-tensioning ducts passing through the concrete diaphragm.

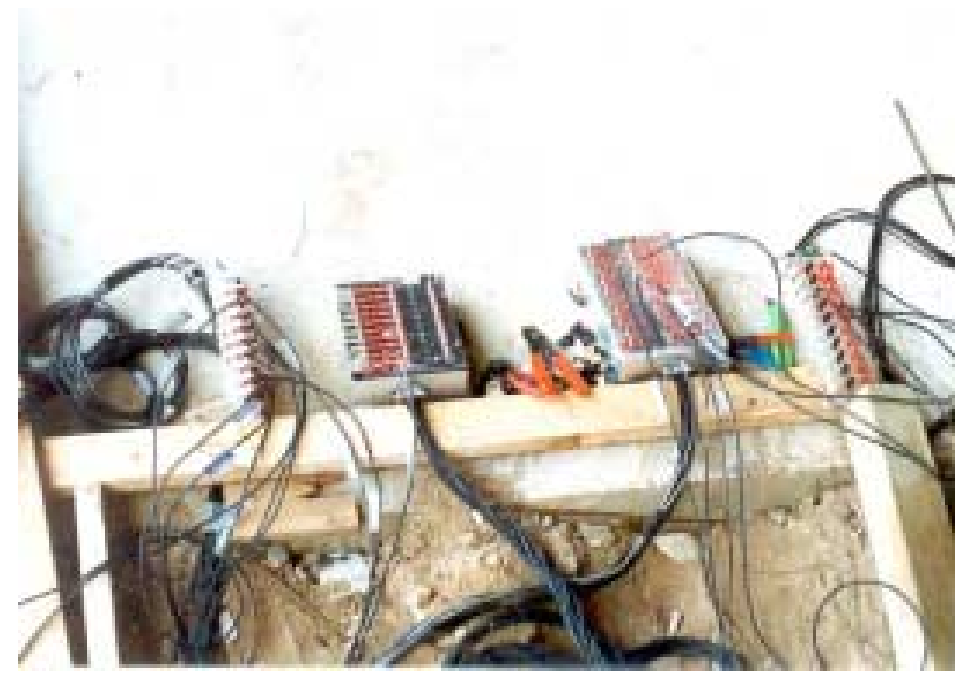

Figure A.20 Boxes for connecting strain gage wires and data acquisition system. 


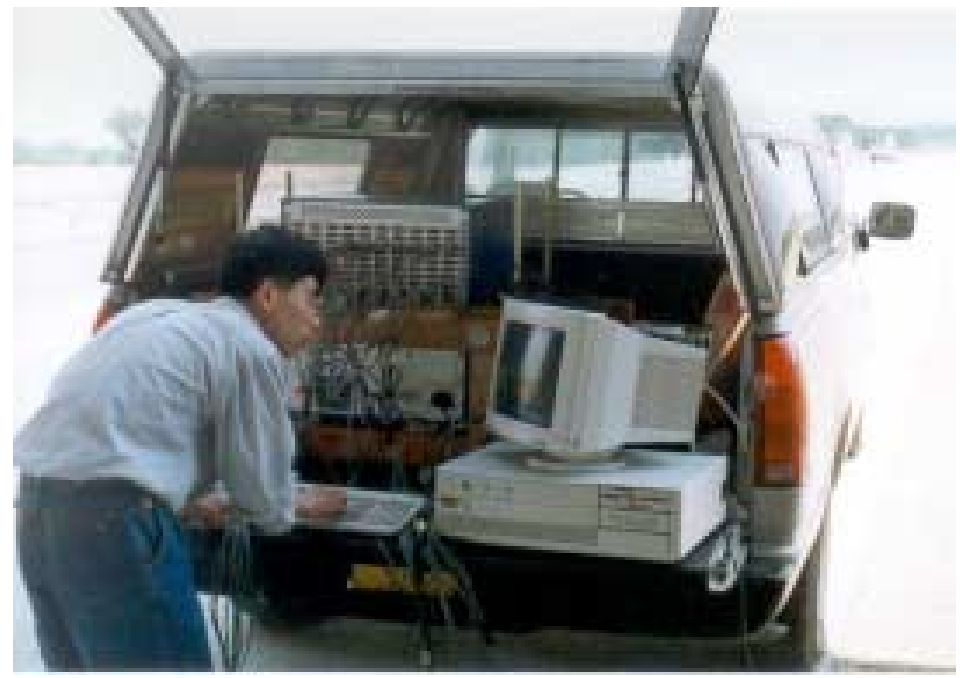

Figure A.21 Operation of data acquisition system used in the field measurement. 


\section{APPENDIX B}

\section{FORTRAN PROGRAM}

The first order analysis conducted throughout the research is primarily based on the FORTRAN program "POSTSTEL". Listed in Table B.1 is the source code of the program. This program is developed to evaluate the short-term (dead load, superimposed dead load and post-tensioning) and long-term (time-dependent factors, including shrinkage and creep in concrete deck and relaxation in steel tendons) structural response of post-tensioned steel-concrete composite plate girder bridges with two equal spans and no skew. The analyzed bridge is assumed symmetric about the middle piers, with only one drape location within each span. Also, it is assumed that no temporary shoring is used during the period of bridge construction. The effects of live load and temperature variation are evaluated elsewhere and are not included in the program.

The algorithm of the program POSTSTEL is based on the methodology described in Chapters 4 and 5. The major part of the parametric study and design examples presented in Chapter 7 is also achieved through using this program. Two input files are required for executing this program. The input data regarding the material as well as geometric properties of the analyzed bridge system (including concrete deck, steel plate girder and post-tensioning tendons), prestress force, superimposed dead load intensity, 
curing information and the load factors are specified in the file "POSTSTEL.INP". The longitudinal and vertical locations for structural response in the composite girder that are of particular interest are stipulated in the input file "GAGELOPS.INP". The explanation for the input arguments can be referred in the comment lines of the source code listed in Table B.1.

Shown in Tables B.2 and B.3 are examples of the input files. The data given in the example input files correspond to the first design case presented in Chapter 7 (an unstiffened post-tensioned steel-concrete composite interior bridge girder for positive moment region). The composite section can be referred in Fig. 7.41(b). All of the load factors are specified to be unity. The comments shown in the input files following the input data are not necessary for executing the program, but are provided only for a clearer demonstration.

The program POSTSTEL generates three major output files: (1) SHCRRE.OUT: the output file containing prestress variation and losses due to time-dependent factors (creep, shrinkage and relaxation); (2) STRESUM.OUT: the output file for longitudinal stresses at specified locations in the composite girder due to different types of loading (dead load, superimposed dead load, post-tensioning and time-dependent effects); and (3) SHEARSUM.OUT: the output file for the shear forces and average shear stresses in the web at specified longitudinal locations along the span, resulting from different types of loading. Respectively shown in Tables B.4, B.5 and B.6 are examples for the above three output files which are generated by executing the program POSTSTEL with the input files as demonstrated in Tables B.2 and B.3. 


\section{Table B.1 Source code of FORTRAN program POSTSTEL}

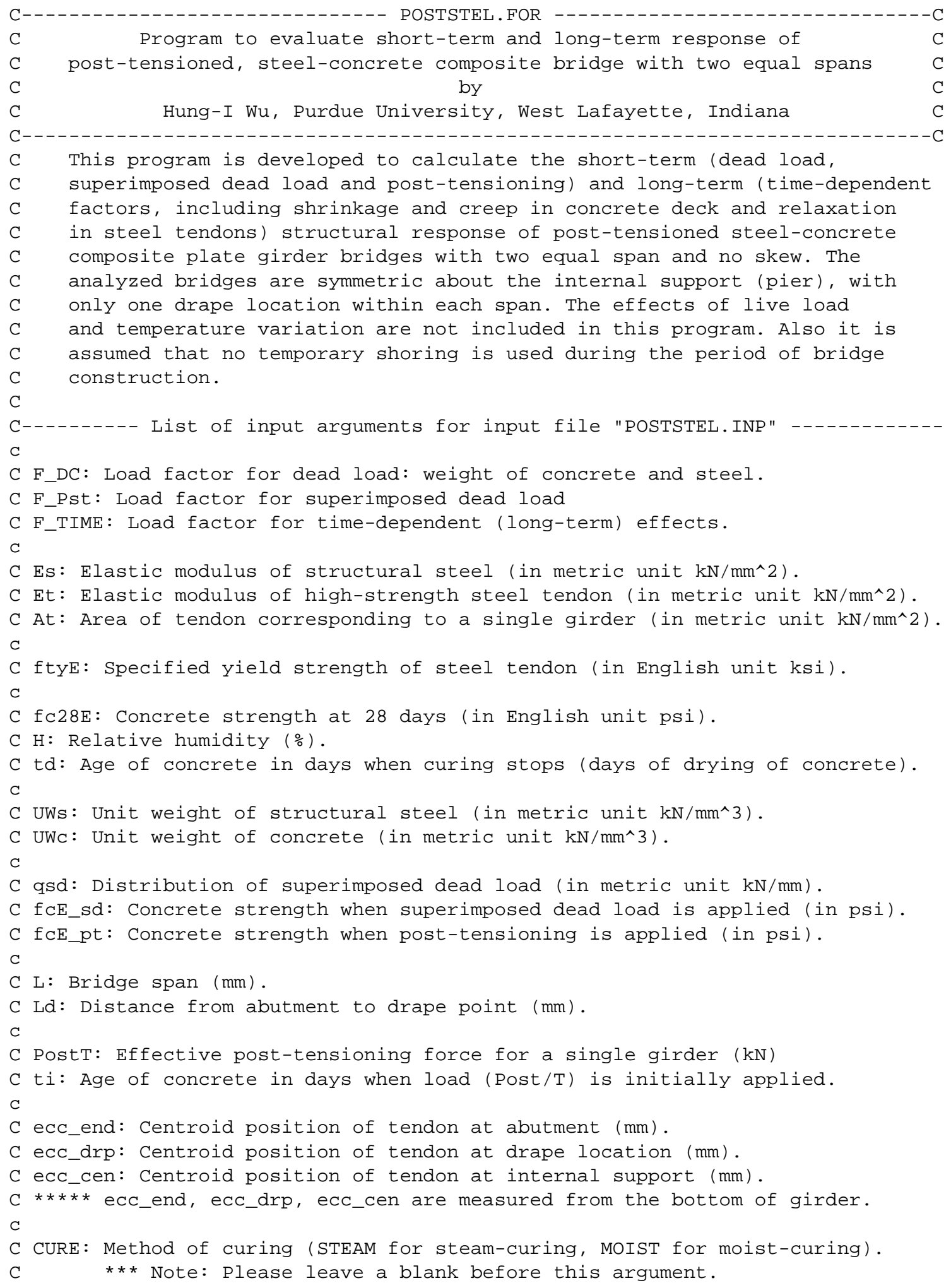


APPROX: Use of approximate method for calculating prestress losses ( $\mathrm{Y}$ or $\mathrm{N}$ ).

C $\quad \star \star \star$ Note: Please leave a blank before this argument.

C

C SIDLT: Indicator to determine the contribution from concrete deck to carry

C superimposed dead load (PN: concrete deck carries load; or

C NM: concrete deck carries no load).

C $\quad \star \star \star$ Note: Please leave a blank before this argument.

$\mathrm{C}$

C N_BKS: Number of steel plates (usually N_BKS $=3$ ).

C N_BKC: Number of blocks of concrete deck (usually N_BKC = 3).

C

C BKS_b(1..3): Array of the horizontal dimension of steel plates (mm).

C BKS_h(1..3): Array of the vertical dimension of steel plates (mm).

$\mathrm{C}$

C BKC_b(1..3): Array of the horizontal dimension of concrete blocks (mm).

C BKC_h(1..3): Array of the vertical dimension of concrete blocks (mm).

$\mathrm{C}$

C NTIMESTP: Number of time step (interval) for time-dependent analysis.

C

C t1: Days after the casting of concrete at the beginning of a time interval.

C t2: Days after the casting of concrete at the end of a time interval.

C

C fcle: Strength of concrete at day "t1" (in English unit psi).

C fcE: Strength of concrete at day "t2" (in English unit psi).

$\mathrm{C}$

C--------- List of input arguments for input file "GAGELOPS.INP" ------------

$\mathrm{C}$

C YstA: Vertical position in concrete where structural response is desired.

C YstB: Vertical position (1) in steel where structural response is desired.

C YstC: Vertical position (2) in steel where structural response is desired.

C

C Npos: Number of longitudinal locations where structural response in desired.

C LOCG(1..NPOs): Array of the longitudinal distance between the interested

C location and the abutment (mm).

$\mathrm{C}$

C-------------------------- Operational arguments -----------------------------

C

C t: Days after the casting of concrete.

C Ec1: Elastic modulus of concrete at day "t2" (in metric unit $\mathrm{kN} / \mathrm{mm}^{\wedge} 2$ ).

C Ec: Elastic modulus of concrete at day "t2" (in metric unit $\mathrm{kN} / \mathrm{mm}^{\wedge} 2$ ).

$\mathrm{C}$

$\mathrm{C}$ - - ${ }^{-}-{ }^{-}-{ }^{-}-{ }^{-}-{ }^{-}-{ }^{-}-{ }^{-}-{ }^{-}-{ }^{-}-{ }^{-}-{ }^{-}-{ }^{-}-{ }^{-}-{ }^{-}-{ }^{-}-{ }^{-}-{ }^{-}-{ }^{-}-{ }^{-}-{ }^{-}-{ }^{-}-{ }^{-}-{ }^{-}-{ }^{-}-{ }^{-}-{ }^{-}-{ }^{-}-{ }^{-}-{ }^{-}-{ }^{-}-{ }^{-}-{ }^{-}-{ }^{-}-{ }^{-}-{ }^{-}-{ }^{-}$

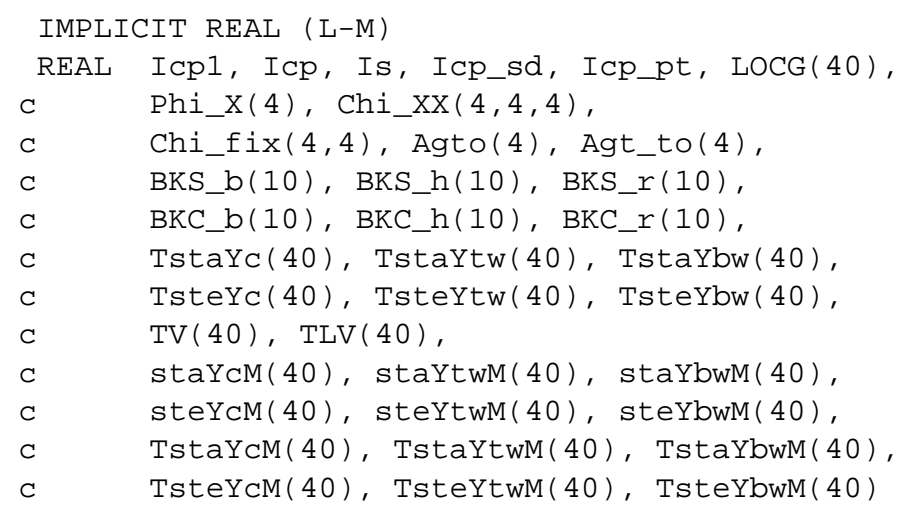




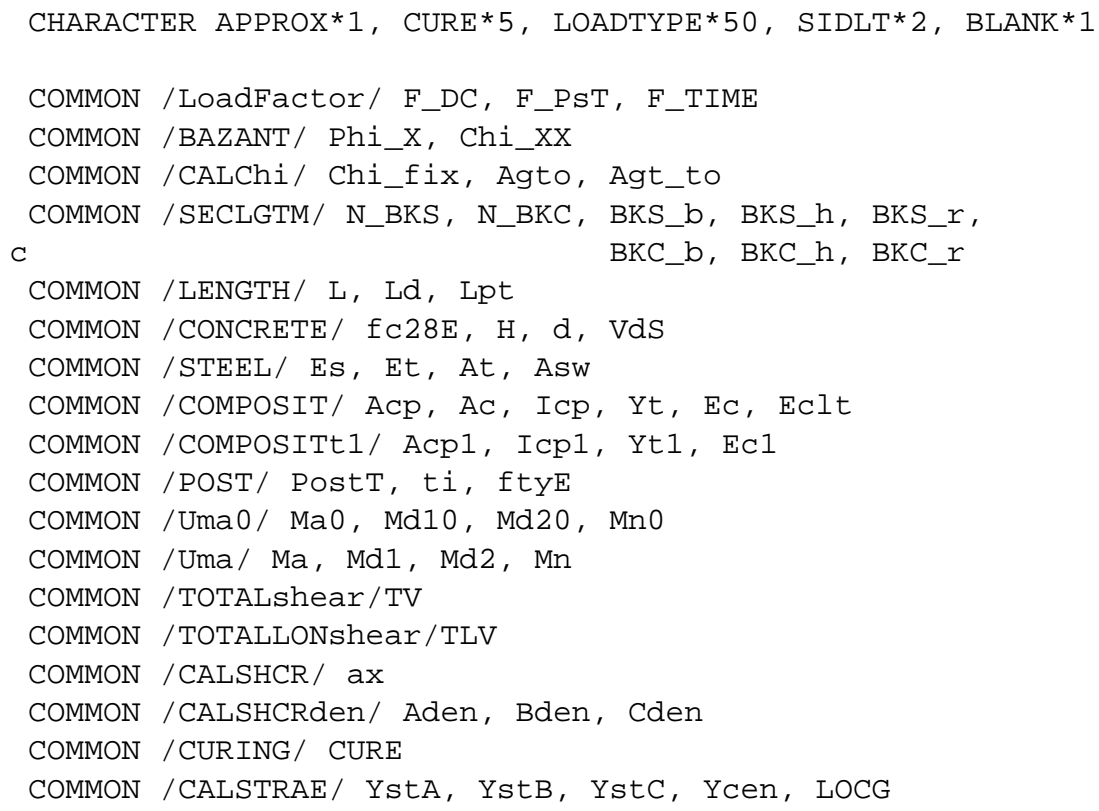




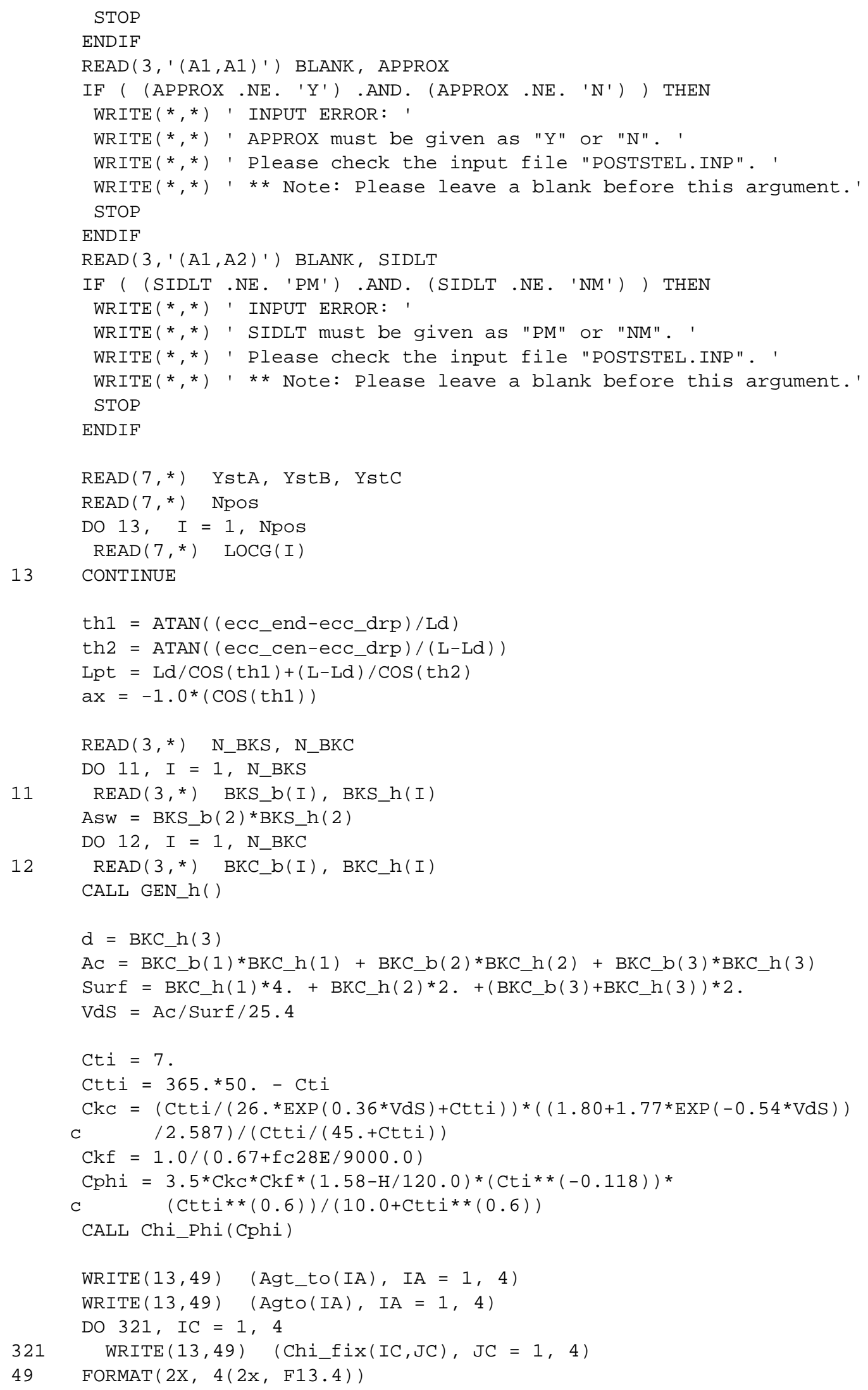




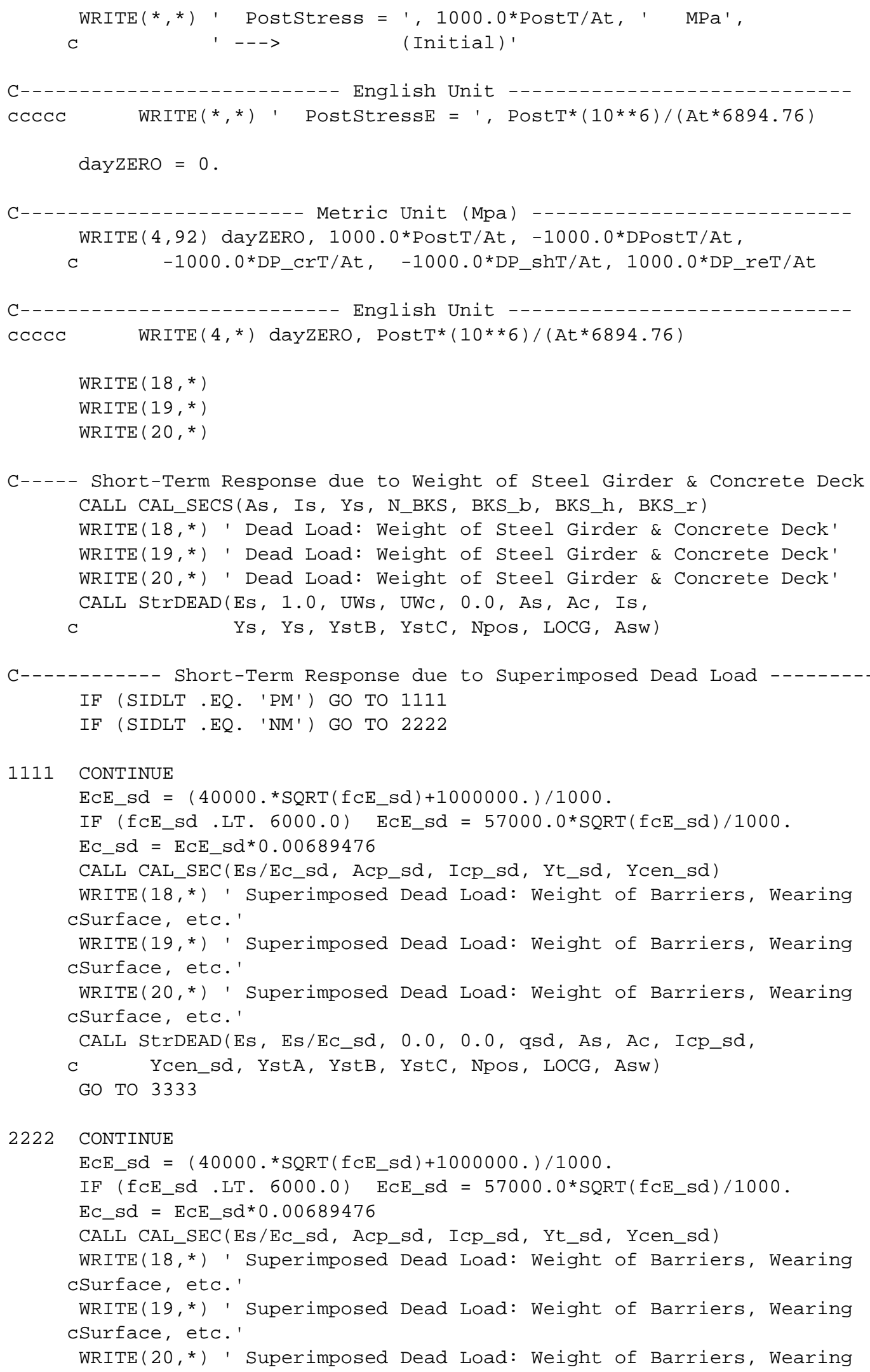


CSurface, etc.'

CALL StrDEAD (Es, 1.0, 0.0, 0.0, qsd, As, Ac, Is,

C

Ys, Ys, YstB, YstC, Npos, LOCG, Asw)

3333 CONTINUE

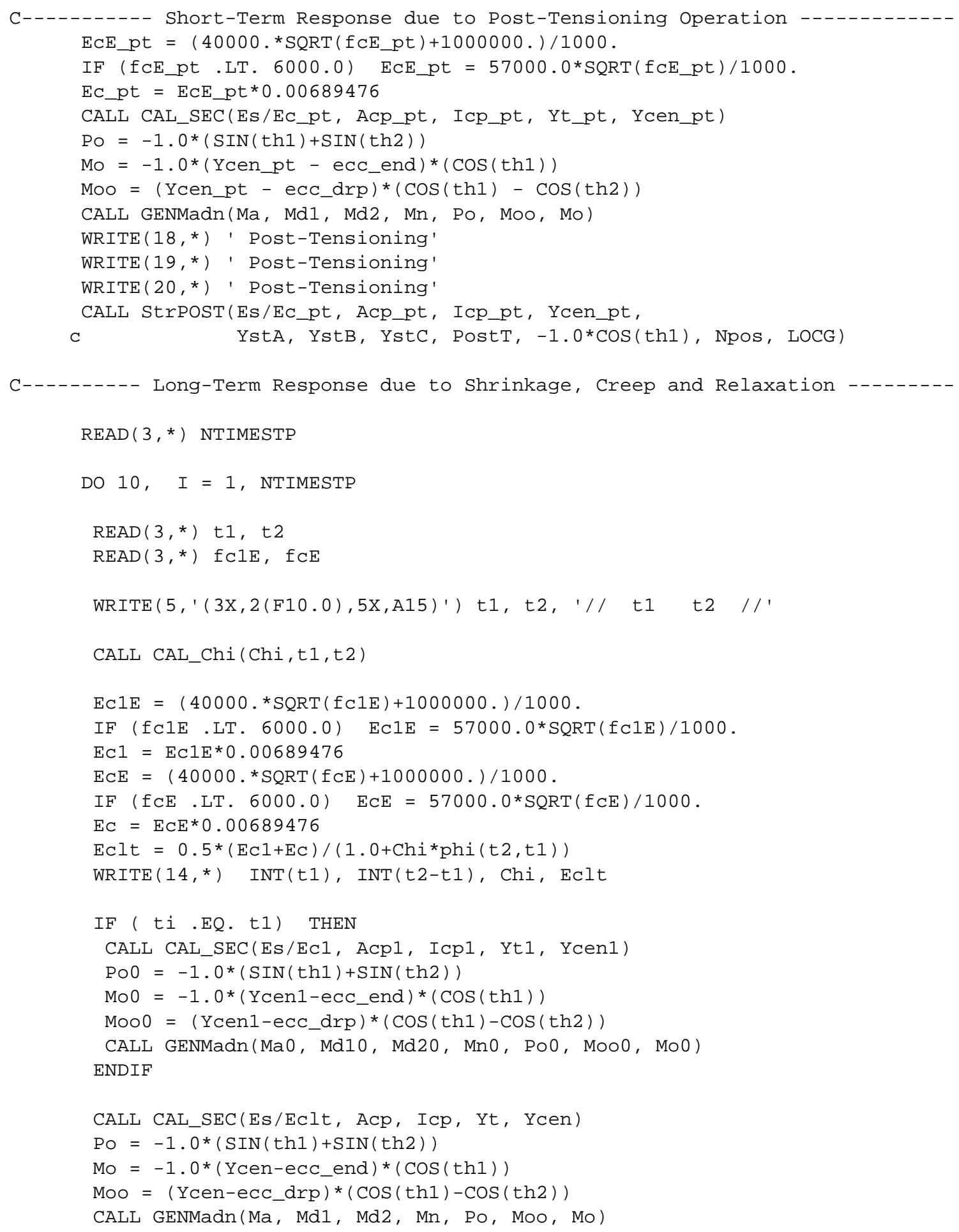




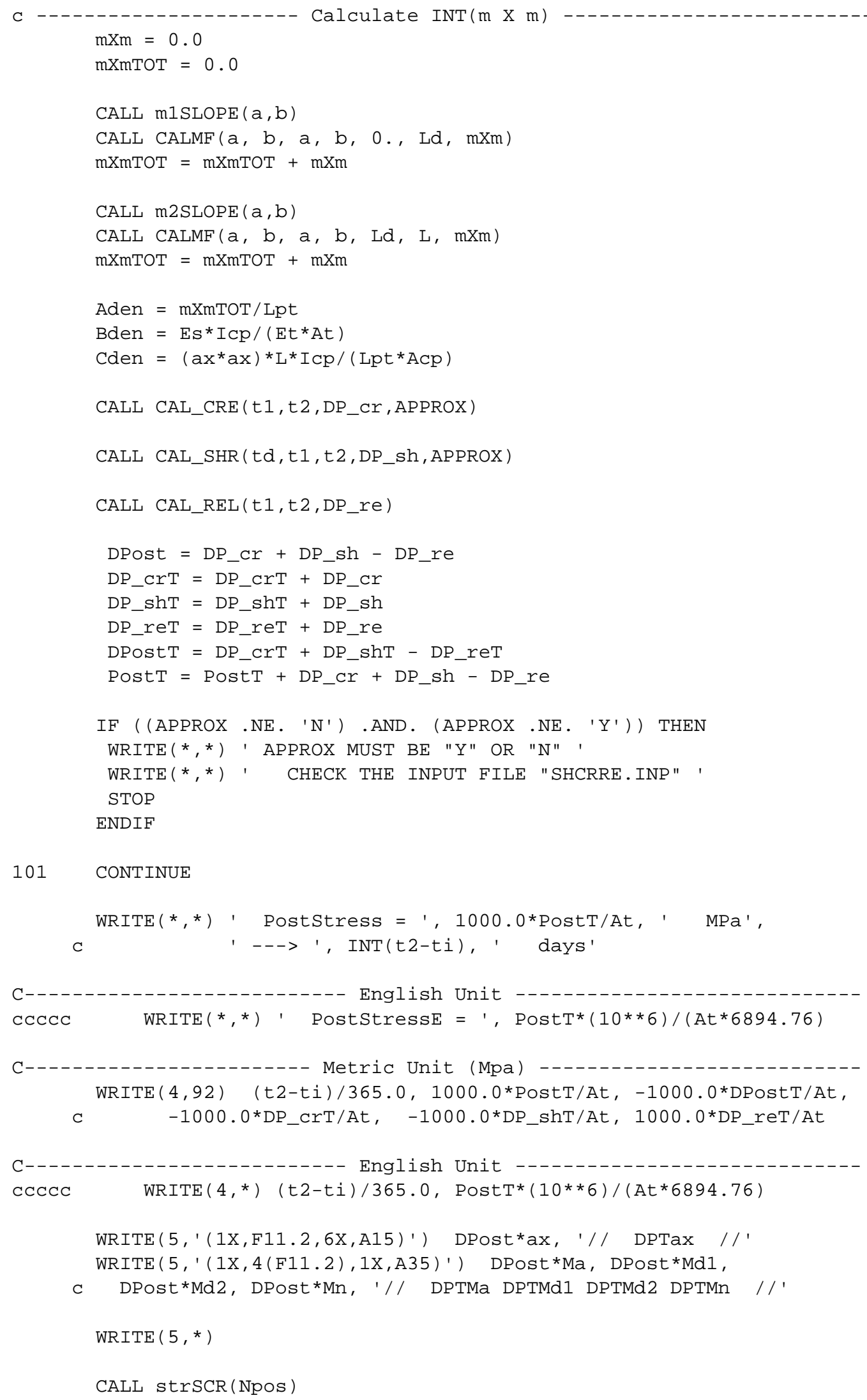




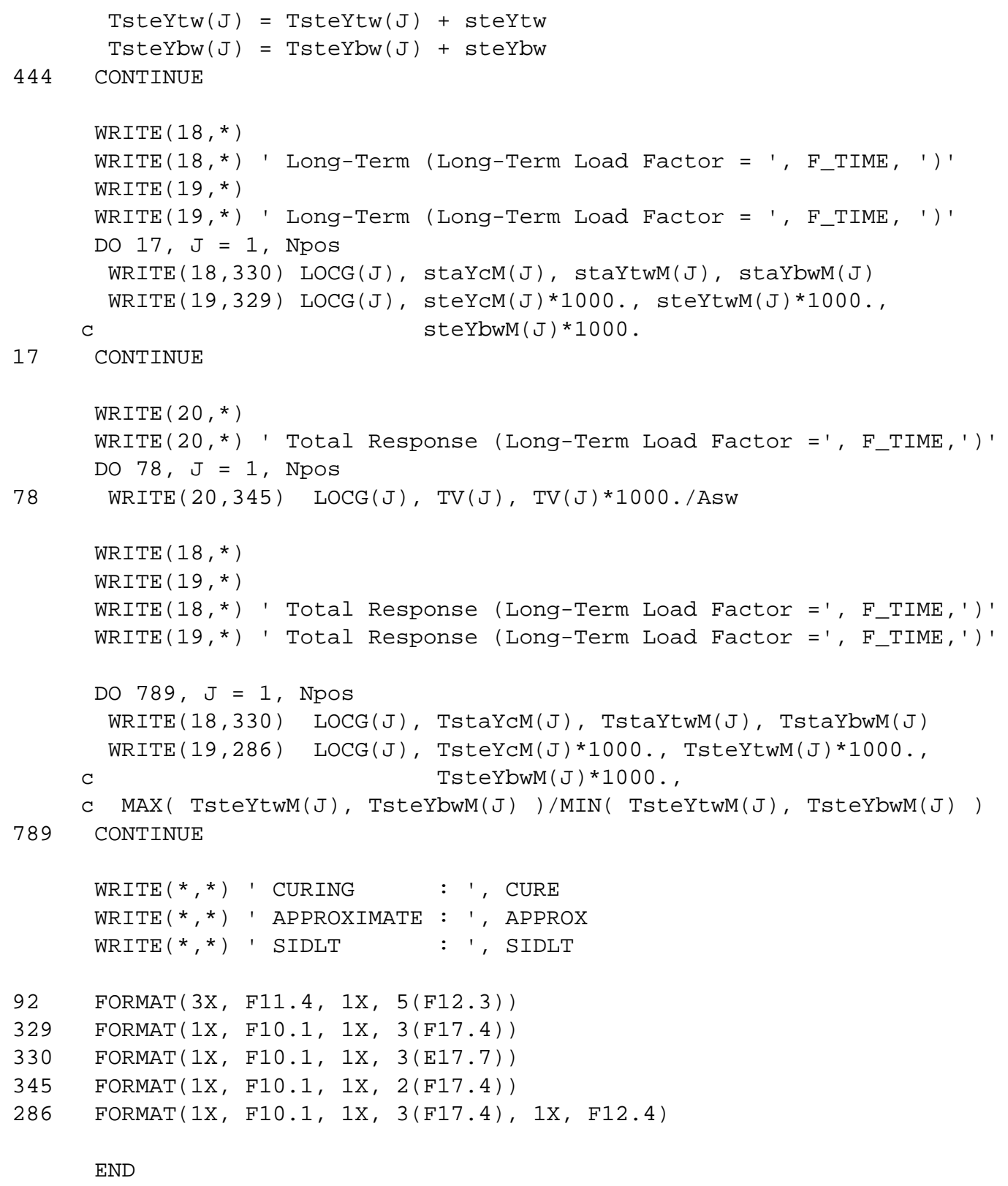




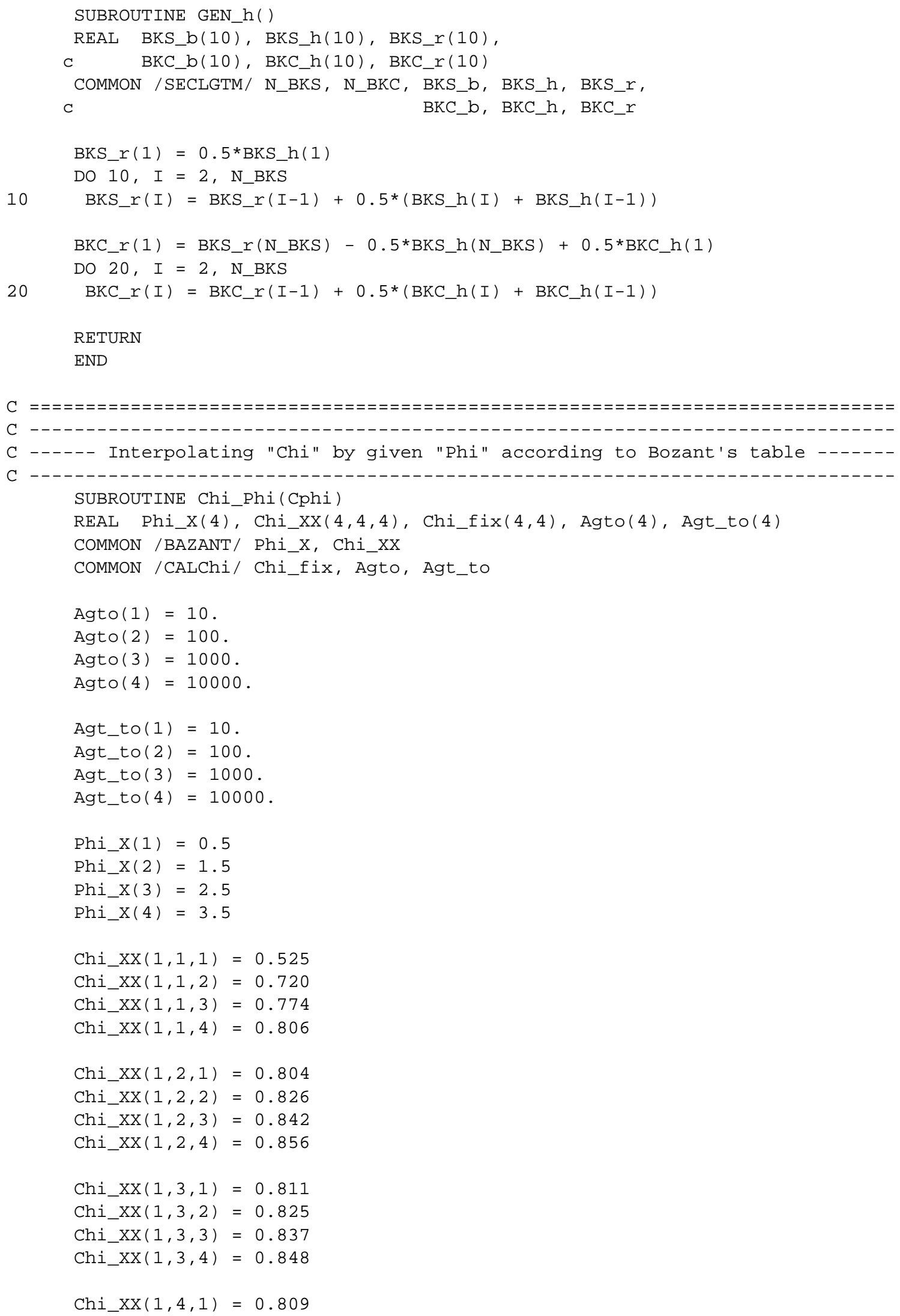




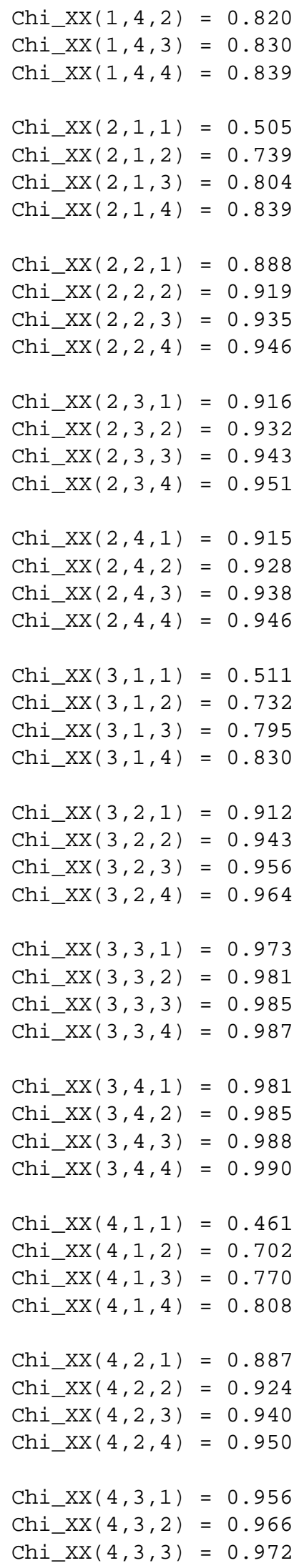




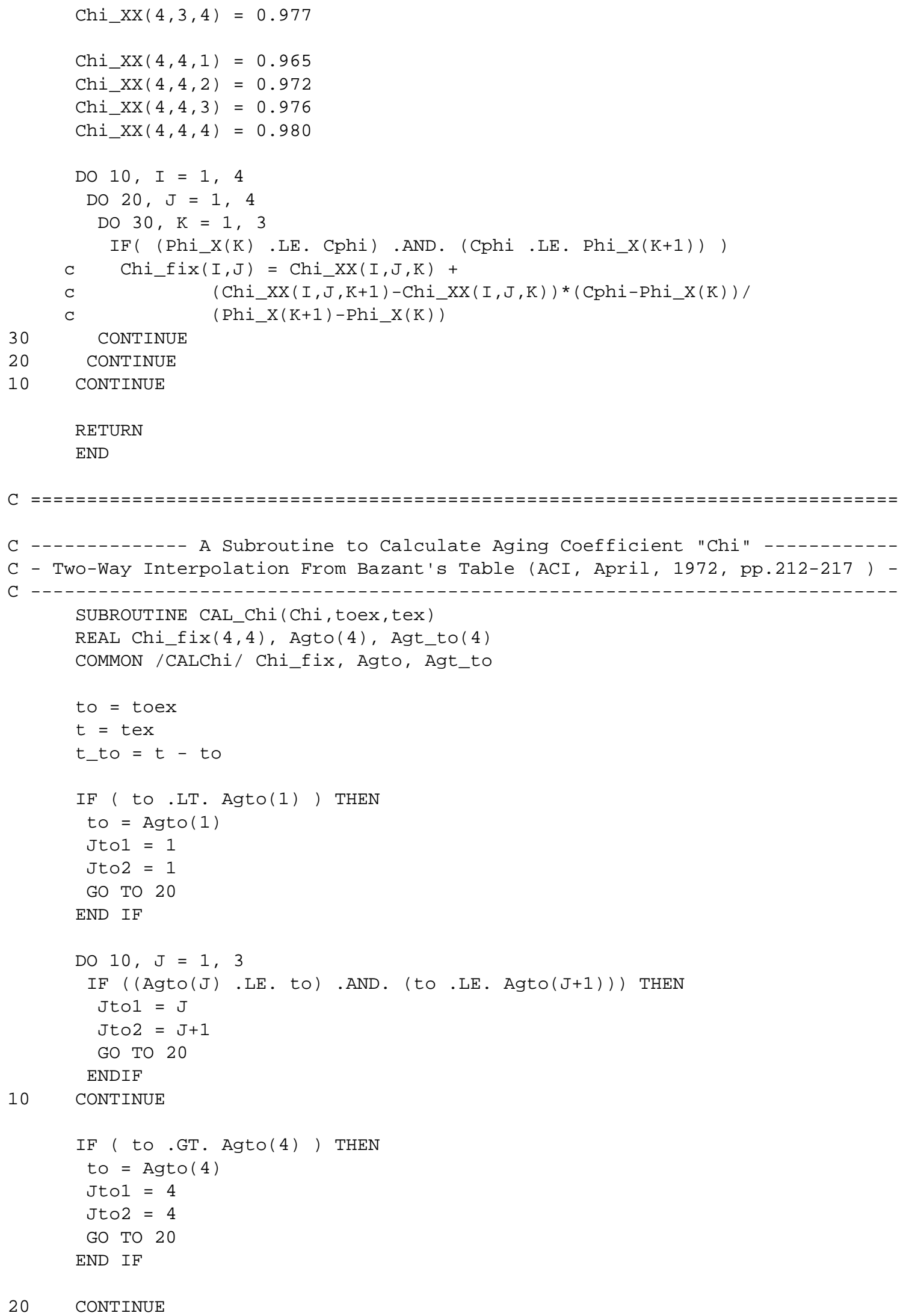




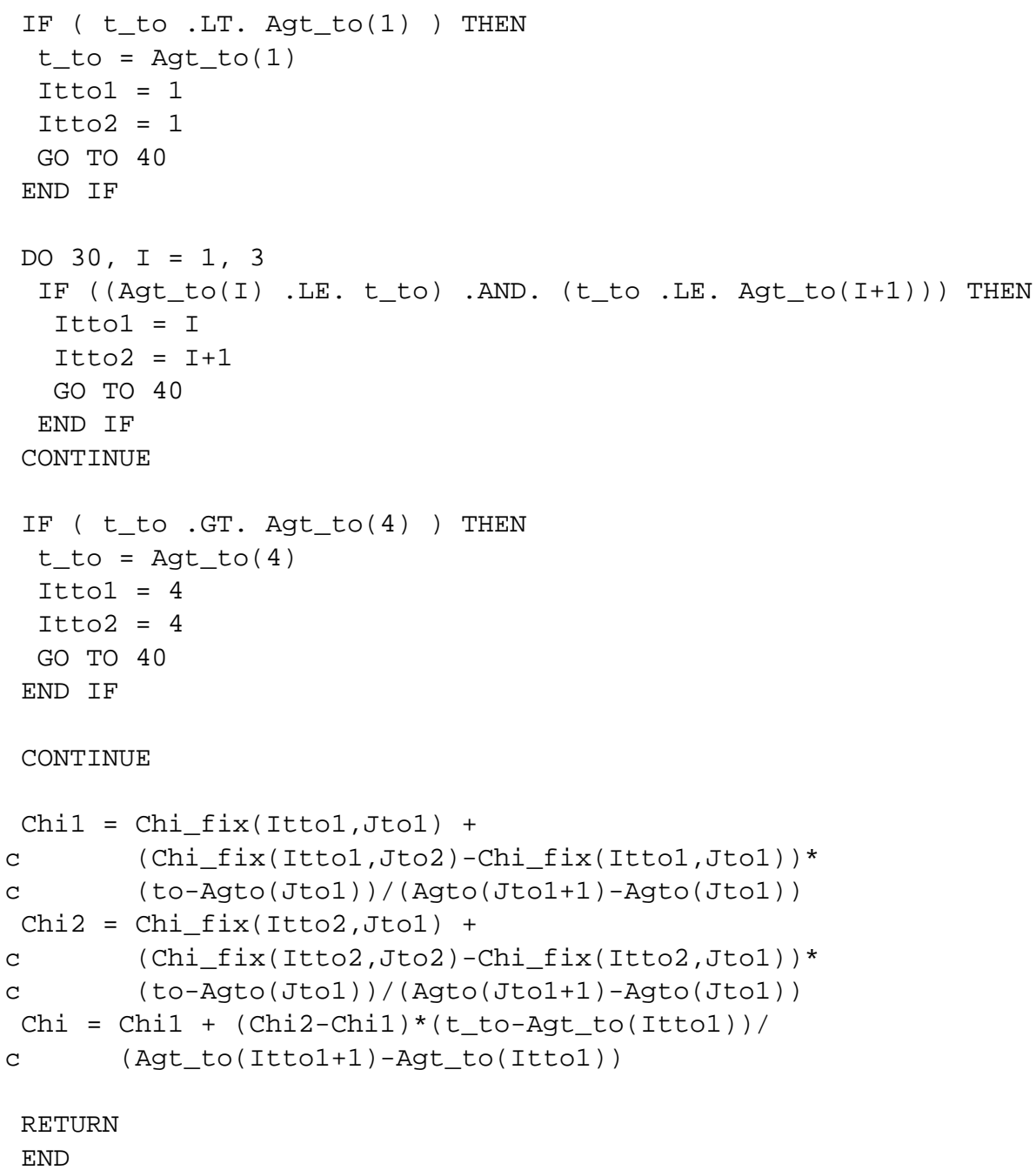


RETURN

END

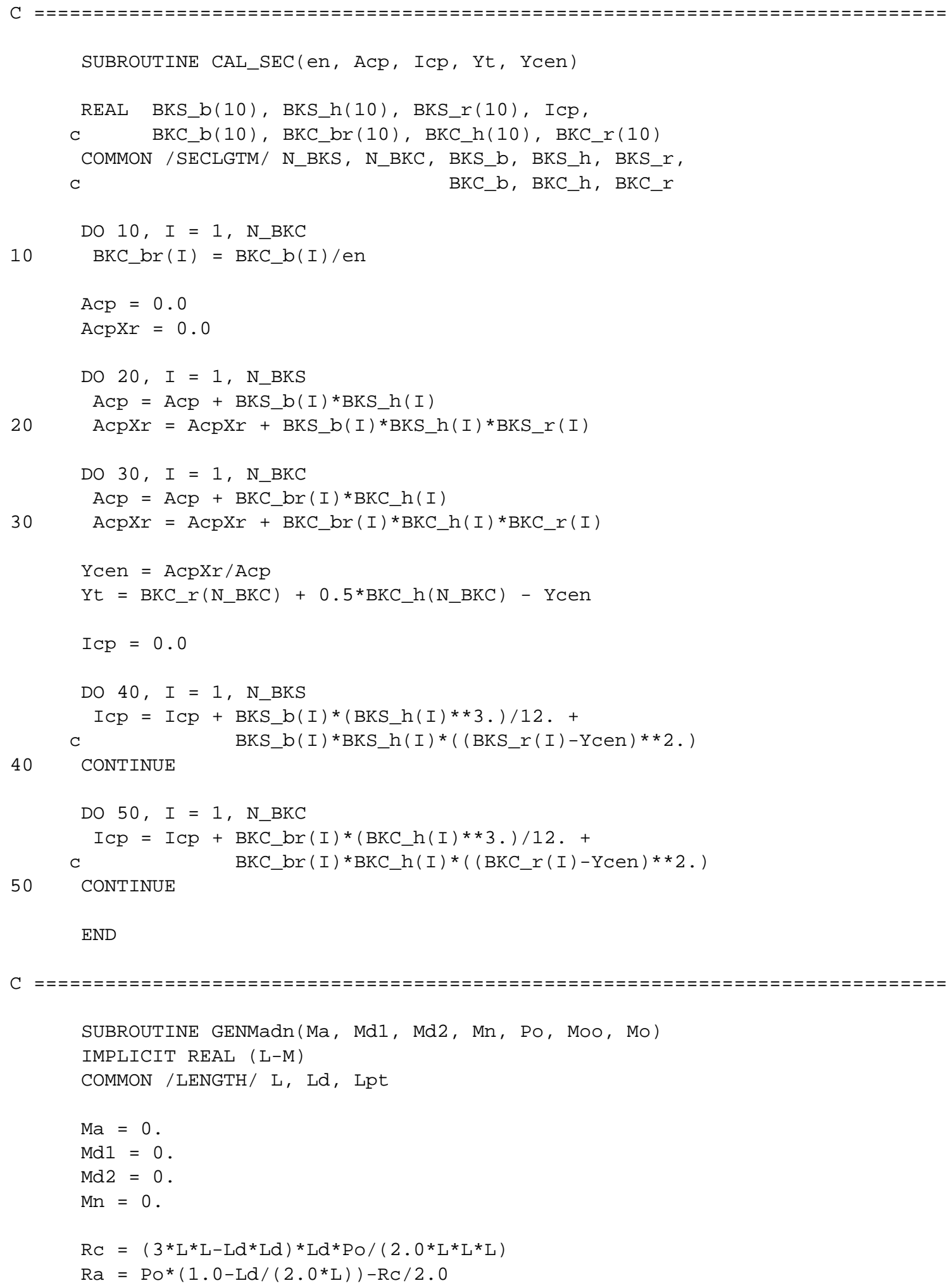




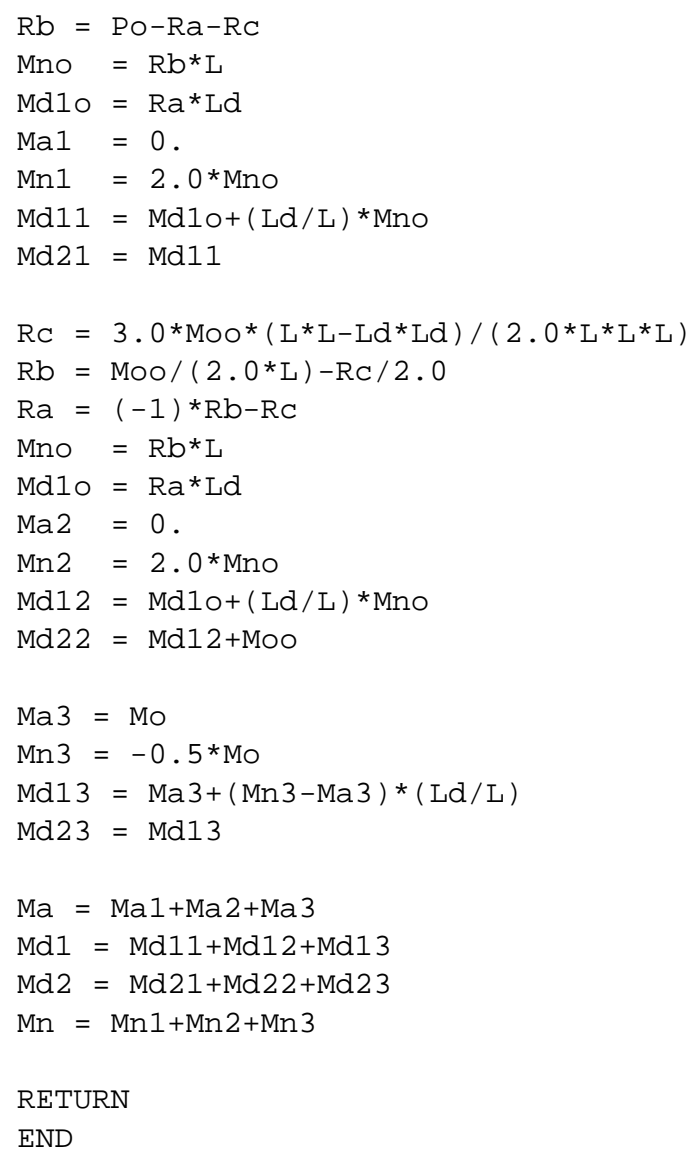




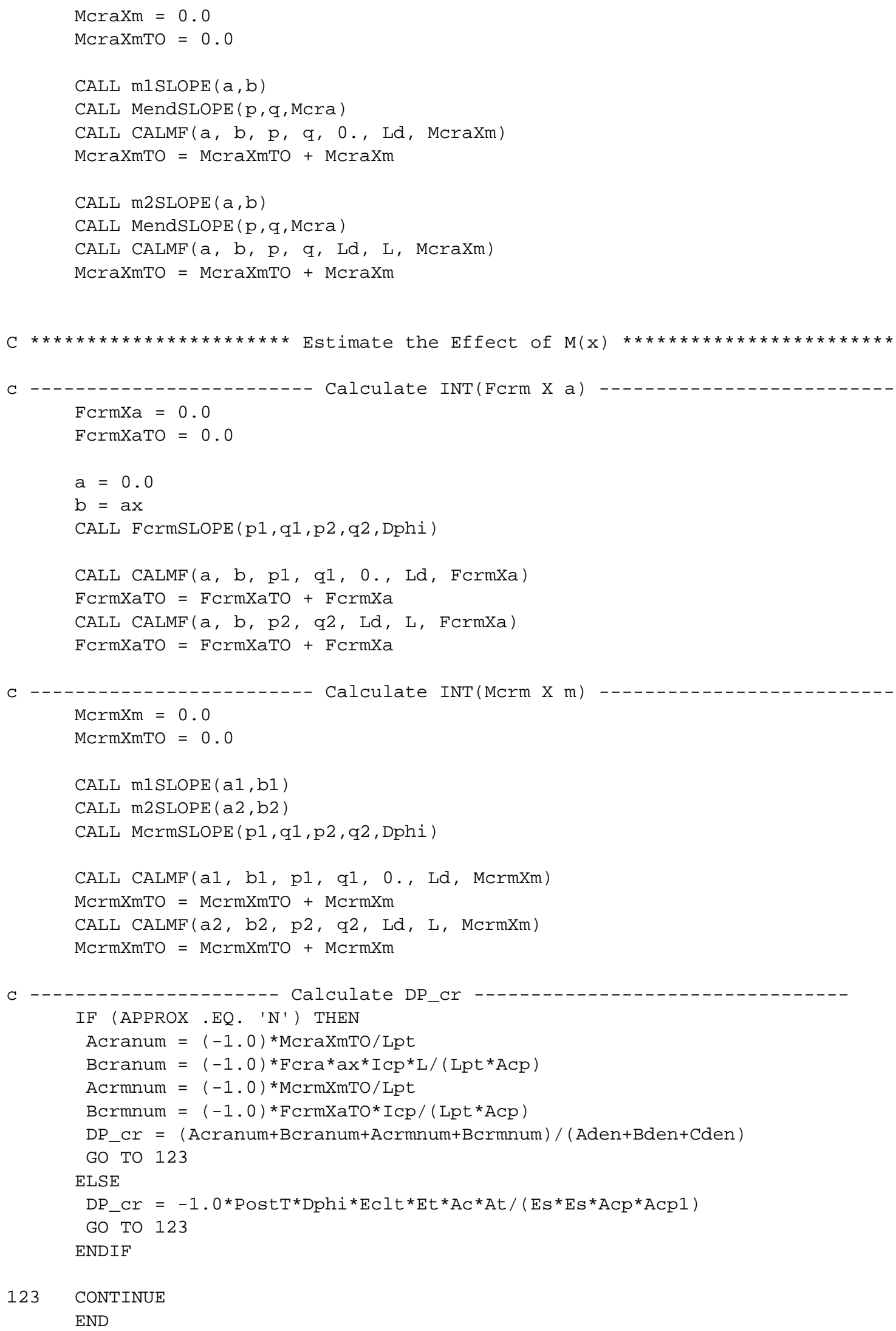




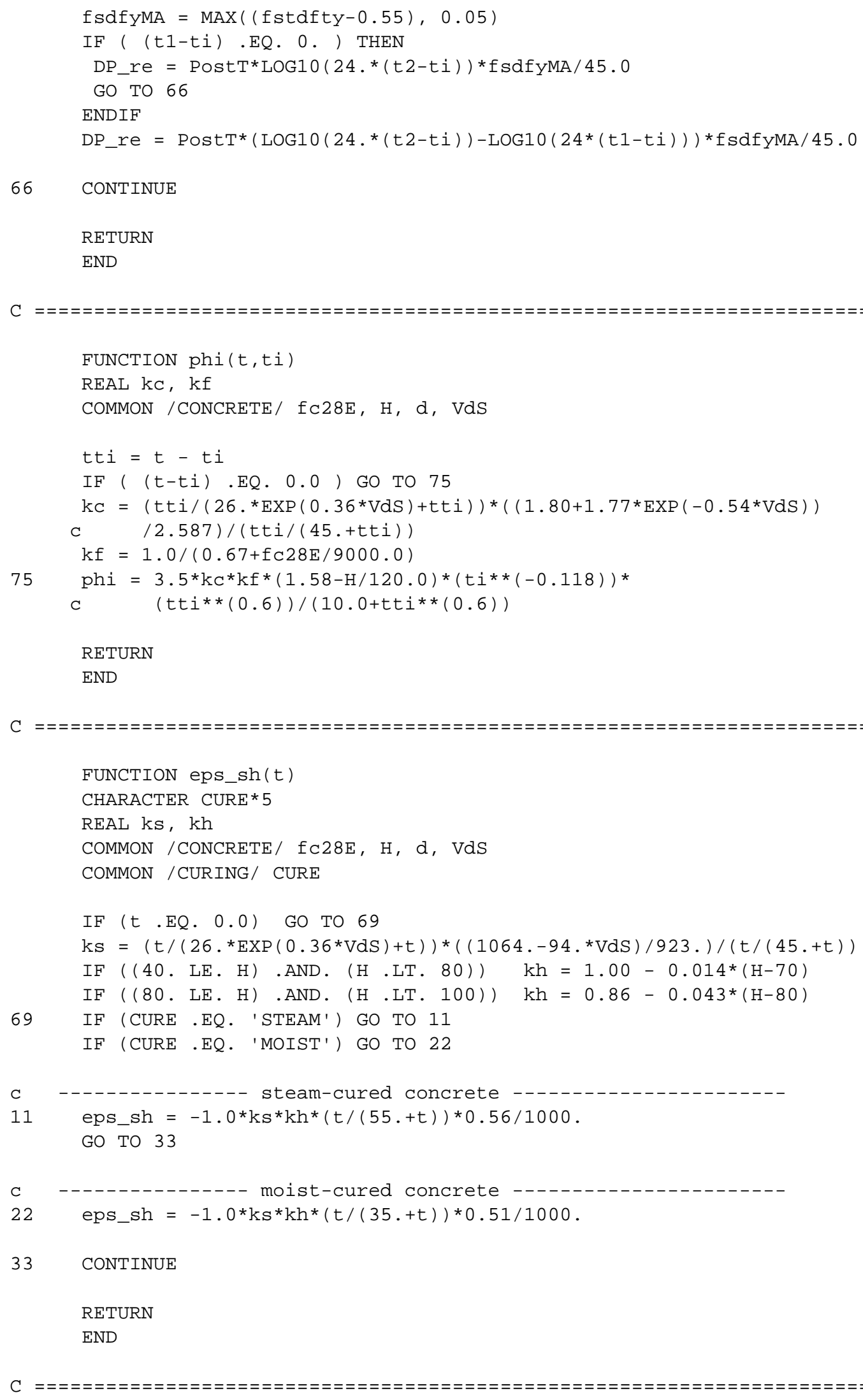


SUBROUTINE CALMF ( $w, \mathrm{x}, \mathrm{y}, \mathrm{z}, \mathrm{L} 1, \mathrm{~L} 2, \mathrm{XXX})$

REAL L1, L2, XXX

$\mathrm{XXX}=0.0$

$\mathrm{XXX}=\mathrm{w}^{*} \mathrm{Y} *(\mathrm{~L} 2 * \star 3-\mathrm{L} 1 * * 3) / 3 .+\left(\mathrm{w}^{\star} \mathrm{z}+\mathrm{x} * \mathrm{y}\right) *(\mathrm{~L} 2 * \mathrm{~L} 2-\mathrm{L} 1 * \mathrm{~L} 1) / 2 .+\mathrm{X}^{*} \mathrm{z} *(\mathrm{~L} 2-\mathrm{L} 1)$

RETURN

END

$\mathrm{C}$

SUBROUTINE m1SLOPE $(a, b)$

IMPLICIT REAL (L-M)

COMMON /LENGTH/ L, Ld, Lpt

COMMON /Uma/ Ma, Md1, Md2, Mn

$\mathrm{a}=(\mathrm{Md} 1-\mathrm{Ma}) / \mathrm{Ld}$

$\mathrm{b}=\mathrm{Ma}$

RETURN

END

SUBROUTINE m2SLOPE $(a, b)$

IMPLICIT REAL (L-M)

COMMON /LENGTH/ L, Ld, Lpt

COMMON /Uma/ Ma, Md1, Md2, Mn

$\mathrm{a}=(\mathrm{Mn}-\mathrm{Md} 2) /(\mathrm{L}-\mathrm{Ld})$

$\mathrm{b}=-1.0 * \mathrm{Ld} *(\mathrm{Mn}-\mathrm{Md} 2) /(\mathrm{L}-\mathrm{Ld})+\mathrm{Md} 2$

RETURN

END

SUBROUTINE FCrmSLOPE (p1, q1, p2, q2, Dphi)

IMPLICIT REAL (L-M)

REAL Icp1, Icp

COMMON /LENGTH/ L, Ld, Lpt

COMMON /CONCRETE/ fC28E, H, d, VdS

COMMON /STEEL/Es, Et, At, Asw

COMMON /COMPOSIT/Acp, Ac, Icp, Yt, Ec, Eclt

COMMON/COMPOSITt1/Acp1, Icp1, Yt1, Ec1

COMMON /POST/ PostT, ti, ftyE

COMMON /UmaO/ Ma0, Md10, Md20, Mn0

COMMON /CALSHCRden/ Aden, Bden, Cden

Fcrma $=-1.0 * \operatorname{PostT} * \operatorname{Ma} 0 * \operatorname{Dphi} *($ Yt 1-0.5*d)*AC*Eclt $/($ Es $*$ Icp 1$)$

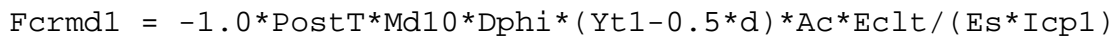

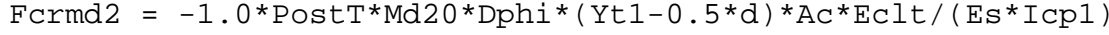

Fcrmn $=-1.0 *$ Post $T * M n 0 * D p h i *($ Yt $1-0.5 * d) * A C * E c l t /(E s * I c p 1)$

WRITE (5,' (1X, 4(F11.2), 2X,A34)') FCrma, Fcrmd1, Fcrmd2, Fcrmn,

$\mathrm{C}$

'// Fcrma Fcrmd1 Fcrmd2 Fcrmn //'

$\mathrm{p} 1=($ F crmd1-FCrma $) / \mathrm{Ld}$

$q 1=$ Fcrma

$\mathrm{p} 2=(\mathrm{Fcrmn}-\mathrm{Fcrmd} 2) /(\mathrm{L}-\mathrm{Ld})$ 
$\mathrm{q} 2=-1 \cdot 0 * L d *(F \mathrm{crmn}-\mathrm{F} \mathrm{crmd} 2) /(\mathrm{L}-\mathrm{Ld})+\mathrm{F} \mathrm{crmd} 2$

RETURN

END

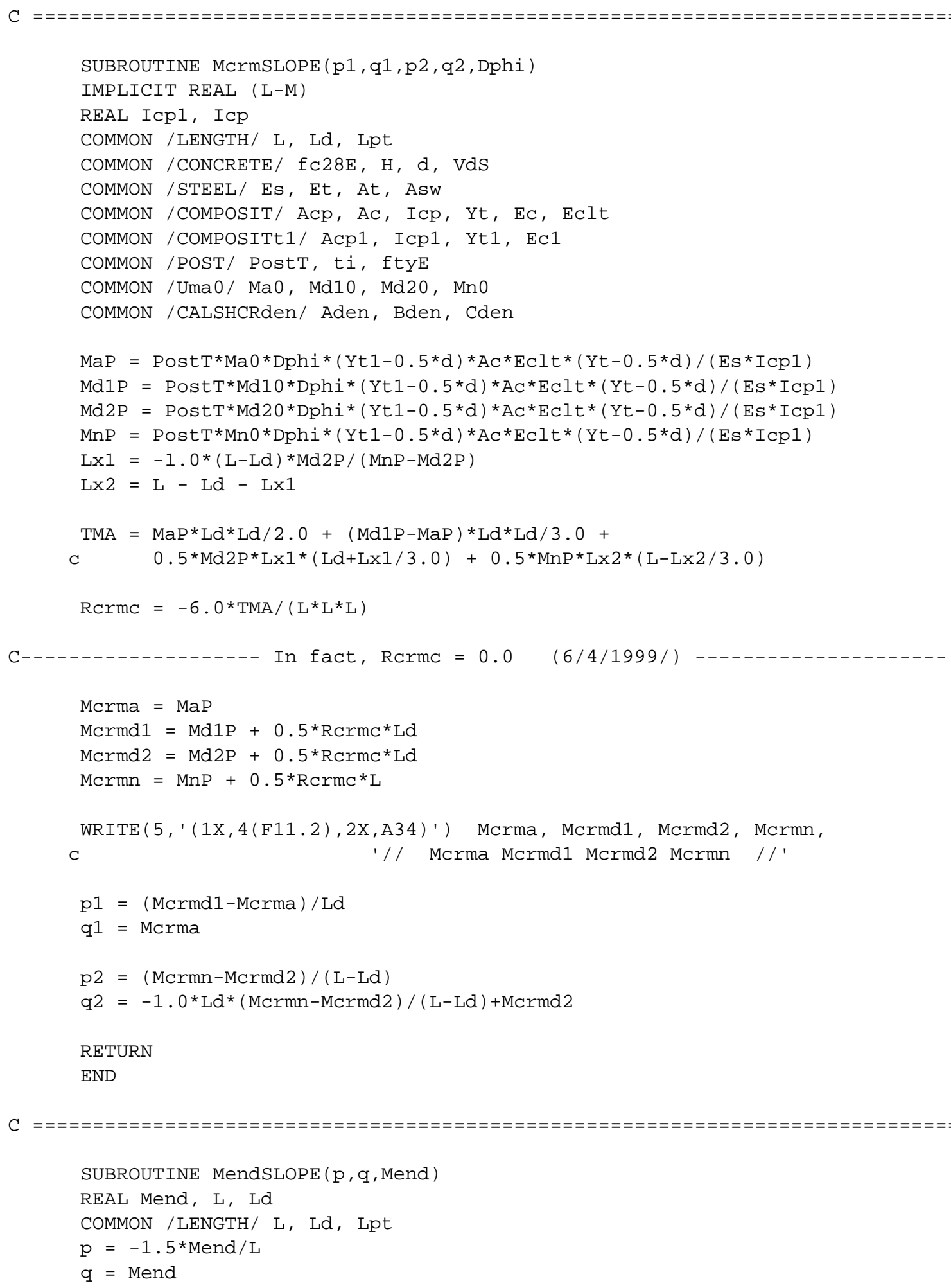


RETURN

END

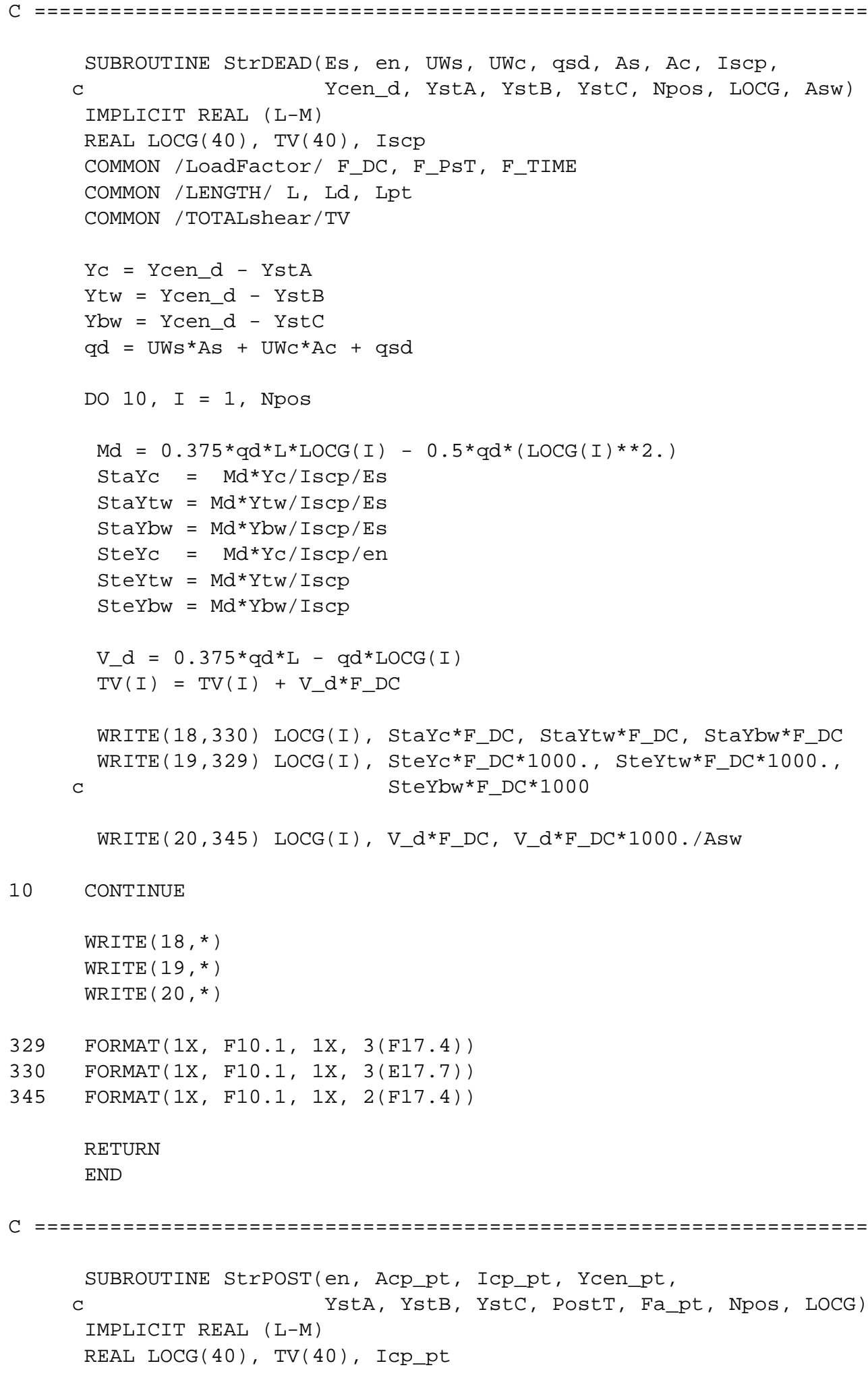




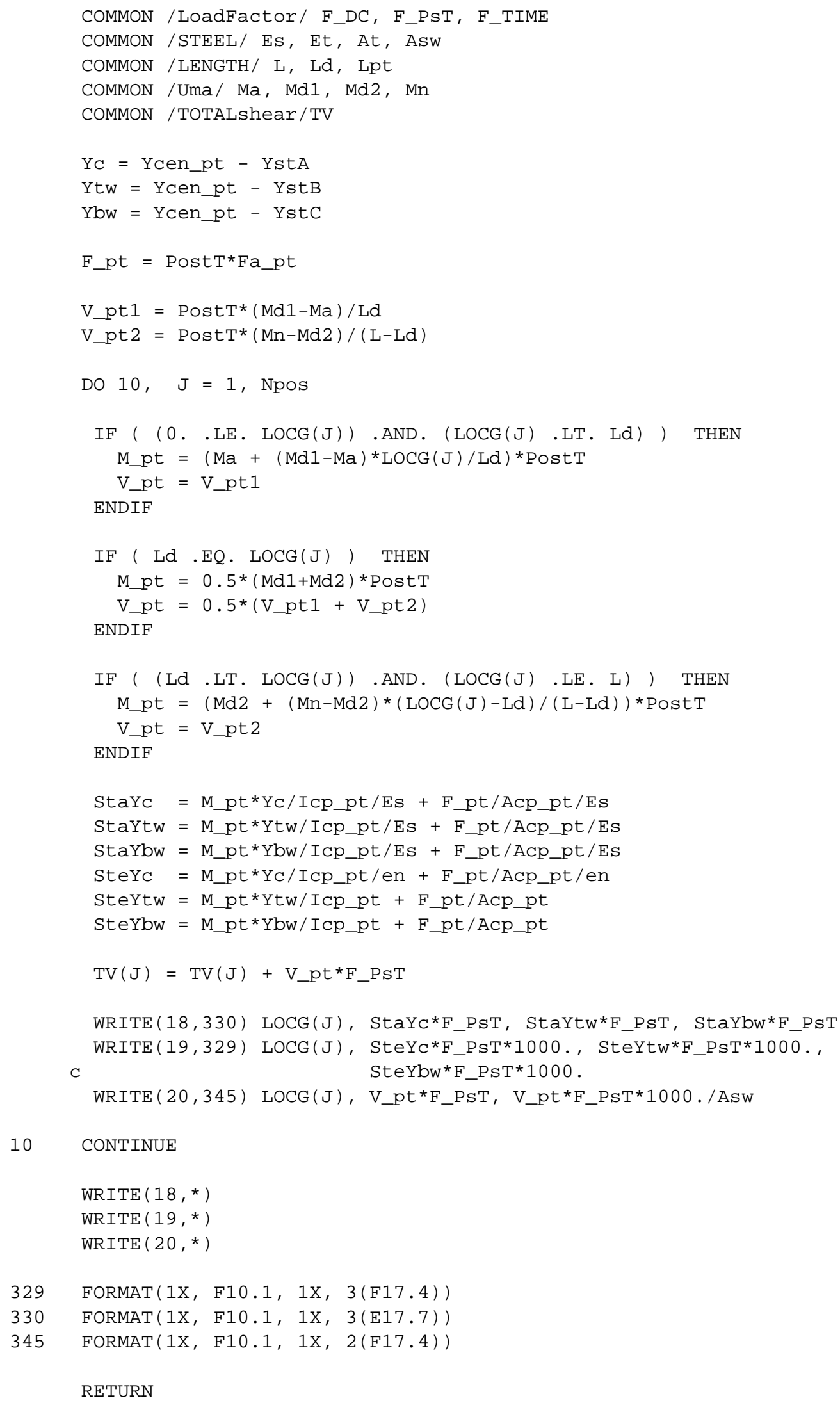


END

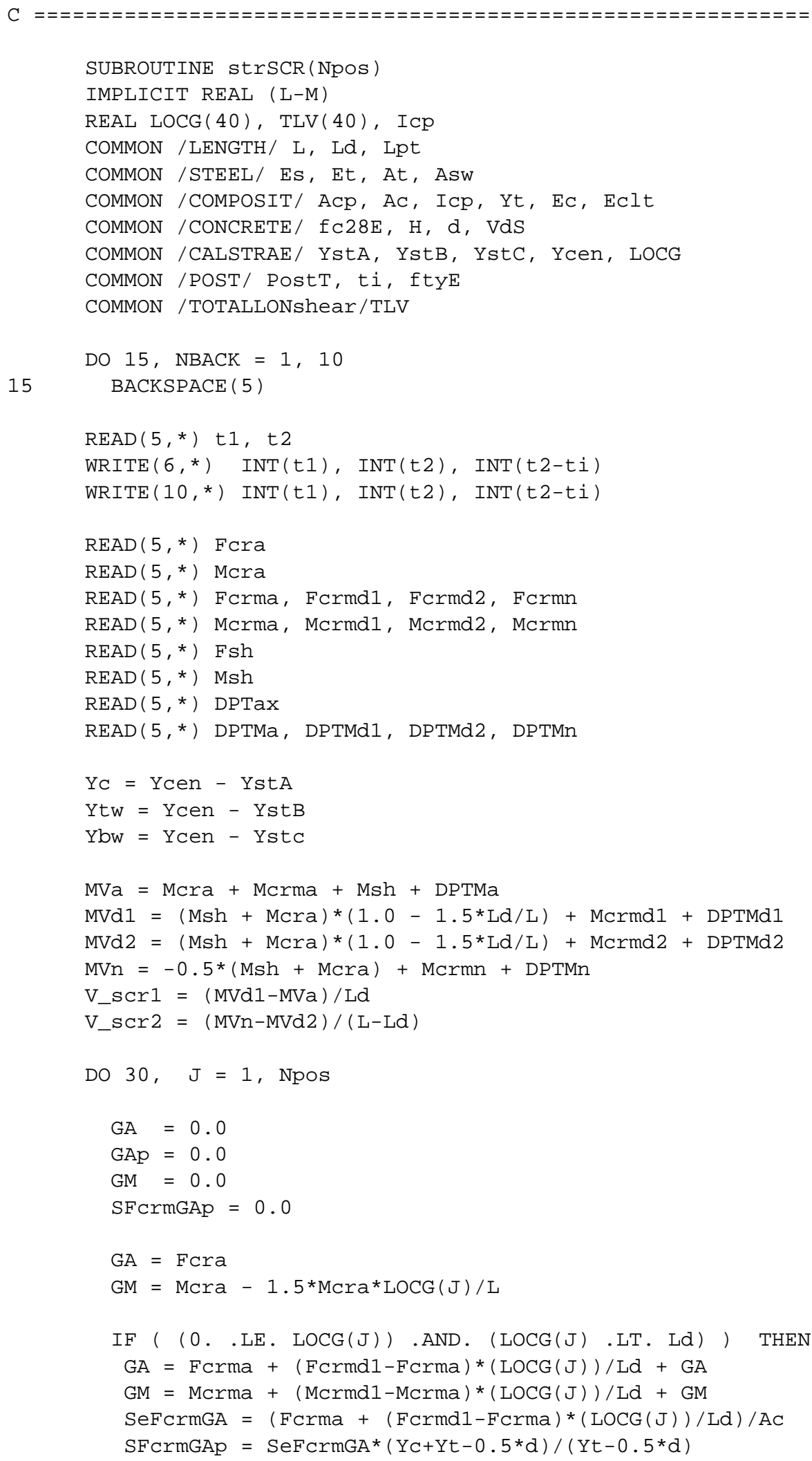




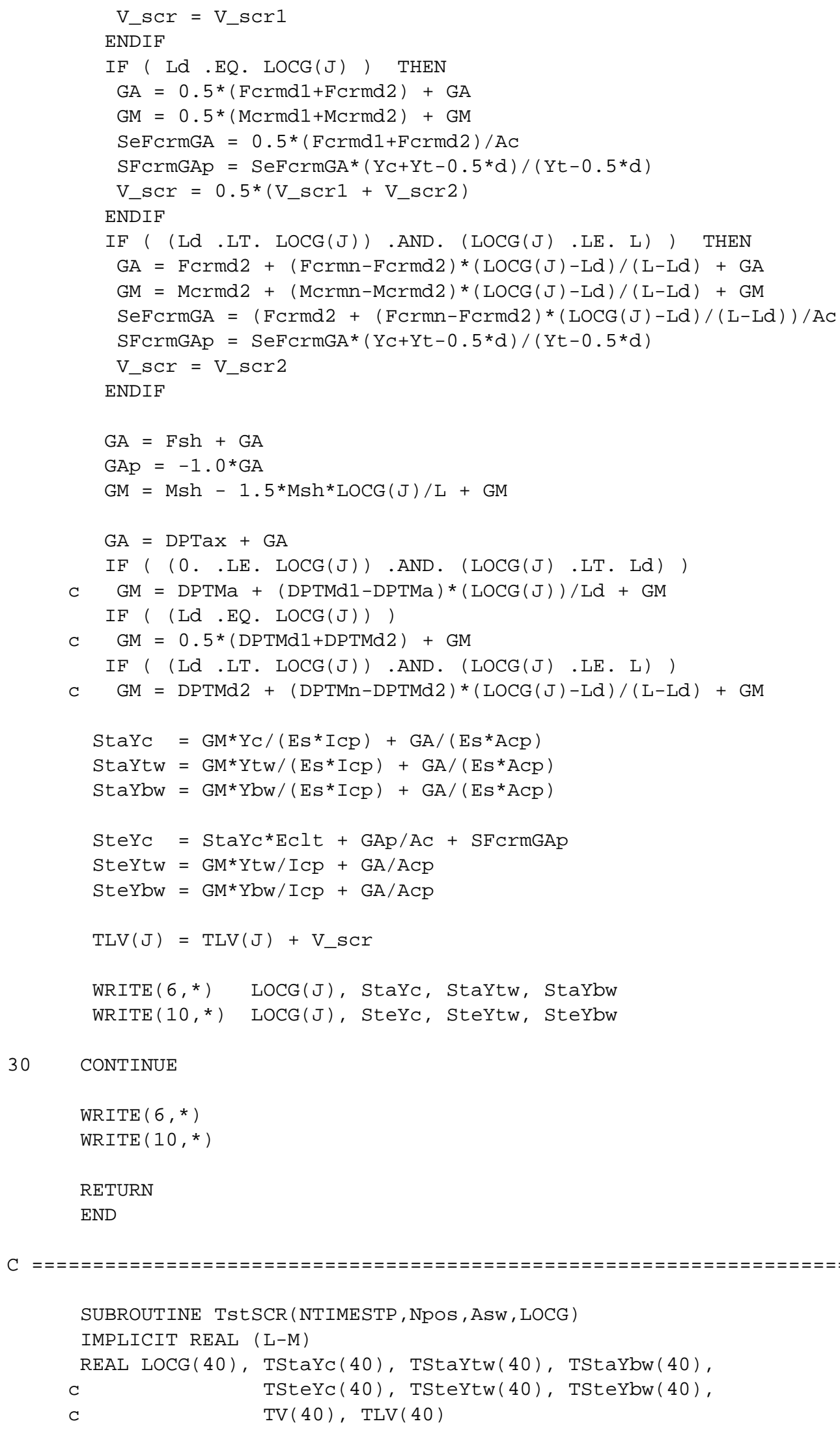




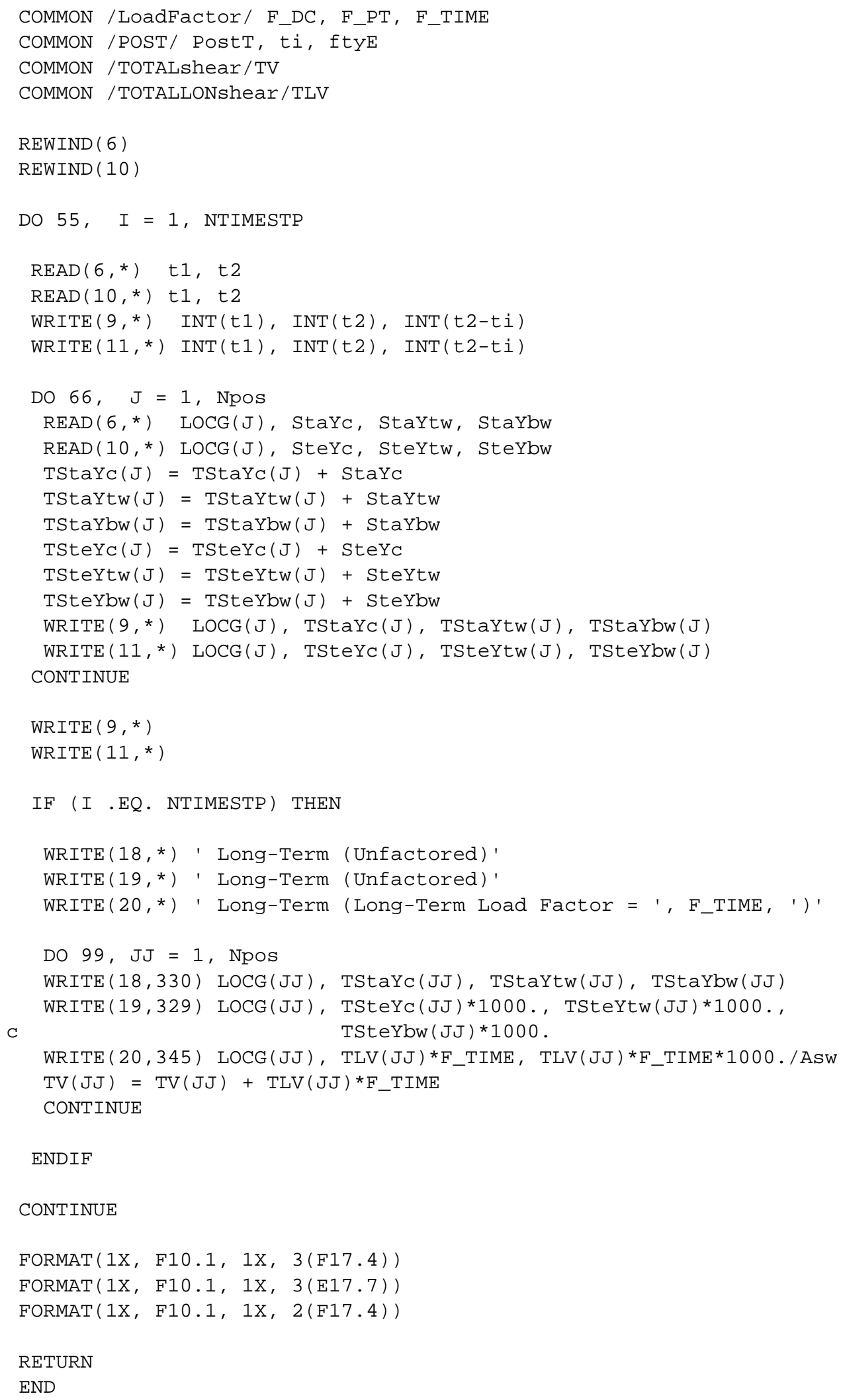




\section{Table B.2 Example of input file POSTSTEL.INP}

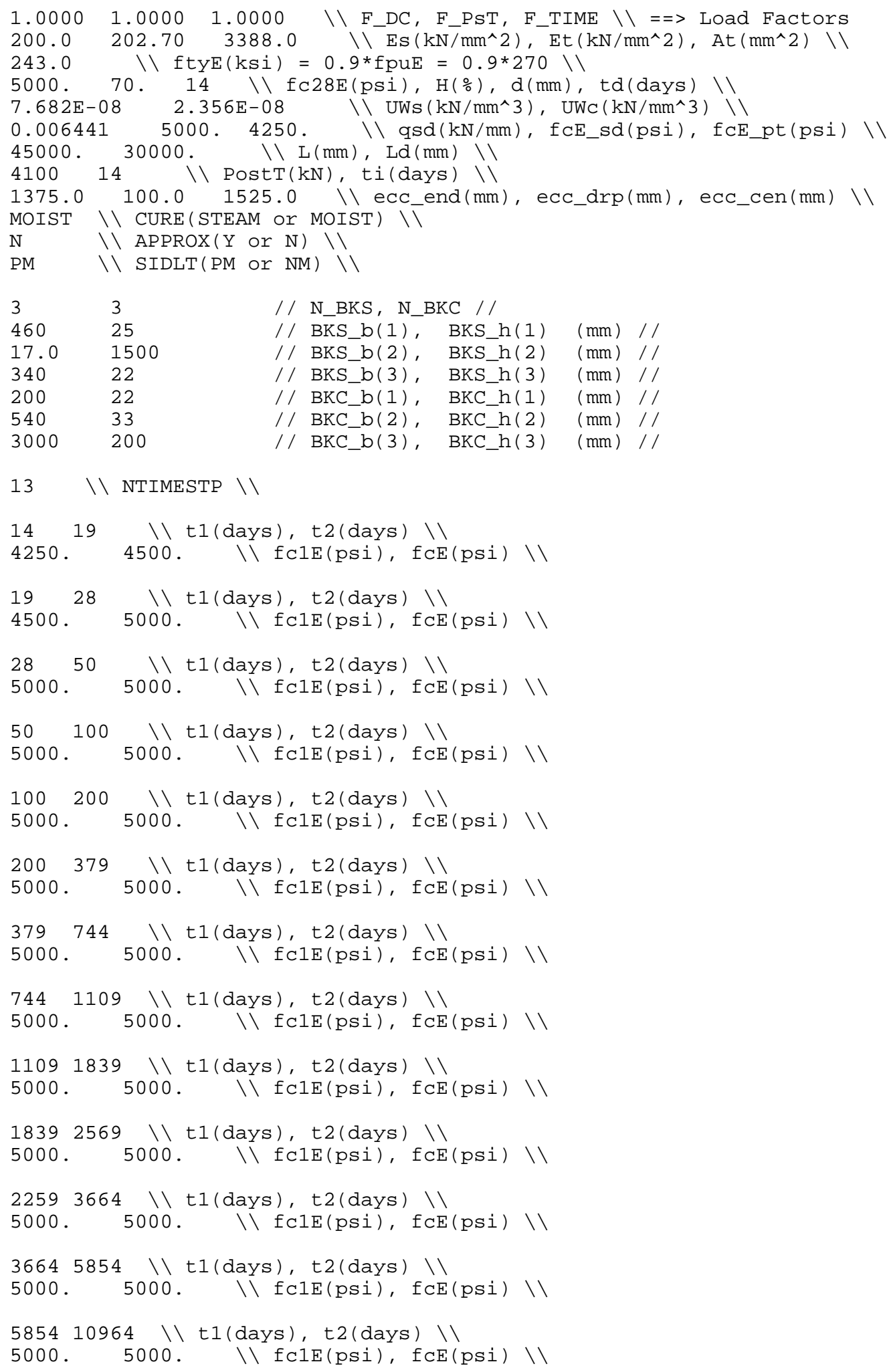




\section{Table B.3 Example of input file GAGELOPS.INP}

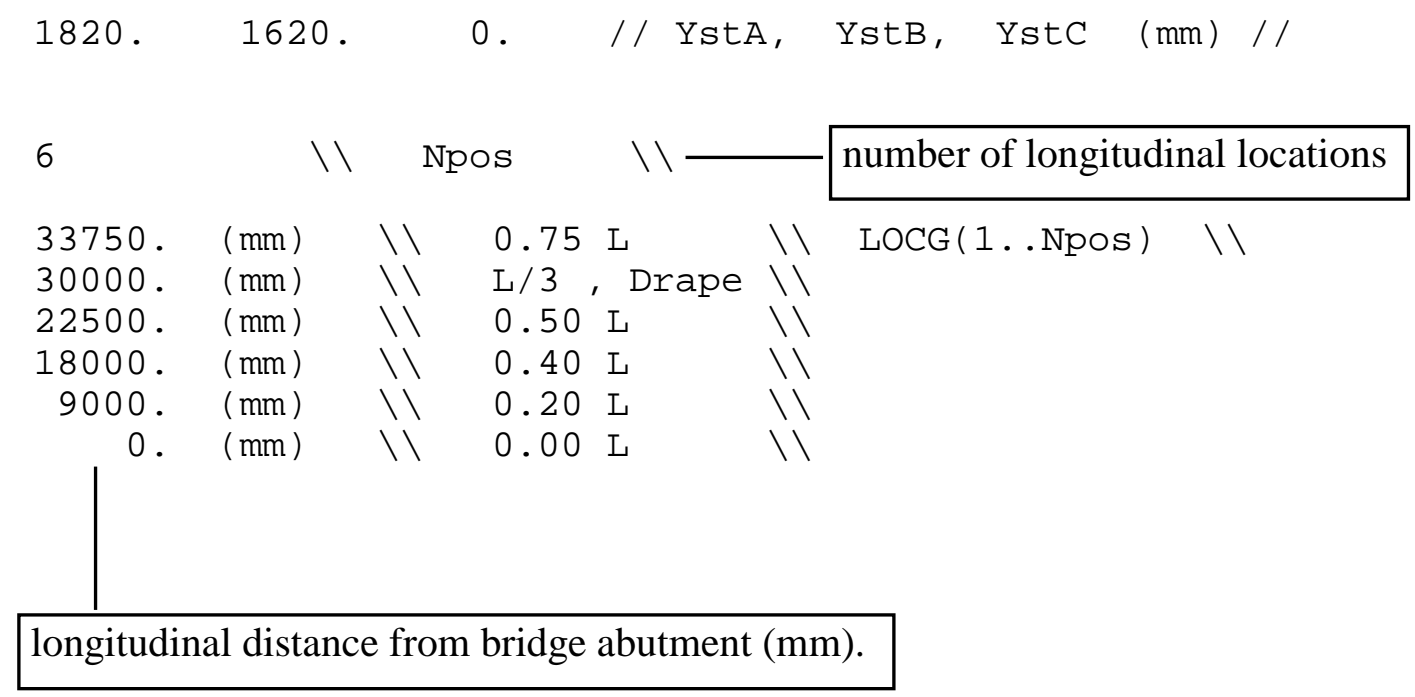

Note:

YstA : vertical position in concrete deck $(\mathrm{mm})$

YstB : vertical position (1) in steel girder (mm)

YstC : vertical position (2) in steel girder (mm)

YstA, YstB and YstC are all measured from the bottom of the girder. 


\section{Table B.4 Example of output file SHCRRE.OUT}

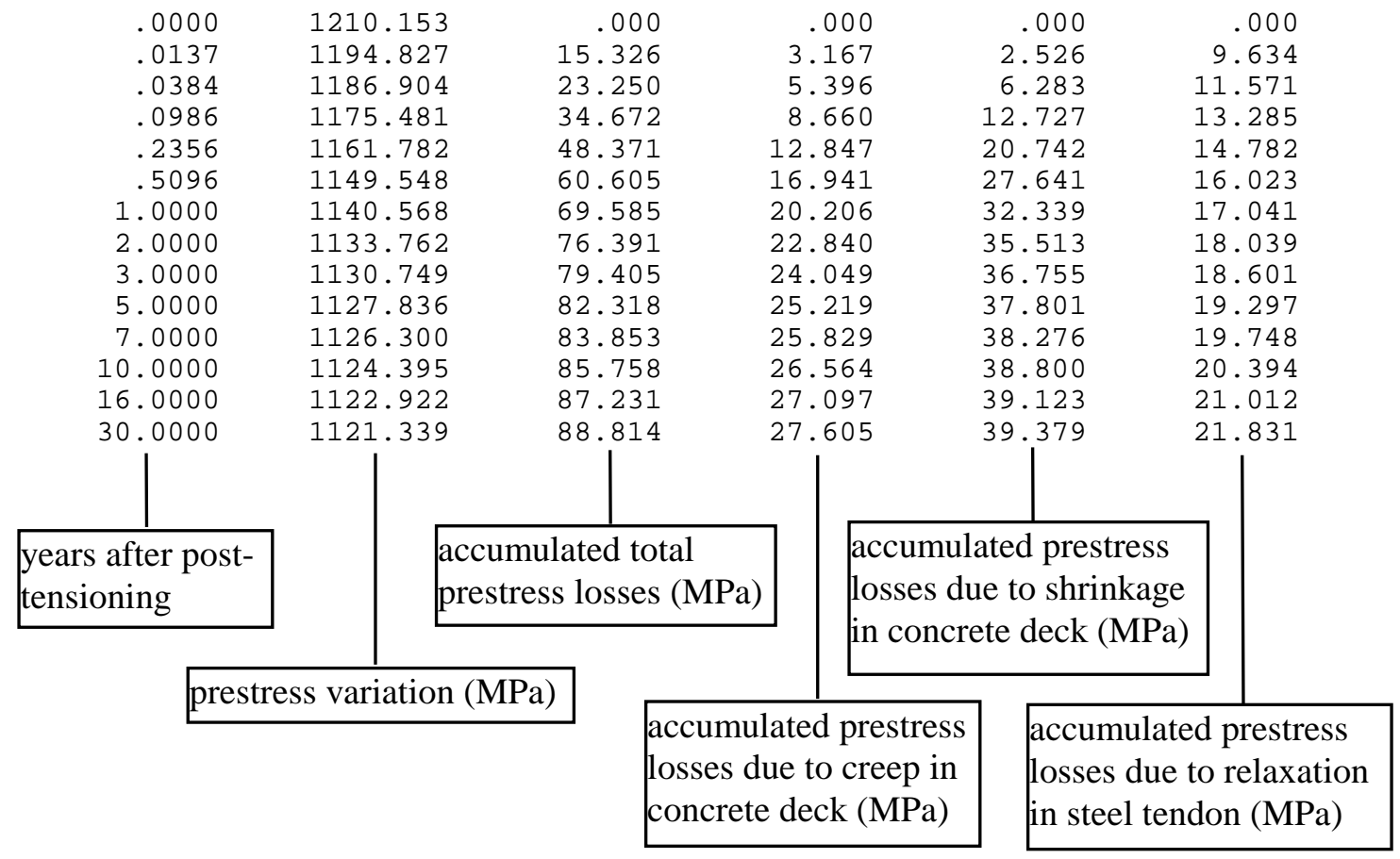




\section{Table B.5 Example of output file STRESUM.OUT}

$\begin{array}{rrrr}\text { Dead Load: Weight of Steel Girder \& Concrete Deck } & \\ 33750.0 & .0000 & .0000 & .0000 \\ 30000.0 & .0000 & -59.6332 & 46.0433 \\ 22500.0 & .0000 & -134.1746 & 103.5974 \\ 18000.0 & .0000 & -150.2756 & 116.0291 \\ 9000.0 & .0000 & -118.0737 & 91.1657 \\ .0 & .0000 & .0000 & .0000\end{array}$

Superimposed Dead Load: Weight of Barriers, Wearing Surface, etc.

$\begin{array}{rrrr}33750.0 & .0000 & .0000 & .0000 \\ 30000.0 & -.5479 & -2.2788 & 11.2037 \\ 22500.0 & -1.2328 & -5.1272 & 25.2083 \\ 18000.0 & -1.3807 & -5.7425 & 28.2333 \\ 9000.0 & -1.0849 & -4.5120 & 22.1833 \\ .0 & .0000 & .0000 & .0000\end{array}$

Post-Tensioning

$\begin{array}{rrrr}33750.0 & -3.1650 & -28.0737 & -55.3466 \\ 30000.0 & -.5434 & -15.9331 & -110.6289 \\ 22500.0 & -1.5245 & -20.4763 & -89.9413 \\ 18000.0 & -2.1211 & -23.2392 & -77.3606 \\ 9000.0 & -3.3142 & -28.7649 & -52.1992 \\ .0 & -4.5074 & -34.2907 & -27.0378\end{array}$

Long-Term (Unfactored)

$\begin{array}{rrrr}33750.0 & 5.6640 & -60.6281 & -83.9172\end{array}$

$\begin{array}{llll}30000.0 & 4.0585 & -47.6020 & -71.9891\end{array}$

$\begin{array}{llll}22500.0 & 3.3764 & -61.0041 & -46.3613\end{array}$

$\begin{array}{llll}18000.0 & 2.9703 & -69.0960 & -30.9817\end{array}$

$\begin{array}{rrrr}9000.0 & 2.1581 & -85.2799 & -.2225\end{array}$

$\begin{array}{llll}.0 & 1.3459 & -101.4638 & 30.5367\end{array}$

Long-Term (Long-Term Load Factor $=$

$\begin{array}{llll}33750.0 & 5.6640 & -60.6281 & -83.9172\end{array}$

$\begin{array}{llll}30000.0 & 4.0585 & -47.6020 & -71.9891\end{array}$

$\begin{array}{llll}22500.0 & 3.3764 & -61.0041 & -46.3613\end{array}$

$\begin{array}{llll}18000.0 & 2.9703 & -69.0960 & -30.9817\end{array}$

$\begin{array}{rrrr}9000.0 & 2.1581 & -85.2799 & -.2225\end{array}$

$\begin{array}{llll}.0 & 1.3459 & -101.4638 & 30.5367\end{array}$

Total Response (Long-Term Load Factor $=1.000000$ )

$\begin{array}{rrrrr}33750.0 & 2.4990 & -88.7018 & -139.2638 & .6369 \\ 30000.0 & 2.9672 & -125.4471 & -125.3710 & .9994 \\ 22500.0 & .6191 & -220.7822 & -7.4969 & .0340 \\ 18000.0 & -.5315 & -248.3533 & 35.9201 & -.1446 \\ 9000.0 & -2.2410 & -236.6305 & 60.9273 & -.2575 \\ .0 & -3.1615 & -135.7545 & 3.4989 & -.0258\end{array}$

\begin{tabular}{|l|l|l|l|}
\hline $\begin{array}{l}\text { distance from } \\
\text { the bridge } \\
\text { abutment }(\mathrm{mm})\end{array}$ & $\begin{array}{l}\text { stress response in } \\
\text { the concrete deck } \\
\text { at position YstA } \\
(\mathrm{MPa})\end{array}$ & $\begin{array}{l}\text { stress response in } \\
\text { the steel girder at } \\
\text { position YstB } \\
(\mathrm{MPa})\end{array}$ \\
\hline
\end{tabular}

stress response in the steel girder at position YstC (MPa) column 3/column 4 or column 4/column 3 


\section{Table B.6 Example of output file SHEARSUM.OUT}

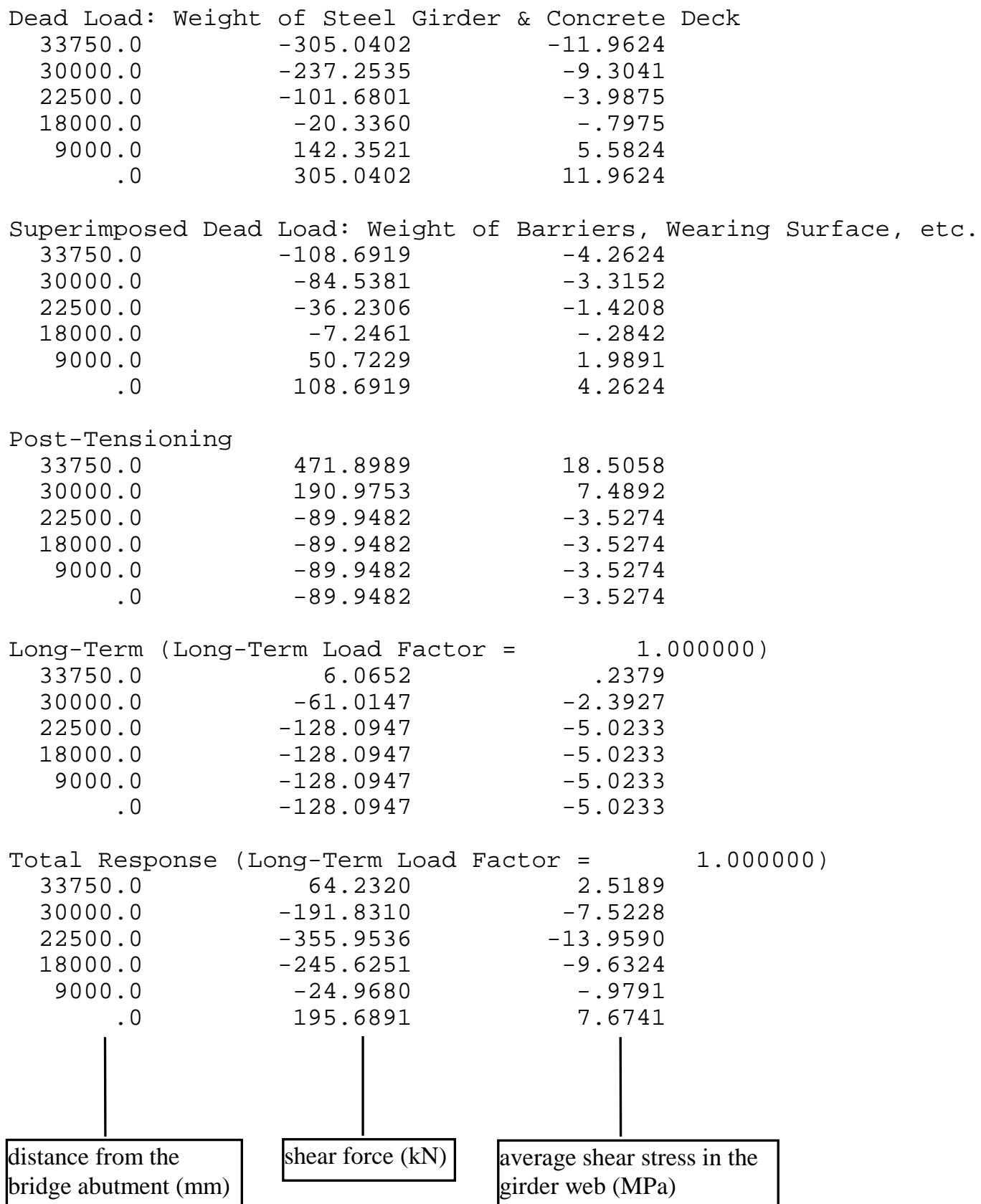

shear force $(\mathrm{kN})$

average shear stress in the girder web $(\mathrm{MPa})$ 


\section{APPENDIX C ORIGINAL COST DATA}

The cost comparison presented in Chapter 6 is based on the data provided by the Toll Road Division of Indiana Department of Transportation. The original cost data for the conventional $(\mathrm{CV})$ and post-tensioned $(\mathrm{PT})$ bridge are illustrated in Tables B.1 and B.2, respectively. 
Table C.1 Original cost data for construction items of bridge (CV)

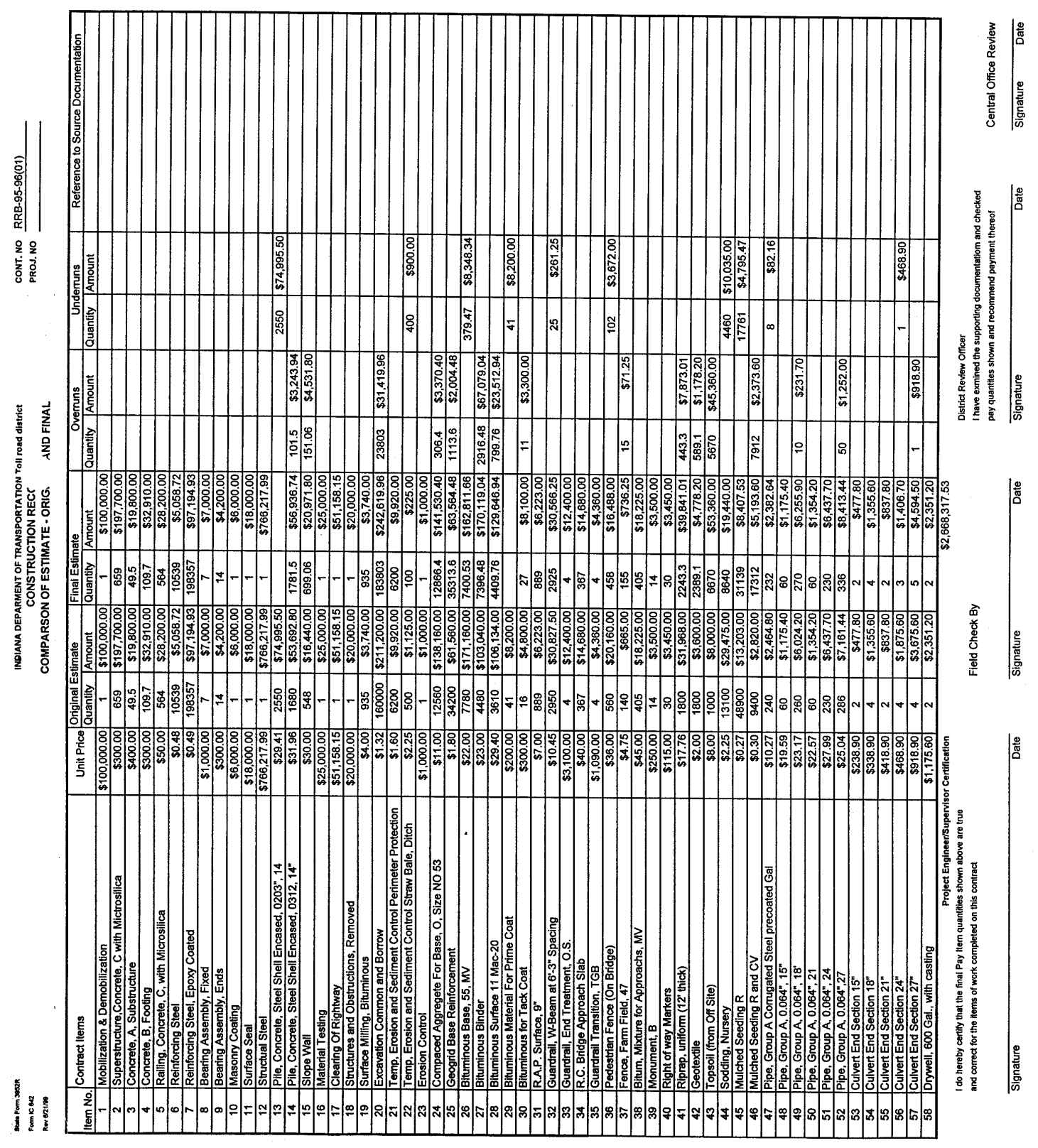




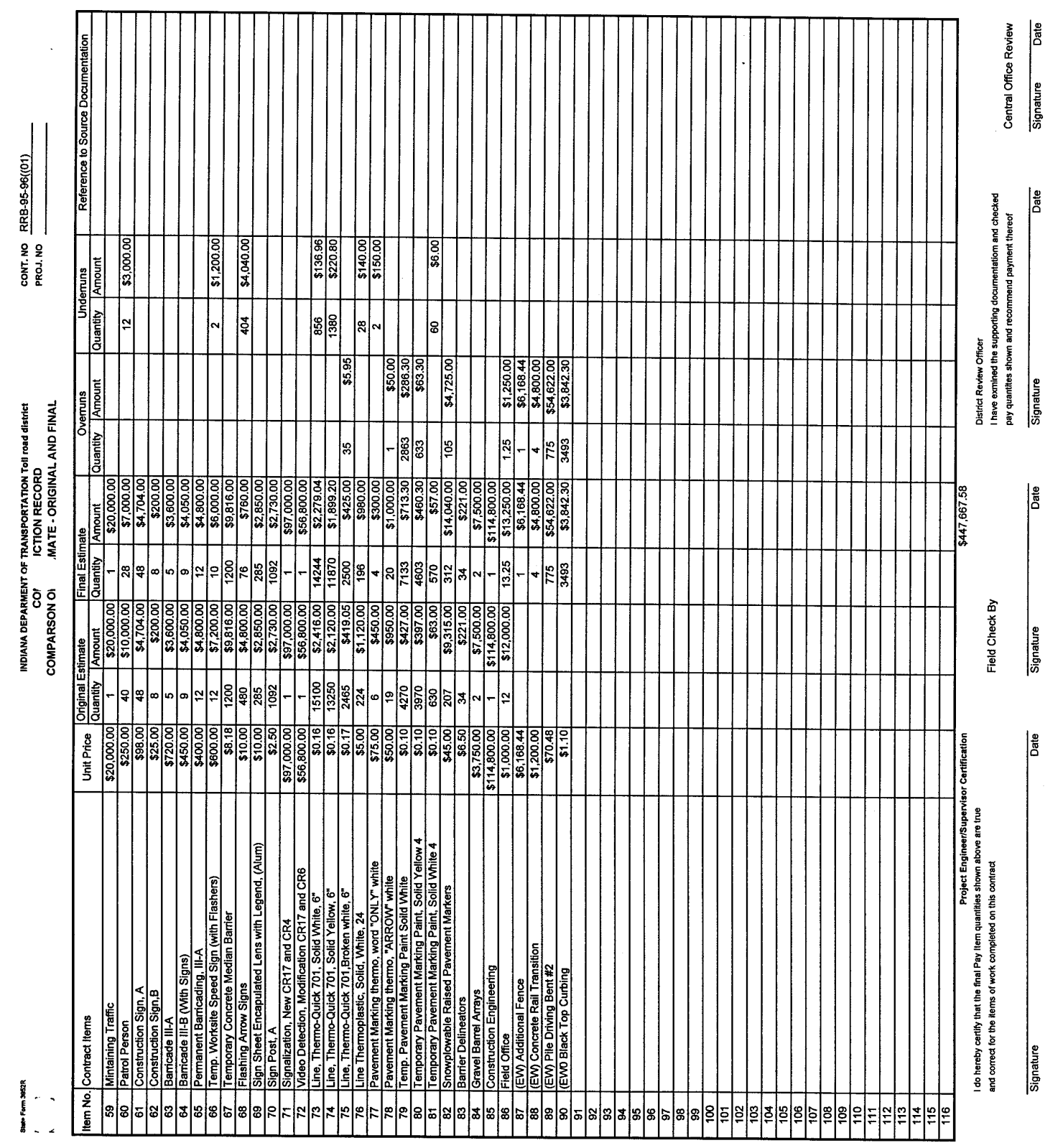




\section{OVERRUN/UNDERRUN EXPLANATIONS}

\#13 PULE, CONCRETE, STEEL SHELL ENCASEI $0.203^{\prime \prime}, 14$ '

This Pay Item was not used. In the original proposal it was estimated that 2,550 feet of piling would be needed for bent \# 2 . Bearing was reached at a average of 15 feet. Only using 30 percent of quantity of Pay Item \#13. This causing a underrun for this Pay Item.

\#14 PILE, CONCRETE, STEEL SHELL ENCASED, 0.312, 14"

To receive bearing of 70 ton. The piling had to be driven further than pianned depth. Causing a overrun of this Pay Item.

\#15 SLOPE WALLL

State Standard MB2 slopewall it shows that a toe to be constructed at each end of the slope wall and a curb to be constructed on sides. The Plan Quantity was only for the square yards of actual slope wall. Causing a overrun of this Pay Item.

\#20 EXCAVATION COMMON AND BORRON

After final cuts and fill was determined it was found the original quantity was low. Causing a overrrun of this Pay Item.

\#21 TEMP EROSION CONTROL PERIMETER

All the quantity of this Item was not used @ plans. Causing a underrun of this Pay Item.

\#22 TEMP EROSION CONTROL STRAW

Same as Pay I tem $\# 21$

\#24 Compacted aggregate for base type "O" Type \#5.3.

Due to design of project more 53 material was needed than original plan quantity. Causing a overrun of this Pay Item.

\#25 Geogrid Base Reinforcement

(e Sam Wolfe/Kevin $0^{\prime}$ Connor had the Geogrid extended over into shoulder area through out the project. Causing a Overrun of This Pay Item.

\#26 Bituminous Base Less Than Plan Quantity was used. Causing a underrun of this Pay Item.

$\$ 27$ Bituminous Binder

Additional Material was used at intersections of CR. 4 and CR.17, Also at the intersection of CR. 104 and CR.17. to maintain proper elevation. Causing a overrun of this Pay Item.

\#28 Bituminous SURFACE 11 HV, MAC-20

Additional Material was used at intersections of CR. 4 and CR.17, Also at the intersection of CR.104 and CR.17. to maintain proper elevation. Causing a overrun of this Pay Item. 
There was no need for this material on this project. Causing a underrun of this Pay Item.

$=29$ BITUMINOUS MATERIAL FOR PRIME COAT

Same as pay Item \#28

¿32 GUARDRALL, W-BEAM AT 6'-3" SPACING

At station \#243 on west side of CR.17, Plan Quantity for Guardrail was 600 $\mathrm{ft}$. There was only space for 575 feet. Causing a underrun of this Pay Item.

\#36 PEDESTRIAN FEÑCE

The Plans called for Pedestrian Fence to extend 280 feet on both barrier raiis. Field change was made for placement of fence causing a underrun of this Pay Item.

$=37 \quad$ EARM FENCE

The Plan Quantity called for $140.0 \mathrm{ft}$ of fence to be piaced 70 feet in each area. It was found due to the design of the structure and embankment the $\mathrm{NW}$ corner needed $66 \mathrm{ft}$ and the $\mathrm{SW}$ corner needed 89 feet of actual quantity. Causing a overrun of this Pay Item.

\#38 BLTUM. MIX FOR APPROACHES, MV.

After measurement was taken on Material piaced it was found that Plan Quantity was not needed for this Pay Item. Causing a underrun of this Pay Item. This item was changed back to the orginal quantity after needing more quantity further into contract.

-41 RIP RAP UNIFORM 12" THICK

The Plans called for Rip kap to be placed at the inlets of the drainage structures. @ Kevin $0^{\prime}$ Connor the Contractor was directed to piace it at both the inlets and outlets. Causing a overrun of this Pay Item.

\section{$\because 42$ GEOTEXTILE}

Same as Pay Item \#41

$=43$ TOP SOLL (OFESITE)

It was decided that the Top Soil onsite was not of good qualits and the quantity was not sufficient. Offsite Top Soil was brought in for the embankment areas.

\section{$\because 44$ SODDING} The quantity for this Pay Item was estimated to high. Causing a underrun
of this Item.

$=45$ MULCHED SEEDING TYPE $\mathrm{R}$

The quantity for this Pay Item was estimated to high. Causing a underrun of this Item.

46 MULCHED SEEDING R AND CV

Due to length and steepness of the slope more quantity of this Item was used than original planned. Causing a overrun of this Pay Item. 
$\div 4$ PIPE GROUP A

Due to the design of the structure all the quantity pianned for this Fay Item was not needed. Causing a underrun of this Pay Item.

$\$ 49$ PIPE GROUP A $0.064^{\prime \prime}, 18^{\prime \prime}$

At structure \#4 a $10 \mathrm{ft}$ section was added to the plan quantity to achieve proper drainage. Causing a overrun of this Pay Item.

\#52 PIPE GROUP A $0.064 " .27^{\prime \prime}$

At structure $\# 7$ a $50 \mathrm{ft}$ section was added to the plan quantity. The driveway at this station was wider than the plan showed. Causing a need for more pipe. Causing a overrun of this Pay Item.

\#56 CULVERT END SECTION, SAEETY METAL, 6:1, 24"

See explanation for Pay Item \#57 Causing a underrun of this Pay item.

\$57 CULVERT END SECTION, SAEETY METAL, $6: 1,27 "$

At structure $\# 7$ extra pipe was added. Contractor had on site extra 27 " pipe so they splice onto the $24^{\prime \prime}$ pipe causing a need for a extra $27^{\prime \prime}$ end instead of the 24 " required by the pians. Causing a overrun of this pay
item.

\section{$\because 60$ PÁTROL PERSON}

All the quantity for this Pay Item was not needed to complete the work. Causing a underrun of this Item.

$=66$ TEMP, WORKSITE SPEED LIMIT SIGÑS

Only 10 Temp. Speed Limit Signs were used in the traffic setup for this project. Causing a underrun of this Pay Item.

$=68$ EIASHING ARROW BOARDS

Same as Pay Item $\# 66$

$=73$ LINE, THERMO-QUICK 701, SOLID WHITE

It was determine that all of the Plan Quantity of this Pay Item was not needed. Causing a underrun of this Pay Item.

\#4 LINE, THERMO-QUICK TO1, SOLID YELLOW

It was determine that all of the PIan Quantity of this Pay Item was not needed. Causing a underrun of this Pay Item.

$=75$ LINE THERMO-QUICK 701 BROKEN, WHITE

It was determined that more skips were need than plan quantity. Causing a overrun of this Pay Item.

\#76 IINE THERMO PLASTIC

It was determine that all of the Plan Quantity of this Pay Item was not needed. Causing a underrun of this Pay Item. 
-77 PERMANENT MESSAGE MARKING WORD ONLY

It was determine that all of the Plan Quantity of this Pay Item was not needed. Causing a underrun of this Pay Item.

$=78$ PERMANENT MESSAGE MARKING THERMO ARROW

One extra arrow was added for better marking of intersection. Causing a overrun of this Pay Item.

$=79$ TEMPORARY PAVEMENT MARKIÑG, PAINT, SOEID, WHITE $4 "$

Due to the shape of intersections additional temporary stripping was needed above planed quality. Causing a overrun of this Pay Item.

$=80$ TEMPORARY PAVEMENT MARKING. PAINT, SOLID, YELLOW $4 "$

Due to the shape of intersections additional temporary stripping was needed above planed quality. Causing a overrun of this Pay Item.

$=81$ TEMEORARY PAVEMENT MARKING, PAINT, BROKEN, WHITE 4 "

Due to the shape of intersections additional temporary stripping was needed above planed quality. Causing a overrun of this Pay Item.

$=82$ SNOWPLOWÄBIE RAISED PALEMENT MARKERS

Severai more pavement markers were added from plan quantity. To better mark out the roadway and distinguish the turn ìnes. Causing a overrun of this Pay Item.

$=86$ EIELD OFEICF

The Field office was needed longer than Plan Quantity. Causing a overrun of this Pay Item.

$=87$ (EW) ADDITIONAL FENCE)

Cr. 17 Station 235 In the Land Acquisition of Carl and Mary wight it was agreed that new chain link fence would be installed from there driveway to the south property Iine also from there driveway to the North property line regular farm fence would be installed. The actual accrual of cost is for Farm Fence, Chain link Fence, $48^{\prime \prime} X 48^{\prime \prime}$ Gate, $48^{\prime \prime} X 112^{\prime \prime}$ gate and obstruction removal.

$=88$ (EW) CONCRETE RAIL TRAÑSITION TYPE TGE

In the original Itemized Proposal there is no Pay Item for a Concrete Rail Transition type TGB. There is a need for 4 of them on this guardrail design. There for causing a need for a Extra Work Agreement.

$=89 \quad$ (EW) PILE DRIVING BENT \#2

In the original proposal it was estimated that 2,550 feet of piling would be needed for bent $\# 2$. Bearing was reached at a average of 15 feet. Oniy using 30 percent of quantity of Pay Item \#13. This Extra Work Agreement was created to compensate for the lesser quantity.

$=90$ (EW) ASPHALT CURBING

A asphalt curb was needed along the bridge approaches to keep embankments from eroding. and to channel and direct the flow of the run off water. 
Table C.2 Original cost data for construction items of bridge (PT)

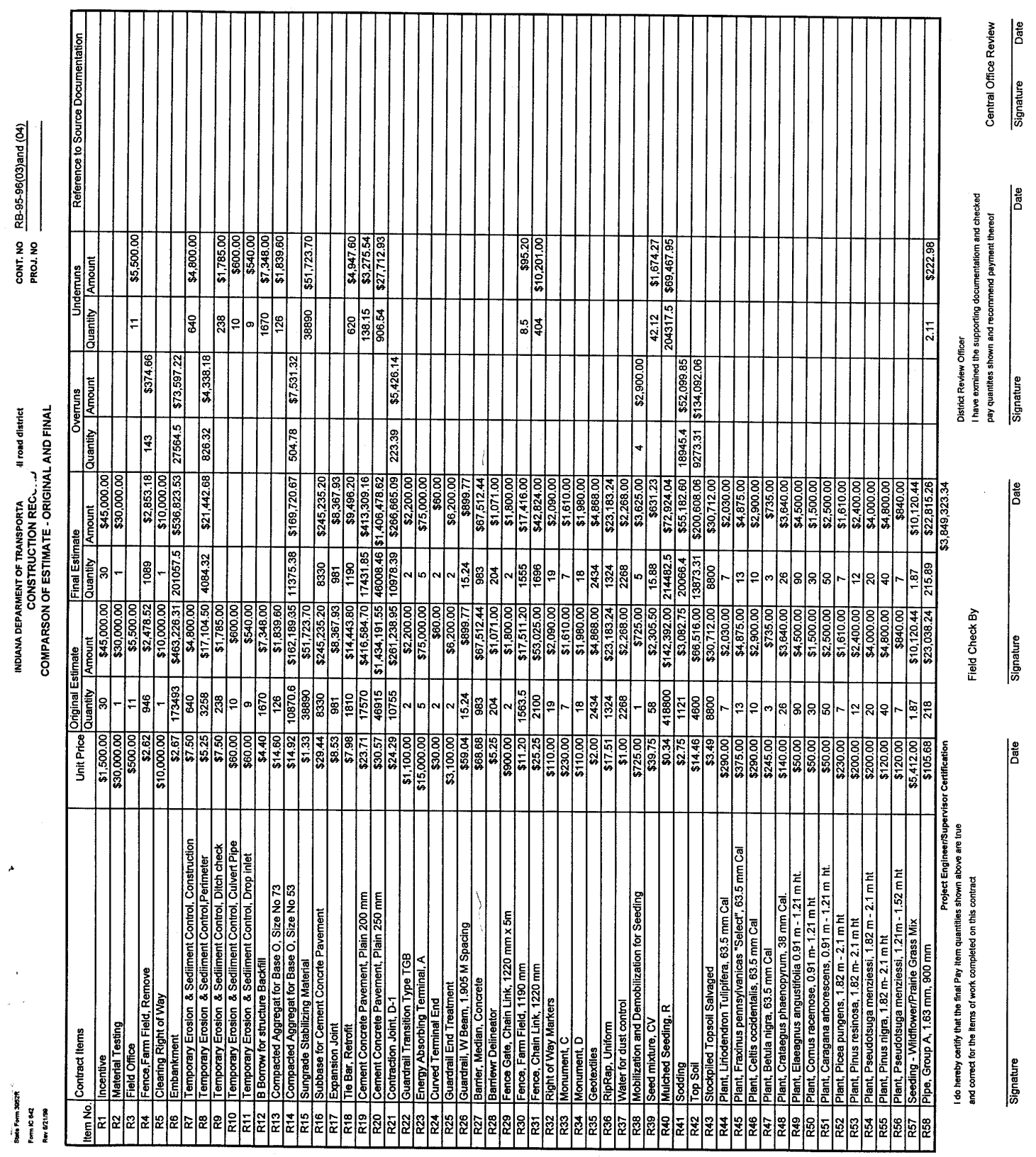




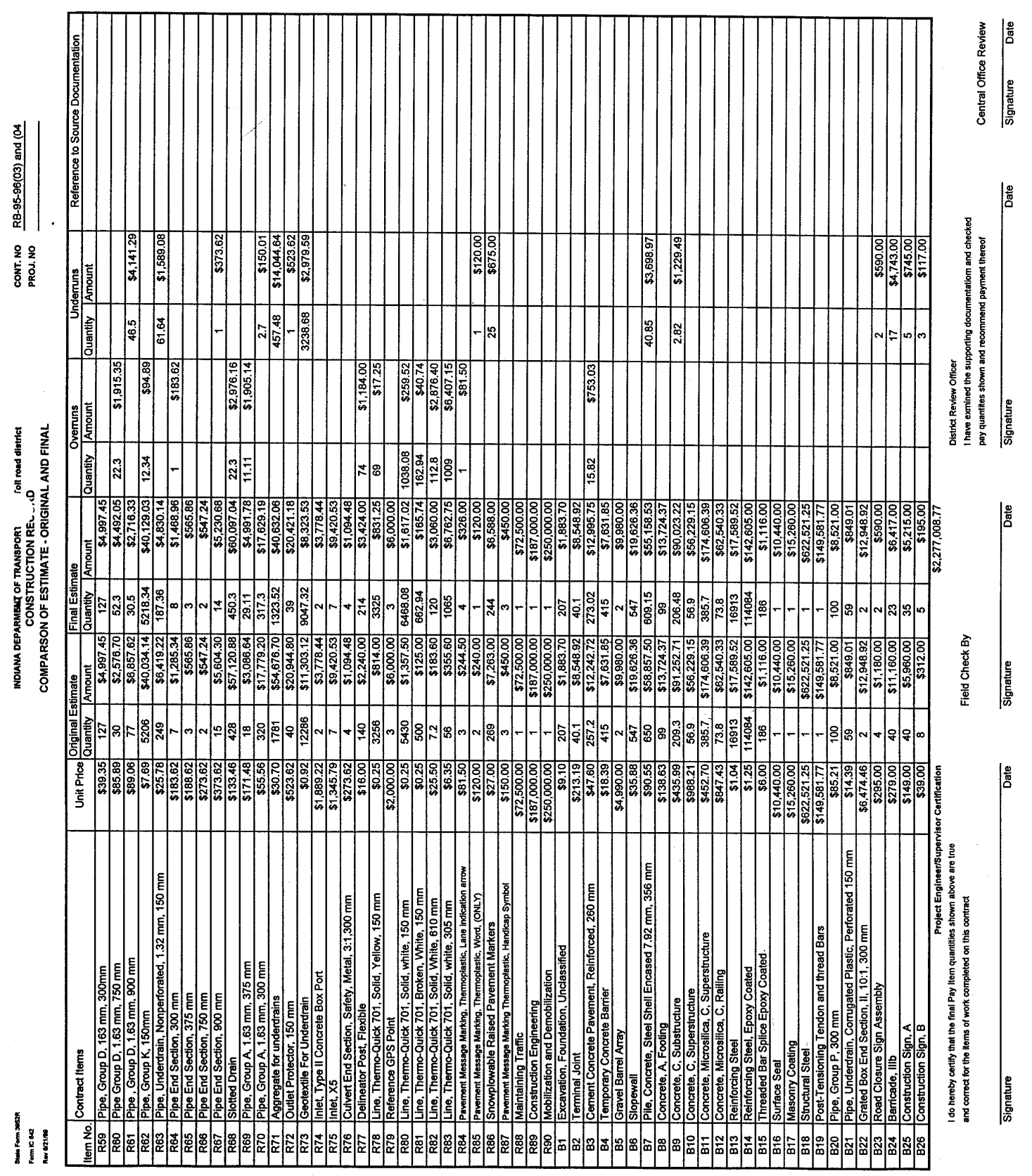




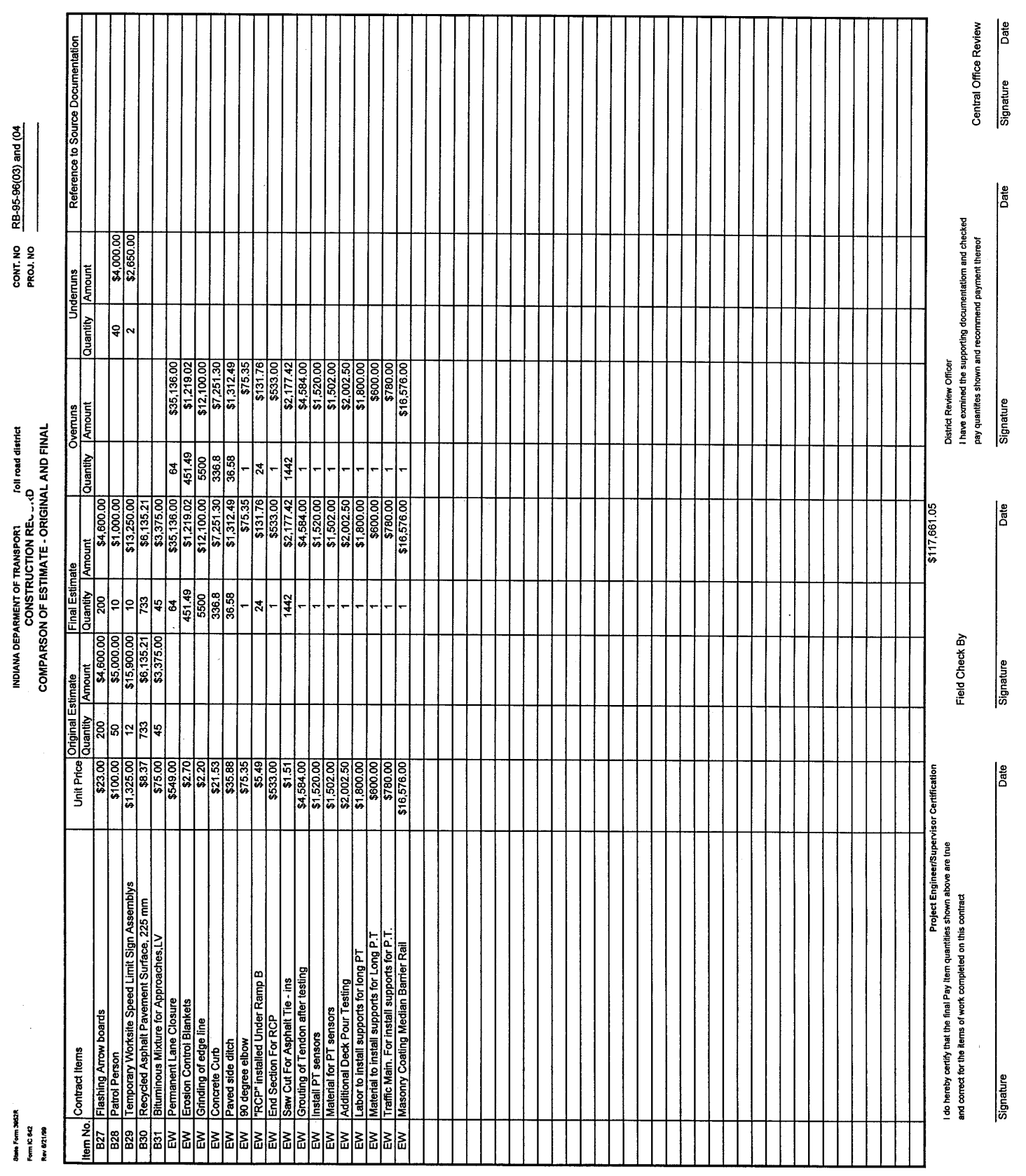




\section{EXPLANATION OF OVERRUN AND UNDERRUN}

\#3

$\# 7$

\#10 Sediment Control Drop Inlet

Perimeter sediment control was used for all sediment control. Causing an under run of this Pay Item.

\#11 B Borrow for Structure Backfil

Existing material was B Borrow causing no need for this Pay Item.

$\# 13$

compacted aggregate for base, $\mathrm{O}$

Extra Compacted Aggregate was needed at the shoulder areas for the placing of concrete pavement.

\#17 Tie Bar, Retrofit

All of the original quantity was not needed to complete this Item. Causing an under run of this Pay Item.

\#29 Farm Field Fence

All of the original quantity was not needed to complete this Item. Causing an under nun of this Pay Item.

\#30 Chain Link Fence

The line of chain link fence running from the county line fence to back of Toll Building was deleted causing a under nun of this Pay Item.

\section{\#38 Sodding}

Extra sod was used in several areas to help in control of erosion control of the side banks. Causing an over run of this Pay Item.

\#39 Top Soil

The original quantity was estimated to low for this Pay Item. Causing an over run of this Pay Item.

\#55 900mm pipe, group A

All of the original quantity was not needed to complete this Item. Causing an under nun of this Pay Item.

\#57 750mm pipe, group

Extra Quantities of this Item was added to the structure' $s$ to direct the water flow for better control of erosion. Causing an Over run of this Pay Item.

\#58 900mm pipe group D

All of the original quantity was not needed to complete this Item. Causing an under run of this Pay Item. 
Pipe Group K, $150 \mathrm{~mm}$

Extra Quantities of this Item was added to the structure' $s$ to direct the water flow for better control of erosion. Causing an Over run of this Pay Item.

$\because 60 \quad$ Pipe underdrain non-perf

All of the original quantity was not needed to complete this Item. Causing an under run of this Pay Item.

$\because 61 \quad$ Pipe end Section

An extra Structure was added to direct the water flow for better control of erosion. Causing an Over run of this Pay Item.

$\because 65 \quad$ Slotted Drain

Extra slotted was used in the paved side slope to aid in the control of erosion. Causing an Over run of this Pay Item.

$=67$ Pipe, Group A, 300 $\mathrm{mm}$

All of the original quantity was not needed to complete this Item. Causing a under run of this Pay Item

$=68 \quad$ Aggregate for underdrains

All of the original quantity was not needed to complete this Item. Causing a under run of this Pay Item

$\$ 18$ Concrete pavement plain

All of the original quantity was not needed to complete this Item. Causing a under run of this Pay Item

\#19 Concrete pavement plain $35 \mathrm{~mm}$

All of the original quantity was not needed to complete this Item. Causing a under run of this Pay Item

$=20 \quad$ D-1 Joints

Extra D-1 joints were added at all existing pavement cracks in mainline. Causing an over run of this Pay Item.

$=75 \quad$ Solid yellow Thermo Quick

Extra lines were added to aid in the directional control of the traffic. @ Bob Mortensen.

\#77 Solid White Thermo Quick

Extra lines were added to aid in the directional control of the traffic. @ Bob Mortensen.

478 Broken White

Extra lines were added to aid in the directional control of the traffic. @ Bob Mortensen.

$\because 79 \quad$ Solid White $610 \mathrm{MM}$

Extra lines were added to aid in the directional control of the traffic. @ Bob Mortensen.

$\because 80 \quad$ Solid White $305 \mathrm{MM}$

Extra lines were added to aid in the directional control of the traffic. @ Bob Mortensen.

$\because 81$ Thermos-Plastic lane indication Arrow

Extra Arrows were added to aid in the directional control of the traffic. @ Bob Mortensen.

\#82 THERMO WORD (ONLY)

All of the original quantity was not needed to complete this Item. Causing a under run of this Pay Item 
$=88 \quad($ EW) Erosion Control Blankets

Erosion Control Blankets were needed in some area to stabilize the ground. Keeping the side banks from eroding.

$=89 \quad$ (EW) Grinding of edge line.

The existing main line edge needed to be ground an asphalt placed back. Causing a need for this Pay Item.

$=90 \quad$ (EW) Concrete Curb

A concrete curb was needed at the makeup backup parking area to direct the run off to a specified spillway. To aid in the control of the erosion. Causing a need for this Pay Item.

$=91 \quad$ (EW) Paved Side Ditch

A paved side ditch was needed to direct the run off to a specified spillway. To aid in the control of the erosion. Causing a need for this Pay Item.

$=92$ (EW) 90 degree elbow

An elbow was needed in the construction of the Paved Side Ditch. Causing a need for this Pay Item.

$\# 03$ (EW) "RCP" INSTALLED UNDER RAMP "B"

While sub-contractors was trenching for underdrain the new metal culvert for this structure was hit. After further investigation it was determined that the structure needed "RCP" because of the shallow invert elevation. It was agreed that we would pay the difference between the "RCP" and metal corrugated.

$=94$ (EW) END SECTION FOR "RCP"

A new end section was needed for the "RCP".

$=95$ (EW) Saw cut for asphalt tie-ins

The existing asphalt was in such bad shape that we had the edge line saw cut so when the existing shoulder was removed and new installed there was a neat line to work to. 


\section{EXPLANATION OF OVERRUN AND UNDERRUN}

\#74 FLEXIBLE DELINEATORS

Additional quantity of this item was needed to properly delineate the ramps at Elkhart East. Causing a over run of this Pay Item.

\#96 (EW) GROUTING OF TENDON USED FOR TESTING A tendon was left ungrouted for Purdue University to do load testing on. They have completed the testing.
Causing a need for a Pay Item to grout the tendon 
EXPLANATION OF OVERRUN AND LNDERRUN

\#36 SEED MIXTLRE, CV

Plan quantity was not needed to finish the job. Causing a under run of this Pay Item.

$\# 37$ MULCHED SEEDING, R

Plan quantity was not needed to finish the job. Causing a under run of this Pay Item

$\because$ :64 PIPE END SECTION

Plan quantity was not needed to finish the job. Causing a under run of this Pay Item

$\# 69$ OUTLET PROTECTOR

Plan quantity was not needed to finish the job. Causing a under run of this Pay Item

\#0. GEOTEXTILE FOR UNDER DRAINS

Plan quantity was not needed to finish the job. Causing a under run of this Pay Item

$=\quad$ TEMPORARY EROSION CONTROL PERIMETER

This material was used along all ramps at the finish of the job to help channel the surface water from eroding the side banks. Causing a over run of this Pay Item.

$=35$ SEEDING MOBILIZATION

In the original proposal it only called for 1 seeding mobilization. With the amount of work called for there was a need for several more mobilizations to complete the job. 
The Original plan quantity was under estimated for the amount of fence

that was needed to be removed. Causing a overrun of this Pay Item.

$=8$ TEMP, EROSION CONTROL (perimeter)

It was determine that all of the Plan Quantity of this Pay Item was not needed. Causing a underrun of this Pay Item.-

*13 Compacted agogregate Trpe 73

This material was to be used on the boat launch. The Geothermo pond was deleted making no need for a boat launch. Causing a underrun of this Pay. I tem.

$=15$ Subgrade Stabilizing Material

This Pay Item was not needed. The borrow material used on this job was the same material as this Pay Item. Causing a Unown of this Item.

$\div 69 \quad 37 \mathrm{NM}$ CMP Pipe

opo

Structure $\# 25$ and 26 was extended for proper drainage. Causing a overrun of this Pay Item

$=122$ (EW) Permanent lane Closure

It was determined that Permanent Lane closure's where needec during the excavation and placement of the new ramps adjacent to the mainline. For the Safety of both Construction worker and the traveling motorist. 
EMBANKMENT

Walsh Construction shot cross section before and after the job. Computer program PC Survey was used by the Contractor to establish the proper quantity for this Item. Causing an over run of this Pay Item.

\#7 CEMENT CONCRETE PAVEMENT, REINF

The actual approach slabs were increased slightly to better join the concrete pavement to the new bridge structure. Causing an over nun of this Pay Item.

\#11 Pipe Concrete Shell

All of the original quantity was not needed to complete this Item. Causing an under nun of this Pay Item.

\#13 Concrete C Substructure

All of the original quantity was not needed to complete this Item. Causing an under nun of this Pay Item.

\#27 Road Closure Sign Assembly

All of the original quantity was not needed to complete this Item. Causing an under run of this Pay Item.

\#28 Barricade IIIB

All of the original quantity was not needed to complete this Item. Causing an under run of this Pay Item.

\#29 Construction Sign, A

All of the original quantity was not needed to complete this Item. Causing an under run of this Pay Item.

\#30 Construction Sign. B

All of the original quantity was not needed to complete this Item. Causing an under run of this Pay Item.

\#32 Patrol Person

All of the original quantity was not needed to complete this Item. Causing an under nan of this Pay Item.

\#33 Temp Worksite Speed Sign Ass'y

All of the original quantity was not needed to complete this Item. Causing an under run of this Pay Item.

\#36 (EW) Install PT Sensors

Sensors were placed for testing purposes in the bridge deck. Purdue requested that the contractor do the actual placing. Causing a need for a Pay Item.

\#37 (EW) Material for PT Sensors

The Contractor supplied the Sensors. . Causing a need for a Pay Item

\#38 (EW) Additional Deck Pour Testing

Additional test cylinders were request by Purdue University. . Causing a need for a Pay Item

\#39 (EW) Labor for installing supports for long. PT

Additional supports were needed to attach the "PT" tendons to the structure. . Causing a need for a Pay Item

\#40 (EW) Material for Long. PT

Additional supports were needed to attach the "PT" tendons to the structure. . Causing a need for a Pay Item

\#42 (EW) Masonry Coating Median Rail

The original Contract called for only the bridge structure median barrier wall to be Masonry Coated with the beige coating it was decided that the remaining wall should be done. Causing a
need for a Pay Item. 